\title{
\begin{tabular}{|l|l}
\hline$\Sigma$ & $\begin{array}{l}\text { Mathematical } \\
\text { and Computational } \\
\text { Applications }\end{array}$
\end{tabular}
}

\section{Mesh-Free and Finite Element- Based Methods for Structural Mechanics Applications}

Mathematical and Computational Applications 
Mesh-Free and Finite Element-Based Methods for Structural Mechanics Applications 



\section{Mesh-Free and Finite Element-Based Methods for Structural Mechanics Applications}

Editor

Nicholas Fantuzzi 
Editor

Nicholas Fantuzzi

University of Bologna

Italy

Editorial Office

MDPI

St. Alban-Anlage 66

4052 Basel, Switzerland

This is a reprint of articles from the Special Issue published online in the open access journal Mathematical and Computational Applications (ISSN 2297-8747) (available at: https:/ /www.mdpi.com/ journal/mca/special_issues/str_mech_appl).

For citation purposes, cite each article independently as indicated on the article page online and as indicated below:

LastName, A.A.; LastName, B.B.; LastName, C.C. Article Title. Journal Name Year, Volume Number, Page Range.

ISBN 978-3-0365-0136-9 (Hbk)

ISBN 978-3-0365-0137-6 (PDF)

(C) 2021 by the authors. Articles in this book are Open Access and distributed under the Creative Commons Attribution (CC BY) license, which allows users to download, copy and build upon published articles, as long as the author and publisher are properly credited, which ensures maximum dissemination and a wider impact of our publications.

The book as a whole is distributed by MDPI under the terms and conditions of the Creative Commons license CC BY-NC-ND. 


\section{Contents}

About the Editor $\ldots \ldots \ldots \ldots \ldots \ldots \ldots \ldots \ldots \ldots \ldots$ vii

Nicholas Fantuzzi

Special Issue "Mesh-Free and Finite Element-Based Methods for Structural Mechanics Applications"

Reprinted from: Math. Comput. Appl. 2020, 25, 75, doi:10.3390/mca25040075 . . . . . . . . . . 1

Ana F. Mota, Maria Amélia R. Loja, Joaquim I. Barbosa and José A. Rodrigues

Porous Functionally Graded Plates: An Assessment of the Influence of Shear Correction Factor on Static Behavior

Reprinted from: Math. Comput. Appl. 2020, 25, 25, doi:10.3390/mca25020025 . . . . . . . . . . 5

Davide Bellora and Riccardo Vescovini

A Continuation Procedure for the Quasi-Static Analysis ofMaterially and Geometrically Nonlinear Structural Problems

Reprinted from: Math. Comput. Appl. 2019, 24, 94, doi:10.3390/mca24040094 . . . . . . . . . . . 31

Abouzar Ebrahimi, Mohammad Saeed Seif and Ali Nouri-Borujerdi

Hydrodynamic and Acoustic Performance Analysis of Marine Propellers by Combination of Panel Method and FW-H Equations

Reprinted from: Math. Comput. Appl. 2019, 24, 81, doi:10.3390/mca24030081 . . . . . . . . . . 57

Michele Bacciocchi and Angelo Marcello Tarantino

Natural Frequency Analysis of Functionally Graded Orthotropic Cross-Ply Plates Based on the Finite Element Method

Reprinted from: Math. Comput. Appl. 2019, 24, 52, doi:10.3390/mca24020052 . . . . . . . . . 75

Reena Patel, Guillermo Riveros, David Thompson, Edward Perkins, Jan Jeffery Hoover,

John Peters and Antoinette Tordesillas

A Transdisciplinary Approach for Analyzing Stress Flow Patterns in Biostructures

Reprinted from: Math. Comput. Appl. 2019, 24, 47, doi:10.3390/mca24020047 . . . . . . . . . . 97

Büşra Uzun and Ömer Civalek

Nonlocal FEM Formulation for Vibration Analysis of Nanowires on Elastic Matrix with

Different Materials

Reprinted from: Math. Comput. Appl. 2019, 24, 38, doi:10.3390/mca24020038 . . . . . . . . . . 117

Slimane Ouakka and Nicholas Fantuzzi

Trustworthiness in Modeling Unreinforced and Reinforced T-Joints with Finite Elements

Reprinted from: Math. Comput. Appl. 2019, 24, 27, doi:10.3390/mca24010027 . . . . . . . . . . 131

Yansong Li and Shougen Chen

A Complex Variable Solution for Lining Stress and Deformation in a Non-Circular Deep Tunnel II Practical Application and Verification

Reprinted from: Math. Comput. Appl. 2018, 23, 43, doi:10.3390/mca23030043 . . . . . . . . . . 161

Serge Dumont, Franck Jourdan and Tarik Madani

4D Remeshing Using a Space-Time Finite Element Method for Elastodynamics Problems

Reprinted from: Math. Comput. Appl. 2018, 23, 29, doi:10.3390/mca23020029 . . . . . . . . . . . 175 


\section{Herbert Moldenhauer}

Integration of Direction Fields with Standard Options in Finite Element Programs

Reprinted from: Math. Comput. Appl. 2018, 23, 24, doi:10.3390/mca23020024 . . . . . . . . . . . 193 


\section{About the Editor}

Nicholas Fantuzzi is a Senior Assistant Professor at the University of Bologna. He graduated with honors in Civil Engineering in 2009 and obtained his PhD in Structural Engineering and Hydraulics in 2013. He is the owner of the Italian National Academic Qualification as Associate Professor in Mechanics of Solids and Structures. He has been the teacher of the courses "Modelling of Offshore Structures" and "Advanced Structural Mechanics" in the International Master in Offshore Engineering since 2017. His research interests are mechanics of solids and structures, fracture mechanics, implementation of numerical methods for the design of structures, application of composite materials in offshore engineering, and design and strengthening of offshore components with numerical simulations. He is currently working on the application of finite element and mesh-free methods in solids mechanics and mechanics of materials. He is the Visiting Scholar at City University of Hong Kong (2018) and Visiting Professor at Zhejiang University (2019), Chongqing University (2019, 2020), and currently at University of Rijeka (2020). He has won international awards, as well as having been a Keynote speaker at four international conferences. Moreover, he has co-organized 14 international conferences on composite materials, composite structures and computational methods. He is also the Section Editor-in-Chief of "Mathematical and Computational Applications", MDPI Publishing, as well as being a reviewer for more than 90 international journals. He has written more than 100 international peer reviewed journal papers, 8 books and more than 70 abstracts in national and international conferences. 



\title{
Editorial
}

\section{Special Issue "Mesh-Free and Finite Element-Based Methods for Structural Mechanics Applications"}

\author{
Nicholas Fantuzzi \\ Department of Civil, Chemical, Environmental, and Materials Engineering, University of Bologna, Viale del \\ Risorgimento 2, 40136 Bologna, Italy; nicholas.fantuzzi@unibo.it
}

Received: 1 December 2020; Accepted: 2 December 2020; Published: 2 December 2020

Authors of the present Special Issue are gratefully acknowledged for writing papers of very high standard. Moreover, the reviewers are also acknowledged for providing punctual and detailed reviews that improved the original manuscripts. Finally, the Guest Editor would like to thank Professors Oliver Schütze and Gianluigi Rozza (Editors-in-Chief of the MCA) for the opportunity to publish this Special Issue and Everett Zhu for accurately managing the editorial process.

The present Special Issue aimed to present relevant and innovative research works in the field of numerical analysis. The problem of solving complex engineering problems has been always a major topic in all industrial fields, such as aerospace, civil and mechanical engineering. The use of numerical methods increased exponentially in the last few years due to modern computers in the field of structural mechanics. Moreover, a wide-range of numerical methods has been presented in the literature for solving such problems. Structural mechanics problems are dealt with by using partial differential systems of equations that might be solved by using the two main classes of methods: Domain-decomposition methods, or the so-called finite element methods, and mesh-free methods where no decomposition is carried out. Both methodologies discretize a partial differential system into a set of algebraic equations that can be easily solved by computer implementation. The aim of the present Special Issue was to present a collection of recent works on these themes and a comparison of the novel advancements of both worlds in structural mechanics applications.

This Special Issue collects 10 (ten) contributions from several countries and topics all within the field of numerical analysis. This Special Issue is devoted to scientists, mathematicians and engineers who are investigating recent developments in analysis and state-of-the-art techniques on mathematical applications in numerical analysis.

Mota et al. [1] presented an assessment on porous functionally graded plates. In particular, this work aimed to assess the influence of different porosity distribution approaches on the shear correction factor, used in the context of the first-order shear deformation theory, which in turn may introduce significant effects in a structure's behavior. To this purpose, porous functionally graded plates with varying composition through their thickness were carried out. The bending behavior of these plates was studied using the finite element method with two quadrilateral plate element models.

Bellora and Vescovini [2] discussed the implementation of a continuation technique for the analysis of nonlinear structural problems, which is capable of accounting for geometric and dissipative requirements. The present strategy can be applied for solving quasi-static problems, where nonlinearities can be due to geometric or material response. The present procedure has been demonstrated to be robust and able to trace the equilibrium path in structures characterized by complex responses. Several examples are presented and discussed for a combination of material and geometry nonlinearities.

The noise emitted by ships is one of the most important noises in the ocean, and propeller noise is one of the major components of ship noise. Ebrahimi et al. [3] carried out a calculation of propeller noise using numerical methods because evaluation of propeller noise in the laboratory, despite the high accuracy and good reliability, has high costs and is very time-consuming. Software for numerical 
calculation of propeller noise, based on $\mathrm{FW}-\mathrm{H}$ equations, was developed and the results were validated by experimental results.

Bacciocchi and Tarantino [4] presented a work on the study of natural frequencies of functionally graded orthotropic laminated plates using a finite element formulation. The main novelty of the research was the modeling of the reinforcing fibers of the orthotropic layers assuming a nonuniform distribution in the thickness direction. The Halpin-Tsai approach was employed to define the overall mechanical properties of the composite layers starting from the features of the two constituents (fiber and epoxy resin). The analyses were carried out in the theoretical framework provided by the first-order shear deformation theory (FSDT) for laminated thick plates. Nevertheless, the same approach was used to deal with the vibration analysis of thin plates, neglecting the shear stiffness of the structure. This objective was achieved by properly choosing the value of the shear correction factor, without any modification in the formulation.

Patel et al. [5] presented a trans-disciplinary, integrated approach that used computational mechanics experiments with a flow network strategy to gain fundamental insights into the stress flow of high-performance, lightweight, structured composites by investigating the rostrum of paddlefish. The evolution of the stress in the rostrum was formulated as a network flow problem, which was generated by extracting the node and connectivity information from the numerical model of the rostrum. The changing kinematics of the system was provided as input to the mathematical algorithm that computes the minimum cut of the flow network. The flow network approach was verified using two simple classical problems.

Uzun and Civalek [6] presented the free vibration behaviors of various embedded nanowires made of different materials. The investigation was carried out by using Eringen's nonlocal elasticity theory. Silicon carbide nanowire (SiCNW), silver nanowire (AgNW) and gold nanowire (AuNW) were modeled as Euler-Bernoulli nanobeams with various boundary conditions such as simply supported (S-S), clamped simply supported (C-S), clamped-clamped (C-C) and clamped-free (C-F). The interactions between nanowires and medium were simulated by the Winkler elastic foundation model. The Galerkin weighted residual method was applied to the governing equations to gain stiffness and mass matrices. In addition, the influence of temperature change on the vibrational responses of the nanowires were also pursued as a case study.

As required by regulations, finite element analysis can be used to investigate the behavior of joints that might be complex to design due to the presence of geometrical and material discontinuities. The static behavior of such problems is mesh dependent; therefore, these results must be calibrated by using laboratory tests or reference data. Once the finite element model is correctly setup, the same settings can be used to study joints for which no reference is available. The work by Ouakka and Fantuzzi [7] analyzed the static strength of reinforced T-joints and sheds light on the following aspects: shell elements are a valid alternative to solid modeling; the best combination of element type and mesh density for several configurations is shown; the ultimate static strength of joints can be predicted, as well as when mechanical properties are roughly introduced for some FE topologies.

$\mathrm{Li}$ and Chen [8] presented a new complex variable method for stress and displacement problems in a noncircular deep tunnel with certain given boundary conditions at infinity. In order to overcome the complex problems caused by noncircular geometric configurations and the multiply-connected region, a complex variable method and continuity boundary conditions were used to determine stress and displacement within the tunnel lining and within the surrounding rock. The coefficients in the conformal mapping function and stress functions werre determined by the optimal design and complex variable method, respectively. The new method was validated by FLAC3D finite difference software through an example.

A Space-Time Finite Element Method (STFEM) is proposed by Dumont et al. [9] for the resolution of mechanical problems involving three dimensions in space and one in time. Special attention was paid to the nonseparation of the space and time variables because this kind of interpolation is well suited to mesh adaptation. For that purpose, a $4 \mathrm{D}$ mesh generation was adopted for space-time remeshing. 
This original technique does not require coarse-to-fine and fine-to-coarse mesh-to-mesh transfer operators and does not increase the size of the linear systems to be solved, compared to traditional finite element methods. Computations were carried out in the context of the continuous Galerkin method. The present method was tested on linearized elastodynamics problems. The convergence and stability of the method were studied and compared with existing methods.

Finally, Moldenhauer [10] investigated two-dimensional differential equations of the kind $y^{\prime}=f(x, y)$ that can be interpreted as a direction fields. Commercial finite element programs can be used for this integration task without additional programming, provided that these programs have options for the calculation of orthotropic heat conduction problems. The differential equation to be integrated with arbitrary boundaries was idealized as an finite element model with thermal 2D elements. Possibilities for application in the construction of fiber-reinforced plastics (FRP) arise, since fiber courses, which follow the local principal stress directions, make use of the superior stiffness and strength of the fibers.

Conflicts of Interest: The author declares no conflict of interest.

\section{References}

1. Mota, A.F.; Loja, M.A.R.; Barbosa, J.I.; Rodrigues, J.A. Porous Functionally Graded Plates: An Assessment of the Influence of Shear Correction Factor on Static Behavior. Math. Comput. Appl. 2020, 25, 25.

2. Bellora, D.; Vescovini, R. A Continuation Procedure for the Quasi-Static Analysis of Materially and Geometrically Nonlinear Structural Problems. Math. Comput. Appl. 2019, $24,94$.

3. Ebrahimi, A.; Seif, M.S.; Nouri-Borujerdi, A. Hydrodynamic and Acoustic Performance Analysis of Marine Propellers by Combination of Panel Method and FW-H Equations. Math. Comput. Appl. 2019, $24,81$. [CrossRef]

4. Bacciocchi, M.; Tarantino, A.M. Natural Frequency Analysis of Functionally Graded Orthotropic Cross-Ply Plates Based on the Finite Element Method. Math. Comput. Appl. 2019, 24, 52. [CrossRef]

5. Patel, R.; Riveros, G.; Thompson, D.; Perkins, E.; Hoover, J.J.; Peters, J.; Tordesillas, A. A Transdisciplinary Approach for Analyzing Stress Flow Patterns in Biostructures. Math. Comput. Appl. 2019, 24, 47. [CrossRef]

6. Uzun, B.; Civalek, O. Nonlocal FEM Formulation for Vibration Analysis of Nanowires on Elastic Matrix with Different Materials. Math. Comput. Appl. 2019, 24, 38. [CrossRef]

7. Ouakka, S.; Fantuzzi, N. Trustworthiness in Modeling Unreinforced and Reinforced T-Joints with Finite Elements. Math. Comput. Appl. 2019, 24, 27. [CrossRef]

8. Li, Y.; Chen, S. A Complex Variable Solution for Lining Stress and Deformation in a Non-Circular Deep Tunnel II Practical Application and Verification. Math. Comput. Appl. 2018, 23, 43. [CrossRef]

9. Dumont, S.; Jourdan, F.; Madani, T. 4D Remeshing Using a Space-Time Finite Element Method for Elastodynamics Problems. Math. Comput. Appl. 2018, 23, 29. [CrossRef]

10. Moldenhauer, H. Integration of Direction Fields with Standard Options in Finite Element Programs. Math. Comput. Appl. 2018, 23, 24. [CrossRef]

(C) 2020 by the author. Licensee MDPI, Basel, Switzerland. This article is an open access article distributed under the terms and conditions of the Creative Commons Attribution (CC BY) license (http:/ / creativecommons.org/licenses/by/4.0/). 



\title{
Porous Functionally Graded Plates: An Assessment of the Influence of Shear Correction Factor on Static Behavior
}

\author{
Ana F. Mota ${ }^{1,2, *}$, Maria Amélia R. Loja ${ }^{1,2,3, *}$, Joaquim I. Barbosa ${ }^{1,3}$ and José A. Rodrigues ${ }^{1}$ \\ 1 CIMOSM, ISEL, Centro de Investigação em Modelação e Optimização de Sistemas Multifuncionais, \\ 1959-007 Lisboa, Portugal; joaquim.barbosa@tecnico.ulisboa.pt (J.I.B.); jose.rodrigues@isel.pt (J.A.R.) \\ 2 Escola de Ciência e Tecnologia, Universidade de Évora, 7000-671 Évora, Portugal \\ 3 IDMEC, IST -Instituto Superior Técnico, Universidade de Lisboa, 1049-001 Lisboa, Portugal \\ * Correspondence: anafilipa.s.mota@gmail.com (A.F.M.); amelia.loja@isel.pt (M.A.R.L.)
}

Received: 6 March 2020; Accepted: 20 April 2020; Published: 24 April 2020

\begin{abstract}
The known multifunctional characteristic of porous graded materials makes them very attractive in a number of diversified application fields, which simultaneously poses the need to deepen research efforts in this broad field. The study of functionally graded porous materials is a research topic of interest, particularly concerning the modeling of porosity distributions and the corresponding estimations of their material properties-in both real situations and from a material modeling perspective. This work aims to assess the influence of different porosity distribution approaches on the shear correction factor, used in the context of the first-order shear deformation theory, which in turn may introduce significant effects in a structure's behavior. To this purpose, we evaluated porous functionally graded plates with varying composition through their thickness. The bending behavior of these plates was studied using the finite element method with two quadrilateral plate element models. Verification studies were performed to assess the representativeness of the developed and implemented models, namely, considering an alternative higher-order model also employed for this specific purpose. Comparative analyses were developed to assess how porosity distributions influence the shear correction factor, and ultimately the static behavior, of the plates.
\end{abstract}

Keywords: functionally graded materials; porosity distributions; first-order shear deformation theory; shear correction factor; higher-order shear deformation theory; equivalent single-layer approach

\section{Introduction}

Material science has undergone great evolution in recent years, representing an extremely important field for the development of many technological areas for several reasons, such as those related to a sustainable economic and environmental nature. The introduction of the functional gradient concept in the context of composite particulate materials has contributed to the design of advanced materials able to meet specific objectives, through spatial variation in composition and/or microstructure [1,2].

Functional gradient materials (FGMs) were developed in Japan in the late 1980s for thermal insulation coatings [2]. With more than three decades of history, and being a part of a wide variety of composite materials, materials with functional gradients continue to be the object of attention. This is due to their tailorability, arising from a gradual and continuous microstructure evolution and, consequently, of locally varying material properties in one or more spatial directions. Therefore, FGMs can be appropriately idealized to meet certain specifications [3,4].

Composite materials can generally be described as systems composed of a matrix and a reinforcement, the material properties of which surpass those presented by each constituent 
individually [5]. The gradual and continuous evolution of their material properties provide FGMs with better mechanical behavior than traditional composite materials [6]. Among other characteristics possessed by some FGMs, such as low thermal conductivity and low residual stresses, these materials also make it possible to minimize the stress discontinuities of conventional laminated composites $[5,7,8]$. FGMs' high resistance to temperature and the absence of interface problems gives them great importance in several engineering applications, which is why these composite materials have been extensively studied and used in the past two decades in a wide range of science fields requiring improved mechanical and thermal performances [5-7]. This broad range of applications justifies the need to study and predict the responses of FGMs' components [9]. In the past year, Li et al. developed several studies involving, among other topics, a stability and buckling analysis of functionally graded structures such as cylinders and arches [10-14].

Considering the continuous mixture concept behind their composition, FGMs can be constituted of two or more material phases. Hence, the resulting local material properties depend on this mixture evolution, allowing for a design according to the desired functions and specifications [15]. From the literature, the most common FGMs are constituted of two material phases, often one ceramic and one metal $[6,11]$.

The manufacturing process of sintering, which is common in the production of FGMs, is responsible for the formation of micro voids or porosities within the materials, making important the introduction of porosity effects at the structures' design stage [9]. The literature contains various studies which include dynamic and static analyses of functionally graded porous plates and beams [16-21]. In addition, there are several studies considering ring and arch structures, especially those developed by Li et al. in the past year [13,22-27].

Functionally graded porous materials (FGPs) combine both porosity and functional gradient characteristics, where the porosity may have a graded evolution across the volume, providing desirable properties for some applications (as in biomedical implants), and undesirable in others where voids may cause serious problems (as in the aeronautical sector). The change in porosity in one or more directions can be caused by local density effects or pore size alteration. Functionally graded porous materials possess a cell-based structure, which can be classified as open or closed (i.e., containing interconnected or isolated pores, respectively) [15].

Porous gradient materials also present a multifunctional character, where, among other aspects, one may highlight a high performance-to-weight ratio and resistance to shock; nevertheless, it is important to note that pores imply a local loss of stiffness. The latest advances in manufacturing processes allow the consideration of the development of porous materials with a functional gradient, using methods such as additive $3 \mathrm{D}$ printing. Thus, it becomes possible to design porous structures with designed variable stiffness, which can be customized for specific engineering applications, optimizing performance and minimizing weight [28]. Due to the relevant role that such materials have in a range of applications, it is important to have a wider perspective of the contexts where one can find them.

Mechanical or more generically structural components made of porous materials, bioinspired materials, can be designed for sensitive and very precise operating conditions - for example, robotic, prosthetic, and aeronautical components, among others [28]. Most of the materials used in engineering are dense; however, porous materials are also of great interest and applicability in fields such as in membranes [3]. Bioinspired materials thus have great potential for current technologies, as their unique characteristics allow them to meet various design requirements [28]. Natural or man-made, porous compacts or foams - the types of porous materials are many and diverse. Bones, wood, ceramics, and aluminum foams are just a few examples [29]. In the field of biomaterials, the inclusion of porosities allows diversification of their applicability, with ceramic and polymeric scaffolds being examples of this [3].

In the biomedical field, a bone implant must guarantee a functional gradient that mimics real bone stiffness variations. With functionally graded porous scaffolds it is possible to obtain the variations in mechanical and biological properties required for bone implants, as the presence of a porosity 
gradient is imperative for bone regeneration. Thus, functionally graded porous scaffolds for bone implants are designed to achieve the ideal balance between porosity and mechanical properties [30]. Since bone implants have various requirements, it is important to have ceramic porous materials with a wide and diversified range of pore sizes and morphologies in order to accomplish these requirements. Therefore, different porous hydroxyapatite structures have been developed to mimic natural bone's bimodal structure [31]. Since implants with no porosity show weak tissue regeneration and implant fixation capability, the introduction of a pore distribution in these alloys' structures leads to bone-like mechanical properties, allowing cellular activity [32].

In another field [33], membranes produced according to the Fuji process present a structure with relatively wide pore surface, followed by a gradually tightening pore size and a clearing of pores, finishing with an isotropic structure. Thus, these membranes can be mentioned as an example of an asymmetric structure from the porosity perspective, being implemented in several applications, with filtration and medical diagnosis being some examples. Porous membranes are the object of research related to materials and structure optimization [34].

Membranes can also be used for gas separation applications, typically possessing a microporous substrate, a mesoporous intermediate layer (or more), and a microporous top layer (or more). Regarding materials, $\alpha-\mathrm{Al}_{2} \mathrm{O}_{3}$ is the most frequent, but $\mathrm{TiO}_{2}, \mathrm{ZrO}_{2}, \mathrm{SiC}$, and their combinations are also very common [35]. For industrial wastewater treatment applications, ceramic microfiltration membranes made of $\mathrm{Al}_{2} \mathrm{O}_{3}, \mathrm{SiO}_{2}, \mathrm{ZrO}_{2}, \mathrm{TiO}_{2}$, and their composites present excellent behavior. These specific membranes possess multi-layered asymmetric structures, with a macroporous support followed by intermediate layers of graded porosity [36]. The use of ceramic membranes extends to catalytic membrane reactors due to the huge resistance to high temperatures and aggressive chemical environments. In this case, the membranes usually present disk-like, planar, tubular, and hollow fiber designs [37]. According to Sopyan et al., the material properties of porous ceramics (e.g., Young's modulus and flexural strength) depend exponentially on the ceramics' total porosity [31].

Secondary batteries technology uses porous membranes to isolate cathode and anode from each other, preventing a short circuit, and to allow the charge transport. Therefore, these membranes should be simultaneously excellent electric insulators and good ion conductors, presenting a great safety level. In this field of action there are microporous, ultrafiltration. and nanofiltration membranes, with organic polymers being the most used materials [34].

Metallic foams are another example of materials whose mechanical properties depend on the porosity characteristics. Recently, they have been gaining use among applications of aluminum and other alloys since the combination of properties intrinsic to metal alloys with the effects of porosity are of great interest, highlighting the low density and high energy absorption. The change in the porosity characteristics of these materials (e.g., pore size) makes it possible to obtain properties suitable for specific applications. Aluminum foams find use in structural applications, as well as automotive and aeronautics industries, as examples [38].

A well-defined spatial porosity gradient is a requirement of solid foams for some specific applications like filtration, energy adsorption, and tissue engineering. Therefore, control over porosity in terms of morphology, pore size, and pores' connectivity is a challenge in the development of fabrication processes, since these parameters have a great influence on the porous materials' performance [39]. In their work, Costantini et al. mentioned that a pore size gradient confers an increased strength and energy absorption to a material, and that this kind of material needs a more precise characterization of the porosity gradient concerning the mechanical properties [39].

Since pores can have different dimensions and distributions, porous materials can appear with different porosity gradients. In a typical rectangular plate, there are several possible porosity gradient configurations. Regardless of the specific distribution, the relevance should be placed on the correspondence to the design requirements [3], knowing that the heterogeneity and spatial gradient characteristic of porous materials will play an extremely important role in the resulting mechanical properties [40]. 
The Young modulus and shear modulus are strongly influenced by several factors, from the manufacturing process, to the size, shape, and distribution of the pores. Consequently, the analytical prediction of porous materials' properties is not simple because of the randomness present in their structures, and the need of a knowledge of the microstructure that is as accurate as possible in order to obtain a significant numerical prediction [29].

Concerning porosity distributions, Nguyen et al. [41] studied the mechanical behavior of porous FGPs. To this purpose they took into account two different porosity distributions, varying both through the thickness direction (namely, the so-called even and uneven distributions). Zhang and Wang [15] produced eight different porous material structures with different pore distributions, including gradient distributions, and subjected them to some mechanical tests in order to evaluate important materials properties like Young's modulus. With this work they developed techniques to estimate the effective Young's modulus of functionally gradient porous materials. Having verified that there is an obvious relation between this material property and porosity, the relationship between both is not necessarily linear. However, the experimental results constitute a good basis for validating material properties obtained through theoretical models.

With this introductory section, the importance of porous materials becomes clear, particularly regarding the development of porosity distribution models that best represent the effects on the respective effective material properties.

Hence, the present work presents three porosity distribution models, two of which are based on the reference literature, and respective estimates of material properties. For these cases, we performed a set of parametric studies focused on the static behavior of porous plates with a functional gradient in order to characterize the influence of the shear correction factor, associated with the use of the first-order shear deformation theory. These studies were performed via the finite element method considering an equivalent single layer approach. To the best of our knowledge, there are no previously published works focusing on the assessment of the influence of the shear correction factor in the static bending behavior of porous plates. Hence, this study addresses this, considering the characterization of the neutral surface deviation from the mid-plane surface, which also provides an illustrative measure of the medium's heterogeneity, typical of graded mixture and porous materials.

\section{Materials and Methods}

Considering the wide and varied number of applications of porous materials briefly discussed in the introductory section, the prediction of their mechanical properties is very relevant. Several models to predict the Young modulus of porous materials have recently been proposed, including linear, quadratic, and exponential models, although these are not suitable for porosities which are too low or too high [29]. Carranza et al. evaluated the Young modulus of metallic foams with fractal porosity distribution, and the respective estimates were close to the experimental results. In this way, they proved the expected effect, and verified that the appropriate choice of the porosity distribution model is an important factor [38].

\subsection{Functionally Graded Materials}

The flexibility in tailoring material properties makes FGMs very interesting for many applications in diverse engineering and science fields like bioengineering, mechanics, and aerospace.

The procedures for manufacturing FGMs are designed in order to obtain a specific spatial distribution of the constitutive phases. The continuous and gradual spatial distribution is responsible for the unique morphology and properties of these materials that make them stand out from others [1]. The gradual evolution of the phases can be varied, and there may even be different variations in more than one direction in the same FGM [2]. Figure 1 illustrates one example of material distribution though one direction of a dual-phase FGM. 


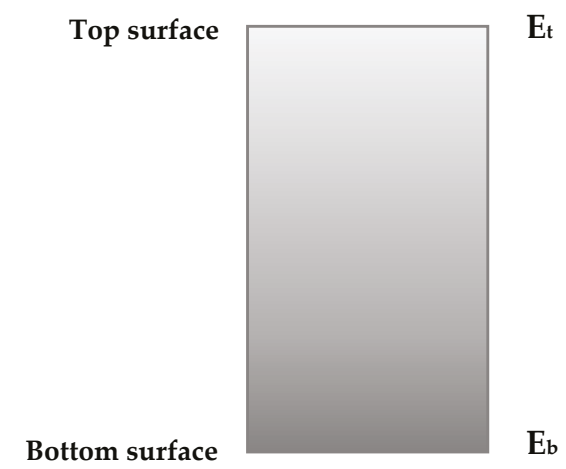

Figure 1. Example of dual-phase functional gradient material (FGM) mixture, with variation in one direction.

As a consequence of the gradual evolution of the bulk fractions and microstructure of FGMs, the respective macroscopic properties also present gradual changes. The gradual evolution of the material properties characteristic of FGMs makes it possible to design them in order to achieve specific mechanical, physical, or biological properties [1]. Since the volume fraction of the constituent phases can vary gradually in one or more directions, the present work considers the particular case of a dual-phase FGM plate in which this variation occurs in one only direction-specifically the thickness direction, as in [42]. In this case, the evolution of the volume fraction according to the direction of the $z$ axis occurs according to the following power law:

$$
V_{f}=\left(\frac{z}{h}+\frac{1}{2}\right)^{p}
$$

where $h$ and $z$ represent, respectively, the plate's thickness and the thickness coordinate, where the origin corresponds to the FGM plate's middle surface, thus $z \in[-h / 2, h / 2]$. Representing this material distribution, Figure 2 presents the volume fraction evolution through the thickness for some power law exponent values. As can be seen, the exponent $p$ dictates the volume fraction behavior along the plate's thickness.

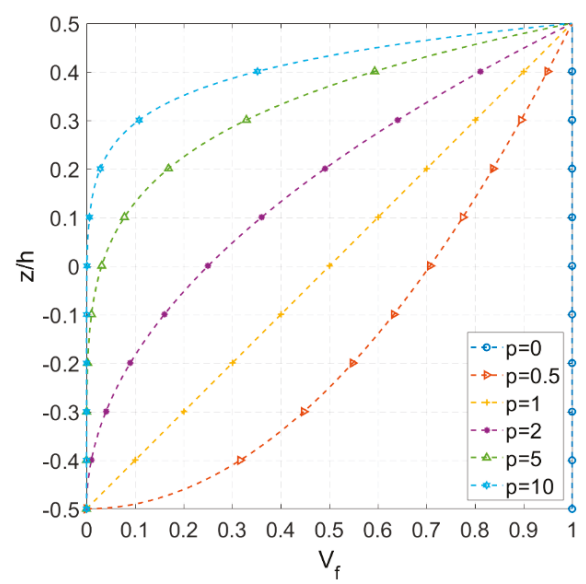

Figure 2. Evolution of the volume fraction through the thickness direction. 
As the volume fraction of the phases of the constituents of FGMs is a function of the $z$ coordinate, so too are the corresponding material properties. The effective material properties of an FGM can be estimated by the Voigt rule of mixtures, according to:

$$
P_{F G M}=\left(P_{t}-P_{b}\right) V_{f_{t}}+P_{b}
$$

where $P_{F G M}$ is the FGM property, and $P_{t}$ and $P_{b}$ represent the corresponding property at top and bottom surfaces, respectively. This approach is suitable for FGMs whose phases are not very different from each other [43].

\subsection{Porosity Distributions}

Functionally graded porous materials combine characteristics of both FGMs and porous materials. Beyond the great rigidity-weight ratio, the incomparable mechanical properties they present explains why these distinctive materials are widely used in a wide range of diverse fields [44].

Despite great developments in manufacturing processes, the formation of micro-voids or porosities is still a fact [4], and in some specific applications this can be even desirable and designed for. Regardless of the specific case, as a consequence of these pores, the material's strength will become lower, and this should be included in mechanical behavior studies [44].

The present work considers three types of porosity distributions through the thickness, the first one being proposed by Kim et al. [45] and applied in several studies, such as those developed by Coskun et al. [46] and by Li and Zheng [22]. The last two were inspired in the uniform distribution mentioned by Du et al. [47], whose studies focus on open-cell metal foams rectangular plates considering different porosity distributions through the thickness.

- $\quad$ Porosity Model M1:

Concerning porous FGMs, Kim et al. [45] considered, among other things, a porosity distribution through the thickness given by:

$$
\Phi(z)=\phi \cos \left[\frac{\pi}{2}\left(\frac{z}{h}-\frac{1}{2}\right)\right]
$$

where $z$ represents the thickness coordinate, $h$ represents the plate's thickness, and $\phi$ is the maximum porosity value.

Thus, the rule of mixtures is affected by this distribution and the effective Young modulus $(E)$ and Poisson's ratio $(v)$ can be estimated as follows:

$$
\begin{aligned}
& E(z)=\left[\left(E_{t}-E_{b}\right)\left(\frac{z}{h}+\frac{1}{2}\right)^{p}+E_{b}\right](1-\Phi(z)), \\
& v(z)=\left[\left(v_{t}-v_{b}\right)\left(\frac{z}{h}+\frac{1}{2}\right)^{p}+v_{b}\right](1-\Phi(z)) .
\end{aligned}
$$

In both Equations (4) and (5), the indexes $t$ and $b$ indicate the top and bottom surfaces, respectively.

Figure 3 illustrates, in a normalized form, the porosity distribution through the thickness, showing an evolution from an absence of pores in the bottom surface to a maximum porosity in the top surface, and the normalized Young's modulus evolution through the thickness for different maximum porosity values and power law exponents, elucidating how this model of porosity distribution affects this material property.

The next two porosity distribution models were based on the uniform distribution referred to in [47].

In both models, the porosity coefficient $\left(e_{0}\right)$ is given by Equation (6), and parameter $\beta$ can be calculated through the relation in Equation (7).

$$
e_{0}=1-\frac{E_{b}}{E_{t}}
$$




$$
\beta=\frac{1}{e_{0}}-\frac{1}{e_{0}}\left(\frac{2}{\pi} \sqrt{1-e_{0}}-\frac{2}{\pi}+1\right)^{2}
$$

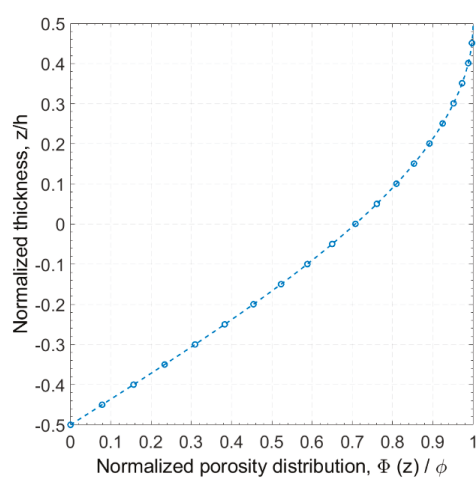

(a)

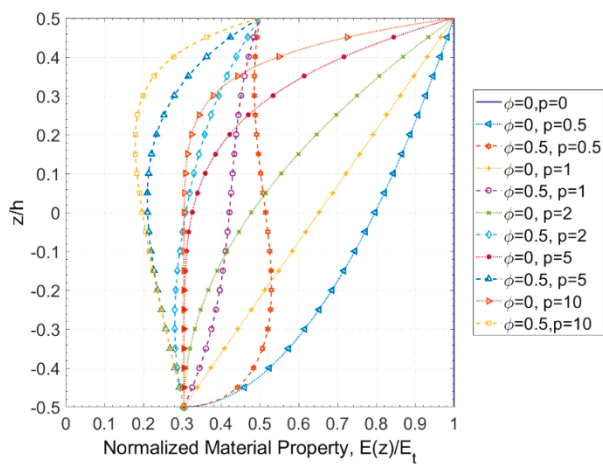

(b)

Figure 3. Normalized: (a) porosity model M1 and (b) Young's modulus (E(z)) through the thickness direction.

- $\quad$ Porosity Model M2:

In this model, the material properties, namely Young's modulus and Poisson's ratio, are estimated by Equations (8) and (9), and the graph of the evolution of the normalized Young modulus through the thickness is presented in Figure 4.

$$
\begin{aligned}
& E(z)=\left[\left(E_{t}-E_{b}\right)\left(\frac{z}{h}+\frac{1}{2}\right)^{p}+E_{b}\right]\left(1-e_{0} \beta\right), \\
& v(z)=\left[\left(v_{t}-v_{b}\right)\left(\frac{z}{h}+\frac{1}{2}\right)^{p}+v_{b}\right]\left(1-e_{0} \beta\right) .
\end{aligned}
$$

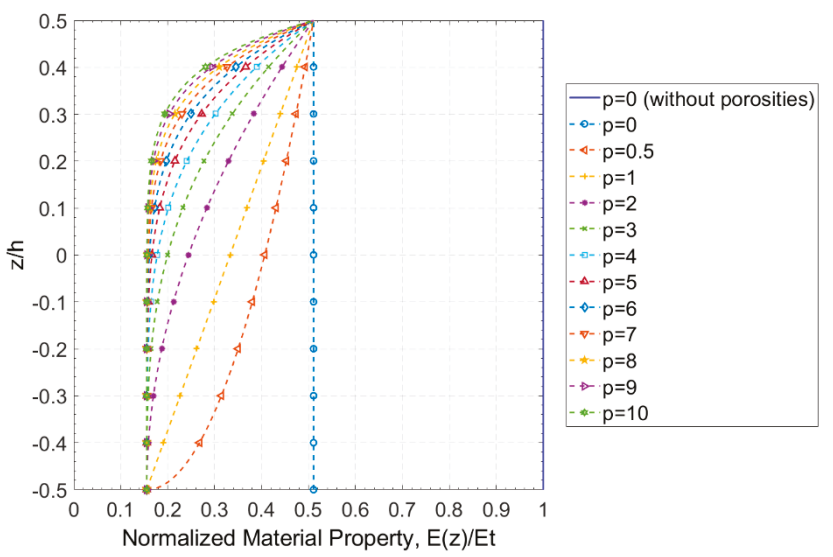

Figure 4. Variation of the normalized Young's modulus (E(z)) through the thickness for porosity model M2. 
- $\quad$ Porosity Model M3:

This model introduces to the previous one a porosity gradient through the thickness, with the respective effective materials properties being given by:

$$
\begin{aligned}
& E(z)=\left[\left(E_{t}-E_{b}\right)\left(\frac{z}{h}+\frac{1}{2}\right)^{p}+E_{b}\right]\left[1-e_{0} \beta \cos ^{2}\left(2 \pi \frac{z}{h}\right)\right], \\
& v(z)=\left[\left(v_{t}-v_{b}\right)\left(\frac{z}{h}+\frac{1}{2}\right)^{p}+v_{b}\right]\left[1-e_{0} \beta \cos ^{2}\left(2 \pi \frac{z}{h}\right)\right] .
\end{aligned}
$$

Figure 5 shows the variation of the normalized Young's modulus through the thickness. Note that the porosity distribution fluctuates between maxima and minima along the thickness.

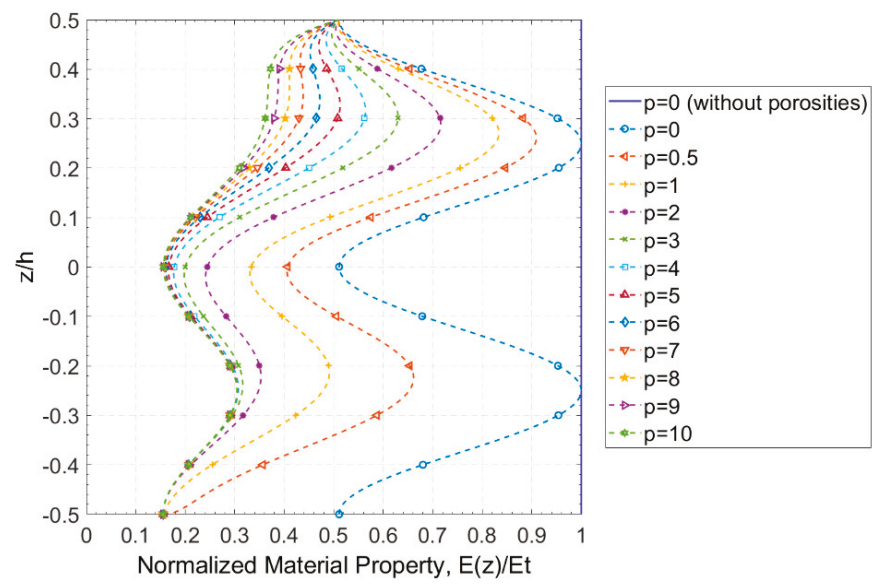

Figure 5. Porosity model M3: normalized Young's modulus (E(z)) through the thickness.

Note that on the upper surface the normalized Young's modulus takes the same value for all power law exponents evaluated once the equality $E(z)=E_{t}\left(1-e_{0} \beta\right)$ is verified. On the opposite surface, Young's modulus is given by $E(z)=E_{b}\left(1-e_{0} \beta\right)$, which justifies the fact that the corresponding $p=0$ curve constitutes an exception from the others, since this particular case corresponds to a plate made up of a single phase whose Young's modulus is given by $E_{t}$, while in the remaining cases the bottom face corresponds to the phase with Young's modulus given by $E_{b}$.

In all three porosity distribution models, the shear modulus is estimated by:

$$
G(z)=\frac{E(z)}{2(1+v(z))} .
$$

In Figures 3b, 4 and 5, the curve corresponding to the case of null power law exponent without porosity is also presented. In this case the represented material property is given by Equation (2), taking the value of $E_{t}$. Thus, the corresponding normalized form takes the value 1 through the entire plate thickness.

\subsection{Constitutive Relation and Finite Element Models}

The static behavior of porous plates with functional classification was evaluated for a set of studies through finite element analysis based on the first-order shear deformation theory (FSDT) and on third-order shear deformation theory (HSDT). According to FSDT the displacement field can be described by:

$$
u(x, y, z)=u^{0}(x, y)+z \cdot \theta_{x}^{0}(x, y)
$$




$$
\begin{gathered}
v(x, y, z)=v^{0}(x, y)+z \cdot \theta_{y}^{0}(x, y), \\
w(x, y, z)=w^{0}(x, y),
\end{gathered}
$$

where $u(x, y, z), v(x, y, z)$, and $w(x, y, z)$ are the displacements of a certain plate coordinate point, and $u^{0}(x, y)$, $v^{0}(x, y)$ and $w^{0}(x, y)$ represent the displacements of a mid-plane point associated to them. The normals to the mid-plane rotations are represented by $\theta_{x}^{0}$ and $\theta_{y}^{0}$, respectively. Thus, this model considers a total of five degrees of freedom per node, $q=\left[u^{0}, v^{0}, w^{0}, \theta_{x}^{0}, \theta_{y}^{0}\right]$. Considering small deformations, the elasticity kinematic relation leads to the following strain field, omitting dependencies for simplicity reasons:

$$
\begin{aligned}
& \varepsilon_{x}=\varepsilon_{x}^{0}+\mathrm{z} \cdot k_{x}^{0} \\
& \varepsilon_{y}=\varepsilon_{y}^{0}+\mathrm{z} \cdot k_{y}^{0} \\
& \gamma_{x y}=\gamma_{x y}^{0}+z \cdot k_{x y}^{0} \\
& \varepsilon_{x}^{0}=\frac{\partial u^{0}}{\partial x} \\
& \varepsilon_{y}^{0}=\frac{\partial v^{0}}{\partial y} \\
& \gamma_{x y}^{0}=\frac{\partial u^{0}}{\partial y}+\frac{\partial v^{0}}{\partial x} \\
& k_{x}^{0}=\frac{\partial \theta_{x}^{0}}{\partial x} \\
& k_{y}^{0}=\frac{\partial \theta_{y}^{0}}{\partial y} \\
& k_{x y}^{0}=\frac{\partial \theta_{y}^{0}}{\partial x}+\frac{\partial \theta_{x}^{0}}{\partial y} \\
& \gamma_{x z}=\gamma_{x z}^{0} \\
& \gamma_{y z}=\gamma_{y z}^{0} \\
& \gamma_{x z}^{0}=\theta_{x}^{0}+\frac{\partial w^{0}}{\partial x} \\
& \gamma_{y z}^{0}=\theta_{y}^{0}+\frac{\partial w^{0}}{\partial y}
\end{aligned}
$$

where $\varepsilon_{\mathrm{x}}, \varepsilon_{\mathrm{y}}$, and $\gamma_{\mathrm{xy}}$ correspond to the two in-plane normal strains and to the in-plane total shear strain, respectively. The interlaminar transverse shear strains are represented by $\gamma_{\mathrm{yz}}$ and $\gamma_{\mathrm{xz}}$. This strain field is characterized by a null normal transverse strain $\left(\varepsilon_{z}=0\right)$, denoting thickness inextensibility. Since the FGM considered in the studies carried out can be considered as an isotropic material, the constitutive law governing the relationship between the stress and strain fields is given by:

$$
\left[\begin{array}{c}
\sigma_{x} \\
\sigma_{y} \\
\sigma_{x y} \\
\sigma_{y z} \\
\sigma_{x z}
\end{array}\right]=\left[\begin{array}{ccccc}
Q_{11} & Q_{12} & 0 & 0 & 0 \\
Q_{12} & Q_{22} & 0 & 0 & 0 \\
0 & 0 & Q_{66} & 0 & 0 \\
0 & 0 & 0 & k \cdot Q_{44} & 0 \\
0 & 0 & 0 & 0 & k \cdot Q_{55}
\end{array}\right] \cdot\left[\begin{array}{c}
\varepsilon_{x} \\
\varepsilon_{y} \\
\gamma_{x y} \\
\gamma_{y z} \\
\gamma_{x z}
\end{array}\right] .
$$

The coefficients $Q_{\mathrm{ij}}$, provided by [48], stand for the elastic coefficients, which in the present work depend on the $z$ coordinate due to the variation in material properties through the thickness direction:

$$
\begin{aligned}
& Q_{11}=Q_{22}=\frac{E_{F G M}(z)}{1-v_{F G M}^{2}(z)} \\
& Q_{12}=\frac{v_{F G M}(z) \cdot E_{F G M}(z)}{1-v_{F G M}^{2}(z)}, \\
& Q_{44}=Q_{55}=Q_{66}=G_{F G M}(z) .
\end{aligned}
$$

The HSDT displacement field also considered in this work for comparison purposes was proposed by Kant et al. [49], and is given by:

$$
\begin{gathered}
u(x, y, z)=u^{0}(x, y)+z \cdot \theta_{x}^{0}(x, y)+z^{3} \cdot \theta_{x}^{*}(x, y), \\
v(x, y, z)=v^{0}(x, y)+z \cdot \theta_{y}^{0}(x, y)+z^{3} \cdot \theta_{y}^{*}(x, y), \\
w(x, y, z)=w^{0}(x, y)+z^{2} \cdot w^{*}(x, y) .
\end{gathered}
$$

As can be observed, this higher-order displacement field not only allows for in-plane displacements that vary as cubic functions of the thickness coordinate, but it also allows for thickness extensibility. This higher-order theory was implemented for comparison purposes, but was not the main focus of the present work.

The finite element method was used for the study presented in this work. This method is widely used due to its great versatility, able to solve a wide range of physical problems ruled by differential equations. In this numerical method, the domain of a certain problem is discretized into elementary 
subdomains, which obey continuity and equilibrium requirements between adjacent subdomains. The resolution of a problem by the finite element method can generically be described by three steps [49,50], as follows. After the pre-processing stage, where all aspects related to the domain discretization and topology, material and geometrical characteristics, loading and boundary conditions are accomplished, one proceeds to the analysis phase where the intended analysis is performed and primary variables are obtained. The results of this analysis can then be post-processed in order to obtain other derived physical quantities of interest in the third and final phase of this method. The plates analyzed in the present work had a rectangular geometry configuration (Figure 6) with a graded material distribution, as already mentioned.

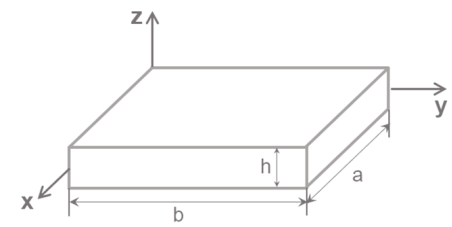

Figure 6. Plate geometry.

Taking into consideration their geometry, the plates were discretized into quadrilateral finite elements. To enable a more comprehensive study, we considered bilinear and biquadratic quadrilateral plate finite elements from the Lagrange family, as illustrated in Figure 7. The bilinear element (a) contained one node at each vertex (four nodes in total), while the biquadratic element (b) additionally contained a center node and one node at the midpoint of each side, resulting in a total of nine nodes. Each node possessed five degrees of freedom-three translations and two rotations associated to the plate mid-plane, as previously mentioned.

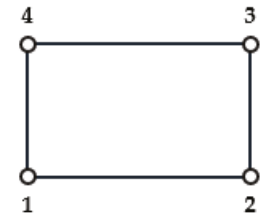

(a)

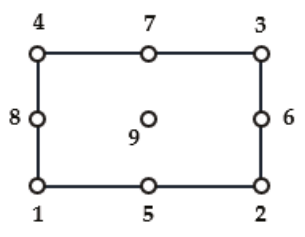

(b)

Figure 7. Plate elements from Lagrange family: (a) bilinear element; (b) biquadratic element.

As the main aim of the present work is related to the assessment of the influence of the shear correction factor on the static bending of FGP plates, the procedure considered to achieve the global equilibrium equations to be solved was based on the minimization of the plates' potential energy. This mathematical formulation yields for each element a set of equilibrium equations. Considering the whole discretized domain where continuity and equilibrium aspects ensure the discretized model will be representative, we finally obtain the global equilibrium equation:

$$
\{f\}^{\text {global }}=[K]^{\text {global }} \cdot\{u\}^{\text {global }},
$$

where $\{u\}^{\text {global }}$ denotes the problem solution (i.e., the generalized nodal displacements), $\{f\}^{\text {global }}$ represents the generalized forces applied to the plates, and $[K]^{\text {global }}$ is the global stiffness matrix. The solution of the problem is obtained after the imposition of the plates' boundary conditions. 


\subsection{Shear Correction Factor}

The studies developed in this work are based on FSDT, which requires the introduction of a shear correction factor on the transverse shear components of the elastic stiffness coefficients matrix in order to redress the uniform transverse shear stresses/strains arising from the deformation kinematics based on the displacement field. Despite this need, FSDT-based models continue to be widely used in the modeling of structures, due to their lower computational cost when compared to other theories and also due to their applicability domain. It is possible to conclude that, even considering a bilinear element, results were very good when compared to other authors' alternative solutions and with biquadratic elements, while also having a lower computational cost. Regarding the homogeneous plates, the shear correction value considered was 5/6. However, the overall heterogeneity of FGMs due to the gradual evolution of their properties makes it desirable to calculate the correction factor for each specific case $[6,43,51]$.

Shear correction factors determined through predictor-corrector procedures that use iteration processes depend on plate geometry, as well as boundary and load conditions. Therefore, the factors thus determined are limited to a given system and cannot be applied to different configurations. The use of energy considerations for the calculation of shear correction coefficients is quite common in studies involving laminated composite materials and FGMs [51]. Nguyen et al. [51] worked on this subject concerning FGMs, comparing the strain energies obtained from the average shear stresses and from the equilibrium to calculate the shear correction factors. Efraim and Eisenberg developed a shear correction factor depending on Poisson's ratio and the volume fractions of both material phases present in a functionally graded plate [52]. Working on FGPs, Li et al. [53] calculated the shear correction factor as a function of the power law exponent, thickness-to-length ratio $(a / h)$, and of some constant coefficients that depend on the material phases involved.

The shear correction factor used in the present work (here represented by $k$ ) was estimated using a formulation similar to the one used by Singha et al. [54]. Accordingly, the reference surface used for the shear correction factor calculation was the neutral one and not the mid-surface as usual. To this purpose we considered an FGP with thickness $h$ as illustrated in Figure 8, where $z_{m s}$ and $z_{n s}$ are the coordinates of a point in the thickness direction relative to the medium and neutral surfaces, respectively.

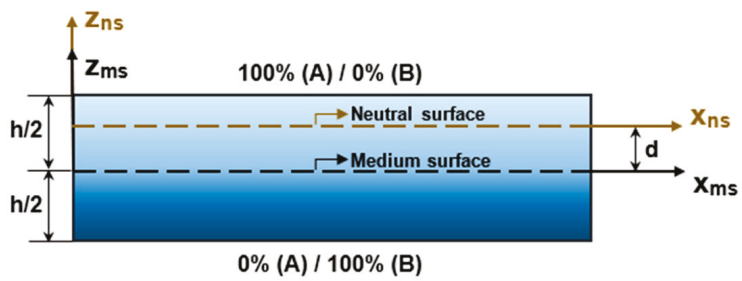

Figure 8. Medium and neutral surfaces.

According to this formulation, the shift of the neutral surface $(d)$ is given by:

$$
d=\frac{\int_{-h / 2}^{h / 2} E\left(z_{m s}\right) \cdot z_{m s} d z_{m s}}{\int_{-h / 2}^{h / 2} E\left(z_{m s}\right) d z_{m s}}
$$

The shear correction factor is then derived from the equivalent energy principle, and can be determined by Equation (20) [54]: 


$$
k=\frac{\left(\int_{-\frac{h}{2}-d}^{\frac{h}{2}-d}\left(\int_{-\frac{h}{2}-d}^{z_{n s}} E(\xi) \cdot \xi d \xi\right) d z_{n s}\right)^{2}}{\int_{-\frac{h}{2}-d}^{z_{n s}} \bar{Q}_{55} d z_{n s} \cdot \int_{-\frac{h}{2}-d}^{\frac{h}{2}-d} \frac{\left(\int_{-\frac{h}{2}-d}^{z_{n s}} E(\xi) \cdot \xi d \xi\right)^{2}}{G\left(z_{n s}\right)}} .
$$

As applied to determine shear correction factor, all calculations carried out a posteriori were simply affected by a referential translation according to the coordinate vector $(0,0,-d)$.

\section{Results}

The present section, dedicated to the presentation of results and their preliminary discussion, comprises a first verification study, after which a set of case studies considering different porosity distributions models are presented and analyzed concerning their influence on neutral surface shift, shear correction factor, and maximum transverse displacement.

\subsection{Verification Studies}

\subsubsection{Shear Correction Factor}

In the course of the literature review, no studies were found on the correction factor in the context of FGMs considering porosity distributions. For this reason, this first verification study regarding the approach applied to the correction factor determination was carried out considering a metal/ceramic functionally graded plate where the volume fraction of the ceramic phase is given by Equation (1). To this purpose, a square plate with unitary length and aspect ratio of 100 was considered, according to [43]. The results obtained for different power law exponents and Young's modulus ratios between both ceramic and metallic phases are presented in Table 1, showing an excellent agreement with the reference results for all parameter values considered.

Table 1. Shear correction factor for different material parameters.

\begin{tabular}{|c|c|c|c|c|c|c|c|c|}
\hline \multirow{3}{*}{$p$} & \multicolumn{8}{|c|}{$E_{c} / E_{m}$} \\
\hline & \multicolumn{2}{|c|}{1} & \multicolumn{2}{|c|}{2} & \multicolumn{2}{|c|}{4} & \multicolumn{2}{|c|}{6} \\
\hline & $k[43]$ & $k$ & $k[43]$ & $k$ & $k[43]$ & $k$ & $k[43]$ & $k$ \\
\hline 0 & $5 / 6$ & 0.8333 & $5 / 6$ & 0.8333 & $5 / 6$ & 0.8333 & $5 / 6$ & 0.8333 \\
\hline 1 & $5 / 6$ & 0.8333 & 0.8320 & 0.8320 & 0.8305 & 0.8305 & 0.8305 & 0.8305 \\
\hline 2 & $5 / 6$ & 0.8333 & 0.8095 & 0.8095 & 0.7804 & 0.7804 & 0.7662 & 0.7662 \\
\hline 5 & $5 / 6$ & 0.8333 & 0.7891 & 0.7891 & 0.7175 & 0.7175 & 0.6643 & 0.6641 \\
\hline 10 & $5 / 6$ & 0.8333 & 0.7989 & 0.7989 & 0.7316 & 0.7318 & 0.6743 & 0.6746 \\
\hline \multirow{3}{*}{$p$} & \multicolumn{8}{|c|}{$E_{c} / E_{m}$} \\
\hline & \multicolumn{2}{|c|}{8} & \multicolumn{2}{|c|}{10} & \multicolumn{2}{|c|}{15} & \multicolumn{2}{|c|}{20} \\
\hline & $k[43]$ & $k$ & $k[43]$ & $k$ & $k[43]$ & $k$ & $k[43]$ & $k$ \\
\hline 0 & $5 / 6$ & 0.8333 & $5 / 6$ & 0.8333 & $5 / 6$ & 0.8333 & $5 / 6$ & 0.8333 \\
\hline 1 & 0.8308 & 0.8308 & 0.8312 & 0.8312 & 0.8319 & 0.8319 & 0.8323 & 0.8323 \\
\hline 2 & 0.7593 & 0.7594 & 0.7563 & 0.7563 & 0.7555 & 0.7556 & 0.758 & 0.7580 \\
\hline 5 & 0.6238 & 0.6235 & 0.5923 & 0.5919 & 0.5381 & 0.5377 & 0.5046 & 0.5043 \\
\hline 10 & 0.6262 & 0.6266 & 0.5856 & 0.5860 & 0.5073 & 0.5080 & 0.4513 & 0.4521 \\
\hline
\end{tabular}

\subsubsection{Porosity Distribution Model 1 (M1)}

The first porosity model (M1) was submitted to verification studies. For this, and according to reference [6], an FGM square plate with $17.6 \mu \mathrm{m}$ thickness and an aspect ratio of 20 was considered. Regarding the constituent materials, the Young moduli of the top and bottom surfaces were assumed 
to be $E_{t}=14.4 \mathrm{GPa}$ and $E_{b}=1.44 \mathrm{GPa}$ respectively, and in this particular study, the Poisson ratio was considered constant, assuming the value of 0.38 . The plate, simply supported, was considered under a uniformly distributed load with $100 \mathrm{kPa}$ magnitude and was discretized in a $10 \times 10$ mesh of four node finite elements (Q4).

The normalized transverse displacement and the respective relative deviation to the reference, presented in Table 2, were obtained by Equations (21) and (22), respectively.

$$
\begin{gathered}
\bar{w}=\frac{E_{b} \cdot h^{3}}{q_{0} \cdot a^{4}} w\left(\frac{a}{2}, \frac{b}{2}, 0\right), \\
\delta(\%)=\frac{\bar{w}^{\text {present }}-\bar{w}^{\text {reference }}}{\bar{w}^{\text {reference }}} \cdot 100(\%) .
\end{gathered}
$$

Table 2. Results of the porosity distribution model 1 (M1) verification study.

\begin{tabular}{cccc}
\hline $\boldsymbol{p}$ & $\boldsymbol{\phi}$ & $\overline{\boldsymbol{w}}$ & $\boldsymbol{\delta} \mathbf{( \% )}$ \\
\hline & 0 & $4.220 \times 10^{-3}$ & 1.49 \\
0 & 0.2 & $4.798 \times 10^{-3}$ & 0.43 \\
& 0.5 & $6.242 \times 10^{-3}$ & -0.57 \\
1 & 0.5 & $1.452 \times 10^{-2}$ & 0.26 \\
5 & 0.5 & $2.685 \times 10^{-2}$ & -0.18 \\
\hline
\end{tabular}

The small relative deviations observed make it possible to conclude that there is very good agreement between the present model and the reference results, although a different approach was applied. In particular, the higher relative deviation occurs when considering an isotropic homogeneous material without porosities $(p=0, \phi=0)$.

\subsection{Case Studies}

In order to perform a set of parametric studies relevant for this work's objectives, we carried out simulations on a set of case studies, in which FGM square plates with $25 \mathrm{~mm}$ thickness and aspect ratio 20 were considered. The material properties involved in the studies presented in this section are given in Table 3, and the volume fraction of $\mathrm{TiO}_{2}$ is given by Equation (1). The plates were considered simply supported and submitted to a uniform load with $100 \mathrm{kPa}$ of magnitude.

Table 3. Material properties.

\begin{tabular}{ccc}
\hline Material & Young's Modulus (GPa) & Poisson's Ratio \\
\hline Aluminum $(\mathrm{Al})$ & 70 & 0.33 \\
Titanium Oxide $\left(\mathrm{TiO}_{2}\right)$ & 230 & 0.27 \\
\hline
\end{tabular}

The case studies show the influence of the different porosity distribution models in the neutral surface shift, shear correction factor, and maximum transverse displacement for a range of power law exponents. The influence of finite element type used was also studied.

\subsubsection{Influence on Neutral Surface Shift and Shear Correction Factor}

In this subsection we present the studies performed to explore the effects of the porosity distribution on the neutral surface shift and shear correction factor.

- $\quad$ Porosity Distribution Model 1 (M1):

When considering porosities distributed according to model M1, it is important to evaluate how the maximum porosity value combined with the power law exponent affects the neutral surface shift and the 
shear correction factor. For this purpose, the following sets of values were respectively considered for the maximum porosity value and for the exponent of the power law: $\phi=\{0,0.1,0.2,0.3,0.4,0.5,0.6\}$ and $p=\{0,1,2,5,10\}$. The results obtained are presented in Table 4, Figures 9 and 10 .

Table 4. Porosities distribution M1: neutral surface' shift and shear correction factor.

\begin{tabular}{|c|c|c|c|c|}
\hline$\phi$ & $p$ & $d(\mathrm{~m})$ & $d / h$ & $k$ \\
\hline \multirow{5}{*}{0} & 0 & 0 & 0 & $5 / 6$ \\
\hline & 1 & $2.2222 \times 10^{-3}$ & 0.0889 & 0.8279 \\
\hline & 2 & $2.7027 \times 10^{-3}$ & 0.1081 & 0.7828 \\
\hline & 5 & $2.4631 \times 10^{-3}$ & 0.0985 & 0.7344 \\
\hline & 10 & $1.7921 \times 10^{-3}$ & 0.0717 & 0.7499 \\
\hline \multirow{5}{*}{0.1} & 0 & $-2.3222 \times 10^{-4}$ & -0.0093 & 0.8316 \\
\hline & 1 & $2.0233 \times 10^{-3}$ & 0.0809 & 0.8289 \\
\hline & 2 & $2.4955 \times 10^{-3}$ & 0.0998 & 0.7843 \\
\hline & 5 & $2.2257 \times 10^{-3}$ & 0.0890 & 0.7359 \\
\hline & 10 & $1.5422 \times 10^{-3}$ & 0.0617 & 0.7505 \\
\hline \multirow{5}{*}{0.2} & 0 & $-4.9832 \times 10^{-4}$ & -0.0199 & 0.8297 \\
\hline & 1 & $1.7903 \times 10^{-3}$ & 0.0716 & 0.8304 \\
\hline & 2 & $2.2520 \times 10^{-3}$ & 0.0901 & 0.7865 \\
\hline & 5 & $1.9481 \times 10^{-3}$ & 0.0779 & 0.7378 \\
\hline & 10 & $1.2519 \times 10^{-3}$ & 0.0501 & 0.7513 \\
\hline \multirow{5}{*}{0.3} & 0 & $-8.0630 \times 10^{-4}$ & -0.0323 & 0.8275 \\
\hline & 1 & $1.5138 \times 10^{-3}$ & 0.0606 & 0.8325 \\
\hline & 2 & $1.9620 \times 10^{-3}$ & 0.0785 & 0.7894 \\
\hline & 5 & $1.6190 \times 10^{-3}$ & 0.0648 & 0.7403 \\
\hline & 10 & $9.1039 \times 10^{-4}$ & 0.0364 & 0.7522 \\
\hline \multirow{5}{*}{0.4} & 0 & $-1.1669 \times 10^{-3}$ & -0.0467 & 0.8248 \\
\hline & 1 & $1.1805 \times 10^{-3}$ & 0.0472 & 0.8353 \\
\hline & 2 & $1.6107 \times 10^{-3}$ & 0.0644 & 0.7934 \\
\hline & 5 & $1.2227 \times 10^{-3}$ & 0.0489 & 0.7436 \\
\hline & 10 & $5.0299 \times 10^{-4}$ & 0.0201 & 0.7534 \\
\hline \multirow{5}{*}{0.5} & 0 & $-1.5948 \times 10^{-3}$ & -0.0638 & 0.8217 \\
\hline & 1 & $7.7050 \times 10^{-4}$ & 0.0308 & 0.8390 \\
\hline & 2 & $1.1761 \times 10^{-3}$ & 0.0470 & 0.7988 \\
\hline & 5 & $7.3631 \times 10^{-4}$ & 0.0295 & 0.7477 \\
\hline & 10 & $8.5279 \times 10^{-6}$ & 0.0003 & 0.7546 \\
\hline \multirow{5}{*}{0.6} & 0 & $2.1109 \times 10^{-3}$ & 0.0844 & 0.8177 \\
\hline & 1 & $2.5421 \times 10^{-4}$ & 0.0102 & 0.8439 \\
\hline & 2 & $6.2495 \times 10^{-4}$ & 0.0250 & 0.8059 \\
\hline & 5 & $1.2505 \times 10^{-4}$ & 0.0050 & 0.7529 \\
\hline & 10 & $-6.0425 \times 10^{-4}$ & -0.0242 & 0.7560 \\
\hline
\end{tabular}

Independent of the $\phi$ value, the neutral surface shift increased from $p=0$ to $p=2$, where it had its maximum value, then decreasing from $p=2$ to $p=10$. Additionally, for all maximum porosity values considered, except for the case where $\phi=0.6$, the shear correction factor decreased for power law exponents between 0 and 5 , increasing for the last transition from $p=5$ to $p=10$. This is explained by the fact that, for higher values of the power law exponent, the functionally graded plate approaches a homogeneous isotropic composition, and at the limit where $p$ tends to infinity, the plate will consist of a single phase (aluminum in this case), so the value of the shear correction factor tends to the typical value $5 / 6$.

When the maximum porosities took the values 0 or 0.1 , the shear correction factor decreased from $p=0$ to $p=5$, increasing for higher values of power law exponent. For the remaining values of 
maximum porosities this factor increased in the transition from $p=0$ to $p=1$, decreased from $p=1$ to $p=5$, and increased again until $p=10$.

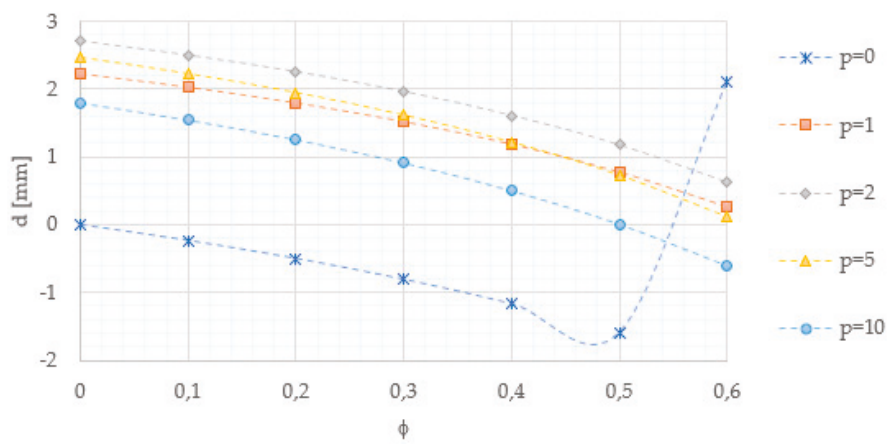

Figure 9. Porosity distribution model M1: evolution of neutral surface shift.

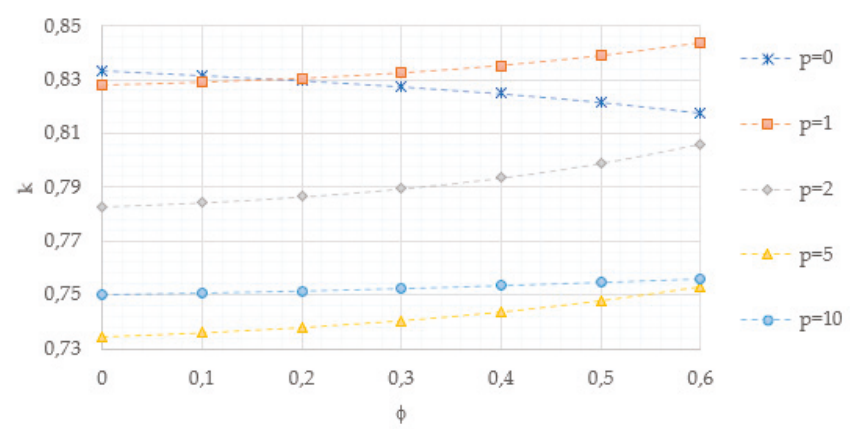

Figure 10. Porosity distribution model M1: evolution of shear correction factor.

In the case of an isotropic homogeneous plate $(p=0)$, the shear correction factor decreased when the maximum porosity values increased. This was also true for the neutral surface shift, excepting for the abrupt increase in the transition between $\phi=0.5$ and $\phi=0.6$. For other power law exponents, as the maximum porosity values increased the neutral surface shift decreased and the shear correction factor increased.

\section{- $\quad$ Porosity Distribution Models 2 and 3 (M2 and M3):}

Concerning these models, we evaluated the influence of the power law exponent, taking the set of integers from 0 to 10 . Figures 11 and 12 show the evolution of neutral surface shift and shear correction factor, respectively, for both distribution models M2 and M3. The curves show a neutral surface shift behavior similar to the one observed in the previous study and a shear correction factor with a decreasing behavior up to $p=5$, after which it increased with the exponent of the power law. This behavior was similar in both models. The porosity distribution M3 led to smaller neutral surface shifts, and the difference between both models increased with increasing power law exponent.

The porosity distribution model M3 generated lower values for the shear correction factor, with the difference between the two models decreasing with the increase in the power law exponent, as opposed to what was observed for the neutral surface shift. 


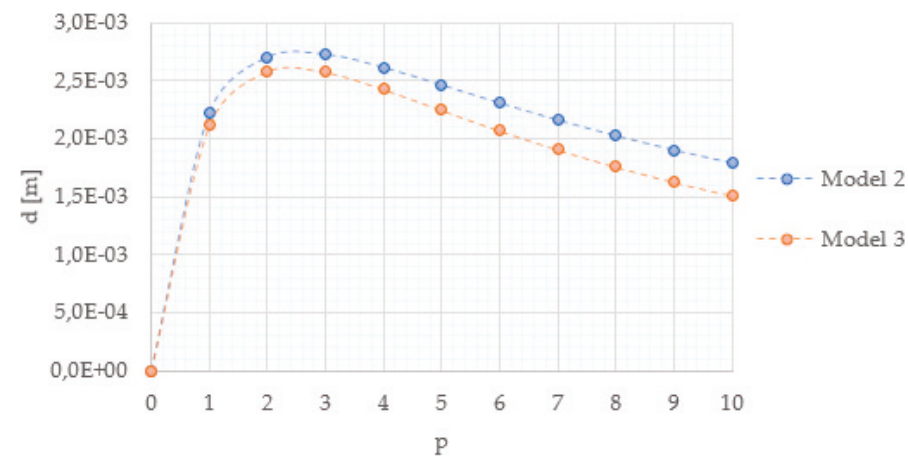

Figure 11. Porosity distribution models M2 and M3: evolution of neutral surface shift.

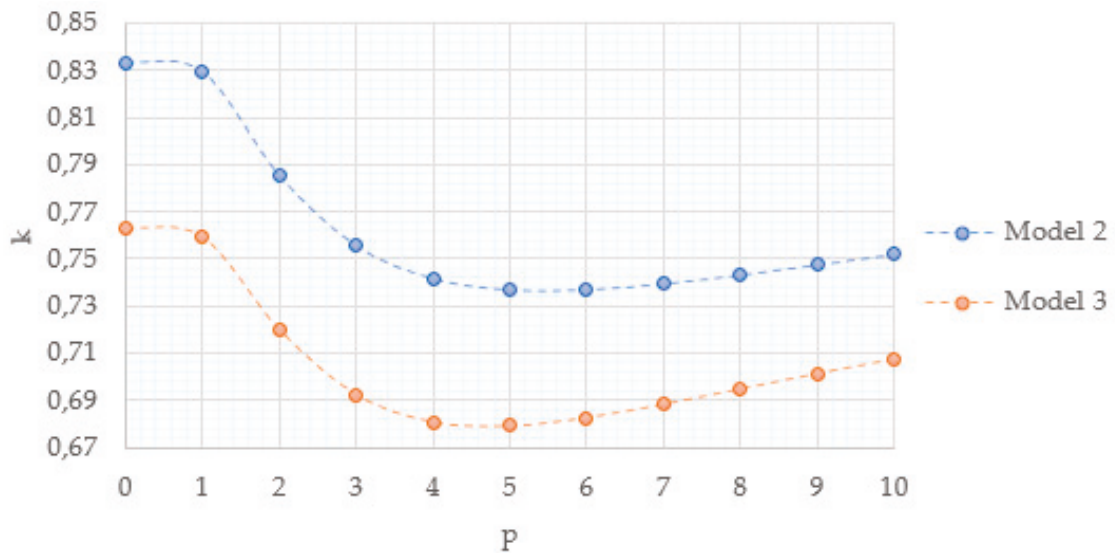

Figure 12. Porosity distribution models M2 and M3: evolution of the shear correction factor.

\subsubsection{Influence on Absolute Maximum Transverse Displacement}

In the studies presented in this section, the absolute maximum transverse displacement corresponds to the magnitude of the plate's center displacement given the considered boundary and load conditions. The results include both 4-node (Q4) and 9-node (Q9) plate finite element results, using the calculated shear correction factor (Section 2.4) here denoted by $k^{\text {calc }}$ and the often used value of 5/6. The relative deviations presented were determined by the following equations:

$$
\begin{aligned}
& \delta_{Q_{4}}(\%)=\frac{w_{\left(k=\frac{5}{6}\right)}^{Q_{4}}-w_{\left(k^{\text {calc }}\right)}^{Q_{4}}}{w_{\left(k^{\text {calc }}\right)}^{Q_{4}}} \cdot 100(\%), \\
& \delta_{Q_{9}}(\%)=\frac{w_{\left(k=\frac{5}{6}\right)}^{Q_{9}}-w_{\left(k^{\text {calc }}\right)}^{Q_{9}}}{w_{\left(k^{\text {calc }}\right)}^{Q_{9}}} \cdot 100(\%) .
\end{aligned}
$$

In the convergence study presented for porosity distribution model 1 , the relative deviations were determined by Equation (25), where the index $i$ denotes the order of the data in the respective results table.

$$
\delta_{\text {mesh }}(\%)=\frac{w_{i}-w_{i-1}}{w_{i-1}} \cdot 100(\%), i \geq 2
$$


- $\quad$ Porosity Distribution Model 1 (M1):

We developed convergence studies in order to evidence the reason for the discretization selection. To illustrate them, we present the results obtained for two sets of distinct parameterizations of maximum porosities and power law exponent values, considering Q4 finite elements and FSDT. The results achieved and presented in Table 5 show that the mesh refinement from $10 \times 10$ elements presents a deviation of less than $0.2 \%$. Based on this evidence, the following studies were performed considering a mesh of $10 \times 10$ elements.

Table 5. Porosity distribution model M1: convergence study.

\begin{tabular}{|c|c|c|c|c|}
\hline$\phi$ & $p$ & Mesh & $w\left(\frac{a}{2}, \frac{b}{2}, 0\right)(\mathrm{m})$ & $\delta_{m e s h}(\%)$ \\
\hline \multirow{9}{*}{0.1} & \multirow{9}{*}{1} & $4 \times 4$ & $-1.3855 \times 10^{-4}$ & - \\
\hline & & $6 \times 6$ & $-1.4035 \times 10^{-4}$ & 1.30 \\
\hline & & $8 \times 8$ & $-1.4091 \times 10^{-4}$ & 0.39 \\
\hline & & $10 \times 10$ & $-1.4116 \times 10^{-4}$ & 0.18 \\
\hline & & $12 \times 12$ & $-1.4129 \times 10^{-4}$ & 0.10 \\
\hline & & $14 \times 14$ & $-1.4137 \times 10^{-4}$ & 0.06 \\
\hline & & $16 \times 16$ & $-1.4142 \times 10^{-4}$ & 0.04 \\
\hline & & $18 \times 18$ & $-1.4146 \times 10^{-4}$ & 0.03 \\
\hline & & $20 \times 20$ & $-1.4149 \times 10^{-4}$ & 0.02 \\
\hline \multirow{9}{*}{0.5} & \multirow{9}{*}{5} & $4 \times 4$ & $-2.5263 \times 10^{-4}$ & - \\
\hline & & $6 \times 6$ & $-2.5597 \times 10^{-4}$ & 1.32 \\
\hline & & $8 \times 8$ & $-2.5701 \times 10^{-4}$ & 0.40 \\
\hline & & $10 \times 10$ & $-2.5747 \times 10^{-4}$ & 0.18 \\
\hline & & $12 \times 12$ & $-2.5773 \times 10^{-4}$ & 0.10 \\
\hline & & $14 \times 14$ & $-2.5788 \times 10^{-4}$ & 0.06 \\
\hline & & $16 \times 16$ & $-2.5798 \times 10^{-4}$ & 0.04 \\
\hline & & $18 \times 18$ & $-2.5804 \times 10^{-4}$ & 0.03 \\
\hline & & $20 \times 20$ & $-2.5809 \times 10^{-4}$ & 0.02 \\
\hline
\end{tabular}

The results obtained regarding transverse displacement at the plate's center are presented in Table 6. In this study, the displacement increased with increasing maximum porosity for all power law exponents considered, as expected.

Table 6. Porosity distribution model M1: plates center transverse displacement for different finite models and shear correction factor approaches.

\begin{tabular}{|c|c|c|c|c|c|}
\hline \multirow{3}{*}{$\phi$} & \multirow{3}{*}{$p$} & \multicolumn{2}{|c|}{ Q4 } & \multicolumn{2}{|c|}{ Q9 } \\
\hline & & $k^{\text {calc }}$ & $k=5 / 6$ & $k^{\text {calc }}$ & $k=5 / 6$ \\
\hline & & $w\left(\frac{a}{2}, \frac{b}{2}, 0\right)(\mathrm{m})$ & $\delta_{Q 4}(\%)$ & $w\left(\frac{a}{2}, \frac{b}{2}, 0\right)(\mathrm{m})$ & $\delta_{Q 9}(\%)$ \\
\hline \multirow{5}{*}{0} & 0 & $-7.9330 \times 10^{-5}$ & 0.00 & $-7.9625 \times 10^{-5}$ & 0.00 \\
\hline & 1 & $-1.3168 \times 10^{-4}$ & -5.14 & $-1.3215 \times 10^{-4}$ & -4.96 \\
\hline & 2 & $-1.5267 \times 10^{-4}$ & -7.17 & $-1.5321 \times 10^{-4}$ & -6.94 \\
\hline & 5 & $-1.7054 \times 10^{-4}$ & -5.51 & $-1.7114 \times 10^{-4}$ & -5.33 \\
\hline & 10 & $-1.8510 \times 10^{-4}$ & -2.95 & $-1.8574 \times 10^{-4}$ & -2.85 \\
\hline \multirow{5}{*}{0.3} & 0 & $-9.9540 \times 10^{-5}$ & -0.94 & $-9.9930 \times 10^{-5}$ & -0.93 \\
\hline & 1 & $-1.6493 \times 10^{-4}$ & -1.81 & $-1.6556 \times 10^{-4}$ & -1.70 \\
\hline & 2 & $-1.9003 \times 10^{-4}$ & -3.06 & $-1.9075 \times 10^{-4}$ & -2.91 \\
\hline & 5 & $-2.1256 \times 10^{-4}$ & -1.83 & $-2.1335 \times 10^{-4}$ & -1.73 \\
\hline & 10 & $-2.3281 \times 10^{-4}$ & -0.50 & $-2.3367 \times 10^{-4}$ & -0.46 \\
\hline \multirow{5}{*}{0.6} & 0 & $-1.3788 \times 10^{-4}$ & -5.65 & $-1.3845 \times 10^{-4}$ & -5.55 \\
\hline & 1 & $-2.2603 \times 10^{-4}$ & 0.13 & $-2.2695 \times 10^{-4}$ & 0.14 \\
\hline & 2 & $-2.5832 \times 10^{-4}$ & 0.03 & $-2.5936 \times 10^{-4}$ & 0.07 \\
\hline & 5 & $-2.9115 \times 10^{-4}$ & -0.07 & $-2.9231 \times 10^{-4}$ & -0.06 \\
\hline & 10 & $-3.2350 \times 10^{-4}$ & -0.91 & $-3.2479 \times 10^{-4}$ & -0.91 \\
\hline
\end{tabular}


Comparing the results with 4-node elements and different shear correction factor approaches, the relative deviation increased from $p=0$ to $p=2$, decreasing with increasing power law exponents. Moreover, the increase in maximum porosity value resulted in a relative deviation increase for $p=$ 0 - the opposite behavior to that verified for the intermediate power law exponents. When considering $\mathrm{p}=10$, the relative deviations showed an oscillating behavior with increasing maximum porosity value. However, for maximum porosity values of 0.5 and 0.6 and intermediate power law exponents these deviations were nearly zero, except for the case of null power law exponent. The same behavior was verified when doing the same comparative study with 9-node elements, except for the specific case considering a maximum porosity value of 0.6 .

Additionally, we verified that when considering the common value of $5 / 6$ for the shear correction factor, the relative deviation between the results observed for Q4 and Q9 elements was very low, presenting a decreasing behavior with increasing maximum porosity value, except for $\mathrm{p}=0$, where the opposite was verified. With the other approach, this relative deviation also presented very low values, although the increase of maximum porosity value resulted in higher deviations for all power law exponents considered.

Figure 13 shows the plate's center displacement for different power law exponents and maximum porosity values, obtained considering Q4 finite elements. In the figure, it is clear that higher maximum porosity values corresponded to higher maximum transverse displacement magnitudes for all exponents, with the effect being more pronounced when considering larger exponents.

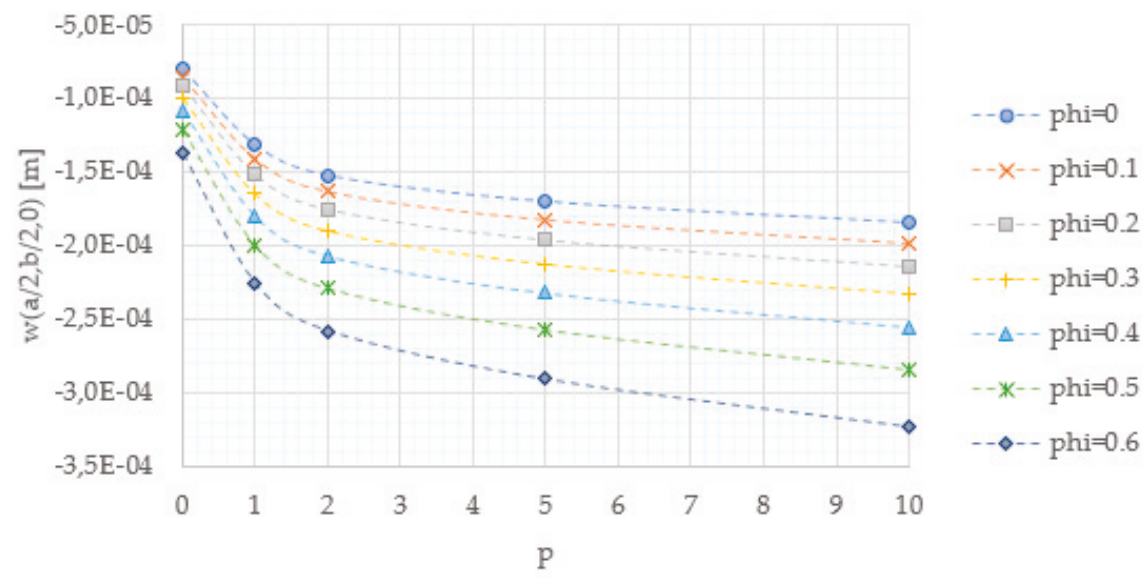

Figure 13. Porosity distribution model M1: evolution of plate's center transverse displacement.

In order to compare the results obtained by applying different shear deformation theories, keeping the configurations considered above, the plate's center displacement was determined by applying the third-order shear deformation theory (HSDT) proposed by [49], and also implemented by the authors for this purpose. The results obtained are presented in Table 7, with the relative deviations determined by:

$$
\delta_{H S D T}(\%)=\frac{w_{H S D T}-w_{F S D T}}{w_{F S D T}} \cdot 100(\%) .
$$

When considering a homogeneous isotropic material $(p=0)$, the deviation between the results obtained was very small for low maximum porosity values, increasing with the increase in porosity. Fixing the power law exponent, the increase of maximum porosities values led to a decrease in the relative deviations, except for the exponent $p=10$ where the transition between $\phi=0.4$ and $\phi=0.5$ led to an increase in the relative deviation. For all the maximum porosity values presented, there were slighter relative deviations in cases where the evolution of the material's constitution through the 
thickness was smoother (i.e., for lower and higher power law exponents), except for the case where $\phi=0.6$, for which the highest power law exponent presented a relative deviation higher than the other non-nulls.

Table 7. Porosity distribution model M1: plate's center transverse displacement for different shear deformation theories.

\begin{tabular}{|c|c|c|c|c|}
\hline$\phi$ & $p$ & $w_{F S D T}\left(\frac{a}{2}, \frac{b}{2}, 0\right)(\mathrm{m})$ & $w_{H S D T}\left(\frac{a}{2}, \frac{b}{2}, 0\right)(\mathrm{m})$ & $\delta_{H S D T}(\%)$ \\
\hline \multirow{5}{*}{0} & 0 & $-7.9330 \times 10^{-5}$ & $-7.9359 \times 10^{-5}$ & 0.04 \\
\hline & 1 & $-1.3168 \times 10^{-4}$ & $-1.2363 \times 10^{-4}$ & -6.11 \\
\hline & 2 & $-1.5267 \times 10^{-4}$ & $-1.3980 \times 10^{-4}$ & -8.43 \\
\hline & 5 & $-1.7054 \times 10^{-4}$ & $-1.5964 \times 10^{-4}$ & -6.39 \\
\hline & 10 & $-1.8510 \times 10^{-4}$ & $-1.7875 \times 10^{-4}$ & -3.43 \\
\hline \multirow{5}{*}{0.1} & 0 & $-8.5029 \times 10^{-5}$ & $-8.4957 \times 10^{-5}$ & -0.08 \\
\hline & 1 & $-1.4116 \times 10^{-4}$ & $-1.3402 \times 10^{-4}$ & -5.05 \\
\hline & 2 & $-1.6335 \times 10^{-4}$ & $-1.5175 \times 10^{-4}$ & -7.10 \\
\hline & 5 & $-1.8250 \times 10^{-4}$ & $-1.7308 \times 10^{-4}$ & -5.16 \\
\hline & 10 & $-1.9861 \times 10^{-4}$ & $-1.9351 \times 10^{-4}$ & -2.57 \\
\hline \multirow{5}{*}{0.2} & 0 & $-9.1656 \times 10^{-5}$ & $-9.1228 \times 10^{-5}$ & -0.47 \\
\hline & 1 & $-1.5208 \times 10^{-4}$ & $-1.4588 \times 10^{-4}$ & -4.08 \\
\hline & 2 & $-1.7562 \times 10^{-4}$ & $-1.6538 \times 10^{-4}$ & -5.83 \\
\hline & 5 & $-1.9628 \times 10^{-4}$ & $-1.8833 \times 10^{-4}$ & -4.05 \\
\hline & 10 & $-2.1425 \times 10^{-4}$ & $-2.1017 \times 10^{-4}$ & -1.91 \\
\hline \multirow{5}{*}{0.3} & 0 & $-9.9540 \times 10^{-5}$ & $-9.8380 \times 10^{-5}$ & -1.17 \\
\hline & 1 & $-1.6493 \times 10^{-4}$ & $-1.5965 \times 10^{-4}$ & -3.20 \\
\hline & 2 & $-1.9003 \times 10^{-4}$ & $-1.8120 \times 10^{-4}$ & -4.64 \\
\hline & 5 & $-2.1256 \times 10^{-4}$ & $-2.0596 \times 10^{-4}$ & -3.10 \\
\hline & 10 & $-2.3281 \times 10^{-4}$ & $-2.2936 \times 10^{-4}$ & -1.48 \\
\hline \multirow{5}{*}{0.4} & 0 & $-1.0919 \times 10^{-4}$ & $-1.0672 \times 10^{-4}$ & -2.27 \\
\hline & 1 & $-1.8050 \times 10^{-4}$ & $-1.7601 \times 10^{-4}$ & -2.49 \\
\hline & 2 & $-2.0744 \times 10^{-4}$ & $-2.0002 \times 10^{-4}$ & -3.58 \\
\hline & 5 & $-2.3237 \times 10^{-4}$ & $-2.2687 \times 10^{-4}$ & -2.36 \\
\hline & 10 & $-2.5551 \times 10^{-4}$ & $-2.5202 \times 10^{-4}$ & -1.37 \\
\hline \multirow{5}{*}{0.5} & 0 & $-1.2147 \times 10^{-4}$ & $-1.1671 \times 10^{-4}$ & -3.92 \\
\hline & 1 & $-2.0008 \times 10^{-4}$ & $-1.9602 \times 10^{-4}$ & -2.03 \\
\hline & 2 & $-2.2932 \times 10^{-4}$ & $-2.2304 \times 10^{-4}$ & -2.74 \\
\hline & 5 & $-2.5747 \times 10^{-4}$ & $-2.5241 \times 10^{-4}$ & -1.97 \\
\hline & 10 & $-2.8446 \times 10^{-4}$ & $-2.7959 \times 10^{-4}$ & -1.71 \\
\hline \multirow{5}{*}{0.6} & 0 & $-1.3788 \times 10^{-4}$ & $-1.2913 \times 10^{-4}$ & -6.35 \\
\hline & 1 & $-2.2603 \times 10^{-4}$ & $-2.2146 \times 10^{-4}$ & -2.02 \\
\hline & 2 & $-2.5832 \times 10^{-4}$ & $-2.5238 \times 10^{-4}$ & -2.30 \\
\hline & 5 & $-2.9115 \times 10^{-4}$ & $-2.8491 \times 10^{-4}$ & -2.14 \\
\hline & 10 & $-3.2350 \times 10^{-4}$ & $-3.1455 \times 10^{-4}$ & -2.77 \\
\hline
\end{tabular}

- $\quad$ Porosity Distribution Models M2 and M3:

The results presented in Tables 8 and 9 were obtained considering porosity distribution models M2 and M3, for Q4 and Q9 finite element discretizations and different shear correction factor approaches, respectively. 
Table 8. Porosity distribution model M2: plate's center transverse displacement for different finite elements and shear correction factor approaches.

\begin{tabular}{ccccc}
\hline & \multicolumn{2}{c}{$\mathrm{Q} 4$} & \multicolumn{2}{c}{$\mathbf{Q 9}$} \\
\cline { 2 - 5 }$p$ & $k^{\text {calc }}$ & $k=5 / 6$ & $\boldsymbol{k}^{\text {calc }}$ & $k=5 / 6$ \\
\cline { 2 - 5 } & $w\left(\frac{a}{2}, \frac{b}{2}, 0\right)(\mathrm{m})$ & $\delta_{\mathbf{Q}^{4}}(\%)$ & $w\left(\frac{a}{2}, \frac{b}{2}, 0\right)(\mathrm{m})$ & $\delta_{Q^{9}}(\%)$ \\
\hline 0 & $-1.6403 \times 10^{-4}$ & 0.00 & $-1.6472 \times 10^{-4}$ & 0.00 \\
1 & $-2.7631 \times 10^{-4}$ & -5.20 & $-2.7747 \times 10^{-4}$ & -5.01 \\
2 & $-3.2144 \times 10^{-4}$ & -7.21 & $-3.2278 \times 10^{-4}$ & -6.95 \\
3 & $-3.4037 \times 10^{-4}$ & -7.01 & $-3.4178 \times 10^{-4}$ & -6.76 \\
4 & $-3.5137 \times 10^{-4}$ & -6.28 & $-3.5282 \times 10^{-4}$ & -6.05 \\
5 & $-3.5972 \times 10^{-4}$ & -5.50 & $-3.6120 \times 10^{-4}$ & -5.31 \\
6 & $-3.6697 \times 10^{-4}$ & -4.81 & $-3.6848 \times 10^{-4}$ & -4.64 \\
7 & $-3.7359 \times 10^{-4}$ & -4.22 & $-3.7512 \times 10^{-4}$ & -4.06 \\
8 & $-3.7975 \times 10^{-4}$ & -3.72 & $-3.8131 \times 10^{-4}$ & -3.58 \\
9 & $-3.8552 \times 10^{-4}$ & -3.29 & $-3.8710 \times 10^{-4}$ & -3.17 \\
10 & $-3.9093 \times 10^{-4}$ & -2.94 & $-3.9254 \times 10^{-4}$ & -2.83 \\
\hline
\end{tabular}

Table 9. Porosity distribution model M3: plate's center transverse displacement for different finite elements and shear correction factor approaches.

\begin{tabular}{ccccc}
\hline & \multicolumn{2}{c}{$\mathbf{Q} 4$} & \multicolumn{2}{c}{ Q9 } \\
\cline { 2 - 5 } & $k^{\text {calc }}$ & $k=5 / 6$ & $k^{\text {calc }}$ & $k=5 / 6$ \\
\cline { 2 - 5 } & $w\left(\frac{a}{2}, \frac{b}{2}, 0\right)(\mathrm{m})$ & $\delta_{Q 4}(\%)$ & $w\left(\frac{a}{2}, \frac{b}{2}, 0\right)(\mathrm{m})$ & $\delta_{Q^{9}}(\%)$ \\
\hline 0 & $-1.1377 \times 10^{-4}$ & -0.10 & $-1.1422 \times 10^{-4}$ & -0.10 \\
1 & $-1.8911 \times 10^{-4}$ & -4.98 & $-1.8984 \times 10^{-4}$ & -4.80 \\
2 & $-2.2387 \times 10^{-4}$ & -7.09 & $-2.2473 \times 10^{-4}$ & -6.85 \\
3 & $-2.3900 \times 10^{-4}$ & -6.79 & $-2.3991 \times 10^{-4}$ & -6.56 \\
4 & $-2.4781 \times 10^{-4}$ & -5.91 & $-2.4875 \times 10^{-4}$ & -5.71 \\
5 & $-2.5454 \times 10^{-4}$ & -5.01 & $-2.5551 \times 10^{-4}$ & -4.84 \\
6 & $-2.6041 \times 10^{-4}$ & -4.24 & $-2.6140 \times 10^{-4}$ & -4.09 \\
7 & $-2.6576 \times 10^{-4}$ & -3.59 & $-2.6677 \times 10^{-4}$ & -3.46 \\
8 & $-2.7070 \times 10^{-4}$ & -3.07 & $-2.7173 \times 10^{-4}$ & -2.96 \\
9 & $-2.7529 \times 10^{-4}$ & -2.64 & $-2.7633 \times 10^{-4}$ & -2.54 \\
10 & $-2.7953 \times 10^{-4}$ & -2.29 & $-2.8059 \times 10^{-4}$ & -2.21 \\
\hline
\end{tabular}

In general, both porosity distribution models led to the same verifications about relative deviations. Like in the previous study, the relative deviations $\delta_{Q 4}$ and $\delta_{Q 9}$ increased for increasing power law exponents between $p=0$ and $p=2$, after which these deviations presented a decreasing behavior. Additionally, comparing Q4 and Q9 results denoted by $k^{\text {cal }}$ we verified that the relative deviation was maximal when $p=0$, and presented a decreasing behavior with increasing power law exponent. When $k=5 / 6$, the relative deviation of Q4 and Q9 to Q4 with $k^{\text {calc }}$ also increased from $p=0$ to $p=2$, then decreased with increasing power law exponent in both comparative analyses.

Figure 14 depicts the behavior of the plate's center transverse displacement, using Q4 finite elements, for the power law exponents considered, evidencing the fact that porosity distribution model M3 provided smaller displacements than model M2, and the increase in the exponent of the power law induced a greater difference between the results obtained with each model. 


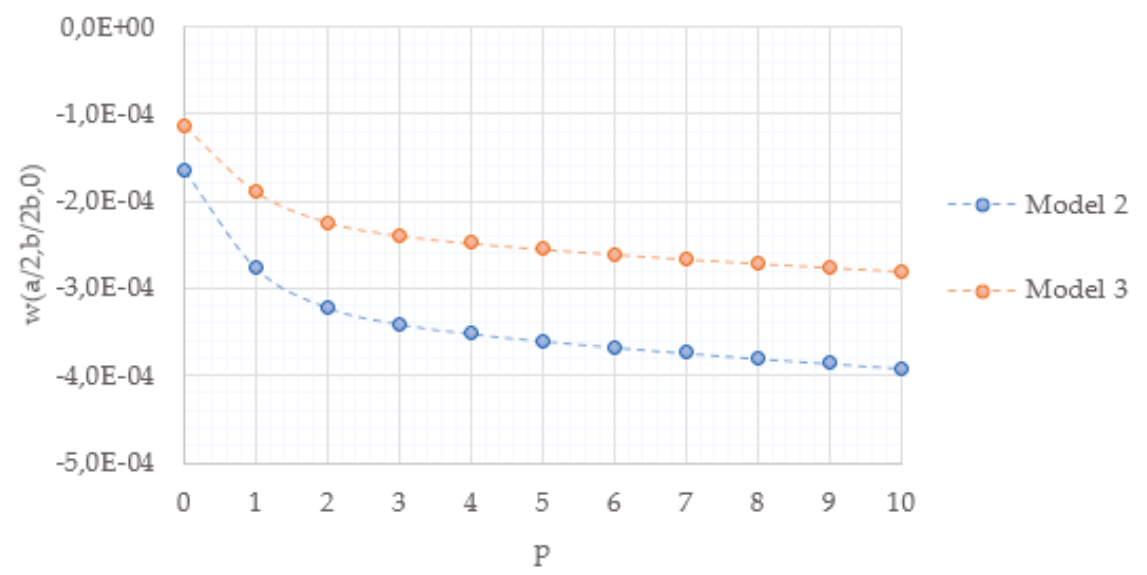

Figure 14. Evolution of the plate's center transverse displacement with the power law exponent.

Similar to the M1 porosity distribution model, the plate's center displacements for models M2 and M3 were also obtained, considering the same third-order shear deformation theory. The results obtained for both porosity distribution models with the different shear deformation theories are shown in Table 10, where the relative deviations presented were determined by Equation (26). The results show a behavior similar to the one observed and described for model M1 about the effect of the power law exponent on the relative deviation.

Table 10. Porosity distribution models M2 and M3: plate's center transverse displacement for different shear deformation theories.

\begin{tabular}{ccccccc}
\hline \multirow{2}{*}{$p$} & \multicolumn{5}{c}{ M2 } & M3 \\
\cline { 2 - 6 } & $w_{\text {FSDT }\left(\frac{a}{2}, \frac{b}{2}, 0\right)(\mathrm{m})}$ & $w_{H S D T}\left(\frac{a}{2}, \frac{b}{2}, 0\right)(\mathrm{m})$ & $\delta_{\text {HSDT }}(\%)$ & $w_{H S D T}\left(\frac{a}{2}, \frac{b}{2}, 0\right)(\mathrm{m})$ & $w_{H S D T}\left(\frac{a}{2}, \frac{b}{2}, 0\right)(\mathrm{m})$ & $\delta_{H S D T}(\%)$ \\
\hline 0 & $-1.6403 \times 10^{-4}$ & $-1.6407 \times 10^{-4}$ & 0.03 & $-1.1377 \times 10^{-4}$ & $-1.1308 \times 10^{-4}$ & -0.60 \\
1 & $-2.7631 \times 10^{-4}$ & $-2.6157 \times 10^{-4}$ & -5.34 & $-1.8911 \times 10^{-4}$ & $-1.7729 \times 10^{-4}$ & -6.25 \\
2 & $-3.2144 \times 10^{-4}$ & $-2.9782 \times 10^{-4}$ & -7.35 & $-2.2387 \times 10^{-4}$ & $-2.0457 \times 10^{-4}$ & -8.62 \\
3 & $-3.4037 \times 10^{-4}$ & $-3.1621 \times 10^{-4}$ & -7.10 & $-2.3900 \times 10^{-4}$ & $-2.1909 \times 10^{-4}$ & -8.33 \\
4 & $-3.5137 \times 10^{-4}$ & $-3.2917 \times 10^{-4}$ & -6.32 & $-2.4781 \times 10^{-4}$ & $-2.2945 \times 10^{-4}$ & -7.41 \\
5 & $-3.5972 \times 10^{-4}$ & $-3.3992 \times 10^{-4}$ & -5.50 & $-2.5454 \times 10^{-4}$ & $-2.3807 \times 10^{-4}$ & -6.47 \\
6 & $-3.6697 \times 10^{-4}$ & $-3.4941 \times 10^{-4}$ & -4.79 & $-2.6041 \times 10^{-4}$ & $-2.4565 \times 10^{-4}$ & -5.67 \\
7 & $-3.7359 \times 10^{-4}$ & $-3.5798 \times 10^{-4}$ & -4.18 & $-2.6576 \times 10^{-4}$ & $-2.5247 \times 10^{-4}$ & -5.00 \\
8 & $-3.7975 \times 10^{-4}$ & $-3.6582 \times 10^{-4}$ & -3.67 & $-2.7070 \times 10^{-4}$ & $-2.5862 \times 10^{-4}$ & -4.46 \\
9 & $-3.8552 \times 10^{-4}$ & $-3.7302 \times 10^{-4}$ & -3.24 & $-2.7529 \times 10^{-4}$ & $-2.6421 \times 10^{-4}$ & -4.02 \\
10 & $-3.9093 \times 10^{-4}$ & $-3.7967 \times 10^{-4}$ & -2.88 & $-2.7953 \times 10^{-4}$ & $-2.6928 \times 10^{-4}$ & -3.67 \\
\hline
\end{tabular}

In the present work, the three porosity distributions analyzed showed some similarities regarding the estimation of effective material properties. Noting that for the materials considered, the product $e_{0} \beta$ assumed an approximate value of 0.49 , we can then compare this case for models 2 and 3 (M2 and M3) to the case where in model 1 (M1) the maximum porosity value took the value 0.5 . In this sense, Table 11 presents the results obtained under these conditions, considering a Q4 plate model and a shear correction factor determined with Equation (20), with respect to neutral surface shift, shear correction factor, and plate's center displacement. 
Table 11. Results obtained with the three porosity distribution models considered.

\begin{tabular}{|c|c|c|c|c|c|c|c|c|c|}
\hline \multirow{2}{*}{$p$} & \multicolumn{3}{|c|}{ Model $1(\phi=0.5)$} & \multicolumn{3}{|c|}{ Model 2} & \multicolumn{3}{|c|}{ Model 3} \\
\hline & $d / h$ & $k$ & $w\left(\frac{a}{2}, \frac{b}{2}, 0\right)(\mathrm{m})$ & $d / h$ & $k$ & $w\left(\frac{a}{2}, \frac{b}{2}, 0\right)(\mathrm{m})$ & $d / h$ & $k$ & $w\left(\frac{a}{2}, \frac{b}{2}, 0\right)(\mathrm{m})$ \\
\hline 0 & -0.0638 & 0.8217 & $-1.215 \times 10^{-4}$ & 0 & $5 / 6$ & $-1.640 \times 10^{-4}$ & $2.6 \times 10^{-25}$ & 0.7632 & $-1.138 \times 10^{-4}$ \\
\hline 1 & 0.0308 & 0.8390 & $-2.001 \times 10^{-4}$ & 0.0889 & 0.8291 & $-2.763 \times 10^{-4}$ & 0.0845 & 0.7590 & $-1.891 \times 10^{-4}$ \\
\hline 2 & 0.0470 & 0.7988 & $-2.293 \times 10^{-4}$ & 0.1081 & 0.7853 & $-3.214 \times 10^{-4}$ & 0.1033 & 0.7199 & $-2.239 \times 10^{-4}$ \\
\hline 5 & 0.0295 & 0.7477 & $-2.575 \times 10^{-4}$ & 0.0985 & 0.7371 & $-3.597 \times 10^{-4}$ & 0.0899 & 0.6791 & $-2.545 \times 10^{-4}$ \\
\hline 10 & 0.0003 & 0.7546 & $-2.845 \times 10^{-4}$ & 0.0717 & 0.7519 & $-3.909 \times 10^{-4}$ & 0.0604 & 0.7074 & $-2.795 \times 10^{-4}$ \\
\hline
\end{tabular}

These results indicate that porosity distribution M2 promoted greater displacement of the plate's center, whereas model M3 led to smaller displacements. Regarding the neutral surface shift, in the case of model M1, it started to assume a negative value when $p=0$ (the highest shift for the exponents evaluated). Then, the neutral surface approached the medium surface and moved away, presenting this behavior from $p=0$ to $p=2$, after which the neutral surface shift assumed smaller and smaller values in the sense of a new approximation between the neutral and medium surfaces as the exponent of the power law increased. In models M2 and M3 the neutral surface shift presented a monotonic increasing behavior from $p=0$ to $p=2$, starting with coincident neutral and medium surfaces when $p$ $=0$, then decreasing with increasing power law exponents, presenting higher neutral surface shifts than those verified with model 3.

Regarding the shear correction factor, there was an oscillating behavior observed in model M1, with an increasing trend between $p=0$ and $p=1$, a decreasing one between $p=2$ and $p=5$, and finishing with an increasing trend again from $p=5$ to $p=10$. In models M2 and M3, this factor presented a decreasing behavior with increasing power law exponents until $p=5$, and then increases from $p=5$ to $p=10$. It is important to note that in the results presented in Table 11 the null power law exponent corresponds to a material constituted only by titanium oxide, but with the presence of a porosity distribution.

In the particular case of model M3 considering $p=0$, the neutral surface presented a shift from the medium surface that can numerically be considered as zero. However, it is important to highlight that this result does not lead to the expected shear correction factor value of 5/6, as verified with model M2. For this reason, the authors decided to maintain the value of $2.6 \times 10^{-25}$ for the respective normalized neutral surface shift, $\mathrm{d} / \mathrm{h}$.

\section{Discussion and Conclusions}

The analyses carried out on the various case studies led to the following conclusions:

- In all porosity distributions models studied, the neutral surface shift increased for power law exponents between 0 and 2, and for larger exponents it showed a decreasing behavior.

- The studies of the influence of porosity distribution model M1 on the shear correction factor showed that for a certain power law exponent the shear correction factor increased with increasing maximum porosity values, except for the case in which $p=0$ which corresponds to a plate constituted only by $\mathrm{TiO}_{2}$. The increasing behavior was more pronounced when the power law exponent took the value 2 , and was less pronounced for the higher power law exponents considered.

- Concerning the influence of the porosity distribution models on the deviation from the neutral surface, models M2 and M3 resulted in behaviors similar to each other and similar to those verified in the corresponding analysis carried out on model M1, with model M2 leading to lower neutral surface shifts. The difference between the models increased with increasing power law exponents.

- With respect to the shear correction factor, porosity distribution models M2 and M3 showed a similar behavior, with M3 presenting lower values (i.e., leading to higher corrections of the transverse shear stiffness coefficients). In this case, the difference between both models decreased with increasing power law exponents. 
- Regarding the maximum transverse displacement, and in correspondence to the expected trend, all case studies showed an increasing behavior with the increase in maximum porosity values and power law exponents. In the case of model M1, for a certain power law exponent the increase in the plate's center displacement became more pronounced as the maximum porosity value increased. The increase in the magnitude of the maximum transverse displacement with the increase of power law exponent is explained by the fact that higher exponents correspond to a smaller volume fraction of the stiffer phase, $\mathrm{TiO}_{2}$.

- The last study carried out to promote a comparison between the three porosity distributions demonstrated that model M2 led to higher displacements of the plate's center, while model M3 led to lower ones. Except for the case of null power law exponent, in which model M2 presented the higher shear correction factor and model M3 the lower one, in the remaining power law exponents considered model M1 and model M3 presented the higher and the lower shear correction factors, respectively. Moreover, the shear correction factor presented a similar behavior with the increase of power law exponent in both models M2 and M3, decreasing from $p=0$ to $p=5$, and increasing in the last transition between $p=5$ and $p=10$. The first model had a different behavior concerning the shear correction factor and power law exponent relation, first presenting an increase in the transition between $p=0$ and $p=1$, decreasing for the intermediate exponents, and increasing in the transition between the two largest exponents presented.

- The results regarding the maximum absolute plate displacement obtained with the plate elements Q4 and Q9 were quite close to each other in most of the studied configurations. Therefore, depending on the application, in order to obtain a shorter computing time the Q4 model may be a good choice, resulting in an accuracy similar to the one obtained with the Q9 model.

- When comparing the relative deviations between the results obtained with the present FSDT and HSDT, model M1 showed an increasing relative deviation with increasing maximum porosity values when considering a null power law exponent. For all three porosity distribution models, the lower relative deviations obtained, corresponded to the lower and to the highest power law exponents (i.e., to smoother material evolutions through the thickness).

- In the future, it is important to give continuity to the development of theoretical models to describe the real porosity distributions and how they influence the resulting material properties and, consequently, the constitutive relations. Obtaining more accurate predictions of porous functionally graded material properties and/or designed ones, will continue to be an important research topic in order to allow for higher-quality predictions of structures' behavior.

- Another important aspect and a limitation of the present work is related to the behavior prediction of thicker plate structures with different porosity models, wherein higher-order models are expected to behave better. However, it will be important to compare the response achieved via higher-order models with the present results under comparable situations.

Author Contributions: A.F.M.: Data curation, Conceptualization, Methodology, Software, Verification, Writing-original draft, Writing-review and editing. M.A.R.L.: Conceptualization, Methodology, Formal analysis, Supervision, Project administration. J.I.B.: Methodology, Writing-review and editing. J.A.R.: Data curation, Writing - review and editing. All authors have read and agreed to the published version of the manuscript.

Funding: This research was funded by Project IDMEC, LAETA UIDB/50022/2020, and Project IPL/2019/MOCHVar/ISEL.

Acknowledgments: The authors wish to acknowledge the support given by FCT/MEC through Project IDMEC, LAETA UIDB/50022/2020. The authors also acknowledge the support of Project IPL/2019/MOCHVar/ISEL.

Conflicts of Interest: The authors declare no conflicts of interest.

\section{References}

1. Naebe, M.; Shirvanimoghaddam, K. Functionally graded materials: A review of fabrication and properties. Appl. Mater. Today 2016, 5, 223-245. [CrossRef] 
2. Sola, A.; Bellucci, D.; Cannillo, V. Functionally graded materials for orthopedic applications-An update on design and manufacturing. Biotechnol. Adv. 2016, 34, 504-531. [CrossRef] [PubMed]

3. Miao, X.; Sun, D. Graded/Gradient Porous Biomaterials. Materials 2009, 3, 26-47. [CrossRef]

4. Kiran, S.C.; Kattimani, M.C. Assessment of porosity influence on vibration and static behavior of functionally graded magneto-electro-elastic plate: A finite element study. Eur. J. Mech. A Solids 2018, 71, 258-277. [CrossRef]

5. Zhang, Z.; Li, Y.; Wu, H.; Zhang, H.; Wu, H.; Jiang, S.; Chai, G. Mechanical analysis of functionally graded graphene oxide-reinforced composite beams based on the first-order shear deformation theory. Mech. Adv. Mater. Struct. 2018, 27, 3-11. [CrossRef]

6. Hosseini-Hashemi, S.; Taher, H.R.D.; Akhavan, H.; Omidi, M. Free vibration of functionally graded rectangular plates using first-order shear deformation plate theory. Appl. Math. Model. 2010, 34, 1276-1291. [CrossRef]

7. Thai, H.-T.; Choi, D.-H. A simple first-order shear deformation theory for the bending and free vibration analysis of functionally graded plates. Compos. Struct. 2013, 101, 332-340. [CrossRef]

8. Sayyad, A.; Ghugal, Y. Modeling and analysis of functionally graded sandwich beams: A review. Mech. Adv. Mater. Struct. 2018, 26, 1776-1795. [CrossRef]

9. Keddouri, A.; Hadji, L.; Tounsi, A. Static analysis of functionally graded sandwich plates with porosities. Adv. Mater. Res. South Korea 2019, 8, 155-177.

10. Li, Z.; Zheng, J.; Chen, Y.; Zhang, Z. Collapse mechanism of the thin-walled functionally graded cylinders encased in the saturated permeable mediums. Eng. Struct. 2019, 198. [CrossRef]

11. Li, Z.; Tang, F.; Chen, Y.; Zheng, J. Material distribution optimization of functionally graded arch subjected to external pressure under temperature rise field. Thin-Walled Struct. 2019, 138, 64-78. [CrossRef]

12. Li, Z.; Zheng, J.; Chen, Y. Nonlinear buckling of thin-walled FGM arch encased in rigid confinement subjected to external pressure. Eng. Struct. 2019, 186, 86-95. [CrossRef]

13. Li, Z.; Zheng, J.; Zhang, Z.; He, H. Nonlinear stability and buckling analysis of composite functionally graded arches subjected to external pressure and temperature loading. Eng. Struct. 2019, 199, 109606. [CrossRef]

14. Li, Z.; Zheng, J.; Sun, Q.; He, H. Nonlinear structural stability performance of pressurized thin-walled FGM arches under temperature variation field. Int. J. Non-linear Mech. 2019, 113, 86-102. [CrossRef]

15. Zhang, Y.; Wang, J. Fabrication of Functionally Graded Porous Polymer Structures using Thermal Bonding Lamination Techniques. Procedia Manuf. 2017, 10, 866-875. [CrossRef]

16. Heshmati, M.; Daneshmand, F. A study on the vibrational properties of weight-efficient plates made of material with functionally graded porosity. Compos. Struct. 2018, 200, 229-238. [CrossRef]

17. Nguyen, L.B.; Thai, C.H.; Zenkour, A.M.; Nguyen-Xuan, H. An isogeometric Bézier finite element method for vibration analysis of functionally graded piezoelectric material porous plates. Int. J. Mech. Sci. 2019, 158, 165-183. [CrossRef]

18. Gao, K.; Li, R.; Yang, J. Dynamic characteristics of functionally graded porous beams with interval material properties. Eng. Struct. 2019, 197, 109441. [CrossRef]

19. Fahsi, B.; Bouiadjra, R.B.; Mahmoudi, A.; Benyoucef, S.; Tounsi, A. Assessing the Effects of Porosity on the Bending, Buckling, and Vibrations of Functionally Graded Beams Resting on an Elastic Foundation by Using a New Refined Quasi-3D Theory. Mech. Compos. Mater. 2019, 55, 219-230. [CrossRef]

20. Zhao, J.; Wang, Q.; Deng, X.; Choe, K.; Zhong, R.; Shuai, C. Free vibrations of functionally graded porous rectangular plate with uniform elastic boundary conditions. Compos. Part B Eng. 2019, 168, 106-120. [CrossRef]

21. Merdaci, S.; Belghoul, H. High-order shear theory for static analysis of functionally graded plates with porosities. Comptes Rendus Mécanique 2019, 347, 207-217. [CrossRef]

22. Li, Z.; Zheng, J. Analytical consideration and numerical verification of the confined functionally graded porous ring with graphene platelet reinforcement. Int. J. Mech. Sci. 2019, 162. [CrossRef]

23. Li, Z.; Zheng, J.; Chen, Y.; Sun, Q.; Zhang, Z. Effect of temperature variations on the stability mechanism of the confined functionally graded porous arch with nanocomposites reinforcement under mechanical loading. Compos. Part B Eng. 2019, 176. [CrossRef]

24. Li, Z.; Zheng, J.; Zhang, Z. Mechanics of the confined functionally graded porous arch reinforced by graphene platelets. Eng. Struct. 2019, 201, 109817. [CrossRef] 
25. Li, Z; Zheng, J. Nonlinear stability of the encased functionally graded porous cylinders reinforced by graphene nanofillers subjected to pressure loading under thermal effect. Compos. Struct. 2020, 233, 111584. [CrossRef]

26. Li, Z. Exploration of the encased nanocomposites functionally graded porous arches: Nonlinear analysis and stability behavior. Appl. Math. Model. 2020, 82,1-16. [CrossRef]

27. Li, Z.; Zheng, J. Structural failure performance of the encased functionally graded porous cylinder consolidated by graphene platelet under uniform radial loading. Thin-Walled Struct. 2020, 146, 106454. [CrossRef]

28. Masjedi, P.K.; Maheri, A.; Weaver, P.M. Large deflection of functionally graded porous beams based on a geometrically exact theory with a fully intrinsic formulation. Appl. Math. Model. 2019, 76, 938-957. [CrossRef]

29. Kovacik, J. Correlation between Elastic Modulus, Shear Modulus, Poisson's Ratio and Porosity in Porous Materials. Adv. Eng. Mater. 2008, 10, 250-252. [CrossRef]

30. Han, C.; Li, Y.; Wang, Q.; Wen, S.; Wei, Q.; Yan, C.; Hao, L.; Liu, J.; Shi, Y. Continuous functionally graded porous titanium scaffolds manufactured by selective laser melting for bone implants. J. Mech. Behav. Biomed. Mater. 2018, 80, 119-127. [CrossRef]

31. Sopyan, I.; Mel, M.; Ramesh, S.; Khalid, K. Porous hydroxyapatite for artificial bone applications. Sci. Technol. Adv. Mater. 2007, 8, 116-123. [CrossRef]

32. Yılmaz, E.; Gökçe, A.; Findik, F.; Gülsoy, H.; Özkan; Iyibilgin, O. Mechanical properties and electrochemical behavior of porous Ti-Nb biomaterials. J. Mech. Behav. Biomed. Mater. 2018, 87, 59-67. [CrossRef] [PubMed]

33. Wang, I.-F.; Ditter, J.F.; Morris, R. Microfiltration membranes having high pore density and mixed isotropic and anisotropic structure. U.S. Patent EP0846024B1, 22 August 2001.

34. Lu, W.; Yuan, Z.; Zhao, Y.; Zhang, H.; Zhang, H.; Li, X. Porous membranes in secondary battery technologies. Chem. Soc. Rev. 2017, 46, 2199-2236. [CrossRef]

35. Meulenberg, W.A.; Schulze-Küppers, F.; Deibert, W.; van Gestel, T.; Baumann, S. Ceramic Membranes: Materials-Components-Potential Applications. ChemBioEng Rev. 2019, 6, 198-208. [CrossRef]

36. Yu, L.; Kanezashi, M.; Nagasawa, H.; Tsuru, T. Phase inversion/sintering-induced porous ceramic microsheet membranes for high-quality separation of oily wastewater. J. Membr. Sci. 2020, 595, 117477. [CrossRef]

37. Zhang, G.; Jin, W.; Xu, N. Design and Fabrication of Ceramic Catalytic Membrane Reactors for Green Chemical Engineering Applications. Engineering 2018, 4, 848-860. [CrossRef]

38. Carranza, J.C.; Pérez, L.; Ganesan, R.; Casas, B.Y.; Drew, R.A.L.; Ruiz-Aguilar, C.; Figueroa, I.A.; Alfonso, I. Effect of fractal distribution of the porosity on mechanical properties of Al foams manufactured by infiltration. J. Braz. Soc. Mech. Sci. Eng. 2019, 41, 379. [CrossRef]

39. Costantini, M.; Jaroszewicz, J.; Kozon, L.; Szlazak, K.; Swieszkowski, W.; Garstecki, P.; Stubenrauch, C.; Barbetta, A.; Guzowski, J. 3D-Printing of Functionally Graded Porous Materials Using On-Demand Reconfigurable Microfluidics. Angewandte Chemie 2019, 131, 7702-7707. [CrossRef]

40. Legrand, A.; Craig, G.A.; Bonneau, M.; Minami, S.; Urayama, K.; Furukawa, S. Understanding the multiscale self-assembly of metal-organic polyhedra towards functionally graded porous gels. Chem. Sci. 2019, 10, 10833-10842. [CrossRef]

41. Nguyen, N.V.; Nguyen, H.X.; Lee, S.; Nguyen-Xuan, H. Geometrically nonlinear polygonal finite element analysis of functionally graded porous plates. Adv. Eng. Softw. 2018, 126, 110-126. [CrossRef]

42. Bernardo, G.; Damásio, F.; Silva, T.; Loja, A. A study on the structural behaviour of FGM plates static and free vibrations analyses. Compos. Struct. 2016, 136, 124-138. [CrossRef]

43. Nguyen, T.-K.; Sab, K.; Bonnet, G. First-order shear deformation plate models for functionally graded materials. Compos. Struct. 2008, 83, 25-36. [CrossRef]

44. Mota, A.F.; Loja, A. Mechanical Behavior of Porous Functionally Graded Nanocomposite Materials. C J. Carbon Res. 2019, 5, 34. [CrossRef]

45. Kim, J.; Żur, K.K.; Reddy, J. Bending, free vibration, and buckling of modified couples stress-based functionally graded porous micro-plates. Compos. Struct. 2019, 209, 879-888. [CrossRef]

46. Coskun, S.; Kim, J.; Toutanji, H. Bending, Free Vibration, and Buckling Analysis of Functionally Graded Porous Micro-Plates Using a General Third-Order Plate Theory. J. Compos. Sci. 2019, 3, 15. [CrossRef]

47. Du, Y.; Wang, S.; Sun, L.; Shan, Y. Free Vibration of Rectangular Plates with Porosity Distributions under Complex Boundary Constraints. Shock. Vib. 2019, 2019, 1-16. [CrossRef]

48. Reddy, J.N. Mechanics of Laminated Composite Plates and Shells; Informa UK Limited: Colchester, UK, 2004. 
49. Kant, T.; Swaminathan, K. Analytical solutions for free vibration of laminated composite and sandwich plates based on a higher-order refined theory. Compos. Struct. 2001, 53, 73-85. [CrossRef]

50. Zienkiewicz, O.C.; Taylor, R.L. The Finite Element Method, 5th ed.; Butterworth-Heinemann: Oxford, UK, 2000; Volume 1.

51. Nguyen, T.-K.; Sab, K.; Bonnet, G. Shear Correction Factors for Functionally Graded Plates. Mech. Adv. Mater. Struct. 2007, 14, 567-575. [CrossRef]

52. Lu, H.; Yao, Y.; Yin, J.; Lin, L. Functionally graded carbon nanotube and nafion/silica nanofibre for electrical actuation of carbon fibre reinforced shape memory polymer. Pigment. Resin Technol. 2016, 45, 93-98. [CrossRef]

53. Li, H.; Liu, N.; Pang, F.; Du, Y.; Li, S. An Accurate Solution Method for the Static and Vibration Analysis of Functionally Graded Reissner-Mindlin Rectangular Plate with General Boundary Conditions. Shock. Vib. 2018, 2018, 1-21. [CrossRef]

54. Singha, M.; Prakash, T.; Ganapathi, M. Finite element analysis of functionally graded plates under transverse load. Finite Elements Anal. Des. 2011, 47, 453-460. [CrossRef]

(C) 2020 by the authors. Licensee MDPI, Basel, Switzerland. This article is an open access article distributed under the terms and conditions of the Creative Commons Attribution (CC BY) license (http://creativecommons.org/licenses/by/4.0/). 


\title{
A Continuation Procedure for the Quasi-Static Analysis of Materially and Geometrically Nonlinear Structural Problems
}

\author{
Davide Bellora and Riccardo Vescovini * \\ Dipartimento di Scienze e Tecnologie Aerospaziali, Politecnico di Milano, Via La Masa 34, 20156 Milano, Italy; \\ davide.bellora@mail.polimi.it \\ * Correspondence: riccardo.vescovini@polimi.it; Tel.: +39-02-2399-8332
}

Received: 14 October 2019; Accepted: 30 October 2019; Published: 2 November 2019

\begin{abstract}
Discussed is the implementation of a continuation technique for the analysis of nonlinear structural problems, which is capable of accounting for geometric and dissipative requirements. The strategy can be applied for solving quasi-static problems, where nonlinearities can be due to geometric or material response. The main advantage of the proposed approach relies in its robustness, which can be exploited for tracing the equilibrium paths for problems characterized by complex responses involving the onset and propagation of cracks. A set of examples is presented and discussed. For problems involving combined material and geometric nonlinearties, the results illustrate the advantages of the proposed hybrid continuation technique in terms of efficiency and robustness. Specifically, less iterations are usually required with respect to similar procedures based on purely geometric constraints. Furthermore, bifurcation plots can be easily traced, furnishing the analyst a powerful tool for investigating the nonlinear response of the structure at hand.
\end{abstract}

Keywords: continuation methods; bifurcations; limit points; cohesive elements

\section{Introduction}

Nonlinear analyses are commonly performed during the design phases of advanced, modern constructions. An example can be found in the design process of lightweight aerospace structures, where the capability of accurately predicting the behaviour in the post-buckling range is a task of paramount importance. The problem can often be studied by considering just nonlinearties of geometrical nature, where analytical and semi-analytical approaches can be successfully employed [1-5]. However, a proper assessment of damage tolerance demands for numerical simulations capable of predicting also the propagation of cracks, thus including nonlinear effects related to the material response. Meaningful examples illustrating the relevance of nonlinear phenomena in structural problems can be found, e.g., in the works of Refs. [6,7], while qualitative aspects on branching of cracks are reported in Ref. [8]. Due to the inherent complexity of responses involving both geometrical and material nonlinearities, the finite element approach is often the most straightforward one for performing the analysis. In this context, many numerical techniques were developed in the years for handling the peculiar aspects that characterize the response of structures in the nonlinear field. Arc-length strategies have been widely used for solving problems characterized by the presence of limit points. The presence of the load parameter as additional unknown of the problem allows the load to be increased or decreased throughout the iterative process. Pioneering work in this field is due to Riks [9] and Wemper [10]. Noteworthy are the successive efforts to develop the updated normal path method by Ramm and the spherical arc-length by Crisfield [11]. Despite the efficiency in capturing the elastic response of the structure, classical arc-length approaches can be inadequate in the presence of delamination phenomena, as the constraint equation is based on global quantities, 
while the failure process tends to involve few degrees of freedom. To overcome these limitations, different strategies were proposed based on the modification of the constraint equation to consider the local nodes involved in the delamination process [12-14] or by enriching the constraint equation with information regarding the energy dissipated during the process [15-17]. An example is found in Ref. [18], where path following techniques were discussed in the framework of Embedded Finite Element Method (E-FEM).

Another class of numerical solution techniques refers to the so-called continuation approach. These path-following techniques were originally introduced by Davidenko $[19,20]$ in the early fifties. They rely on the idea of transforming a nonlinear system of equations into a differential one. The system is parametrized with respect to a solution parameter, and is solved as an initial value problem [21-23]. The application of continuation methods to nonlinear structural analyses offers several interesting features. It allows to trace the primary equilibrium path of the structure, also in presence of snaps [24], to detect and compute the critical points [24-26] and to trace the bifurcation branches [27,28]. As a result, continuation methods enable to trace the complete bifurcation diagram of the structure, which is of particular interest for gathering deeper insight into the behavior of the structure.

While a consistent number of examples concerning the use of continuation methods for the nonlinear analysis of elastic structures are available in the literature, relatively few applications can be found for handling problems characterized by the presence of crack propagation phenomena. To the best of the authors' knowledge, no continuation methods were specifically developed for problems involving strain-softening responses.

In this article a novel continuation approach is proposed, offering the capability of handling structural responses characterized by geometrically and materially nonlinear problems. The continuation is performed referring to a modified version of the the Riks' method [24] in conjunction with a constraint regarding the dissipated energy $[15,16]$. The approach tries to combine the efficiency of geometric-based procedures in the elastic field, with that of the dissipative ones in the presence of damage. The resulting approach can accurately trace the equilibrium paths and shows excellent robustness on bifurcation branches dominated by dissipative phenomena.

The performance of the approach is demonstrated by means of five numerical examples. The first two ones illustrate the ability of the methods to compute the bifurcation diagram of simple elastic problems. Then, test cases characterized by mixed geometric-material nonlinearities are assessed. Finally, the application of the methods as quasi-static solution strategies is demonstrated in a challenging example dominated by material nonlinearities.

The paper is organized as follows. Introductory aspects on continuation methods are presented in Section 2, where the formulation of the nonlinear finite element approximation is briefly presented. The detection of critical points is discussed in Section 3 by introducing algorithmic aspects for classifying bifurcations and limit points, while the technique for implementing branch-switching is the subject of Section 4. Geometric and dissipative constraint equations are introduced in Section 5, and an overview of the method is provided in Section 6. The application of the method to a set of nonlinear problems is illustrated in Section 7, where test cases involving geometrically and materially nonlinear responses are presented.

\section{Preliminaries}

Continuation methods are a class of numerical techniques for computing the approximate solution of a system of parametrized nonlinear equations $[19,20]$. Denoting with $\tilde{\lambda}$ the quantity adopted for the parametrization, the procedure consists in constructing an approximation $x_{i}$ of $x\left(\tilde{\lambda}_{i}\right)$ for a sequence of parameter values $\tilde{\lambda}_{1}, \ldots, \tilde{\lambda}_{N}$ in the interval of definition of the parameter itself.

Within the context of the finite element approach, the nonlinear equilibrium equations of a structure can be represented, in their general form, as:

$$
\mathbf{f}_{\text {int }}(\mathbf{a})-\lambda \hat{\mathbf{f}}=0
$$


where $\mathbf{f}_{\text {int }}$ defines the vector of the internal forces, and $\hat{\mathbf{f}}$ provides the shape of the external forces. Nodal displacements are denoted as $\mathbf{a}$, while $\lambda$ is the load parameter.

The system of Equation (1) can be seen in terms of continuation techniques after noticing that the set of equations defines implicitly a curve $\mathbf{c}$ of solution points that can be continuously parametrized by means of the parameter $\tilde{\lambda}$. Starting from an initial equilibrium solution condition defined by $\left[\mathbf{a}_{0}, \lambda_{0}\right]$-which, in turn, corresponds to an initial value $\tilde{\lambda}_{0}$ of the parameter $\tilde{\lambda}$ 一the continuation problem consists in calculating the locus of solutions given by the branch $\mathbf{c}(\tilde{\lambda})$. The process is terminated when a target point is reached.

The choice of the parametrization is a focal aspect in the development of a continuation technique. This choice is, in general, not unique and, according to its definition, different methods can be obtained. One possibility is given by the adoption of the load factor $\lambda$, leading to a force-control approach. Another choice consists in considering the displacements of a subset of nodes, which leads to a displacement-based control. These two kinds of parametrization are inadequate for handling turning points, where the surfaces $\lambda=$ const and $a_{i}=$ const are tangent to the equilibrium path, and no intersections can be found. On the contrary, a more suitable approach consists in adopting a parametrization that is always nearly orthogonal to the equilibrium path, so that an intersection between the surface and the path is always ensured. This is the case of a parametrization based on the arc-length of the curve $s$ [24]. By introducing a parametrization based on $s$, the load factor $\lambda$ becomes an additional unknown and a scalar constraint equation must be added to Equation (1). The augmented system is then obtained as:

$$
\mathbf{H}=\left\{\begin{array}{l}
\mathbf{f}_{\text {int }}(\mathbf{a})-\lambda \hat{\mathbf{f}}=0 \\
g(\mathbf{a}, \lambda, s)=0
\end{array}\right.
$$

where $g(\mathbf{a}, \lambda, s)$ is the general expression of the additional constraint equation.

Starting from the initial solution $\left[\mathbf{a}_{0}, \lambda_{0}\right]$, the equilibrium path $\mathbf{c}(s)$ can be regarded as the solution of the initial value problem obtained by differentiating the augmented system in Equation (2) with respect to $s$ :

$$
\frac{\mathrm{d} \mathbf{H}(\mathbf{c})}{\mathrm{d} \mathbf{c}} \mathbf{c}^{\prime}=0, \quad\left\|\mathbf{c}^{\prime}\right\|=1, \quad \mathbf{c}(0)=\left[\mathbf{a}_{0}, \lambda_{0}\right]
$$

where $(\cdot)^{\prime}$ denotes the derivative with respect to the path parameter $s$.

The solution of Equation (3) can be achieved by using predictor-corrector techniques: the differential problem is initially integrated coarsely, then an iterative method is used as a stabilizer for solving locally $\mathbf{H}(\mathbf{c}(s))=0$.

It is worth observing that predictor-corrector continuation methods are considerably different if compared to the predictor-corrector techniques for the numerical integration of initial value problems. In fact, despite the similarity between the two strategies, the corrector process in the continuation methods thrives on the powerful contractive properties of the solution set $\mathbf{H}^{-1}(0)$ for iterative methods such as Newton's method [21]. This property is not valid for the solution curves of general initial value problems, where the corrector process converges, in the limit, to an approximating point, whose quality depends on the size of the step.

One can observe that the system of Equation (3) involves the presence of derivatives with respect to the parameter $s$, which is one of the main differences between arc-length and continuation methods. This is an essential feature of continuation methods and allows to easily detect critical points along the equilibrium path and to trace bifurcation branches. The main drawback is given by the need for solving a linear system for computing the derivatives, and to perform an eigenvalue decomposition for detecting critical points.

With this regard, the development of a continuation algorithm capable of tracing the complete bifurcation diagram relies on the definition of: 
- a method for detecting and computing critical points, and capable of distinguishing between limit and bifurcation points

- a method for moving from the primary path to a bifurcational branch

- a strategy to follow the equilibrium path along the solution branches

Different continuation methods can be realized by referring to different parametrizations and constraint equations. The performance of the method can be modified by adopting different strategies to perform the prediction or the correction. In addition, a consistent number of procedures can be adopted to perform branch-switching or detect critical points. As result, it is possible to create a wide range of methods characterized by different performance.

In the implementation proposed here, critical points are detected looking at the positive eigenvalues of the stiffness matrix, while their classification is based on the analysis of the stiffness parameter; the eigenvector injection method is adopted for switching from the primary to the bifurcational path, while a novel energy-based path-following technique is proposed for performing the continuation.

\section{Critical Points}

In this section, the procedure for detecting and approximating the critical points is described. Critical points are equilibrium points corresponding to the singularity of the tangent stiffness matrix $\mathbf{K}$. According to the theory of stability of conservative systems, the stability of an equilibrium configuration is ensured by the positive definiteness of the quadratic form $\mathcal{Q}$, defined as:

$$
\mathcal{Q}(\mathbf{a})=\frac{1}{2} \mathbf{a}^{T} \mathbf{K a}
$$

where $\mathbf{K}$ is the tangent stiffness matrix. The transition state between a stable and an unstable equilibrium configuration is characterized by the appearance of a singularity in the matrix $\mathbf{K}$. It follows that critical points define the limits of stability of the equilibrium path. The $k$-th critical point can be defined by means of the following generalized eigenvalue problem:

$$
\left(\mathbf{K}-\omega_{k} \mathbf{I}\right) \mathbf{v}_{k}=0
$$

where $\omega_{k}$ and $\mathbf{v}_{k}$ are the $k$-th eigenvalue and eigenvector of the stiffness matrix, respectively, whereas I is the identity matrix.

The eigenvalues $\omega_{k}$ are supposed to be arranged according to the sequence

$$
\omega_{1} \leq \omega_{2} \leq \cdots \leq \omega_{N}
$$

where $N$ is the dimension of the stiffness matrix. When the critical state is reached, one or more eigenvalues $\omega_{k}$ in the sequence of Equation (6) are zero.

Critical points can be divided into limit and bifurcation points. In particular the following two conditions are considered:

$$
\begin{cases}\lambda^{\prime}=0, & \text { Limit Point } \\ \hat{\mathbf{f}}^{T} \mathbf{v}_{k}=0, & \text { Bifurcation Point }\end{cases}
$$

The first condition of Equation (7) states that the load does not vary with respect to the path parameter in the neighborhood of a limit point. This also means that limit points are equilibrium configurations associated with a local maximum or minimum of the applied load. In most cases, limit points separate stable and unstable equilibrium paths, although this is not a rule. The presence of a limit point is commonly associated with snap-through phenomena, causing sudden snaps to a stable configuration and not adjacent to the original one. In the neighborhood of limit points there exists one unique branch, thus no further equilibrium branches need to be traced. 
On the contrary, multiple equilibrium configurations exist in the neighbourhood of a bifurcation point, each of these solutions belonging to a different equilibrium branch and characterized by a different tangent. During the solution process, it is then necessary to distinguish between limit and bifurcation points to establish whether the continuation should be carried out along the emanating branches or not. For this purpose, a procedure based on the evaluation of the stiffness parameter $S_{p}$ is adopted.

\subsection{Stiffness Parameter}

The stiffness parameter is a scalar quantity defined as [26]:

$$
S_{p}=\frac{\left.\frac{\mathrm{d} \mathbf{a}}{\mathrm{d} \lambda}\right|_{0} ^{T} \hat{\mathbf{f}}}{\frac{\mathrm{d} \mathbf{a}}{\mathrm{d} \lambda} \hat{\mathbf{f}}}
$$

where the subscript 0 defines the initial state.

The value of $S_{p}$ provides a relative measure of the incremental stiffness along the direction in which the solution is moving. During the incremental solution process, this parameter is computed through substitution of the increments into the derivatives, leading to the incremental expression:

$$
S_{p}=\frac{\frac{1}{\Delta \lambda_{0}} \Delta \mathbf{a}_{0}^{T} \hat{\mathbf{f}}}{\frac{1}{\Delta \lambda_{j}} \Delta \mathbf{a}_{j}^{T} \hat{\mathbf{f}}}=\frac{\Delta \lambda_{j}}{\Delta \lambda_{0}} \frac{\Delta \mathbf{a}_{0}^{T} \hat{\mathbf{f}}}{\Delta \mathbf{a}_{j}^{T} \hat{\mathbf{f}}}
$$

where $\Delta \mathbf{a}_{0}$ and $\Delta \lambda_{0}$ are the increments computed in the first load step, and $\Delta \mathbf{a}_{j}$ and $\Delta \lambda_{j}$ are the total increments computed at the current load step. As seen from Equation (9), the initial value of $S_{p}$ is equal to one. Whenever the structure loses stiffness with respect to the initial value, viz. $\Delta \mathbf{a}_{j}^{T} \hat{\mathbf{f}}>\Delta \mathbf{a}_{0}^{T} \hat{\mathbf{f}}$ and $\Delta \lambda_{j}<\Delta \lambda_{0}$, the parameter $S_{p}$ becomes smaller than one. On the other hand, if the structure undergoes a stiffening response, i.e., $\Delta \mathbf{a}_{j}^{T} \hat{\mathbf{f}}<\Delta \mathbf{a}_{0}^{T} \hat{\mathbf{f}}$ and $\Delta \lambda_{j}>\Delta \lambda_{0}, S_{p}$ is greater than the unity. Furthermore, the stiffness parameter is positive on stable equilibrium branches and negative on unstable branches. As a matter of fact, $S_{p}$ turns out to be negative for unstable branches because, in such cases, the increment of the load parameter is negative.

An important feature of the stiffness parameter is that, in correspondence of the limit points, it satisfies the condition:

$$
S_{p}=0
$$

as implicit in the definition of limit point itself. On the other hand, the relative stiffness is different from zero in the presence of a bifurcation point and, after crossing the point, the sign of $S_{p}$ does not change.

It is worth observing that the condition of Equation (10) is hardly met during the numerical solution of the problem, as the solution is computed for a discrete set of points only. For this reason, the detection of the limit point is performed referring to a numerical approach, described in the next section, for checking the condition given by Equation (10).

\subsection{Detection and Classification}

Among the various strategies for the computation of critical points [23,25], the approach proposed here is based on the analysis of the sign of the eigenvalues of the tangent stiffness matrix. This approach is simple and robust, and is associated with a relatively small computational effort. It is preliminarly assumed that the eigenvalues of $\mathbf{K}$ are well separated, which implies that multiple or clustered bifurcations cannot be handled.

The tangent stiffness matrix $\mathbf{K}$ of a structure, evaluated in its initial, undeformed state, is a positive definite quantity. When a critical point is reached, one eigenvalue, at least, becomes null and, just after crossing the point, the eigenvalue becomes negative. Based on this consideration, it is straightforward to implement the condition:

$$
\operatorname{sign}\left(\omega_{k}\left(s_{i}\right)\right) \neq \operatorname{sign}\left(\omega_{k}\left(s_{i-1}\right)\right)
$$


The critical points subsequent to the first one are captured by monitoring the sign of the lowest positive eigenvalue. In particular, the following condition is employed:

$$
N_{\text {pos }}\left(s_{i}\right)<N_{\text {pos }}\left(s_{i-1}\right)
$$

where $N_{p o s}$ is the number of positive eigenvalues of the tangent stiffness matrix $\mathbf{K}$.

This strategy is particularly simple as the counting of the positive eigenvalues of $\mathbf{K}$ is just needed.

Once a critical point is detected, the classification between limit and bifurcation points relies upon the valuation of the stiffness parameter (see Equation (10) as outlined in the pseudo-code reported in Algorithm 1.

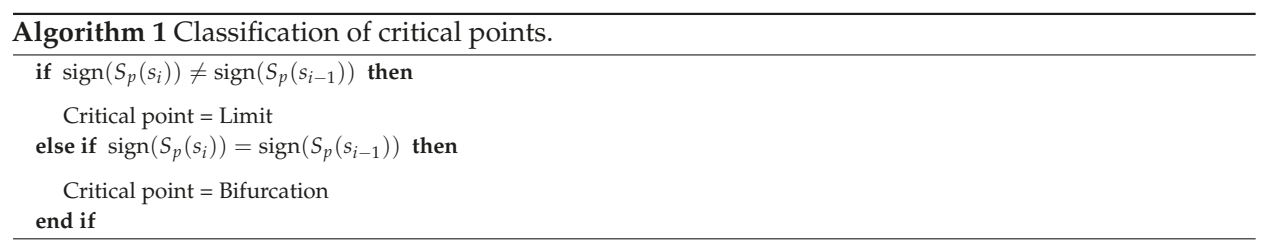

Note, $s_{i-1}$ and $s_{i}$ are the values of the path parameter $s$ just before and just after the critical point, respectively. The criterion summarized in Algorithm 1 is based on the fact that the stiffness parameter undergoes a change of sign whenever a limit point is crossed. Hence, the critical point is a limit point if a change of sign is detected in $S_{p}$, otherwise it is a bifurcation point.

\subsection{Approximation}

Once critical points are identified and classified, an approximation of the critical state is needed. In continuation methods, bifurcation points are used as initial solutions for tracing bifurcation branches. It follows that the quality of the prediction affects the convergence of the solution along the bifurcation branches. An accurate prediction is then sought. On the other hand, the requirements on the accuracy of the limit point predictions are less strict, as no equilibrium branches emanate from them.

Based on these observations, limit points are determined by means of a linear approximation seeking for the zero of $S_{p}(s)$. Given the quantities $S_{p}\left(s_{i-1}\right), S_{p}\left(s_{i}\right)$, the linear approximation reads:

$$
\begin{aligned}
S_{p}(s) & =S_{p}\left(s_{i-1}\right)+\frac{S_{p}\left(s_{i}\right)-S_{p}\left(s_{i-1}\right)}{\Delta \lambda}\left[\lambda(s)-\lambda\left(s_{i-1}\right)\right] \\
\mathbf{a}(s) & =\mathbf{a}\left(s_{i-1}\right)+\frac{\Delta \mathbf{a}}{\Delta \lambda}\left[\lambda(s)-\lambda\left(s_{i-1}\right)\right]
\end{aligned}
$$

where $s_{i-1}$ and $s_{i}$ are the values of the path parameter in the converged equilibrium points before and after the critical point, respectively, and:

$$
\begin{aligned}
\Delta \lambda & =\lambda\left(s_{i}\right)-\lambda\left(s_{i-1}\right) \\
\Delta \mathbf{a} & =\mathbf{a}\left(s_{i}\right)-\mathbf{a}\left(s_{i-1}\right)
\end{aligned}
$$

Setting $S_{p}(s)=0$ in Equation (13), and denoting with an asterisk the critical state, the critical load $\lambda\left(s^{*}\right)=\lambda^{*}$ is obtained as:

$$
\lambda^{*}=\lambda\left(s_{i-1}\right)-\frac{\Delta \lambda}{S_{p}\left(s_{i}\right)-S_{p}\left(s_{i-1}\right)} S_{p}\left(s_{i-1}\right)
$$


while the deformed configuration $\mathbf{a}\left(s^{*}\right)=\mathbf{a}^{*}$ is obtained after substituting Equation (15) into the second of Equation (13), and is obtained as:

$$
\mathbf{a}^{*}=\mathbf{a}\left(s_{i-1}\right)+\frac{\Delta \mathbf{a}}{\Delta \lambda}\left(\lambda^{*}-\lambda\left(s_{i-1}\right)\right)
$$

This strategy is fast and tends to guarantee satisfactory precision for obtaining an estimate of the limit points.

Regarding the approximation of the bifurcation points, an iterative procedure is implemented to achieve improved accuracy. It is based on the polynomial approximation of the critical point $\tilde{\mathbf{x}}\left(s^{*}\right)$, and seeks the zero of $\omega_{k}(s)$. In turn, the polynomial approximation is obtained through a Taylor expansion of $\omega_{k}(s)$ as:

$$
\omega_{k}(s)=\omega_{k}\left(s_{i}\right)+\omega_{k}^{\prime}\left(s_{i}\right)\left(s-s_{i}\right)
$$

where $s_{i}$ corresponds to the value of the path parameter at the converged equilibrium solution before the critical point.

A first estimate of the critical state $\tilde{\mathbf{x}}^{*}=\tilde{\mathbf{x}}\left(s^{*}\right)$, relative to the point $\tilde{\mathbf{x}}\left(s_{i}\right)$, is given by:

$$
\tilde{\mathbf{x}}^{*}=\tilde{\mathbf{x}}\left(s_{i}\right)+\tilde{\mathbf{x}}^{\prime}\left(s_{i}\right) \Delta s^{*}
$$

where the vector of the unknowns $\tilde{\mathbf{x}}$ and its derivative $\tilde{\mathbf{x}}^{\prime}$ are defined as:

$$
\tilde{\mathbf{x}}=\left\{\begin{array}{ll}
\mathbf{a}^{\mathrm{T}} & \lambda
\end{array}\right\}^{\mathrm{T}} \quad \tilde{\mathbf{x}}^{\prime}=\left\{\begin{array}{ll}
\mathbf{a}^{\mathrm{T}} & \lambda^{\prime}
\end{array}\right\}^{\mathrm{T}}
$$

and:

$$
\Delta s^{*}=s^{*}-s_{i}=-\frac{\omega_{k}\left(s_{i}\right)}{\omega_{k}^{\prime}\left(s_{i}\right)}
$$

and:

$$
\omega^{\prime}\left(s_{i}\right) \cong \frac{\omega\left(s_{i}\right)-\omega\left(s_{i-1}\right)}{s_{i}-s_{i-1}}
$$

Improved robustness can be achieved by modifying Equation (18) as:

$$
\tilde{\mathbf{x}}\left(s_{i+1}\right)=\tilde{\mathbf{x}}\left(s_{i}\right)+\eta \Delta s^{*} \tilde{\mathbf{x}}^{\prime}\left(s_{i}\right)
$$

where $\eta$ is a scale factor, chosen while keeping in mind that small values are associated with high accuracy, but high costs, while the opposite holds for high values of $\eta$. In the present study, a proper tradeoff between quality and computational costs was found by taking $\eta$ equal to 0.1 .

A new value of $\omega_{k}$, corresponding to the point $\tilde{\mathbf{x}}\left(s_{i+1}\right)$, can be computed and, according to Equations (18) and (20), a new estimate of $\tilde{\mathbf{x}}^{*}$ and $\Delta s^{*}$ is also available. The procedure is repeated until convergence is met according to the criterion:

$$
\left|\frac{\Delta s^{*}}{\Delta s_{0}}\right| \leq \text { tol }_{\Delta s}
$$

where $\Delta s_{0}$ is the step length adopted to start the analysis, where tol ${ }_{\Delta s}$ is the tolerance defined by the user. Based on an extensive set of preliminary investigations, a value of $\operatorname{tol}_{\Delta s}=5 \times 10^{-3}$ is set by default in the algorithms proposed in this work. To prevent excessively long loops, the criterion of Equation (23) is accompanied by a check on the maximum number of iterations.

\section{Branch-Switching Method}

After detecting the critical points, a numerical strategy is needed to compute the initial solution, which is then used to start the continuation procedure along the bifurcation branches. An extensive review of branch-switching methods is available in [25]. In the present method, the eigenvector 
injection method is adopted. It relies on the idea of perturbing the configuration at the critical state by using the eigenvector $\mathbf{v}_{k}$ associated with the eigenvalue $\omega_{k}\left(s^{*}\right)=0$ [27].

The perturbation is introduced according to:

$$
\mathbf{a}_{0}=\mathbf{a}^{*}+\xi \frac{\mathbf{v}_{k}}{\left\|\mathbf{v}_{k}\right\|}
$$

where $\xi$ is a scale factor defined as:

$$
\xi= \pm \frac{\left\|\mathbf{a}^{*}\right\|}{\tilde{\tau}}
$$

and $\tilde{\tau}$ is a scalar variable prescribed by the user and referred to as buckling mode scale factor, while the sign of $\xi$ determines the side of the bifurcation branch to be traced.

The initial solution is completed by:

$$
\lambda_{0}=\lambda^{*}
$$

The tangent vector in $\left[\mathbf{a}_{0}, \lambda_{0}\right]$ is approximated as:

$$
\begin{aligned}
& \mathbf{a}_{0}^{\prime}=\operatorname{sign}(\xi) \frac{\mathbf{v}_{k}}{\left\|\mathbf{v}_{\mathbf{k}}\right\|} \\
& \lambda_{0}^{\prime}=0
\end{aligned}
$$

After computing the initial solution, it is possible to start the continuation process, and the equilibrium points along the branch are so identified.

It is highlighted that a proper choice of $\tilde{\tau}$ is needed for guaranteeing convergence on the desired branch. If this value is too small, the eigenvector injection method is likely to diverge, whereas a too large value may determine a perturbation size incapable of deviating the solution on the bifurcation branch, and the continuation process is likely to converge on the primary path again [27].

Usually, suitable values of $\tilde{\tau}$ are in the range between 10 and 100. However, no general rules exist for properly selecting the value of $\tilde{\tau}$, and a trial and error strategy is, generally, the only viable solution.

To deal with this problem, it is necessary that the branch-switching algorithm allows the restart of the analysis of the bifurcation branch with a different value of $\tilde{\tau}$.

\section{Path-Following Continuation Technique}

In most cases, continuation techniques are developed by adopting a geometric constraint in Equation (2), an example of which is given by the Riks continuation technique [24]. This kind of constraint is well-suited for those problems dominated by nonlinearities of geometric type. On the contrary, the adoption of a geometric constraint can be source of convergence difficulties whenever the nonlinearities are mainly due to the material response that would lead to localized failure [16]. For instance, this is the case of delamination phenomena, where the strain field is localized in the surroundings of the crack tip. It follows that geometric constraints based on global quantities can be inadequate to capture the deformation process.

Starting from a recent arc-length technique proposed by the authors [17], the continuation technique proposed here tries to overcome the above mentioned difficulties by adopting a modified version of the Riks technique in conjunction with an approach based on the evaluation of the dissipated energy, as outlined in the next sections.

\subsection{Modified Riks Method}

The modified Riks continuation method is a modified version of the technique described in [24] and relies on a constraint equation involving the displacement vector $\mathbf{a}$ and its derivative $\mathbf{a}^{\prime}$.

As opposed to original implementation proposed by Riks, the equation is adopted here in a modified form, where the contribution due to the load parameter is prevented from entering the equation. In particular, the constraint equation is formulated as: 


$$
g(\mathbf{a}, s)=\mathbf{a}_{1}^{\prime}\left(\mathbf{a}-\mathbf{a}_{1}\right)-\left(s-s_{1}\right)=0
$$

where $\mathbf{a}_{1}$ denotes an equilibrium point on the path, while $s_{1}$ is the value of the arc-length at this point; the terms a defines an arbitrary point on the surface defined by the constraint equation.

An extensive set of preliminary analyses revealed that Equation (29) offers the main advantage of preventing the doubling back of the solution, a problem often encountered when adopting the classical Riks formulation.

The continuation procedure is performed referring to a predictor-corrector technique, where the increments $\Delta \mathbf{a}$ and $\Delta \lambda$ are decomposed as:

$$
\Delta \mathbf{a}=\Delta \mathbf{a}_{j}+\mathrm{d} \mathbf{a}, \quad \Delta \lambda=\Delta \lambda_{j}+\mathrm{d} \lambda
$$

where $\Delta \mathbf{a}$ and $\Delta \lambda$ are the total increments at the current step, $\Delta \mathbf{a}_{j}$ and $\Delta \lambda_{j}$ are the iterative increments at the last iteration $j$, and da and $\mathrm{d} \lambda$ are the iterative increments referred to the current $(j+1)$ iteration.

The predictor solution is computed according to:

$$
\Delta \mathbf{a}_{0}=\mathbf{a}_{1}+\mathbf{a}_{1}^{\prime} \Delta s \quad \Delta \lambda_{0}=\lambda_{1}+\lambda_{1}^{\prime} \Delta s
$$

The corrector phase is performed referring to the Newton's method, which is applied to solve the augmented system of Equation (2) using Equation (29) as constraint, so:

$$
\left\{\begin{array}{l}
\mathbf{K d} \mathbf{a}-\hat{\mathbf{f}} \mathrm{d} \lambda=\mathbf{r} \\
\mathbf{a}_{1}^{\prime} \Delta \mathbf{a}=\Delta s
\end{array}\right.
$$

where $\mathbf{r}$ is the residual at the end of the $j$-th iteration, defined as:

$$
\mathbf{r}=\Delta \lambda_{j} \hat{\mathbf{f}}-\mathbf{f}_{i n t}\left(\Delta \mathbf{a}_{j}, \Delta \lambda_{j}\right)
$$

Substituting Equation (30) in Equation (32), the iterative increments da and $\mathrm{d} \lambda$ are obtained as:

$$
\begin{aligned}
& \mathrm{d} \mathbf{a}=\mathbf{a}_{I} \mathrm{~d} \lambda+\mathbf{a}_{I I} \\
& \mathrm{~d} \lambda=-\frac{\mathbf{a}_{1}^{\prime T} \mathbf{a}_{I I}}{\mathbf{a}_{1}^{\prime T} \mathbf{a}_{I}}
\end{aligned}
$$

having assumed that $\Delta s \approx \mathbf{a}_{1}^{\prime} \Delta \mathbf{a}_{j}$, and

$$
\mathbf{a}_{I}=\mathbf{K}^{-1} \hat{\mathbf{f}} \quad \mathbf{a}_{I I}=\mathbf{K}^{-1} \mathbf{r}
$$

It is worth noting that Equation (34)b resembles the expression of $\mathrm{d} \lambda$ of the Riks arc-length method [24]. However, the two equations differ in the term $\mathbf{a}_{1}^{\prime}$ of Equation (34)b, viz., the derivative of the displacement vector in the last converged equilibrium point, which is replaced by the displacement increment of the predictor step $\Delta \mathbf{a}_{1}$ in the Riks formulation.

To gather insight into the constraint equation developed here, it is possible to compare Equations (34)b and (29), and re-write the constraint as:

$$
\mathbf{a}_{1}^{\prime \mathrm{T}} \mathrm{d} \mathbf{a}=0
$$

The expression of Equation (36) defines a hyperplane in the subspace of displacements, which is normal to the vector $\mathbf{a}_{1}^{\prime}$. In addition, Equation (36) can be thought as the projection of the Riks constraint equation onto the subspace of displacements. 
After reaching convergence, the tangent vector $\tilde{\mathbf{x}}^{\prime}=\left[\mathbf{a}^{\prime}, \lambda^{\prime}\right]$ is computed by solving the linear system obtained from the differentiation of Equation (32) with respect to $s$. The linear system is derived as:

$$
\left\{\begin{array}{l}
\mathbf{K} \mathbf{a}^{\prime}-\hat{\mathbf{f}} \lambda^{\prime}=0 \\
\mathbf{a}_{1}^{\prime T} \mathbf{a}^{\prime}-1=0
\end{array}\right.
$$

The vector $\left[\mathbf{a}^{\prime}, \lambda^{\prime}\right]$ defines the direction of the tangent vector, but not its orientation [22]. To this aim the scalar $\beta$ is introduced as:

$$
\beta=\frac{\Delta \mathbf{a}^{\mathrm{T}} \mathbf{a}^{\prime}}{\left\|\Delta \mathbf{a}^{\mathrm{T}} \mathbf{a}^{\prime}\right\|}
$$

On the basis of the sign assumed by $\beta$, the orientation is defined by reversing $\tilde{\mathbf{x}}^{\prime}$ when $\beta<0$. This criterion imposes that the tangent vector points towards the direction that forms an angle smaller than $90^{\circ}$ with respect to the last computed displacement increment $\Delta \mathbf{a}$.

\subsection{Dissipated Energy Method}

The adoption of a purely geometric constraint equation can be inadequate for analyzing those probelms characterized by material nonlinearities, especially in the presence of strain-softening responses. To overcome these difficulties, a second constraint, to be used in conjunction with the previous geometric one, is introduced.

The method makes use of the energy release rate $G$. In the context of the finite element approximation, it is obtained as [16]:

$$
G=\frac{1}{2}\left(\lambda \dot{\mathbf{a}}^{\mathrm{T}}-\dot{\lambda} \mathbf{a}^{\mathrm{T}}\right) \hat{\mathbf{f}}
$$

where the symbol () denotes differentation with respect to time.

Applying a forward Euler approximation of Equation (39), the expression of the energy dissipated in the current step, $\Delta \tau$, is obtained as:

$$
\Delta \tau=\left(\tau-\tau_{1}\right)=\frac{1}{2}\left(\lambda_{1} \Delta \mathbf{a}^{\mathrm{T}}-\Delta \lambda \mathbf{a}_{1}^{\mathrm{T}}\right) \hat{\mathbf{f}}
$$

where $\tau$ is the total dissipated energy and $\tau_{1}$ the amount of energy dissipated up to the last converged equilibrium condition. From inspection of Equation (40), a constraint equation for the continuation method can be derived as:

$$
g(\Delta \mathbf{a}, \Delta \lambda)=\frac{1}{2}\left(\lambda_{1} \Delta \mathbf{a}^{\mathrm{T}}-\Delta \lambda \mathbf{a}_{1}^{\mathrm{T}}\right) \hat{\mathbf{f}}-\left(\tau-\tau_{1}\right)=0
$$

where the parametrization is now performed with respect to the parameter $\tau$, i.e., the dissipated energy, instead of the arc-length $s$. More specifically, the constraint specified by Equation (41) imposes that the energy dissipated in the current load step is equal to the amount $\left(\tau-\tau_{1}\right)$.

As done for the modified Riks continuation, a predictor-corrector method is employed for solving the governing equations. However, derivatives are now taken with respect to the parameter $\tau$. The predictor step is then:

$$
\Delta \mathbf{a}_{0}=\mathbf{a}_{1}+\mathbf{a}_{1}^{\prime} \Delta \tau \quad \Delta \lambda_{0}=\lambda_{1}+\tau \lambda_{1}^{\prime} \Delta \tau
$$

while the corrector phase is based upon the Newton solution of Equation (2) together with Equation (41), leading to the linearized system:

$$
\left\{\begin{array}{l}
\mathbf{K d} \mathbf{a}-\hat{\mathbf{f}} \mathrm{d} \lambda=\mathbf{r} \\
\frac{1}{2} \lambda_{1} \Delta \mathbf{a}-\frac{1}{2} \mathbf{a}_{1}^{\mathrm{T}} \Delta \lambda=\Delta \tau
\end{array}\right.
$$


By substituting Equation (30) into Equation (43), the system can be represented in matrix form as:

$$
\left[\begin{array}{cc}
\mathbf{K} & -\hat{\mathbf{f}} \\
\mathbf{h}^{\mathrm{T}} & w
\end{array}\right]\left\{\begin{array}{l}
\mathrm{d} \mathbf{a} \\
\mathrm{d} \lambda
\end{array}\right\}=\left\{\begin{array}{c}
\mathbf{r} \\
-g_{1}
\end{array}\right\}
$$

where:

$$
\mathbf{h}^{\mathrm{T}}=\frac{\partial g}{\partial \mathbf{a}} \quad w=\frac{\partial g}{\partial \lambda}
$$

and:

$$
g_{1}=g\left(\Delta \mathbf{a}_{1}, \Delta \lambda_{1}\right)
$$

The iterative increments $\mathrm{da}$ and $\mathrm{d} \lambda$ are computed from Equation (44) applying the Sherman-Morrison formula [16]):

$$
\left\{\begin{array}{l}
\mathrm{d} \mathbf{a} \\
\mathrm{d} \lambda
\end{array}\right\}=\left\{\begin{array}{c}
\mathbf{d}_{I} \\
-g_{1}
\end{array}\right\}-\frac{1}{\mathbf{h}^{\mathrm{T}} \mathbf{d}_{I I}-w}\left\{\begin{array}{c}
\left(\mathbf{h}^{\mathrm{T}} \mathbf{d}_{I}+g_{1}\right) \mathbf{d}_{I I} \\
-\mathbf{h}^{\mathrm{T}} \mathbf{d}_{I}-g_{1}\left(1+\mathbf{h}^{\mathrm{T}} \mathbf{d}_{I I}-w\right)
\end{array}\right\}
$$

where the terms $\mathbf{d}_{I}$ and $\mathbf{d}_{I I}$ are the solutions of the following linear systems:

$$
\mathbf{K d}_{I}=\mathbf{r}, \quad \mathbf{K d}_{I I}=-\hat{\mathbf{f}}
$$

After reaching convergence, the tangent vector $\left[\mathbf{a}^{\prime}, \lambda^{\prime}\right]$ is computed through the solution of the linear system obtained by taking the derivative with respect to $\tau$ of Equation (43):

$$
\left\{\begin{array}{l}
\mathbf{K} \mathbf{a}^{\prime}-\hat{\mathbf{f}} \lambda^{\prime}=0 \\
\frac{1}{2} \lambda_{1} \mathbf{a}^{\prime} \mathrm{T} \hat{\mathbf{f}}-\frac{1}{2} \lambda^{\prime} \mathbf{a}^{\mathrm{T}} \hat{\mathbf{f}}-1=0
\end{array}\right.
$$

As observed in the previous section, the orientation of the tangent vector is not available from the solution of Equation (49), and the evaluation of the parameter $\beta$ of Equation (38) is thus performed.

\subsection{Switching from Geometric to Dissipation-Based Constraints}

Aiming at combining the advantages (and ranges of employment) of the techniques previously outlined, a single continuation approach is developed based on the geometric and dissipation strategies. As a matter of fact, the strategy based on the dissipated energy cannot be adopted in the context of purely elastic conditions, as it would fail to converge to the equilibrium solution. Similarly, convergence issues could arise when the modified Riks approach is adopted in the presence of damage phenomena. For this reason, the two approaches are combined referring to amount of energy dissipated. In particular, two threshold values are defined, namely $\Delta \tau_{\text {switch }}$ and $\Delta \tau_{\text {sback }}$.

The analysis is started by adopting the modified Riks continuation method to trace the first load steps, which are always elastic, provided their size is chosen to be sufficiently small. When the energy dissipated in the current step exceeds $\Delta \tau_{\text {switch }}$ the dissipative continuation technique is activated. Then, as soon as the energy dissipated during the current step falls below $\Delta \tau_{\text {sback, }}$ the solution strategy is switched back to the modified Riks continuation. In absence of specific motivations, the two thresholds can be generally be taken equal each other. The approach is summarized in Algorithm 2.

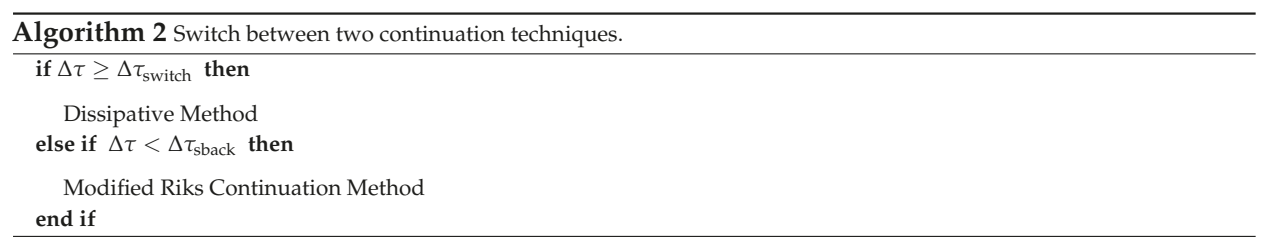


It is remarked that the adoption of the two mentioned strategies determines the presence of a double parametrization, as the modified Riks continuation method is parametrized with respect to the arc-length $s$, and the dissipated energy constraint equation relies on the parameter $\tau$. This peculiarity may be source of difficulties whenever a critical point is detected between two equilibrium points computed by means of different strategies (e.g., one adopting the modified Riks continuation method and the other adopting the dissipative procedure). This aspect has to be carefully monitored during the solution process, and possible critical points so obtained should be recomputed.

\section{Implementation}

To clarify the various steps involved in the solution process, an overview of the method is provided in the flowchart of Figure 1. The relevant equations adopted at each steps are reported by observing that they vary according to the continuation strategy currently active - this can be either the modified Riks or the dissipation-based method.

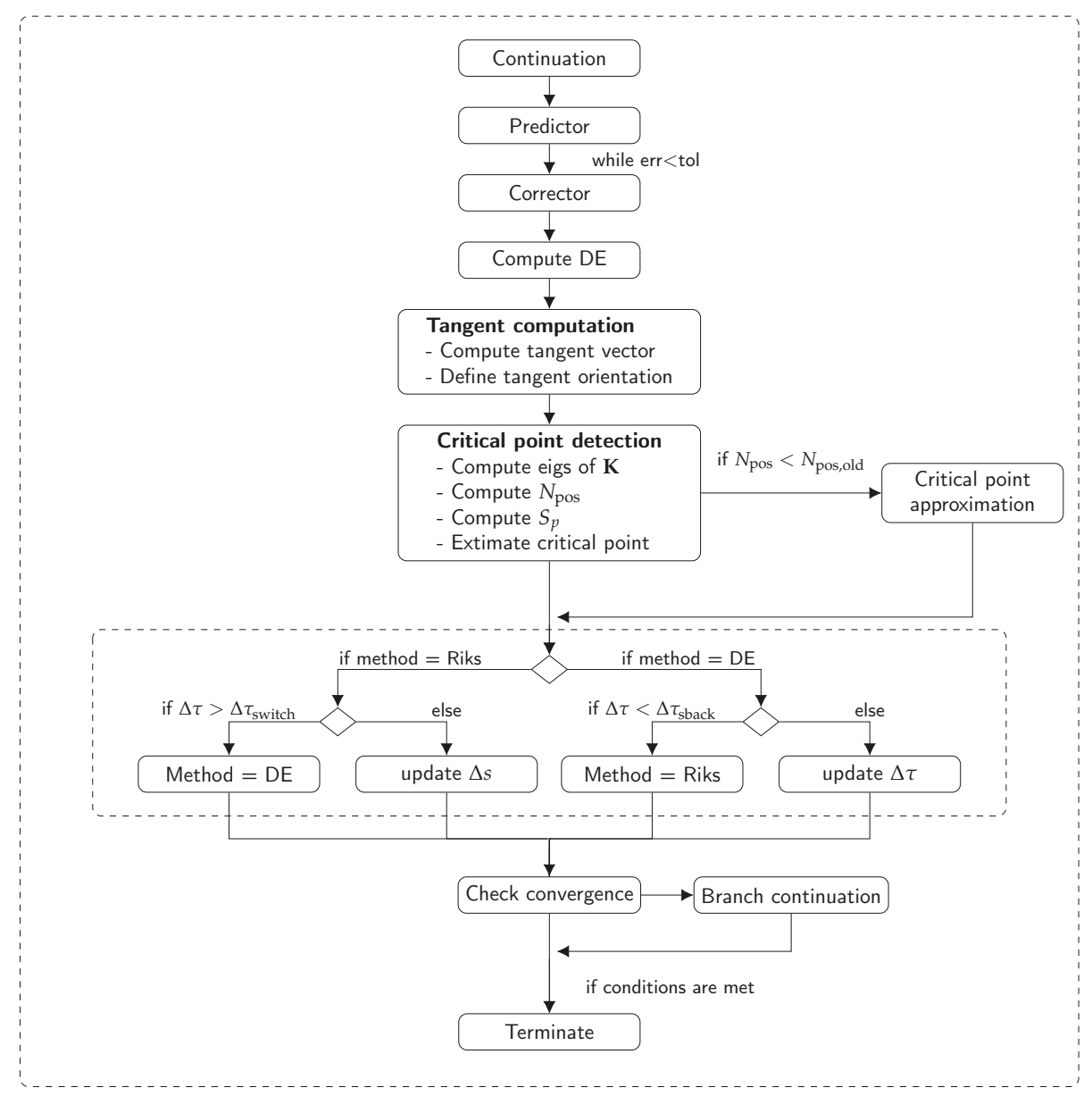

Figure 1. Flowchart of the continuation procedure.

At the beginning of the procedure, the modified Riks method is set by default, so all the equations reported henceforth are those relative to the Riks approach during the first loop. The initial predictor 
step is performed on the basis of Equation (31) or (31), while Newton-Raphson iterations are repeated during the corrector phase referring to Equation (37) or (43). The procedure is arrested when the error is below the threshold defined by the tolerance. The successive step consists in the evaluation of the dissipated energy, which is used subsequently to define the continuation algorithm. The evaluation of the tangent vector requires the solution of the linear systems according to Equation (37) or (49), while the parameter $\beta$ of Equation (38) is adopted for defining the orientation.

The successive phase consists in detecting the critical points, which is performed by evaluating the eigenvalues of the stiffness matrix $\mathbf{K}$. Depending on the number of positive eigenvectors and the value of the stiffness parameter, it can be established whether a critical point is obtained, and its nature, i.e., bifurcation or limit. After detecting a critical point, the approximation procedure is activated following the procedure outlined in Section 3.3.

The method to be used in the subsequent step is defined by comparing the dissipated energy $\Delta \tau$ with the threshold values defined by the user. In the presence of dissipation phenomena, the method is set as DE, otherwise the Riks continuation is maintained. Note that the switch between the two strategies is controlled by the algorithm outlined in Algorithm 2.

The step length is finally updated following the approach presented in Ref. [17], and convergence is finally checked. Whenever one or more bifurcation points are detected throughout the iteration process, the continuation along the secondary equilibrium path is performed.

\section{Results}

In this section, the continuation strategy is applied to the analysis of problems involving both geometric or material nonlinearities. These kind of nonlinearities can be present independently or in combination. All the simulations are performed using the open source finite element code PyFem [29], that was used as underlying framework for implementing the numerical procedure. The elements used for the analyses are nonlinear four-node, plane-strain continuum elements. To model delamination phenomena, the cohesive element proposed by Camanho et al. [30] was implemented in PyFem. Mesh sizes were defined on the basis of preliminary convergence studies. In those analyses involving the use of cohesive elements, the mesh size was chosen to guarantee a proper description of the process zone, referring to the length $l_{\mathrm{p}}$ defined by [31,32]:

$$
l_{\mathrm{p}}=\gamma \frac{E G_{c}}{N^{2}}
$$

where $\gamma$ is a scalar nondimensional parameter, here taken as $0.884, E$ denotes the Young's modulus, $G_{c}$ is the fracture toughness in mode I, and $N$ is the interfacial strength.

It is noted that the ability of the method to converge to the correct solution and to successfully trace the bifurcation diagrams is strongly affected by the choice of the parameter $\tilde{\tau}$. To this aim, preliminary tests were carried out to properly choose the value of the parameter.

The section of results is organized as follows. Two preliminary test cases, an axially compressed column and a simple one-degree-of-freedom (one-DOF) problem, are discussed in Sections 7.1 and 7.2 to illustrate the application of the method to geometrically nonlinear problems. The application of the continuation approach is then presented for two problems, a beam and an arch embedding initial cracks, in Sections 7.3 and 7.4, where materially nonlinear responses are of concern. Bifurcation plots are presented for these two cases, and the robustness of the approach is illustrated against an alternative formulation based on a purely geometric constraint equation. Finally, the procedure is presented as a mean for solving quasi-static problems for a relatively complex nonlinear example, a perforated beam loaded in mode I, which is discussed in Section 7.5.

\subsection{Subcritical Bifurcation (One-DOF Problem)}

This first example deals with the nonlinear response of a simple 1-DOF problem characterized by the presence of a sub-critical bifurcation point. It follows that equilibrium branches emanating 
from the bifurcation are, in this case, unstable. The structure is composed by a rigid truss and a linear spring, as illustrated in Figure 2. The length $l$ is equal to $10 \mathrm{~mm}$, while the stiffness of the spring is $k_{\mathrm{g}}=100 \mathrm{~N} / \mathrm{mm}$. An horizontal load of intensity $P$ is applied at the free end of the rigid bar.

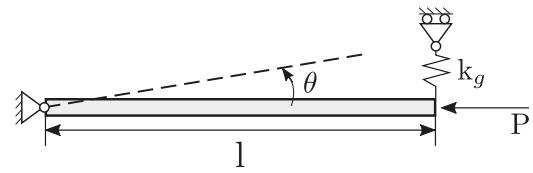

Figure 2. Sketch of the truss-spring system.

The numerical model is obtained by using a linear spring element and truss with a very high stiffness value to simulate the ideal infinite value.

The numerical predictions obtained with the proposed continuation method are compared with the analytical solution of the problem, which is given by:

$$
P(\theta)=k_{\mathrm{g}} l \cos (\theta)
$$

where $\theta$ is the rotation angle and $P$ the applied load. The results are summarized in Figure 3.

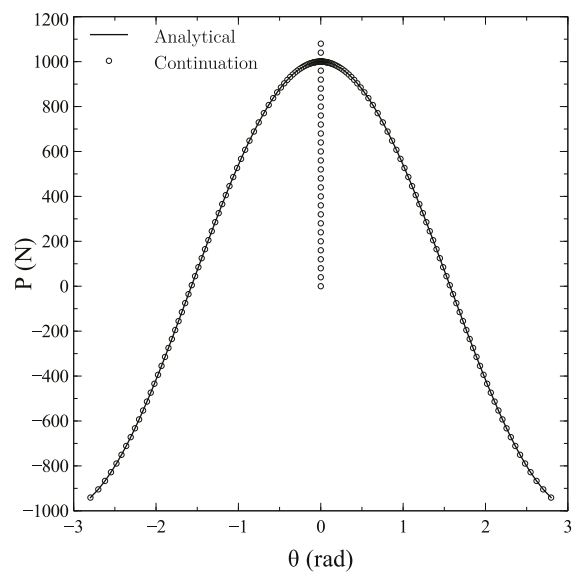

Figure 3. Bifurcation plot for the spring-truss example.

As observed, perfect matching is achieved when comparing the analytical and the numerical solution. The continuation procedure correctly identifies the overall bifurcation plot, and provides an accurate description of the three equilibrium paths emanating from the bifurcation point. Note that the solution process is carried out without activation of the dissipative continuation technique as the response is purely elastic.

\subsection{Supercritical Bifurcation (Axially Compressed Column)}

The second assessment regards the analysis of a column subjected to a compressive end load. The bifurcation is defined as supercritical as both sides of the bifurcation branch are locally stable equilibrium paths characterized by increasing values of the load factor [23]. A sketch of the structure is provided in Figure 4. The column is fixed at one end and free at the second one, while a concentrated force is applied at the free end. The overall length $l$ is equal to $10 \mathrm{~mm}$ and the thickness $h$ is $1 \mathrm{~mm}$. An isotropic homogeneous material of modulus $E=230 \mathrm{GPa}$ and $v=0.25$ is assumed. Ten finite elements are used for discretization. 


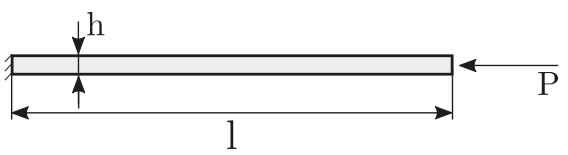

Figure 4. Cantilever beam with end compressive load.

The problem does not involve any kind of dissipative phenomena, so the modified Riks continuation approach is maintained throughout the solution process. The results are compared against those obtained by using the Crisfield arc-length approach, whose implementation in the PyFem code was already assessed in Ref. [17]. It is worth noting that the Crisfield method is not developed for tracing bifuraction diagrams in one single run. For this reason, two distinct nonlinear analyses were performed by assuming initial imperfections with opposite sign and shape equal to the first buckling mode. On the contrary, the continuation method allows to plot the bifuraction diagram in one single run, with no need for introducing initial imperfections.

The results are summarized in Figure 5, where the axial displacement of the bottom-most corner of the column is reported against the axial load.

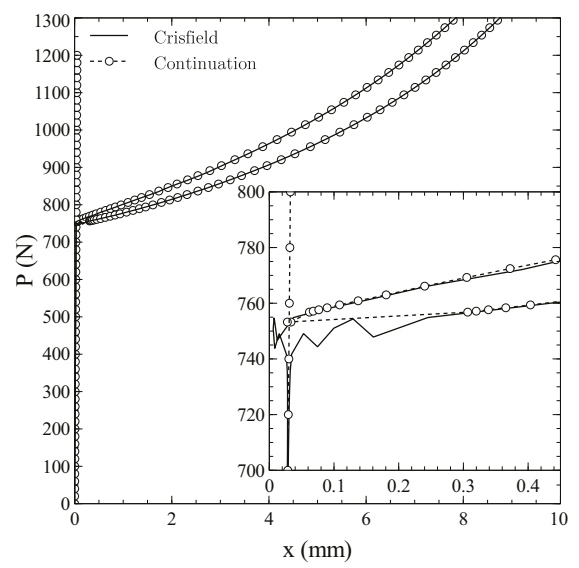

Figure 5. Bifurcation plot for axially compressed column.

The close matching with the results obtained using the Crisfield method demonstrates the ability of the continuation method to deal with the solution of equilibrium branches arising from supercritical bifurcation points.

In addition, the quality of the prediction can be noted by inspection of the region in the neighborhood of the bifurcation. As seen from the zoom of the plot, the continuation approach provides a precise description of the equilibrium branches departing from the critical point. In contrast, the Crisfield approach undergoes small oscillations, and convergence to spurious equilibrium configurations is observed in proximity of the bifurcation. Again, it is remarked that no initial imperfections are needed within the context of the continuation approach, so the bifurcation is precisely detected.

\subsection{Beam Containing an Initial Crack}

Previous test cases are restricted to geometrically nonlinear phenomena, and material nonlinearities are not accounted for. In the example presented here, a beam containing an initial delamination is considered and the propagation of the crack is accounted for by making use of cohesive elements characterized by a bilinear cohesive law. Further details regarding the implementation of these elements in the PyFem code are available in [33]. A sketch of the structure is reported in Figure 6. 


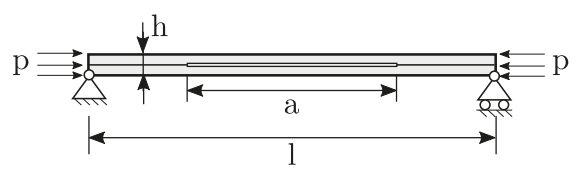

Figure 6. Beam with initial crack.

The beam's length and thickness are denoted with $l$ and $h$, respectively. The central portion of the beam is characterized by the presence of an initial crack, whose length is equal to $a$. The material is isotropic with modulus $E=230 \mathrm{GPa}$ and Poisson's ratio 0.25 , and a plane strain constitutive law is assumed.

The structure is hinged at the bottom left vertex, while the translation along the direction normal to the beam axis is constrained at the bottom right end. The load is introduced in the form of a compressive force per unit length of magnitude $p$.

As seen from Figure 6, the structure is divided into two equal parts with respect to the midline running parallel to the beam axis. The two outer portions are connected by means of two layers of cohesive elements. The fracture toughnesses in mode I and II are taken as $0.5 \mathrm{~N} / \mathrm{mm}$, while the interfacial strengths are fixed at $1.0 \mathrm{~N} / \mathrm{mm}^{2}$. The penalty stiffness of the cohesive elements is $5000 \mathrm{~N} / \mathrm{mm}^{3}$.

The centrally located pre-damaged area is simulated by means of cohesive elements with reduced properties, whose aim is to avoid interpenetration between the upper and lower parts of the structure during the deformation process [32].

The finite element model is composed of 200 nonlinear shell elements and 20 cohesive elements. Note, the mesh size is chosen in order to guarantee the presence of $4 / 5$ elements in the process zone, whose size is estimated referring to Equation (50).

In the first part of the procedure, the primary equilibrium path is computed. Starting from the undeformed configuration, the load is progressively increased, and the corresponding equilibrium points are found. During this first phase, the eigenvalues of the stiffness matrix are determined at each step of the iterative process, and the presence of critical points is verified. Whenever a critical point is detected, it is approximated referring to the procedure outlined in Section 3, depending on whether it is a limit or a bifurcation point. As an example, the initial part of the primary path is reported in Figure $7 \mathrm{a}$, just after the detection of the first bifurcation point. The procedure is then switched to the continuation procedure, and the tracing of the equilibrium path restarts from the last equilibrium point available. The complete primary path up to a load of $300 \mathrm{~N}$ is presented in Figure $7 \mathrm{~b}$ together with the four bifurcation points detected.

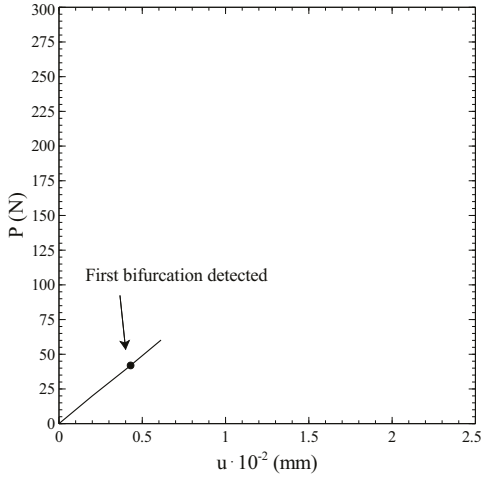

(a)

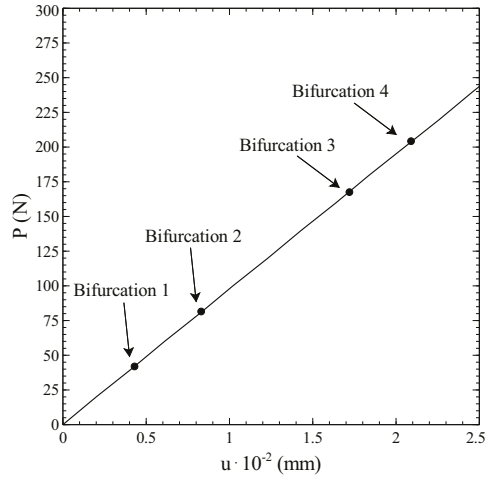

(b)

Figure 7. Primary equilibrium path: (a) detection of the first critical point, (b) full equilibrium path. 
As reported in Figure $7 \mathrm{~b}$, the primary path is characterized by an almost linear behaviour, consisting of a progressive shortening of the beam with no bending deflections. After the first bifurcation point, the primary equilibrium path becomes unstable. However, the absence of initial imperfections and the symmetry of the problem make its computation possible. It is also noted that no damage phenomena are associated with this primary equilibrium configurations.

In the second phase of the solution process, the equilibrium branches departing from the bifurcation points are computed. The branch associated with a positive sign of the first buckling mode is plotted in Figure 8a, while the branch obtained by assuming a negative sign is reported in Figure $8 c$. The two corresponding deformed shapes are presented in in Figure 8b,d, respectively.

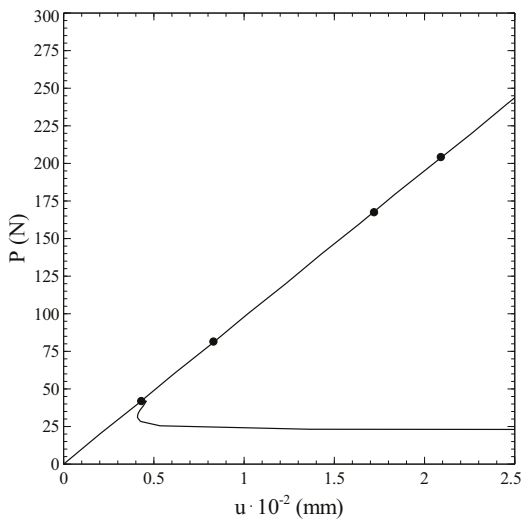

(a)

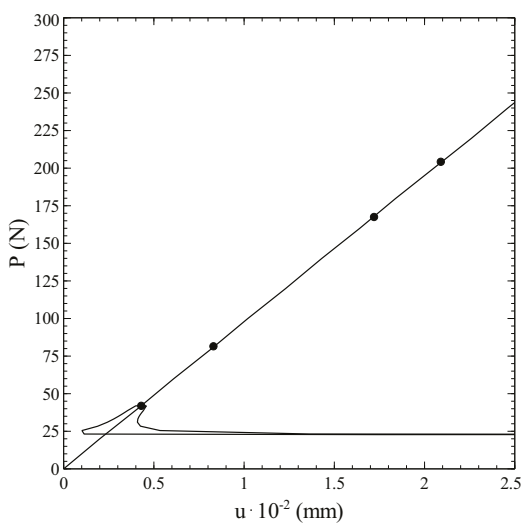

(c)

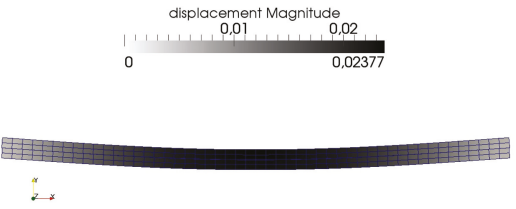

(b)

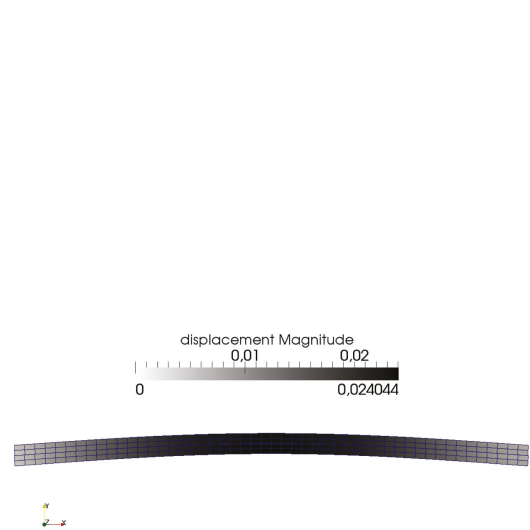

(d)

Figure 8. Computation of the first bifurcation branch: (a) positive side of the branch, (b) corresponding deformed shape, (c) negative side of the branch, (d) corresponding deformed shape.

In a similar fashion, the procedure is repeated for all the bifurcations points. The results of Figure 9 illustrate the force-displacement curves and the corresponding deformed shapes during the evaluation of the fourth equilibrium branch.

The curve of Figure 9c corresponds also with the complete bifurcation diagram, comprehensive of all the equilibrium paths departing from the bifurcation points detected. 


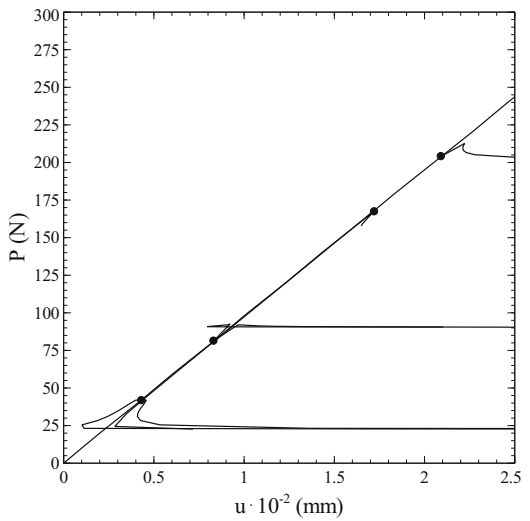

(a)

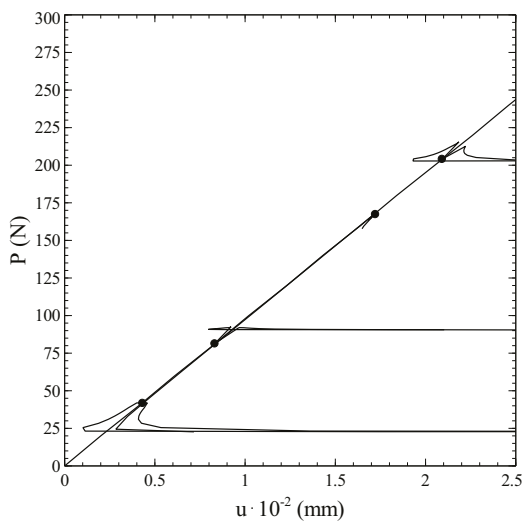

(c)

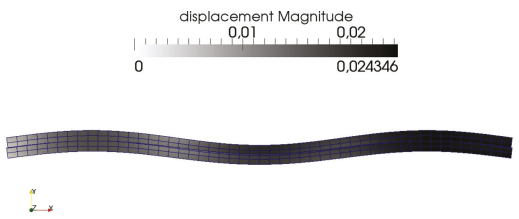

(b)

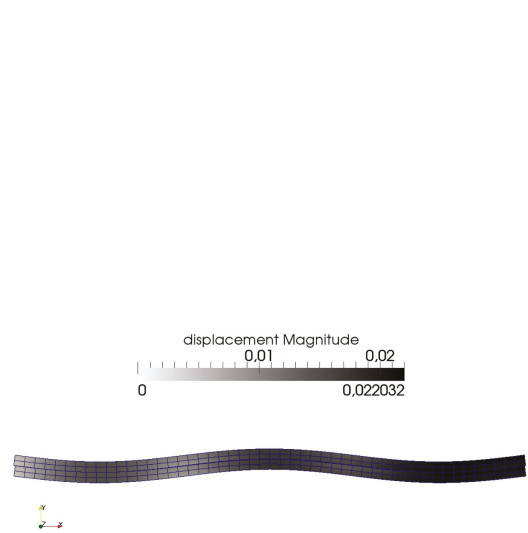

(d)

Figure 9. Computation of the fourth bifurcation branch: (a) positive side of the branch, (b) corresponding deformed shape, (c) negative side of the branch, (d) corresponding deformed shape.

A summary of the deformed configurations obtained at the end of the loading process along the four equilibrium branches is reported in Figure 10.

From Figure 10 it can be noted that all the deformed shapes but the third one exhibit the typical buckled pattern of pristine beams. In this case, the deformed configurations are characterized by one to three half-waves, and a partial damaging of the cohesive elements is observed due to the shear transfer mechanism between the upper and lower portion of the beam. As result, the fracture is dominated by a mode II mechanism. As observed from Figure 9c, the corresponding load-deflection curves are associated with a slight drop of load just after the bifurcation point, motivated by the contemporary onset of the buckling half-waves and damage mechanism in mode II. Afterwards, no additional load is carried by the structure, and the shortening happens at a constant load level.

A different response is observed for the third equilibrium branch, whose post-buckled pattern is reported in Figure 10c. In this case, the damage mechanism is governed by mode I component, with a clear opening between the upper and lower portions of the structure. The propagation is unstable and sudden, and is responsible for a drastic drop of load, not clearly visible from the complete bifurcation diagram. For clarity purposes, the force-displacement curve is reported in Figure 11 by restricting the plot to the third equilibrium branch, where the collapse induced by the onset of the buckling phenomenon can be fully appreciated. 


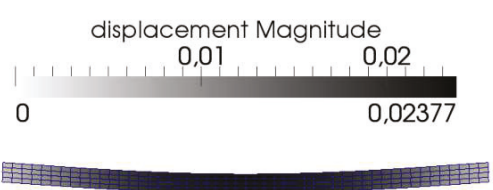

(a)

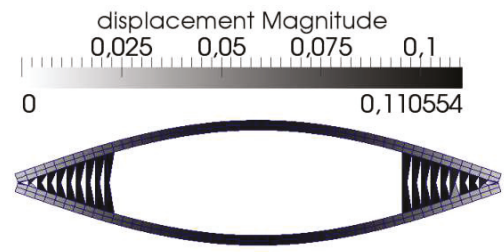

(c)

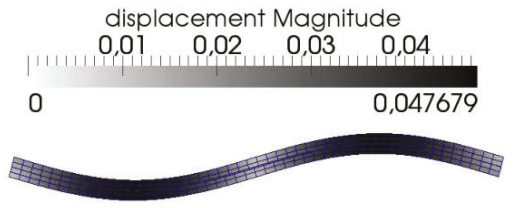

(b)

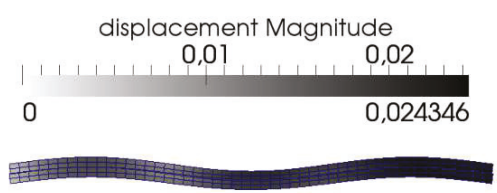

(d)

Figure 10. Post-critical deformed shapes along the equilibrium paths 1 to 4 : (a) path 1, (b) path 2, (c) path $3,(\mathbf{d})$ path 4 .

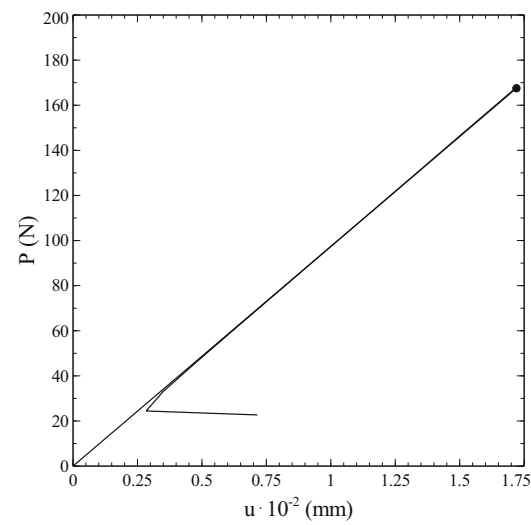

Figure 11. Detail of the force-displacment curve for the equilibrium branch 3.

The unloading phase happens in a steep manner, along a path which is close, but distinct, from loading one. After the drop of load, all the cohesive elements are completely damaged, thus the solution is arrested.

It is interesting to investigate the performance of the method in terms of number of iterations along the equilibrium branches previously considered. The results are summarized in Table 1 with regard to two different solution procedures. In one case, denoted with hybrid, the procedure refers to the combined geometric-dissipative approach summarized in Figure 1; in the other case, denoted with geometric, the dissipative constraint is removed, and the procedure is forced to adopt the purely geometric Riks constraint. As far as the number of iterations is a function of the parameter $\tilde{\tau}$ of Equation (25), a preliminary assessment was performed to obtain the values of $\tilde{\tau}$-reported in the brackets-guaranteeing the smallest number of iterations. 
Table 1. Number of increments using different constraint equations. ( $\tilde{\tau}$ in the parenthesis).

\begin{tabular}{|c|c|c|c|c|c|c|c|}
\hline \multirow[t]{2}{*}{ Constraint } & \multicolumn{6}{|c|}{ N. of Incr. } & \multirow{2}{*}{$\frac{\text { N. of Iter. }}{\text { Tot }}$} \\
\hline & Primary & Branch 1 & Branch 2 & Branch 3 & Branch 4 & Tot & \\
\hline Hybrid & 10 & $18(10)$ & $90(10)$ & $35(50)$ & $40(20)$ & 193 & 332 \\
\hline Geometric & 15 & $40(10)$ & $22(10)$ & $38(20)$ & $31(30)$ & 146 & 150 \\
\hline
\end{tabular}

As summarized in Table 1, the adoption of the dissipative constraint determines an increase of the total number of iterations with respect to the modified Riks case. The increase is motivated by the mainly geometric nature of the post-critical configurations associated with the equilibrium branches 1,2 and 4 . In these cases, the sources of nonlinearity can be mostly attributed to the deflected patterns, as seen from Figure 10, thus the advantages offered by a dissipation based-criterion cannot be fully appreciated. On the contrary, the number of iterations is slightly smaller when the combined dissipative-geometric constraint is adopted for tracing the third equilibrium branch, i.e., the one characterized by the opening of the initial crack. While the comparison in terms of iterations seems to suggest the adoption of a purely geometric constraint, it is important to notice that the quality of the predictions in the two cases is not equal. In particular, the adoption of a geometric constraint tends to determine relatively large increments, and the step size can be hardly controlled. It follows that the equilibrium path is not accurately described. On the other hand, the adoption of a dissipative constraint provides an excellent control of the step size, so enabling to trace the equilibrium branches with improved degree of refinement.

\subsection{Fixed-Fixed Arch}

An additional example is discussed regarding the configuration illustrated in Figure 12.

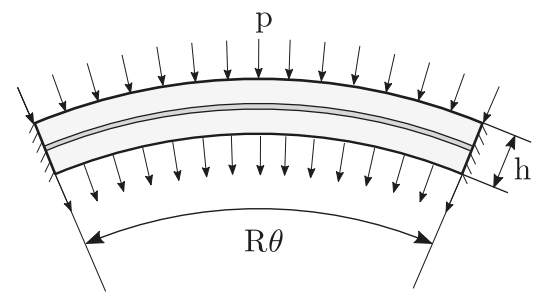

Figure 12. Arch subjected to distributed pressure.

The structure is an arch characterized by a radius $R$ equal to $10 \mathrm{~mm}$, an arc-length angle $\theta$ of $\pi / 4$ radians, and a radius-to-thickness ratio of 100 . The material elastic properties are those considered in the previous example. The two ends of the beam are fixed, while a uniformly distributed force per unit length $p$ is applied along the radial direction at the upper and lower surface of the arch, as illustrated in Figure 6. In order to account for material nonlinear responses, a layer of cohesive elements is introduced along the midline of the arch. In this case the fracture toughnesses in mode I and II are $0.5 \mathrm{~N} / \mathrm{mm}$, while the interfacial strengths are equal to $15 \mathrm{~N} / \mathrm{mm}^{2}$. The mesh is made of 100 nonlinear shell elements and 50 cohesive elements.

The bifurcation plot is presented in Figure 13 by reporting the nodal displacement of the central upper node versus the nodal force applied at the same node. 


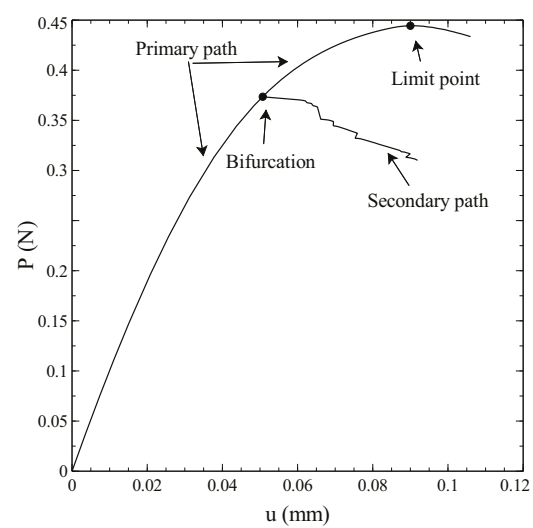

Figure 13. Bifurcation plot of the arch subjected to distributed pressure.

In this case, two critical points are detected along the primary path. The first one is a bifurcation point, as seen from the equilibrium path emanating from it. The second one is a limit point, and corresponds to a local maximum of the load carried by the structure. The primary path is now characterized by a nonlinear behaviour, where a progressive reduction of the linear stiffness is observed. No damage phenomena are observed along the major part of this path, as cohesive elements display the initiation of damage mechanisms just in the surroundings of the limit point.

The secondary equilibrium branch is characterized by an unloading phase, associated with a progressive failure of all the cohesive elements in mode II. From the curve of Figure 13, it can be observed the step-wise shape of the secondary equilibrium path, which is related to the failure of the interface elements. In any case, the layer of cohesive elements is distributed along all the overall structure, thus the role played by dissipative phenomena is significant.

To further address the relevance of dissipative phenomena, a comparison is presented between the performances achieved by considering the geometric-dissipative constraint, and those obtained with a run based on the adoption of a purely geometric constraint. The number of increments and iterations to complete the analysis are summarized in Table 2, where the optimal value of the parameter $\tilde{\tau}$ is reported in the brackets.

Table 2. Number of increments using different constraint equations. ( $\tilde{\tau}$ in the parenthesis).

\begin{tabular}{|c|c|c|c|c|}
\hline Constraint & \multicolumn{3}{|c|}{ N. of Incr. } & N. of Iter. \\
\hline & Primary & Branch 1 & Total & \\
\hline Hybrid & 47 & $160(20)$ & 207 & 276 \\
\hline Geometric & 47 & $160(30)$ & 207 & 445 \\
\hline
\end{tabular}

In this test, the advantages offered by the use of the hybrid constraint are clearer. While the number of increments along the two paths is, by chance, identical, the total number of iterations is much smaller when the energy control is active. The reduction of iterations determines a gain of approximately $15 \%$ in terms of computational time. In fact, the post-bifurcational response is dominated by the material nonlinearities, so the control over the dissipated energy is the more efficient approach.

\subsection{Perforated Beam}

This final example illustrates the use of the continuation approach as a mean for solving a nonlinear problem characterized by a complex response, where nonlinearites are due to geometric and material behaviour. The numerical solution strategy is not used here for tracing the bifurcation plot, but for obtaining the primary equilibrium path, as commonly done when solving a nonlinear 
quasi-static problem. The problem was analyzed using arc-length hybrid strategies in a previous work form the authors [17].

The analysis regards the mode I opening of a cantilever beam characterized by the presence of five equally spaced, square holes. The beam is loaded at the tip by means of two concentrated forces, as indicated in Figure 14, where all the relevant geometric dimensions are reported. An isotropic material is considered, with modulus equal to $1000 \mathrm{MPa}$ and Poisson's ratio 0.3 .

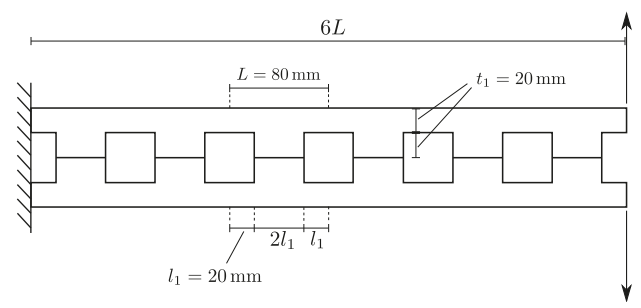

Figure 14. Cantilever perforated beam: geometry and dimensions.

To capture the onset and the propagation of the crack, a layer of cohesive elements is introduced along the horizontal line of symmetry of the structure. The fracture toughnesses in mode I and II are equal to $0.01 \mathrm{~N} / \mathrm{mm}$, while the interfacial tensile and shear strength are taken as $1 \mathrm{~N} / \mathrm{mm}^{2}$. A penalty stiffness of $100 \mathrm{~N} / \mathrm{mm}^{3}$ is assumed. The resulting finite element model is composed of 288 two-dimensional, plane-strain continuum elements, and 24 cohesive elements.

The curves reporting to the vertical displacement of the loaded nodes are plotted against the applied load in Figure 15. The comparison is presented for two different strategies. In one case the continuation method is applied as implemented, thus allowing the automatic switch between geometric and dissipative constraint. In the second case the constraint is forced to be of purely geometric, with the scope of illustrating the advantages due to the hybrid continuation approach presented here.

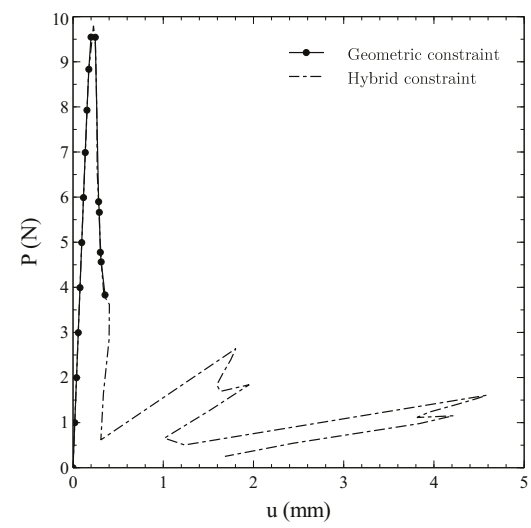

Figure 15. Force-displacement curve using different constraints.

As seen from the figure, the adoption of a purely geometric constraint leads to a premature failure of the analysis, which terminates along the first descending branch of the curve. On the contrary, the hybrid implementation is capable of capturing the subsequent portions of the equilibrium path. Note that each sudden drop is due to the opening of the holes. After the first peak, a snap-back behaviour can be observed, where a secondary snap characterizing the unloading phase. Indeed, a partial recovery of the load-carrying capability is observed in the post-peak region, followed by another snap-back. 
The deformed shapes at the end of the analysis are reported in Figure 16.

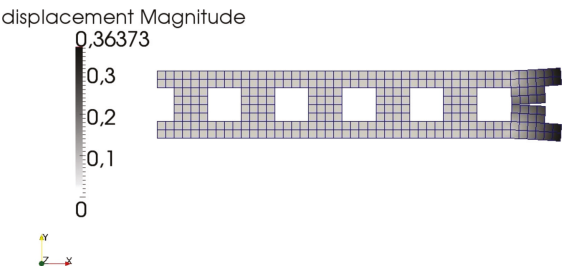

(a)

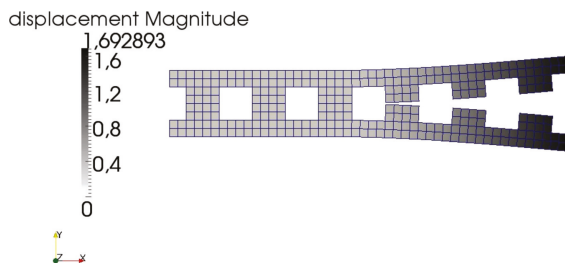

(b)

Figure 16. Deformed configuration at the end of the analysis by using: (a) geometric constraint, (b) hybrid constraint.

While the initiation of the opening of the first crack coincides with the failure of the purely geometric approach (Figure 16a), the improved robustness properties of the hybrid method are clearly visible from Figure 16b.

It is useful to illustrate the comparison between the two strategies in terms of numerical performance of the solution procedure, as done in Table 3.

Table 3. Summary of numerical performance for the perforated cantilever beam.

\begin{tabular}{cccccc}
\hline Constraint & & N. of Incr. & N. of Iter. & Step Cuts & N. of Fully Damaged Coh. Els \\
\cline { 1 - 5 } \cline { 4 - 6 } Geometric & & 17 & 59 & 3 & 2 \\
Hybrid & 35 & 85 & 1 & 12 \\
\hline
\end{tabular}

The results provide a clear picture of what highlighted by inspection of the force-displacement response. In particular, the geometric continuation is capable of obtaining the solution in the initial part of the curve, where 17 increments are performed. At the onset of the first crack, the step length is reduced 3 times, demonstrating the difficulties experienced by the method to obtain the solution. Then, after reaching the complete damaging of two cohesive elements, the analysis is terminated.

On the other hand, the hybrid strategy is capable of reaching the condition characterized by a number of 12 completely damaged cohesive elements. No step length reductions are observed. Clearly, the total time for the analysis and the number of increments are higher.

\section{Conclusions}

This work discussed the development and implementation of a numerical continuation technique for analyzing structural problems characterized by geometric and material nonlinearities. The continuation strategy can be successfully applied for detecting bifurcation and limit points as well as tracing the equilibrium paths, which is a useful mean for gathering insight into the response of the structure. For instance, the complete post-buckling behaviour can be assessed by performing one single quasi-static analysis, with no needs to introduce initial imperfections in the finite element model.

The effectiveness of the current implementation was checked by means of illustrative examples, where cohesive elements were employed for capturing the onset and propagation of cracks. Main advantage of the approach is the robustness of the numerical solution strategy, which is based upon a hybrid constraint equation that includes both geometric and dissipative considerations. Thus crack propagation phenomena can be accurately predicted, whilst standard geometric-based approaches tend to undergo converge issues. In addition, the method can be used as a technique for solving quasi-static nonlinear problems, when the analysis of secondary equilibrium paths is not of concern. The effectiveness of the approach was illustrated with a test case characterized by the presence of sharp snap-backs, and secondary snap-backs. The analyst can benefit for the adoption of 
a unique, integrated solution scheme, which can be used for solving the structural boundary value problem, as well as exploring multiple equilibrium paths with improved robustness.

Acknowledgments: This study did not receive funds.

Author Contributions: The two authors equally contributed to the present paper.

Conflicts of Interest: The authors declare no conflict of interest.

\section{References}

1. Buermann, P.; Rolfes, R.; Tessmer, J.; Schagerl, M. A Semi-Analytical Model for Local Post-Buckling Analysis of Stringer- and Frame-Stiffened Cylindrical Panels. Thin Walled Struct. 2006, 44, 102-114. [CrossRef]

2. Brubak, L.; Hellesland, J. Semi-analytical Postbuckling and Strength Analysis of Arbitrarily Stiffened Plates in Local and Global Bending. Thin Walled Struct. 2007, 45, 620-633. [CrossRef]

3. Vescovini, R.; Bisagni, C. Two-step procedure for fast post-buckling analysis of composite stiffened panels. Comput. Struct. 2013, 128, 38-47. [CrossRef]

4. Barriére, L.; Marguet, S.; Castanié, B.; Cresta, P.; Passieux, J.C. An adaptive model reduction strategy for post-buckling analysis of stiffened structures. Thin Walled Struct. 2013, 73, 81-93. [CrossRef]

5. Milazzo, A.; Oliveri, V. Post-buckling analysis of cracked multilayered composite plates by pb-2 Rayleigh-Ritz method. Compos. Struct. 2015, 132, 75-86. [CrossRef]

6. Cricrì, G.; Perrella, M.; Calì, C. Experimental and numerical post-buckling analysis of thin aluminium aeronautical panels under shear load. Strain 2014, 50, 208-222. [CrossRef]

7. Citarella, R.; Giannella, V.; Lepore, M. DBEM crack propagation for nonlinear fracture problems. Frattura ed Integrità Strutturale 2015, 34, 514-523.

8. Pook, L. Determination of fatigue crack paths. Int. J. Bifurc. Chaos 1997, 7, 469-475. [CrossRef]

9. Riks, E. The Application of Newton's Method to the Problem of Elastic Stability. J. Appl. Mech. 1972, 39, 1060-1065. [CrossRef]

10. Wempner, G. Discrete approximations related to nonlinear theories of solids. Int. J. Solids Struct. 1971, 7, 1581-1599. [CrossRef]

11. Crisfield, M. A fast incremental/iterative solution procedure that handles "snap-through". Comput. Struct. 1981, 13, 55-62. [CrossRef]

12. May, I.; Duan, Y. A local arc-length procedure for strain softening. Comput. Struct. 1997, 64, $297-303$. [CrossRef]

13. Schellekens, J.; Borst, R.D. A non-linear finite element approach for the analysis of mode-I free edge delamination in composites. Int. J. Solids Struct. 1993, 30, 1239-1253. [CrossRef]

14. Borst, R.D. Computation of post-bifurcation and post-failure behavior of strain-softening solids. Comput. Struct. 1987, 25, 211-224. [CrossRef]

15. Gutierrez, M. Energy release control for numerical simulations of failure in quasi-brittle solids. Commun. Numer. Methods Eng. 2004, 20, 19-29. [CrossRef]

16. Verhoosel, C.; Remmers, J.; Gutierrez, M. A dissipation-based arc-length method for robust simulation of brittle and ductile failure. Int. J. Numer. Methods Eng. 2009, 77, 1290-1321. [CrossRef]

17. Bellora, D.; Vescovini, R. Hybrid geometric-dissipative arc-length methods for the quasi-static analysis of delamination problems. Comput. Struct. 2016, 175, 123-133. [CrossRef]

18. Rastiello, G.; Riccardi, F.; Richard, B. Discontinuity-scale path-following methods for the embedded discontinuity finite element modeling of failure in solids. Comput. Methods Appl. Mech. Eng. 2019, 349, 431-457. [CrossRef]

19. Davidenko, D. On a new method of numerical solution of systems of nonlinear equations. Doklady Akademii Nauk SSSR 1953, 4, 601-602. [in Russian].

20. Davidenko, D. On approximate solution of systems of nonlinear equations. Ukrainian Math. J. 1953, 5, $196-206$. [in Russian].

21. Allgower, E.; Georg, K. Introduction to Numerical Continuation Method; SIAM: Philadelphia, PA, USA, 2003.

22. Rheinboldt, W. Numerical Analysis of Continuation Methods for Nonlinear Structural Problems. Comput. Struct. 1981, 13, 103-113. [CrossRef]

23. Seyde, R. Practical Bifurcation and Stability Analysis; Springer: New York, NY, USA, 2009. 
24. Riks, E. An incremental approach to the solution of snapping and buckling problems. Int. J. Solids Struct. 1979, 2, 529-551. [CrossRef]

25. Shi, J. Computing critical points and secondary paths in nonlinear structural stability analysis by the finite element method. Comput. Struct. 1996, 58, 203-220. [CrossRef]

26. Bergan, P.; Horrigmoe, G.; Bråkeland, B.; Søreide, T. Solution techniques for non- linear finite element problems. Int. J. Numer. Methods Eng. 1978, 12, 1677-1696. [CrossRef]

27. Wagner, W.; Wriggers, P. A simple method for the calculation of postcritical branches. Eng. Comput. 1988, 5, 103-109. [CrossRef]

28. Magnusson, A.; Svensson, I. Numerical treatment of complete load-deflection curves. Int. J. Numer. Methods Eng. 1998, 41, 955-971. [CrossRef]

29. Borst, R.D.; Crisfield, M.; Remmers, J.; Verhoosel, C. Non-Linear Finite Element Analysis of Solids and Structures; John Wiley \& Sons: New York, NY, USA, 2012.

30. Camanho, P.; Dávila, C.G.; Moura, M.D. Numerical simulation of mixed-mode progressive delamination in composite materials. J. Compos. Mater. 2003, 37, 1415-1424. [CrossRef]

31. Turon, A.; Dávila, C.; Camanho, P.; Costa, J. An engineering solution for using coarse meshes in the simulation of delamination with cohesive zone models. Available online: https:/ /ntrs.nasa.gov/search.jsp? $\mathrm{R}=20050160472$ (accessed on 30 October 2019).

32. Vescovini, R.; Dávila, C.; Bisagni, C. Failure analysis of composite multi-stringer panels using simplified models. Compos. B Eng. 2013, 45, 939-951. [CrossRef]

33. Bellora, D. Quasi-static solution procedures for the finite element analysis of delaminations. M.Sc. Thesis, Politecnico di Milano, Milano, Italy, 2015.

(C) 2019 by the authors. Licensee MDPI, Basel, Switzerland. This article is an open access article distributed under the terms and conditions of the Creative Commons Attribution (CC BY) license (http:/ / creativecommons.org/licenses/by/4.0/). 



\title{
Hydrodynamic and Acoustic Performance Analysis of Marine Propellers by Combination of Panel Method and FW-H Equations
}

\author{
Abouzar Ebrahimi ${ }^{1}$, Mohammad Saeed Seif ${ }^{1, *}$ and Ali Nouri-Borujerdi ${ }^{2}$ \\ 1 Center of Excellence in Hydrodynamic and Dynamic of Marine Vehicles, Sharif University of Technology, \\ Tehran, Iran \\ 2 Mechanical Engineering Department, Sharif University of Technology, Tehran, Iran \\ * Correspondence: seif@sharif.edu
}

Received: 13 August 2019; Accepted: 5 September 2019; Published: 9 September 2019

\begin{abstract}
The noise emitted by ships is one of the most important noises in the ocean, and the propeller noise is one of the major components of the ship noise. Measuring the propeller noise in a laboratory, despite the high accuracy and good reliability, has high costs and is very time-consuming. For this reason, the calculation of propeller noise using numerical methods has been considered in recent years. In this study, the noise of a propeller in non-cavitating conditions is calculated by the combination of the panel method (boundary element method) and solving the Ffowcs Williams-Hawkings (FW-H) equations. In this study, a panel method code is developed, and the results are validated by the experimental results of the model tests carried out in the cavitation tunnel of the Sharif University of Technology. Software for numerical calculation of propeller noise, based on FW-H equations, is also developed and the results are validated by experimental results. This study shows that the results of the panel method code have good agreement with experimental results, and that the maximum error of this code for the thrust and torque coefficients is $4 \%$ and $7 \%$, respectively. The results of the FW-H noise code are also in good agreement with the experimental data.
\end{abstract}

Keywords: panel method; marine propeller; noise; FW-H equations; experimental test

\section{Introduction}

In recent years, the use of numerical methods has become very common in modeling the flow around marine propellers. Although the experimental methods have a higher accuracy and reliability than numerical methods, the higher costs and the need for special equipment has caused numerical methods to be preferred.

The boundary element method (BEM) is one of the methods used to solve the governing equations of fluid mechanics, acoustics, electromagnetics, etc. In this method, using mathematical theorems, the equations from the interior of the solution domain are transferred to the boundaries of the domain. Therefore, compared to other numerical methods such as finite element or finite volume method which interior of domain should be considered, BEM exhibits less solution time and computation cost and therefore it is preferred to other methods.

The panel method is one of boundary element methods. In this method, the surface of the body is divided into small panels and a combination of source and doublets is distributed to each panel. After calculating the strength of sources and doublets, velocity and pressure distribution on surface panels can be obtained. For lifting bodies, a wake surface should be assumed at the trailing edge of body, and the effect of panels of this surface on body panels should be considered.

In recent decades, the panel method has been widely used to solve the flow around the bodies, especially marine propellers. A literature review shows that a lot of research has been done on the 
use of the panel method for the hydrodynamic analysis of objects. For instance, Hess and Smith [1], in 1962, invented the panel method for analyzing the motion of three dimensional bodies in fluid. The formulation of these researchers was presented for the arbitrary three dimensional bodies and could not be used for lifting bodies. In 1972, Hess [2] developed this method for lifting bodies and calculated the pressure distribution around an aircraft model and lift force. The panel method was first used in 1985 by Hess and Valarezo [3] to model the flow around the ship propeller. In this study, they obtained the pressure distribution around a propeller using quadrilateral panels and calculated the thrust and torque coefficients. In 2010, Baltazar et al. [4] modeled cavitation around a propeller using the panel method. The results of this study show that the panel method can model cavitation on propeller blades with good accuracy. Also, several papers have been published between the years 1985 to 2010, but most have done similar work.

In 2010, Gaggero et al. [5] modeled the flow around the propeller using the panel method and computational fluid dynamics. The results of the panel method show that this method has a good and acceptable accuracy compared to the CFD method.

According to the importance of propeller noise, various methods have been proposed to reduce it. Methods used to reduce the noise of marine propellers can be divided into two main methods, namely:

\section{- $\quad$ Using equipment \\ - $\quad$ Modification of the geometry}

Each of these methods is fully described in a review paper published by Ebrahimi et al. [6]. In recent years, calculating the noise of marine propellers using numerical methods has also been developed by researchers. For numerical calculation of noise, a numerical method such as panel method is required to obtain flow quantities and then these quantities are used as input for calculation of noise for a receiver at the desired location. Seol and Park [7] carried out several experiments on a propeller model in MOERI cavitation tunnel and measured the noise of propeller in cavitating and non-cavitating condition. Also, they used combination of time domain acoustic analogy and panel method for the numerical investigation of the propeller noise.

In another study by Kellett et al. [8] in 2013, the noise generated by the propeller of a LNG carrier ship was measured numerically and experimentally. Bagheri et al. [9] conducted a research on a three bladed propeller of the Gawn series and used the ANSYS Fluent commercial software for calculation of the propeller noise. Testa et al. [10] calculated the noise of E779A propeller model by using the FW-H equations in presence of unsteady sheet cavitation. They also used potential-based panel method for modelling flow around propeller. Gorji et al. [11] performed a research on calculation of noise of a marine propeller using a RANS solver in the low frequencies band.

Bagheri et al. [12] carried out some numerical and experimental tests on the noise of marine propellers and the effects of cavitation on the sound pressure level of propeller. They used viscous flow with FW-H solver for noise calculation.

In present research, a boundary element method is used for hydrodynamic analysis of marine propellers. Also, the FW-H equations are used for acoustic performance analysis of these propellers. For this purpose, two codes have been developed by authors and their results validated using experimental tests carried out in cavitation tunnel of Sharif University of Technology. The most significant novelty of our paper is that we used experimental noise measurements to validate the results of the FW-H equations code.

\section{Formulation of the Panel Method}

For development of the panel method formulation for marine propeller, the flow around propeller in computational domain of $\Omega$ is assumed to be irrotational, incompressible and inviscid. By these assumptions, the flow governing equations (Navier-Stokes equation) are simplified into 
the three-dimensional Laplace equation where is $\nabla^{2} \phi$. By using Green's second identity, the general solution of this equation is as follows [13]:

$$
\int_{\Omega}\left(\phi_{1} \nabla^{2} \phi_{2}-\phi_{2} \nabla^{2} \phi_{1}\right) d V=\int_{S}\left(\phi_{1} \nabla \phi_{2}-\phi_{2} \nabla \phi_{1}\right) \cdot \vec{n} d S
$$

On the right-hand side of the Equation (1), integrating is done on the surface $S$, which includes the body surface, Kutta strip and wake surfaces of the object (Figure 1). If the points $P, Q$ and $O$ are defined as the control point, source point and the origin of the coordinates, respectively, the distance vector $\vec{R}$ is defined as follows:

$$
\vec{R}=\overrightarrow{O Q}-\overrightarrow{O P} \quad r=|\vec{R}|
$$

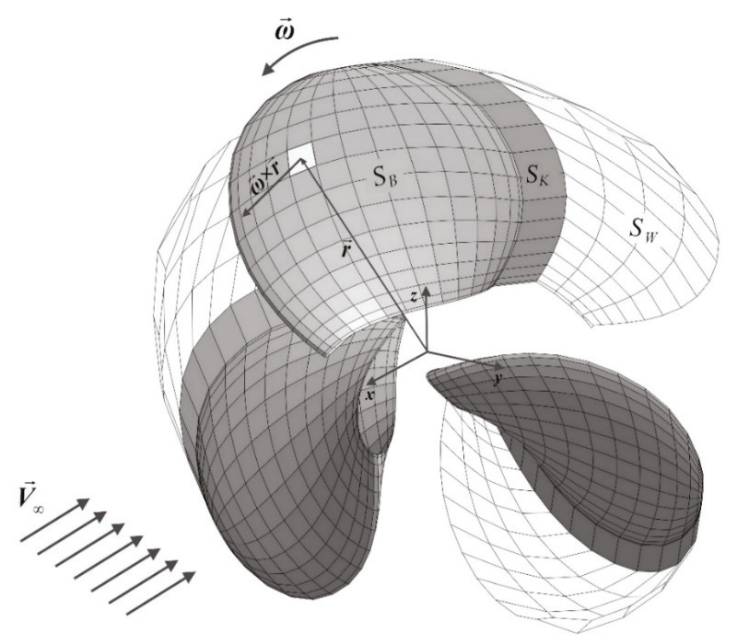

Figure 1. Body surface, Kutta strip and wake surface.

Now, if we suppose $\phi_{1}=1 / r$ and $\phi_{2}=\phi$, where $\phi$ is potential of flow, Equation (1) becomes:

$$
\int_{\Omega}\left(\frac{1}{r} \nabla^{2} \phi\right) d V-\int_{\Omega}\left(\phi \nabla^{2} \frac{1}{r}\right) d V=\int_{S}\left(\frac{1}{r} \nabla \phi-\phi \nabla \frac{1}{r}\right) \cdot \vec{n} d S
$$

In the case where $P$ is outside of the domain $\Omega$, then $\phi_{1}$ and $\phi_{2}$ satisfy Laplace's equation [13]. Therefore, the first and second terms on the left-hand side of Equation (3) will be zero. Then:

$$
\int_{S}\left(\frac{1}{r} \nabla \phi-\phi \nabla \frac{1}{r}\right) \cdot \vec{n} d S=0
$$

In the case of flow around the propeller, the point $P$ is located on the body surface. When point $P$ approaches to point $Q$ and $r \rightarrow 0$, the above integral is singular $(1 / r \rightarrow \infty)$. Therefore, the point $P$ should be excluded from $S$ using a small hemisphere of radius $\varepsilon$. Therefore, the integration region will change to $S+S_{\varepsilon}$ :

$$
\int_{S}\left(\frac{1}{r} \nabla \phi-\phi \nabla \frac{1}{r}\right) \cdot \vec{n} d S+\int_{S_{\varepsilon}}\left(\frac{1}{r} \nabla \phi-\phi \nabla \frac{1}{r}\right) \cdot \vec{n} d S=0
$$


Using mathematical methods, the value of second integral is $-2 \pi \phi(P)$. Then Equation (5) becomes:

$$
\phi(P)=\frac{1}{2 \pi} \int_{S}\left(\frac{1}{r} \nabla \phi-\phi \nabla \frac{1}{r}\right) \cdot \vec{n} d S
$$

This equation gives the potential of flow $(\phi)$ at any point of body surface in terms of $\nabla \phi$ and $\phi$ on the boundaries of domain. In Equation (6), the first and second terms of the integral can be interpreted as a dipole with strength of $\phi$ and a source with strength of $\nabla \phi$, respectively. To expand the above equation, as shown in Figure 1, the integration surface $S$ is divided to body surface $\left(S_{B}\right)$, Kutta strip $\left(S_{K}\right)$, and wake surface $\left(S_{W}\right)$. Also, $N_{B}, N_{K}$ and $N_{W}$ are number of panels on body surface, Kutta strip, and wake surface, respectively.

Therefore, Equation (6) can written as below:

$$
\phi(P)=\frac{1}{2 \pi} \int_{S_{B}}\left(\frac{1}{r} \nabla \phi-\phi \nabla \frac{1}{r}\right) \cdot \vec{n} d S+\frac{1}{2 \pi} \int_{S_{K}+S_{W}}\left(\frac{1}{r} \nabla \phi-\phi \nabla \frac{1}{r}\right) \cdot \vec{n} d S
$$

The thickness of Kutta and wake surfaces are assumed to be zero. Then, $\nabla \phi \cdot \vec{n}$ for these surfaces becomes zero. So:

$$
\phi(P)=\frac{1}{2 \pi} \int_{S_{B}}\left(\frac{1}{r} \nabla \phi-\phi \nabla \frac{1}{r}\right) \cdot \vec{n} d S-\frac{1}{2 \pi} \int_{S_{K}+S_{W}} \phi \nabla \frac{1}{r} \cdot \vec{n} d S
$$

This formulation is equivalent to distributing a source with strength of $\partial \phi / \partial n$ on each panel of the body surface and a doublet with strength of $\phi$ on each panel of the body surface, Kutta strip and wake surface. For greater simplicity, the strength of doublets on the Kutta strip and wake surface are displayed by $\mu$. The final form of Equation (8) is as follows:

$$
\phi(P)=-\frac{1}{2 \pi} \int_{S_{B}} \frac{\vec{n} \cdot \nabla \phi}{r} d S+\frac{1}{2 \pi} \int_{S_{B}} \phi \frac{\vec{n} \cdot \vec{r}}{r^{3}} d S+\frac{1}{2 \pi} \int_{S_{K}} \mu \frac{\vec{n} \cdot \vec{r}}{r^{3}} d S+\frac{1}{2 \pi} \int_{S_{W}} \mu \frac{\vec{n} \cdot \vec{r}}{r^{3}} d S
$$

\subsection{Boundary Conditions}

Applying boundary conditions is one of the most important steps in solving problems by numerical methods. The first boundary condition that is applied is the non-entrance condition. If the propeller assumed to be rigid, the fluid particles cannot enter into the surface and the normal velocity will be zero on the surface of the propeller. If the propeller is operating in a uniform axial flow with velocity of $\vec{V}_{\infty}$ and rotates with angular velocity of $\vec{\omega}$, as shown in Figure 1 , the total velocity of point $A$ on propeller blade is:

$$
\vec{v}_{A}=\vec{V}_{\infty}+\vec{r} \times \vec{\omega}
$$

Then, the non-entrance condition for all panels of body surface is [14]:

$$
(\nabla \phi \cdot \vec{n})_{i}=\left(\vec{v}_{A} \cdot \vec{n}\right)_{i} i=1, \ldots, N_{B}
$$

Then, Equation (9) becomes:

$$
\phi(P)=-\frac{1}{2 \pi} \int_{S_{B}} \frac{\vec{v}_{A} \cdot \vec{n}}{r} d S+\frac{1}{2 \pi} \int_{S_{B}} \phi \frac{\vec{n} \cdot \vec{r}}{r^{3}} d S+\frac{1}{2 \pi} \int_{S_{K}} \mu \frac{\vec{n} \cdot \vec{r}}{r^{3}} d S+\frac{1}{2 \pi} \int_{S_{W}} \mu \frac{\vec{n} \cdot \vec{r}}{r^{3}} d S
$$


We can rewrite the above equation in series form as follows:

$$
\begin{aligned}
& \phi_{i}+ \sum_{\substack{j=1 \\
i \neq j}}^{N_{B}} A_{i j} \phi_{j}+\sum_{j=1}^{N_{K}} A_{i j} \mu_{j}=\sum_{j=1}^{N_{B}} B_{i j}\left(\vec{v}_{A} \cdot \vec{n}\right)_{j}-\sum_{j=1}^{N_{W}} A_{i j} \mu_{j} \quad i=1, \ldots, N_{B} \\
& A_{i j}=-\frac{1}{2 \pi} \int_{\text {panel } j} \frac{\vec{n} \cdot \overrightarrow{P Q}}{|\overrightarrow{P Q}|^{3}} d s \\
& B_{i j}=-\frac{1}{2 \pi} \int_{\text {panel } j} \frac{1}{|\overrightarrow{P Q}|} d s
\end{aligned}
$$

where the points $P$ and $Q$ are assumed to be the center of panel $i$ and $j$, respectively. $A_{i j}$ and $B_{i j}$ are known as influence coefficients and can be calculated according to Delhommeau [15]. The first and second terms in right-hand side of Equation (13) are obtained from non-entrance boundary condition and from previous time step, respectively. Therefore, the unknowns of the above equation are the strength of doublets on body panels and doublets on Kutta strip, hence $N_{B}+N_{K}$ unknowns. Another boundary condition that is used to solve this problem is the Kutta condition. This condition for lifting bodies states that the flow should leave trailing edge smoothly and the velocity of flow there be finite [13]. One of the best ways to apply this condition is presented by Morino [16]:

$$
\mu_{i}=\left(\phi_{i}\right)^{u}-\left(\phi_{i}\right)^{l} i=1, \ldots, N_{K}
$$

This means that strength of Kutta strip doublets is equal to difference of strength of upper $(\phi)^{u}$ and lower $(\phi)^{l}$ doublets. Equation (13) will give $N_{B}$ equations and $N_{K}$ equations will be added to system of equations by applying Kutta condition. Finally, by solving this set of $N_{B}+N_{K}$ equations, all $N_{B}+N_{K}$ unknowns will be obtained.

\subsection{Hydrodynamic Forces}

After the calculation of velocity potential, the total velocity on each panel can be calculated using numerical differentiation methods. Then, the unsteady Bernoulli's equation was used for calculation of total pressure [17]:

$$
\frac{p-p_{\infty}}{\rho}=-\frac{1}{2}\left|\overrightarrow{\nabla \phi}-\vec{v}_{A}\right|^{2}+\frac{1}{2}\left|\vec{v}_{A}\right|^{2}-\frac{\partial \phi}{\partial t}
$$

where $\overrightarrow{\nabla \phi}$ and $\partial \phi / \partial t$ denote the surface gradient and time derivate of velocity potential. Propeller thrust can be calculated by integration of pressure forces on panels of propeller surface. Also, viscous forces should be obtained by using empirical formulas for surface friction coefficient $\left(C_{D}\right)$ of each panel [18]:

$$
C_{D}=\frac{0.0986}{\left(\log _{10} R e_{l}-1.22\right)^{2}}
$$

where $R e_{l}$ is local Reynolds number based on radial location of panel $\left(x_{r}\right)$ and is $U x_{r} / v$. The viscous drag of each panel is calculated as below [17]:

$$
\vec{F}_{D}=\frac{1}{2} \rho \cdot C_{D} \cdot\left|\vec{v}_{t o t}\right| \vec{v}_{t o t}
$$


where $\vec{v}_{t o t}=\vec{v}+\vec{v}_{r e f}$ and $\vec{v}_{r e f}=-\vec{v}_{A}$. Also, $\vec{v}$ is blade surface perturbation velocity and can be obtained by taking surface gradient of velocity potential on blade panels. Then, total force and torque of propeller are obtained as [19]:

$$
\begin{aligned}
& \vec{F}=\int\left(p \cdot \vec{n}+\vec{F}_{D}\right) d S \\
& \vec{M}=\int \vec{r} \times\left(p \cdot \vec{n}+\vec{F}_{D}\right) d S
\end{aligned}
$$

Flowchart of panel method for calculation of hydrodynamic coefficient of propeller is shown in Figure 2. Initially, geometry of propeller and panels are generated by a function. A Kutta strip is also created due to geometric considerations, propeller rotation and flow velocity. In the next step, a function calculates the influence coefficients for the sources and doublets. Then, the systems of linear equations of Equation (13) is formed and then solved using iterative methods. By solving the equations, the velocity potential is obtained on the panels of body surface. With numerical derivation of potentials, velocity and pressure and on each panel are calculated and hydrodynamic coefficients of propeller are obtained. This process repeated until the solution converges. In this flowchart, $\vec{v}_{m}$ is the mean perturbation velocity of the points located on wake and Kutta surface. If these points are located in $X_{\text {old }}$ position in time $t$, new position $\left(X_{\text {new }}\right)$ of points in time $t+d t$ can be obtained using the following equation:

$$
X_{\text {new }}=X_{\text {old }}+\vec{v}_{m} \cdot d t
$$

where $\vec{v}_{m}$ is calculated as below [19]:

$$
\vec{v}_{m}=\frac{1}{4 \pi} \sum_{S_{B}}\left(\vec{n} \cdot \vec{v}_{A}\right) \frac{\vec{r}}{r^{3}} d S+\frac{1}{4 \pi} \sum_{S_{B}} \phi \frac{\overrightarrow{d l} \times \vec{r}}{r^{3}}+\frac{1}{4 \pi} \sum_{S_{W}+S_{K}} \mu \frac{\overrightarrow{d l} \times \vec{r}}{r^{3}}
$$

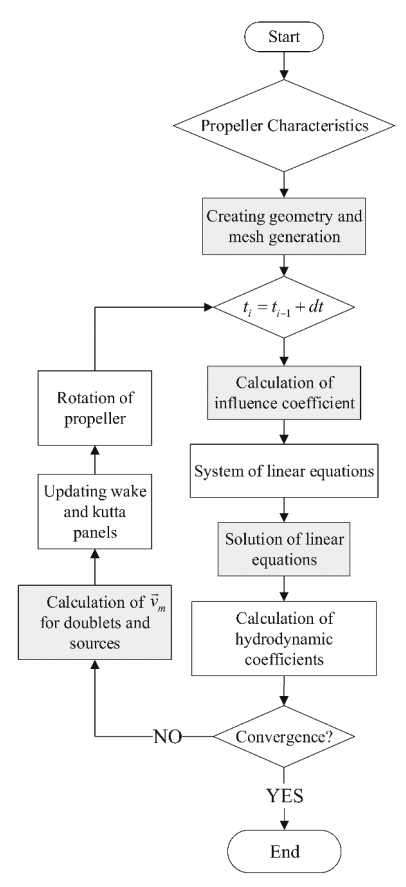

Figure 2. Flowchart of panel method. 


\section{Validation of Results of Panel Method}

One of the most common propellers that has been used for the validation of numerical methods is the DTMB 4119 propeller. This propeller is shown in Figure 3. Also, the main particulars and details of geometry are given in Tables 1 and 2, respectively.

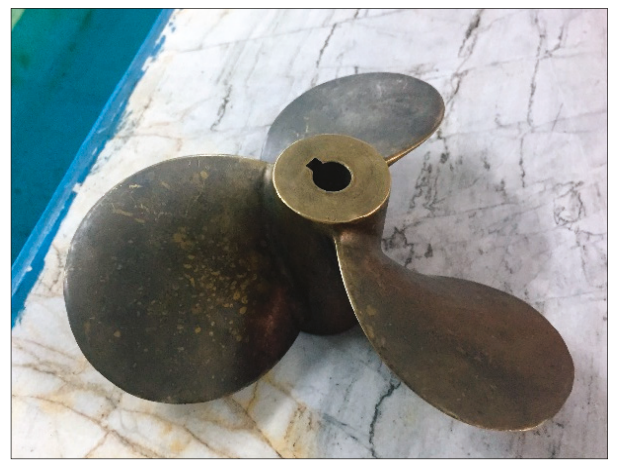

Figure 3. DTMB 4119 propeller.

Table 1. Main Particulars of DTMB 4119 Propeller [20].

\begin{tabular}{cc}
\hline Number of Blades & 3 \\
Diameter & $0.20 \mathrm{~m}$ \\
Blade Sections Profile & NACA 66 modified $(\mathrm{a}=0.8)$ \\
Design Advance Ratio $(J)$ & 0.833 \\
\hline
\end{tabular}

Table 2. Geometry of DTMB 4119 Propeller [20].

\begin{tabular}{ccccccc}
\hline $\mathbf{r} / \mathbf{R}$ & $\mathbf{c} / \mathbf{D}$ & $\mathbf{P} / \mathbf{D}$ & Skew & Rake & $\mathbf{t}_{\text {max }} / \mathbf{c}$ & $\mathbf{f}_{\text {max }} / \mathbf{c}$ \\
\hline 0.2 & 0.3200 & 1.1050 & 0 & 0 & 0.2055 & 0.0143 \\
0.3 & 0.3635 & 1.1022 & 0 & 0 & 0.1553 & 0.0232 \\
0.4 & 0.4048 & 1.0983 & 0 & 0 & 0.1180 & 0.023 \\
0.5 & 0.4392 & 1.0932 & 0 & 0 & 0.0916 & 0.0218 \\
0.6 & 0.4610 & 1.0879 & 0 & 0 & 0.0696 & 0.0207 \\
0.7 & 0.4622 & 1.0839 & 0 & 0 & 0.0542 & 0.0200 \\
0.8 & 0.4347 & 1.0811 & 0 & 0 & 0.0421 & 0.0197 \\
0.9 & 0.3613 & 1.0785 & 0 & 0 & 0.0332 & 0.0182 \\
0.95 & 0.2775 & 1.0770 & 0 & 0 & 0.0323 & 0.0163 \\
1 & 0.0800 & 1.0750 & 0 & 0 & 0.0316 & 0.0118 \\
\hline
\end{tabular}

$\mathrm{P}$ is propeller pitch, $\mathrm{r}$ is radius of each section, $\mathrm{R}$ is propeller radius, $\mathrm{c}$ is chord of each section, $\mathrm{D}$ is propeller diameter, $\mathrm{t}_{\max }$ is Maximum thickness of each section and $\mathrm{f}_{\max }$ is maximum camber. For the validation of the results of numerical modeling for hydrodynamics and noise of DTMB 4119 propeller, some experiments were carried out with a model propeller in a cavitation tunnel of the Marine Engineering Laboratory of Sharif University of Technology (Figure 4). For measuring the thrust and torque of propeller model, a H29 dynamometer has been used in this tunnel. 


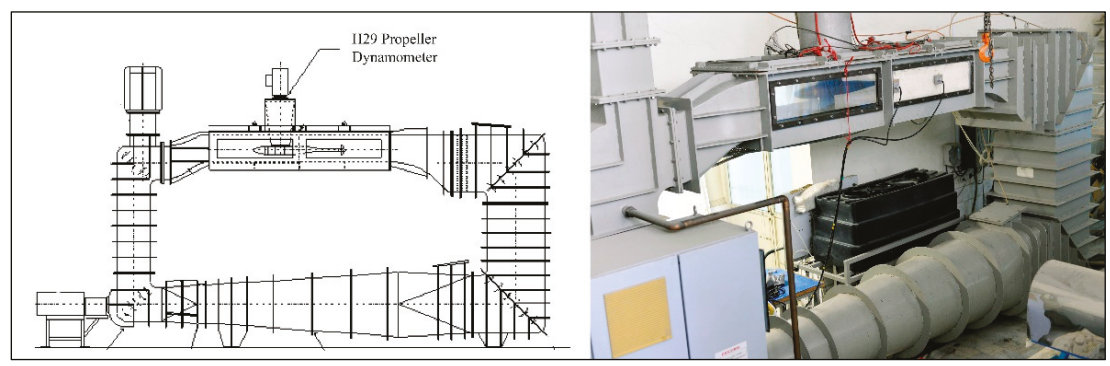

Figure 4. Cavitation Tunnel of Sharif University of Technology.

The developed code can also create the geometry of the propeller and generate mesh for it. For this purpose, the profile of propeller blades, the number of blades, the rake and skew angle and the desired grid size should be specified as input. In the panel method, it is not common to specify mesh dimensions. Because of the cosine spacing of panels in chordwise and spanwise directions, the exact size of panels cannot be specified. Grid size is shown as $m \times n$ which $m$ and $n$ are number of elements in chordwise and spanwise, respectively. For optimization of the grid size, thrust coefficient for design advance ratio $(J=0.833)$ is calculated for some different grids, and error of numerical results compared with experimental results for each grid size are presented in Table 3 and Figure 5. All simulations are carried out using Intel Core i7 2.6 GHz and 8 GB of RAM. Also, the CPU times for the panel method are given in Table 3.

Table 3. CPU Time and error for different grids $(J=0.833)$.

\begin{tabular}{ccccc}
\hline Grid & Numerical Result & Experimental Result & Error (\%) & CPU Time (sec.) \\
\hline $10 \times 10$ & 0.128 & & 14.6 & 242 \\
$10 \times 15$ & 0.132 & 0.15 & 12 & 521 \\
$20 \times 20$ & 0.144 & & 4 & 1224 \\
$30 \times 30$ & 0.146 & & 2.6 & 2545 \\
\hline
\end{tabular}

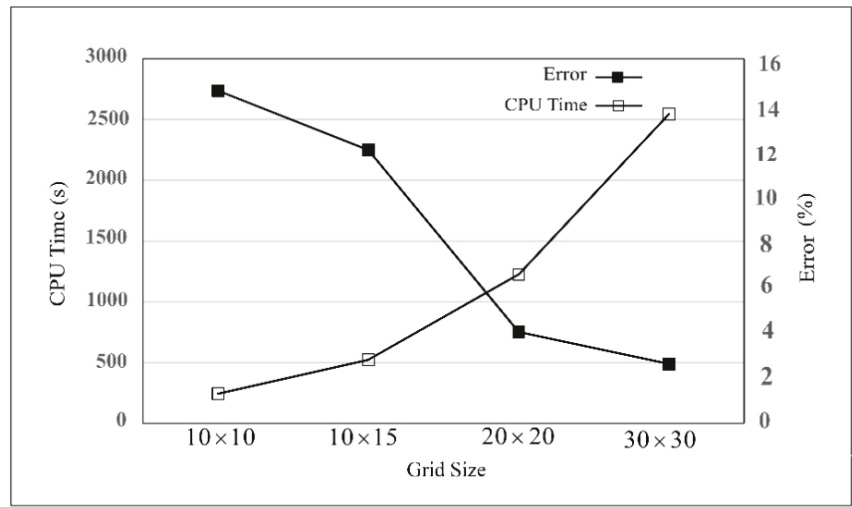

Figure 5. CPU Time and error for different grids $(J=0.833)$.

It can be seen from Figure 5 that the grid size of $20 \times 20$ compared to other grids has more appropriate CPU time and error, and then the grid is used to simulate propeller. After simulation of flow around DTMB 4119 propeller, wake surface and hydrodynamic coefficients of propeller (Thrust, torque and efficiency) are obtained. In Figure 6, the wake surface of the propeller is shown for different advance ratios. 


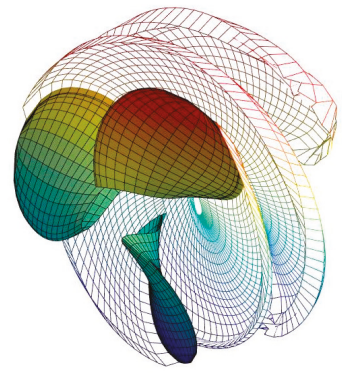

$(J=0.5)$

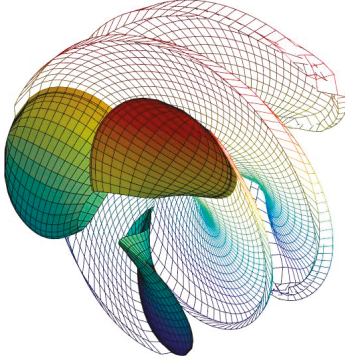

$(J=0.7)$

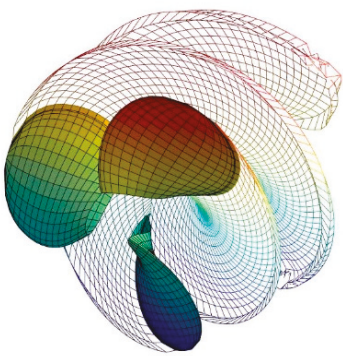

$(J=0.833)$

Figure 6. Wake surface of propeller for different advance ratios.

As shown in Figure 6, in low advance ratios which ship speed is low and propeller load is high (heavy duty conditions of propeller), disks of wake surface are closer together and wake rollup is intense but in higher advance ratios, disks of wake surface are more spacious and rollup is very low. Numerical simulation of propeller is performed by developed code for various advance ratios and Figure 7 compares the hydrodynamic coefficients of the propeller obtained by the panel method with experimental data.

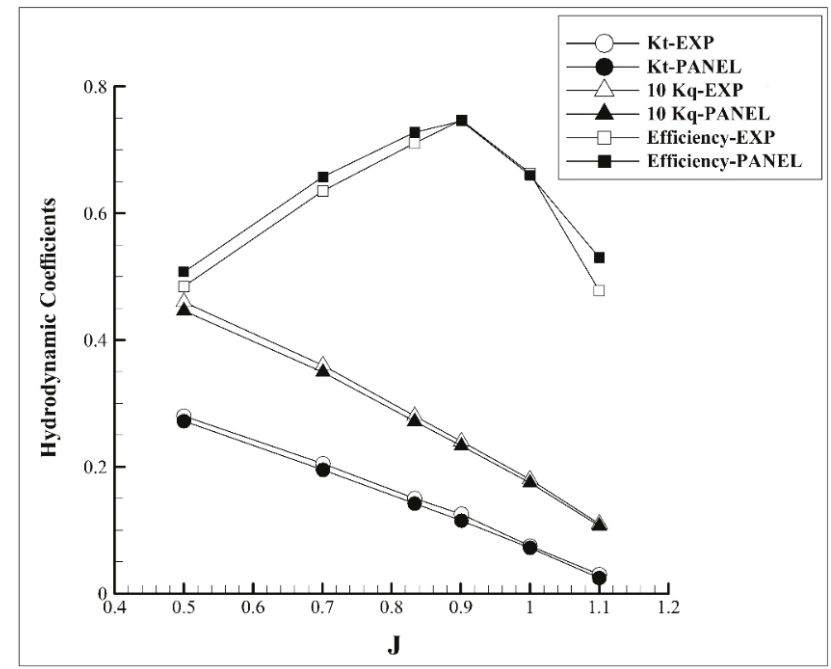

Figure 7. Hydrodynamic coefficients of DTMB 4119 propeller.

The pressure distribution in the advance ratio of $J=0.833$ for different radial sections of the propeller blade is compared with experimental measurements [21] in Figure 8. 


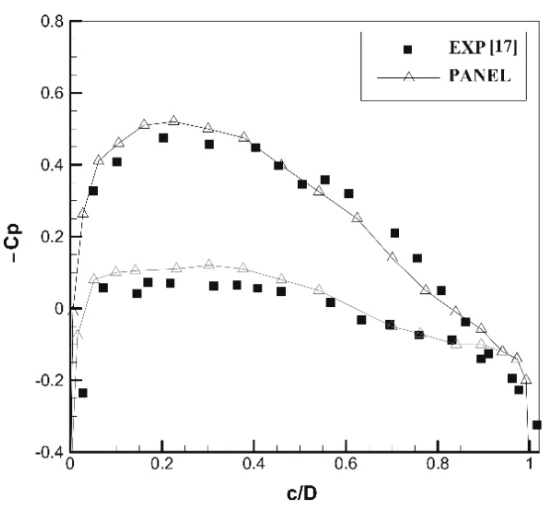

(a)

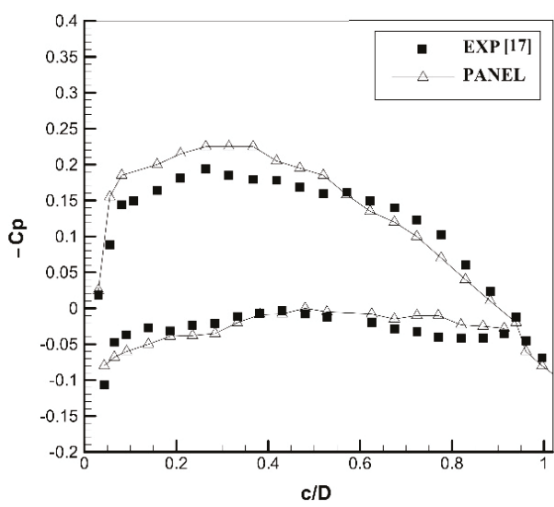

(b)

Figure 8. Experimental and panel method results for pressure distribution at (a) $r / R=0.3$ and (b) $r / R=0.7$.

According to Figure 7, there is satisfactory agreement between the hydrodynamic coefficients obtained from the panel method and experimental results. The maximum error of the trust coefficient calculated by the panel method is about $4 \%$ and for torque coefficient is about $7 \%$. As can be seen in Figure 8, pressure distribution obtained from the panel method is in good agreement with the experimental data. This shows that the accuracy of the panel method results is acceptable. One reason for the greater error of the torque coefficient is the use of empirical formulas in the calculation of the friction resistance of each panel. According to the above results, the panel method can be used for hydrodynamic performance analysis of marine propellers.

\section{Acoustic Formulations}

In most acoustic problems, rigid bodies located in the fluid flow have very important effects on the generated noise. For this reason, Williams and Hawkings [22] in 1969 developed the Lighthill equations [23] with the assumption of rigid bodies. The equations presented by these researchers in acoustic science are known as the FW-H model. For solving the FW-H equation, Farassat et al. [24] presented a method that can predict the generated noise of moving object with arbitrary geometry. According to this formulation, total sound pressure can $\left(p^{\prime}\right)$ expressed as below [24]:

$$
p^{\prime}(\vec{x}, t)=p_{T}^{\prime}(\vec{x}, t)+p_{L}^{\prime}(\vec{x}, t)
$$

where $p^{\prime}{ }_{T}$ and $p^{\prime}{ }_{L}$ denote the thickness and loading pressure, respectively. Thickness pressure is due to the fluid displacement caused by movement of the body within the fluid and classified as monopole noise source. Another phenomenon caused by the motion of the non-symmetric body in fluid is the distribution of positive and negative pressure on the face and back of the body. In fact, this pressure distribution is the main source of trust force in propellers. Pressure difference on both sides of the body create a dipole noise source which is referred as loading pressure. This solution is obtained with assumption of low Mach number, and hence the quadrupole noise can be neglected. These components are calculated through the following relations [24]:

$$
\begin{aligned}
& 4 \pi p^{\prime} T(\vec{x}, t)=\int_{S}\left[\frac{\rho\left(\dot{v}_{n}+v_{\dot{n}}\right)}{r\left(1-M_{r}\right)^{2}}\right]_{r e t} d S+\int_{S}\left[\frac{\rho v_{n}\left(r \dot{M}_{r}+c M_{r}-c M^{2}\right)}{r^{2}\left(1-M_{r}\right)^{3}}\right]_{r e t} d S \\
& 4 \pi p^{\prime}(\vec{x}, t)=\frac{1}{c} \int_{S}\left[\frac{\dot{L}_{r}}{r\left(1-M_{r}\right)^{2}}\right]_{r e t} d S+\frac{1}{c} \int_{S}\left[\frac{L_{r}-L_{M}}{r^{2}\left(1-M_{r}\right)^{2}}\right]_{r e t} d S \\
&+\frac{1}{c} \int_{S}\left[\frac{L_{r}\left(r \dot{M}_{r}+c M_{r}-c M^{2}\right)}{r^{2}\left(1-M_{r}\right)^{3}}\right]_{r e t} d S
\end{aligned}
$$


where $\rho$ is fluid density in $\mathrm{kg} / \mathrm{m}^{3}, c$ is sound speed in $\mathrm{m} / \mathrm{s}, \vec{v}$ is the noise source velocity in $\mathrm{m} / \mathrm{s}, \vec{r}$ is distance vector from noise source to receiver in $m, r$ is magnitude of $\vec{r}$ in $m$, Mach number is $\vec{M}=\vec{v} / c$, $L=p \cdot \vec{n}$ is pressure force in $N$ and $p$ is hydrodynamic pressure in $P a$. The dot over variables means time derivate of the variable. The ret subscript in this equation means that the calculations must be done using values at the retarded time.

In the first step of noise calculation, the flow around the body is simulated using the panel method and flow quantities such as pressure and velocity are obtained for panels on the body surface. Then, for receiver in position of $\vec{x}$ and time of $t_{i}$, retarded time $\left(\tau_{i, j}\right)$ is obtained for panel $j$. After this step, all quantities required in Equation (20) must be calculated for each panel at the retarded time, numerical integrals of the equation are solved for each panel, and the acoustic pressure of each element is obtained. By summing up the acoustic pressures of all the elements, a total acoustic pressure is obtained for the specified receiver. Flowchart of noise calculation using FW-H equations is shown in Figure 9.

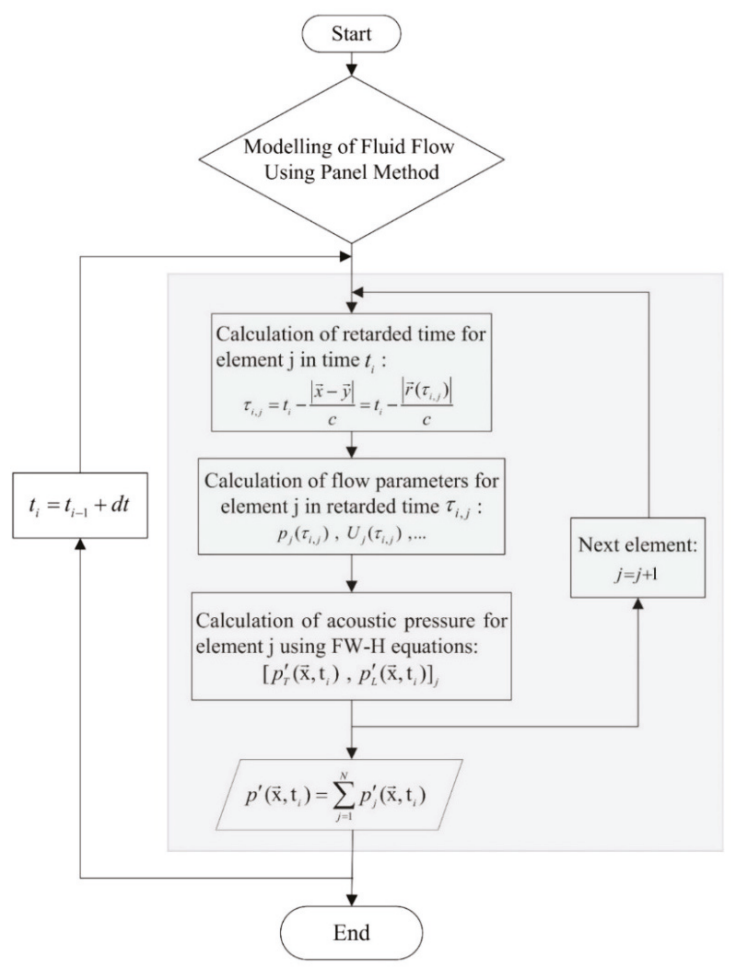

Figure 9. Flowchart of noise calculation using FW-H equations.

\section{Noise of DTMB 4119 Propeller}

The noise generated by the propeller is one of the most important components of the vessels noise [6]. Therefore, measuring and calculating the propeller noise is one of the interesting research subjects in naval engineering. In the first step, the results of the developed FW-H code for noise calculation should validated using experimental results obtained in cavitation tunnel. For measuring the noise of propeller, a TC4042 hydrophone has been used in cavitation tunnel of Sharif University of Technology. The arrangement of this validation hydrophone is shown in Figure 10. Also, the location of this hydrophone is given in Figure 11. 


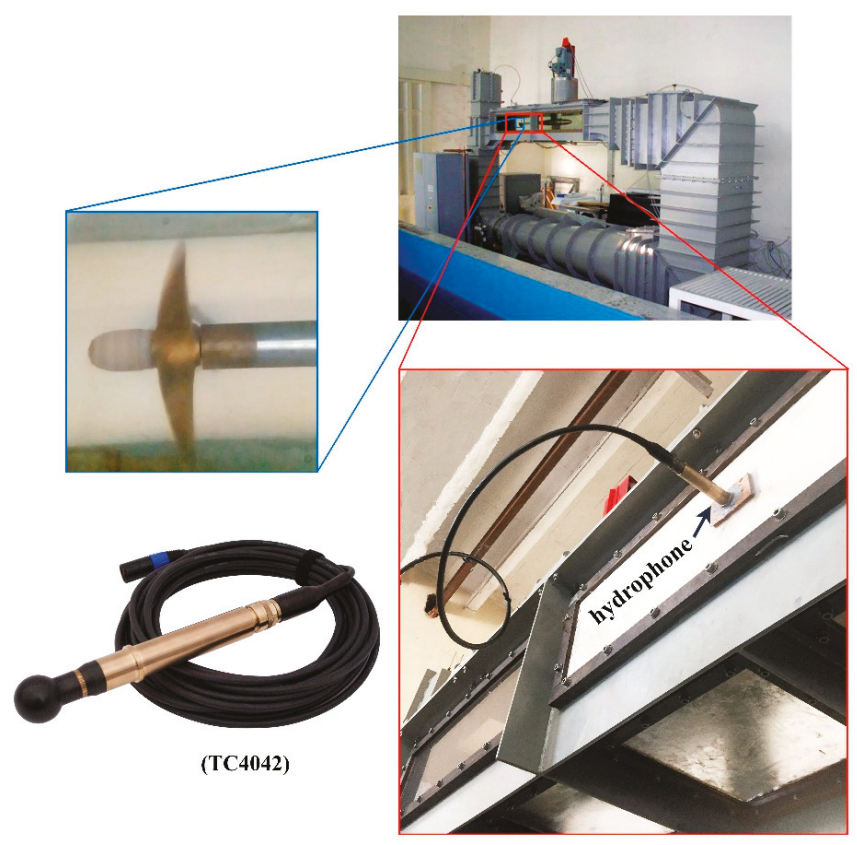

Figure 10. Arrangement of TC4042 hydrophone on cavitation tunnel.

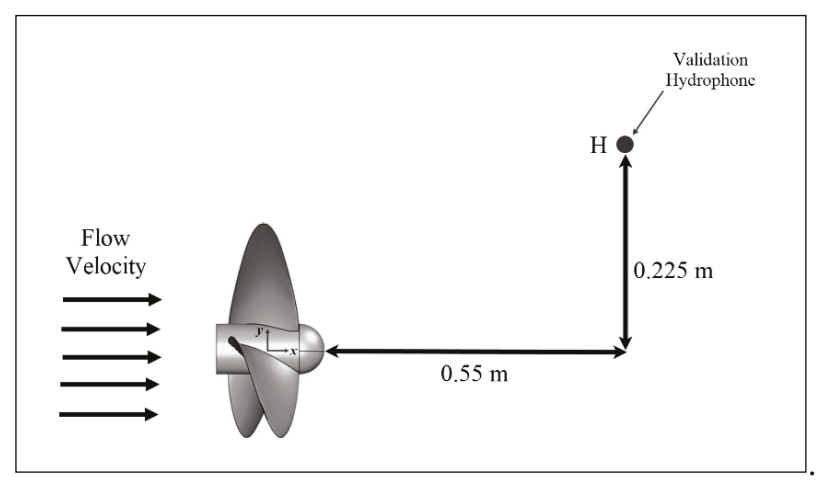

Figure 11. Location of validation hydrophone.

For validation of results of FW-H code, noise measurements done in cavitation tunnel under below conditions:

- $\quad$ Flow velocity: $2.2 \mathrm{~m} / \mathrm{s}$

- $\quad$ Propeller RPM: 792

- $\quad$ Advance ratio $(J): 0.833$

The time history of acoustic pressure as measured by validation hydrophone is shown in Figure 12. 


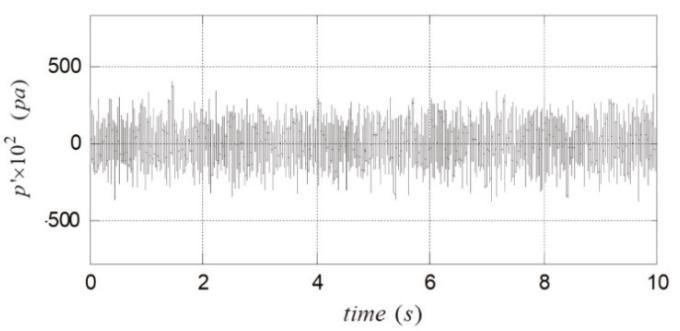

Figure 12. Time history of acoustic pressure measured by validation hydrophone.

In the post-processing stage, acoustic pressure from time domain in transferred to frequency domain by using the fast Fourier transform (FFT) and the sound pressure level is calculated using the following formula [25]:

$$
\operatorname{SPL}(\vec{x}, t)=20 \log _{10}\left(p^{\prime} / p_{\text {ref }}\right)
$$

where $p_{r e f}$ is the reference pressure and related to threshold of a normal human hearing for frequency of $1 \mathrm{kHz}$ which for water is $1 \mu \mathrm{Pa}$ [26]. The numerical results of noise obtained by own developed code compared by experimental results in Figure 13. Propeller and flow conditions are the same as the experimental setup.

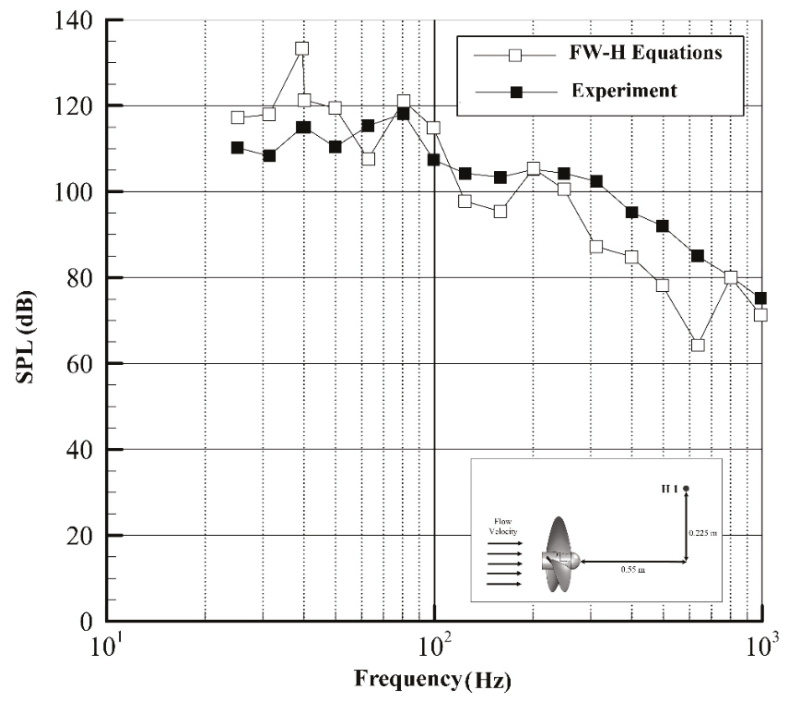

Figure 13. Validation of FW-H code results.

It can be seen that there is good agreement between the numerical and experimental results of propeller noise, especially in frequencies under $250 \mathrm{~Hz}$. It seems that main sources of error of numerical results are:

- $\quad$ Error of panel method code for calculation of pressure and flow velocity: As mentioned in the previous sections, the results of the thrust and torque of the panel method have $4 \%$ and $7 \%$ error in comparison with the experimental results. Since the FW-H equations use panel method outputs, the above error causes an error in calculating the noise of the impeller.

- The error of FW-H code for noise calculation: FW-H equations include some integrals that are calculated numerically on the source of noise. Therefore, the numerical calculation error of these 
equations can cause error in the value of noise. Also, in the solution presented in this study, the quadrupole sources of noise are neglected, which could cause a slight error in the value of propeller noise.

- The reflection of sound inside the cavitation tunnel is another reason for the difference between the experimental and numerical results. The restricted space of the cavitation tunnel causes the reverberation of sound emitted by the propeller, which results in an error in the measured noise values. For investigating the effects of sound reverberation on noise data, special equipment is needed which is not available in our laboratory.

Seol et al. [27] and Yang et al. [28] carried out some numerical studies on DTMB 4119 propeller, but since the specifications of the propeller used are not the same as the propeller model in our laboratory, the results cannot be compared.

By looking more precisely into results, it founds that both experimental and numerical results have peaks in some frequencies. It seems that these are Blade Passing Frequencies (BPF) of propeller. For the propeller, the rate at which the blades pass by a fixed position is called the BPF. This frequency is known as the blade passing frequency $(B P F)$ of the blade. As regards the propeller, the BPF and its higher harmonics can be calculated as:

$$
B P F=Z \times r p s \times h(\mathrm{~Hz})
$$

where $\mathrm{Z}$ is number of propeller blades, $r p s$ is the frequency of propeller rotation in $\mathrm{Hz}$ and $h$ is no of harmonic. When receiver is located in an asymmetric location relative to blades, the noise of propeller in BPFs will have local peaks in comparison to other frequencies. For the receiver, which is located in the hub plane of the propeller, at an equal and constant distance from the blades at all times, BPF could not be seen in the SPL diagram. For noise diagrams in Figure 13, rps is 13.2 and $Z$ is 3 . Therefore, BPF for three first harmonics of propeller is about $39.6,79.2$ and $118.8 \mathrm{~Hz}$ and noise results have peaks in the first two frequencies.

After the validation of FW-H code, propeller noise calculations were made for four hydrophones (receivers). Two hydrophones located in the propeller rotation plane and two hydrophones located in front of propeller hub. The location of hydrophones and their coordinates are given in Figure 14 and Table 4.

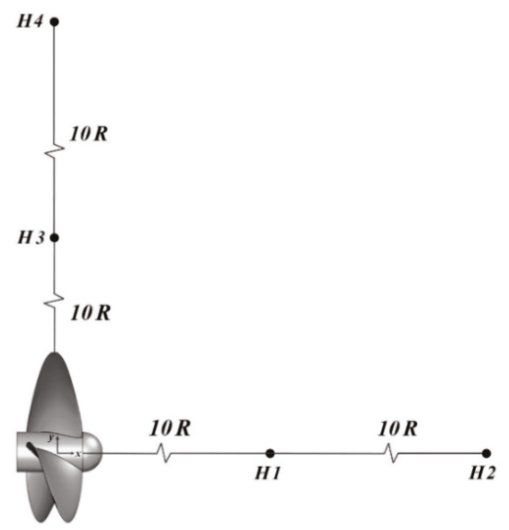

Figure 14. Location of hydrophones. 
Table 4. Coordinates of hydrophones.

\begin{tabular}{cccc}
\hline & $X(m)$ & $Y(m)$ & $Z(m)$ \\
\hline H1 & 1 & 0 & 0 \\
H2 & 2 & 0 & 0 \\
H3 & 0 & 1 & 0 \\
H4 & 0 & 2 & 0 \\
\hline
\end{tabular}

The noise of the propeller as calculated by the FW-H code for hydrophones $\mathrm{H} 1$ and $\mathrm{H} 2$ are presented in Figure 15. Noise predictions were made at a flow speed of $2.2 \mathrm{~m} / \mathrm{s}$ and RPM of 792 where $J=0.833$.

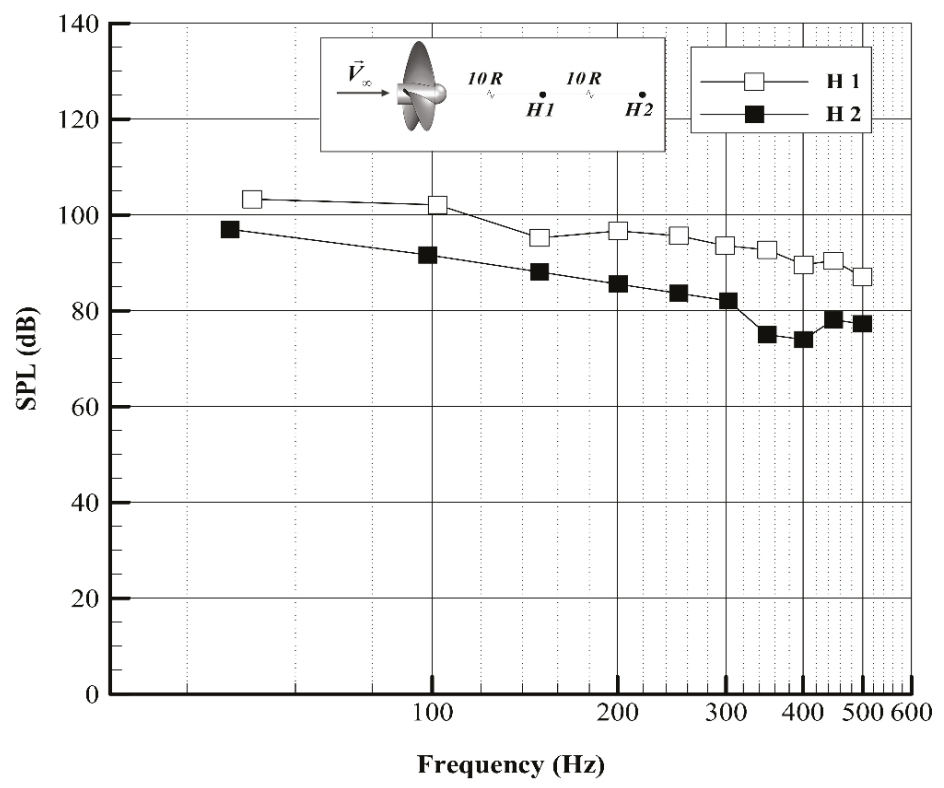

Figure 15. Sound pressure level of propeller in hydrophones $\mathrm{H} 1$ and $\mathrm{H} 2$.

Results shown in Figure 15 reveals that by increasing the distance of hydrophone from propeller, noise of $\mathrm{H} 2$ decreased significantly in comparison with H1. Also, in Figure 16, the propeller noise in hydrophones $\mathrm{H} 3$ and $\mathrm{H} 4$ are presented. 


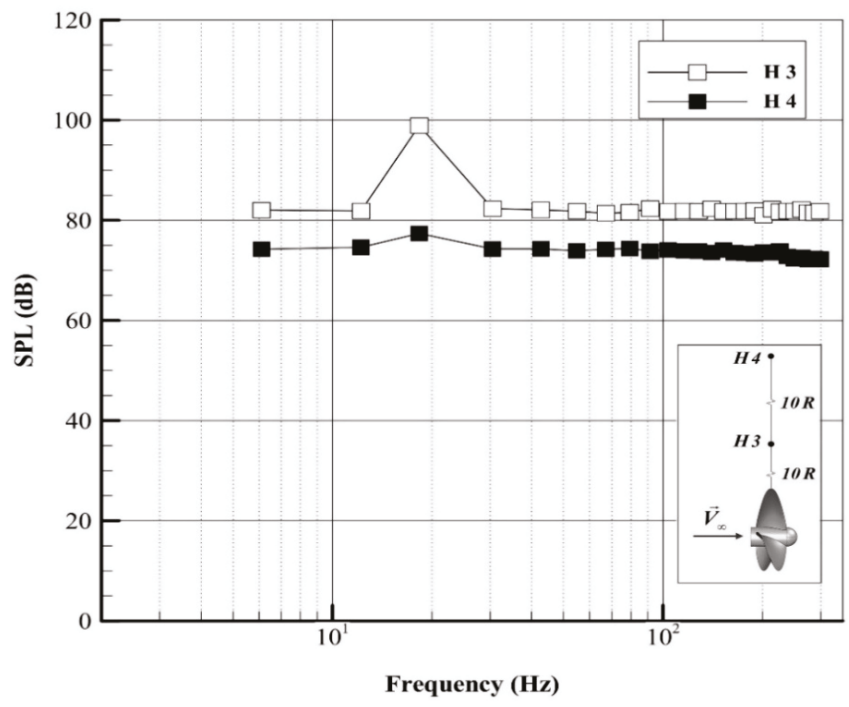

Figure 16. Sound pressure level of propeller in hydrophones $\mathrm{H} 3$ and $\mathrm{H} 4$.

As can be seen in Figure 16, with an increase of distance from the propeller, noise decreased significantly. Also, in both hydrophones, at a blade passing frequency of $18.22 \mathrm{~Hz}$, the noise has a peak value which is also indicated in the Figure 16. By comparing Figures 15 and 16, it can be seen that the SPL in front of the propeller hub is higher than the SPL in the propeller rotational plane.

\section{Conclusions}

Measuring and calculating the noise of marine propellers is very important. In this research, the combination of the panel method and FW-H equations has been used to calculate the non-cavitating noise of marine propellers. In the first step, a numerical panel method code is developed to solve the flow around the propeller and calculate the propeller hydrodynamic coefficients. The results of this code in comparison with the experimental results had maximum error of $4 \%$ and $7 \%$ for thrust and torque coefficients, respectively.

Then, a noise calculation code based on FW-H acoustic equations was developed, which uses the outputs of the panel method code as inputs and calculates the noise of the propeller for the desired receiver. The results of this code are in good agreement with the noise of the propeller obtained using results from a test carried out in cavitation tunnel of Sharif University. Using this code, the propeller noise is measured for four hydrophones with different distances and orientations. With increasing distance from the propeller, the noise of the propeller has been significantly reduced. For the hydrophones located on the rotation plane of the propeller, as predicted by theory and experiments on propeller noise, the maximum noise has occurred at the blade passing frequency. The results show that the SPL in front of the propeller hub is higher than SPL in the propeller rotational plane.

According to the above results, the combination of the panel method and FW-H equations can be used for hydrodynamic and acoustic analysis of marine propellers.

Author Contributions: All authors contributed equally in this work.

Funding: This research received no external funding.

Conflicts of Interest: The authors declare no conflict of interest. 


\section{References}

1. Hess, J.L.; Smith, A.M.O. Calculation of Nonlifting Potential Flow about Arbitrary Three-Dimensional Bodies; Douglas Aircraft Co.: Long Beach, CA, USA, 1962.

2. Hess, J.L. Calculation of Potential Flow about Arbitrary Three-Dimensional Lifting Bodies; Douglas Aircraft Co.: Long Beach, CA, USA, 1972.

3. Hess, J.; Valarezo, W. Calculation of steady flow about propellers by means of a surface panel method. J. Propuls. Power 1985, 1, 470-476. [CrossRef]

4. Baltazar, J.M.R.D.C.; De Campos, J.A.C.F. An iteratively coupled solution of the cavitating flow on marine propellers using BEM. J. Hydrodyn. 2010, 22, 796-801. [CrossRef]

5. Gaggero, S.; Villa, D.; Brizzolara, S. RANS and PANEL method for unsteady flow propeller analysis. J. Hydrodyn. 2010, 22, 547-552. [CrossRef]

6. Ebrahimi, A.; Razaghian, A.; Seif, M.; Zahedi, F.; Nouri-Borujerdi, A. A comprehensive study on noise reduction methods of marine propellers and design procedures. Appl. Acoust. 2019, 150, 55-69. [CrossRef]

7. Seol, H.; Park, C. Numerical and Experimental Study on the Marine Propeller Noise. In Proceedings of the 19th International Congress on Acoustics, Madrid, Spain, 2-7 September 2007; pp. 2-7.

8. Kellett, P.; Turan, O.; Incecik, A. A study of numerical ship underwater noise prediction. Ocean Eng. 2013, 66, 113-120. [CrossRef]

9. Bagheri, M.; Seif, M.; Mehdigholi, H. Numerical Simulation of underwater propeller noise. J. Ocean Mech. Aerosp. 2014, 4, 4-9.

10. Testa, C.; Ianniello, S.; Salvatore, F. A Ffowcs Williams and Hawkings formulation for hydroacoustic analysis of propeller sheet cavitation. J. Sound Vib. 2018, 413, 421-441. [CrossRef]

11. Gorji, M.; Ghassemi, H.; Mohamadi, J. Calculation of sound pressure level of marine propeller in low frequency. J. Low Freq. Noise Vib. Act. Control. 2018, 37, 60-73. [CrossRef]

12. Bagheri, M.R.; Seif, M.S.; Mehdigholi, H.; Yaakob, O. Analysis of noise behaviour for marine propellers under cavitating and non-cavitating conditions. Ships Offshore Struct. 2017, 12, 1-8. [CrossRef]

13. Katz, J.; Plotkin, A. Low-Speed Aerodynamics; Cambridge University Press: Cambridge, UK, 2001.

14. Politis, G. Unsteady Wake Rollup Modeling Using a Mollifier Based Filtering Technique. Dev. Appl. Ocean. Eng. 2016, 5, 1. [CrossRef]

15. Delhommeau, G. Amélioration des performances des codes de calcul de diffraction-radiation au premier ordre. In Proceedings of the 2nd Journées de l'Hydrodynamique, Nantes, France, 13-15 February 1989.

16. Morino, L.; Kuo, C. Subsonic potential aerodynamics for complex configurations: A general theory. AIAA J. 1974, 12, 191-197.

17. Politis, G.K. Unsteady Rollup Modeling for Wake-Adapted Propellers Using a Time-Stepping Method. J. Ship Res. 2005, 49, 216-231.

18. Blevins, R.D. Applied Fluid Dynamics Handbook; Van Nostrand Reinhold Co.: New York, NY, USA, 1984; p. 568.

19. Najafi, S.; Abbaspoor, M. Numerical investigation of flow pattern and hydrodynamic forces of submerged marine propellers using unsteady boundary element method. Proc. Inst. Mech. Eng. Part M J. Eng. Marit. Environ. 2017, 233, 67-79. [CrossRef]

20. Boumediene, K.; Belhenniche, S. Numerical Analysis of the Turbulent Flow around DTMB 4119 Marine Propeller. Int. J. Mech. Aerosp. Ind. Mechatron. Manuf. Eng. 2016, 10, 347-351.

21. Jessup, S. An Experimental Investigation of Viscous Aspects of Propeller Blade Flow. Ph.D. Thesis, The Catholic University of America, Washington, DC, USA, 1989.

22. Williams, J.E.F.; Hawkings, D.L. Sound Generation by Turbulence and Surfaces in Arbitrary Motion. Philos. Trans. R. Soc. A Math. Phys. Eng. Sci. 1969, 264, 321-342. [CrossRef]

23. Lighthill, M.J. On sound generated aerodynamically II. Turbulence as a source of sound. Proc. R. Soc. Lond. Ser. A. Math. Phys. Sci. 1954, 222, 1-32.

24. Farassat, F. Derivation of Formulations 1 and 1 A of Farassat; NASA Langley Research Center: Hampton, VA, USA, 2007.

25. Kinsler, L.A.; Frey, A.R.; Coppens, A.B.; Sanders, J.V. Fundamentals of Acoustics; John Wiley \& Sons: New York, NY, USA, 2000.

26. Council, N.R. Low-Frequency Sound and Marine Mammals: Current Knowledge and Research Needs; National Academies Press: Washington, DC, USA, 1994. 
27. Seol, H.; Suh, J.-C.; Lee, S. Development of hybrid method for the prediction of underwater propeller noise. J. Sound Vib. 2005, 288, 345-360. [CrossRef]

28. Yang, Q.; Wang, Y.; Zhang, M. Calculation of propeller's load noise using LES and BEM numerical acoustics coupling methods. WIT Trans. Model. Simul. Bound. Elem. Other Reduct. Methods XXXIII 2011, 52, 85-97.

(C)

(C) 2019 by the authors. Licensee MDPI, Basel, Switzerland. This article is an open access article distributed under the terms and conditions of the Creative Commons Attribution (CC BY) license (http://creativecommons.org/licenses/by/4.0/). 


\title{
Natural Frequency Analysis of Functionally Graded Orthotropic Cross-Ply Plates Based on the Finite Element Method
}

\author{
Michele Bacciocchi ${ }^{1,2, *}$ and Angelo Marcello Tarantino ${ }^{3}$ \\ 1 DICAM Department, University of Bologna, Viale del Risorgimento, 2, 40136 Bologna BO, Italy \\ 2 DESD Department, University of San Marino, Via Consiglio dei Sessanta, 99, 47891 Repubblica Di San \\ Marino, San Marino \\ 3 DIEF Department, University of Modena and Reggio Emilia, Via Vivarelli, 10, 41125 Modena MO, Italy; \\ angelomarcello.tarantino@unimore.it \\ * Correspondence: michele.bacciocchi@unibo.it or michele.bacciocchi@unirsm.sm; Tel.: +39-051-209-3494
}

Received: 30 April 2019; Accepted: 17 May 2019; Published: 19 May 2019

\begin{abstract}
This paper aims to present a finite element (FE) formulation for the study of the natural frequencies of functionally graded orthotropic laminated plates characterized by cross-ply layups. A nine-node Lagrange element is considered for this purpose. The main novelty of the research is the modelling of the reinforcing fibers of the orthotropic layers assuming a non-uniform distribution in the thickness direction. The Halpin-Tsai approach is employed to define the overall mechanical properties of the composite layers starting from the features of the two constituents (fiber and epoxy resin). Several functions are introduced to describe the dependency on the thickness coordinate of their volume fraction. The analyses are carried out in the theoretical framework provided by the first-order shear deformation theory (FSDT) for laminated thick plates. Nevertheless, the same approach is used to deal with the vibration analysis of thin plates, neglecting the shear stiffness of the structure. This objective is achieved by properly choosing the value of the shear correction factor, without any modification in the formulation. The results prove that the dynamic response of thin and thick plates, in terms of natural frequencies and mode shapes, is affected by the non-uniform placement of the fibers along the thickness direction.
\end{abstract}

Keywords: finite element modelling; laminated composite plates; non-uniform mechanical properties

\section{Introduction}

The finite element (FE) method currently represents the most-utilized computational approach to solve several engineering problems and in applications whose solutions cannot be obtained analytically [1]. The technological advancements in computer sciences have allowed a fast and easy diffusion of this technique, especially in terms of structural mechanics problems. The key to the success of the FE method lies in the reduction of complex problems into simpler ones in which the reference domain is made of several discrete elements, and in its easy computational implementation. This idea was first highlighted by Duncan and Collar [2,3], and successively emphasized by Hrennikoff [4], Courant [5], Clough [6], and Melosh [7].

The approximate solutions that can be obtained by means of the FE approach are accurate and representative of the physical problem under consideration [8,9]. To the best of the authors' knowledge, the progression and development of this technique are well-described in many pertinent books, such as the ones by Oden [10], Oden and Reddy [11], Hinton [12], Zienkiewicz [13], Reddy [14], Onate [15], Hughes [16], and Ferreira [17]. These books should be used as references for the theoretical background of the numerical approach at issue. For completeness purposes, it should be recalled that various and 
alternative approaches have been developed in past decades to obtain approximated but accurate solutions to several complex structural problems, not only based on the FE method [18-21].

An intriguing application that is efficiently solved by means of the FE methodology is about the structural response of plates and panels made of composite materials [22-24]. With respect to an isotropic and conventional medium, a composite material can reach superior performance by combining two (or more) constituents. A typical example of this category are fiber-reinforced composites, in which the high-strength fibers are the main load-carrying elements, whereas the matrix has the task of keeping them together and protecting the reinforcing phase from the environment [25-28]. In general, a micromechanical approach should be employed to evaluate the overall mechanical properties of these materials, starting from the features of the single constituents. The review paper by Chamis and Sendeckyj represents a fundamental contribution is this direction [29]. One of the most effective approaches that can be used toward this aim is the one proposed by Halpin [30] and Tsai [31,32], who developed a semi-empirical method and expressed the mechanical properties of the constituents in terms of Hill's elastic moduli [33,34]. Further details concerning the micromechanics of fiber-reinforced composite materials can be found in [35].

The use of a versatile numerical method also allows us to investigate the structural response of composite structures with non-uniform mechanical properties. In particular, in the present paper the reinforcing fibers are characterized by a gradual variation of their volume fraction along the plate thickness, following the same idea of functionally graded materials [36-52]. With respect to this class of materials, in which the composites turn out to be isotropic, the layers of the plate assume orthotropic features and can also be oriented. This topic clearly falls within the aim of the optimal design of composite structures [53-58]. It should be mentioned that a similar approach is followed in the design of functionally graded carbon-nanotube-reinforced composites, due to the advancements in nanostructures and nanotechnologies [59-68].

In this paper, the research is organized in two main sections. After this brief introduction, the FE formulation for laminated thick and thin plates is presented in Section 2. Here, the theoretical framework is based on the well-known first-order shear deformation theory (FSDT) for laminated composite structures $[69,70]$. The effect of the shear correction factor is also discussed in order to deal with thin plates [71]. In addition, the micromechanics approach based on the Halpin-Tsai model is described in detail, by also introducing the topic of variable mechanical properties. Section 3 presents the results of the numerical applications. As a preliminary test, the accuracy and convergence features of the numerical approach are discussed by means of the comparison with the semi-analytical solutions available in the literature for thin and thick laminated composite plates. Then, the natural frequencies of functionally graded orthotropic cross-ply plates are presented for several mechanical configurations. Finally, Appendix A is added to define the terms of the fundamental operators of the proposed FE formulation.

\section{Finite Element (FE) Formulation for Laminated Thick and Thin Plates}

The theoretical framework of the current research is based on the first-order shear deformation theory (FSDT). The governing equations are presented in this section by developing the corresponding FE formulation. The following kinematic model is assumed within each discrete element of the plate [69]:

$$
\begin{aligned}
& U_{x}^{(e)}(x, y, z, t)=u_{x}^{(e)}(x, y, t)+z \phi_{x}^{(e)}(x, y, t) \\
& U_{y}^{(e)}(x, y, z, t)=u_{y}^{(e)}(x, y, t)+z \phi_{y}^{(e)}(x, y, t) \\
& U_{z}^{(e)}(x, y, z, t)=u_{z}^{(e)}(x, y, t)
\end{aligned}
$$

where $U_{x}^{(e)}, U_{y}^{(e)}, U_{z}^{(e)}$ are the three-dimensional displacements of the structure, whereas the degrees of freedom of the problem are given by three translations $u_{x}^{(e)}, u_{y}^{(e)}, u_{z}^{(e)}$ and two rotations $\phi_{x}^{(e)}, \phi_{y}^{(e)}$ defined 
on the plate middle surface. These quantities can be conveniently collected in the corresponding vector $\mathbf{u}^{(e)}$, defined below

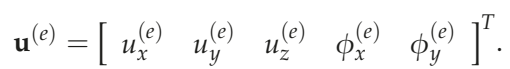

The coordinates $x, y, z$ specify the local reference system of the plate and $t$ is the time variable. The superscript $(e)$ clearly specifies that this model is valid for each element. The geometry of the plate is fully described once the lengths $L_{x}, L_{y}$ of its sides and its overall thickness $h$ are defined. It should be recalled that for a laminate structure one gets

$$
h=\sum_{k=1}^{N_{L}}\left(z_{k+1}-z_{k}\right),
$$

in which $z_{k+1}, z_{k}$ stand for the upper and lower coordinates of the $k$-th layer, respectively. The degrees of the freedom (2) are approximated in each element by means of quadratic Lagrange interpolation functions. As can be noted from Figure 1, nine nodes are introduced in each subdomain. As a consequence, the degrees of freedom assume the following aspect:

$$
\begin{aligned}
& u_{x}^{(e)}(x, y, t)=\sum_{i=1}^{9} N_{i}(x, y) u_{x, i}^{(e)}(t)=\overline{\mathbf{N}} \mathbf{u}_{x}^{(e)} \\
& u_{y}^{(e)}(x, y, t)=\sum_{i=1}^{9} N_{i}(x, y) u_{y, i}^{(e)}(t)=\overline{\mathbf{N}} \mathbf{u}_{y}^{(e)} \\
& u_{z}^{(e)}(x, y, t)=\sum_{i=1}^{9} N_{i}(x, y) u_{z, i}^{(e)}(t)=\overline{\mathbf{N}} \mathbf{u}_{z}^{(e)}, \\
& \phi_{x}^{(e)}(x, y, t)=\sum_{i=1}^{9} N_{i}(x, y) \phi_{x, i}^{(e)}(t)=\overline{\mathbf{N}} \phi_{x}^{(e)} \\
& \phi_{y}^{(e)}(x, y, t)=\sum_{i=1}^{9} N_{i}(x, y) \phi_{y, i}^{(e)}(t)=\overline{\mathbf{N}} \phi_{y}^{(e)}
\end{aligned}
$$

where $N_{i}$ represents the $i$-th shape function, whereas $u_{x, i}^{(e)}, u_{y, i}^{(e)}, u_{z, i}^{(e)}, \phi_{x, i}^{(e)}, \phi_{y, i}^{(e)}$ denote the nodal displacements, which can be included in the corresponding vectors

$$
\begin{gathered}
\mathbf{u}_{x}^{(e)}=\left[\begin{array}{ccc}
u_{x, 1}^{(e)} & \cdots & u_{x, 9}^{(e)}
\end{array}\right]^{T}, \mathbf{u}_{y}^{(e)}=\left[\begin{array}{lll}
u_{y, 1}^{(e)} & \cdots & u_{y, 9}^{(e)}
\end{array}\right]^{T}, \mathbf{u}_{z}^{(e)}=\left[\begin{array}{lll}
u_{z, 1}^{(e)} & \cdots & u_{z, 9}^{(e)}
\end{array}\right]^{T} . \\
\boldsymbol{\phi}_{x}^{(e)}=\left[\begin{array}{lll}
\phi_{x, 1}^{(e)} & \cdots & \phi_{x, 9}^{(e)}
\end{array}\right]^{T}, \boldsymbol{\phi}_{y}^{(e)}=\left[\begin{array}{lll}
\phi_{y, 1}^{(e)} & \cdots & \phi_{y, 9}^{(e)}
\end{array}\right]^{T}
\end{gathered}
$$

On the other hand, the shape functions linked to the nine nodes of the finite element are included in the vector $\overline{\mathbf{N}}$, defined below:

$$
\overline{\mathbf{N}}=\left[\begin{array}{lll}
N_{1} & \cdots & N_{9}
\end{array}\right] .
$$

For the sake of clarity, it should be recalled that the nodes are identified in each element by following the numbering specified in Figure 1.

At this point, the nodal degrees of freedom can be collected in a sole vector $\overline{\mathbf{u}}^{(e)}$ to simplify the nomenclature:

$$
\begin{aligned}
& \overline{\mathbf{u}}^{(e)}=\left[\begin{array}{lllll}
\mathbf{u}_{x}^{(e)} & \mathbf{u}_{y}^{(e)} & \mathbf{u}_{z}^{(e)} & \boldsymbol{\phi}_{x}^{(e)} & \boldsymbol{\phi}_{y}^{(e)}
\end{array}\right]^{T} \\
& =\left[\begin{array}{lllllllllllllll}
u_{x, 1}^{(e)} & \cdots & u_{x, 9}^{(e)} & u_{y, 1}^{(e)} & \cdots & u_{y, 9}^{(e)} & u_{z, 1}^{(e)} & \cdots & u_{z, 9}^{(e)} & \phi_{x, 1}^{(e)} & \cdots & \phi_{x, 9}^{(e)} & \phi_{y, 1}^{(e)} & \cdots & \phi_{y, 9}^{(e)}
\end{array}\right]^{T},
\end{aligned}
$$

and to write the definitions (4) by using the following matrix notation: 


$$
\left[\begin{array}{c}
u_{x}^{(e)} \\
u_{y}^{(e)} \\
u_{z}^{(e)} \\
\phi_{x}^{(e)} \\
\phi_{y}^{(e)}
\end{array}\right]=\left[\begin{array}{ccccc}
\overline{\mathbf{N}} & 0 & 0 & 0 & 0 \\
0 & \overline{\mathbf{N}} & 0 & 0 & 0 \\
0 & 0 & \overline{\mathbf{N}} & 0 & 0 \\
0 & 0 & 0 & \overline{\mathbf{N}} & 0 \\
0 & 0 & 0 & 0 & \overline{\mathbf{N}}
\end{array}\right]\left[\begin{array}{c}
\mathbf{u}_{x}^{(e)} \\
\mathbf{u}_{y}^{(e)} \\
\mathbf{u}_{z}^{(e)} \\
\boldsymbol{\phi}_{x}^{(e)} \\
\boldsymbol{\phi}_{y}^{(e)}
\end{array}\right] \Leftrightarrow \mathbf{u}_{5 \times 1}^{(e)}=\underset{5 \times(9 \times 5)(9 \times 5) \times 1}{\mathbf{N}} \underset{\overline{\mathbf{u}}}{(e)} .
$$

The size of each matrix is indicated under the corresponding symbol. It is important to specify that the same approximation is employed for all degrees of freedom (both translational and rotational displacements).

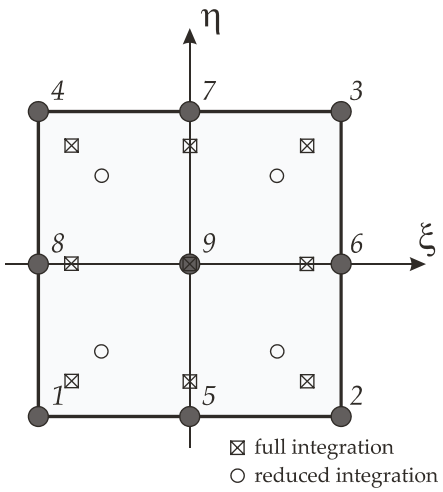

Figure 1. Nine-node quadratic Lagrange rectangular element.

As mentioned in the books by Reddy [14] and Ferreira [17], it is convenient to introduce the natural coordinates $\xi, \eta$ within the reference finite element, which is called the master element (or parent element). In this reference system, which is also depicted in Figure 1, the shape functions $N_{i}=N_{i}(\xi, \eta)$ assume the following definitions:

$$
\begin{array}{llc}
N_{1}=\frac{1}{4}\left(\xi^{2}-\xi\right)\left(\eta^{2}-\eta\right) & N_{2}=\frac{1}{4}\left(\xi^{2}+\xi\right)\left(\eta^{2}-\eta\right) & N_{3}=\frac{1}{4}\left(\xi^{2}+\xi\right)\left(\eta^{2}+\eta\right) \\
N_{4}=\frac{1}{4}\left(\xi^{2}-\xi\right)\left(\eta^{2}+\eta\right) & N_{5}=\frac{1}{2}\left(1-\xi^{2}\right)\left(\eta^{2}-\eta\right) & N_{6}=\frac{1}{2}\left(\xi^{2}+\xi\right)\left(1-\eta^{2}\right) \\
N_{7}=\frac{1}{2}\left(1-\xi^{2}\right)\left(\eta^{2}+\eta\right) & N_{8}=\frac{1}{2}\left(\xi^{2}-\xi\right)\left(1-\eta^{2}\right) & N_{9}=\left(1-\xi^{2}\right)\left(1-\eta^{2}\right)
\end{array}
$$

for $\xi, \eta \in[-1,1]$. The same functions are also used to describe the geometry of each discrete element according to the principles of the isoparametric FE formulation. The coordinate change between the physical domain and the parent element is accomplished through the relations shown below

$$
x^{(e)}=\sum_{i=1}^{9} N_{i}(\xi, \eta) x_{i}^{(e)}, y^{(e)}=\sum_{i=1}^{9} N_{i}(\xi, \eta) y_{i}^{(e)},
$$

where the couple $x_{i}^{(e)}, y_{i}^{(e)}$ defines the coordinates of the $i$-th node of the generic element. For the sake of conciseness, these quantities can be collected in the corresponding vectors $\mathbf{x}^{(e)}, \mathbf{y}^{(e)}$ :

$$
\mathbf{x}^{e}=\left[\begin{array}{lll}
x_{1}^{(e)} & \cdots & x_{9}^{(e)}
\end{array}\right]^{T}, \mathbf{y}^{e}=\left[\begin{array}{lll}
y_{1}^{(e)} & \cdots & y_{9}^{(e)}
\end{array}\right]^{T} .
$$

The Jacobian matrix J related to the coordinate change (10) can be now introduced in order to evaluate the derivatives with respect to the natural coordinates of the parent element: 


$$
\mathbf{J}=\left[\begin{array}{cc}
\frac{\partial x^{(e)}}{\partial \xi} & \frac{\partial y^{(e)}}{\partial \xi} \\
\frac{\partial x^{(e)}}{\partial \eta} & \frac{\partial y^{(e)}}{\partial \eta}
\end{array}\right]=\left[\begin{array}{cc}
\sum_{i=1}^{9} x_{i}^{(e)} \frac{\partial N_{i}}{\partial \xi} & \sum_{i=1}^{9} y_{i}^{(e)} \frac{\partial N_{i}}{\partial \xi} \\
\sum_{i=1}^{9} x_{i}^{(e)} \frac{\partial N_{i}}{\partial \eta} & \sum_{i=1}^{9} y_{i}^{(e)} \frac{\partial N_{i}}{\partial \eta}
\end{array}\right]=\left[\begin{array}{c}
\mathbf{B}_{\xi} \\
\mathbf{B}_{\eta}
\end{array}\right]\left[\begin{array}{ll}
\mathbf{x}^{(e)} & \mathbf{y}^{(e)}
\end{array}\right]=\left[\begin{array}{cc}
\mathbf{B}_{\xi} \mathbf{x}^{(e)} & \mathbf{B}_{\xi} \mathbf{y}^{(e)} \\
\mathbf{B}_{\eta} \mathbf{x}^{(e)} & \mathbf{B}_{\eta} \mathbf{y}^{(e)}
\end{array}\right]
$$

where the vectors $\mathbf{B}_{\xi}, \mathbf{B}_{\eta}$ collect the derivatives of the shape functions (9) with respect to $\xi, \eta$

$$
\mathbf{B}_{\xi}=\left[\begin{array}{lll}
\frac{\partial N_{1}}{\partial \xi} & \cdots & \frac{N_{9}}{\partial \xi}
\end{array}\right], \mathbf{B}_{\eta}=\left[\begin{array}{lll}
\frac{\partial N_{1}}{\partial \eta} & \cdots & \frac{N_{9}}{\partial \eta}
\end{array}\right] .
$$

At this point, the compatibility equations can be presented to define the strain components in each element. In particular, the membrane strains are given by

$$
\varepsilon_{x}^{(e)}=\frac{\partial u_{x}^{(e)}}{\partial x}=\mathbf{B}_{x} \mathbf{u}_{x}^{(e)}, \varepsilon_{y}^{(e)}=\frac{\partial u_{y}^{(e)}}{\partial y}=\mathbf{B}_{y} \mathbf{u}_{y}^{(e)}, \gamma_{x y}^{(e)}=\frac{\partial u_{y}^{(e)}}{\partial x}+\frac{\partial u_{x}^{(e)}}{\partial y}=\mathbf{B}_{x} \mathbf{u}_{y}^{(e)}+\mathbf{B}_{y} \mathbf{u}_{x}^{(e)} .
$$

On the other hand, the bending and twisting curvatures can be defined as follows:

$$
k_{x}^{(e)}=\frac{\partial \phi_{x}^{(e)}}{\partial x}=\mathbf{B}_{x} \boldsymbol{\phi}_{x}^{(e)}, k_{y}^{(e)}=\frac{\partial \phi_{y}^{(e)}}{\partial y}=\mathbf{B}_{y} \boldsymbol{\phi}_{y}^{(e)}, k_{x y}^{(e)}=\frac{\partial \phi_{y}^{(e)}}{\partial x}+\frac{\partial \phi_{x}^{(e)}}{\partial y}=\mathbf{B}_{x} \boldsymbol{\phi}_{y}^{(e)}+\mathbf{B}_{y} \boldsymbol{\phi}_{x}^{(e)} .
$$

Finally, the shear strains assume the following definitions:

$$
\gamma_{x z}^{(e)}=\frac{\partial u_{z}^{(e)}}{\partial x}+\phi_{x}^{(e)}=\mathbf{B}_{x} \mathbf{u}_{z}^{(e)}+\overline{\mathbf{N}} \phi_{x}^{(e)}, \gamma_{y z}^{(e)}=\frac{\partial u_{z}^{(e)}}{\partial y}+\phi_{y}^{(e)}=\mathbf{B}_{y} \mathbf{u}_{z}^{(e)}+\overline{\mathbf{N}} \phi_{y}^{(e)} .
$$

Note that the derivatives of the shape functions with respect to the physical coordinates $x, y$ are introduced and collected in the corresponding vectors $\mathbf{B}_{x}, \mathbf{B}_{y}$. They can be computed as follows by inverting the Jacobian matrix (this procedure is admissible if its determinant is greater than zero):

$$
\left[\begin{array}{l}
\mathbf{B}_{x} \\
\mathbf{B}_{y}
\end{array}\right]=\mathbf{J}^{-1}\left[\begin{array}{l}
\mathbf{B}_{\xi} \\
\mathbf{B}_{\eta}
\end{array}\right]
$$

The following matrix notation can be used to collect and define the strains previously introduced in (14)-(16):

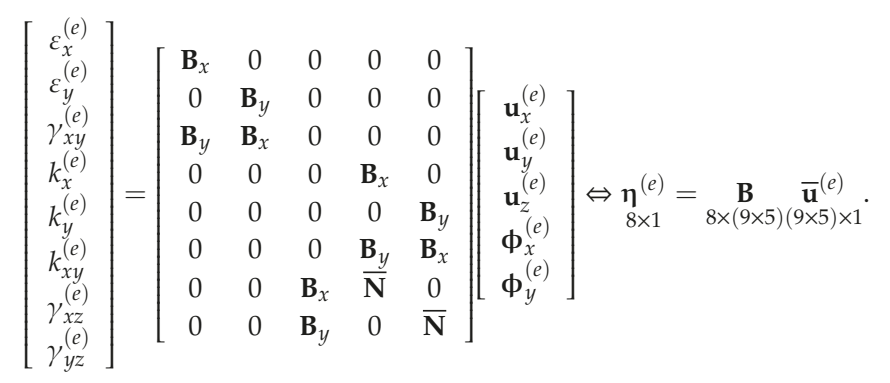

The vector $\boldsymbol{\eta}^{(e)}$ collects the aforementioned strain components. Such terms are needed to compute the stress resultants in each element by means of the constitutive relation shown below in matrix form: 


$$
\left[\begin{array}{c}
N_{x}^{(e)} \\
N_{y}^{(e)} \\
N_{x y}^{(e)} \\
M_{x}^{(e)} \\
M_{y}^{(e)} \\
M_{x y}^{(e)} \\
T_{x}^{(e)} \\
T_{y}^{(e)}
\end{array}\right]=\left[\begin{array}{cccccccc}
A_{11} & A_{12} & A_{16} & B_{11} & B_{12} & B_{16} & 0 & 0 \\
A_{12} & A_{22} & A_{26} & B_{12} & B_{22} & B_{26} & 0 & 0 \\
A_{16} & A_{26} & A_{66} & B_{16} & B_{26} & B_{66} & 0 & 0 \\
B_{11} & B_{12} & B_{16} & D_{11} & D_{12} & D_{16} & 0 & 0 \\
B_{12} & B_{22} & B_{26} & D_{12} & D_{22} & D_{26} & 0 & 0 \\
B_{16} & B_{26} & B_{66} & D_{16} & D_{26} & D_{66} & 0 & 0 \\
0 & 0 & 0 & 0 & 0 & 0 & \kappa A_{44} & \kappa A_{45} \\
0 & 0 & 0 & 0 & 0 & 0 & \kappa A_{45} & \kappa A_{55}
\end{array}\right]\left[\begin{array}{c}
\varepsilon_{x}^{(e)} \\
\varepsilon_{y}^{(e)} \\
\gamma_{x y}^{(e)} \\
k_{x}^{(e)} \\
k_{y}^{(e)} \\
k_{x y}^{(e)} \\
\gamma_{x z}^{(e)} \\
\gamma_{y z}^{(e)}
\end{array}\right]
$$

in which $N_{x}^{(e)}, N_{y}^{(e)}, N_{x y}^{(e)}$ are the membrane forces, $M_{x}^{(e)}, M_{y}^{(e)}, M_{x y}^{(e)}$ the bending and twisting moments, and $T_{x}^{(e)}, T_{y}^{(e)}$ the shear stresses. On the other hand, $\kappa$ stands for the shear correction factor. For moderately thick and thick plates, which are commonly studied through the FSDT, the shear correction factor is generally assumed equal to $5 / 6$. Nevertheless, the same structural model can be employed to accurately investigate the mechanical behavior of thin plates, which are usually analyzed in the theoretical framework provided by the classical laminated plate theory (CLPT), taking the Kirchhoff hypothesis into account. This theory neglects the shear stresses, and the same circumstance can be obtained from the FSDT by setting $10^{6}$ as the shear correction factor. In other words, the effect of shear stresses is negligible if the shear stiffness is extremely large [71].

The stress resultants can be also expressed as follows in extended matrix form in terms of nodal displacements, having in mind the definitions (18)

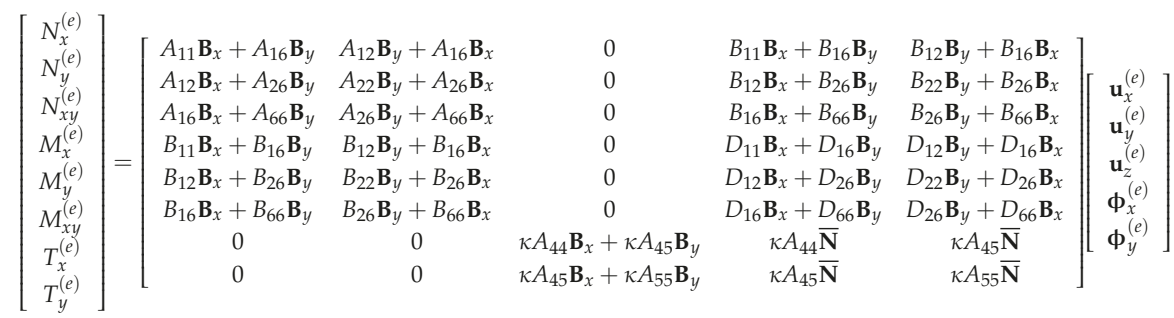

or in compact matrix form

$$
\underset{8 \times 1}{\mathbf{S}^{(e)}}=\underset{8 \times 88 \times(9 \times 5)}{\mathbf{C}} \underset{(9 \times 5) \times 1^{\prime}}{\mathbf{B}} \underset{\mathbf{u}^{(e)}}{ }
$$

where the meaning of the constitutive operator $\mathbf{C}$ can be deduced from Equation (19). It should be observed that the mechanical properties are the same in each element, and the corresponding coefficients are defined as

$$
\left(A_{i j}, B_{i j}, D_{i j}\right)=\sum_{k=1}^{N_{L}} \int_{z_{k}}^{z_{k+1}} \bar{Q}_{i j}^{(k)}\left(1, z, z^{2}\right) d z,
$$

where $\bar{Q}_{i j}^{(k)}$ represents the stiffnesses of the $k$-th orthotropic layer, which can be oriented as $\theta^{(k)}$. Once the orientation of the fibers is defined, the following relations are employed to compute the coefficients $\bar{Q}_{i j}^{(k)}$ : 


$$
\begin{aligned}
& \bar{Q}_{11}^{(k)}=Q_{11}^{(k)} \cos ^{4} \theta^{(k)}+2\left(Q_{12}^{(k)}+2 Q_{66}^{(k)}\right) \sin ^{2} \theta^{(k)} \cos ^{2} \theta^{(k)}+Q_{22}^{(k)} \sin ^{4} \theta^{(k)} \\
& \bar{Q}_{12}^{(k)}=\left(Q_{11}^{(k)}+Q_{22}^{(k)}-4 Q_{66}^{(k)}\right) \sin ^{2} \theta^{(k)} \cos ^{2} \theta^{(k)}+Q_{12}^{(k)}\left(\sin ^{4} \theta^{(k)}+\cos ^{4} \theta^{(k)}\right) \\
& \bar{Q}_{22}^{(k)}=Q_{11}^{(k)} \sin ^{4} \theta^{(k)}+2\left(Q_{12}^{(k)}+2 Q_{66}^{(k)}\right) \sin ^{2} \theta^{(k)} \cos ^{2} \theta^{(k)}+Q_{22}^{(k)} \cos ^{4} \theta^{(k)} \\
& \bar{Q}_{16}^{(k)}=\left(Q_{11}^{(k)}-Q_{12}^{(k)}-2 Q_{66}^{(k)}\right) \sin \theta^{(k)} \cos ^{3} \theta^{(k)}+\left(Q_{12}^{(k)}-Q_{22}^{(k)}+2 Q_{66}^{(k)}\right) \sin ^{3} \theta^{(k)} \cos \theta^{(k)} \\
& \bar{Q}_{26}^{(k)}=\left(Q_{11}^{(k)}-Q_{12}^{(k)}-2 Q_{66}^{(k)}\right) \sin ^{3} \theta^{(k)} \cos \theta^{(k)}+\left(Q_{12}^{(k)}-Q_{22}^{(k)}+2 Q_{66}^{(k)}\right) \sin \theta^{(k)} \cos ^{3} \theta^{(k)}, \\
& \bar{Q}_{66}^{(k)}=\left(Q_{11}^{(k)}+Q_{22}^{(k)}-2 Q_{12}^{(k)}-2 Q_{66}^{(k)}\right) \sin ^{2} \theta^{(k)} \cos ^{2} \theta^{(k)}+Q_{66}^{(k)}\left(\sin ^{4} \theta^{(k)}+\cos ^{4} \theta^{(k)}\right) \\
& \bar{Q}_{44}^{(k)}=Q_{44}^{(k)} \cos ^{2} \theta^{(k)}+Q_{55}^{(k)} \sin ^{2} \theta^{(k)} \\
& \bar{Q}_{45}^{(k)}=\left(Q_{44}^{(k)}-Q_{55}^{(k)}\right) \sin \theta^{(k)} \cos \theta^{(k)} \\
& \bar{Q}_{55}^{(k)}=Q_{55}^{(k)} \cos ^{2} \theta^{(k)}+Q_{44}^{(k)} \sin ^{2} \theta^{(k)}
\end{aligned}
$$

where the quantities $Q_{i j}^{(k)}$ are defined below in terms of the engineering constants of the corresponding layer, which are the Young's moduli $E_{11}^{(k)}, E_{22}^{(k)}$, the shear moduli $G_{12}^{(k)}, G_{13}^{(k)}, G_{23}^{(k)}$, and the Poisson's ratio $v_{12}^{(k)}$ :

$$
Q_{11}^{(k)}=\frac{E_{11}^{(k)}}{1-v_{12}^{(k)} v_{21}^{(k)}}, \quad Q_{22}^{(k)}=\frac{E_{22}^{(k)}}{1-v_{12}^{(k)} v_{21}^{(k)}}, \quad Q_{12}^{(k)}=\frac{v_{12}^{(k)} E_{22}^{(k)}}{1-v_{12}^{(k)} v_{21}^{(k)}}, \quad Q_{66}^{(k)}=G_{12}^{(k)}, \quad Q_{44}^{(k)}=G_{13}^{(k)}, \quad Q_{55}^{(k)}=G_{23}^{(k)} .
$$

It should be recalled that the Poisson's ratio $v_{21}^{(k)}$ can be evaluated by using the well-known relation for orthotropic materials $v_{21}^{(k)}=E_{22}^{(k)} v_{12}^{(k)} / E_{11}^{(k)}$.

The engineering constants are computed by means of the Halpin-Tsai approach, once the mechanical features of the reinforcing fibers and the epoxy resin of the orthotropic fiber-reinforced layers are known. As highlighted in [35], this methodology can be applied by using Hill's elastic moduli and a semi-empirical approach. The reinforcing fibers are modeled as a transversely isotropic material, and the following engineering constants are required to characterize them: the Young's moduli $E_{11}^{F}, E_{22}^{F}$, the shear modulus $G_{12}^{F}$, and the Poisson's ratios $v_{12}^{F}, v_{23}^{F}$. The Hills's elastic moduli of the fibers $k_{F}, l_{F}, m_{F}, n_{F}, p_{F}$ are given by:

$$
\begin{gathered}
k_{F}=\frac{E_{22}^{F}}{2\left(1-v_{23}^{F}-2 v_{21}^{F} v_{12}^{F}\right)}, \quad l_{F}=2 v_{12}^{F} k_{F}, \quad m_{F}=\frac{1-v_{23}^{F}-2 v_{21}^{F} v_{12}^{F}}{1+v_{23}^{F}} k_{F}, \\
n_{F}=2\left(1-v_{23}^{F}\right) \frac{E_{11}^{F}}{E_{22}^{F}} k_{F}, \quad p_{F}=G_{12}^{F}
\end{gathered}
$$

On the other hand, the epoxy resin is modeled as an isotropic medium characterized by its Young's modulus $E^{M}$ and its Poisson's ratio $v^{M}$. The Hill's elastic moduli of the matrix $k_{M}, l_{M}, m_{M}, n_{M}, p_{M}$ are defined below:

$$
\begin{gathered}
k_{M}=\frac{E^{M}}{2\left(1+v^{M}\right)\left(1-2 v^{M}\right)}, \quad l_{M}=2 v^{M} k_{M}, \quad m_{M}=\left(1-2 v^{M}\right) k_{M} . \\
n_{M}=2\left(1-v^{M}\right) k_{M}, \quad p_{M}=\left(1-2 v^{M}\right) k_{M}
\end{gathered}
$$

At this point, the overall mechanical properties of the composite material can be computed in terms of the Hill's elastic moduli $k, l, m, n, p$ : 


$$
\begin{aligned}
& k=\frac{k_{M}\left(k_{F}+m_{M}\right) V_{M}+k_{F}\left(k_{M}+m_{M}\right) V_{F}}{\left(k_{F}+m_{M}\right) V_{M}+\left(k_{M}+m_{M}\right) V_{F}} \\
& l=V_{F} l_{F}+V_{M} l_{M}+\frac{l_{F}-l_{M}}{k_{F}-k_{M}}\left(k-V_{F} k_{F}-V_{M} k_{M}\right) \\
& m=m_{M} \frac{2 V_{F} m_{F}\left(k_{M}+m_{M}\right)+2 V_{M} m_{F} m_{M}+V_{M} k_{M}\left(m_{F}+m_{M}\right)}{2 V_{F}\left(k_{M}+m_{M}\right)+2 V_{M} m_{F} m_{M}+V_{M} k_{M}\left(m_{F}+m_{M}\right)}, \\
& n=V_{F} n_{F}+V_{M} n_{M}+\left(\frac{l_{F}-l_{M}}{k_{F}-k_{M}}\right)^{2}\left(k-V_{F} k_{F}-V_{M} k_{M}\right) \\
& p=\frac{\left(p_{F}+p_{M}\right) p_{M} V_{M}+2 p_{F} p_{M} V_{F}}{\left(p_{F}+p_{M}\right) V_{M}+2 p_{M} V_{F}}
\end{aligned}
$$

where $V_{F}, V_{M}$ are the volume fractions of the fibers and of the matrix, respectively. They are related by the following relation: $V_{M}=1-V_{F}$. In the current research, a non-uniform distribution of the fibers is defined along the plate thickness. Therefore, the volume fraction of the reinforcing fibers turns out to be a function of the thickness coordinate $V_{F}=V_{F}(z)=\widetilde{V}_{F} f^{(k)}(z)$, in which $\widetilde{V}_{F}$ represents a constant value. This idea is representative of functionally graded materials. Several distributions $f^{(k)}(z)$ can be introduced toward this aim, and can be applied in each layer separately. The following functions are used in this paper:

$$
f^{(k)}(z)=\left\{\begin{array}{l}
f_{U D}^{(k)}(z)=1 \\
f_{O}^{(k)}(z)=1-\frac{1}{2}\left|\frac{2\left(z-z_{k}\right)}{z_{k+1}-z_{k}}-\frac{2\left(z_{k+1}-z\right)}{z_{k+1}-z_{k}}\right| \\
f_{X}^{(k)}(z)=\frac{1}{2}\left|\frac{2\left(z-z_{k}\right)}{z_{k+1}-z_{k}}-\frac{2\left(z_{k+1}-z\right)}{z_{k+1}-z_{k}}\right| \\
f_{V}^{(k)}(z)=\frac{z-z_{k}}{z_{k+1}-z_{k}} \\
f_{A}^{(k)}(z)=\frac{z_{k+1}-z}{z_{k+1}-z_{k}}
\end{array} .\right.
$$

For the sake of completeness, these functions are depicted in Figure 2.

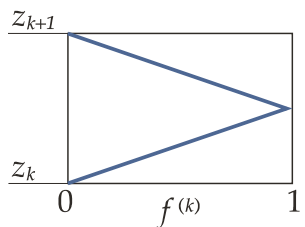

(a)

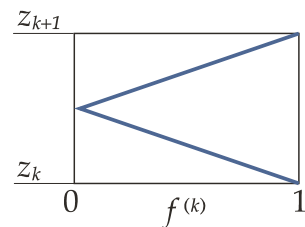

(b)

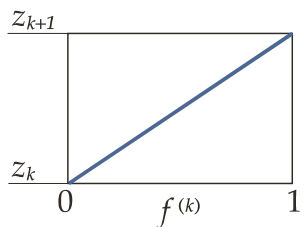

(c)

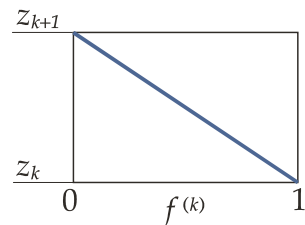

(d)

Figure 2. Through-the-thickness variation of $f^{(k)}$ : (a) $f_{O}^{(k)} ;(\mathbf{b}) f_{X}^{(k)} ;(\mathbf{c}) f_{V}^{(k)} ;(\mathbf{d}) f_{A}^{(k)}$.

Once the Hill's elastic moduli (27) are computed, the engineering constants of the $k$-th fiber reinforced composite layer can be evaluated as well. The definitions shown below are required for this purpose:

$$
E_{11}^{(k)}=n-\frac{l^{2}}{k}, E_{22}^{(k)}=\frac{4 m\left(k n-l^{2}\right)}{k n-l^{2}+m n}, v_{12}^{(k)}=\frac{l}{2 k}, G_{12}^{(k)}=G_{13}^{(k)}=p, G_{23}^{(k)}=m .
$$

It should be noted that these quantities are all functions of the thickness coordinate $z$ due to the relations (28). As a consequence, the material properties $\bar{Q}_{i j}^{(k)}$ defined in (23) depend also on the coordinate $z$, and the integrals in (22) have to be computed numerically. The function "trap $z$ " embedded in MATLAB was employed toward this aim. 
At this point, the Hamilton's variational principle can be applied to obtain the equations of motion and the corresponding weak form [23]. As a result, it is possible to write the dynamic fundamental system in each element as follows:

$$
\underset{(9 \times 5) \times(9 \times 5)(9 \times 5) \times 1}{\mathbf{K}^{(e)}} \underset{(9 \times 5) \times(9 \times 5)(9 \times 5) \times 1}{\overline{\mathbf{u}}^{(e)}}+\underset{\overline{\mathbf{u}}}{\mathbf{M}^{(e)}}=0,
$$

where the stiffness matrix of the element is denoted by $\mathbf{K}^{(e)}$, whereas the mass matrix is identified .. (e)

by $\mathbf{M}^{(e)}$. On the other hand, the vector $\overline{\mathbf{u}} \quad$ collects the second-order derivatives with respect to the time variable $t$ of the nodal displacements (7). By definition, the stiffness matrix $\mathbf{K}^{(e)}$ assumes the following aspect:

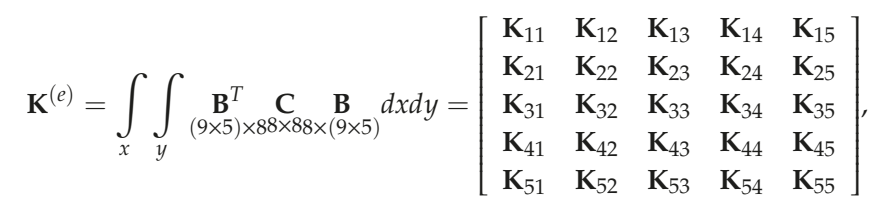

where the operators $\mathbf{K}_{i j}$ of size $9 \times 9$ are illustrated in Appendix A. Analogously, the mass matrix $\mathbf{M}^{(e)}$ can be written as follows:

$$
\mathbf{M}^{(e)}=\int_{x} \int_{y} \underset{(9 \times 5) \times 55 \times 55 \times(9 \times 5)}{\mathbf{N}^{T}} \underset{\mathbf{N}}{\mathbf{N}} d x d y=\left[\begin{array}{ccccc}
\mathbf{M}_{11} & 0 & 0 & \mathbf{M}_{14} & 0 \\
0 & \mathbf{M}_{22} & 0 & 0 & \mathbf{M}_{25} \\
0 & 0 & \mathbf{M}_{33} & 0 & 0 \\
\mathbf{M}_{41} & 0 & 0 & \mathbf{M}_{44} & 0 \\
0 & \mathbf{M}_{52} & 0 & 0 & \mathbf{M}_{55}
\end{array}\right],
$$

where the operators $\mathbf{M}_{i j}$ of size $9 \times 9$ are also illustrated in Appendix A. The matrix $\mathbf{m}$ instead collects the inertia terms and assumes the definition shown below:

$$
\mathbf{m}=\left[\begin{array}{ccccc}
I_{0} & 0 & 0 & I_{1} & 0 \\
0 & I_{0} & 0 & 0 & I_{1} \\
0 & 0 & I_{0} & 0 & 0 \\
I_{1} & 0 & 0 & I_{2} & 0 \\
0 & I_{1} & 0 & 0 & I_{2}
\end{array}\right]
$$

in which

$$
I_{i}=\sum_{k=1}^{N_{L}} \int_{z_{k}}^{z_{k+1}} \rho^{(k)} z^{i} d z
$$

where $\rho^{(k)}$ is the density of the $k$-th layer. Its value can be obtained by means of the rule of mixture, combining the densities of the reinforcing fibers $\rho_{F}^{(k)}$ and of the matrix $\rho_{M}^{(k)}$ :

$$
\rho^{(k)}=V_{F} \rho_{F}^{(k)}+V_{M} \rho_{M}^{(k)}
$$

Note that the density is also a function of the thickness coordinate $z$ due to the through-the-thickness variation of the volume fraction of the fibers. Therefore, the integrals in (34) have to be computed numerically as well. 


\subsection{Numerical Evaluation of the Fundamental Operators}

It is well-known that the integrals in definitions (31) and (32) require a tool to be computed numerically. In the current research, the Gauss-Legendre rule is used. According to this approach, the infinitesimal area $d x d y$ is evaluated in the master element as follows, through the determinant of the Jacobian matrix: $d x d y=\operatorname{det} J d \xi d \eta$. Consequently, the integral of a generic two-dimensional function $F(x, y)$ can be written as

$$
\int_{x} \int_{y} F(x, y) d x d y=\int_{-1}^{1} \int_{-1}^{1} F(\xi, \eta) \operatorname{det} \mathbf{J} d \xi d \eta
$$

At this point, the integral is converted into a weighted linear sum by introducing the roots of Legendre polynomials $\xi_{I}, \eta_{J}$ and the corresponding weighting coefficients $W_{I}, W_{J}$ :

$$
\left.\int_{-1}^{1} \int_{-1}^{1} F(\xi, \eta) \operatorname{det} J d \xi d \eta \approx \sum_{I=1}^{M} \sum_{J=1}^{N} F\left(\xi_{I}, \eta_{J}\right) \operatorname{det} \mathbf{J}\right|_{\xi_{I}, \eta_{J}} W_{I} W_{J}
$$

The values of the roots of Legendre polynomials and the corresponding weighting coefficients used in the numerical integration are listed in Table 1 . Recall that the full integration is performed by setting $N=M=3$. On the other hand, the reduced integration is accomplished for $N=M=2$ as far as the shear terms are concerned. In other words, the elements of the stiffness matrix which involve the mechanical properties $A_{44}, A_{45}, A_{55}$ are computed by means of the reduced integration. This procedure aims to avoid the shear locking problem as highlighted in the book by Reddy [14]. For the sake of completeness, the roots of Legendre polynomials are also depicted in Figure 1, for both the full and reduced integrations.

Table 1. Roots of Legendre polynomials and weighting coefficients for the numerical integration.

\begin{tabular}{ccc}
\hline$N, M$ & $\xi_{I}, \eta_{J}$ & $W_{I}, W_{J}$ \\
\hline 2 & $\pm 1 / \sqrt{3}$ & 1 \\
\hline 3 & $\pm \sqrt{3 / 5}$ & $5 / 9$ \\
\hline & 0 & $8 / 9$
\end{tabular}

Finally, the assembly procedure is performed to enforce the $C^{0}$ compatibility conditions among the elements in which the reference domain is divided. In other words, the model is characterized by continuous displacements at the interfaces of the elements. The global discrete system of governing equations assumes the following aspect:

$$
\underset{N_{\text {dofs }} \times N_{\text {dofs }} N_{\text {dofs }} \times 1}{\mathbf{u}}+\underset{N_{\text {dofs }} \times N_{\text {dofs }} N_{\text {dofs }} \times 1}{\mathbf{u}} \underset{\ddot{u}}{\ddot{\mathbf{u}}}=0,
$$

where the number of degrees of freedom is given by $N_{\text {dofs }}=5 \times N_{P}, N_{P}$ being the number of nodes of the discrete domain. With reference to Equation (38), K, $\mathbf{M}$ clearly stand for the global stiffness and mass matrices, whereas $\mathbf{u}$ is the vector of the nodal displacements of the global system defined below:

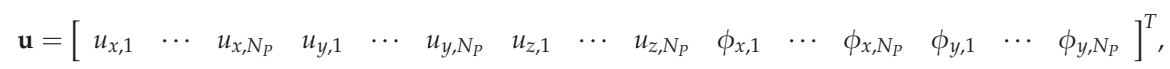

in which the numbering is performed following the scheme in Figure 3. Finally, $\ddot{\mathbf{u}}$ is the vector that collects the second-order time derivatives of the nodal displacements. 


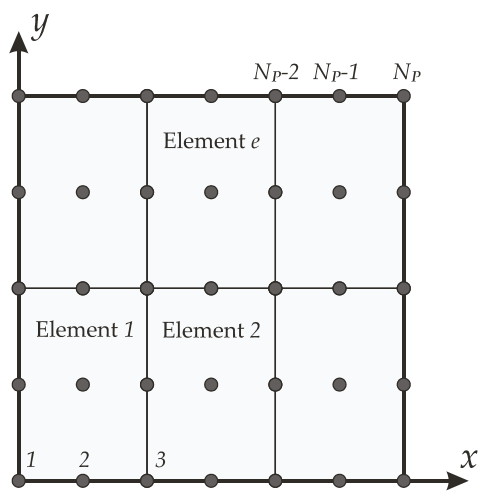

Figure 3. Example of a discrete domain and node numbering.

\subsection{Natural Frequency Analysis}

Once the discrete fundamental system (38) is defined and the proper boundary conditions are enforced, the separation of variables provide the following relation:

$$
\left(\mathbf{K}-\omega^{2} \mathbf{M}\right) \mathbf{d}=0,
$$

in which $\omega$ represents the circular frequencies of the structural system, whereas the vector $\mathbf{d}$ collects the corresponding modal amplitudes. The natural frequencies of the plate can be evaluated as $f_{n}=\omega / 2 \pi$. It can be observed that the expression (40) is a generalized eigenvalue problem. In the present research, the function "eigs" embedded in MATLAB was employed to obtain the natural frequencies and the mode shapes of the laminated composite plates.

\section{Numerical Applications}

The formulation illustrated in the previous section was implemented in a MATLAB code. The current approach was first validated by means of the comparison with the semi-analytical solutions provided by Reddy in his book [23], for both thin and thick simply-supported plates with an antisymmetric cross-ply layup. In these circumstances, a uniform distribution of the fiber was assumed along the plate thickness.

The convergence analysis was also performed for the sake of completeness. Subsequently, the natural frequencies of functionally graded orthotropic laminated plates are discussed. The geometry of the plates considered in the numerical applications was defined by $L_{x}=L_{y}=1 \mathrm{~m}$, whereas their lamination scheme was given by $\left(0^{\circ} / 90^{\circ} / 0^{\circ} / 90^{\circ}\right)$. The four layers were characterized by the same value of $\widetilde{V}_{F}=0.6$, whereas their thickness was assumed as $2.5 \times 10^{-3} \mathrm{~m}$ for thin plates and as $2.5 \times$ $10^{-2} \mathrm{~m}$ for the thick ones. The mechanical properties of the constituents (Carbon fibers and epoxy resin) are listed in Table 2.

Table 2. Mechanical properties of the layer constituents.

\begin{tabular}{ccccc}
\hline Constituent & Young's Moduli & Shear Moduli & Poisson's Ratios & Density \\
\hline \multirow{2}{*}{ Carbon fibers } & $E_{11}^{F}=230 \mathrm{GPa}$ & \multirow{2}{*}{$G_{12}^{F}=50 \mathrm{GPa}$} & $\begin{array}{l}v_{12}^{F}=0.20 \\
v_{23}^{F}=0.25\end{array}$ & $\rho^{F}=1800 \mathrm{~kg} / \mathrm{m}^{3}$ \\
& $E_{22}^{F}=15 \mathrm{GPa}$ & - & $v^{M}=0.38$ & $\rho^{M}=1200 \mathrm{~kg} / \mathrm{m}^{3}$ \\
\hline Epoxy resin & $E^{M}=3.27 \mathrm{GPa}$ & - &
\end{tabular}




\subsection{Convergence and Accuracy}

The convergence analysis was performed by increasing the number of discrete elements up to 256, which means 16 elements along each principal direction. The results of this test are presented in Table 3 for a thin plate and in Table 4 for the thicker ones, in terms of the first ten natural frequencies. A very good accuracy was obtained by using only eight finite elements per side, for both cases under consideration. In particular, the percentage error for the first mode shapes was lower than $0.4 \%$ if 64 elements were used. Therefore, the formulation and the numerical approach were validated. Only the bending mode shapes were considered in the analyses.

Table 3. Convergence features of the numerical approach and comparison of the first ten natural frequencies $(\mathrm{Hz})$ with the semi-analytical solutions provided by Reddy [23] for a simply-supported thin plate with a through-the-thickness uniform distribution of the reinforcing fibers. CLPT: classical laminated plate theory.

\begin{tabular}{cccccc}
\hline Mode & CLPT Ref. [23] & $\begin{array}{c}\text { 4 Elements } \\
\boldsymbol{N}_{\text {dofs }}=125\end{array}$ & $\begin{array}{c}\text { 16 Elements } \\
\boldsymbol{N}_{\text {dofs }}=405\end{array}$ & $\begin{array}{c}\text { 64 Elements } \\
\boldsymbol{N}_{\text {dofs }}=1445\end{array}$ & $\begin{array}{c}\text { 256 Elements } \\
\boldsymbol{N}_{\text {dofs }}=5445\end{array}$ \\
\hline 1 & 43.9262 & 44.4153 & 43.9592 & 43.9284 & 43.9265 \\
2 & 123.1041 & 135.1515 & 124.3804 & 123.1900 & 123.1096 \\
3 & 123.1041 & 135.1515 & 124.3804 & 123.1901 & 123.1096 \\
4 & 175.6547 & 192.6267 & 177.6096 & 175.7865 & 175.6632 \\
5 & 265.0021 & 505.8042 & 278.5590 & 265.9668 & 265.0653 \\
6 & 265.0021 & 505.8084 & 278.5590 & 265.9668 & 265.0653 \\
7 & 300.0618 & 533.0928 & 313.2389 & 300.9965 & 300.1230 \\
8 & 300.0618 & 533.0928 & 313.2389 & 300.9965 & 300.1230 \\
9 & 395.0350 & 751.5685 & 415.3231 & 396.4841 & 395.1299 \\
10 & 465.3946 & 838.4999 & 515.4317 & 470.6168 & 465.7466 \\
\hline
\end{tabular}

Table 4. Convergence features of the numerical approach and comparison of the first ten natural frequencies $(\mathrm{Hz})$ with the semi-analytical solutions provided by Reddy [23] for a simply-supported thick plate with a through-the-thickness uniform distribution of the reinforcing fibers. FSDT: first-order shear deformation theory.

\begin{tabular}{cccccc}
\hline Mode & FSDT Ref. [23] & $\begin{array}{c}\text { 4 Elements } \\
N_{\text {dofs }}=125\end{array}$ & $\begin{array}{c}\text { 16 Elements } \\
\boldsymbol{N}_{\text {dofs }}=405\end{array}$ & $\begin{array}{c}\text { 64 Elements } \\
\boldsymbol{N}_{\text {dofs }}=1445\end{array}$ & $\begin{array}{c}\text { 256 Elements } \\
\boldsymbol{N}_{\text {dofs }}=5445\end{array}$ \\
\hline 1 & 397.3772 & 400.9285 & 397.6161 & 397.3928 & 397.3782 \\
2 & 939.4637 & 987.3930 & 946.0547 & 939.9116 & 939.4924 \\
3 & 939.4637 & 987.3930 & 946.0547 & 939.9116 & 939.4924 \\
4 & 1285.7309 & 1295.8113 & 1293.1889 & 1286.2465 & 1285.7646 \\
5 & 1640.7304 & 2202.4349 & 1687.2607 & 1644.1525 & 1640.9552 \\
6 & 1640.7304 & 2219.9298 & 1687.2607 & 1644.1525 & 1640.9552 \\
7 & 1869.3853 & 2219.9298 & 1905.9136 & 1872.1146 & 1869.5668 \\
8 & 1869.3853 & 2224.7306 & 1905.9136 & 1872.1146 & 1869.5668 \\
9 & 2313.9852 & 2486.7150 & 2349.5762 & 2316.8723 & 2314.1827 \\
10 & 2372.3369 & 3354.9399 & 2442.9101 & 2385.5578 & 2373.2355 \\
\hline
\end{tabular}

For completeness, the convergence features of the proposed approach are presented in graphical form in Figure 4, where the relative error $e_{r}=f_{n} / f_{n \text {,exact }}-1$ was computed for increasing values of the degrees of freedom $\left(N_{d o f_{s}}\right)$. The graphs are presented in logarithmic scale. It can be observed that a good convergence was reached for both thin and thick plates. 


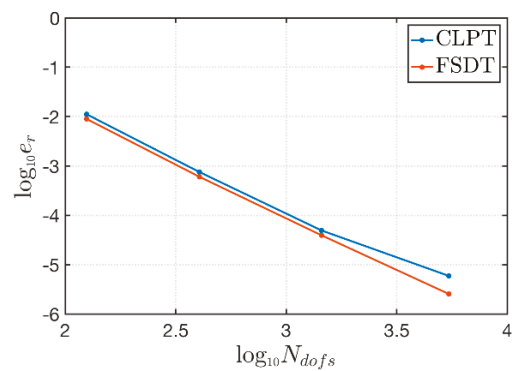

(a)

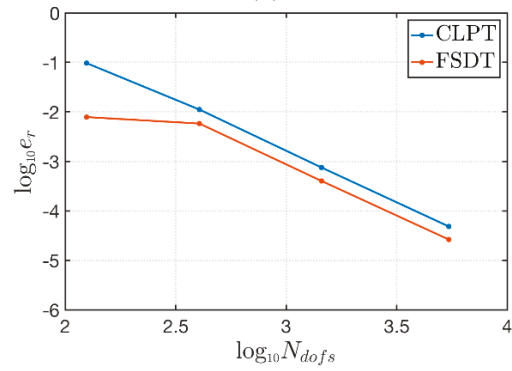

(c)

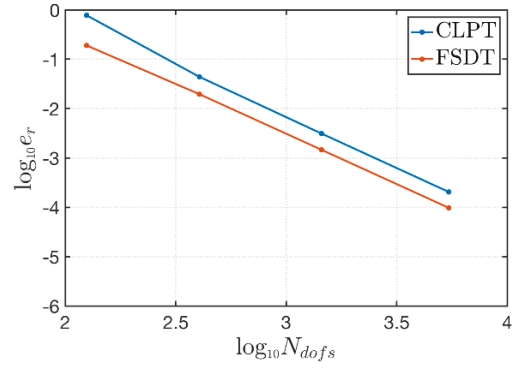

(e)

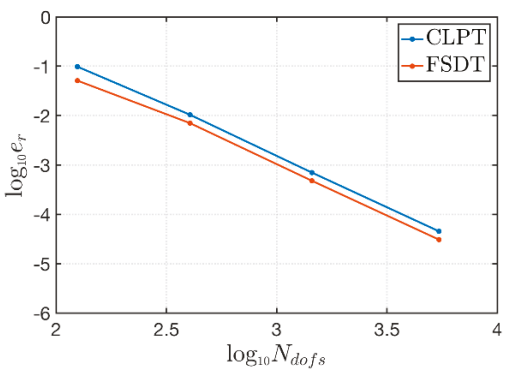

(b)

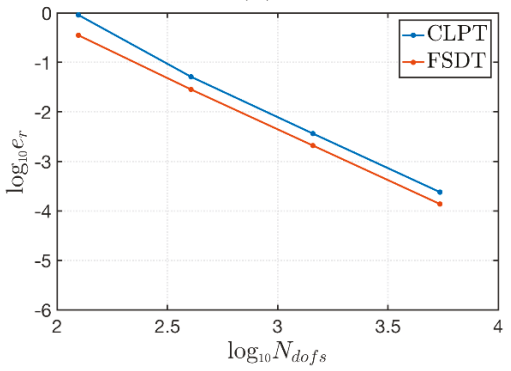

(d)

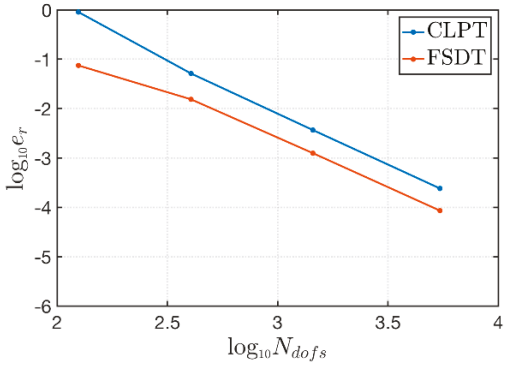

(f)

Figure 4. Convergence graphs for thin (CLPT) and thick (FSDT) laminated plates in terms of natural frequencies: (a) first frequency; (b) second frequency; (c) third frequency; (d) fourth frequency; (e) fifth frequency; (f) sixth frequency.

\subsection{Natural Frequency Analysis of Functionally Graded Orthotropic Plates}

In this section, four different through-the-thickness fiber distributions are analyzed. These four schemes, as well as the functions $f^{(k)}$ employed in each layer, are summarized in Table 5. The layers were numbered from the bottom to the top surface of the plate. As far as the mechanical and geometric features of the plates are concerned, the same values of the previous section were used. Due to the results of the convergence analyses, the plates were discretized by using ten finite elements per side. The first fourteen natural frequencies of a simply-supported thin plate for the various through-the-thickness distributions of the reinforcing fibers specified in Table 5 are presented in Table 6, whereas Table 7 collects the same results for a simply-supported thick plate. Finally, the first six mode shapes are also depicted in graphical form. In particular, Figure 5 presents the mode shapes related to the thin plates, whereas the same results for the thick plates are shown in Figure 6. Note that the mode shapes assumed different aspects by varying the through-the-thickness distributions of the fibers in the four layers, keeping their orientation constant. Analogously, the values of natural frequencies were 
affected by the non-uniform distribution of the fibers along the thickness of the structures, for both thin and thick configurations.

Table 5. Definition of the through-the-thickness distribution of the reinforcing fibers.

\begin{tabular}{ccccc}
\hline Scheme & Layer $\mathbf{1}$ & Layer 2 & Layer 3 & Layer 4 \\
\hline Scheme 1 & $f_{\text {UD }}^{(1)}$ & $f_{U D}^{(2)}$ & $f_{U D}^{(3)}$ & $f_{U D}^{(4)}$ \\
Scheme 2 & $f_{O}^{(1)}$ & $f_{O}^{(2)}$ & $f_{O}^{(3)}$ & $f_{O}^{(4)}$ \\
Scheme 3 & $f_{X}^{(1)}$ & $f_{X}^{(2)}$ & $f_{X}^{(3)}$ & $f_{X}^{(4)}$ \\
Scheme 4 & $f_{V}^{(1)}$ & $f_{U D}^{(2)}$ & $f_{U D}^{(3)}$ & $f_{A}^{(4)}$ \\
\hline
\end{tabular}

Table 6. First fourteen natural frequencies $(\mathrm{Hz})$ of a simply-supported thin plate for several through-the-thickness distributions of the reinforcing fibers.

\begin{tabular}{ccccc}
\hline Mode & Scheme 1 & Scheme 2 & Scheme 3 & Scheme 4 \\
\hline 1 & 43.9271 & 33.5339 & 35.0923 & 34.5204 \\
2 & 123.1397 & 93.9281 & 97.6817 & 96.4943 \\
3 & 123.1397 & 93.9281 & 97.6817 & 96.4994 \\
4 & 175.7093 & 134.1367 & 140.3692 & 138.0894 \\
5 & 265.4059 & 202.3576 & 209.7406 & 207.6677 \\
6 & 265.4059 & 202.3576 & 209.7406 & 207.6780 \\
7 & 300.4527 & 229.2791 & 239.2330 & 235.8151 \\
8 & 300.4527 & 229.2791 & 239.2330 & 235.8250 \\
9 & 395.6415 & 302.0350 & 316.0631 & 310.9587 \\
10 & 467.6067 & 356.4637 & 368.9774 & 365.6794 \\
11 & 467.6067 & 356.4637 & 368.9774 & 365.6944 \\
12 & 494.2235 & 376.9847 & 392.0326 & 387.3268 \\
13 & 494.2235 & 376.9847 & 392.0326 & 387.3477 \\
14 & 565.8001 & 431.8446 & 451.1608 & 444.3823 \\
\hline
\end{tabular}

Table 7. First fourteen natural frequencies $(\mathrm{Hz})$ of a simply-supported thick plate for several through-thethickness distributions of the reinforcing fibers.

\begin{tabular}{ccccc}
\hline Mode & Scheme 1 & Scheme 2 & Scheme 3 & Scheme 4 \\
\hline 1 & 397.3836 & 306.5341 & 318.4900 & 319.8942 \\
2 & 939.6493 & 734.9017 & 753.9989 & 780.5089 \\
3 & 939.6493 & 734.9017 & 753.9989 & 780.5337 \\
4 & 1285.9465 & 1008.8635 & 1036.0206 & 1077.1948 \\
5 & 1642.1651 & 1300.8952 & 1322.6235 & 1405.6371 \\
6 & 1642.1651 & 1300.8952 & 1322.6235 & 1405.6638 \\
7 & 1870.5349 & 1480.8980 & 1510.3561 & 1600.6668 \\
8 & 1870.5349 & 1480.8980 & 1510.3561 & 1600.6865 \\
9 & 2315.2138 & 1839.1055 & 1872.3302 & 1997.7076 \\
10 & 2377.9654 & 1899.9655 & 1921.4452 & 2077.7168 \\
11 & 2377.9654 & 1899.9655 & 1921.4452 & 2077.7322 \\
12 & 2545.0693 & 2031.1334 & 2059.3510 & 2219.4198 \\
13 & 2545.0693 & 2031.1334 & 2059.3510 & 2219.4394 \\
14 & 2888.3228 & 2306.5360 & 2339.2521 & 2523.7454 \\
\hline
\end{tabular}



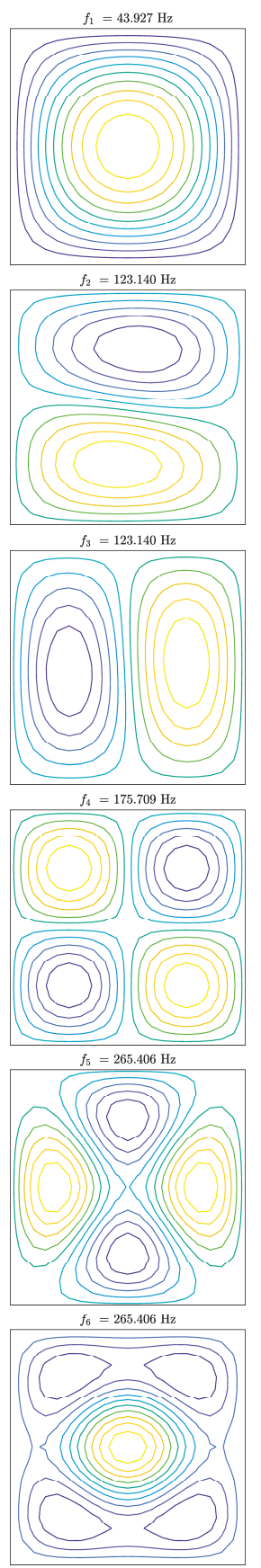

(a)
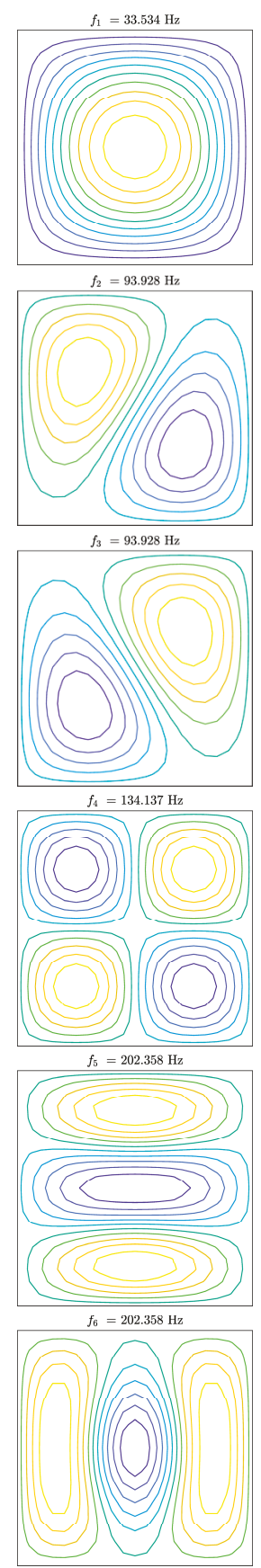

(b)
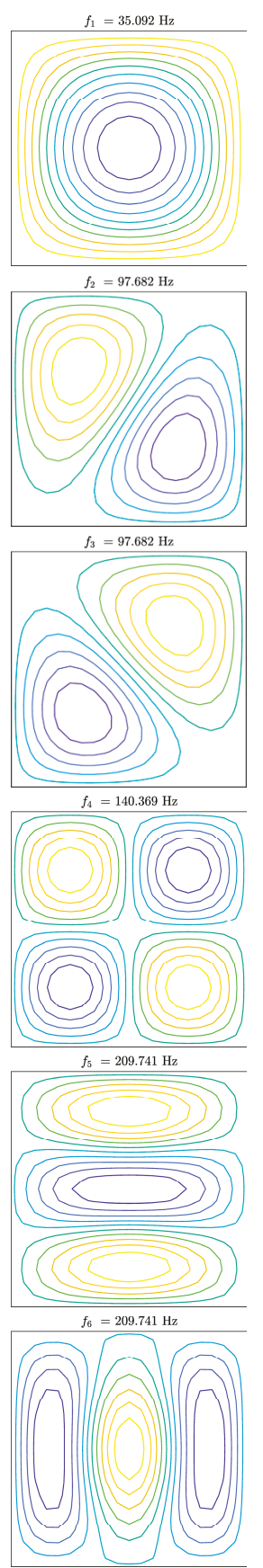

(c)
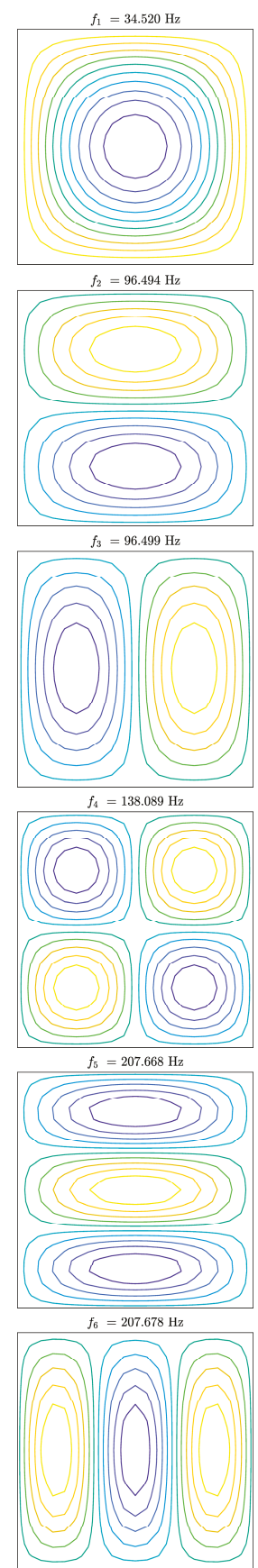

(d)

Figure 5. First six mode shapes for a simply-supported laminated thin plate with different fiber distributions: (a) Scheme 1 (uniform); (b) Scheme 2; (c) Scheme 3; (d) Scheme 4. 

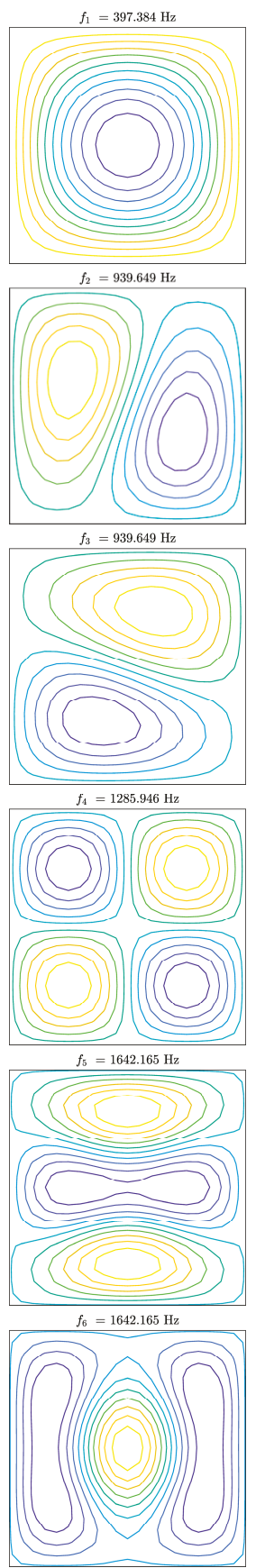

(a)
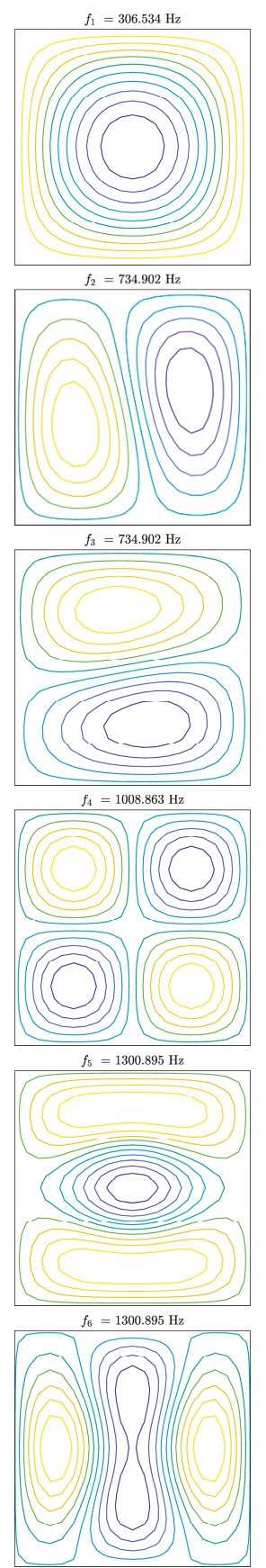

(b)
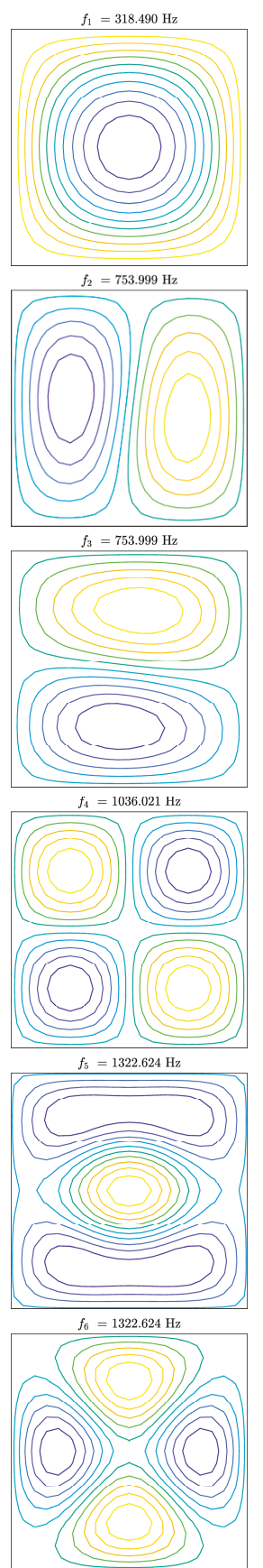

(c)
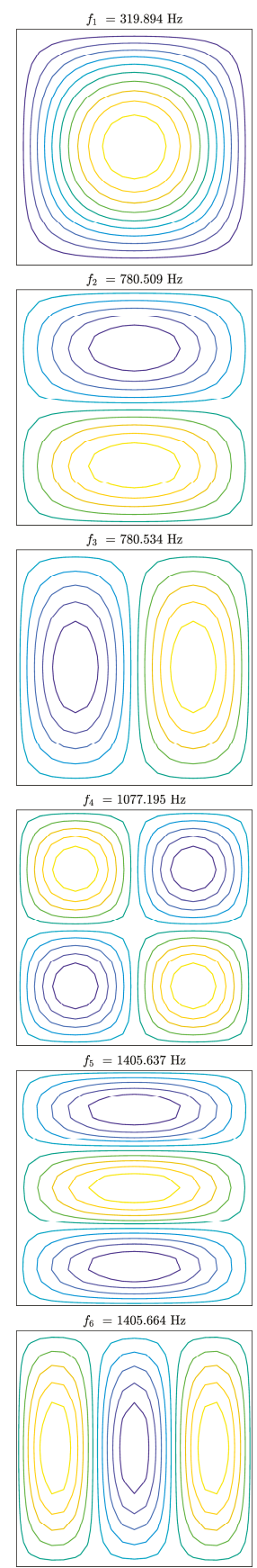

(d)

Figure 6. First six mode shapes for a simply-supported laminated thick plate with different fiber distributions: (a) Scheme 1 (uniform); (b) Scheme 2; (c) Scheme 3; (d) Scheme 4. 


\section{Conclusions}

A FE formulation was presented and implemented to investigate the natural frequencies of functionally graded orthotropic thin and thick plates with cross-ply layups. The layers of the structures were modeled as fiber-reinforced materials with orthotropic features. The fibers were characterized by a gradual variation of their volume fraction along the thickness of the plates. Toward this aim, several functions depending on the thickness coordinate were introduced. Their effects on the free vibrations were discussed. The research proved that the natural frequencies, as well as the corresponding mode shapes, were affected by the non-uniform placement of the fibers in the thickness direction. In particular, the dynamic response of laminated plates could be changed by varying the through-the-thickness distributions of the volume fraction of the reinforcing fibers, keeping the fiber orientation and the thickness of the various layers constant. The same considerations were deduced for thin and thick plates.

Author Contributions: Conceptualization, M.B. and A.M.T.; methodology, M.B. and A.M.T.; software, M.B. and A.M.T.; validation, M.B. and A.M.T.; formal analysis, M.B. and A.M.T.; investigation, M.B. and A.M.T.; resources, M.B. and A.M.T.; data curation, M.B. and A.M.T.; Writing-Original Draft preparation, M.B. and A.M.T.; Writing-Review and Editing, M.B. and A.M.T.; visualization, M.B. and A.M.T.; supervision, M.B. and A.M.T.; project administration, M.B. and A.M.T.

Funding: This research received no external funding.

Conflicts of Interest: The authors declare no conflict of interest.

\section{Appendix A}

The following definitions are required to compute the terms of the element stiffness matrix $\mathbf{K}^{(e)}$ introduced in Equation (31). Recall that the operator at issue is symmetrical. The submatrices $\mathbf{K}_{i j}^{(e)}$ for $i, j=1,2, \ldots, 5$ assume the following aspects:

$$
\begin{aligned}
& \mathbf{K}_{11}=\int_{x} \int_{y}\left(\mathbf{B}_{x}^{T}\left(A_{11} \mathbf{B}_{x}+A_{16} \mathbf{B}_{y}\right)+\mathbf{B}_{y}^{T}\left(A_{16} \mathbf{B}_{x}+A_{66} \mathbf{B}_{y}\right)\right) d x d y \\
& \mathbf{K}_{12}=\int_{x} \int_{y}\left(\mathbf{B}_{x}^{T}\left(A_{12} \mathbf{B}_{y}+A_{16} \mathbf{B}_{x}\right)+\mathbf{B}_{y}^{T}\left(A_{26} \mathbf{B}_{y}+A_{66} \mathbf{B}_{x}\right)\right) d x d y \\
& \mathbf{K}_{13}=0 \\
& \mathbf{K}_{14}=\int_{x} \int_{y}\left(\mathbf{B}_{x}^{T}\left(B_{11} \mathbf{B}_{x}+B_{16} \mathbf{B}_{y}\right)+\mathbf{B}_{y}^{T}\left(B_{16} \mathbf{B}_{x}+B_{66} \mathbf{B}_{y}\right)\right) d x d y \\
& \mathbf{K}_{15}=\int_{x} \int_{y}\left(\mathbf{B}_{x}^{T}\left(B_{12} \mathbf{B}_{y}+B_{16} \mathbf{B}_{x}\right)+\mathbf{B}_{y}^{T}\left(B_{26} \mathbf{B}_{y}+B_{66} \mathbf{B}_{x}\right)\right) d x d y \\
& \mathbf{K}_{21}=\mathbf{K}_{12}^{T} \\
& \mathbf{K}_{22}=\int_{x} \int_{y}\left(\mathbf{B}_{y}^{T}\left(A_{22} \mathbf{B}_{y}+A_{26} \mathbf{B}_{x}\right)+\mathbf{B}_{x}^{T}\left(A_{26} \mathbf{B}_{y}+A_{66} \mathbf{B}_{x}\right)\right) d x d y \\
& \mathbf{K}_{23}=0 \\
& \mathbf{K}_{24}=\int_{x} \int_{y}\left(\mathbf{B}_{y}^{T}\left(B_{12} \mathbf{B}_{x}+B_{26} \mathbf{B}_{y}\right)+\mathbf{B}_{x}^{T}\left(B_{16} \mathbf{B}_{x}+B_{66} \mathbf{B}_{y}\right)\right) d x d y \\
& \mathbf{K}_{25}=\int_{x} \int_{y}\left(\mathbf{B}_{y}^{T}\left(B_{22} \mathbf{B}_{y}+B_{26} \mathbf{B}_{x}\right)+\mathbf{B}_{x}^{T}\left(B_{26} \mathbf{B}_{y}+B_{66} \mathbf{B}_{x}\right)\right) d x d y \\
& \mathbf{K}_{31}= \mathbf{K}_{13}^{T} \\
& \mathbf{K}_{32}= \mathbf{K}_{23}^{T} \\
& \mathbf{K}_{33}=\int_{x} \int_{y}\left(\mathbf{B}_{x}^{T}\left(\kappa A_{44} \mathbf{B}_{x}+\kappa A_{45} \mathbf{B}_{y}\right)+\mathbf{B}_{y}^{T}\left(\kappa A_{45} \mathbf{B}_{x}+\kappa A_{55} \mathbf{B}_{y}\right)\right) d x d y \\
& \mathbf{K}_{34}=\int_{x} \int_{y}\left(\mathbf{B}_{x}^{T}\left(\kappa A_{44} \overline{\mathbf{N}}\right)+\mathbf{B}_{y}^{T}\left(\kappa A_{45} \overline{\mathbf{N}}\right)\right) d x d y \\
& \mathbf{K}_{35}=\int_{x} \int_{y}\left(\mathbf{B}_{x}^{T}\left(\kappa A_{45} \overline{\mathbf{N}}\right)+\mathbf{B}_{y}^{T}\left(\kappa A_{55} \overline{\mathbf{N}}\right)\right) d x d y
\end{aligned}
$$




$$
\begin{aligned}
& \mathbf{K}_{41}=\mathbf{K}_{14}^{T} \\
& \mathbf{K}_{42}=\mathbf{K}_{24}^{T} \\
& \mathbf{K}_{43}=\mathbf{K}_{34}^{T} \\
& \mathbf{K}_{44}=\int_{x} \int_{y}\left(\mathbf{B}_{x}^{T}\left(D_{11} \mathbf{B}_{x}^{e}+D_{16} \mathbf{B}_{y}\right)+\mathbf{B}_{y}^{T}\left(D_{16} \mathbf{B}_{x}+D_{66} \mathbf{B}_{y}\right)\right) d x d y+\int_{x} \int_{y} \overline{\mathbf{N}}^{T} \kappa A_{44} \overline{\mathbf{N}} d x d y \\
& \mathbf{K}_{45}=\int_{x} \int_{y}\left(\mathbf{B}_{x}^{T}\left(D_{12} \mathbf{B}_{y}+D_{16} \mathbf{B}_{x}\right)+\mathbf{B}_{y}^{T}\left(D_{26} \mathbf{B}_{y}+D_{66} \mathbf{B}_{x}\right)\right) d x d y+\int_{x} \int_{y} \overline{\mathbf{N}}^{T} \kappa A_{45} \overline{\mathbf{N}} d x d y \\
& \mathbf{K}_{51}=\mathbf{K}_{15}^{T} \\
& \mathbf{K}_{52}=\mathbf{K}_{25}^{T} \\
& \mathbf{K}_{53}=\mathbf{K}_{35}^{T} \\
& \mathbf{K}_{54}=\mathbf{K}_{45}^{T} \\
& \mathbf{K}_{55}=\int_{x} \int_{y}\left(\mathbf{B}_{y}^{T}\left(D_{22} \mathbf{B}_{y}+D_{26} \mathbf{B}_{x}\right)+\mathbf{B}_{x}^{T}\left(D_{26} \mathbf{B}_{y}+D_{66} \mathbf{B}_{x}\right)\right) d x d y+\int_{x} \int_{y} \overline{\mathbf{N}}^{T} \kappa A_{55} \overline{\mathbf{N}} d x d y
\end{aligned}
$$

Analogously, the following definitions are needed to evaluate the terms of the element mass matrix $\mathbf{M}^{(e)}$ introduced in Equation (32), which also turns out to be symmetrical. The submatrices $\mathbf{M}_{i j}^{(e)}$, for $i, j=1,2, \ldots, 5$ assume the following aspects:

$$
\begin{aligned}
\mathbf{M}_{11} & =\int_{x} \int_{y} \overline{\mathbf{N}}^{T} I_{0} \overline{\mathbf{N}} d x d y \\
\mathbf{M}_{14} & =\int_{x} \int_{y} \overline{\mathbf{N}}^{T} I_{1} \overline{\mathbf{N}} d x d y \\
\mathbf{M}_{22} & =\int_{x} \int_{y} \overline{\mathbf{N}}^{T} I_{0} \overline{\mathbf{N}} d x d y \\
\mathbf{M}_{25} & =\int_{x} \int_{y} \overline{\mathbf{N}}^{T} I_{1} \overline{\mathbf{N}} d x d y \\
\mathbf{M}_{33} & =\int_{x} \int_{y} \overline{\mathbf{N}}^{T} I_{0} \overline{\mathbf{N}} d x d y \\
\mathbf{M}_{41} & =\mathbf{M}_{14}^{T} \\
\mathbf{M}_{44} & =\int_{x} \int_{y} \overline{\mathbf{N}}^{T} I_{2} \overline{\mathbf{N}} d x d y \\
\mathbf{M}_{52} & =\mathbf{M}_{25}^{T} \\
\mathbf{M}_{55} & =\int_{x} \int_{y} \overline{\mathbf{N}}^{T} I_{2} \overline{\mathbf{N}} d x d y
\end{aligned}
$$

\section{References}

1. Kardestuncer, H.; Norrie, D.H. Finite Element Handbook; McGraw-Hill: New York, NY, USA, 1987.

2. Duncan, W.J.; Collar, A.R. A method for the solution of oscillations problems by matrices. Lond. Edinb. Dublin Philos. Mag. J. Sci. 1934, 17, 865-909. [CrossRef]

3. Duncan, W.J.; Collar, A.R. Matrices applied to the motions of damped systems. Lond. Edinb. Dublin Philos. Mag. J. Sci. 1935, 19, 197-219. [CrossRef]

4. Hrennikoff, A. Solution of Problems of Elasticity by the Frame-Work Method. ASME J. Appl. Mech. 1941, 8, A619-A715.

5. Courant, R. Variational methods for the solution of problems of equilibrium and vibration. Bull. Am. Math. Soc. 1943, 49, 1-23. [CrossRef]

6. Clough, R.W. The finite element method in plane stress analysis. In Proceedings of the 2nd A.S.C.E. Conference in Electronics Computation, Pittsburgh, PA, USA, 8-9 September 1960.

7. Melosh, R.J. Basis for derivation of matrices for the direct stiffness method. AIAA J. 1963, 1, 1631-1637. 
8. Ouakka, S.; Fantuzzi, N. Trustworthiness in Modeling Unreinforced and Reinforced T-Joints with Finite Elements. Math. Comput. Appl. 2019, 24, 27. [CrossRef]

9. Uzun, B.; Civalek, Ö. Nonlocal FEM Formulation for Vibration Analysis of Nanowires on Elastic Matrix with Different Materials. Math. Comput. Appl. 2019, 24, 38. [CrossRef]

10. Oden, J.T. Finite Elements of Nonlinear Continua; McGraw-Hill: New York, NY, USA, 1972.

11. Oden, J.T.; Reddy, J.N. An Introduction to the Mathematical Theory of Finite Elements; John Wiley: New York, NY, USA, 1976.

12. Hinton, E. Numerical Methods and Software for Dynamic Analysis of Plates and Shells; Pineridge Press: Swansea, UK, 1988.

13. Zienkiewicz, O.C. The Finite Element Method; McGraw-Hill: New York, NY, USA, 1991.

14. Reddy, J.N. An Introduction to the Finite Element Method; McGraw-Hill: New York, NY, USA, 1993.

15. Onate, E. Calculo de Estruturas por el Metodo de Elementos Finitos; CIMNE: Barcelona, Spain, 1995.

16. Hughes, T.J.R. The Finite Element Method_Linear Static and Dynamic Finite Element Analysis; Dover Publications: New York, NY, USA, 2000.

17. Ferreira, A.J.M. MATLAB Codes for Finite Element Analysis; Springer: New York, NY, USA, 2008.

18. Dezi, L.; Menditto, G.; Tarantino, A.M. Homogeneous structures subjected to successive structural system changes. J. Eng. Mech. ASCE 1990, 116, 1723-1732. [CrossRef]

19. Dezi, L.; Tarantino, A.M. Time dependent analysis of concrete structures with variable structural system. ACI Mater. J. 1991, 88, 320-324.

20. Dezi, L.; Menditto, G.; Tarantino, A.M. Viscoelastic heterogeneous structures with variable structural system. J. Eng. Mech. ASCE 1993, 119, 238-250. [CrossRef]

21. Dezi, L.; Tarantino, A.M. Creep in continuous composite beams. Part I: Theoretical treatment. J. Struct. Eng. ASCE 1993, 119, 2095-2111. [CrossRef]

22. Reddy, J.N.; Miravete, A. Practical Analysis of Composite Laminates; CRC Press: Boca Raton, FL, USA, 1995.

23. Reddy, J.N. Mechanics of Laminated Composite Plates and Shells-Theory and Analysis, 2nd ed.; CRC Press: Boca Raton, FL, USA, 2004.

24. Tornabene, F.; Bacciocchi, M. Anisotropic Doubly-Curved Shells. Higher-Order Strong and Weak Formulations for Arbitrarily Shaped Shell Structures; Esculapio: Bologna, Italy, 2018.

25. Vinson, J.R. The Behavior of Shells Composed of Isotropic and Composite Materials; Springer: New York, NY, USA, 1993.

26. Jones, R.M. Mechanics of Composite Materials, 2nd ed.; Taylor \& Francis: Philadelphia, PA, USA, 1999.

27. Christensen, R.M. Mechanics of Composite Materials; Dover Publications: New York, NY, USA, 2005.

28. Barbero, E.J. Introduction to Composite Materials Design; CRC Press: Boca Raton, FL, USA, 2011.

29. Chamis, C.C.; Sendeckyj, G.P. Critique on Theories Predicting Thermoelastic Properties of Fibrous Composites. J. Compos. Mater. 1968, 2, 332-358. [CrossRef]

30. Halpin, J.C. Effects of Environmental Factors on Composite Materials. Available online: http://citeseerx.ist. psu.edu/viewdoc/download?doi=10.1.1.844.575\&rep=rep1\&type=pdf (accessed on 18 May 2019).

31. Tsai, S.W. Structural Behavior of Composite Materials; NASA: Washington, DC, USA, 1964.

32. Tsai, S.W. Strength Characteristics of Composite Materials; NASA: Washington, DC, USA, 1965.

33. Hill, R. Theory of Mechanical Properties of Fibre-Strengthened Materials: I. Elastic Behavior. J. Mech. Phys. Solids 1964, 12, 199-212. [CrossRef]

34. Hill, R. Theory of Mechanical Properties of Fibre-Strengthened Materials: II. Inelastic Behavior. J. Mech. Phys. Solids 1964, 12, 213-218. [CrossRef]

35. Tornabene, F.; Bacciocchi, M.; Fantuzzi, N.; Reddy, J.N. Multiscale Approach for Three-Phase CNT/Polymer/ Fiber Laminated Nanocomposite Structures. Polym. Compos. 2019, 40, E102-E126. [CrossRef]

36. Reddy, J.N.; Chin, C.D. Thermomechanical Analysis of Functionally Graded Cylinders and Plates. J. Therm. Stresses 1998, 21, 593-626. [CrossRef]

37. Reddy, J.N. Analysis of functionally graded plates. Int. J. Numer. Methods Eng. 2000, 47, 663-684. [CrossRef]

38. Reddy, J.N. Microstructure-dependent couple stress theories of functionally graded beams. J. Mech. Phys. Solids 2011, 59, 2382-2399. [CrossRef]

39. Reddy, J.N.; Kim, J. A nonlinear modified couple stress-based third-order theory of functionally graded plates. Compos. Struct. 2012, 94, 1128-1143. [CrossRef] 
40. Kim, J.; Reddy, J.N. A general third-order theory of functionally graded plates with modified couple stress effect and the von Kármán nonlinearity: Theory and finite element analysis. Acta Mech. 2015, 226, 2973-2998. [CrossRef]

41. Kim, J.; Reddy, J.N. Modeling of functionally graded smart plates with gradient elasticity effects. Mech. Adv. Mater. Struct. 2017, 24, 437-447. [CrossRef]

42. Kim, J.; Zur, K.K.; Reddy, J.N. Bending, free vibration, and buckling of modified couples stress-based functionally graded porous micro-plates. Compos. Struct. 2019, 209, 879-888. [CrossRef]

43. Gutierrez Rivera, M.; Reddy, J.N. Stress analysis of functionally graded shells using a 7-parameter shell element. Mech. Res. Commun. 2016, 78, 60-70. [CrossRef]

44. Lanc, D.; Vo, T.P.; Turkalj, G.; Lee, J. Buckling analysis of thin-walled functionally graded sandwich box beams. Thin Wall. Struct. 2015, 86, 148-156. [CrossRef]

45. Lanc, D.; Turkalj, G.; Vo, T.; Brnic, J. Nonlinear buckling behaviours of thin-walled functionally graded open section beams. Compos. Struct. 2016, 152, 829-839. [CrossRef]

46. Sofiyev, A.H.; Kuruoglu, N. Dynamic instability of three-layered cylindrical shells containing an FGM interlayer. Thin Wall. Struct. 2015, 93, 10-21. [CrossRef]

47. Alibeigloo, A. Thermo elasticity solution of sandwich circular plate with functionally graded core using generalized differential quadrature method. Compos. Struct. 2016, 136, 229-240. [CrossRef]

48. Tornabene, F. Free Vibration Analysis of Functionally Graded Conical, Cylindrical Shell and Annular Plate Structures with a Four-parameter Power-Law Distribution. Comput. Method. Appl. Mech. Eng. 2009, 198, 2911-2935. [CrossRef]

49. Tornabene, F.; Viola, E. Free Vibration Analysis of Functionally Graded Panels and Shells of Revolution. Meccanica 2009, 44, 255-281. [CrossRef]

50. Tornabene, F.; Fantuzzi, N.; Viola, E.; Batra, R.C. Stress and strain recovery for functionally graded free-form and doubly-curved sandwich shells using higher-order equivalent single layer theory. Compos. Struct. 2015, 119, 67-89. [CrossRef]

51. Mercan, K.; Baltacioglu, A.K.; Civalek, Ö. Free vibration of laminated and FGM/CNT composites annular thick plates with shear deformation by discrete singular convolution method. Compos. Struct. 2018, 186, 139-153. [CrossRef]

52. Civalek, Ö.; Baltacioglu, A.K. Free vibration analysis of laminated and FGM composite annular sector plates. Compos. Part B Eng. 2019, 157, 182-194. [CrossRef]

53. Mróz, Z. Optimal design of structures of composite materials. Int. J. Solids Struct. 1970, 6, 859-870. [CrossRef]

54. Bert, C.W. Optimal design of a composite-material plate to maximize its fundamental frequency. J. Sound Vib. 1977, 50, 229-237. [CrossRef]

55. Bruyneel, M. A general and effective approach for the optimal design of fiber reinforced composite structures. Compos. Sci. Technol. 2006, 66, 1303-1314. [CrossRef]

56. Pelletier, J.L.; Vel, S.S. Multi-objective optimization of fiber reinforced composite laminates for strength, stiffness and minimal mass. Comput. Struct. 2006, 84, 2065-2080. [CrossRef]

57. Dong, C.; Davies, I.J. Optimal design for the flexural behaviour of glass and carbon fibre reinforced polymer hybrid composites. Mater. Design. 2012, 37, 450-457. [CrossRef]

58. Ganguli, R. Optimal design of composite structures: a historical review. J. Indian I. Sci. 2013, 93, 557-570.

59. Ke, L.-L.; Yang, J.; Kitipornchai, S. Nonlinear free vibration of functionally graded carbon nanotube-reinforced composite beams. Compos. Struct. 2010, 92, 676-683. [CrossRef]

60. Shen, H.-S. Thermal buckling and postbuckling behavior of functionally graded carbon nanotube-reinforced composite cylindrical shells. Compos. Part B Eng. 2012, 43, 1030-1038. [CrossRef]

61. Zhang, L.W.; Lei, Z.X.; Liew, K.M.; Yu, J.L. Static and dynamic of carbon nanotube reinforced functionally graded cylindrical panels. Compos. Struct. 2014, 111, 205-212. [CrossRef]

62. Liew, K.M.; Lei, Z.X.; Zhang, L.W. Mechanical analysis of functionally graded carbon nanotube reinforced composites: A review. Compos. Struct. 2015, 120, 90-97. [CrossRef]

63. Alibeigloo, A. Elasticity solution of functionally graded carbon nanotube-reinforced composite cylindrical panel subjected to thermo mechanical load. Compos. Part B Eng. 2016, 87, 214-226. [CrossRef]

64. Civalek, Ö. Free vibration of carbon nanotubes reinforced (CNTR) and functionally graded shells and plates based on FSDT via discrete singular convolution method. Compos. Part B Eng. 2017, 111, 45-59. [CrossRef] 
65. Thang, P.T.; Nguyen, T.-T.; Lee, J. A new approach for nonlinear buckling analysis of imperfect functionally graded carbon nanotube-reinforced composite plates. Compos. Part B Eng. 2017, 127, 166-174. [CrossRef]

66. Zhao, J.; Choe, K.; Shuai, C.; Wang, A.; Wang, Q. Free vibration analysis of functionally graded carbon nanotube reinforced composite truncated conical panels with general boundary conditions. Compos. Part B Eng. 2019, 160, 225-240. [CrossRef]

67. Civalek, Ö.; Baltacioglu, A.K. Vibration analysis of circular cylindrical panels with CNT reinforced and FGM composites. Compos. Struct. 2018, 202, 374-388.

68. Civalek, Ö.; Baltacioglu, A.K. Vibration of carbon nanotube reinforced composite (CNTRC) annular sector plates by discrete singular convolution method. Compos. Struct. 2018, 203, 458-465. [CrossRef]

69. Bacciocchi, M.; Tarantino, A.M. Time-dependent behavior of viscoelastic three-phase composite plates reinforced by Carbon nanotubes. Compos. Struct. 2019, 216, 20-31. [CrossRef]

70. Fantuzzi, N.; Tornabene, F.; Bacciocchi, M.; Neves, A.M.A.; Ferreira, A.J.M. Stability and accuracy of three Fourier expansion-based strong form finite elements for the free vibration analysis of laminated composite plates. Int. J. Numer. Methods Eng. 2017, 111, 354-382. [CrossRef]

71. Tornabene, F.; Fantuzzi, N.; Bacciocchi, M. On the mechanics of laminated doubly-curved shells subjected to point and line loads. Int. J. Eng. Sci. 2016, 109, 115-164. [CrossRef]

(C) 2019 by the authors. Licensee MDPI, Basel, Switzerland. This article is an open access article distributed under the terms and conditions of the Creative Commons Attribution (CC BY) license (http://creativecommons.org/licenses/by/4.0/). 



\title{
A Transdisciplinary Approach for Analyzing Stress Flow Patterns in Biostructures
}

\author{
Reena Patel ${ }^{1, *}$, Guillermo Riveros ${ }^{1}$, David Thompson ${ }^{2}$, Edward Perkins ${ }^{1}$, Jan Jeffery Hoover ${ }^{1}$, \\ John Peters ${ }^{3}$ and Antoinette Tordesillas ${ }^{4}$ \\ 1 US Army Engineer Research and Development Center, Vicksburg, MS 39180, USA; \\ Guillermo.A.Riveros@usace.army.mil (G.R.); Edward.J.Perkins@usace.army.mil (E.P.); \\ Jan.J.Hoover@usace.army.mil (J.J.H.) \\ 2 Department of Aerospace Engineering, Mississippi State University, Starkville, MS 39762, USA; \\ dst@ae.msstate.edu \\ 3 Center for Advanced Vehicular Systems (CAVS), Mississippi State University, Starkville, MS 39762, USA; \\ john@cavs.msstate.edu \\ 4 School of Mathematics and Statistics, University of Melbourne, Parkville, Victoria 3010, Australia; \\ atordesi@unimelb.edu.au \\ * Correspondence: Reena.R.Patel@usace.army.mil; Tel.: +1-601-634-5430
}

Received: 27 March 2019; Accepted: 24 April 2019; Published: 26 April 2019

\begin{abstract}
This work presents a transdisciplinary, integrated approach that uses computational mechanics experiments with a flow network strategy to gain fundamental insights into the stress flow of high-performance, lightweight, structured composites by investigating the rostrum of paddlefish. Although computational mechanics experiments give an overall distribution of stress in the structural systems, stress flow patterns formed at nascent stages of loading a biostructure are hard to determine. Computational mechanics experiments on a complex model will involve a high degree of freedom thereby making the extraction of finer details computationally expensive. To address this challenge, the evolution of the stress in the rostrum is formulated as a network flow problem generated by extracting the node and connectivity information from the numerical model of the rostrum. The flow network is weighted based on the parameter of interest, which is stress in the current research. The changing kinematics of the system is provided as input to the mathematical algorithm that computes the minimum cut of the flow network. The flow network approach is verified using two simple classical problems. When applied to the model of the rostrum, the flow network approach identifies strain localization in tensile regions, and buckling/crushing in compressive regions.
\end{abstract}

Keywords: biostructure; rostrum; paddlefish; Polyodon spathula; maximum-flow/minimum-cut; stress patterns

\section{Introduction}

A fundamental understanding of the response of a structural system to external loading conditions is important in determining if the design is able to withstand the prescribed external load. Numerous methods are available to quantify the response of a structural system to external loads. Existing methods, such as acoustic emission [1-7] and digital image correlation [8-17], offer the capability to experimentally measure/quantify/determine strains/deformations. From a modelling perspective, there are continuum-based [18-20] and discrete- or lattice-based [21-23] approaches that not only satisfy the physical laws governing the systems, but also provide abundant information required for structural analysis. Finite element analysis has been used to study the mechanical properties of bioinspired structures. Flores-Johnson et al. [24] carried out simulations on bioinspired, nacre-like composite plates. Nacre, found in mollusk shells, is a biological material that shows remarkable mechanical performance 
because of its hierarchical geometry spanning multiple length scales [25]. Flores-Johnson et al. [24] found that the nacre-like plates demonstrated superior performance as compared to standard bulk plates under blast loadings. Tran et al. [26] conducted a study to analyze the response of a bioinspired composite plate based on nacre structure subjected to underwater impulsive loading. They discovered that the bioinspired composite structure spread the damage over a larger area, thereby decreasing stress. Although a considerable amount of research is performed on biostructures and bioinspired structures, there is yet a lack of understanding on what imparts superior performance to biostructures. Numerical analysis provides the overall response of the structural system under an external loading condition. However, additional information is required to understand the influence of the local topology on the global structural response and overall performance of the system. To our knowledge, advanced mathematical algorithms have not been used with continuum-based models to gain fundamental insight into the structural response of biostructures. Since biostructures are geometrically optimized at the coarsest level of hierarchy, the information obtained at the local topology level provides insights that could be used to identify optimal geometrical configurations that impart superior characteristics to biostructures.

Any conceivable problem dealing with the transmission of information can be solved by abstraction to a network or a graph [27]. These abstractions involve representing the system in terms of a transmission medium and the internal details that block the information flow, i.e., flow bottlenecks. From this viewpoint, maximum flow and minimum cut are two of the most widely used algorithmic approaches in network flow theory [27]. Maximum flow governs the amount of information that can be transmitted through the network. Minimum cut embodies a set of edges in the network that form the bottlenecks to transmission. Tordesillas et al. examined the formation of bottlenecks in transmission of force through the contact network by applying network flow theory [27-29]. The location and formation of the bottlenecks are influenced by the local as well as global characteristics of the structural system. The early identification of bottlenecks in force transmission opens new avenues for detection, as well as manipulation of the approaching failure in granular systems.

Lefort et al. [30] used image analysis that employed an integration of a numerical model with Ripley's function [31] to identify patterns in the fracture process zone (FPZ) in a quasibrittle material. Macrocracks in a quasibrittle material are formed by the combination of microcrack propagation, interaction, and coalescence, situated within the FPZ. Ripley's function is used to characterize randomness in the spatial spreading of point distributions and to identify the spreading and development of diverse patterns, e.g., cell migration [31], tree [32] and plant [33] dissemination, and disease transmission [34]. Lefort's research could open up new paths that would result in nonlocal continuum modeling at the macroscale level.

The mathematical approaches used for dense granular systems and quasibrittle materials demonstrate that local topological interactions are a major contributor to the global structural response. To gain an understanding of failure mechanisms, it is essential to capture the activity around the failure site at the nascent stage of loading. Obtaining this insight at the onset of loading may provide insight into failure mechanisms and possibly suggest novel approaches to alter the process and location of failure. Additionally, if the location of the failure is identified earlier, there is a possibility of developing a relationship that aids in quantifying the external force required for the structural system to fail at that location. This knowledge can be used for designing new structural systems, and for the assessment of existing critical infrastructure.

Biostructures have, in general, demonstrated superior characteristics as they evolved to serve multiple and specific functions [35]. The biostructure of interest here is the snout, or rostrum, of the paddlefish (Polyodon spathula). This large North American freshwater fish is related to sturgeons. Unlike those fish with their short thick rostra, the paddlefish has an elongated, flattened rostrum that in adults may be in the range of $29-59 \%$ of its body length, and in breadth in the range of $8-17 \%$ of body length [36]. The rostrum was once believed to be a simple digging or stirring implement, but is now known to be a complex hydrodynamic and electrosensory structure [37]. This prominent structure is easily observed and is supported internally by a distinctive skeleton of ossified cartilage comprised of 
large axial rods with two posterior buttresses at the base and with numerous small stellate elements lateral to the axis (Figure 1).

The rostrum's lattice-like architecture with complex geometry appears to contribute to its unique strength and resilience. This research is motivated by an earlier feasibility study that concluded that the nonuniform geometry of the rostrum is one of its toughening mechanisms used to mitigate failure [38]. Since biostructures in general and the paddlefish rostrum in particular are made of heterogeneous materials and also have hierarchical geometry, it is difficult to identify the major contributor to their superior structural response. A fundamental understanding of how this lattice-like architecture functions has the potential to provide novel insights into applications over a wide range of disciplines (e.g., protective panels, novel building materials, body and vehicle armor, and ship design, among other possible uses). This research seeks to use an integrated transdisciplinary approach that feeds information from computational mechanics experiments on biostructures to a mathematical algorithm to identify the patterns formed at the early stages of loading of the biostructure.

Patel et al. developed the methodology of using network theory with finite element analysis in their previous work [39], which used a single source and sink combination to analyze the stress flow pattern on the rostrum. They also presented the procedure of preparing the data in an appropriate format from finite element binary database files to an abstract mathematical domain in their recent publication [40]. Patel et al. have also developed bioinspired structural systems to analyze the relationship of structural resiliency and geometrical complexity [39]. This work extends their previous research to include a multiple combination of source and sink. Since the material is in the linearly elastic regime, the solution from the mathematical algorithm can be superimposed. The addition of this aspect to the current analysis assisted in capturing the shear and flexure pattern in a four point bending of a simply supported concrete beam.
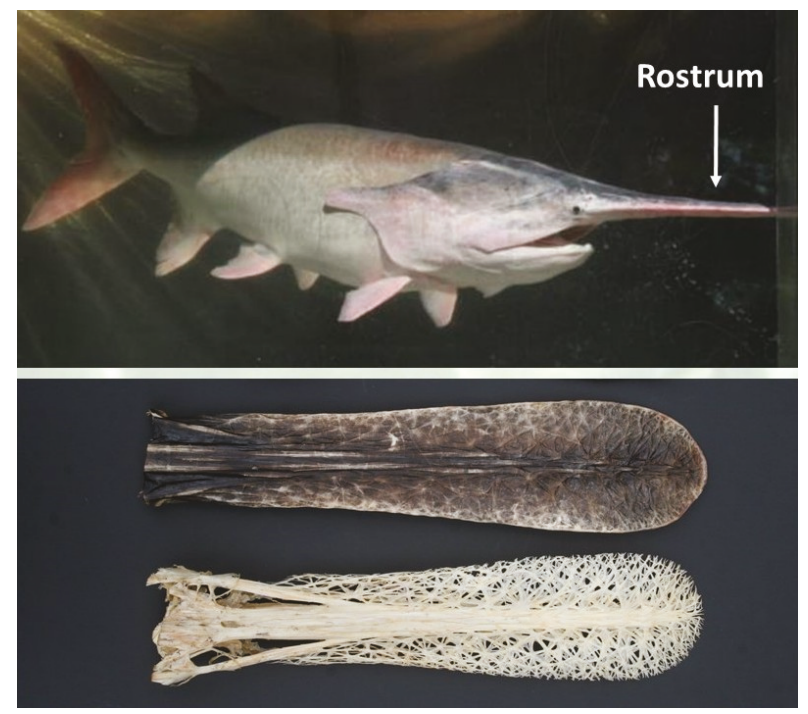

Figure 1. Paddlefish from the lower Mississippi River showing the unique rostrum of the species. (Upper photo) Adult fish $1 \mathrm{~m}$ long contained in a mobile swim tunnel; (lower photo) dried and skeletonized rostrum. Photo taken by Dr. Jan Jeffrey Hoover, USACE.

The paper is organized as follows. In Section 2, a brief introduction to network flow is provided, and Section 3 describes the procedure to formulate the biostructure as a network flow problem. In Section 4, the proposed approach is verified using two classical problems. Details of the computational 
mechanics experiments are discussed in Section 5, followed by results in Section 6, and conclusions follow in Section 7.

\section{Network Flow}

A brief introduction of the basic concepts of network flow is now provided. A flow network is a directed graph with two distinguished vertices, called a source and a sink, coupled with a non-negative real-valued function called the capacity function [27,41,42].

A network $N$ is defined as a set comprising:

1. directed graph $G(V, E)$, where $V$ is a finite set of vertices, and $E$ is a subset of ordered pairs of vertices representing the edges;

2. vertex $s \in V$ that has only outgoing edges represented as the source node;

3. vertex $t \in V$ that has only incoming edges represented as the sink node; and

4. positive function $c: E \rightarrow R^{+}$called the capacity function.

Flow $f$ on a network $N$ is defined by real-valued function $f: E \rightarrow R^{+}$. Flow $\mathrm{f}$ is a feasible flow vector if it satisfies the following constraints:

1. Capacity constraint $\forall u, v \in V$ requires that $f(u, v) \leq c(u, v)$; flow cannot exceed the capacity of the respective edge.

2. Conservation of flow $\forall u, v \in V-(s, t)$ requires that

(a) $\quad \sum_{v \epsilon V} f(u, v)=0$,

(b) the total flow entering a node must equal the total flow leaving that node provided the node is not a source or sink node, and

3. the total flow leaving the source node $s$ must be equal to the total flow entering sink node $t$.

Given a flow network $G(V, E)$, with source $s$ and sink $t$, such that there are no incoming edges at the source and no outgoing edges at the sink, the maximum flow problem involves finding a function $f$ that satisfies the capacity and conservation constraints described above; its value is defined as follows:

$$
\operatorname{val}(f)=\max (f)
$$

A cut of a flow network $G(V, E)$ is defined as a set of vertices $\left(E_{1}, E_{2}\right)$ that partition $V$ into $E_{1}$ and $E_{2}=V-E_{1}$, such that $s \in E_{1}$ and $t \in E_{2}$. If $\mathrm{f}$ is a flow, then the net flow across the cut $\left(E_{1}, E_{2}\right)$ is defined as $\mathrm{f}\left(E_{1}, E_{2}\right)$. The capacity of the cut $\left(E_{1}, E_{2}\right)$ is $\mathrm{c}\left(E_{1}, E_{2}\right)$.

A minimum $E_{1}-E_{2}$ cut problem involves minimizing $\mathrm{c}\left(E_{1}, E_{2}\right)$. That would mean the identification of E1 and E2 in such a manner so as to find the minimal capacity of the $\left(E_{1}, E_{2}\right)$ cut.

In combinatorial optimization theory, the maximum-flow/minimum-cut theorem states that in a flow network $G(V, E)$, the maximum quantity of flow $f$ travelling from source node $s$ to sink node $t$ is identical to the total weight of the edges in the minimum cut. Essentially speaking, the maximum flow in the network is equal to the smallest total weight of the edges that, if removed, would cause the source to be totally disconnected from the sink.

The network flow approach has previously been applied to characterize stress transmission in various synthetic and natural materials with complex microstructures, for example, sand [29,43], photoelastic disks [29], and concrete [5]. In these studies, microstructural data on the internal connectivity and edge capacities were obtained from high-resolution imaging experiments (e.g., X-ray CT, birefringence analysis) and discrete-element (DEM) models.

\section{Formulation of the Biostructure as Network Flow Problem}

The flow of stress in a structural system can be interpreted as a directed graph that facilitates the investigation of failure mechanisms. Intuitively, the transmission of information at a given point in the 
structural system is the rate at which the information (i.e., variables such as stress, displacement, kinetic energy, elastic or plastic strain in the structural system) travels. Each edge in a directed graph can be compared to a channel through which information is transmitted. Each edge has a corresponding capacity that is representative of the maximum rate at which information can be passed through the edge. Vertices of the graph are the points where the edges connect. Two special nodes identified as source and sink govern the flow. The edges connected to these special nodes are assigned infinite capacity $[27,29]$. The selection of these special nodes is dependent on the type of boundary conditions implemented on the model. For example, if the model is subjected to constant uniform pressure in the vertical direction, the nodes on the top or bottom surface of the model are chosen to act as the source and sink, respectively. Since the flow of stress is symmetric, altering the assignment of source and sink does not change the flow pattern.

A flow network $\mathrm{N}(V, E, A, U, s, t)[27,29]$ is constructed from the computational-mechanics model, such that:

1. $V$ represents the nodes obtained from the finite element model of the biostructure. As shown in Figure $2 \mathrm{a}$, for a hexahedral element in a finite element model, nodes are $1,2, \ldots, 8$.

2. E represents the edges, connecting the nodes in $V$, indicating connectivity A between the nodes. The edges of a hexahedral element, as shown in Figure $2 a$, are $\{(1,2),(2,3),(3,4),(4,1)\}$ for Face 1 .

3. Each edge $(u, v) \in$ Ehas a capacity $U$ associated with it that is representative of the maximum amount of flow that could be transmitted through the edge. Capacity calculation for Edge $(1,2)$ is shown in Figure 2c. Capacity for Edge $(1,2)$ is the average of the von Mises stresses at Nodes 1 and 2.

4. Von Mises stresses (as shown in Figure 3) at each node are calculated using the average of the integration points shown in Figure 2d.

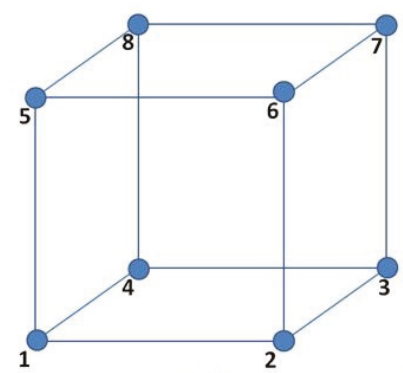

(a)

$$
\begin{gathered}
U_{1}=\frac{\left(\sigma_{1}+\sigma_{2}\right)}{2} \\
U_{1}=\text { Capacity of the edge }(1,2) \\
\sigma_{1}=\text { Von Mises stress at node } 1 \\
\sigma_{2}=\text { Von Mises stress at node } 2
\end{gathered}
$$

(c)

$$
\begin{array}{ll}
\text { Face } 1 & 1-2-3-4 \text { face } \\
\text { Face 2 } & 5-8-7-6 \text { face } \\
\text { Face 3 } & 1-5-6-2 \text { face } \\
\text { Face 4 } & 2-6-7-3 \text { face } \\
\text { Face 5 } & 3-7-8-4 \text { face } \\
\text { Face 6 } & 4-8-5-1 \text { face }
\end{array}
$$

(b)

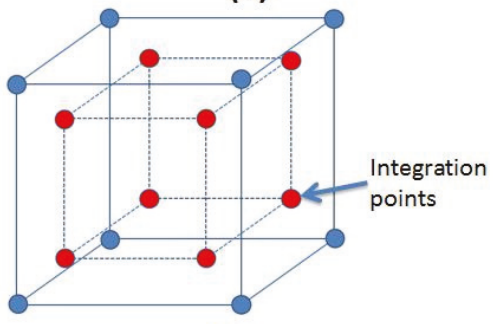

(d)

Figure 2. (a) Node numbering in an eight-node hexahedral element. (b) Hexahedral element faces in an eight-node hexahedral element. (c) Capacity calculation for edges in flow network graph. (d) Integration points on an eight-node hexahedral. 


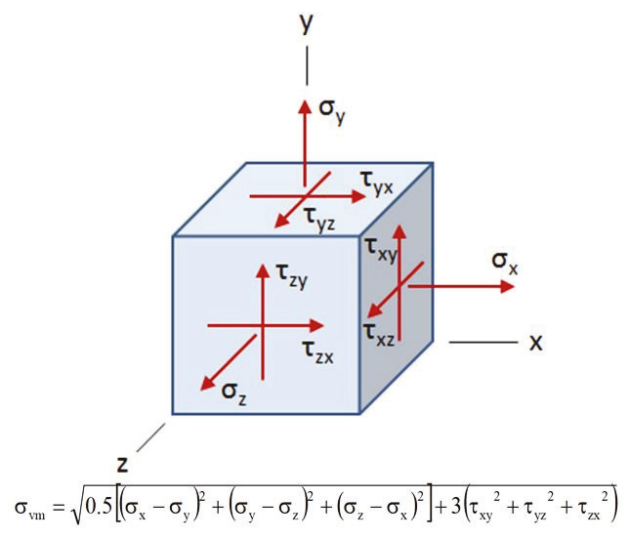

Figure 3. Von Mises stress equation expressed by the six stress components.

Each time frame from the computational-mechanics experiment is constructed as a network flow graph, where each graph depicts the flow of the variable of interest in response to the external loading condition. For each network, the maximum flow and minimum cut is calculated. As stated by the maximum-flow/minimum-cut theorem, the value of the maximum flow is equal to the capacity of the minimum cut.

The procedure outlined above for the proposed flow network approach is outlined in the flowchart shown in Figure 4. As shown in Figure 4, the flow network is constructed using the nodes and connectivity information from the computational mechanics experiment. Capacity $C$ of the flow network was obtained from the Von Mises stresses produced from the binary output database files of the computational-mechanics experiment. The mathematical algorithm was executed on the abstract domain. The solution obtained from the mathematical algorithm was mapped back to the physical domain using the coordinate information of the nodes. Paraview was used for visualizing the solution of the mathematical algorithm. As mentioned above, the procedure of formulating the solution from finite element analysis as a flow network graph was carried out in four stages. As shown in Figure 4, in Stage 1 , the flow network was constructed using the nodes and connectivity information from finite element analysis. In Stage 2, the weight of the flow network was obtained using the parameter of interest (stresses, in the current study, were also acquired from finite element analysis). In Stage 3, the flow network graph was created. In Stage 4, the flow network graph was provided as input to the mathematical algorithm that computes the minimum cut of the flow network graph. The details of the procedure involved in the four stages mentioned above is described in the previous work of Patel et al. [40].

The flow network graph in the current study was developed using data obtained from finite element simulation. The Abaqus/Standard solver was used for conducting static analysis. The choice of Abaqus/Standard for performing these analyses is justified based on the algorithm that it employs for static and low-speed dynamic events, where highly accurate stress solutions are required. Analysis was executed in two steps. Gravity load (self-weight) is applied to the models in the initialization stage. Pressure load was applied to the models in the second stage. The pressure load applied to the model was obtained by multiplying the total load with the time increment of the finite element simulation. Geometric nonlinearity was taken into account in the simulations. 


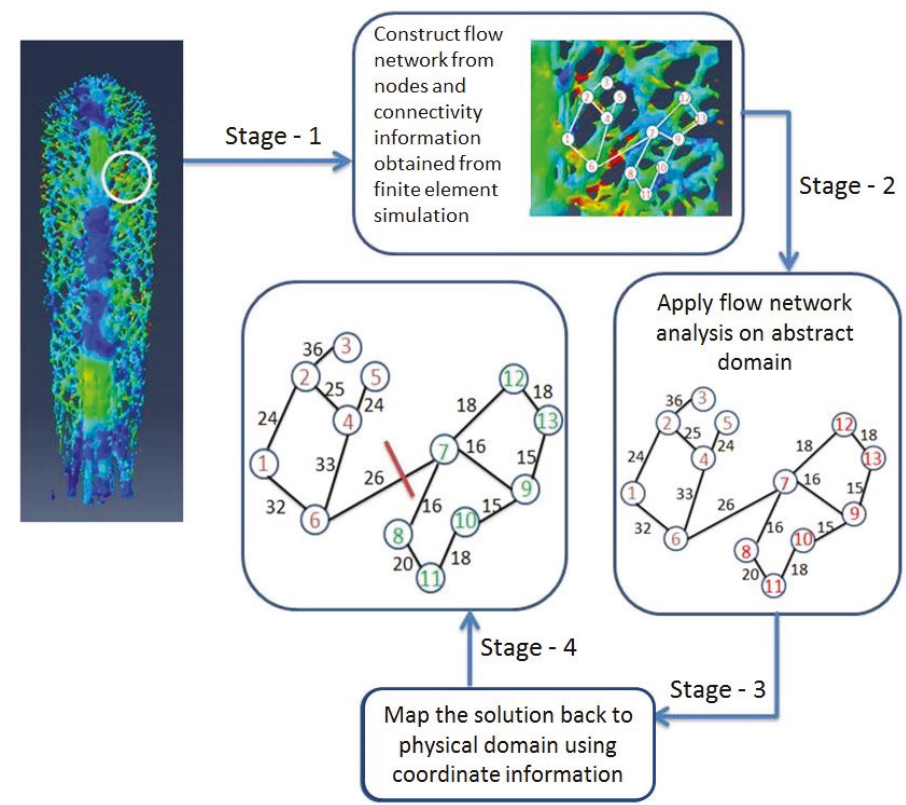

Figure 4. Flowchart depicting the steps involved in formulating a small part of rostrum as a network flow problem.

\section{Verification of Approach on Known Datasets}

The customary method for verifying a new approach is to use a problem that is simple enough to have an analytical solution and similar enough to the phenomena that are being simulated so that a meaningful extrapolation to the actual problem is feasible. By doing so, numerical and analytical solutions can be compared, and the fundamental shortcomings of the numerical approach used to solve the problem can be identified. To verify the current research methodology, two simple problems were chosen. The problem descriptions and methodology implementations follow in the subsections below.

\subsection{Problem 1: Three-Point Bending of a Simply Supported Beam}

The Abaqus/Standard solver [44] was used to carry out a computational-mechanics experiment on a simply supported concrete beam. The length of the beam was 216 inches, the width was 36 inches, and the thickness was 6 inches. A mass density of $8.67 \times 10^{-11} \mathrm{slug} / \mathrm{in}^{3}$, Young's modulus of $3 \times 10^{6} \mathrm{psi}$, and Poisson's ratio of 0.3 were used for the concrete material model $[45,46]$. The Abaqus mesh consisted of 73,278 quadratic tetrahedral elements comprising 117,709 nodes. The schematic of the loading condition on the simply supported concrete beam is displayed in Figure 5.

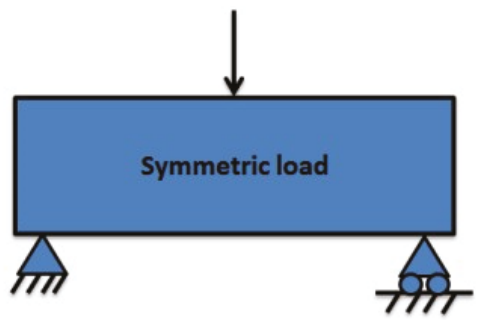

Figure 5. Schematics of three-point bending of a simply supported concrete beam. 
The maximum principal stresses obtained from the computational-mechanics experiment are plotted in Figure 6, which gives the overall stress distribution in the beam subjected to a concentrated load of $100 \mathrm{MPa}$ at the center. The simulation was carried out using the dynamic, explicit method in Abaqus for a total time of $0.005 \mathrm{~s}$. Geometric nonlinearity effects were included. The node and connectivity information were extracted from the computational mechanics model to construct a flow network. The network was weighted based on the Von Mises stress values from the Abaqus output database file. The von Mises stresses are equivalent tensile stresses derived from the stress deviator [47].

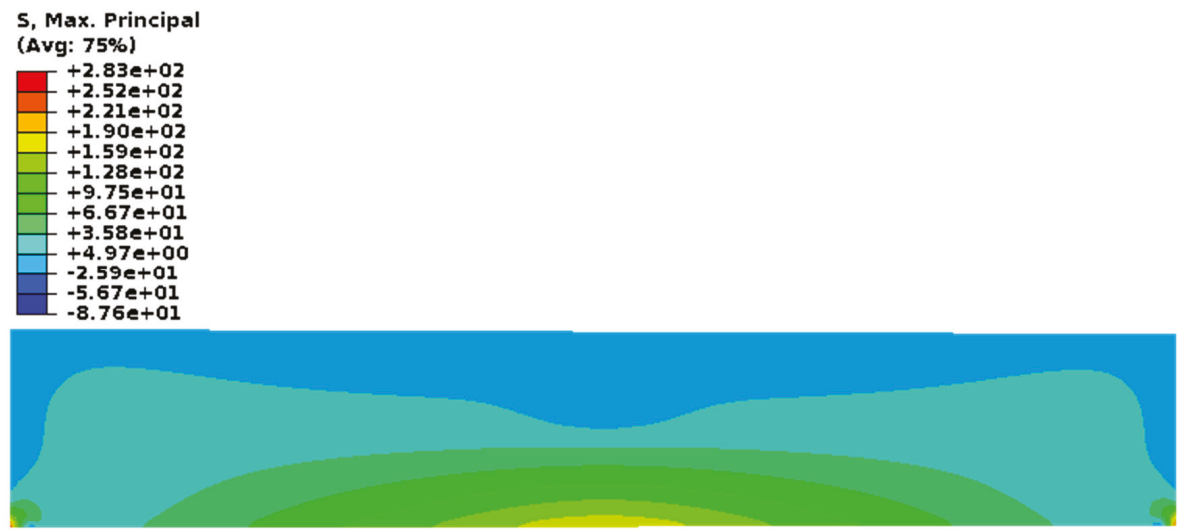

Figure 6. Maximum principal stresses for three-point bending of a simply supported concrete beam.

Source and sink combinations were selected from high-stress areas. The result of the network flow analysis is shown in Figure 7. The source in this case was chosen in the area where the load was applied. The sink was selected in the area where maximum deformation occurs for this classical problem (i.e., the midpoint of the beam parallel to where the source was selected). Figure 7 shows the result of network flow analysis. The minimum cut identified by the mathematical algorithm is represented by the blue line in Figure 7 . The minimum cut denotes a set of edges in the flow network that inhibit flow transmission or form transmission bottlenecks, i.e., flow network analysis highlights the members of the beam where the failure mechanism initiates. For this problem, network analysis shows the typical behavior of a beam subjected to a concentrated load at the center line. A failure for this classical problem initiates with a crack at the bottom face sheet at the beams midspan and continues to grow as the load increases. Hence, the proposed methodology for detecting the failure mechanisms at the nascent stage of loading from the computational-mechanics experiment and flow network approach was verified using a simple problem with a known solution.

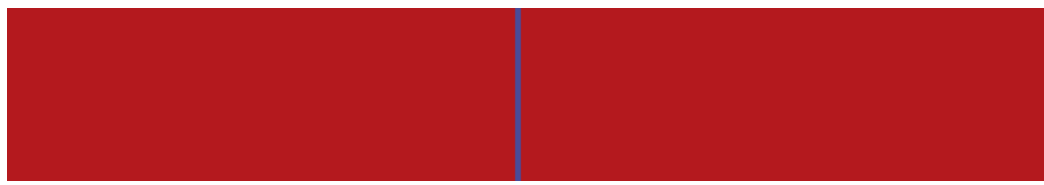

Figure 7. Flow network analysis for three-point bending of a simply supported concrete beam.

\subsection{Problem 2: Four-Point Bending of a Simply Supported Beam}

The dimensions and material of the model used for Problem 2 are identical to the concrete beam used in Problem 1. For this problem, the concrete beam was a simply supported beam with two equal forces applied equidistant from the supports, as shown in Figure 8 [45,46]. 


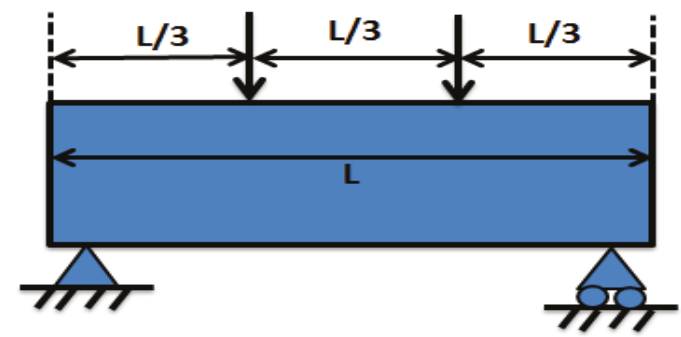

Figure 8. Schematics of four-point bending of a simply supported concrete beam.

The maximum-flow/minimum-cut algorithm was executed in seven scenarios. The source node in each scenario was chosen to be a vertex where the force is applied while the sink node in each scenario was chosen to be a point in an area where a stress transition was visually identified. The sink nodes were selected by looking at the maximum principal stresses, shown in Figure 9. Seven sink nodes were selected. The mathematical algorithm was executed seven times using this source-sink combination. The results were superimposed as the material is in the linear elastic regime.

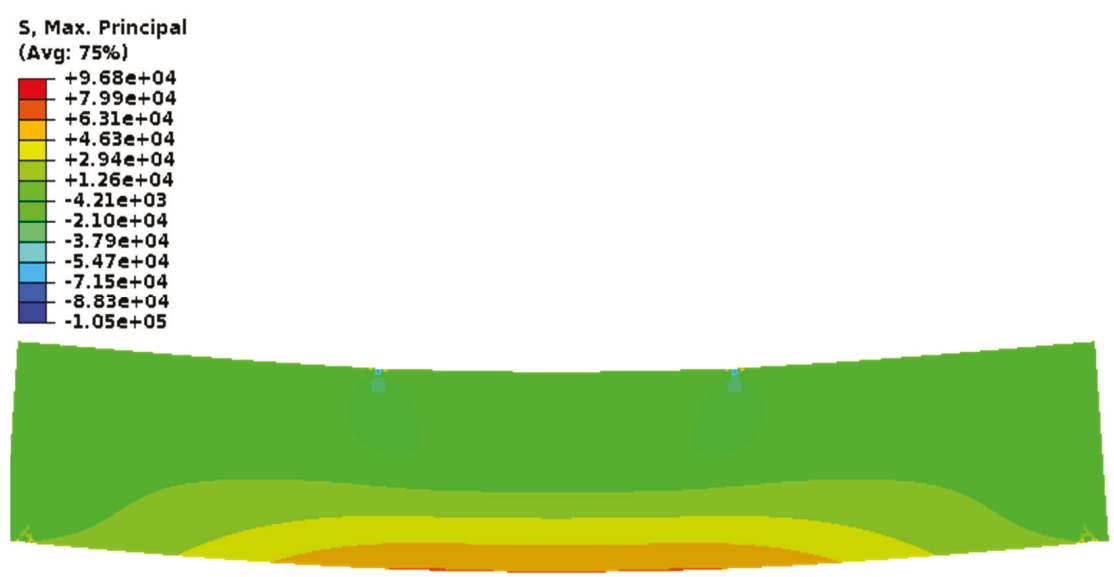

Figure 9. Maximum principal stresses for four-point bending of a simply supported concrete beam.

Figure 10 shows the superimposed result obtained by running the maximum-flow/minimum-cut algorithm. The minimum cut clearly identified the shear patterns on the left and right corners, and flexure patterns in the center are highlighted by the white lines in Figure 10. These shear and flexure patterns govern the phenomena of the failure mechanism in this classical problem. The network flow strategy identified these patterns at the onset of loading when the material was still in the linear elastic regime.

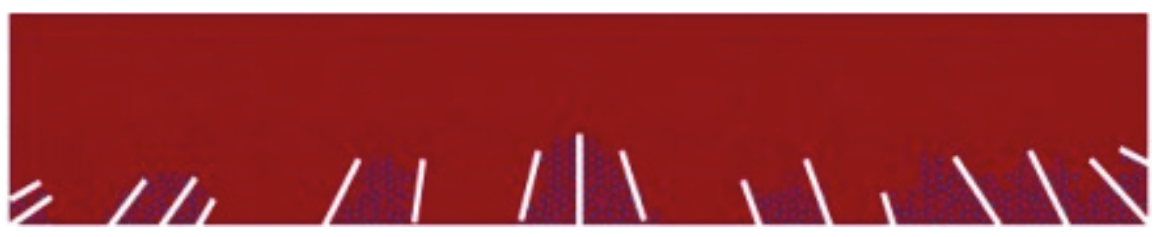

Figure 10. Flow network analysis of four-point bending of a simply supported concrete beam. 


\section{Computational Mechanics Experiments on the Rostrum}

Computational mechanics experiments were carried out on the rostrum of the paddlefish. Simulations were performed in the U.S. Army Engineer Research and Development Centers High Performance Computing facilities located in Vicksburg, MS. The computational model was generated from the tomography (CT scan) of the paddlefish rostrum. The grey tones of the scanned image of the rostrum helped identify the three parts of the rostrum (tissue, hard cartilage, and soft cartilage). Figure 11 shows the tissue component of the rostrum. The tissue is the outermost layer of the rostrum that encompasses the hard cartilage, shown in Figure 11. The soft cartilage is located in the central portion of the rostrum. The mesh assembly cross-section shows the location of the three components of the rostrum in the actual model. The component parts of the rostrum were individually imported into commercial software Abaqus. The parts were meshed using a combination of hexahedral and tetrahedral elements. The model used in the current research comprises 119,712 hexahedral elements, 1,300,451 tetrahedral elements, 375,361 nodes, and 1,126,083 total degrees of freedom.

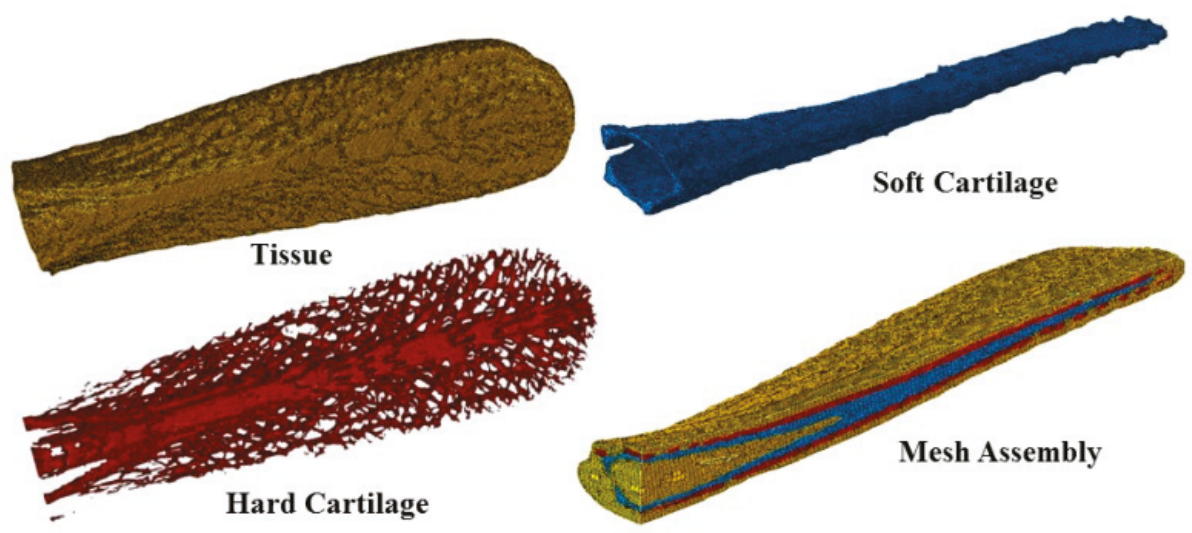

Figure 11. Abaqus mesh model of the rostrum. Dorsal view of tissue and hard cartilage on the left, lateral view of soft cartilage and oblique lateral sagittal section of mesh assembly on the right.

\subsection{Material Properties}

Nano-indentation experiments carried out on the rostrum revealed the material properties of the components of the rostrum [48]. The three parts of the rostrum, as shown in Figure 11, have considerably different material properties [49]. Taking this into consideration, a similar variation in material properties was maintained when selecting the materials for finite element analysis. Table 1 shows the three commercially available materials selected to represent the rostrum components shown in Figure 11.

Table 1. Commercial materials selected to model the rostrum.

\begin{tabular}{ccc}
\hline Part & Commercial Material & Elastic Modulus \\
\hline Tissue & Vinyl ester epoxy & $2.9 \mathrm{GPa}$ \\
Hard cartilage & Polyethylene fibers & $66 \mathrm{GPa}$ \\
Soft cartilage & Polyethylene/epoxy(as isotropic) & $49,762 \mathrm{MPa}$ \\
\hline
\end{tabular}

\subsection{Force and Displacement Boundary Conditions}

A uniform pressure was applied on the top surface of the rostrum, displayed in purple shading in Figure 12. A fixed-plate boundary condition was implemented on the rostrum by restraining the three components of displacement. A uniform pressure of $50 \mathrm{MPa}$ was applied in the loading direction, 
shown in Figure 12. Pressure was applied perpendicular to each element face on the top surface of the rostrum, displayed in purple shading in Figure 12, thereby following the surface contour.

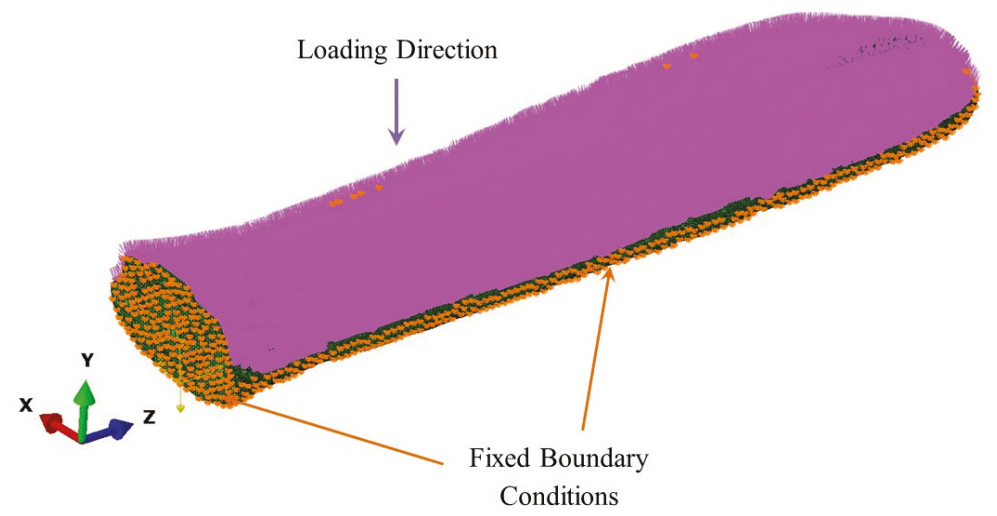

Figure 12. Force and displacement boundary conditions implemented on the rostrum.

\section{Results}

Although computational-mechanics experiments provide the overall distribution of stresses in the structural system, information about stress-flow patterns formed at nascent stages of loading is missing. Identification of the stress-flow patterns in the linear elastic regime may lead to novel insight into failure mechanisms through identification of the location of stress concentration areas developed due to change in geometry and material properties. To obtain these stress patterns, the rostrum is formulated as a network flow model by utilizing node and connectivity information from the computational-mechanics model. The network is weighted based on the Von Mises stresses obtained from the output database file produced from the computational-mechanics experiments on the rostrum. The maximum-flow/minimum-cut algorithm described in Reference [39] was used to identify the stress-flow patterns when the rostrum is subjected to a uniform pressure load. A seamless, platform-independent interface was developed to formulate the rostrum as a network flow problem and compute the maximum flow/minimum cut of the network.

Source and sink nodes were identified and provided as input to the maximum-flow/minimum-cut algorithm. For the rostrum, these nodes were selected based on the displacement contours shown in Figure 13. The source and sink nodes were identified at the edges and center of the maximum displacement area on the top and bottom surfaces of the rostrum, represented by red coloring in Figure 13. As verified earlier in Problem 2, the mathematical algorithm was executed three times using the source/sink combination shown in Figure 13. The results were superimposed, as the material was in the linear elastic regime. Reversing the selection of source and sink nodes on the top and bottom surfaces does not change the result obtained using the maximum-flow/minimum-cut algorithm. 

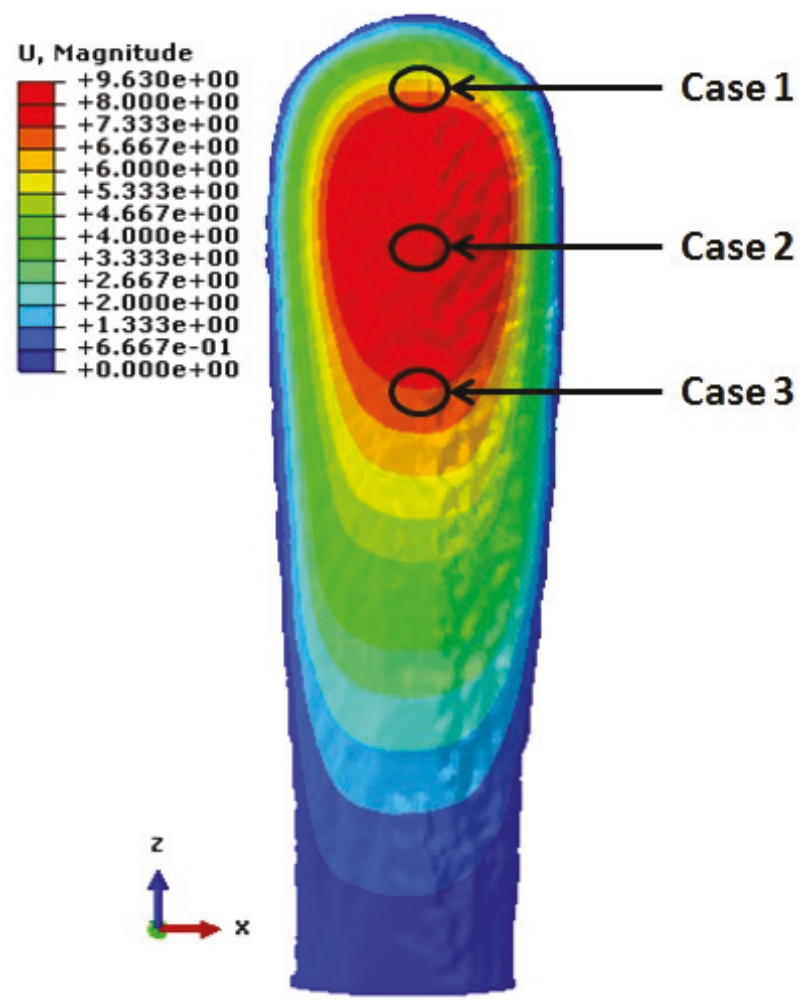

Figure 13. Displacement contours of the rostrum subjected to uniform pressure loading with a fixed plate boundary condition showing the location of source/sink. Cases 1 and 3 were chosen at the edges, and Case 2 in the center of the maximum displacement contour. Dorsal view of rostrum.

\subsection{Flow Network Analysis on Soft Cartilage of the Rostrum}

Figure 14a shows the maximum principal stresses on the bottom surface of the soft-cartilage part of the rostrum. Although stresses progressively increased in the early stages of loading, they were evenly distributed across the entire bottom surface, thereby providing no insight into the phenomena of the failure mechanism. In contrast, Figure $14 \mathrm{~b}$ displays the output obtained by executing the maximum-flow/minimum-cut algorithm using the source/sink combination, shown earlier in Figure 9, and superimposing the results as validated in Problem 2. The minimum cut embodies a set of vertices that inhibit the transmission of information, i.e., stresses in the current analysis. The minimum cut on the bottom surface of the soft cartilage is along the edges where there is a change in material properties. The failure initiates at this position owing to change in geometry and material properties. The red regions at the tip and base of the bottom surface are also located at the areas where material properties change. The maximum-flow/minimum-cut algorithm detected the failure sites at nascent stages of loading. 

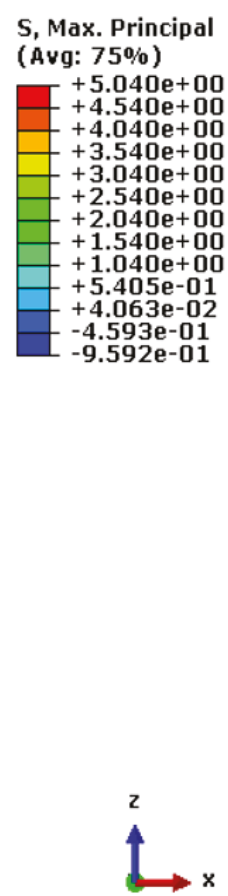

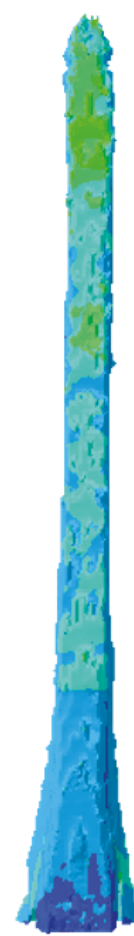

(a)

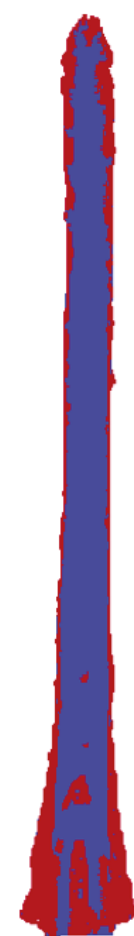

(b)

Figure 14. Soft cartilage of paddlefish rostrum. (a) Maximum principal stress obtained from finite element analysis. (b) Minimum cut obtained from mathematical analysis on bottom surface of rostrum's soft cartilage. Nodes are colored by their respective locations on the source (red) or sink (blue) side of the minimum cut.

Figure 15a shows the maximum principal stresses on the top surface of the soft-cartilage part of the rostrum. As observed earlier in Figure 14a, finite element analysis shows stresses progressively increasing as the external load increases, as expected. On the other hand, Figure 15b shows the minimum cut obtained by running the maximum-flow/minimum-cut algorithm. The interface between the red and blue nodes is the location of the failure site. The failure for the top surface of the rostrum initiates along the edges where there is a change in the material properties. The red region at the base of the top surface falls in areas where there is contact between components with varying material properties. Hence, the flow network strategy successfully identified the local regions where failure mechanisms were expected to initiate. 

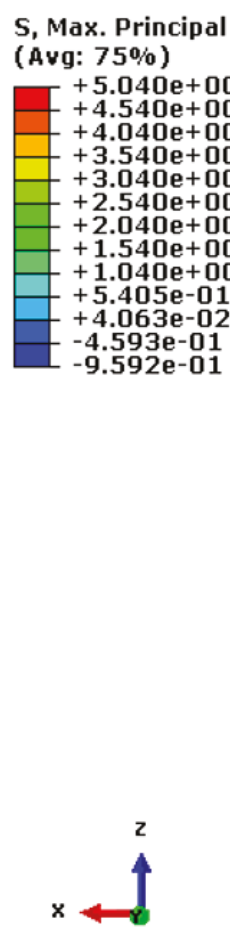

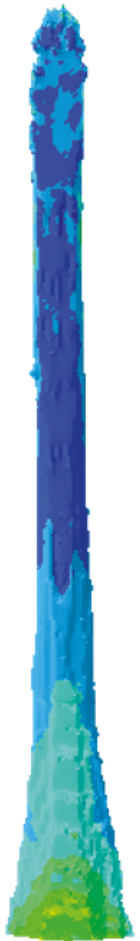

(a)

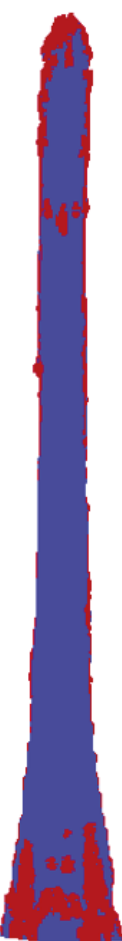

(b)

Figure 15. Soft cartilage of paddlefish rostrum. (a) Maximum principal stress obtained from finite element analysis. (b) Minimum cut obtained from mathematical analysis on top surface of rostrum's soft cartilage. Nodes are colored by their respective locations on the source (red) or sink (blue) side of the minimum cut.

\subsection{Flow Network Analysis on Hard Cartilage of Rostrum}

Figure 16a shows the maximum principal stresses on the bottom surface of the hard-cartilage part of the rostrum. Overall stresses progressively increased with the increase in external loading, as expected. Figure 16b displays the minimum cut obtained from the maximum-flow/minimum-cut algorithm. Nodes are colored by their respective locations on the source (red) or sink (blue) side of the minimum cut. The failure sites are located in the interface between the red and blue nodes. As seen in Figure 16b, these regions are located in the center part of the hard cartilage. Since the lattice region of the hard cartilage is not a continuous pattern, failure does not travel though the lattice region. The minimum cut picks up a region in the lattice where stress concentration is high and nodes are prone to failure. The center part of Figure 16b clearly displays strain localization, which is a typical mode of failure of a structural system under tensile loading. Hence, the research strategy implemented in the current work successfully identified the failure mechanisms at the nascent stages of loading. 


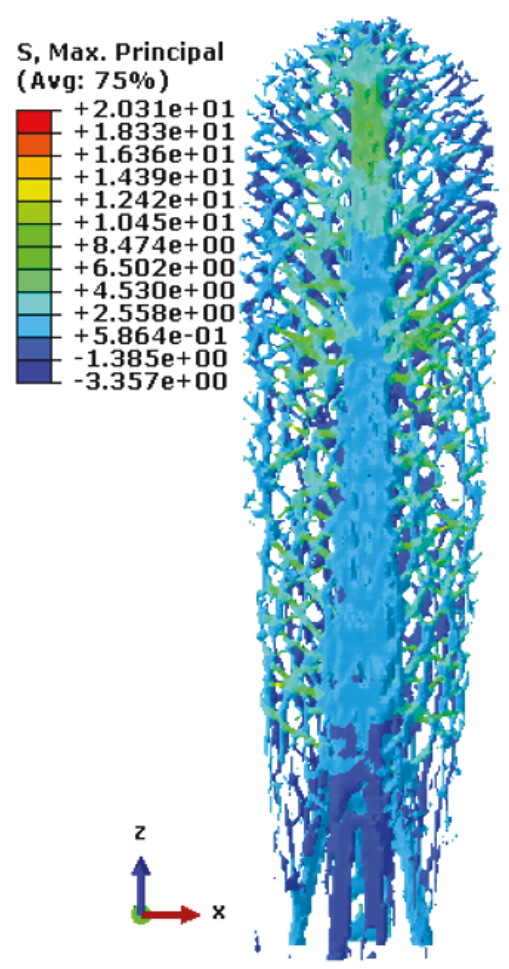

(a)

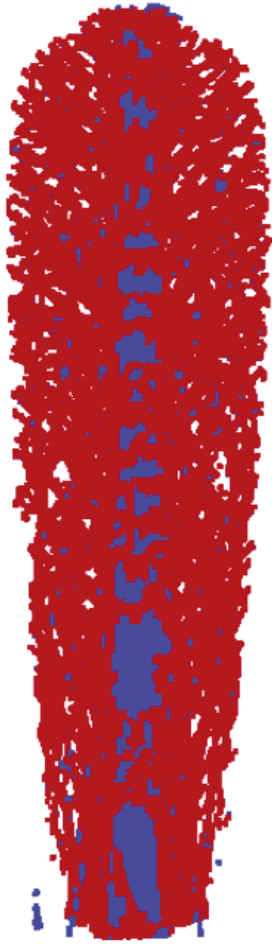

(b)

Figure 16. Hard cartilage of paddlefish rostrum. (a) Maximum principal stress obtained from finite element analysis. (b) Minimum cut obtained from mathematical analysis on bottom surface of rostrum's hard cartilage. Nodes are colored by their respective locations on the source (red) or sink (blue) side of the minimum cut.

Figure 17a displays the maximum principal stresses on the top surface of the hard-cartilage part of the rostrum. Although finite element analysis gives the overall distribution of stresses in the structural system, the underlying details that govern the phenomena of failure mechanism are hard to determine from the stresses. Figure $17 \mathrm{~b}$, on the contrary, shows the minimum cut obtained by executing the maximum-flow/minimum-cut algorithm. The central part of Figure $17 \mathrm{~b}$ clearly highlights the crushing/buckling behavior that is typically seen in compressive failure. A major part of the failure sites in this case were also located in the region where there was a change in geometry and material properties, which is a typical region where failure initiates. Again, the current methodology successfully identified the sites of failure at the nascent stages of loading. 


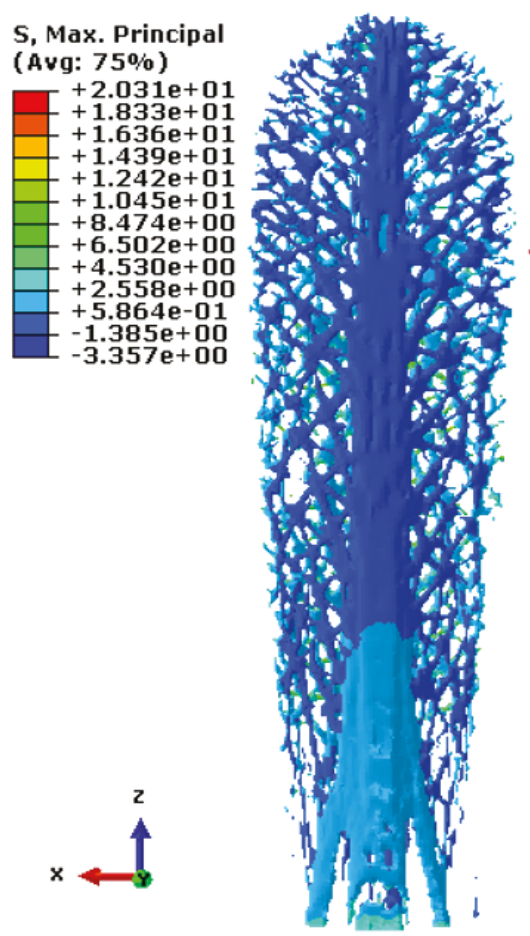

(a)

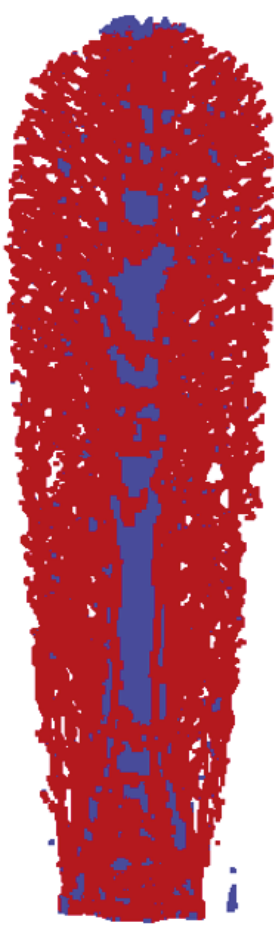

(b)

Figure 17. Hard cartilage of paddlefish rostrum. (a) Maximum principal stress obtained from finite element analysis. (b) Minimum cut obtained from mathematical analysis on top surface of rostrum's hard cartilage. Nodes are colored by their respective locations on the source (red) or sink (blue) side of the minimum cut.

\subsection{Flow Network Analysis on Rostrum Tissue}

Figure 18a shows the minimum cut obtained from flow network analysis on the bottom surface of the rostrum using the source-sink combination shown Figure 9. As seen in Figure 18a, the flow network captured the failure patterns at much earlier stages of loading that were not evident in the computational-mechanics results shown in Figure 18b. At low pressure levels, the strain localization patterns are captured on the tip of the central region of Figure 18a. As pressure increases, the strain-localization patterns are formed all along the central region of the rostrum tissue. Trusslike patterns are captured on the tissue part of the rostrum, as seen in Figure 18a. In contrast, the maximum principal stresses seen in Figure 18b did not show evidence of such patterns. The strain localization patterns captured through the flow network strategy are typically observed in a structural system under tensile loading. At a pressure of 7.143 MPa, Figure 18a also does not show any failure sites at the base of the tissue part of the rostrum indicative of it being stronger than the remaining part of the rostrum. This is the region that is attached to the mouth of the paddlefish, and this particular structural system is optimized for performance in this area. The flow network strategy captured this pattern at nascent stages, when the material was still in the linearly elastic regime.

Figure 19a displays the minimum cut obtained by using the flow network approach on the top surface of the rostrum. As seen in Figure 19a, at lower stresses the minimum cut is clearly formed in the right and left side of the rostrum. This is the region where the material properties of this component of the rostrum drastically change. As pressure increases, patterns are formed in the center and base 
region. As pressure increases in Figure 19a, there is also an absence of failure sites at the left and right corners of the base region of the rostrum. This behavior is similar to the one observed on the top surface of the rostrum. This is indicative of the identification of the stronger part of the rostrum at the nascent stage of loading.

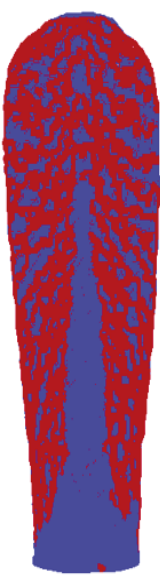

$1.428 \mathrm{MPa}$

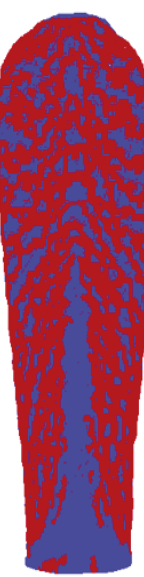

$2.857 \mathrm{MPa}$

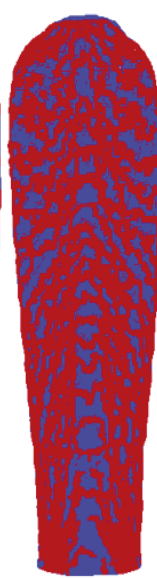

$4.285 \mathrm{MPa}$

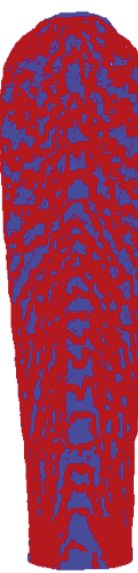

$5.714 \mathrm{MPa}$

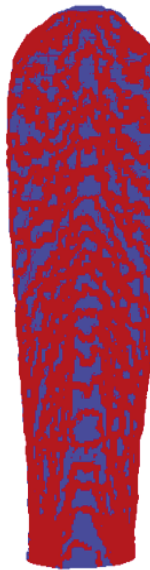

$7.143 \mathrm{MPa}$

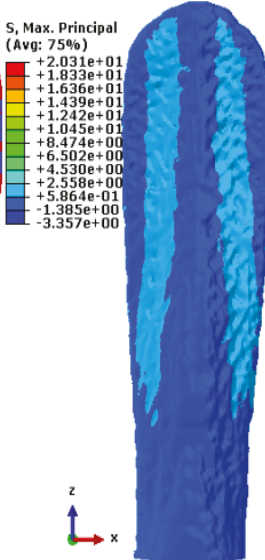

$7.143 \mathrm{MPa}$

(b)

Figure 18. Tissue of paddlefish rostrum. (a) Network flow analysis on the bottom surface of rostrum subjected to uniform pressure loading with a fixed plate boundary condition. Nodes are colored by their respective locations on the source (red) or sink (blue) side of the minimum cut. (b) Maximum principal stresses on the bottom surface of rostrum.

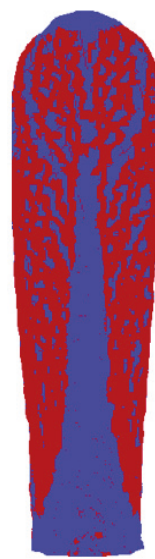

1.428 MPa

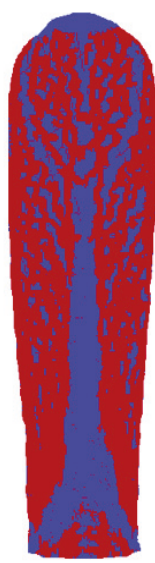

2.857 MPa

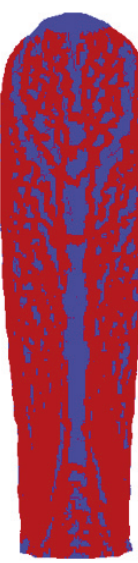

4.285 $\mathrm{MPa}$

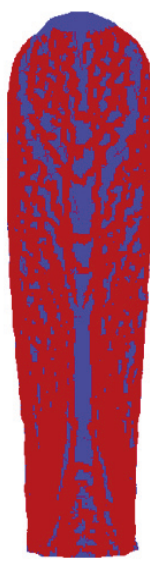

$5.714 \mathrm{MPa}$

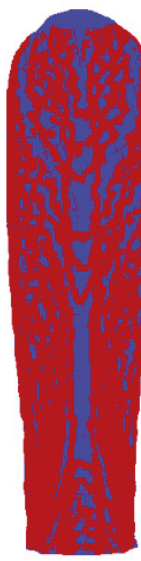

7.143 MPa

(a)

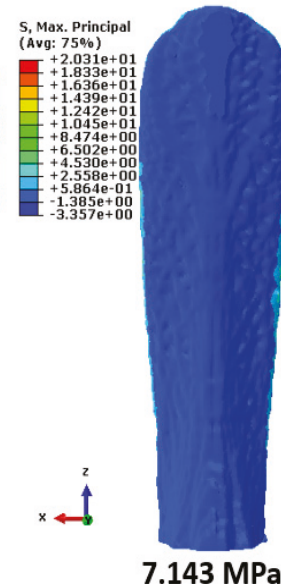

(b)

Figure 19. Tissue of paddlefish rostrum. (a) Network flow analysis on the top surface of the rostrum subjected to uniform pressure loading with a fixed plate boundary condition. Nodes are colored by their respective locations on the source (red) or sink (blue) side of the minimum cut. (b) Maximum principal stresses on the top surface of rostrum. 


\section{Conclusions}

This work successfully used the maximum-flow/minimum-cut algorithm for early detection of failure mechanisms in the paddlefish rostrum under uniform pressure loading and a fixed plate boundary condition. The transdisciplinary approach proposed in the current study was verified using two classical problems before implementing it on the complex biostructure.

The flow network approach was able to identify failure for the problem involving the three-point bending of a simply supported concrete beam. The proposed approach was able to identify the shear and flexure patterns for four-point bending of a simply supported concrete beam. The shear and flexure patterns identified by the flow network approach govern the phenomena of the failure mechanism in this classical problem. Failure patterns were identified at the onset of loading when the material was still in the linear elastic regime.

When applied to a complex biostructure, the flow network strategy was able to identify the strain localization in the tensile region, and the crushing behavior in the compressive region of the rostrum. Additionally, the flow network approach was able to identify the failure sites at locations where the material properties as well as the geometry of the component parts of the rostrum change, i.e., at the interface regions. The interface regions are typically where the failure initiates. This information can be used to efficiently design smart structural systems. The guidance regarding the selection of the source and sink nodes was obtained from the stress and deformation contours obtained from the computational mechanics experiments.

\section{Comments on the Transdisciplinary Approach}

This work successfully identified the stress-flow patterns in a complex biostructure through efficient use of flow network strategy. The approach used in the current work reduces the computational time, and cost since it is not required to run finite element analysis to failure. Stress patterns were identified when the material was in the linearly elastic regime. This approach laid the foundation of an efficient design-test-build cycle for the rapid prototyping of bioinspired structures. The flow network approach reduces the size of the problem since we concentrate on the parameter of interest, i.e., stress in the current study.

Author Contributions: Investigation, R.P.; resources, A.T.; supervision, G.R.; writing-review and editing, G.R., D.T., E.P., J.J.H., and J.P.

Acknowledgments: The authors acknowledge the financial support provided by U.S. Army Engineer Research and Development Center (ERDC) under PE 0601102, Project T22 "Research in Soil and Rock Mechanics", Task 01. The support and resources from the Engineer Research and Development Center Department of Defense Supercomputing Resource Center (ERDC DSRC) under the subproject Environmental Quality Modeling and Simulation are gratefully acknowledged. Permission was granted by the Director of the Information Technology Laboratory to publish this information.

Conflicts of Interest: The authors declare no conflict of interest.

\section{References}

1. Aggelis, D.G. Classification of cracking mode in concrete by acoustic emission parameters. Mech. Res. Commun. 2011, 38, 153-157. [CrossRef]

2. Carpinteri, A.; Lacidogna, G.; Niccolini, G.; Puzzi, S. Critical defect size distributions in concrete structures detected by the acoustic emission technique. Meccanica 2008, 43, 349-363. [CrossRef]

3. Farhidzadeh, A.; Salamone, S.; Singla, P. A probabilistic approach for damage identification and crack classification in reinforced concrete structures. J. Intell. Mater. Syst. Struct. 2013, 24, 1722-1735. [CrossRef]

4. Farhidzadeha, A.; Mpalaskasb, A.C.; Matikasb, T.E.; Farhidzadehc, H.; Aggelis, D.G. Fracture mode identification in cementitious materials using supervised pattern recognition of acoustic emission features. Constr. Build. Mater. 2014, 67, 129-138. [CrossRef] 
5. Kageyama, K.; Murayama, H.; Ohsawa, I.; Kanai, M.; Nagata, K.; Machijima, Y. Acoustic emission monitoring of a reinforced concrete structure by applying new fiber-optic sensors. Smart Mater. Struct. 2005, 14, s52-s59. [CrossRef]

6. Ohtsu, M.; Uchida, M.; Okamoto, T.; Yuyama, S. Damage assessment of reinforced concrete beams qualified by acoustic emission. ACI Struct. J. 2002, 99, 411-417.

7. Shiotani, T.; Aggelis, D.G.; Makishima, O. Global monitoring of large concrete structures using acoustic emission and ultrasonic techniques: Case study. J. Bridge Eng. 2009, 14, 188-192. [CrossRef]

8. Bahlouli, N.; Guil, S.M.; Ahzi, S.; Laberge, M. Stress-strain response of biomaterials by a digital image correlation method: Application to tecoflex. J. Mater. Sci. Technol. 2004, 20, 114-116.

9. Carroll, J.; Efstathiou, C.; Lambros, J.; Sehitoglu, H.; Hauber, B.; Spottswood, S.; Chona, R. Investigation of fatigue crack closure using multiscale image correlation experiments. Eng. Fract. Mech. 2009, 76, 2384-2398. [CrossRef]

10. Fazzini, M.; Mistou, S.; Karama, M. Identification of elastomers by digital image correlation. In Proceedings of the 5th European Conference on Constitutive Models for Rubber, Paris, France, 4-7 September 2007.

11. Goh, C.P.; Ratnam, M.M.; Ismail, H. Large in-plane deformation mapping and determination of young's modulus of rubber using scanner-based digital image correlation. Exp. Tech. 2015, 40, 1117-1127. [CrossRef]

12. Gonzáles, G.L.; González, J.A.; Castro, J.T.; Freire, J.L. A J-integral approach using digital image correlation for evaluating stress intensity factors in fatigue cracks with closure effects. Theor. Appl. Fract. Mech. 2017, 90, 14-21. [CrossRef]

13. Lopez-Crespo, P.; Shterenlikht, A.; Yates, J.; Patterson, E.; Withers, P. Some experimental observations on crack closure and crack-tip plasticity. Fatigue Fract. Eng. Mater. Struct. 2009, 32, 418-429. [CrossRef]

14. Mudassar, A.A.; Butt, S. Improved digital image correlation method. Opt. Lasers Eng. 2016, 87, $156-167$. [CrossRef]

15. Sutton, M.A.; McNeill, S.R.; Helm, J.D.; Chao, Y.J. Advances in Two-Dimensional and Three Dimensional Computer Vision. In Photomechanics; Springer: Berlin/Heidelberg, Germany, 2000; pp. 323-372.

16. Sutton, M.A.; Orteu, J.J.; Schreier, H. Image Correlation for Shape, Motion and Deformation Measurements: Basic Concepts, Theory and Applications; Springer Science and Business Media: New York, NY, USA, 2009.

17. Vasco-Olmo, J.; Díaz, F.; García-Collado, A.; Dorado-Vicente, R. Experimental evaluation of crack shielding during fatigue crack growth using digital image correlation. Fatigue Fract. Eng. Mater. Struct. 2015, 38, 223-237. [CrossRef]

18. Hallgren, M.; Bjerke, M. Non-linear finite element analyses of punching shear failure of column footings. Cem. Concr. Compos. 2002, 24, 491. [CrossRef]

19. Mamede, N.F.; Ramos, A.P.; Faria, D.M. Experimental and parametric 3D nonlinear finite element analysis on punching of flat slabs with orthogonal reinforcement. Eng. Struct. 2013, 48, 442-457. [CrossRef]

20. Shu, J.; Plos, M.; Zandi, K.; Johansson, M.; Nilenius, F. Prediction of punching behavior of RC slabs using continuum non-linear FE analysis. Eng. Struct. 2016, 15, 15-25. [CrossRef]

21. Borderie, C.L.; Lawrence, C.; Menou, A. Approche mésoscopique du comportement du béton: Apport de la représentation géométrique. Revue Européenne de Génie Civil 2007, 11, 407-421. [CrossRef]

22. Grassl, P.; Grégoire, D.; Rojas-Solano, L.B.; Pijaudier-Cabot, G. Meso-scale modelling of the size effect on the fracture process zone of concrete. Int. J. Solids Struct. 2012, 49, 1818-1827. [CrossRef]

23. Grégoire, D.; Verdon, L.B.; Lefort, V.; Grassl, P.; Saliba, J.; Regoin, J.P.; Loukili, A.; Pijaudier-Cabot, G. Mesoscale analysis of failure in quasi-brittle materials: Comparison between lattice model and acoustic emission data. Int. J. Numer. Anal. Methods Geomech. 2015, 39, 1639-6164. [CrossRef]

24. Flores-Johnson, E.; Shen, L.; Guiamatsia, I.; Nguyen, G.D. A numerical study of bioinspired nacre-like composite plates under blast loading. Compos. Struct. 2015, 126, 329-336. [CrossRef]

25. Flores-Johnson, E.; Shen, L.; Guiamatsia, I.; Nguyen, G.D. Numerical investigation of the impact behavior of bioinspired nacre-like aluminum composite plates. Compos. Sci. Technol. 2014, 96, 13-22. [CrossRef]

26. Tran, P.; Ngo, T.D.; Mendis, P. Bioinspired composite structures subjected to under water impulsive loading. Comput. Mater. Sci. 2014, 82, 134-139. [CrossRef]

27. Bertsekas, D.P. Network Optimization: Continuous and Discrete Models (Optimization, Computation, and Control); Athena Scientific: Nashua, NH, USA, 1998.

28. Lin, Q.; Tordesillas, A. Towards an optimization theory for deforming dense granular materials: Minimum cost maximum flow solutions. J. Ind. Manag. Optim. 2014, 10, 337. [CrossRef] 
29. Tordesillas, A.; Tobin, S.T.; Cil, M.; Alshibli, K.; Behringer, R.P. Network flow model of force transmission in unbonded and bonded granular media. Phys. Rev. E 2015, 91, 062204. [CrossRef]

30. Lefort, V.; Pijaudier-Cabot, G.; Grégoire, D. Analysis by Ripley's function of the correlations involved during failure in quasi-brittle materials: Experimental and numerical investigations at the mesoscale. Eng. Fract. Mech. 2015, 147, 449-467. [CrossRef]

31. Ripley, B.D. Modelling spatial patterns. J. R. Stat. Soc. Ser. B Methodol. 1977, 39, 172-212. [CrossRef]

32. Duncan, R.P. Flood disturbance and the coexistence of species in a lowland podocarp forest, south Westland, New Zealand. J. Ecol. 1993, 81, 403-416. [CrossRef]

33. Stamp, N.E.; Lucas, J.R. Spatial Patterns and Dispersal Distances of Explosively Dispersing Plants in Florida Sandhill Vegetation. J. Ecol. 1990, 78, 589-600. [CrossRef]

34. Diggle, P.J.; Chetwynd, A.G. Second-Order Analysis of Spatial Clustering for Inhomogeneous Populations. Biometrics 1991, 47, 1155-1163. [CrossRef]

35. Weiner, S.; Wagner, H.D. The material bone: Structure-Mechanical Function Relation. Annu. Rev. Mater. Sci. 1998, 28, 271-298. [CrossRef]

36. Hoover, J.J.; George, S.G.; Killgore, K.J. Rostrum size of paddlefish (Polyodon spathula) (Acipenseiformes: Polyodontidae) from the Mississippi Delta. Copeia 2000, 1, 288-290. [CrossRef]

37. Kuhajda, B.R. Polyodontidae: Paddlefishes; Johns Hopkins University Press: Baltimore, MD, USA, 2014.

38. Riveros, G.A.; Patel, R.R.; Hoover, J.J. Swimming and Energy Dissipation Enhancement Induced by the Rostrum of the Paddlefish (Polyodon spathula): A Multiphysics, Fluid-Structure Interaction Analysis. In Proceedings of the Materials Research Society Fall Meeting, Boston, MA, USA, 29 November-4 December 2015.

39. Patel, R.R.; Riveros, G.A.; Thompson, D.S.; Acosta, F.J.; Perkins, E.J.; Hoover, J.J.; Peters, J.F.; Tordesillas, A. Early Detection of Failure Mechanisms in Resilient Biostructures: A Network Flow Study; ERDC: Vicksburg, MS, USA, 2017.

40. Patel, R.R.; Valles, D.; Riveros, G.A.; Thompson, D.S.; Perkins, E.J.; Hoover, J.J.; Peters, J.F.; Tordesillas, A. Stress flow analysis of biostructures using the finite element method and the flow network approach. Finite Elem. Anal. Des. 2018, 152, 46-54. [CrossRef]

41. Bondy, J.A.; Murty, U.S.R. Graph Theory. In Graduate Texts in Mathematics; Springer: New York, NY, USA, 2008.

42. Jungnickel, D. Graphs, Networks and Algorithms. In Algorithms and Computation in Mathematics 5, 3rd ed.; Springer: Berlin, Germany, 2008.

43. Tordesillas, A.; Pucilowski, S.; Tobin, S.; Kuhn, M.R.; Ando, E.; Viggiani, G.; Druckrey, A.; Alshibli, K. Shear bands as bottlenecks in force transmission. Eur. Phys. Lett. 2015, 110, 58005. [CrossRef]

44. Dassault Systems. Abaqus 6.13 Using Abaqus Online Documentation. Available online: http://dsk.ippt.pan. pl/docs/abaqus/v6.13/pdf_books/HELP.pdf (accessed on 26 April 2019).

45. Riveros, G.A.; Gopalaratnam, V.S. Fracture response of reinforced concrete deep beams finite element investigation of strength and beam size. Appl. Math. 2013, 4, 1568. [CrossRef]

46. Riveros, G.A.; Gopalaratnam, V.S. Shear Response of Reinforced Concrete Deep Beam: Validating Fracture Mechanics Based Numerical Modelling with Experiments; American Concrete Institute: Farmington Hills, MI, USA, 2015.

47. Segalman, D.J.; Fulcher, C.W.G.; Reese, G.M.; Field, R.V., Jr. An Efficient Method for Calculating RMS von Mises Stress in a Random Vibration Environment. J. Sound Vib. 1998, 230, 393-410. [CrossRef]

48. Deang, J.; Horstemeyer, M.; Williams, L.; Perkins, E.; Allison, P.; Riveros, G. Paddlefish rostrum as a structure for bioinspiration: Analysis and modeling of the of the stress state and strain rate dependence behavior of cartilage. In Proceedings of the TMS Annual Meeting and Exhibition, Nashville, TN, USA, 14-18 February 2016.

49. Allison, P.G.; Deang, J.F.; Diaz, A.J.; Poda, A.R.; Hoover, J.J.; Horstemeyer, M.F.; Perkins, E.J. Characterization of paddlefish (Polyodon spathula) rostrum stellate bones. Bioinspired Biomim. Nanobiomater. 2013, 3, 63-68. [CrossRef]

(C) 2019 by the authors. Licensee MDPI, Basel, Switzerland. This article is an open access article distributed under the terms and conditions of the Creative Commons Attribution (CC BY) license (http://creativecommons.org/licenses/by/4.0/). 


\title{
Nonlocal FEM Formulation for Vibration Analysis of Nanowires on Elastic Matrix with Different Materials
}

\author{
Büşra Uzun ${ }^{1}$ and Ömer Civalek ${ }^{2, *}$ \\ 1 Civil Engineering Department, Uludağ University, 16059 Bursa, Turkey; uzunbusra34@gmail.com \\ 2 Civil Engineering Department, Akdeniz University, 07070 Antalya, Turkey \\ * Correspondence: civalek@yahoo.com
}

Received: 12 February 2019; Accepted: 4 April 2019; Published: 6 April 2019

\begin{abstract}
In this study, free vibration behaviors of various embedded nanowires made of different materials are investigated by using Eringen's nonlocal elasticity theory. Silicon carbide nanowire (SiCNW), silver nanowire (AgNW), and gold nanowire (AuNW) are modeled as Euler-Bernoulli nanobeams with various boundary conditions such as simply supported (S-S), clamped simply supported (C-S), clamped-clamped (C-C), and clamped-free (C-F). The interactions between nanowires and medium are simulated by the Winkler elastic foundation model. The Galerkin weighted residual method is applied to the governing equations to gain stiffness and mass matrices. The results are given by tables and graphs. The effects of small-scale parameters, boundary conditions, and foundation parameters on frequencies are examined in detail. In addition, the influence of temperature change on the vibrational responses of the nanowires are also pursued as a case study.
\end{abstract}

Keywords: nonlocal elasticity theory; Galerkin weighted residual FEM; silicon carbide nanowire; silver nanowire; gold nanowire

\section{Introduction}

Nanoscale structures/materials have very different characteristics. Nanostructures/nanomaterials have attracted great attention because their extraordinary features, such as high strength, low density, high elasticity modulus, and high hardness [1-4], have become the focus of researchers. The aforementioned unique properties of such structures, materials, and rapid developments in nanotechnology has led to use of these structural elements in designing micro- and nanoelectro mechanical systems (MEMS and NEMS) such as resonators, atomic force microscopes, switches, actuators, and sensors.

Some experimental studies have revealed the deformation behaviors of micro-/nanosized structures $[5,6]$. However, experiments are very difficult and quite expensive on these scales because high precision test devices are needed. On the other hand, atomistic modeling such as molecular dynamic simulations is computationally expensive and requires a long period of time. Consequently, this option is limited to structures that have only a few atoms [7].

To understand and accurately interpret the mechanical properties and behaviors of nanoscale structures, use of models based on continuum mechanics may be a better alternative than experiments and atomistic modelling. Unfortunately, classical continuum theories are not sufficient to predict and estimate size dependency because they lack internal/additional material length scale parameters. In ultrasmall scales (micrometer dimension, nanometer dimension) interactions between atoms and molecules have increasing importance and cannot be neglected. Therefore, the solution to classical continuum theories, which does not take into account size effects, does not give accurate results. In order to obtain more accurate results, higher-order continuum theories such as couple stress theory [8-10], modified couple stress theory [11], strain gradient theory [12], modified strain gradient theory [13], and nonlocal elasticity theory [14] have been developed and contain various length scale 
parameters. These theories were used by many researchers for various analyses such as buckling [15], bending [16,17], free vibration [18,19], forced vibration [20], and nonlinear vibration [21].

Rahmanian et al. [22] presented free vibrations of single-walled carbon nanotubes (SWCNT) on a Winkler elastic foundation via nonlocal elasticity theory. In this study, SWCNT was modeled as both beam and shell structures. Demir and Civalek [23] reported thermal vibration formulation of a nonlocal Euler-Bernoulli beam embedded in an elastic matrix. Finite element formulation for Eringen's nonlocal elasticity theory was employed via Hermitian cubic shape functions. Thermal vibrational behaviors of silicon carbide nanowire on an elastic matrix were investigated for simply supported (S-S) and clamped-clamped (C-C) boundary conditions. Finite element formulations of nonlocal elastic Euler-Bernoulli and Timoshenko beam theories were achieved by Pradhan [24]. Vibration, buckling, and bending analyses of carbon nanotubes with four different boundary conditions were performed by the Galerkin finite element technique. Rajasekaran and Bakhshi Khaniki [25] reported static deformation, stability, and free vibration responses of small-scale beams. A finite element model of axial, functionally graded, nonuniform small-scale beams was investigated by using nonlocal strain gradient theory. Eltaher et al. [26] pursued free vibration analysis of functionally graded Euler-Bernoulli nanobeams by using Eringen's nonlocal elasticity theory. Finite element results were given for a dynamic analysis of the nanobeam. Nejad and Hadi [27] studied bending analysis of non-homogeneous nanobeams. Eringen's nonlocal elasticity theory was utilized in Euler-Bernoulli nanobeams made of bi-directional, functionally graded material. Murmu and Pradhan [28] studied the thermo-mechanical vibration response of embedded carbon nanotubes surrounded by an elastic matrix based on nonlocal elasticity theory. Reddy [29] developed nonlocal beam models based on four different beam theories. Static bending, free vibration, and buckling analyses of nanobeams are performed in this study. Tornabene et al. [30] presented a multiscale approach for three-phase carbon nanotube (CNT)/polymer/fiber-laminated nanocomposite structures. Detailed formulations can be found in the literature [31-35] about CNT-reinforcement or the finite element method (FEM). More recently, Uzun et al. [36] investigated the free vibration responses of carbon nanotubes and boron nitride nanotubes based on nonlocal elasticity theory. Nonlocal natural frequencies are obtained for various cross-section geometries.

In the present study, free vibration analysis of three kinds of nanowires resting on a Winkler elastic foundation with various boundary conditions are performed. Simply supported (S-S), clamped simply supported (C-S), clamped-clamped (C-C), and clamped-free (C-F) boundary conditions are selected. Silicon carbide nanowire (SiCNW), silver nanowire (AgNW), and gold nanowire (AuNW) are modeled as nonlocal Euler-Bernoulli beams, and their vibration behaviors are investigated using the finite element method (FEM). A Galerkin weighted residual method is utilized to govern equations and matrices, and the Winkler foundation parameter and small-scale parameter are gained. Effects of boundary conditions, temperature rise, and small-scale and Winkler foundation parameters of frequency values are investigated and compared for three kinds of nanowires.

\section{Euler-Bernoulli Nanobeam Resting on a Winkler Elastic Foundation}

The nonlocal stress tensor $\sigma_{i j}$ at point $x$ is expressed as follows [14]:

$$
\begin{gathered}
\sigma_{i j, j}=0, \\
\left.\sigma_{i j}(x)=\int_{\Omega} K\left(\left|x^{\prime}-x\right|\right), \tau\right) C_{i j k l} \varepsilon_{k l} d \Omega\left(x^{\prime}\right),
\end{gathered}
$$

where $K\left(\left|x^{\prime}-x\right|, \tau\right)$ is the Kernel function, $\left|x^{\prime}-x\right|$ is the distance in the Euclidean form, $\tau=e_{0} a / l$ is a material constant that depends upon the internal characteristic lengths $(a)$ and external characteristic length $(l)$, and $e_{0}$ is a material constant that is determined experimentally. $C_{i j k l}$ and $\varepsilon_{k l}$ represent the fourth-order elasticity and the strain tensors, respectively, and $\Omega$ is the region occupied by the body. 
The nonlocal constitutive formulation is [28]:

$$
\left[1-\tau^{2} l^{2} \nabla^{2}\right] \sigma_{i j}=C_{i j k l} \varepsilon_{k l} .
$$

$x, y, z$ depict length, width, and height of the beam, respectively and $u_{1}, u_{2}, u_{3}$ are the displacements in the $x, y, z$ directions. The displacements for a Bernoulli-Euler beam can be written as below [17]:

$$
u_{1}(x, z, t)=-z \frac{\partial w(x, t)}{\partial x}, u_{2}(x, z, t)=0, u_{3}(x, z, t)=w(x, t) .
$$

$\varepsilon_{i j}$ is the strain tensor, expressed as:

$$
\varepsilon_{i j}=0.5\left(\partial u_{i, j}+\partial u_{j, i}\right) .
$$

From Equation (5) we find the strains of the Euler-Bernoulli beam as follows:

$$
\varepsilon_{x x}=-z \frac{\partial^{2} w(x, t)}{\partial x^{2}}, \varepsilon_{x y}=\varepsilon_{y x}=\varepsilon_{x z}=\varepsilon_{z x}=\varepsilon_{y y}=\varepsilon_{y z}=\varepsilon_{z y}=\varepsilon_{z z}=0 .
$$

Stress $\sigma$ for the linear elastic materials is expressed as follows:

$$
\sigma=E \varepsilon,
$$

where $E$ is the elastic modulus of the material. If $\varepsilon_{x x}$, the only nonzero component of strain, is written in Equation (7), $\sigma_{x x}$ is obtained as:

$$
\sigma_{x x}=-E z \frac{\partial^{2} w(x, t)}{\partial x^{2}}
$$

Moment $(M)$ and the moment of inertia $(I)$ are given by:

$$
M=\int_{A} z \sigma_{x x} d A, I=\int_{A} z^{2} d A,
$$

where $A$ represents the cross-section area.

For the transverse vibration of an Euler-Bernoulli beam (shown in Figure 1) resting on a Winkler elastic foundation, the equilibrium conditions are:

$$
\begin{gathered}
\frac{\partial V(x, t)}{\partial x}=-q(x, t)+\rho A \frac{\partial^{2} w(x, t)}{\partial x^{2}}+k_{w} w(x, t), \\
V(x, t)=\frac{\partial M(x, t)}{\partial x}, \\
\frac{\partial^{2} M(x, t)}{\partial x^{2}}=-q(x, t)+\rho A \frac{\partial^{2} w(x, t)}{\partial x^{2}}+k_{w} w(x, t),
\end{gathered}
$$

where $\rho, q(x, t)$, and $k_{w}$ are the mass density, distributed load, and Winkler foundation parameter, respectively.

The nonlocal constitutive relations can be simplified in the following form for a one-dimensional case [14,29]:

$$
\sigma_{x x}-\left(e_{0} a\right)^{2} \frac{\partial^{2} \sigma_{x x}}{\partial x^{2}}=E \varepsilon_{x x} .
$$

By multiplying $z$ on both sides of Equation (13) and integrating the cross-sectional area of the beam, we obtain:

$$
\int_{A} z \sigma d A-\left(e_{0} a\right)^{2} \int_{A} z \frac{\partial^{2} \sigma}{\partial x^{2}} d A=\int_{A} z E \varepsilon d A=0 .
$$


Substituting Equations (6) and (9) into (14), we get:

$$
M(x, t)-\left(e_{0} a\right)^{2} \frac{\partial^{2} M(x, t)}{\partial x^{2}}=-E I \frac{\partial^{2} w(x, t)}{\partial x^{2}} .
$$

By differentiating Equation (15) twice with respect to variable $x$ and substituting Equation (12) into Equation (15), we obtain the governing equation for the vibration of an Euler-Bernoulli nanobeam resting on a Winkler elastic foundation, as below:

$$
E I \frac{\partial^{4} w(x, t)}{\partial x^{4}}+\rho A \frac{\partial w^{2}(x, t)}{\partial t^{2}}+k_{w} w-\left(e_{0} a\right)^{2} \frac{\partial^{2}}{\partial x^{2}}\left[\rho A \frac{\partial^{2} w(x, t)}{\partial t^{2}}+k_{w} w\right]=0 .
$$

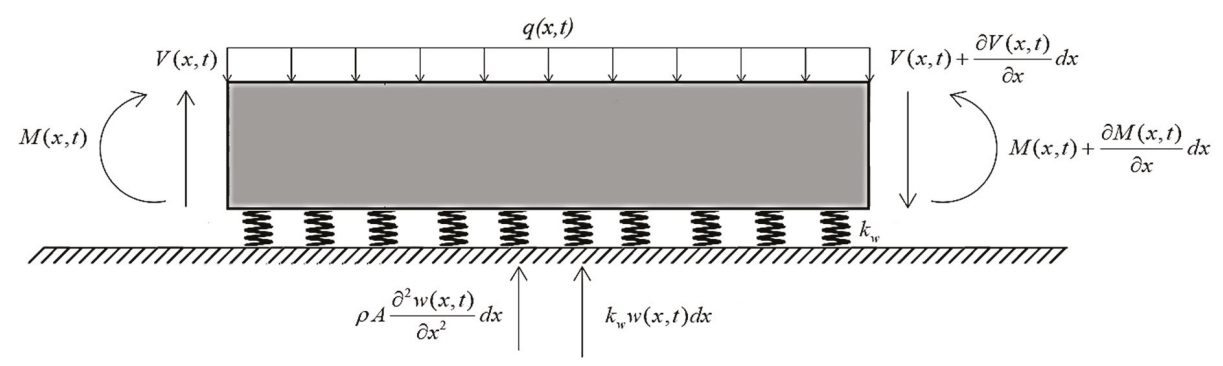

Figure 1. Euler-Bernoulli beam resting on a Winkler foundation.

\section{Solution to the Vibration Problem}

\subsection{Galerkin Weighted Residual Method}

The shape function for beam $\varphi$ is as follows $[37,38]$ :

$$
\varphi=\left\{\begin{array}{c}
\varphi_{1} \\
\varphi_{2} \\
\varphi_{3} \\
\varphi_{4}
\end{array}\right\}=\left\{\begin{array}{c}
1-3 \xi^{2}+2 \xi^{3} \\
L\left(\xi-2 \xi^{2}+\xi^{3}\right) \\
3 \xi^{2}-2 \xi^{3} \\
L\left(-\xi^{2}+\xi^{3}\right)
\end{array}\right\},
$$

where $\xi=x / L$ is a non-dimensional local coordinate. In order to obtain the weak form of the governing equation of an Euler-Bernoulli nanobeam resting on a Winkler elastic foundation, the residue can be expressed as:

$$
\left[E I \frac{\partial^{4} w(x, t)}{\partial x^{4}}+\rho A \frac{\partial w^{2}(x, t)}{\partial t^{2}}+k_{w} w-\left(e_{0} a\right)^{2} \frac{\partial^{2}}{\partial x^{2}}\left(\rho A \frac{\partial^{2} w(x, t)}{\partial t^{2}}+k_{w} w\right)\right]=\text { residue. }
$$

To determine the weighted residue, Equation (18) is multiplied by a weighting function $\varphi$. When the weighted residual is integrated over the length:

$$
\int_{0}^{L}\left[\varphi E I \frac{\partial^{4} w(x, t)}{\partial x^{4}}+\varphi \rho A \frac{\partial w^{2}(x, t)}{\partial t^{2}}+\varphi k_{w} w-\varphi\left(e_{0} a\right)^{2} \frac{\partial^{2}}{\partial x^{2}}\left(\rho A \frac{\partial^{2} w(x, t)}{\partial t^{2}}+k_{w} w\right)\right] d x=0 .
$$

Equation (19) is integrated by parts. According to the chain rule, in the general form:

$$
\int_{0}^{L}\left[E I \frac{\partial^{2} \varphi}{\partial x^{2}} \frac{\partial^{2} \varphi^{T}}{\partial x^{2}}+\rho A \varphi \varphi^{T} \ddot{w}+k_{w} \varphi \varphi^{T}-\left(e_{0} a\right)^{2} \rho A \frac{\partial \varphi}{\partial x} \frac{\partial \varphi^{T}}{\partial x} \ddot{w}-\left(e_{0} a\right)^{2} k_{w} \frac{\partial \varphi}{\partial x} \frac{\partial \varphi^{T}}{\partial x}\right] d x=0 .
$$


By using the shape function in Equation (17) and the non-dimensional local coordinate, bending stiffness matrix $K^{b}$, Winkler foundation stiffness matrix $K^{w}$, and the mass matrix $M$, the following are obtained:

$$
\begin{aligned}
& K^{b}=E I \int_{0}^{L}\left\{\begin{array}{c}
\varphi_{1}^{\prime \prime} \\
\varphi_{2}^{\prime \prime} \\
\varphi_{3}^{\prime \prime} \\
\varphi_{4}^{\prime \prime}
\end{array}\right\}\left\{\begin{array}{llll}
\varphi_{1}^{\prime \prime} & \varphi_{2}^{\prime \prime} & \varphi_{3}^{\prime \prime} & \varphi_{4}^{\prime \prime}
\end{array}\right\} d x ; \\
& K^{b}=\frac{E I}{L^{3}}\left[\begin{array}{cccc}
12 & 6 L & -12 & 6 L \\
6 L & 4 L^{2} & -6 L & 2 L^{2} \\
-12 & -6 L & 12 & -6 L \\
6 L & 2 L^{2} & -6 L & 4 L^{2}
\end{array}\right] \\
& K^{w 1}=k_{w} \int_{0}^{L}\left\{\begin{array}{c}
\varphi_{1} \\
\varphi_{2} \\
\varphi_{3} \\
\varphi_{4}
\end{array}\right\}\left\{\begin{array}{llll}
\varphi_{1} & \varphi_{2} & \varphi_{3} & \varphi_{4}
\end{array}\right\} d x ; \\
& K^{w 1}=\frac{k_{w}}{420}\left[\begin{array}{cccc}
156 L & 22 L^{2} & 54 L & -13 L^{2} \\
22 L^{2} & 4 L^{3} & 13 L^{2} & -3 L^{3} \\
54 L & 13 L^{2} & 156 L & -22 L^{2} \\
-13 L^{2} & -3 L^{3} & -22 L^{2} & 4 L^{3}
\end{array}\right] \text {; } \\
& K^{w 2}=\left(e_{0} a\right)^{2} k_{w} \int_{0}^{L}\left\{\begin{array}{c}
\varphi_{1}^{\prime} \\
\varphi_{2}^{\prime} \\
\varphi_{3}^{\prime} \\
\varphi_{4}^{\prime}
\end{array}\right\}\left\{\begin{array}{cccc}
\varphi_{1}^{\prime} & \varphi_{2}^{\prime} & \varphi_{3}^{\prime} & \varphi_{4}^{\prime}
\end{array}\right\} d x ; \\
& K^{w 2}=\frac{\left(e_{0} a\right)^{2} k_{w}}{30 L}\left[\begin{array}{cccc}
36 & 3 L & -36 & 3 L \\
3 L & 4 L^{2} & -3 L & -L^{2} \\
-36 & -3 L & 36 & -3 L \\
3 L & -L^{2} & -3 L & 4 L^{2}
\end{array}\right] \text {; } \\
& M^{1}=\rho A \int_{0}^{L}\left\{\begin{array}{c}
\varphi_{1} \\
\varphi_{2} \\
\varphi_{3} \\
\varphi_{4}
\end{array}\right\}\left\{\begin{array}{cccc}
\varphi_{1} & \varphi_{2} & \varphi_{3} & \varphi_{4}
\end{array}\right\} d x \\
& M^{1}=\frac{\rho A}{420}\left[\begin{array}{cccc}
156 L & 22 L & 54 L & -13 L^{2} \\
22 L^{2} & 4 L^{3} & 13 L^{2} & -3 L^{3} \\
54 L & 13 L^{2} & 156 L & -22 L^{2} \\
-13 L^{2} & -3 L^{3} & -22 L^{2} & 4 L^{3}
\end{array}\right] \text {; } \\
& M^{2}=\left(e_{0} a\right)^{2} \rho A \int_{0}^{L}\left\{\begin{array}{c}
\varphi_{1}^{\prime} \\
\varphi_{2}^{\prime} \\
\varphi_{3}^{\prime} \\
\varphi_{4}^{\prime}
\end{array}\right\}\left\{\begin{array}{llll}
\varphi_{1}^{\prime} & \varphi_{2}^{\prime} & \varphi_{3}^{\prime} & \varphi_{4}^{\prime}
\end{array}\right\} d x \text {; } \\
& M^{2}=\frac{\left(e_{0} a\right)^{2} \rho A}{30 L}\left[\begin{array}{cccc}
36 & 3 L & -36 & 3 L \\
3 L & 4 L^{2} & -3 L & -L^{2} \\
-36 & -3 L & 36 & -3 L \\
3 L & -L^{2} & -3 L & 4 L^{2}
\end{array}\right] \text {. }
\end{aligned}
$$


The vibration of the Euler-Bernoulli beam is found as follows:

$$
\left|K-\omega^{2} M\right|=0
$$

where $K=K^{\mathrm{b}}+K^{w 1}+K^{w 2}, M=M^{1}+M^{2}$, and $\omega$ is frequency.

\subsection{Thermal Effect on the Vibrational Response of Embedded Nanowires}

Here, the effect of temperature change on the natural frequencies of embedded nanowires in a thermal environment is investigated. Equation (16) can be rewritten in the presence of thermal loading as:

$$
\left(E I-N^{T}\left(e_{0} a\right)^{2}\right) \frac{\partial^{4} w}{\partial x^{4}}+k_{w} w+\left(N^{T}-k_{w}\left(e_{0} a\right)^{2}\right) \frac{\partial^{2} w}{\partial x^{2}}+\rho A \frac{\partial^{2} w}{\partial t^{2}}-\rho A\left(e_{0} a\right)^{2} \frac{\partial^{4} w}{\partial x^{2} \partial t^{2}}=0
$$

where the additional term $N^{T}$ is the axial load resulting from the temperature change and can be defined as [28]:

$$
N^{T}=\frac{E A}{1-2 v} \alpha \Delta T,
$$

in which $\alpha$ is the thermal expansion coefficient in the axial direction, $\Delta T$ is the temperature change, and $v$ is Poisson's ratio. It is notable that only an axial load resulting from temperature change exists for the nanowires in this study [28].

The following Navier's solution procedure is applied to achieve simply supported (S-S) nanowires as an illustrative example:

$$
w(x, t)=\sum_{n=1}^{\infty} W_{n} \sin \beta e^{i \omega_{n} t},
$$

where $n$ is the mode number, $W_{n}$ is the unknown Fourier coefficient, and $\beta=\frac{n \pi x}{L}$. Using Equation (29) in Equation (27) yields the following relation for small-scale-dependent natural frequencies of embedded S-S nanowires that includes the thermal effect:

$$
\omega_{n}=\sqrt{\frac{\beta^{4}\left(E I-N^{T}\left(e_{0} a\right)^{2}\right)+k_{w}-\beta^{2}\left(N^{T}-k_{w}\left(e_{0} a\right)^{2}\right)}{\rho A\left(1+\beta^{2}\left(e_{0} a\right)^{2}\right)}} .
$$

\section{Results and Discussion}

In this section, frequency values of nanowires were obtained with various non-dimensional small-scale parameters $\left(e_{0} a / L\right)$, different non-dimensional Winkler foundation parameters $\left(K_{W}\right)$, different boundary conditions, and different number of elements $(N)$. The material properties for the three nanowires are listed in Table 1 . The results obtained were shown in tables and graphs. The dimensionless Winkler parameter used for the results is expressed as the formula below:

$$
K_{W}=\frac{k_{w} L^{4}}{E I}
$$

Table 1. Material properties of the nanowires.

\begin{tabular}{cccc}
\hline Material Properties & SiCNW & AuNW & AgNW \\
\hline$E(\mathrm{GPa})$ & 524.8 & 79 & 82.7 \\
$v$ & 0.14 & 0.42 & 0.37 \\
$\rho\left(\mathrm{kg} / \mathrm{m}^{3}\right)$ & 3100 & 19,320 & 10,490 \\
$\alpha(1 /)$ & $3.7 \times 10^{-6}$ & $14.2 \times 10^{-6}$ & $19.68 \times 10^{-6}$ \\
\hline
\end{tabular}


Table 2 presents the natural frequencies of nanowires with C-C, C-S, S-S, and C-F boundary conditions. Finite element solutions for the three nanowires were compared with each other. It was clearly observed from the table that the highest frequency value occurred for both SiCNW and the C-C boundary condition, while the lowest value was seen in both AuNW and C-F boundary conditions.

Table 2. The first three natural frequencies $(\mathrm{GHz})$ of isolated nanowires for four different boundary conditions $\left(K_{W}=0, e_{0} a / L=0.1\right)$.

\begin{tabular}{ccccc}
\hline \multirow{2}{*}{ Mode Number } & \multicolumn{4}{c}{ SiCNW } \\
\cline { 2 - 5 } & C-C & C-S & S-S & C-F \\
\hline $\mathbf{1}$ & 21.8286 & 15.0969 & 9.7369 & 3.5540 \\
$\mathbf{2}$ & 52.7211 & 43.2195 & 34.5672 & 19.7887 \\
$\mathbf{3}$ & 88.6384 & 77.4034 & 66.8450 & 48.0785 \\
\hline \multirow{4}{c}{ AuNW } \\
Mode Number & C-C & C-S & S-S & C-F \\
\cline { 2 - 5 } & 3.3525 & 2.3186 & 1.4954 & 0.5458 \\
$\mathbf{1}$ & 8.0970 & 6.6378 & 5.3089 & 3.0392 \\
$\mathbf{2}$ & 13.6133 & 11.8878 & 10.2662 & 7.3840 \\
$\mathbf{3}$ & \multicolumn{4}{c}{ AgNW } \\
\cline { 2 - 5 } Mode Number & C-C & C-S & S-S & C-F \\
\hline \multirow{2}{*}{$\mathbf{1}$} & 4.6550 & 3.2195 & 2.0764 & 0.7579 \\
$\mathbf{2}$ & 11.2430 & 9.2167 & 7.3716 & 4.2200 \\
$\mathbf{3}$ & 18.9025 & 16.5066 & 14.2550 & 10.2529 \\
\hline
\end{tabular}

Tables 3-6 show natural frequencies of embedded simply supported nanowires for $K_{W}=1$, $K_{W}=10, K_{W}=100$, and $K_{W}=1000$, respectively, with different $e_{0} a / L$ values. It was found from these tables that frequency values increased as $K_{W}$ values increased, but frequencies decreased by increasing $e_{0} a / L$. Moreover, it was evident that small-scale effects became more considerable for higher modes.

Table 3. The first three natural frequencies $(\mathrm{GHz})$ of embedded nanowires corresponding to various values of $e_{0} a / L\left(K_{W}=1\right)$.

\begin{tabular}{|c|c|c|c|c|}
\hline \multirow{3}{*}{ Mode Number } & \multicolumn{4}{|c|}{ SiCNW } \\
\hline & \multicolumn{4}{|c|}{$e_{0} a / L$} \\
\hline & 0.0 & 0.1 & 0.2 & 0.3 \\
\hline 1 & 10.2583 & 9.7916 & 8.7034 & 7.4989 \\
\hline 2 & 40.8373 & 34.5827 & 25.4413 & 19.1602 \\
\hline 3 & 91.8603 & 66.8530 & 43.0600 & 30.6452 \\
\hline \multirow{3}{*}{ Mode Number } & \multicolumn{4}{|c|}{ AuNW } \\
\hline & \multicolumn{4}{|c|}{$e_{0} a / L$} \\
\hline & 0.0 & 0.1 & 0.2 & 0.3 \\
\hline 1 & 1.5755 & 1.5038 & 1.3367 & 1.1517 \\
\hline 2 & 6.2719 & 5.3113 & 3.9073 & 2.9427 \\
\hline 3 & 14.1081 & 10.2675 & 6.6133 & 4.7066 \\
\hline \multirow{3}{*}{ Mode Number } & \multicolumn{4}{|c|}{ AgNW } \\
\hline & \multicolumn{4}{|c|}{$e_{0} a / L$} \\
\hline & 0.0 & 0.1 & 0.2 & 0.3 \\
\hline 1 & 2.1876 & 2.0881 & 1.8560 & 1.5992 \\
\hline 2 & 8.7087 & 7.3749 & 5.4255 & 4.0860 \\
\hline 3 & 19.5896 & 14.2567 & 9.1827 & 6.5352 \\
\hline
\end{tabular}


Table 4. The first three natural frequencies $(\mathrm{GHz})$ of embedded nanowires for various values of $e_{0} a / L$ $\left(K_{W}=10\right)$.

\begin{tabular}{|c|c|c|c|c|}
\hline \multirow{3}{*}{ Mode Number } & \multicolumn{4}{|c|}{ SiCNW } \\
\hline & \multicolumn{4}{|c|}{$e_{0} a / L$} \\
\hline & 0.0 & 0.1 & 0.2 & 0.3 \\
\hline 1 & 10.7171 & 10.2713 & 9.2398 & 8.1152 \\
\hline 2 & 40.9550 & 34.7215 & 25.6298 & 19.4097 \\
\hline \multirow[t]{2}{*}{3} & 91.9126 & 66.9249 & 43.1716 & 30.8018 \\
\hline & \multicolumn{4}{|c|}{ AuNW } \\
\hline \multirow[t]{2}{*}{ Mode Number } & \multicolumn{4}{|c|}{$e_{0} a / L$} \\
\hline & 0.0 & 0.1 & 0.2 & 0.3 \\
\hline 1 & 1.6460 & 1.5775 & 1.4191 & 1.2464 \\
\hline 2 & 6.2900 & 5.3326 & 3.9363 & 2.9810 \\
\hline \multirow[t]{2}{*}{3} & 14.1162 & 10.2785 & 6.6304 & 4.7306 \\
\hline & \multicolumn{4}{|c|}{ AgNW } \\
\hline \multirow[t]{2}{*}{ Mode Number } & \multicolumn{4}{|c|}{$e_{0} a / L$} \\
\hline & 0.0 & 0.1 & 0.2 & 0.3 \\
\hline 1 & 2.2855 & 2.1904 & 1.9704 & 1.7306 \\
\hline 2 & 8.7338 & 7.4045 & 5.4656 & 4.1392 \\
\hline 3 & 19.6007 & 14.2720 & 9.2065 & 6.5686 \\
\hline
\end{tabular}

Table 5. The first three natural frequencies $(\mathrm{GHz})$ of embedded nanowires with respect to various values of $e_{0} a / L\left(K_{W}=100\right)$.

\begin{tabular}{|c|c|c|c|c|}
\hline \multirow{3}{*}{ Mode Number } & \multicolumn{4}{|c|}{ SiCNW } \\
\hline & \multicolumn{4}{|c|}{$e_{0} a / L$} \\
\hline & 0.0 & 0.1 & 0.2 & 0.3 \\
\hline 1 & 14.5292 & 14.2035 & 13.4764 & 12.7318 \\
\hline 2 & 42.1135 & 36.0808 & 27.4431 & 21.7480 \\
\hline \multirow[t]{2}{*}{3} & 92.4347 & 67.6401 & 44.2722 & 32.3263 \\
\hline & \multicolumn{4}{|c|}{ AuNW } \\
\hline \multirow[t]{2}{*}{ Mode Number } & \multicolumn{4}{|c|}{$e_{0} a / L$} \\
\hline & 0.0 & 0.1 & 0.2 & 0.3 \\
\hline 1 & 2.2314 & 2.1814 & 2.0697 & 1.9554 \\
\hline 2 & 6.4679 & 5.5414 & 4.2148 & 3.3401 \\
\hline \multirow[t]{2}{*}{3} & 14.1964 & 10.3883 & 6.7994 & 4.9648 \\
\hline & \multicolumn{4}{|c|}{ AgNW } \\
\hline \multirow[t]{2}{*}{ Mode Number } & \multicolumn{4}{|c|}{$e_{0} a / L$} \\
\hline & 0.0 & 0.1 & 0.2 & 0.3 \\
\hline 1 & 3.0984 & 3.0290 & 2.8739 & 2.7151 \\
\hline 2 & 8.9809 & 7.6944 & 5.8524 & 4.6379 \\
\hline 3 & 19.7121 & 14.4245 & 9.4412 & 6.8937 \\
\hline
\end{tabular}


Table 6. The first three natural frequencies $(\mathrm{GHz})$ of embedded nanowires against various values of $e_{0} a / L\left(K_{W}=1000\right)$.

\begin{tabular}{|c|c|c|c|c|}
\hline \multirow{3}{*}{ Mode Number } & \multicolumn{4}{|c|}{ SiCNW } \\
\hline & \multicolumn{4}{|c|}{$e_{0} a / L$} \\
\hline & 0.0 & 0.1 & 0.2 & 0.3 \\
\hline 1 & 34.2564 & 34.1196 & 33.8234 & 33.5336 \\
\hline 2 & 52.3064 & 47.5839 & 41.4190 & 37.8864 \\
\hline \multirow[t]{2}{*}{3} & 97.5017 & 74.4150 & 54.0595 & 44.8040 \\
\hline & \multicolumn{4}{|c|}{ AuNW } \\
\hline \multirow[t]{2}{*}{ Mode Number } & \multicolumn{4}{|c|}{$e_{0} a / L$} \\
\hline & 0.0 & 0.1 & 0.2 & 0.3 \\
\hline 1 & 5.2612 & 5.2402 & 5.1947 & 5.1502 \\
\hline 2 & 8.0333 & 7.3081 & 6.3612 & 5.8187 \\
\hline \multirow[t]{2}{*}{3} & 14.9746 & 11.4288 & 8.3026 & 6.8811 \\
\hline & \multicolumn{4}{|c|}{ AgNW } \\
\hline \multirow[t]{2}{*}{ Mode Number } & \multicolumn{4}{|c|}{$e_{0} a / L$} \\
\hline & 0.0 & 0.1 & 0.2 & 0.3 \\
\hline 1 & 7.3053 & 7.2761 & 7.2130 & 7.1512 \\
\hline 2 & 11.1545 & 10.1475 & 8.8328 & 8.0794 \\
\hline 3 & 20.7926 & 15.8693 & 11.5284 & 9.5546 \\
\hline
\end{tabular}

In Table 7, the frequency values of C-C nanowires are given by analytical and finite element solutions for $e_{0} a / L=0.2$. For the finite element solution, as the element number increased, the results approached the real value.

Effects of both temperature rise and the Winkler parameter on the first three natural frequencies of nanowires are revealed in Table 8. It is apparent from the table that an increase temperature rise led to a decrease in frequency, contrary to the Winkler parameter. Also, it can be emphasized that the frequencies of AuNW were more affected than the other nanowires because of their related material properties, given in Table 1. Moreover, it can be observed that the influence of temperature rise was more prominent for lower modes and smaller Winkler parameters.

Table 7. Convergence of the present results with the analytical results of different element numbers.

\begin{tabular}{|c|c|c|c|c|c|c|c|c|}
\hline \multirow{2}{*}{ Mode Number } & \multicolumn{8}{|c|}{ SiCNW } \\
\hline & $N=4$ & $N=5$ & $N=6$ & $N=7$ & $N=8$ & $N=9$ & $N=10$ & Analytica \\
\hline 1 & 18.9418 & 18.9252 & 18.9189 & 18.9162 & 18.9148 & 18.9141 & 18.9137 & 18.9129 \\
\hline 2 & 38.0339 & 37.8398 & 37.7549 & 37.7155 & 37.6955 & 37.6845 & 37.6781 & 37.6656 \\
\hline 3 & 56.8236 & 57.2104 & 56.8478 & 56.6515 & 56.5467 & 56.4878 & 56.4529 & 56.3826 \\
\hline \multirow{2}{*}{ Mode Number } & \multicolumn{8}{|c|}{ AuNW } \\
\hline & $N=4$ & $N=5$ & $N=6$ & $N=7$ & $N=8$ & $N=9$ & $N=10$ & Analytica \\
\hline 1 & 2.9091 & 2.9066 & 2.9056 & 2.9052 & 2.9050 & 2.9049 & 2.9048 & 2.9047 \\
\hline 2 & 5.8413 & 5.8115 & 5.7985 & 5.7924 & 5.7894 & 5.7877 & 5.7867 & 5.7848 \\
\hline 3 & 8.7271 & 8.7865 & 8.7308 & 8.7007 & 8.6846 & 8.6755 & 8.6702 & 8.6594 \\
\hline \multirow{2}{*}{ Mode Number } & \multicolumn{8}{|c|}{ AgNW } \\
\hline & $N=4$ & $N=5$ & $N=6$ & $N=7$ & $N=8$ & $N=9$ & $N=10$ & Analytica \\
\hline 1 & 4.0394 & 4.0359 & 4.0345 & 4.0340 & 4.0337 & 4.0335 & 4.0334 & 4.0332 \\
\hline 2 & 8.1109 & 8.0695 & 8.0514 & 8.0430 & 8.0387 & 8.0364 & 8.0350 & 8.0323 \\
\hline 3 & 12.1179 & 12.2003 & 12.1230 & 12.0811 & 12.059 & 12.0462 & 12.0388 & 12.0238 \\
\hline
\end{tabular}


Table 8. The first three small-scale-dependent natural frequencies $(\mathrm{GHz})$ of embedded nanowires for different Winkler parameters and temperature changes $\left(e_{0} a / L=0.1, \mathrm{~d}=1 \mathrm{~nm}\right.$, and $\left.L=20 \mathrm{~d}\right)$.

\begin{tabular}{|c|c|c|c|c|c|c|}
\hline \multirow{3}{*}{ Mode Number } & \multicolumn{6}{|c|}{ SiCNW } \\
\hline & \multicolumn{3}{|c|}{$\Delta T=0$} & \multicolumn{3}{|c|}{$\Delta T=30^{\circ} \mathrm{C}$} \\
\hline & $K w=100$ & $K w=500$ & $K w=1000$ & $K w=100$ & $K w=500$ & $K w=1000$ \\
\hline 1 & 17.7768 & 31.4013 & 42.7034 & 17.3120 & 31.1405 & 42.5119 \\
\hline 2 & 45.1580 & 52.0507 & 59.5551 & 44.4297 & 51.4201 & 59.0047 \\
\hline 3 & 84.6569 & 88.5259 & 93.1362 & 83.7854 & 87.6928 & 92.3448 \\
\hline \multirow{3}{*}{ Mode Number } & \multicolumn{6}{|c|}{ AuNW } \\
\hline & \multicolumn{3}{|c|}{$\Delta T=0$} & \multicolumn{3}{|c|}{$\Delta T=30^{\circ} \mathrm{C}$} \\
\hline & $K w=100$ & $K w=500$ & $K w=1000$ & $K w=100$ & $K w=500$ & $K w=1000$ \\
\hline 1 & 2.7628 & 4.8802 & 6.6368 & 0.9103 & 4.1246 & 6.1026 \\
\hline 2 & 7.0182 & 8.0895 & 9.2558 & 4.6945 & 6.1824 & 7.6454 \\
\hline 3 & 13.157 & 13.7583 & 14.4748 & 10.5767 & 11.3159 & 12.177 \\
\hline \multirow{3}{*}{ Mode Number } & \multicolumn{6}{|c|}{ AgNW } \\
\hline & \multicolumn{3}{|c|}{$\Delta T=0$} & \multicolumn{3}{|c|}{$\Delta T=30^{\circ} \mathrm{C}$} \\
\hline & $K w=100$ & $K w=500$ & $K w=1000$ & $K w=100$ & $K w=500$ & $K w=1000$ \\
\hline 1 & 3.8362 & 6.7764 & 9.2153 & 1.8782 & 5.8932 & 8.5868 \\
\hline 2 & 9.7450 & 11.2325 & 12.8519 & 7.0859 & 9.0229 & 10.9734 \\
\hline 3 & 18.2689 & 19.1038 & 20.0987 & 15.2661 & 16.2559 & 17.4143 \\
\hline
\end{tabular}

Effects of nanoscale and foundation parameters on the first five natural frequencies are respectively depicted in Figures 2 and 3. It can be concluded from these figures that size dependency was more pronounced for higher modes, while the natural frequencies in lower modes were more affected from foundation parameters. As stated before, it was clear that the natural frequencies decreased and increased by increasing $e_{0} a / L$ and $K_{W}$, respectively.

Figure 4 displays the variation of fundamental frequencies of the three embedded nanowires with respect to temperature rise for various small-scale parameter values. It can be recognized from the figure that the effect of $e_{0} a / L$ was more prominent for SiCNW than the other ones. On the other hand, the influence of temperature rise was more significant for gold and silver nanowires than the silicon carbide nanowire.

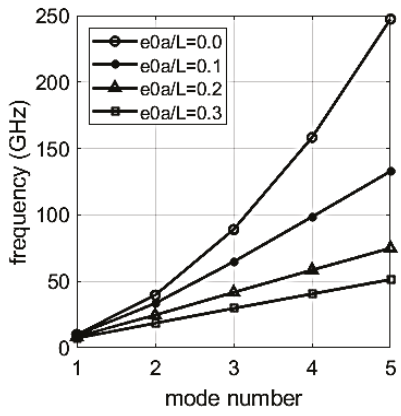

(a) SiCNW

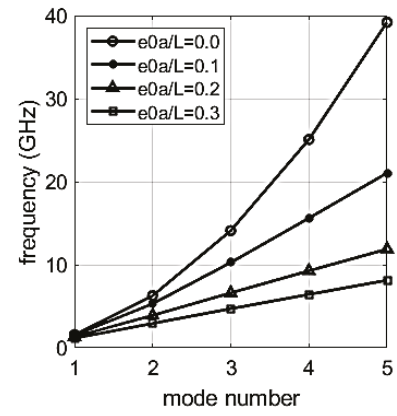

(b) AuNW

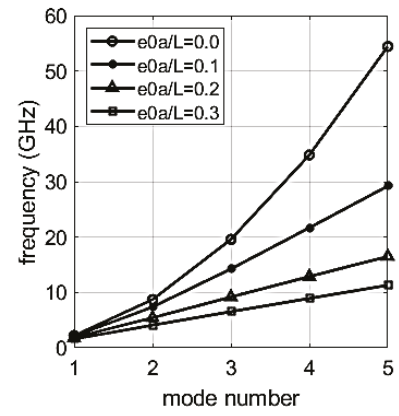

(c) $\mathrm{AgNW}$

Figure 2. Variation of the first five natural frequencies of nanowires with respect to $e_{0} a / L$. 


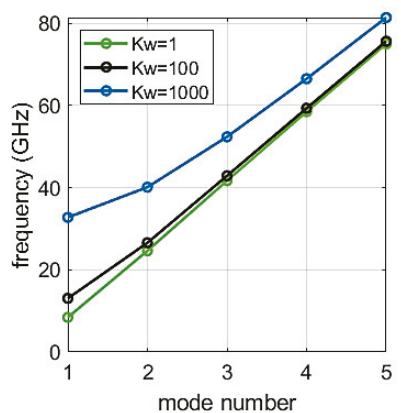

(a) SiCNW

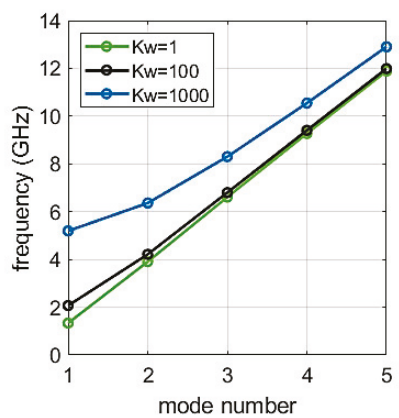

(b) AuNW

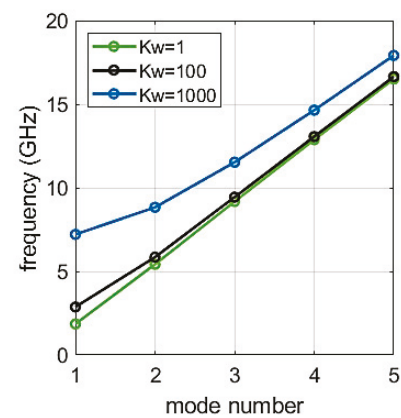

(c) AgNW

Figure 3. Effect of the Winkler parameter on the first five natural frequencies of nanowires.

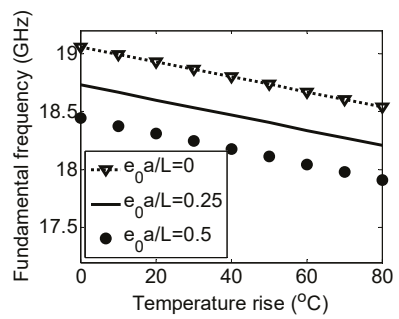

(a) $\mathrm{SiCNW}$

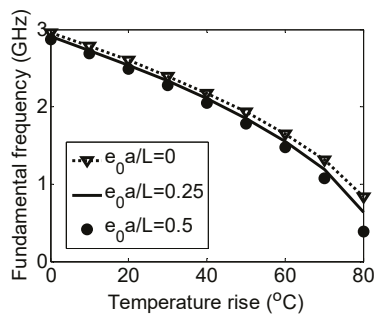

(b) AuNW

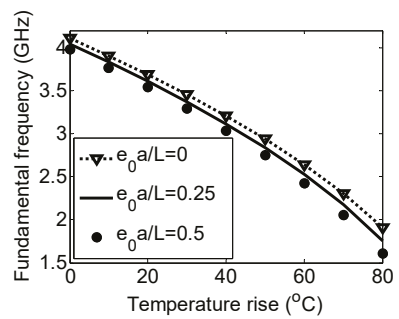

(c) $\mathrm{AgNW}$

Figure 4. Variation of the fundamental frequency for several $e_{0} a / L$ against temperature rise $(L=30 \mathrm{~d}$, $\left.K_{w}=1000\right)$.

\section{Conclusions}

Three types of nanowires, $\mathrm{SiCNW}, \mathrm{AgNW}$, and AuNW, are modeled as nonlocal Euler-Bernoulli nanobeams resting on a Winkler elastic foundation. Frequency values of these nanowires are obtained via a finite element solution, and results are given by tables and graphs. The effect of temperature change on the vibrational responses of simply supported nanowires is also examined as a case study. It can be concluded from the results that among all boundary conditions, $\mathrm{C}-\mathrm{C}$ has the highest frequency values and C-F has the lowest ones. Also, it can be emphasized that by increasing the Winkler parameter value, frequency values increase, while by increasing non-dimensional small-scale parameter $\left(e_{0} a / L\right)$, frequency value decreases. When we compare the frequency values of nanowires, SiCNW has the highest frequency values, while AuNW has the lowest frequency values because of its different material properties. Additionally, it is revealed that the effects of temperature change and small-scale parameters on the frequencies of nanowires are both considerable and negligible depending on the values of the involved material properties.

Author Contributions: All authors contributed extensively to the present study. The derivation of the governing equations, obtaining and discussion of the numerical results, and writing the manuscript have been performed by B.U. and Ö.C.

Acknowledgments: This study was supported by The Scientific and Technological Research Council of Turkey (TÜBITAK) with Project no: 117M495. This support is gratefully acknowledged.

Conflicts of Interest: The authors declare no conflict of interest. 


\section{References}

1. Makeev, M.A.; Srivastava, D.; Menon, M. Silicon carbide nanowires under external loads: An atomistic simulation study. Phys. Rev. B 2006, 74, 165303. [CrossRef]

2. Schulz, M.; Shanov, V.; Yin, Z. (Eds.) Nanotube Superfiber Materials: Changing Engineering Design; William Andrew: Norwich, NY, USA, 2013.

3. Numanoğlu, H.M. Nanoyapıların Kiriş ve Çubuk Modellerinin Yerel Olmayan Elastisite Teorisi Kullanılarak Titreşim Analizi; Akdeniz Üniversitesi: Antalya, Turkey, 2017. (In Turkish)

4. Vazinishayan, A.; Yang, S.; Duongthipthewa, A.; Wang, Y. Effects of cross-section on mechanical properties of Au nanowire. AIP Adv. 2016, 6, 025006. [CrossRef]

5. Poole, W.J.; Ashby, M.F.; Fleck, N.A. Micro-hardness of annealed and work-hardened copper polycrystals. Scr. Mater. 1996, 34, 559-564. [CrossRef]

6. Mc Farland, A.W.; Colton, J.S. Role of material microstructure in plate stiffness with relevance to microcantilever sensors. J. Micromech. Microeng. 2005, 15, 1060-1067. [CrossRef]

7. Zhang, C.-L.; Shen, H.-S. Predicting the elastic properties of double- walled carbon nanotubes by molecular dynamics simulation. J. Phys. D Appl. Phys. 2008, 41, 055404. [CrossRef]

8. Toupin, R.A. Elastic materials with couple-stresses. Arch. Ration. Mech. Anal. 1962, 11, 385-414. [CrossRef]

9. Mindlin, R.D.; Tiersten, H.F. Effects of couple-stresses in linear elasticity. Arch. Ration. Mech. Anal. 1962, 11, 415-448. [CrossRef]

10. Koiter, W.T. Couple stresses in the theory of elasticity, I and II. Proc. K. Ned. Akad. Wet. B 1964, 67, 17-44.

11. Yang, F.; Chong, A.C.M.; Lam, D.C.C.; Tong, P. Couple stress based strain gradient theory for elasticity. Int. J. Solids Struct. 2002, 39, 2731-2743. [CrossRef]

12. Mindlin, R.D. Second gradient of strain and surface-tension in linear elasticity. Int. J. Solids Struct. 1965, 1, 417-438. [CrossRef]

13. Lam, D.C.C.; Yang, F.; Chong, A.C.M.; Wang, J.; Tong, P. Experiments and theory in strain gradient elasticity. J. Mech. Phys. Solids 2003, 51, 1477-1508. [CrossRef]

14. Eringen, A.C. On differential equations of nonlocal elasticity and solutions of screw dislocation and surface waves. J. Appl. Phys. 1983, 54, 4703-4710. [CrossRef]

15. Nejad, M.Z.; Hadi, A.; Rastgoo, A. Buckling analysis of arbitrary two-directional functionally graded Euler-Bernoulli nano-beams based on nonlocal elasticity theory. Int. J. Eng. Sci. 2016, 103, 1-10. [CrossRef]

16. Akgöz, B.; Civalek, Ö. Bending analysis of FG microbeams resting on Winkler elastic foundation via strain gradient elasticity. Compos. Struct. 2015, 134, 294-301. [CrossRef]

17. Akgöz, B.; Civalek, Ö. Bending analysis of embedded carbon nanotubes resting on an elastic foundation using strain gradient theory. Acta Astronaut. 2016, 119, 1-12.

18. Togun, N.; Bağdatli, S.M. The vibration of nanobeam resting on elastic foundation using modified couple stress theory. Tehnički Glasnik 2018, 12, 221-225. [CrossRef]

19. Pradhan, S.C.; Phadikar, J.K. Nonlocal elasticity theory for vibration of nanoplates. J. Sound Vib. 2009, 325, 206-223. [CrossRef]

20. Uymaz, B. Forced vibration analysis of functionally graded beams using nonlocal elasticity. Compos. Struct. 2013, 105, 227-239. [CrossRef]

21. Togun, N. Nonlocal beam theory for nonlinear vibrations of a nanobeam resting on elastic foundation. Bound. Value Probl. 2016, 1, 57. [CrossRef]

22. Rahmanian, M.; Torkaman-Asadi, M.A.; Firouz-Abadi, R.D.; Kouchakzadeh, M.A. Free vibrations analysis of carbon nanotubes resting on Winkler foundations based on nonlocal models. Phys. B Condens. Mat. 2016, 484, 83-94. [CrossRef]

23. Demir, Ç.; Civalek, Ö. A new nonlocal FEM via Hermitian cubic shape functions for thermal vibration of nano beams surrounded by an elastic matrix. Compos. Struct. 2017, 168, 872-884. [CrossRef]

24. Pradhan, S.C. Nonlocal finite element analysis and small scale effects of CNTs with Timoshenko beam theory. Finite Elem. Anal. Des. 2012, 50, 8-20. [CrossRef]

25. Rajasekaran, S.; Bakhshi Khaniki, H. Finite element static and dynamic analysis of axially functionally graded nonuniform small-scale beams based on nonlocal strain gradient theory. Mech. Adv. Mat. Struct. 2018, 1-15. [CrossRef] 
26. Eltaher, M.A.; Emam, S.A.; Mahmoud, F.F. Free vibration analysis of functionally graded size-dependent nanobeams. Appl. Math. Comput. 2012, 218, 7406-7420. [CrossRef]

27. Nejad, M.Z.; Hadi, A. Eringen's non-local elasticity theory for bending analysis of bi-directional functionally graded Euler-Bernoulli nano-beams. Int. J. Eng. Sci. 2016, 106, 1-9. [CrossRef]

28. Murmu, T.; Pradhan, S.C. Thermo-mechanical vibration of a single-walled carbon nanotube embedded in an elastic medium based on nonlocal elasticity theory. Comput. Mat. Sci. 2009, 46, 854-859. [CrossRef]

29. Reddy, J.N. Nonlocal theories for bending, buckling and vibration of beams. Int. J. Eng. Sci. 2007, 45, $288-307$. [CrossRef]

30. Tornabene, F.; Bacciocchi, M.; Fantuzzi, N.; Reddy, J.N. Multiscale Approach for Three-Phase CNT/Polymer/Fiber Laminated Nanocomposite Structures. Polym. Compos. 2019, 40, 102-126. [CrossRef]

31. Fantuzzi, N. New insights into the strong formulation finite element method for solving elastostatic and elastodynamic problems. Curved Layer. Struct. 2014, 1, 93-126.

32. Civalek, O. Free vibration of carbon nanotubes reinforced (CNTR) and functionally graded shells and plates based on FSDT via discrete singular convolution method. Compos. Part B Eng. 2017, 111, 45-59. [CrossRef]

33. Civalek, O. Nonlinear dynamic response of laminated plates resting on nonlinear elastic foundations by the discrete singular convolution-differential quadrature coupled approaches. Compos. Part B Eng. 2013, 50, 171-179. [CrossRef]

34. Civalek, O.; Demir, C. Buckling and bending analyses of cantilever carbon nanotubes using the Euler-Bernoulli beam theory based on non-local continuum model. Asian J. Civ. Eng. 2011, 12, 651-661.

35. Civalek, O. Finite Element Analysis of Plates and Shells; Firat University: Elazı $\breve{g}$, Turkey, 1998. (In Turkish)

36. Uzun, B.; Numanoğlu, H.M.; Civalek, O. Free Vibration Analysis of BNNT with Different Cross-Sections via Nonlocal FEM. J. Comput. Appl. Mech. 2018, 49, 252-260.

37. Omurtag, M.H. Çubuk Sonlu Elemanlar; Birsen Yayınevi: Istanbul, Turkey, 2010. (In Turkish)

38. Işı1k, Ç. Mikro ve Nano Ölçekli Mekanik Sistemlerin Modellenmesinde Yerel Olmayan Sonlu Eleman Formülasyonu; Akdeniz Üniversitesi: Antalya, Turkey, 2018. (In Turkish)

(C) 2019 by the authors. Licensee MDPI, Basel, Switzerland. This article is an open access article distributed under the terms and conditions of the Creative Commons Attribution (CC BY) license (http://creativecommons.org/licenses/by/4.0/). 

Article

\title{
Trustworthiness in Modeling Unreinforced and Reinforced T-Joints with Finite Elements
}

\author{
Slimane Ouakka and Nicholas Fantuzzi * \\ DICAM Department, University of Bologna, Viale del Risorgimento 2, 40136 Bologna, Italy; souakka@gmail.com \\ * Correspondence: nicholas.fantuzzi@unibo.it; Tel.: +39-051-2093494
}

Received: 26 November 2018; Accepted: 16 February 2019; Published: 20 February 2019

\begin{abstract}
As required by regulations, Finite Element Analyses (FEA) can be used to investigate the behavior of joints which might be complex to design due to the presence of geometrical and material discontinuities. The static behavior of such problems is mesh dependent, thus these results must be calibrated by using laboratory tests or reference data. Once the Finite Element (FE) model is correctly setup, the same settings can be used to study joints for which no reference is available. The present work analyzes the static strength of reinforced T-joints and sheds light on the following aspects: shell elements are a valid alternative to solid modeling; the best combination of element type and mesh density for several configurations is shown; the ultimate static strength of joints can be predicted, as well as when mechanical properties are roughly introduced for some FE topologies. The increase in strength of 12 unreinforced and reinforced (with collar or doubler plate) T-joints subjected to axial brace loading is studied. The present studies are compared with the literature and practical remarks are given in the conclusion section.
\end{abstract}

Keywords: numerical modeling; joint static strength; finite element method; parametric investigation; reinforced joint (collar and doubler plate)

\section{Introduction}

Joints in offshore engineering are crucial because they are key components in the design of jacket structures. The study of joints is generally performed after a pre-dimensioning of the complete jacket has been carried out. Jacket structures are investigated using simple lattice models made of beams which simulate the tubular elements of the whole structures. Such simple model is extremely convenient for global structural analysis but does not give enough detail regarding local structural failure such as fatigue cracking at joints. For this reason, several authors in the past and recent years investigated the problem of static and dynamic strength of offshore extensively [1-17]. Among these, Moffat et al. [1] investigated the effect of the chord length and the applied boundary conditions on the static strength of tubular T-joints. Ring stiffened DT-joints (cross shaped) have been studied by Marcus et al. [3]; the dynamic performance of collar plate reinforced joints was studied by Qu et al. [10]. Numerical and experimental studies on the ultimate strength of KT-joints (K shaped) were presented by Li et al. [15].

On the other hand, in the recent studies some researchers have investigated in detail the concertation of the stress and its distribution in critical areas [18-23], where Lostsberg [18], Osawa et al. [19] and Lotfollahi-Yagin et al. [20] have focused on the stress hot spots in tubular joints, whereas, Cheng et al. [21] investigated how the concertation of the stress affect the fatigue.

In addition to the aforementioned researches, other studies that involved joints should be mentioned [24-46], such as the work by Xia et al. [24] regarding the hysteretic behavior of stiffened T-joints. Furthermore, Dong et al. [28] presented long-term fatigue analysis of multi-planar tubular joints. Finally, experimental tests were performed on joints using white light speckle method by 
Liu [32]. Numerical implementation and coupling of finite elements of different topology in the study of joints were proposed by Pey et al. [33], and a good review on finite and boundary element methods was given by Mackerle [34,35]. Regarding the topic of composite structures, the work by Chowdhury et al. [41] and Shen et al. [42] should be cited for their application to practical engineering practices for the static and fatigue strength of joints. Finally, the parametric study on composite joints by Liu et al. [46] is mentioned for their thorough description of this particular problem.

The present work investigates tubular joints made of steel Circular Hollow Sections (CHSs) that are the main structural components of steel off-shore structures such as jackets, compliant towers, jack-ups, etc. In these kinds of structures, the CHSs members are joined in a point, forming a tubular simple joint by welding the profiled ends of the secondary members, the braces, onto the circumference of the main member, the chord.

Due to their crucial rule, some guidelines and methods have been introduced, to guarantee the needed serviceability and to understand the strength behavior of these type of element, the most used are: American Petroleum Institute (API) [47], International Organization for Standardization (ISO) [48], American Bureau of Shipping (ABS) [49], and Eurocode3 (EC3) [50].

Several types of reinforcement are available nowadays in order to enhance the strength of tubular joints. One typology that can be found often in off-shore is schematically shown in the sections depicted in Figure 1, where the collar plate (a) consists in flat surface around the brace-chord intersection, whereas the doubler plate (b) is a surface in between the two elements. These two methods are widely used since they can be an important improvement either for rehabilitation purposes in old structures or for new structures.
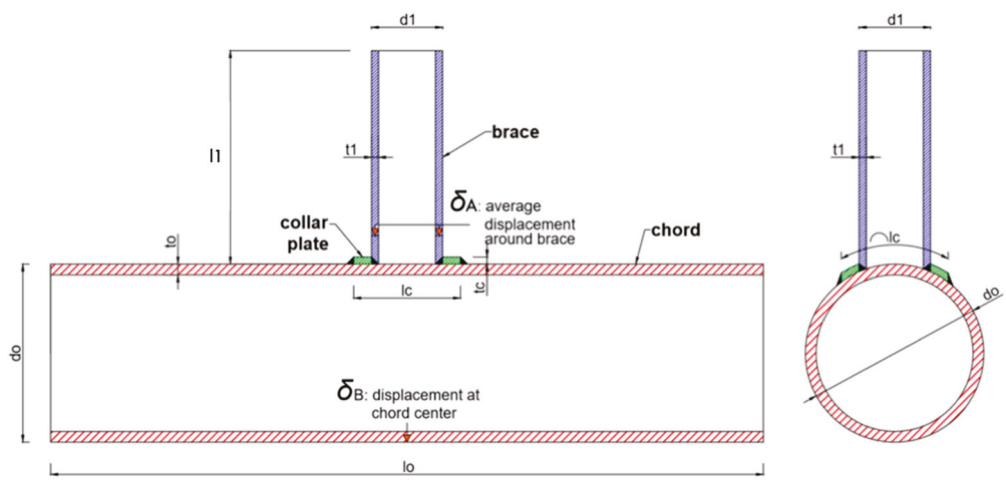

(a)
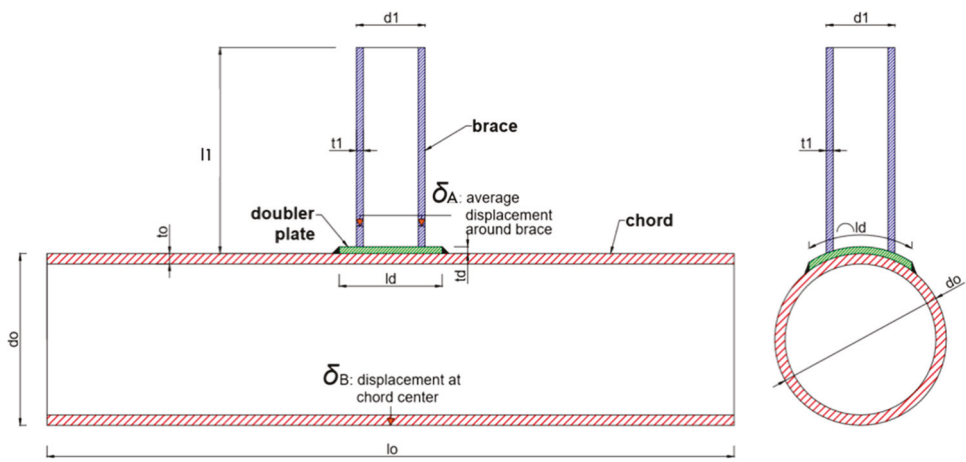

Figure 1. Scheme of specimens: (a) collar plate reinforcement and (b) doubler plate reinforcement. 
Thus, for a better understanding of the static strength of joints with the introduction of the two types of reinforcement, 12 T-Joint specimens subjected to axial brace load (6 in compression and 6 in tension) have been studied, with different geometric and material parameters. T-joints have been selected in this investigation even though several types of joints can be formed in lattice structures such as $\mathrm{Y}, \mathrm{K}$, and $\mathrm{X}$ joints or their combinations. Based on this study, conclusions can be obtained in assessing the reinforcing efficiency.

The aforementioned guidelines [47-50] suggest using FE models whenever new configuration of joint are treated, but the sharp geometric discontinuities (wherein stress concentrations occur) in the tubular joints lead to a mesh dependence for the results and so such FE models need to be calibrated by considering reference data such as laboratory tests. In the present paper, the works $[4,5]$ have been considered as a reference.

The reference paper [5] considers a 3-D solid FE model, on the contrary the present research simplifies the problem by modeling the joints with less degrees of freedom and a much simpler geometric description. Shell elements are used in this research because of their simplicity and the lower cost in terms of computational time with respect to solid elements. Moreover, the geometry of FE models made of solid elements is more difficult to generate and assemble with respect to 2-D shell elements. In the reference 3-D simulations [5] the authors decided to deploy solid elements instead of 2-D elements, mainly because: the contact algorithm will only identify contact between two members if the shell elements (which represent the mid-surfaces of each member) touch each other, thereby incorrectly allowing penetration of one member into the other, in addition to preventing the uncertainty in modeling the welded area of the brace-chord intersection. Therefore, succeeding in solving the aforementioned limits, as will be showed in the following sections, shell elements can be used instead of solid elements as in the present research.

The present work considers the commercial finite element ABAQUS package, since it offers an attractive alternative to generate reliable data, provided that the accuracy of the FE model is firstly verified against test evidence. In particular, ABAQUS 6.14 will be used for all the simulations.

\section{Previous Researches}

The aim of the work, as has been already aforementioned, is to improve the understanding of the static behavior of plate reinforced joints, by building an FE model with shell elements. This is generally required from the regulations when new joint configurations are studied, but since stress concentrations computed by FE are mesh dependent, they need to be calibrated with the help of laboratory tests [4].

The reference papers present an experimental investigation and a numerical simulation. The numerical simulation has been considered here to have the possibility of discussing the improvement that have been obtained by the use of a different element topology. In this section, relevant information will be summarized. However, for an exhaustive description of the tests, the reader is invited to the articles [4,5].

\subsection{Experimental Investigations}

Laboratory tests are one useful way to calibrate numerical simulations. The basic configuration and the T-joint geometries are illustrated in Figure 1. The experimental program consists of 12 tests, of unreinforced and reinforced with collar or doubler plates, as depicted in Figure 1. Joints have been tested under both compression and tension loading. The geometric data indicated in Figure 1 are listed in Table 1, except for the joint lengths that are constant for all twelve specimens, respectively: $l_{0}=2840 \mathrm{~mm}, l_{1}=1100 \mathrm{~mm}$, and $l_{d}=l_{c}=305 \mathrm{~mm}$. 
Table 1. Geometrical details of the twelve T-joint specimens.

\begin{tabular}{cccccccc}
\hline & Type & $\boldsymbol{d}_{0}$ & $\boldsymbol{d}_{1}$ & $\boldsymbol{t}_{0}$ & $\boldsymbol{t}_{1}$ & $\boldsymbol{t}_{\boldsymbol{d}}$ & Brace Loading \\
\hline$E X-01$ & Unreinforced & 409.5 & 221.9 & 8.1 & 6.8 & - & Compression \\
$E X-02$ & Unreinforced & 409.5 & 221.9 & 8.1 & 6.8 & - & Tension \\
$E X-03$ & Collar & 409.5 & 221.9 & 8.1 & 6.8 & 6.4 & Compression \\
$E X-04$ & Collar & 409.5 & 221.9 & 8.5 & 6.8 & 6.4 & Tension \\
$E X-05$ & Collar & 409.5 & 221.9 & 12.8 & 8.4 & 8.3 & Compression \\
$E X-06$ & Collar & 409.5 & 221.9 & 12.8 & 8.4 & 8.3 & Tension \\
$E X-07$ & Doubler & 409.5 & 221.9 & 8.5 & 6.8 & 6.6 & Compression \\
$E X-08$ & Doubler & 409.5 & 221.9 & 8.2 & 6.5 & 6.4 & Tension \\
$E X-09$ & Unreinforced & 409.5 & 114.7 & 8.5 & 5.9 & - & Compression \\
$E X-10$ & Unreinforced & 409.5 & 114.7 & 8.5 & 5.9 & - & Tension \\
$E X-11$ & Doubler & 409.5 & 114.7 & 8.5 & 5.9 & 6.6 & Compression \\
$E X-12$ & Doubler & 409.5 & 114.7 & 8.5 & 5.9 & 6.6 & Tension \\
\hline
\end{tabular}

The materials used in each test are fabricated from carbon steel pipe, using the guidelines given from the API 5L Gr. B/ASTM A106-94A; while the welding procedure follows AWS D1.1. Table 2 indicates the measured yield stress for each steel component of the chord $f_{y, 0}$, brace $f_{y, 1}$, and reinforcement $f_{y, d}$.

Table 2. Material properties and yield stresses of the twelve T-joint specimens.

\begin{tabular}{lccc}
\hline & $\begin{array}{c}f_{y, 0} \\
{\left[N / m^{2}\right]}\end{array}$ & $\begin{array}{c}f_{y, 1} \\
{\left[N / m^{2}\right]}\end{array}$ & $\begin{array}{c}f_{y, d} \\
{\left[N / m^{2}\right]}\end{array}$ \\
\hline$E X-01$ & 285 & 300 & - \\
$E X-02$ & 285 & 300 & - \\
$E X-03$ & 285 & 300 & 461 \\
$E X-04$ & 276 & 300 & 461 \\
$E X-05$ & 276 & 275 & 464 \\
$E X-06$ & 276 & 275 & 464 \\
$E X-07$ & 276 & 300 & 461 \\
$E X-08$ & 312 & 284 & 461 \\
$E X-09$ & 276 & 312 & - \\
$E X-10$ & 276 & 312 & 461 \\
$E X-11$ & 276 & 312 & 461 \\
$E X-12$ & 276 & 312 & \\
\hline
\end{tabular}

Each specimen is pin-supported at the chord ends to minimize the possibility of axial load, and the brace is bolted at the top. The load is applied through a displacement-controlled actuator that has a rated compression capacity of $2000 \mathrm{kN}$, a tension capacity of $1200 \mathrm{kN}$, and range of displacement of $\pm 200 \mathrm{~mm}$. In all tests, the load is applied at initial stage at rate of $0.3 \mathrm{~mm} / \mathrm{min}$ for the linear part and increased up to $1.2 \mathrm{~mm} / \mathrm{min}$ through the Instron 8800 controller [4].

\subsection{Numerical Simulations}

The present section illustrates the details of the numerical simulations performed with 3-D solid elements in the reference [5]. Strength and weaknesses of all the modeling details provided are listed below.

The analysis considers the dimensions of the tubular members reported in Table 1, whereas for the materials proprieties Table 2 has been considered. Major aspects of the reference FE strategy adopted for the T-joints test, such as element type, mesh density, and contact interaction, are described below.

The T-joint specimen is simply supported with symmetric brace axial load [5], therefore the model has been simplified by considering a quarter of the whole model; and solid elements were selected to model the tubular joints, obviously these elements account for the joint dimensions in all three directions. Alternatively, mesh size is composed in such a way to be relatively smaller where the 
stress gradient is more critical, therefore the density of the mesh decreases from the vicinity of the intersection to the end of the brace or chord.

In the reference [5], the authors stated that: "Weld geometry was modeled using a ring of shell elements along the brace-chord" and that: "the penetration weld between the brace and the doubler plate is modeled in accordance with the measured dimensions obtained from the T-joint specimens". However, the graphical representation, presented in the papers [4,5], shows solid elements also for the welded area, instead of the mentioned shell elements. Therefore, a detailed size of the weld is not given in [4] or [5].

Since in this case the plate reinforced joints are loaded by an axial force/displacement, contact shall occur between bottom surface of the reinforcement and the external surface of the chord. Thus, contact plays a main rule as transferring mechanism from the secondary element (brace) to the primary one (chord), and this is a source of nonlinearity in the FE analysis. Due to the fact that both reinforcement and chord walls are deformable bodies, a deformable-deformable contact interaction is defined using a "master-slave" algorithm in the numerical analysis, with no friction between the members is assumed.

According to the experimental investigations, the axial load is applied using the displacement control method by prescribing the vertical displacement of the nodes at the brace tip.

The true stress-strain relationship enforced in these simulations is related to the yielding point of each material, as given in Table 2 , and can be represented by a bilinear relationship. No further hardening in the true stress-true strain behavior is assumed after the peak load in the engineering stress-strain curve, i.e., the true stress-true strain curve is assumed to remain horizontal beyond this point as for elastic-perfectly plastic model, as shown in Figure 2a. Contrastingly, the welds are assumed to have the same material properties as the base metal.

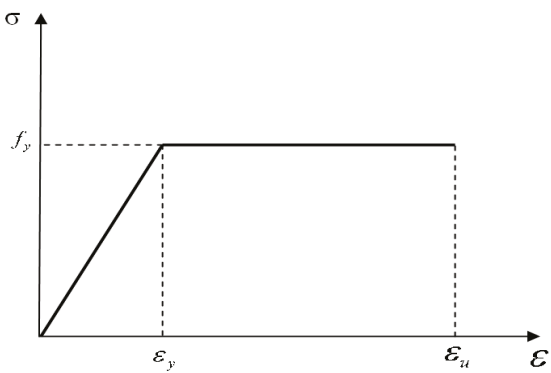

(a)

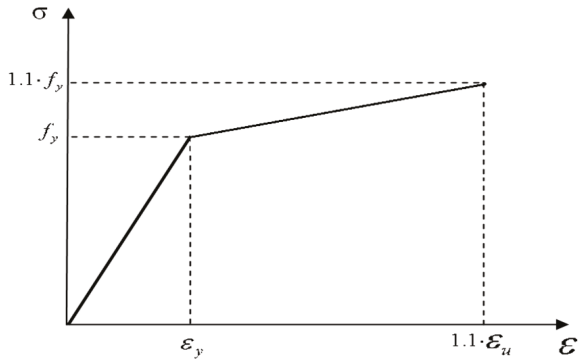

(b)

Figure 2. True stress-strain relationships: (a) without hardening and (b) with $10 \%$ hardening.

The use of this simplification in the true stress-strain relationship is commonly used in the design of structures because allows the designer to stay in the safety size due to the fact that no hardening is considered. This approximation, though, can lead to a result slightly different from the real behavior. For this, in Section 6, in addition to the elastic-perfectly plastic model, a model where the hardening is taken into account, as in Figure 2b, will also be considered.

\subsection{Comparison Setup}

More detailed information about the results of the two investigations will be given in the next section while showing the results of the work made during this research. However, in this section the main point of data collecting and how the results have been shown by the authors of $[4,5]$ will be given. In general, the results of the two papers can be summarized in just one, since they are two parts of the same study. The results given by the references are given in terms of load-ovalization curves and deformed shapes. 
The load-ovalization curves are presented, where the ovalization indicates the deformation of the chord cross section at the center of the T-joint, which is calculated by subtracting the height of point fixed simultaneously at the bottom of the chord and at the center of the joint, $\delta_{B}$, from the average value of four fixed points (in the one quarter FE model of course will be only two) on the brace near the intersection with the chord, $\delta_{A}$. These points are obtained by the transducer reading in case of experimental investigation, whereas for the FE model by using RP (Reference Point) as shown in Figure 1. The ultimate load for each test is identified simply by selecting the peak of each curve, and these values are listed in Table 3, where $F_{u, t e s t}$ is for the experimental investigations [4] while $F_{u, n u m}$ for the numerical simulations [5]. At the same time, the two phases of the joint behavior, such as the linear phase and the plastic one, can be deducted.

Table 3. Ultimate load obtained in the numerical simulation [5].

\begin{tabular}{lccc}
\hline & $\begin{array}{c}\boldsymbol{F}_{\mathbf{u}, \text { test }} \\
{[\mathbf{k} \boldsymbol{N}]}\end{array}$ & $\begin{array}{c}\boldsymbol{F}_{\mathbf{u}, \text { num }} \\
{[\mathbf{k N}]}\end{array}$ & $\boldsymbol{F}_{\mathbf{u}, \text { num }} / \boldsymbol{F}_{\mathbf{u}, \text { test }}$ \\
\hline$E X-01$ & 305.1 & 310.9 & 1.02 \\
$E X-02$ & 543.2 & 557.8 & 1.03 \\
$E X-03$ & 425.6 & 431.4 & 1.01 \\
$E X-04$ & 609.2 & 648.9 & 1.07 \\
$E X-05$ & 780.0 & 798.5 & 1.02 \\
$E X-06$ & 1065.3 & 1069.3 & 1.00 \\
$E X-07$ & 415.8 & 446.4 & 1.07 \\
$E X-08$ & 708.0 & 712.2 & 1.01 \\
$E X-09$ & 200.1 & 193.0 & 0.96 \\
$E X-10$ & 407.8 & 393.7 & 0.97 \\
$E X-11$ & 305.0 & 309.3 & 1.01 \\
$E X-12$ & 520.0 & 506.2 & 0.97 \\
\hline
\end{tabular}

The experimental [4] and numerical [5] results are not only evaluated in terms of load-ovalization curves but also by comparison of deformed sliced "rings", taken at the center of the T-joints after the tests and numerical analyses were completed. Then at the end of the tests, for some of the specimens, sections or "rings" were obtained for the joint elements, chord and brace.

In conclusion, all the results obtained from these two papers will be used in the fundamental step of calibration of the FE model of the present work. In the following section the results of the present FE simulations will be compared to the ones of the previous studies and comments and highlights will be provided.

\section{Present Modeling}

Commercial finite element packages offer an attractive and cheaper alternative to the laboratory experiments, but at the same time they require the use of sophisticated hardware, and also need the help of other software to post-processing the results. Hence, different software have been used, such as Abaqus/CAE 6.14-1 [51], Matlab R2017a, and AutoCAD 2017. The hardware used is the Intel(R) Core(TM) i7-4700MQ CPU @ 2.40GHz 2.40 GHz with 12GB of RAM.

This section addresses various aspects of all the steps that have been performed to study and simulate the state of T-joints subjected to the axial force. The cheaper shell elements have been used in this work, whereas the numerical simulation made in [5] considered solid elements.

The dimensions of the tubular members and reinforcement plates assumed in this research are in accordance with the measured dimensions of the test specimen of the papers as sketched in Figure 1, for which the values are reported in Table 1. In contrast, Table 2 summarizes the measured yield stress for each chord $f_{y, 0}$, brace $f_{y, 1}$, and reinforcement $f_{y, d}$.

Although the type of element material is specified in the reference papers [4,5] as carbon steel, the characteristics of this typology of material are not unique, especially concerning Young's Modulus $(E)$ and Poisson ratio $(v)$, where $E$ ranges from 203 to $210 \mathrm{GPa}$ whereas $v$ ranges from 0.25 to 0.3. 
With the help of preliminary simulations, the more fitting values of these have been demonstrated to be $E=210 \mathrm{GPa}$ and $v=0.3$. These values were obtained by comparing the normalized load $R$, defined as $R=F_{u, t e s t} / f_{y, 0} \cdot t_{0}^{2}$ of the EX-01, where $t_{0}$ is the chord thickness, with the one given by the experimental investigations, as reported in Table 4.

Table 4. Values of $E$ and $v$ of preliminary simulations [17].

\begin{tabular}{ccccc}
\hline Specimen & $\begin{array}{c}\text { Experimental } \\
\text { Investigation }\end{array}$ & $\begin{array}{c}\text { Preliminary } \\
\text { Simulation 1 } \\
\boldsymbol{E}=\mathbf{2 0 3} v=\mathbf{0 . 2 5}\end{array}$ & $\begin{array}{c}\text { Preliminary } \\
\text { Simulation 2 } \\
\boldsymbol{E}=\mathbf{2 1 0} \boldsymbol{v} \mathbf{0 . 2 5}\end{array}$ & $\begin{array}{c}\text { Preliminary } \\
\text { Simulation 3 } \\
\boldsymbol{E}=\mathbf{2 1 0} v=\mathbf{0 . 3 0}\end{array}$ \\
\hline$f_{y, 0}\left[\mathrm{~N} / \mathrm{mm}^{2}\right]$ & 285 & 285 & 285 & 285 \\
$t_{0}[\mathrm{~mm}]$ & 8.1 & 8.1 & 8.1 & 8.1 \\
$R=F_{u, t e s t} / f_{y, 0} \cdot t_{0}^{2}$ & 16.32 & 13.95 & 14.09 & 14.11 \\
\hline
\end{tabular}

AutoCAD 3D was selected to draw the T-joint tubular elements. Although ABAQUS gives the possibility to directly represents different geometries, AutoCAD is a more suitable software for this kind of parametric research since it gives the possibility to easily modify and change elements on it. Afterwards, only a quarter of the joint has been imported in the FE packages, this idealization does not make any differences in the results and reduces the total computational effort.

Starting from the first adjustments of the simulations, the three cases are discussed below: unreinforced, collar plate reinforcement, and doubler plate reinforcement.

Three native parts have been created for the reinforced cases (chord, brace, and reinforcement plate), and only two for the unreinforced case because no reinforcement is present. Some internal parts have been introduced in the part module, with their respective edges. In other words, due to the fact that the joint is made of several parts and contact must be implemented in order to connect the different parts together, construction edges (from CAD) are kept in the FE model also in order to simplify the following step.

Once all the parts are created (in their own coordinate system), they should be assembled in the Abaqus Assembly module [51] that is used to create instances of the parts and to position the instances relative to each other in a global coordinate system. The instances made for all the three cases are one for each part. Then for the unreinforced case, two instances have been generated, one for the chord and the other for the brace; whereas for the reinforced cases a third instance that represents the reinforcement is present.

The displacement induced by the controlled actuator in the experimental investigation [4] is at an initial stroke rate of $0.3 \mathrm{~mm} / \mathrm{min}$, and then progressively increased up to $1.2 \mathrm{~mm} / \mathrm{min}$ through the Instron 8800 controller. During the simulations in the present study, two types of step sequences are analyzed: the first automatic step sequence, and the second fixed step sequence, i.e., varying the load range during the simulations.

The automatic step sequence has the same step range for the whole simulation. While the fixed ones in order to reproduce the experimental investigation have two different ranges, in particular the second step starts at the starting point of the material plasticization which has been identified during the automatic step sequence.

The restraints that have been adopted for the FE model are in accordance with the experimental investigation; where the specimen is pin-supported at the chord ends and the brace end is bolted to the Instron actuator mounted on top of the brace, where the load is obtained as reaction force from the boundary condition at the brace tip. Since one-quarter of the whole specimen has been modeled, along with symmetry planes, peculiar boundary conditions have been considered as XSYMM (along the $x$-axis) and ZSYMM (along the $z$-axis) [51].

Material properties of the elements are modeled in accordance to the experimental investigation as listed in Table 2, whereas the Young's modulus and Poisson ratio are taken from the preliminary simulations already described Table 4 . The true stress-strain curve that has been assumed by var 
der Vegte et al. [5] in his numerical simulations is represented by a multilinear relationship and subsequently converted into a true stress-true strain relationship, and no further hardening in the true stress-true strain behavior is assumed after the peak load, i.e., the curve is assumed horizontal beyond this point.

Since no more detailed information is given about the true strain-stress curve, in this study, the elastic-perfectly plastic model is assumed to fit these characteristics as shown in Figure 2a. Due to the lack in the reference paper [5] for what concerns the latter relationship, in this research some hardening will be considered during the simulations, by introducing a hardening of $10 \%$ for all the materials, as depicted in Figure $2 b$, to verify the reliability of the elastic-perfectly plastic model.

Currently, the following types of constraints have been used in the simulations, the multi-point constraints (MPC) [51], which allows the restriction imposed by the boundary conditions (BCs) along the whole circumference, and the TIE [51], which instead has been used to connect the edges of the elements to simulate the welded connections. However, since the brace is directly welded to the chord in the unreinforced cases, no separation will occur between the two elements and the two parts have been merged (i.e., combine two elements in one instance [51]) into a single one.

During the development of the contact between the chord and the reinforcement made for the reinforced T-joints, contact mechanical properties are imposed by fixing the relevant points, such as tangential and normal behavior in the contact interaction propriety. The tangential behavior was imposed as "Frictionless", while the normal behavior as "Hard" contact. The contact properties are also imposed to "Allow separation after contact" since, in the case of the reinforced plate, separation between the plate and the chord that are linked, by the "tie" constraints, might occur. After the contact property is defined, surface-to-surface contact interaction is set up. Here the sliding is finite, and no adjustments are required since the two surfaces lie in the same plane. A further important feature is "Contact controls" option, which helps the convergence of the simulation without considering the penetration of one member into the other, as after solved using the Top/Bottom surface function [51].

The final results will be given in terms of load-ovalization curves; the ovalization was detected by the use of the transducers, so four reference points around the brace near the brace-chord intersection precisely at $20 \mathrm{~mm}$ distance, and one placed under the chord at the center of the T-joint have been introduced. The load detection is done by inserting an additional point at the tip of the brace, the reaction force at that point is indeed the opposite of the load applied. For the sake of further comparison, the deformed shape at the center of the joint will be considered

The FE mesh is a key point in modeling, since it has a huge influence on the results of the simulations in these kinds of elements, as will be seen when analyzing the results. The mesh module [51] allows generating meshes of parts and assemblies. For each FE model, four mesh densities are generated, and for each of them four types of elements are considered.

The four mesh densities analyzed are:

- $\quad$ Coarse mesh (average size $50 \mathrm{~mm}$ )

- $\quad$ Medium mesh (average size $20 \mathrm{~mm}$ )

- $\quad$ Fine mesh (almost the same number of elements as the medium ones but the size decreases from the ends to the intersection)

- $\quad$ Article mesh (same number of nodes on the edges shown in [5])

The four element types are:

- $\quad$ S4R (4 nodes with reduced integration)

- $\quad$ S4 (4 nodes without reduced integration)

- $\quad$ S8R (8 nodes with reduced integration and six degrees of freedom (DOF))

- $\quad$ S8R5 (8 nodes with reduced integration and five DOF) 
In the "Mesh controls", the "Structured" technique with element shape as "Quad-dominated" [51] was used for all the simulations. These two selections are able to carry out a regular mesh made (mostly) by quadrilaterals.

The four aforementioned meshes are used in this work to better understand the fitting of the results depending on the element size. In three of the four densities cases, the medium, fine, and article one, shown in Figure $3 \mathrm{~b}-\mathrm{d}$, the doubling of the lines in the reinforcement area can be noticed. This is because the contact interaction algorithm needs two different meshes in order to have a reliable result of the contact [51]. In particular, the "slave surface" should have a finer mesh with respect to the "master surface".

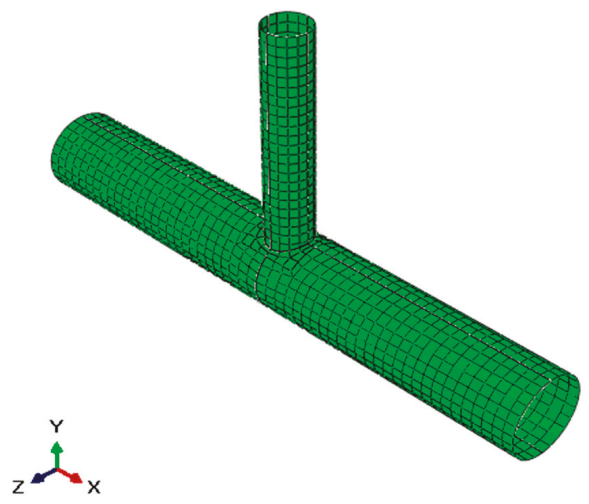

(a)

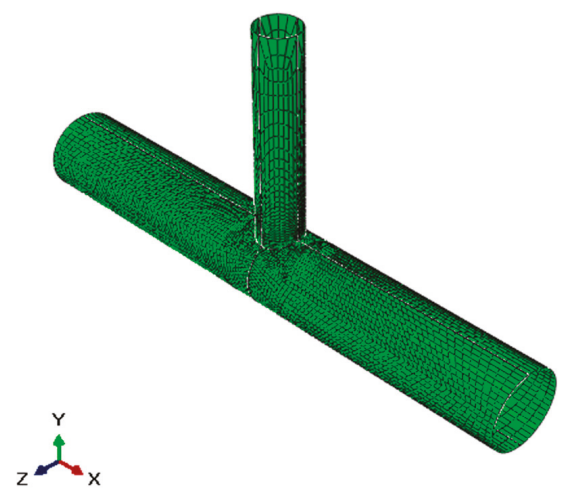

(c)

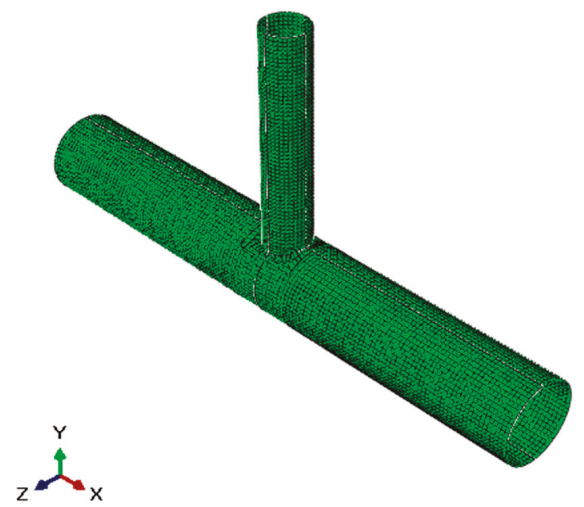

(b)

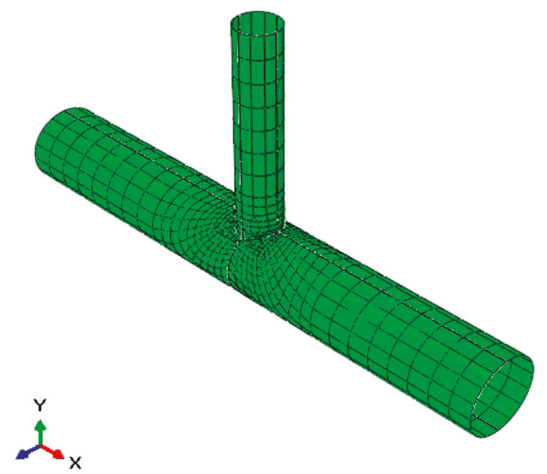

(d)

Figure 3. Meshes used in the simulations and their nomenclature: (a) coarse mesh, (b) medium mesh, (c) fine mesh, and (d) article mesh.

The coarse and medium mesh densities are obtained by imposing a unique size on the instance of the T-joint. The larger mesh has a size of 50, whereas the medium has a global size of 20, as depicted respectively in Figure 3a,b. The total number of elements is equal to 466 in coarse mesh, while 2745 elements are generated for the medium mesh. In the remaining cases, i.e., the fine and the article meshes depicted respectively in Figure $3 c, d$, the element sizes are varied in such a way that relatively smaller elements are used where the stress gradient is more critical. Therefore, the mesh density decreases from the vicinity of the intersection to the end of the brace or chord, and therefore smaller sizes in the vicinity of the brace-chord intersection. This strategy, to decrease the size while 
getting close to the critical area, is frequently used in FE analysis, because it allows to have an accurate study of the area most influential thanks to the small sizes, and at the same time to reduce the computational effort, due to increase of the sizes far from the critical point. Fine mesh has almost the same number of elements of the medium mesh, but their sizes, as aforementioned, are decreasing while going close to the intersection. For the article mesh, as in the fine one, the sizes are smaller near the intersection, this mesh is obtained from the mesh used by var der Vegte et al. [5]. Precisely, an equal number of nodes at each edge with almost the same factor of variation (decrease in size) is considered, so a total of 341 elements is obtained. In particular, by using the proportion between the geometries of the specimens in Table 1 and the graphic dimensions displayed by var der Vegte et al. [5], it was possible to discretize the FE model as depicted in Figure 4, in order to have the same nodes of the paper [5] at edges as previously stated.

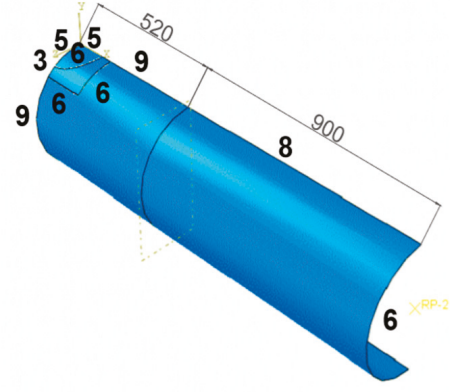

(a)

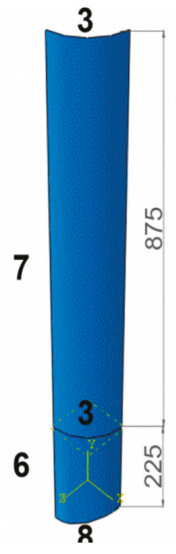

(b)

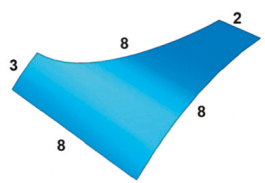

(c)

Figure 4. True article mesh density subdivision: (a) chord, (b) brace, and (c) reinforcement. [3]

From Figure $4 \mathrm{a}-\mathrm{c}$, it can be seen that the reinforcement and its respective area in the chord have different repartition, in particular three of the edges of the chord are increased by two units, i.e., from six to eight spaces. This is done to help the contact interaction as aforementioned. As a result, a total of 341 elements is obtained. For the final view of the specimen in the case of the article mesh please consider Figure 3d.

\section{Results}

The results of the research will be shown in this section, comparing them with the results of the two researches $[4,5]$ discussed in Section 2. Therefore, in order to overlap the outcomes, the numerical results of this research are shown in terms of load-ovalization curves and deformed shapes. The numerical values of both solutions are considered at the end of the loading process (e.g., end of the curves provided by the references).

Due to mesh size and the element type, sixteen different simulations have been performed. The load-ovalization curves obtained from the simulations are presented for all the element types and density sizes of the meshes. These will be compared to the literature research by overlapping the graphs, afterwards the results will be discussed and evaluated. The graphs from the literature are made of black lines and they follow these criteria:

\footnotetext{
- Experimental Investigations, Compression Load, EX-odd number

-a Numerical Simulations, Compression Load, EX-odd number

¿ Experimental Investigations, Tension Load, EX-even number
} 


\section{— Numerical Simulations, Tension Load, EX-even number}

When the results of this work will be presented no specification will be made in between the compression and tension specimens, since each specimen has the same trend to the respective reference case, i.e., the compression case of this research will overlap the reference cases, and so for the tension cases.

The graphs have as $\mathrm{X}$-axis the ovalization that refers to the deformation of the chord cross-section at the center of the T-joint, which is calculated by subtracting a reference point at the chord bottom from average of the points in the brace; whereas the Y-axis, instead, represents the load applied at the brace tip, obtained by the opposite of the reaction force at the same point. Each of the following graphs will be shown with the curves of all the four types of density mesh that have been analyzed.

\subsection{Curves With Automatic Step Sequence}

In this section, the curves are represented in terms of the automatic step sequence. So far, a single step size is applied to the model through the constant step range, and all the four types of elements will be considered (S4R, S4, S8R, and S8R5). For what concerns the figures that will be described, these will represent respectively the Mises stress and PEEQ (Equivalent Plastic Strains) [51] of the specimens.

\subsubsection{S4R Element Type}

The graphs reported in Figure 5 represent the simulations done for meshes using the S4R element type (that are those given by default from the program), with an automatic step range. It is remarked that the lower curve for each graph refers to the compression specimens (i.e., EX-01 to EX-11 with odd numbers), whereas the higher one represents the tension specimens (i.e., EX-02 to EX-12 with even numbers).

Generally, for all the graphs it is easy to see a good fitting between the reference curves and the present work, especially for the unreinforced cases and for the coarse mesh of collar plate EX-03 and EX-04. The coarse mesh of EX-01, EX-02, EX-03, and EX-04 are depicted in Figure 6a-d.

Unreinforced cases show an inferior maximum stress but are distributed in almost all the chord, whereas the case of collar reinforced plate is characterized by higher maximum value but concentrated in the reinforcement. It is obvious that unreinforced cases have higher values in terms of plastic deformations with respect to the reinforced ones.

The above good fitting is not relatively the case of EX-06, where the present curves are slightly below the reference curves, the marked difference is also influenced by the fact that this is the thicker case, which causes higher values. However, this difference is repeated for all the element types with improvements in S8R, so further discussions are left when the results for these elements will be discussed.

From the curves can be seen two irregular trends, one is the temporary variation in the force values such as for the EX-10, EX-12 with the coarse mesh and slightly in EX-07 and EX-08 with the article mesh; and the second trend is the change in direction of the ovalization (where can be seen a turning back of the curves) such as for fine mesh of EX-01, EX-02, EX-04, EX-06, and EX-08. The first trend is not a model problem but is a consequence of the plasticity of the elements, so it is more related to the FE implementation than the actual modeling, in any case this happens after the maximum ovalization of the reference papers is reached. In addition, this behavior does not affect the specimens in compression. Contrastingly, the second trend is due to the fact that a small part of a corner of a quarter's brace rises in an unexpected way, as shown in Figure $7 \mathrm{~b}$ for the fine mesh of the specimen EX-08. It is remarked that this behavior occurs just in the fine mesh, and this might be caused by the fragility of the mesh type (in fact, that does not occur in the S8R, which is the stronger). 


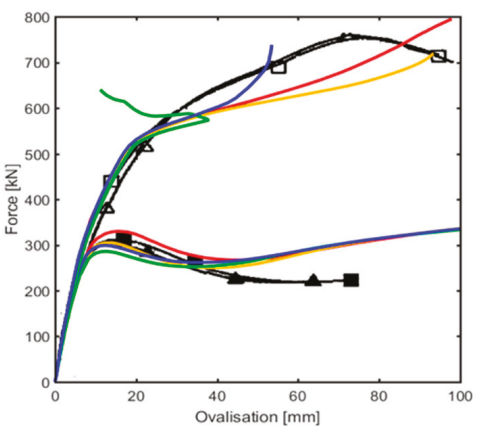

(a)

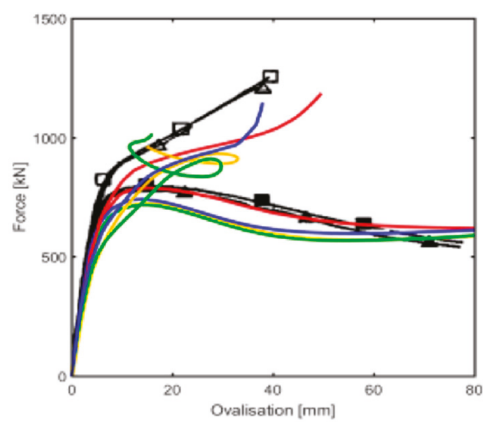

(c)

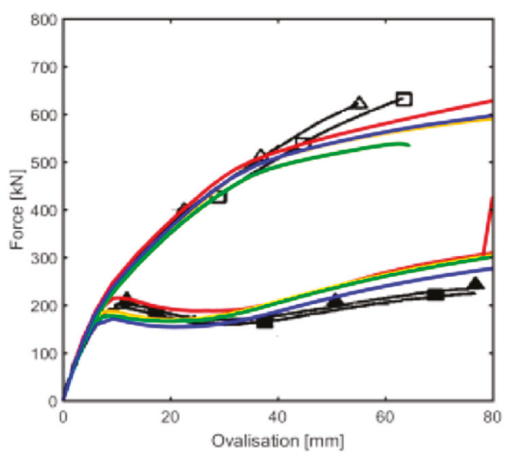

(e)

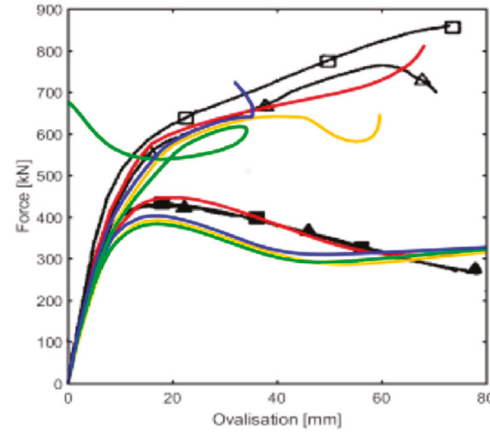

(b)

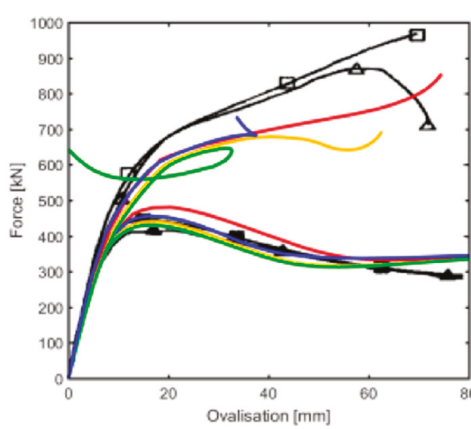

(d)

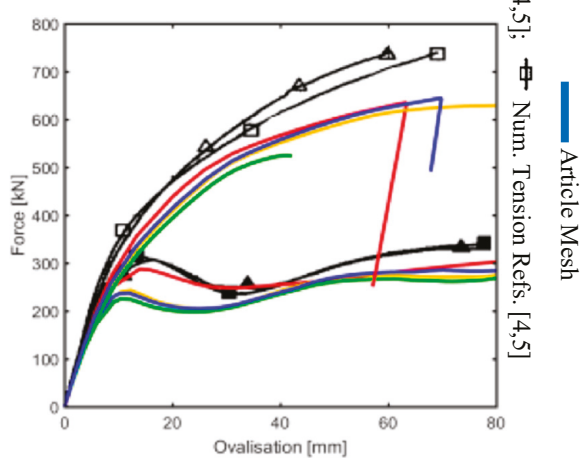

(f)

Figure 5. Load-Ovalization curves with S4R element type: (a) Unreinforced Ex-01-02, (b) Collar Plate Ex-03-04, (c) Collar Plate Ex-05-06, (d) Doubler Plate Ex-07-08, (e) Unreinforced Ex-09-10, and (f) Doubler Plate Ex-11-12. 


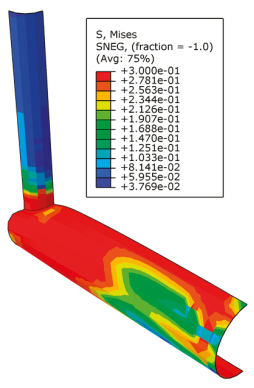

(a)

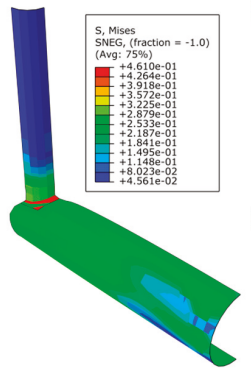

(c)
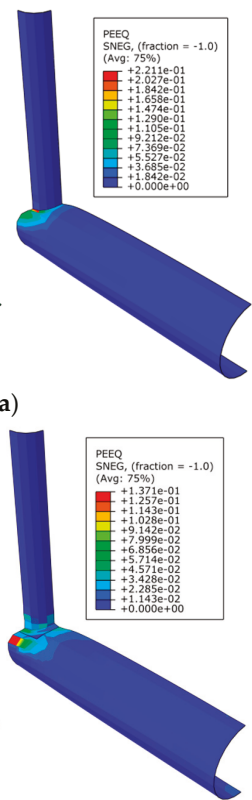
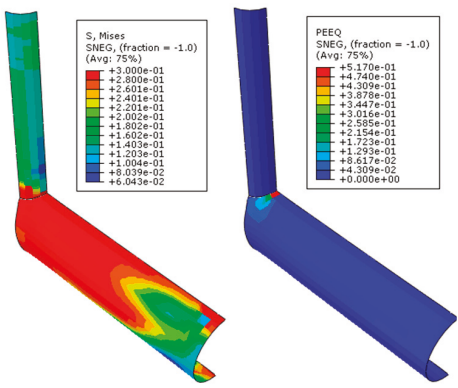

(b)

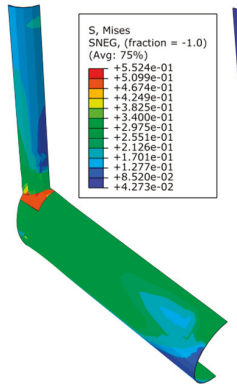

(d)

Figure 6. Mises stress and PEEQ representations: (a) Unreinforced Ex-01, (b) Unreinforced Ex-02, (c) Collar Plate Ex-03, and (d) Collar Plate Ex-04.

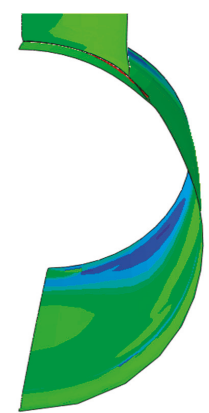

(a)

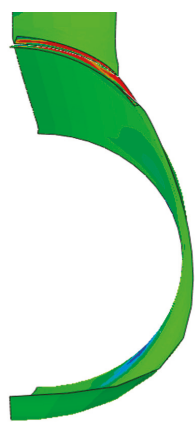

(b)

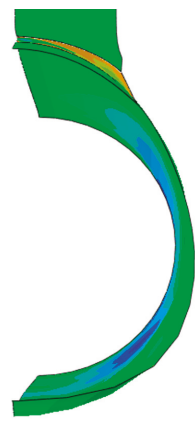

(c)

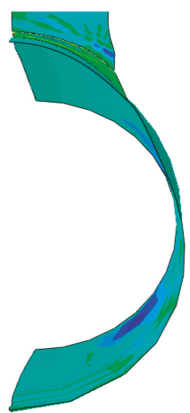

(d)

Figure 7. Ovalization change in fine mesh of Ex-08: (a) before the corner rise, (b) corner rise with S4R, (c) corner rise with S4, and (d) no corner rise S8R.

\subsubsection{S4 Element Type}

The major difference between the S4 element type and the previous, the S4R, is that in S4 elements no reduced integration is excepted. This similarity between the S4 and S4R is directly reflected by the results of the load-ovalization curves depicted in Figure 8, from which can be seen there are no big changes from the load-ovalization curves in Figure 5.

Relevant differences can be observed for fine meshes. In the tension cases EX-04 and EX-08 shown in Figure 8b,d, the "hook" is no longer present at the tail of each curve. Moreover, another improvement can be seen in EX-06 in Figure 8c where the reverse curve trend in terms of ovalization is 
avoided for the medium mesh. While this remains in the fine mesh, such behavior as aforementioned is due to the rise of a part of the chord and this effect is depicted in Figure 7c.

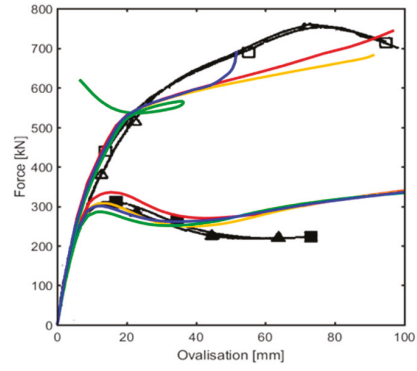

(a)

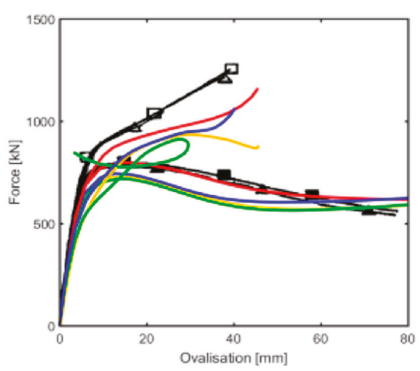

(c)

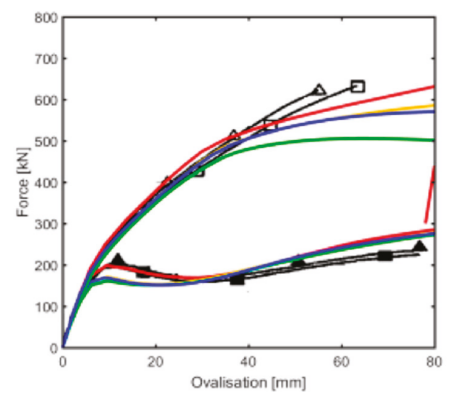

(e)

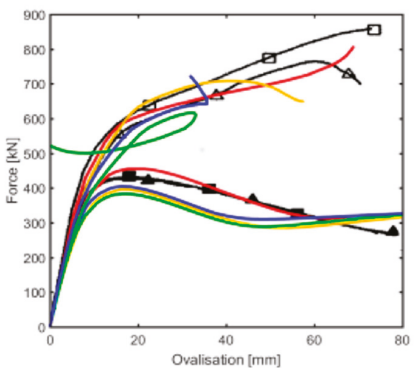

(b)

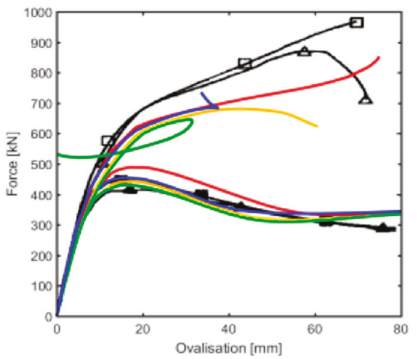

(d)

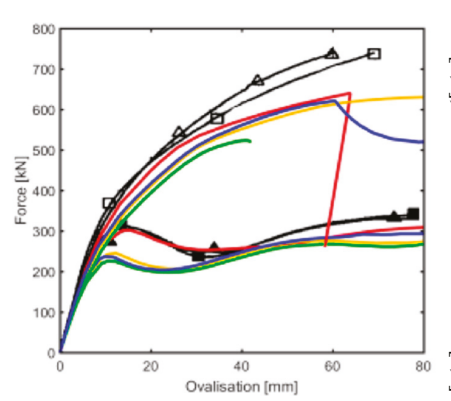

(f)

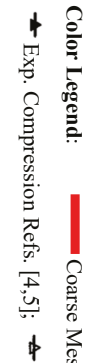

中

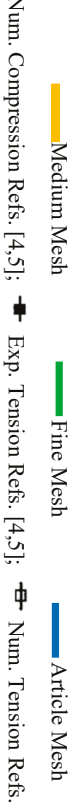

Figure 8. Load-Ovalization curves with S4 element type: (a) Unreinforced Ex-01-02, (b) Collar Plate Ex-03-04, (c) Collar Plate Ex-05-06, (d) Doubler Plate Ex-07-08, (e) Unreinforced Ex-09-10, and (f) Doubler Plate Ex-11-12.

\subsubsection{S8R Element Type}

In Figure 9 the S8R element is considered. With this type of element, the problem of the drop-in force (due to model exceeding plasticization at the end of the process) of the two previous cases is avoided. But several simulations end before the maximum ovalization (around $80 \mathrm{~mm}$ in the references) is reached, especially in the unreinforced case such as the article and coarse mesh of the specimen EX-01, the article mesh of specimen EX-03, and medium and fine mesh of the specimen EX-10. 
In the case of the EX-06, that is the one that defers more from the references, but with the S8R element type the differences are reduced. The article mesh solution in Figure 9c has still a different shape with respect to the references but the curves coincide at their ends.

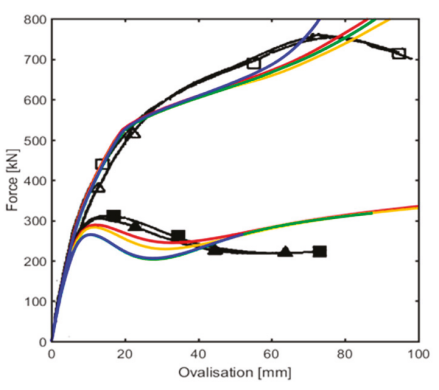

(a)

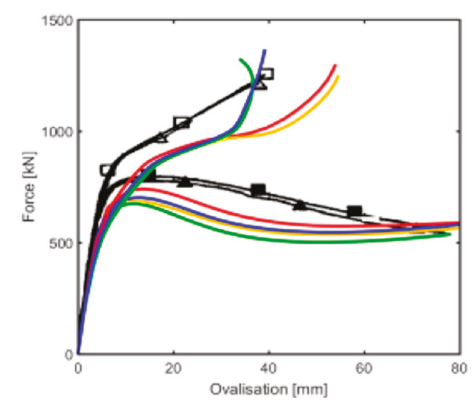

(c)

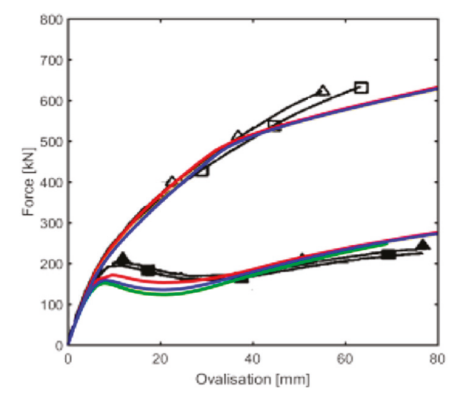

(e)

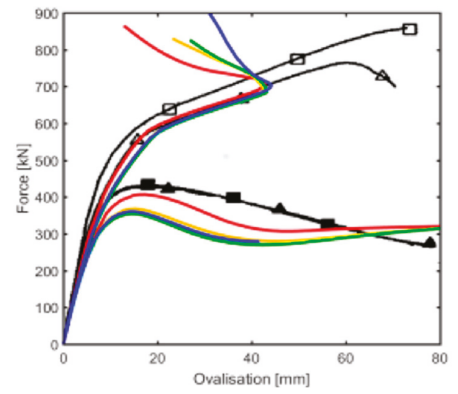

(b)

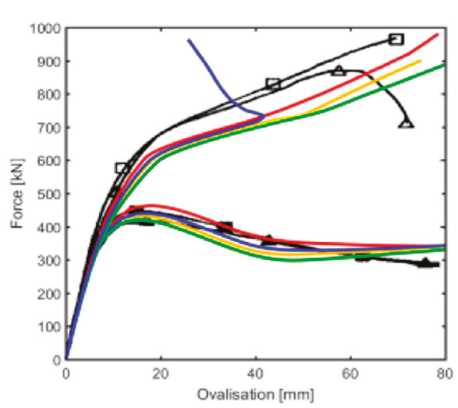

(d)

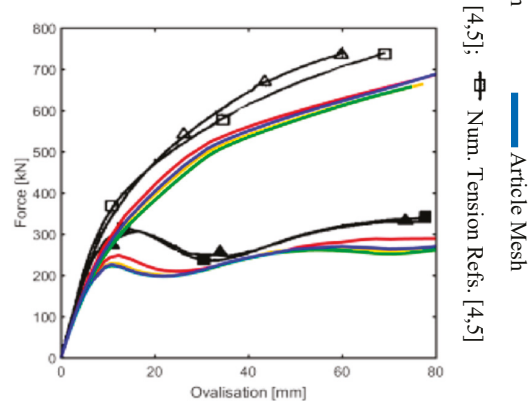

(f)

Figure 9. Load-Ovalization curves with S8R element type: (a) Unreinforced Ex-01-02, (b) Collar Plate Ex-03-04, (c) Collar Plate Ex-05-06, (d) Doubler Plate Ex-07-08, (e) Unreinforced Ex-09-10, and (f) Doubler Plate Ex-11-12.

As results in the EX-06 and EX-07 the smaller stress and plasticization can be seen, this is due to the increase in thickness of all the elements, which almost doubled with respect to the other cases. In addition, to this first improvement, for the specimen EX-08 using the S8R element, in the fine mesh the change in direction of the ovalization is also avoided. See Figure $7 \mathrm{~d}$ where at the end of the simulation no rise of the corner part is present. 


\subsubsection{S8R5 Element Type}

The last element type considered in this research is the S8R5 element type. The S8R5 has just five DOF, this means that there is a simplification respect to the S8R, and this simplification results in the inability to submit all the simulations. The load-ovalization curves of S8R element type depicted in Figure 9 are very close in shape to those of S8R5 reported in Figure 10, but an important issue is solved definitely and no reverse in ovalization is present for all the cases. To notice that was not possible to complete all the simulations because of the complexity of the S8R5 elements type, which does not always allow finding the convergence.

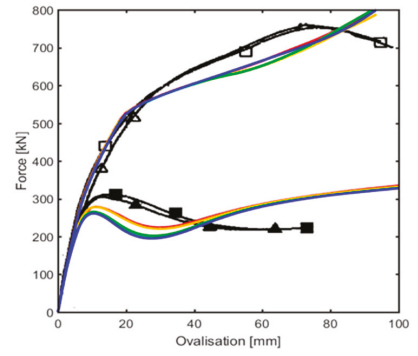

(a)

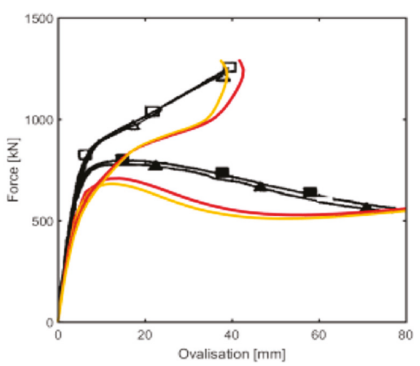

(c)

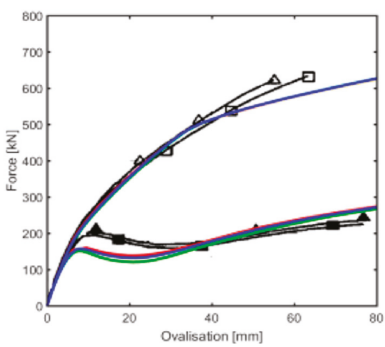

(e)

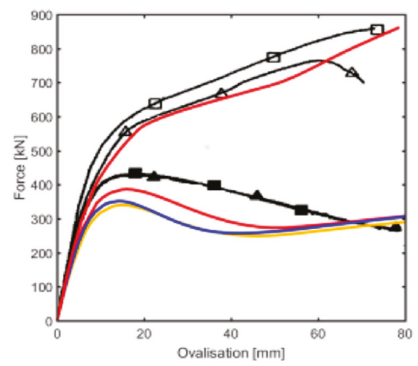

(b)

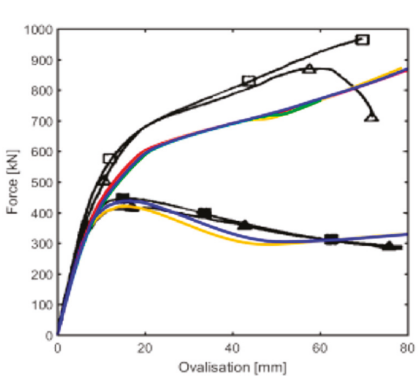

(d)

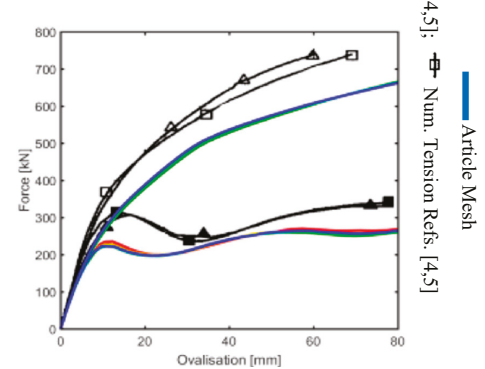

(f)

Figure 10. Load-Ovalization curves with S8R5 element type: (a) Unreinforced Ex-01-02, (b) Collar Plate Ex-03-04, (c) Collar Plate Ex-05-06, (d) Doubler Plate Ex-07-08, (e) Unreinforced Ex-09-10, and (f) Doubler Plate Ex-11-1.

\subsection{Curves With Fixed Step Sequence}

The plasticization of the T-joint is a key point for the fixed step sequence, since the starting point of the plasticization defines the application of the second step range. From the results of the simulations of EX-01 and EX-02 of the automatic step sequence, the starting period of the plastic behavior of the 
material of the T-joint is identified. The set of all these values for the two specimens EX-01 and EX-02 are reported in Table 5, which shows that the plasticization starts at the very early stage of the simulations.

So fixed step sequence introduces one more step respect to the automatic one, as in the experimental investigation. For this reason, the simulations have been done for the first two specimens EX-01 and EX-02 as reported for all the four-element type in Figure 11, and once it emerged that no relevant changes in the results are present, it was understood that for this type of model the range application of the load/displacement makes no differences.

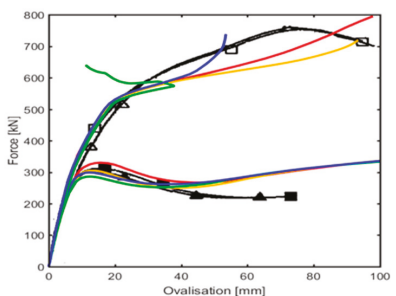

(a)

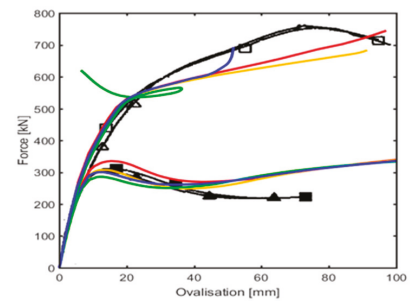

(c)

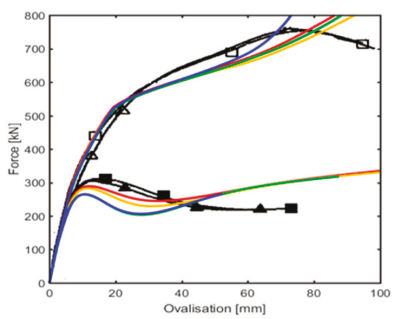

(e)

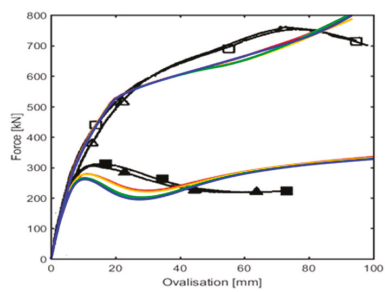

(g)

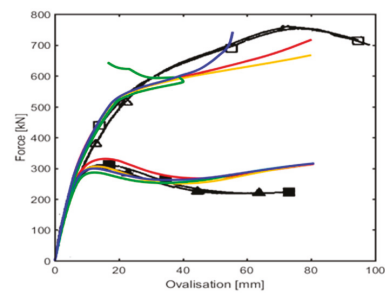

(b)

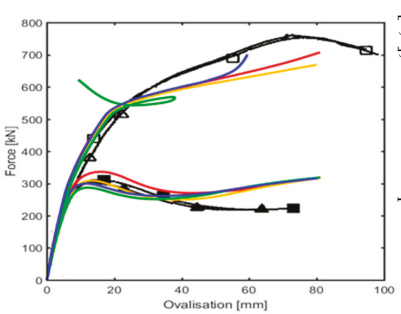

(d)

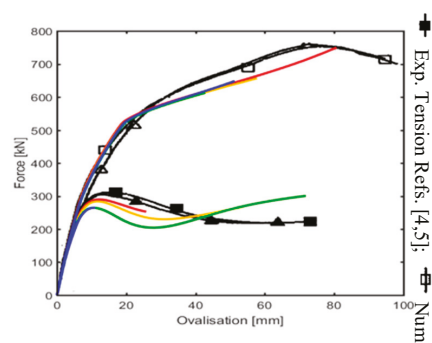

(f)

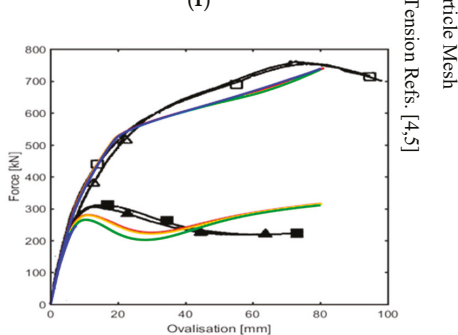

(h)

Figure 11. Load-Ovalization curves unreinforced Ex-01 and Ex-02: (a) automatic step S4R, (b) fixed step S4R, (c) automatic step S4, (d) fixed step S4, (e) automatic step S8R, (f) fixed step S8R, (g) automatic step S8R5, and (h) fixed step S8R5. 
Table 5. Starting point of the plasticization for Ex-01 and Ex-02 [17].

\begin{tabular}{ccc}
\hline Element Type Mesh EX-01 and Ex-02 & Density Mesh & Starting Point of Plastic Behavior \\
\hline \multirow{2}{*}{ S4R } & Coarse & 0.009 \\
& Medium & 0.009 \\
& Fine & 0.009 \\
& Article & 0.009 \\
\hline \multirow{2}{*}{ S4 } & Coarse & 0.009 \\
& Medium & 0.009 \\
& Fine & 0.009 \\
& Article & 0.009 \\
\hline \multirow{2}{*}{ S8R } & Coarse & 0.009 \\
& Medium & 0.006 \\
& Fine & 0.006 \\
& Article & 0.006 \\
\hline \multirow{2}{*}{ S8R5 } & Coarse & 0.009 \\
& Medium & 0.006 \\
& Fine & 0.006 \\
\end{tabular}

\subsection{Deformed Shapes}

Other comparisons between the present research and the references [4,5] are discussed in this section. Through considering sections or rings of the tubular elements, from which it is possible to observe the ovalization and associated yield hinges, as well as the separation between the chord and doubler/collar plate.

In the experimental investigation and the numerical simulation $[4,5]$, after the tests were completed, for some of the specimens, sections or "rings" were obtained from the chord and brace. The results have been presented firstly for the specimens with diameter ratio (where $\beta=d_{1} / d_{0}$ ), i.e., from the EX-01 to EX-08, and then for the specimens with $\beta=0.28$, i.e., from the EX-09 to EX-12. Alternatively, in the numerical simulation of var der Vegte et al. [5] the deformed rings, which have been showed, are just some that have the diameter ratio $\beta=0.54$. Therefore, some gaps can be seen in the representation of the rings for what concern to the reference [4,5].

In the following the ovalization shapes will be shown, compared with the papers' results, where these are present. The same experimental subdivision will be considered. The research simulations exceed the length of this paper's simulation in most cases, whereby in the section will be taken from the same ovalization as literature cases. In addition, in this paper for further investigations of the shapes will be selected the ones that have the best fit in the load-ovalization curves.

\subsubsection{Failure of the Specimens with $\beta=0.54$ under Compression}

Specimens under compression in the brace element with diameter ratio equal to 0.54 are EX-01, EX-03, EX-05, and EX-07. These are all represented in the experimental investigation, whereas in the numerical simulation reference to EX-05 is missing.

\section{Specimen EX-01}

Figure 12a,b shows the overall view of the unreinforced specimens of the Experimental Case (EC) (reference figures from $[4,5]$ ) and the Research Case (RC) (the present simulations). The present contour plots represent the MISES stresses. 


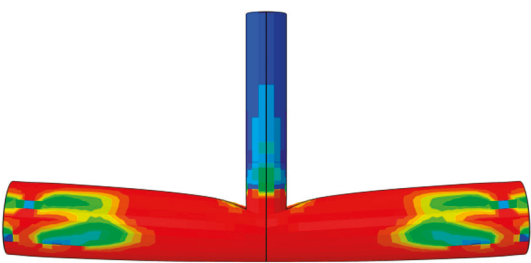

(a) Present

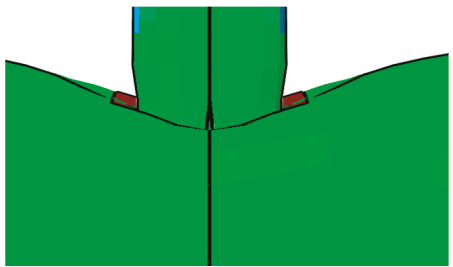

(c) Present

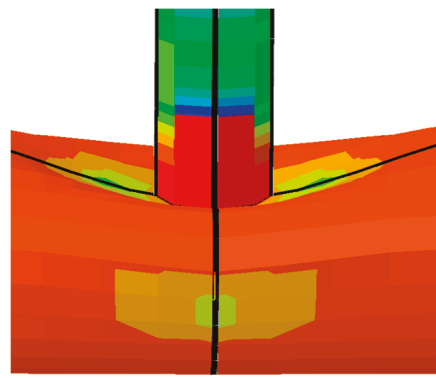

(e) Present

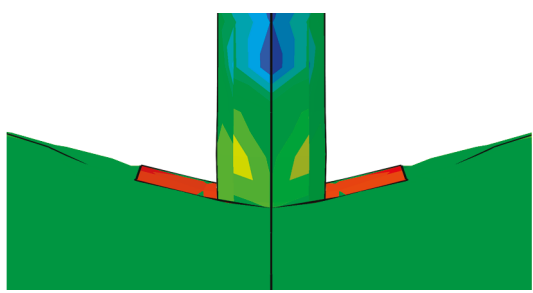

(g) Present

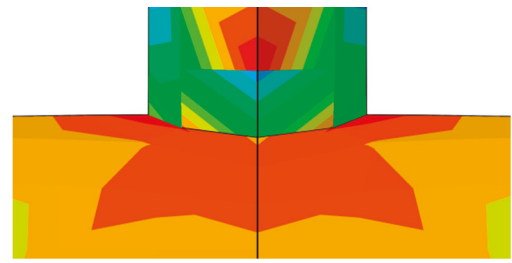

(i) Present

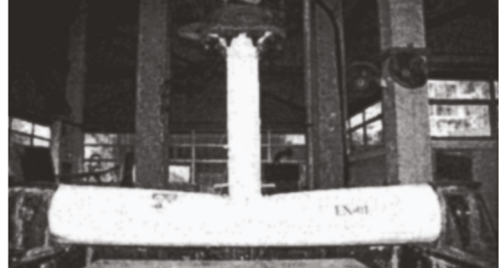

(b) Refs. [4,5]

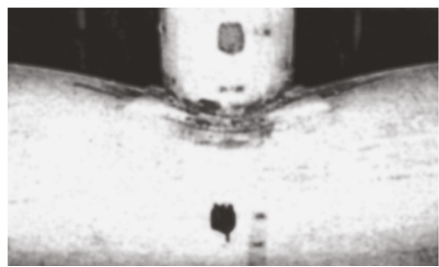

(d) Refs. $[4,5]$

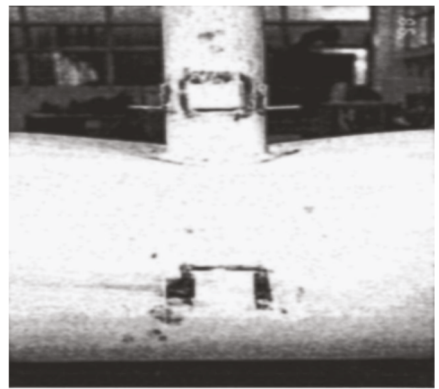

(f) Refs. $[4,5]$

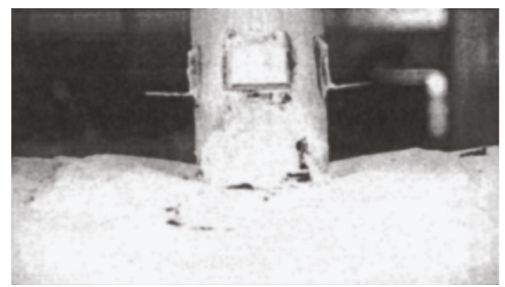

(h) Refs. [4,5]

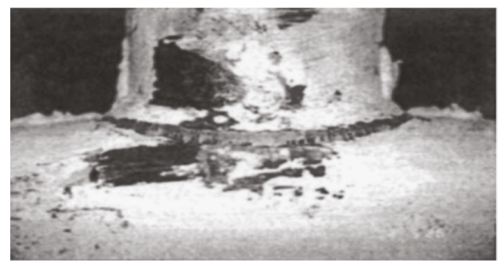

(j) Refs. [4,5]

Figure 12. Overall and detail views: (a) Overall view Ex-01 RC, (b) Overall view Ex-01 EC, (c) Detail view Ex-05 RC, (d) Detail view Ex-05 EC, (e) Detail view Ex-09 RC, (f) Detail view Ex-09 EC, (g) Detail view Ex-11 RC, (h) Detail view Ex-11 EC, (i) Detail view Ex-10 RC, and (j) Detail view Ex-10 EC. (With permission from ASCE) 
Figure 13a-c shows the sliced ring (chord and short portion of brace) from specimen EX-01, and here, different from the overall view, the case of Numerical Case (NC) (FE simulation from the references $[4,5]$ ) is also present. From the paper's results can be seen the two yield hinges adjacent to brace-chord intersection become clear, while in the research case, however, this cannot be seen and the shape does not follow the same trend. As aforementioned the step taken is at ovalization $100 \mathrm{~mm}$, which corresponds to the period 0.672 of 1 Abaqus simulation time period.

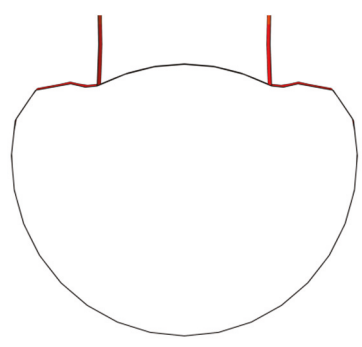

(a) Present

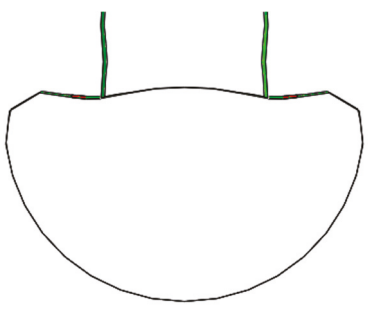

(d) Present

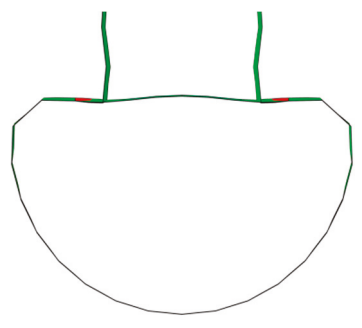

(g) Present

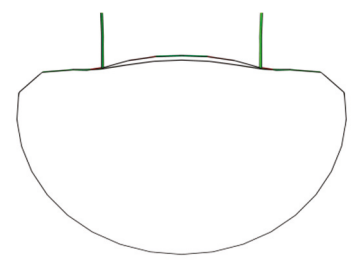

(i) Present

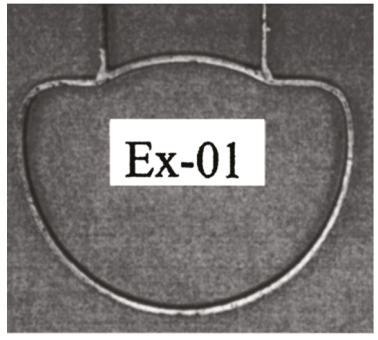

(b) Refs. [4,5]

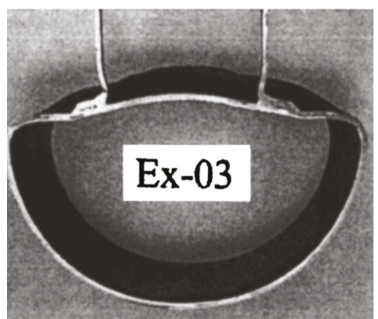

(e) Refs. $[4,5]$

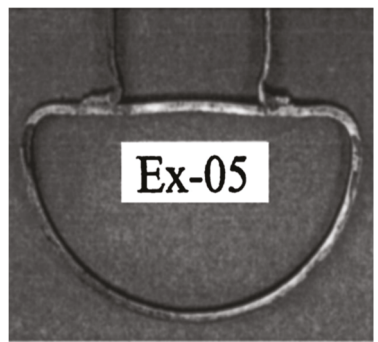

(h) Refs. [4,5]

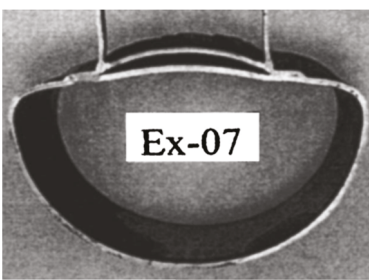

(j) Refs. [4,5]

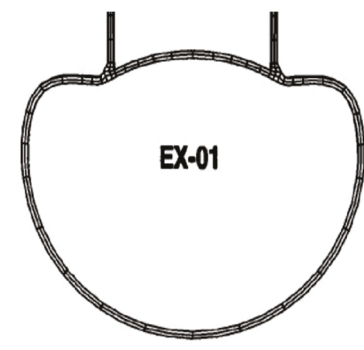

(c) Refs. $[4,5]$

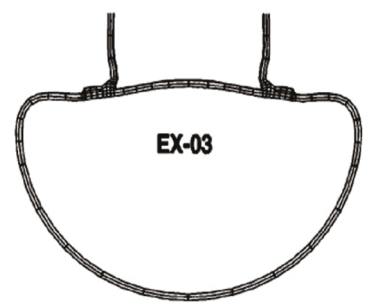

(f) Refs. $[4,5]$

Figure 13. Cont. 


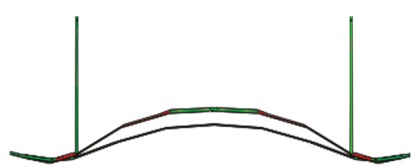

(k) Present

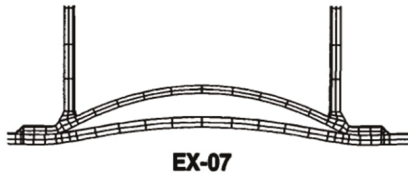

(1) Refs. $[4,5]$

Figure 13. Sliced rings of specimens subjected to compression with $\beta=0.54$ : (a) Ex-01 RC, (b) Ex-01 EC, (c) Ex-01 NC, (d) Ex-03 RC, (e) Ex-03 EC, (f) Ex-03 NC, (g) Ex-05 RC, (h) Ex-05 EC, (i) Ex-07 RC, (j) Ex-07EC, (k) particular Ex-07 RC, and (1) particular Ex-07 EC. (With permission from ASCE)

\section{Specimen EX-03}

For the specimen EX-03 the papers presented only the sliced result. Figure $13 \mathrm{~d}-\mathrm{f}$ shows the sliced ring of specimen EX-03. Between this specimen and the previous one it can be immediately noticed the significant strength enhancement offered by the collar reinforcement to the chord section for EX-03. The step taken here is at ovalization $80 \mathrm{~mm}$, which corresponds to the period 0.476 of 1 Abaqus simulation time period.

\section{Specimen EX-05}

Figure 12c,d shows the detail of the chord indentation side view of the reinforced specimen EX-05; also, here the work case is showed in form of MISES stress, where plastic deformation is near the intersection chord-brace, whereas the chord bottom part remained relatively straight with no deformation and no stress concentration. Figure 13g,h shows the sliced ring from specimen EX-05, here the NC was not available in the results. The bending in this case is more severe than that of specimen EX-03, some results of the relatively higher strength of the chord, as can be seen in load-ovalization curves. Ovalization $80 \mathrm{~mm}$ corresponds to the period 0.614 of 1 Abaqus simulation time period.

\section{Specimen EX-07}

As far as EX-07 is concerned, the papers presented only the sliced result, as depicted in Figure 13i,j. The separation from the chord outer surface of the doubler plate (which at the original shape fits the chord perfectly) can be observed. As can be seen in Figure 13k,l, RC also has this effect but with no such emphasis as in the papers' cases. This behavior is due to the fact while the brace compresses the chord, the wall of the latter has a different rotation with respect to the reinforcement; in the FE model the modeling of the contact and ties have played a fundamental role. In this the ovalization $80 \mathrm{~mm}$ corresponds to the period 0.531 of 1 Abaqus simulation time period.

\subsubsection{Failure of the Specimens with $\beta=0.54$ under Tension}

In the following, the specimens under tension in the brace element with diameter ratio equal to 0.54 will be considered. These specimens are EX-02, EX-04, EX-06, and EX-08. Here the experimental investigation results are given for EX-04, EX-06, and EX-08, while in the numerical simulation just the EX-08 is given.

During the experimental test, some specimens buckle such as EX-02 and EX-04; while for the numerical simulations the buckling was not taken into account. Also, in the present research, the buckling is not covered, but is left as future development.

Specimens with Buckling EX-02, EX-04, and EX-08

Even if in this research the buckling is not taken into account, due to the initial purpose which is that of building a reliable model made of shell elements, a good match has been found in the load-ovalization curves as can be seen also here in the deformed shapes of the specimen where the 
buckling occurred in the physical experiment [4]. However, the buckling was also not considered in the previous numerical simulation [5], and the results in terms of deformed shape from the numerical simulation were not provided.

EX-02, EX-04, and 08 are the three cases where the specimens buckle. The doubler plate specimen EX-08 subjected to brace tension has a similar pattern to the EX-04, with pronounced chord plasticization. In Figure 14a-c the separation of the doubler plate from the chord at the brace-doubler intersection is illustrated. This separation can be seen both in the reinforced and unreinforced case, also without considering the buckling effect.

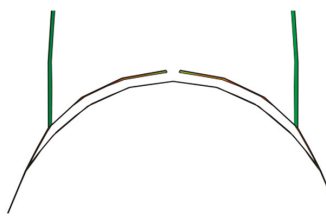

(a) Present

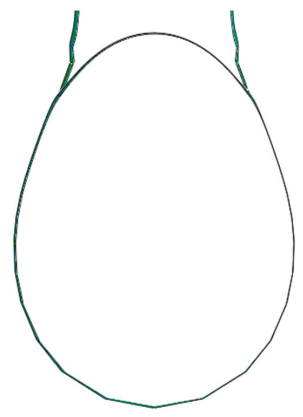

(d) Present

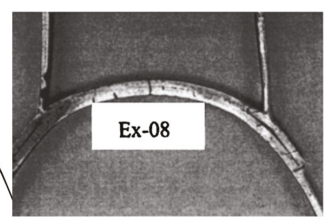

(b) Refs. $[4,5]$

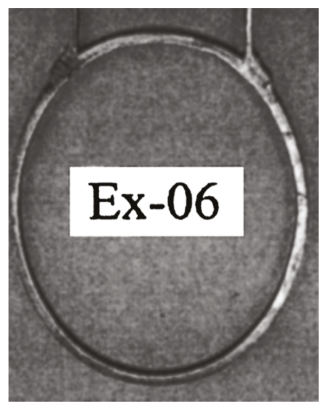

(e) Refs. [4,5]

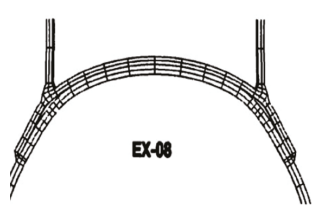

(c) Refs. [4,5]

Figure 14. Sliced rings of specimens subjected to tension with $\beta=0.54$ : (a) Ex-08 RC, (b) Ex-08 EC, (c) Ex-08 NC, (d) Ex-06 RC, and (e) Ex-06 EC. (With permission from ASCE)

\section{Specimen EX-06}

EX-06 is the thicker specimen with collar reinforcement subjected to brace tension. Its deformed shape is shown in Figure 14d,e. For this specimen the experimental test was terminated after the tensile capacity of the actuator was reached at $1200 \mathrm{kN}$, in RC the simulation also stops earlier, and at the end of time period 1 the ovalization is equal to $38 \mathrm{~mm}$ instead of $80 \mathrm{~mm}$ in the references [4,5].

\subsubsection{Failure of the Specimens with $\beta=0.28$ under Compression}

In the following specimens under compression with $\beta=0.28$ are shown, and this is the case of EX-09 and EX-11. For both cases the NC does not show any result, so they will be compared only for the EC with the present research.

\section{Specimen EX-09}

Figure 12e,f shows the localized indentation of the chord of unreinforced specimen EX-09 subjected to compression, which has a similar behavior of the respective specimen with higher diameter ratio EX-01. For the sake of comparison, Figure 15a,b presents its sliced ring. The step taken here is at ovalization $80 \mathrm{~mm}$, which corresponds to the period 0.450 of 1 Abaqus simulation time period. 


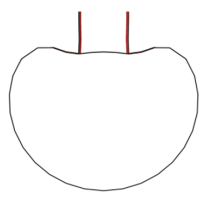

(a) Present

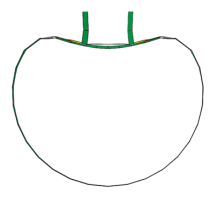

(c) Present

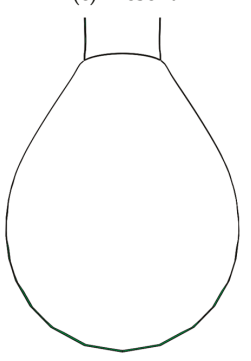

(e) Present

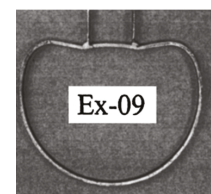

(b) Refs. $[4,5]$

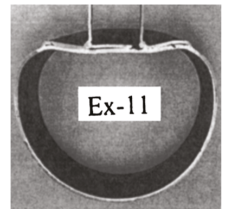

(d) Refs. $[4,5]$

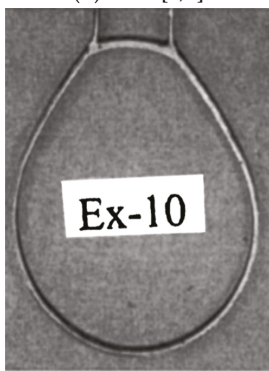

(f) Refs. [4,5]

Figure 15. Sliced rings of specimens subjected to compression/tension with $\beta=0.28$ : (a) Ex-09 RC, (b) Ex-09 EC, (c) Ex-11 RC and (d) Ex-11 EC, (e) Ex-10 RC, and (f) Ex-10 EC. (With permission from ASCE)

\section{Specimen EX-11}

Figure 12g,h shows the localized indentation of the chord of the reinforced specimen EX-11 subjected to compression; besides the appreciable indentation at the intersection area, the doubler plate is observed to bulge out of its plane. Contrastingly, Figure $15 \mathrm{c}, \mathrm{d}$ presents its sliced ring. The step taken here is at ovalization $80 \mathrm{~mm}$, which corresponds to the period 0.537 of 1 Abaqus simulation time period.

\subsubsection{Failure of the Specimens with $\beta=0.28$ under Tension}

In the following the last two specimens examined are EX-10 and EX-12, with small ratio but subjected to tension. Only EX-10 will be showed here, since EX-12 in the experimental investigation was stopped due to brittle failure that occurred through punching shear fracture of the brace out of the doubler plate.

\section{Specimen EX-10}

Figure 12i,j shows a detailed view of the brace-chord intersection of the unreinforced specimen EX-10 subjected to brace tension; also, after severe plasticization, the joint exhibited brittle shear failure of the chord wall at one side of the intersection. In the EC the focus is at the weld zone that is not present in the RC. Alternatively, Figure 15e,f presents its sliced rings, which shows very similar shapes. The time step taken here is at ovalization $80 \mathrm{~mm}$, for a period 0.443 of 1 Abaqus simulation time period. 


\section{Summary and Conclusions}

The FE analyses with the use of shell elements in this research have been directed to simulate the static behavior of a set of 12 unreinforced and reinforced test cases, on uniplanar T-joints under axial brace load carried out by Choo et al. [4]. In addition to the investigation conducted by Choo et al. [4], the numerical simulation of var der Vegte et al. [5] has been taken into account. Even though a FE study is already present, there the authors have decided to use the more computationally expensive 3-D model made by solid elements. The present research tries to solve the issues faced in [5] and sheds light on the importance of the mechanical data in FE modeling. Some peculiar data that are missing in the reference papers were discussed.

The results are presented in terms of restraining force versus ovalization of the chord under the applied force and also in terms of deformed shapes, by considering sections or rings of the tubular elements. Based on the results presented in the previous sections, where several parametric settings have been considered, the following conclusions and considerations can be made.

- The difference in terms of load application does not affect the results in relevant way, since the results obtained by the automatic step sequence (i.e., unique stroke range) and those obtained by the fixed step sequence (i.e., two stroke ranges as in the experimental investigation) for the first two specimens are almost the same, except in some cases of the fixed step where it was not possible to find the convergence for all, due to the complexity caused by the double step. Therefore, the automatic step sequence was considered for the remaining samples that besides being simpler also allows an easier convergence.

- Automatic step sequence and the true stress-strain relationship without hardening after the peak load were considered. For these settings, four different mesh densities (coarse, medium, fine, and article) each one analyzed with four-element types (S4R, S4, S8R, and S8R5) were analyzed, respectively. Generally, it was possible to see that all load-ovalization curves fit quite well in the initial (linear) part with the reference investigation, and then go slightly below in the post-peak, and finally to converge almost in final value. In the first group of element types, i.e., S4R and S4, it can be seen that there is an abnormal shape of the curves, with the reverse of the ovalization, when the elements size of the mesh used are relatively small such as in the medium and fine mesh. This is valid when $\beta=0.54$, i.e., the diameter of the brace is more than half of the chord one. While if this ratio is decreased to $\beta=0.28$ this does not occur. In contrast, the second group of element types with a higher number of nodes solves the issue of the change in direction of the ovalization for all the cases, even though a higher number of nodes leads to no convergence for some specimens.

- Due to the fact that the automatic step sequences, with no hardening simulations, have the relevant and complete data, these were compared to the references by cutting rings from the center of the chord of each specimen. The ring of the research shows a perfect matching to the experimental case under compression. In the research study, as in the numerical reference, the buckling that occurs in some of the tension cases of the experimental research was not considered. However, the shapes obtained by this research kept good accordance, even in the specimens where buckling occurred.

- Although the reliability of shell elements is the object of this research, a 3-D model with the same setting used by var der Vegte et al. [5] was built to verify the reliability of the preliminary parameters considered in the model with those of the numerical simulation [5], since assumptions were done in the first stage due to lack in the data in the reference papers. From the results of the 3-D model of this study, as depicted in Figure 16a, the load-ovalization curves in Figure 16b have been obtained where almost similar shapes and characteristics of the main model made by shells with $\mathrm{S} 4$ element type can be seen. Thus, it is possible to deduce that some relevant data are missing in the paper.

- The model considered in this research is able to show comparable strength characteristics as in $[4,5]$. In fact, the T-joints considered in this study show a great improvement in terms of strength of the specimen reinforced by doubler plate or a collar plate as compared to the strength 
of the unreinforced joints. An enhancement of almost $40 \%$ was reached for $\beta=0.54$ as in the experimental case, but a smaller value was found for $\beta=0.28$ where the research value is five per cent smaller than the experimental one of sixteen percent. Therefore, it can be said that the two types of reinforcement have the capacity to distribute the brace axial load to a larger region of the chord, with consequent improvement in terms of strength enhancement.

- Through the use of shell elements instead of the more expensive (in terms of computational cost) solid elements, the contact problem and the introduction of the element thickness have been solved. It turned out that shell elements with 8 nodes and reduced integration are suitable for this type of structures; this type of elements gives a reliable solution already starting from coarse meshes. Whereas the S4R elements type given by default from the software gives less precise results.

- The missing data in the reference papers have inevitably lead to some differences in the results, partially solved by the use of a different true stress-strain relationship. However, due to the good agreement between the research and the experimental results, the former can be developed through parametric studies to extend the above considerations to different types of joints. Moreover, the present research can be considered for studies involving composite materials as reinforcing phase for damaged or weak joints.

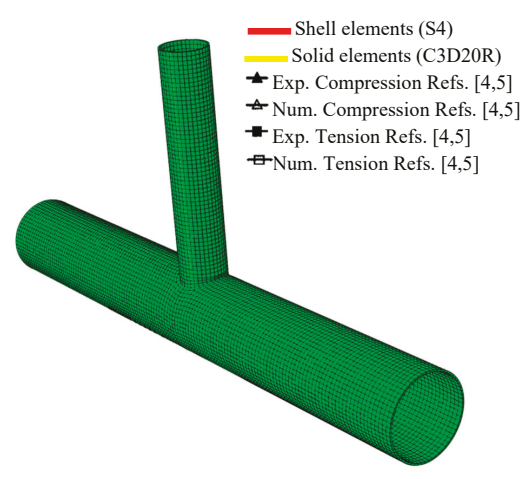

(a)

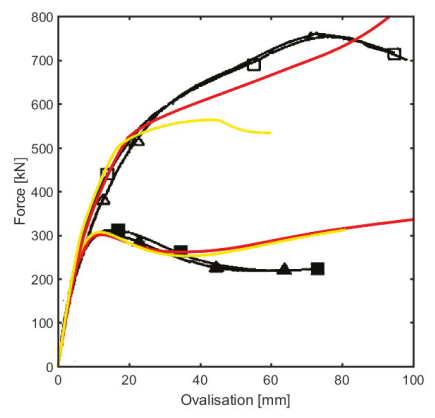

(b)

Figure 16. Three-Dimensional model C3D20R: (a) medium mesh global view and (b) Load-Ovalization curves, shell, and solid elements comparison using for both medium meshes (size 20).

\section{Future Developments}

The authors analyzed, in the first instance, T-joints with shell elements. Since small deviations have been noticed, solid elements models have been generated to test the same joints as in [5]. However, the following conclusions are drawn to a future development of the present paper, which will consider a deep analysis of the mechanical properties used in the experimental testing. As a matter of fact, as a preliminary study the solid models are comparable to the shell ones by considering the same input data.

The differences between the reference studies [4,5] and the present ones are in the "large deformation" part of the curves. The linear elastic branch is so small that it cannot be seen in the plots, but it is very clear that the curves have two segments, and both are nonlinear.

Due to this change, which seemed to be a consequence of the previous assumption done for the material properties, where the true stress-true strain relationship was taken as elastic-perfectly plastic model reported in Figure 2a. Hence, it has been decided to introduce a hardening of $10 \%$ in the true stress-strain relationship for the first two specimens as reported in Figure 2b, differently to what was written in the reference paper [5]. 
The outcome of this setting, as depicted in Figure 17, gives for the tension cases a very close match of the curves for almost $90 \%$ of each simulation, while in compression some differences can still be noted. True strain-stress relationship closer to the real behavior of the steel material (left to future studies) can solve this last deference.

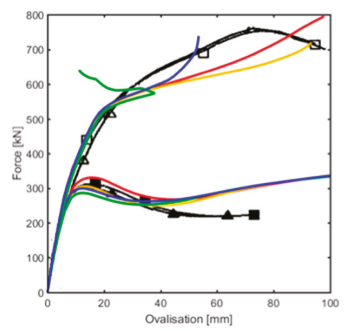

(a)

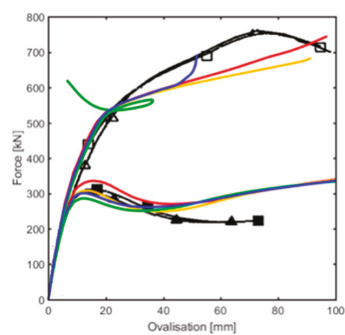

(c)

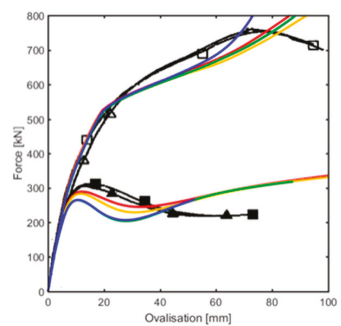

(e)

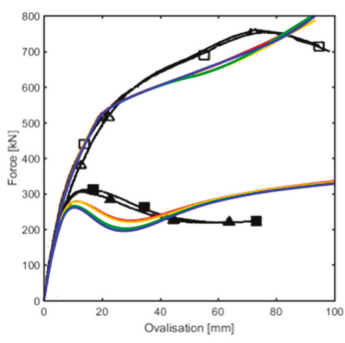

(g)

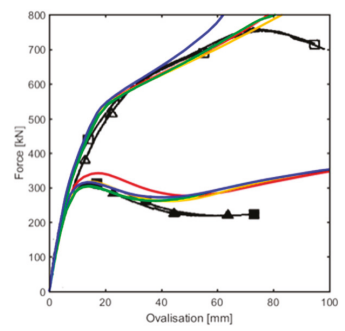

(b)

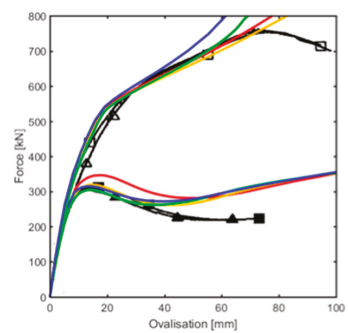

(d)

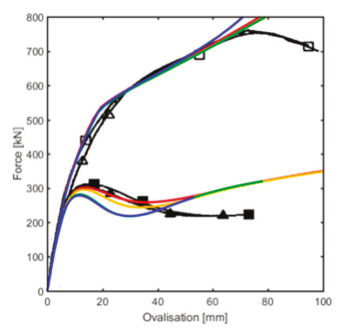

(f)

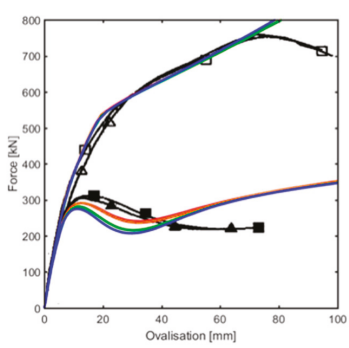

(h)

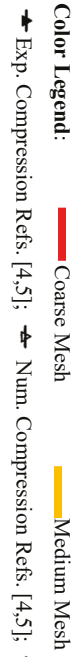

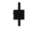

प1

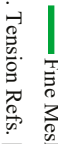

品

中

壳

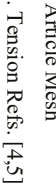

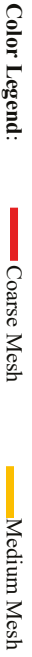

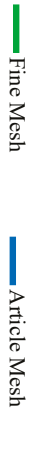

Figure 17. Load-Ovalization curves unreinforced Ex-01 and Ex-02 with $10 \%$ hardening: (a) S4R without hardening, (b) S4R with hardening, (c) S4 without hardening, (d) S4 with hardening, (e) S8R without hardening, (f) S8R with hardening, (g) S8R5 without hardening, and (h) S8R5 with hardening. 
Author Contributions: S.O. and N.F. conceived and designed the experiments; S.O. performed the experiments; S.O. and N.F. analyzed the data; S.O. and N.F. contributed reagents/materials/analysis tools; S.O. and N.F. wrote and review the paper.

Acknowledgments: The authors acknowledge "Fondazione Flaminia" (Ravenna, Italy) for supporting the present research.

Conflicts of Interest: The authors declare no conflict of interest.

\section{References}

1. Moffat, D.G.; Kruzelecki, J.; Blachut, J. The effects of chord length and boundary conditions on the static strength of a tubular T-joint under brace compression loading. Mar. Struct. 1996, 9, 935-947. [CrossRef]

2. Choo, Y.S.; Qian, X.D.; Foo, K.S. Static strength variation of thick-walled CHS X-joints with different included angles and chord stress levels. Mar. Struct. 2004, 17, 311-324. [CrossRef]

3. Lee, M.M.K.; Llewelyn-Parry, A. Strength prediction for ring-stiffened DT-joints in offshore jacket structures. Eng. Struct. 2005, 27, 421-430. [CrossRef]

4. Choo, Y.S.; van der Vegte, G.J.; Zettlemoyer, N.; Li, B.H.; Liew, J.Y.R. Static strength of T-joints reinforced with doubler or collar plates. I: Experimental investigations. J. Struct. Eng. 2005, 131, 119-128. [CrossRef]

5. van der Vegte, G.J.; Choo, Y.S.; Liang, J.X.; Zettlemoyer, N.; Liew, J.Y.R. Static Strength of T-joints reinforced with doubler or collar plates. II: Numerical investigations. J. Struct. Eng. 2005, 131, 129-138. [CrossRef]

6. Yang, J.; Shao, Y.; Chen, C. Static strength of chord reinforced tubular Y-joints under axial loading. Mar. Struct. 2012, 29, 226-245. [CrossRef]

7. Ozyurt, E.; Wang, Y.C.; Tan, K.H. Elevated temperature resistance of welded tubular joints under axial load in the brace member. Eng. Struct. 2014, 59, 574-586. [CrossRef]

8. Chen, Y.; Feng, R.; Wang, C. Tests of steel and composite CHS X-joints with curved chord under axial compression. Eng. Struct. 2015, 99, 423-438. [CrossRef]

9. Lie, S.T.; Li, T.; Shao, Y.B.; Vipin, S.P. Plastic collapse load prediction of cracked circular hollow section gap K-joints under in-plane bending. Mar. Struct. 2016, 50, 20-34. [CrossRef]

10. Qu, H.; Li, A.; Huo, J.; Liu, Y. Dynamic performance of collar plate reinforced tubular T-joint with precompression chord. Eng. Struct. 2017, 141, 555-570. [CrossRef]

11. Zhu, L.; Yang, K.; Bai, Y.; Sun, H.; Wang, M. Capacity of steel CHS X-joints strengthened with external stiffening rings in compression. Thin-Walled Struct. 2017, 115, 110-118. [CrossRef]

12. Đuričić, Đ.; Aleksić, S.; Šćepanović, B.; Lučić, D. Experimental, theoretical and numerical analysis of K-joint made of CHS aluminium profiles. Thin-Walled Struct. 2017, 119, 58-71. [CrossRef]

13. Lan, X.; Huang, Y.; Chan, T.-M.; Young, B. Static strength of stainless steel K- and N-joints at elevated temperatures. Thin-Walled Struct. 2018, 122, 501-509. [CrossRef]

14. Qu, S.; Wu, X.; Sun, Q. Experimental study and theoretical analysis on the ultimate strength of high-strength-steel tubular K-Joints. Thin-Walled Struct. 2018, 123, 244-254. [CrossRef]

15. Li, X.; Zhang, L.; Xue, X.; Wang, X.; Wang, H. Prediction on ultimate strength of tube-gusset KT-joints stiffened by $1 / 4$ ring plates through experimental and numerical study. Thin-Walled Struct. 2018, 123, 409-419. [CrossRef]

16. Feng, R.; Chen, Y.; Wei, J.; He, K.; Fu, L. Behaviour of grouted stainless-steel tubular X-joints with CHS chord under axial compression. Thin-Walled Struct. 2018, 124, 323-342. [CrossRef]

17. Ouakka, S. On the Static Strength of Reinforce Joints. Master's Thesis, University of Bologna, Bologna, Italy, 2017.

18. Lotsberg, I. On stress concentration factors for tubular Y- and T-Joints in frame structures. Mar. Struct. 2011, 24, 60-69. [CrossRef]

19. Osawa, N.; Yamamoto, N.; Fukuoka, T.; Sawamura, J.; Nagai, H.; Maeda, S. Study on the preciseness of hot spot stress of web-stiffened cruciform welded joints derived from shell finite element analyses. Mar. Struct. 2011, 24, 207-238. [CrossRef]

20. Lotfollahi-Yaghin, M.A.; Ahmadi, H. Geometric stress distribution along the weld toe of the outer brace in two-planar tubular DKT-joints: Parametric study and deriving the SCF design equations. Mar. Struct. 2011, 24, 239-260. [CrossRef]

21. Cheng, B.; Qian, Q.; Zhao, X.-L. Stress concentration factors and fatigue behavior of square bird-beak SHS T-joints under out-of-plane bending. Eng. Struct. 2015, 99, 677-684. [CrossRef] 
22. Pradana, M.R.; Qian, X.; Swaddiwudhipong, S. Simplified Effective Notch Stress calculation for non-overlapping circular hollow section K-Joints. Mar. Struct. 2017, 55, 1-16. [CrossRef]

23. Cheng, B.; Li, C.; Lou, Y.; Zhao, X.-L. SCF of bird-beak SHS X-joints under asymmetrical brace axial forces. Thin-Walled Struct. 2018, 123, 57-69. [CrossRef]

24. Xia, J.; Chang, H.; Goldsworthy, H.; Bu, Y.; Lu, Y. Axial hysteretic behavior of doubler-plate reinforced square hollow section tubular T-joints. Mar. Struct. 2017, 55, 162-181. [CrossRef]

25. Gao, F.; Guo, X.-Z.; Long, X.-H.; Guan, X.-Q.; Zhu, H.-P. Hysteretic behaviour of SHS brace-H-shaped chord T-joints with transverse stiffeners. Thin-Walled Struct. 2018, 122, 387-402. [CrossRef]

26. Ahmadi, H.; Nejad, A.Z. Geometrical effects on the local joint flexibility of two-planar tubular DK-joints in jacket substructure of offshore wind turbines under OPB loading. Thin-Walled Struct. 2017, 114, 122-133. [CrossRef]

27. Tong, L.W.; Xu, G.W.; Yang, D.L.; Mashiri, F.R.; Zhao, X.L. Fatigue behavior and design of welded tubular T-joints with CHS brace and concrete-filled chord. Thin-Walled Struct. 2017, 120, 180-190. [CrossRef]

28. Dong, W.; Moan, T.; Gao, Z. Long-term fatigue analysis of multi-planar tubular joints for jacket-type offshore wind turbine in time domain. Eng. Struct. 2011, 33, 2002-2014. [CrossRef]

29. Jiang, W.Q.; Wang, Z.Q.; McClure, G.; Wang, G.L.; Geng, J.D. Accurate modeling of joint effects in lattice transmission towers. Eng. Struct. 2011, 33, 1817-1827. [CrossRef]

30. Cheng, S.; Becque, J. A design methodology for side wall failure of RHS truss X-joints accounting for compressive chord pre-load. Eng. Struct. 2016, 126, 689-702. [CrossRef]

31. Ahmed, A.; Qian, X. A toughness-based deformation limit for fatigue-cracked X-joints under in-plane bending. Mar. Struct. 2015, 42, 33-52. [CrossRef]

32. Liu, B.C. Experimental analysis of tubular joints using white light speckle method. Mar. Struct. 1995, 8, 81-91. [CrossRef]

33. Pey, L.P.; Soh, A.K.; Soh, C.K. Partial implementation of compatibility conditions in modeling tubular joints using brick and shell elements. Finite Elem. Anal. Des. 1995, 20, 127-138. [CrossRef]

34. Mackerle, J. Fastening and joining: Finite element and boundary element analyses-A bibliography (1992-1994). Finite Elem. Anal. Des. 1995, 20, 205-215. [CrossRef]

35. Mackerle, J. Fatigue analysis with finite and boundary element methods a bibliography (1993-1995). Finite Elem. Anal. Des. 1997, 24, 187-196. [CrossRef]

36. Wang, T.; Hopperstad, O.S.; Lademo, O.-G.; Larsen, P.K. Finite element analysis of welded beam-to-column joints in aluminium alloy EN AW 6082 T6. Finite Elem. Anal. Des. 2007, 44, 1-16. [CrossRef]

37. Lee, C.K.; Chiew, S.P.; Lie, S.T.; Nguyen, T.B.N. Adaptive mesh generation procedures for thin-walled tubular structures. Finite Elem. Anal. Des. 2010, 46, 114-131. [CrossRef]

38. Nicol, D.A.C. Creep behaviour of butt-welded joints. Int. J. Eng. Sci. 1985, 23, 541-553. [CrossRef]

39. Kolpakov, A.G. Influence of non-degenerated joint on the global and local behavior of joined plates. Int. J. Eng. Sci. 2011, 49, 1216-1231. [CrossRef]

40. Wu, X.-F.; Jenson, R.A. Stress-function variational method for stress analysis of bonded joints under mechanical and thermal loads. Int. J. Eng. Sci. 2011, 49, 279-294. [CrossRef]

41. Chowdhury, N.M.; Wang, J.; Chiu, W.K.; Chang, P. Static and fatigue testing bolted, bonded and hybrid step lap joints of thick carbon fibre/epoxy laminates used on aircraft structures. Compos. Struct. 2016, 142, 96-106. [CrossRef]

42. Shen, W.; Yan, R.; Luo, B.; Zhu, Y.; Zeng, H. Ultimate strength analysis of composite typical joints for ship structures. Compos. Struct. 2017, 171, 32-42. [CrossRef]

43. Pearce, G.M.; Tao, C.; Quek, Y.H.E.; Chowdhury, N.T. A modified Arcan test for mixed-mode loading of bolted joints in composite structures. Compos. Struct. 2018, 187, 203-211. [CrossRef]

44. Bigaud, J.; Aboura, Z.; Martins, A.T.; Verger, S. Analysis of the mechanical behavior of composite T-joints reinforced by one side stitching. Compos. Struct. 2018, 184, 249-255. [CrossRef]

45. Liu, F.; Lu, X.; Zhao, L.; Zhang, J.; Xu, J.; Hu, N. Investigation of bolt load redistribution and its effect on failure prediction in double-lap, multi-bolt composite joints. Compos. Struct. 2018, 202, 397-405. [CrossRef]

46. Liu, Y.; Lemanski, S.; Zhang, X. Parametric study of size, curvature and free edge effects on the predicted strength of bonded composite joints. Compos. Struct. 2018, 202, 364-373. [CrossRef]

47. API-American Petroleum Institute. Available online: http://www.api.org/ (accessed on 17 February 2019). 
48. ISO-International Organization for Standardization. Available online: https://www.iso.org/ (accessed on 17 February 2019).

49. ABS-American Bureau of Shipping ABS. Available online: https://ww2.eagle.org/en.html (accessed on 17 February 2019).

50. Gardner, L.; Nethercot, D.A. Guida all'Eurocodice 3. Progettazione di Edifici in Acciaio: EN 1993-1-1, -1-3 e -1-8; EPC Editore: Roma, Italy, 2012. (In Italian)

51. Abaqus 6.14 Documentation: Abaqus/CAE User's Guide. Available online: http:/ /ivt-abaqusdoc.ivt.ntnu. no:2080/v6.14/books/usi/default.htm (accessed on 17 February 2019).

(C) 2019 by the authors. Licensee MDPI, Basel, Switzerland. This article is an open access article distributed under the terms and conditions of the Creative Commons Attribution (CC BY) license (http:/ / creativecommons.org/licenses/by/4.0/). 



\title{
Article \\ A Complex Variable Solution for Lining Stress and Deformation in a Non-Circular Deep Tunnel II Practical Application and Verification
}

\author{
Yansong Li and Shougen Chen * \\ MOE Key Laboratory of Transportation Tunnel Engineering, Southwest Jiaotong University, Chengdu 610031, \\ Sichuan, China; liyansong@my.swjtu.edu.cn \\ * Correspondence: shougenChen@163.com
}

Received: 1 July 2018; Accepted: 28 August 2018; Published: 30 August 2018

\begin{abstract}
A new complex variable method is presented for stress and displacement problems in a non-circular deep tunnel with certain given boundary conditions at infinity. In order to overcome the complex problems caused by non-circular geometric configurations and the multiply-connected region, a complex variable method and continuity boundary conditions are used to determine stress and displacement within the tunnel lining and within the surrounding rock. The coefficients in the conformal mapping function and stress functions are determined by the optimal design and complex variable method, respectively. The new method is validated by FLAC3D finite difference software through an example. Both the new method and the numerical simulation obtained similar results for the stress concentration and the minimum radial displacement occurred at a similar place in the tunnel. It is demonstrated that the new complex variable method is reliable and reasonable. The new method also provides another way to solve non-circular tunnel excavation problems in a faster and more accurate way.
\end{abstract}

Keywords: non-circular deep tunnel; complex variables; conformal mapping; elasticity; numerical simulation

\section{Introduction}

With the rapid economic development in China, which has caused the expansion of road and railway networks from east to west and to areas in the northeast that are surrounded by mountains, the construction of tunnels is broadly used to improve existing transportation networks. Lining is the primary support adopted to ensure rock pressure. It has been of high interest in determining stress fields within lining using analytical methods. Analytical solutions for stress distribution within circular lining and around circular and elliptical holes have been proposed by many authors (Bobet [1,2]; Lee and Nam [3]; Timoshenko and Goodier [4]).

Many studies have been carried out to determine the stress and deformation of tunnels by applying numerical methods, which have been generally used to provide an understanding of how the stress and deformation of lining are influenced by different parameters. Numerical methods are largely employed to find the stress and deformation stages of lining in the preliminary stages of design.

Muskhelishvili's [5] complex variable method is one of the useful analytical approaches that fully expounded a basic theory of complex potential functions in order to address some issues of plane elasticity mechanics. Based on this method, Exadaktyol and Stavtopoulou [6,7] proposed a closed-form plane strain solution for stress and displacement around semicircular holes. Verruijt $[8,9]$ calculated the stress and displacement components around a circular tunnel in an elastic half-plane. Zhao and Yang [10] obtained a general solution for deep square tunnels with different pressure coefficients. Kargar et al. [11] made an attempt to study lining stress and deformation in a non-circular deep tunnel 
using the Cauchy integral formula of complex variable methods. Li and Chen [12] obtained analytical solutions for a non-circular tunnel lining in power series forms. However, the analytical solutions for stress and displacement found in the above-mentioned literature have seldom come to an available expression for non-circular deep tunnels, especially non-circular deep tunnels with a lining, except for Kargar et al. [11] and Li [12].

When considering the problem with circular support, the solution is much easier. When a non-circular lining is included, the problem considers a multiply-connected region and conformal mapping, which increases the complexity of the problem. Kargar's [11] method can calculate the stress of a non-circular deep tunnel with a lining, although the method needs to integrate. The integral is too complex and difficult to calculate. Li's [12] method only considered the non-circular tunnel lining without thinking about the surrounding rock around the lining. In order to simplify the calculation, $\mathrm{Li}$ [12] assumed that the surrounding rock has a certain given surface traction applied to the lining. In this study it will be shown that these difficulties can be surmounted at last for the case of a non-circular deep tunnel with certain boundary conditions at infinity, by a new complex variable method in power series forms.

Based on the research findings proposed, an attempt was made in this paper to find the stress distribution and radial displacement of the lining in a non-circular deep tunnel, considering the boundary conditions of the surrounding rocks by applying a complex variable power series method, which is more efficient, simple and accurate. Finally, the new method was validated by the FLAC finite difference software through an example.

\section{Problem Statement and General Consideration}

The problem refers to a non-circular tunnel with lining in an elastic geomaterial. The tunnel is located at great depth compared with the tunnel dimension; the problem is considered a single hole with support in an infinite plane. The infinite plate on the complex plane is divided into the two isotropic homogenous regions of $S_{1}$ and $S_{2}$ bounded by contours $L_{1}$ and $L_{2}$. The regions $S_{1}$ and $S_{2}$ refer to the lining and the surrounding rock, respectively. The boundary of the tunnel lining inside $\left(\mathrm{L}_{1}\right)$ is free of stress, and the rock-lining interface $\left(\mathrm{L}_{2}\right)$ satisfies the continuity boundary conditions. It is assumed that the region $S_{1}$ in the z-plane can be mapped conformally onto a ring $\left(\mathrm{O}_{1}\right)$ in the $\zeta$-plane. The surrounding rock, region $S_{2}$ in the z-plane, can be mapped conformally onto the region $\mathrm{O}_{2}$, which is the area outside the $\mathrm{L}_{2}$ outline in the $\zeta$-plane, see Figure 1 . The general formula of the conformal mapping function is determined based on the Laurent series as follows:

$$
\mathrm{w}(\zeta)=\mathrm{R}\left(\zeta+\sum_{\mathrm{k}=0}^{\infty} \mathrm{J}_{\mathrm{k}} \zeta^{-\mathrm{k}}\right)
$$

where $\mathrm{R}$ is positive real number reflecting the hole's size, and the $\mathrm{J}_{\mathrm{k}}$ are generally complex numbers, which are determined by the shape of the tunnel. In most situations it is accurate enough to only take the first few of $J_{k}$ of the series. $\theta$ and $\rho$ are assumed to be two polar coordinates of point $\zeta$ in the

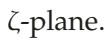

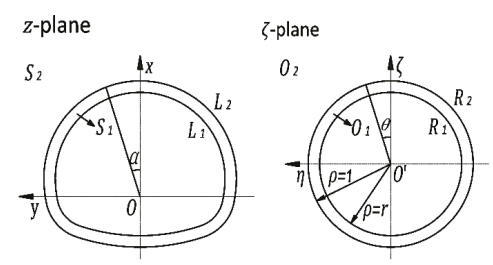

Figure 1. Conformal mapping of the tunnel in the z-plane in to two concentric circles in the $\zeta$-plane. 
In the complex variable method $[5,13,14]$, the solution is expressed in terms of two functions $\varphi$ and $\psi$, which must be analyzed in the region $\mathrm{S}_{1}$ occupied by the elastic material. The stresses are determined based on the stress functions in the equations:

$$
\begin{gathered}
\sigma_{\theta}+\sigma_{\rho}=4 \operatorname{Re}\left[\frac{\varphi^{\prime}(\zeta)}{\mathrm{w}^{\prime}(\zeta)}\right] \\
\sigma_{\theta}-\sigma_{\rho}+\tau_{\rho \theta}=\frac{2 \zeta^{2}}{\rho^{2}} \frac{1}{\overline{\mathrm{w}^{\prime}(\zeta)}}\left[\overline{\mathrm{w}(\zeta)} \frac{\varphi^{\prime \prime}(\zeta) \mathrm{w}^{\prime}(\zeta)-\varphi^{\prime}(\zeta) \mathrm{w}^{\prime \prime}(\zeta)}{\mathrm{w}^{\prime}(\zeta) \mathrm{w}^{\prime}(\zeta)}+\psi^{\prime}(\zeta)\right]
\end{gathered}
$$

where $\sigma_{\rho}, \sigma_{\theta}$ and $\tau_{\rho \theta}$ are the radial, circumferential and tangential stress components, respectively.

The displacements are given by

$$
2 \mathrm{G}\left(\mathrm{u}_{\rho}+\mathrm{iu}_{\theta}\right)=\frac{\bar{\zeta}}{\rho} \frac{\overline{\mathrm{w}^{\prime}(\zeta)}}{|\mathrm{w}(\zeta)|}\left[\kappa \varphi(\zeta)-\frac{\mathrm{w}(\zeta)}{\overline{\mathrm{w}^{\prime}(\zeta)}} \overline{\varphi^{\prime}(\zeta)}-\overline{\psi(\zeta)}\right]
$$

where $G$ is the shear modulus of the elastic material, and $k$ is determined by Poisson's ratio $v$, $\kappa=3-4 v$ and $\kappa=\frac{3-v}{1+v}$ are plane strain and plane stress, respectively. In this paper, plane strain conditions are assumed. Based on the Kargar's [11] method and Chen [15], the stress functions $\varphi_{1}$ and $\psi_{1}$ in the region $\mathrm{O}_{2}$ have the following expansions:

$$
\begin{gathered}
\varphi_{1}(\zeta)=\Gamma \mathrm{w}(\zeta)+\varphi_{0}(\zeta) \\
\psi_{1}(\zeta)=\Gamma^{\prime} \mathrm{w}(\zeta)+\psi_{0}(\zeta)
\end{gathered}
$$

where

$$
\begin{aligned}
\varphi_{0}(\zeta) & =\sum_{\mathrm{k}=0}^{\infty} \mathrm{h}_{\mathrm{k}} \zeta^{-\mathrm{k}} \\
\psi_{0}(\zeta) & =\sum_{\mathrm{k}=0}^{\infty} \mathrm{m}_{\mathrm{k}} \zeta^{-\mathrm{k}}
\end{aligned}
$$

where $h_{k}, m_{k}$ are generally complex numbers, which must be determined from boundary conditions. $\varphi_{0}(\zeta)$ and $\psi_{0}(\zeta)$ are holomorphic functions with $\varphi_{0}(\infty)=0$ and $\psi_{0}(\infty)=0, \Gamma$ and $\Gamma^{\prime}$ are real and complex constants with regard to the stress state at infinity, which are determined as follows:

$$
\begin{gathered}
\Gamma=\frac{1}{4}\left(\sigma_{1}+\sigma_{2}\right)=\frac{1+\mathrm{K}}{4} \gamma \mathrm{H} \\
\Gamma^{\prime}=-\frac{1}{4}\left(\sigma_{1}-\sigma_{2}\right) \mathrm{e}^{-2 \mathrm{i} \alpha}=-\frac{1-\mathrm{K}}{4} \gamma \mathrm{H}
\end{gathered}
$$

where $\sigma_{1}$ and $\sigma_{2}$ are the principal stress components at infinity; $\alpha$ is the angle made between the $\sigma_{1}$ direction and the $\mathrm{x}$ axis; $\mathrm{K}$ is the lateral pressure coefficient; $\gamma$ and $\mathrm{H}$ are the unit weight of the surrounding rock and the depth of tunnel, respectively.

The stress functions $\varphi_{2}(\zeta)$ and $\psi_{2}(\zeta)$, which are represent region $O_{1}$, have the following Laurent series expansions:

$$
\begin{aligned}
& \varphi_{2}(\zeta)=\sum_{\mathrm{k}=0}^{\infty} \mathrm{a}_{\mathrm{k}} \zeta^{\mathrm{k}}+\sum_{\mathrm{k}=1}^{\infty} \mathrm{b}_{\mathrm{k}} \zeta^{-\mathrm{k}} \\
& \psi_{2}(\zeta)=\sum_{\mathrm{k}=0}^{\infty} \mathrm{c}_{\mathrm{k}} \zeta^{\mathrm{k}}+\sum_{\mathrm{k}=1}^{\infty} \mathrm{d}_{\mathrm{k}} \zeta^{-\mathrm{k}}
\end{aligned}
$$

where $a_{k}, b_{k}, c_{k}, d_{k}$ are generally complex numbers that must be determined from boundary conditions. 
Stress functions $\varphi_{1}(\zeta), \psi_{1}(\zeta), \varphi_{2}(\zeta)$ and $\psi_{2}(\zeta)$ should satisfy the continuity boundary conditions on $R_{2}$ circles and satisfy the boundary conditions on $R_{1}$ circles. The displacement at lining and rock should be equal on the boundary $\mathrm{R}_{2}$. It can be concluded as:

$$
u_{\rho 1}^{R 2}+i u_{\theta 1}^{R 2}=u_{\rho 2}^{R 2}+i u_{\theta 2}^{R 2}
$$

where $u_{\rho 1}^{R 2}$ and $u_{\theta 1}^{R 2}$ are the lining displacement components of boundary $R_{2}$ in the $\rho$ and $\theta$ directions, and $u_{\rho 2}^{R 2}$ and $u_{\theta 2}^{R 2}$ are the rock displacement components of boundary $R_{2}$ in the $\rho$ and $\theta$ directions, respectively.

The stress at lining and rock should be equal on boundary $R_{2}$. It can be concluded as:

$$
\mathrm{f}_{1}^{\mathrm{R} 2}=\mathrm{f}_{2}^{\mathrm{R} 2}
$$

where $\mathrm{f}_{1}^{\mathrm{R} 2}$ and $\mathrm{f}_{2}^{\mathrm{R} 2}$ are displacement components of boundary $\mathrm{R}_{2}$ in the lining and rock, respectively.

The stress at the lining on the boundary $R_{1}$ should be 0 . It can be concluded as:

$$
\mathrm{f}_{1}^{\mathrm{R} 1}=0
$$

Based on Chen [15], the boundary conditions (13)-(15) can be rewritten. Equation (13) is expressed as:

$$
\frac{\mathrm{K}_{1}}{\mathrm{G}_{1}} \varphi_{0}\left(\mathrm{t}_{2}\right)-\frac{1}{\mathrm{G}_{1}}\left(\frac{\mathrm{w}\left(\mathrm{t}_{2}\right)}{\overline{\mathrm{w}^{\prime}\left(\mathrm{t}_{2}\right)}} \overline{\varphi_{0}^{\prime}\left(\mathrm{t}_{2}\right)}+\overline{\psi_{0}\left(\mathrm{t}_{2}\right)}\right)=\frac{\mathrm{k}_{2}}{\mathrm{G}_{2}} \varphi_{2}\left(\mathrm{t}_{2}\right)-\frac{1}{\mathrm{G}_{2}}\left(\frac{\mathrm{w}\left(\mathrm{t}_{2}\right)}{\overline{\mathrm{w}^{\prime}\left(\mathrm{t}_{2}\right)}} \overline{\varphi_{2}^{\prime}\left(\mathrm{t}_{2}\right)}+\overline{\psi_{2}\left(\mathrm{t}_{2}\right)}\right)
$$

The stress boundary condition of Equation (14) is expressed as:

$$
\varphi_{1}\left(\mathrm{t}_{2}\right)+\frac{\mathrm{w}\left(\mathrm{t}_{2}\right)}{\overline{\mathrm{w}^{\prime}\left(\mathrm{t}_{2}\right)}} \overline{\varphi_{1}^{\prime}\left(\mathrm{t}_{2}\right)}+\overline{\psi_{1}\left(\mathrm{t}_{2}\right)}=\varphi_{2}\left(\mathrm{t}_{2}\right)+\frac{\mathrm{w}\left(\mathrm{t}_{2}\right)}{\overline{\mathrm{w}^{\prime}\left(\mathrm{t}_{2}\right)}} \overline{\varphi_{2}^{\prime}\left(\mathrm{t}_{2}\right)}+\overline{\psi_{2}\left(\mathrm{t}_{2}\right)}
$$

The stress boundary condition of Equation (15) is expressed as:

$$
\varphi_{2}\left(\mathrm{t}_{1}\right)+\frac{\mathrm{w}\left(\mathrm{t}_{1}\right)}{\overline{\mathrm{w}^{\prime}\left(\mathrm{t}_{1}\right)}} \overline{\varphi_{2}^{\prime}\left(\mathrm{t}_{1}\right)}+\overline{\psi_{2}\left(\mathrm{t}_{1}\right)}=0
$$

where $t_{1}$ and $t_{2}$ are the point of the boundary $R_{1}$ and $R_{2}$ in the $\zeta$-plane, respectively. $u_{\rho 1}$ and $u_{\theta 1}$ are the displacement components of the lining-rock interface in the $\rho$ and $\theta$ directions.

The expressions $\Gamma \mathrm{w}(\zeta)$ and $\Gamma^{\prime} w(\zeta)$ should not be incorporated into continuity Equation (16) since they define initial ground stress and deformation in the surrounding rock when tunnels are excavated. Equations (16) and (17) are concerned with the continuity of deformation and the stress field across the lining-rock interface due to the no-slip condition. Equation (18) is concerned that the tunnel lining inside $\left(L_{1}\right)$ is entirely free of stress.

\section{Solution}

In order to eliminate the difficulties caused by the power series, Equations (16)-(18) are rewritten in the form:

$$
\begin{aligned}
\frac{\mathrm{K}_{1}}{\mathrm{G}_{1}} \varphi_{0}\left(\mathrm{t}_{2}\right) \overline{\mathrm{w}^{\prime}\left(\mathrm{t}_{2}\right)} & -\frac{1}{\mathrm{G}_{1}} \mathrm{w}\left(\mathrm{t}_{2}\right) \overline{\varphi_{0}^{\prime}\left(\mathrm{t}_{2}\right)}-\frac{1}{\mathrm{G}_{1}} \overline{\psi_{0}\left(\mathrm{t}_{2}\right) \mathrm{w}^{\prime}\left(\mathrm{t}_{2}\right)} \\
= & \frac{\mathrm{K}_{2}}{\mathrm{G}_{2}} \varphi_{2}\left(\mathrm{t}_{2}\right) \overline{\mathrm{w}^{\prime}\left(\mathrm{t}_{2}\right)}-\frac{1}{\mathrm{G}_{2}} \mathrm{w}\left(\mathrm{t}_{2}\right) \overline{\varphi_{2}^{\prime}\left(\mathrm{t}_{2}\right)}-\frac{1}{\mathrm{G}_{2}} \overline{\psi_{2}\left(\mathrm{t}_{2}\right) \mathrm{w}^{\prime}\left(\mathrm{t}_{2}\right)} \\
\varphi_{1}\left(\mathrm{t}_{2}\right) \overline{\mathrm{w}^{\prime}\left(\mathrm{t}_{2}\right)}+ & \mathrm{w}\left(\mathrm{t}_{2}\right) \overline{\varphi_{1}^{\prime}\left(\mathrm{t}_{2}\right)}+\overline{\psi_{1}\left(\mathrm{t}_{2}\right) \mathrm{w}^{\prime}\left(\mathrm{t}_{2}\right)} \\
& =\varphi_{2}\left(\mathrm{t}_{2}\right) \overline{\mathrm{w}^{\prime}\left(\mathrm{t}_{2}\right)}+\mathrm{w}\left(\mathrm{t}_{2}\right) \overline{\varphi_{2}^{\prime}\left(\mathrm{t}_{2} \mathrm{t}\right)}+\overline{\psi_{2}\left(\mathrm{t}_{2}\right) \mathrm{w}^{\prime}\left(\mathrm{t}_{2}\right)} \\
\varphi_{2}\left(\mathrm{t}_{1}\right) \overline{\mathrm{w}^{\prime}\left(\mathrm{t}_{1}\right)}+\mathrm{w}\left(\mathrm{t}_{1}\right) \overline{\varphi_{2}^{\prime}\left(\mathrm{t}_{1}\right)} & \overline{\psi_{2}\left(\mathrm{t}_{1}\right) \mathrm{w}^{\prime}\left(\mathrm{t}_{1}\right)}=0
\end{aligned}
$$


The form of an infinite polynomial times another infinite polynomial, such as $\varphi_{0}\left(\mathrm{t}_{2}\right) \overline{\mathrm{w}^{\prime}\left(\mathrm{t}_{2}\right)}$ of Equation (19), is hard to calculated and merge so that the equation cannot be calculated. To acquire a solution for Equations (19)-(21), Li's method needs to be introduced, which converts the form of the multiplication of two infinite polynomials to an infinite matrix, which can be easily calculated and merged for a computer.

From the power series theory of the complex variable method written by Chen [15], the first factor of Equation (19) on the left can be rewritten as follows:

$$
\begin{aligned}
\frac{\mathrm{k}_{1}}{\mathrm{G}_{1}} \varphi_{0}\left(\mathrm{t}_{2}\right) \overline{\mathrm{w}^{\prime}\left(\mathrm{t}_{2}\right)} & =\frac{\mathrm{k}_{1}}{\mathrm{G}_{1}} \sum_{\mathrm{k}=0}^{\infty} \mathrm{h}_{\mathrm{v}} \rho^{-\mathrm{v}} \sigma^{-\mathrm{v}}+\sum_{\mathrm{v}=-\infty}^{+\infty} \mathrm{vL} \mathrm{L}_{\mathrm{v}} \rho^{\mathrm{v}-1} \sigma^{1-\mathrm{v}} \\
& =\frac{\mathrm{k}_{1}}{\mathrm{G}_{1}}\left(\sum_{\mathrm{k}=0}^{\infty} \mathrm{h}_{\mathrm{v}} \rho^{-\mathrm{v}} \sigma^{-\mathrm{v}}+\sum_{\mathrm{v}=-\infty}^{0} \mathrm{vL}_{\mathrm{v}} \rho^{\mathrm{v}-1} \sigma^{1-\mathrm{v}}\right. \\
& \left.+\sum_{\mathrm{k}=0}^{\infty} \mathrm{h}_{\mathrm{v}} \rho^{-\mathrm{v}} \sigma^{-\mathrm{v}}+\sum_{0}^{+\infty} \mathrm{vL} \mathrm{L}_{\mathrm{v}} \rho^{\mathrm{v}-1} \sigma^{1-\mathrm{v}}\right)
\end{aligned}
$$

where $\mathrm{k}$ is substituted by $\mathrm{v}$ to facilitate the calculation. $\sigma$ is the angle of the point of the boundary; $\rho$ is the radius of the boundary circle in the $\zeta$-plane, which equals 1 at the lining-rock interface. $\sigma$ and $\rho$ can be related by $\zeta=\rho \sigma$, where $\sigma=\exp (\mathrm{i} \theta)$. $\mathrm{L}_{\mathrm{v}}$ can be calculated by $\mathrm{R}$ and $\mathrm{J}_{\mathrm{k}}$, and Equation (1) is rewritten in the form:

$$
\mathrm{w}(\zeta)=\sum_{\mathrm{v}=-\infty}^{+\infty} \mathrm{L}_{\mathrm{v}} \zeta^{-\mathrm{v}}
$$

The first item on the right of the Equation (22) must be expanded as follows:

$$
\begin{aligned}
\frac{\mathrm{k}_{1}}{\mathrm{G}_{1}} \sum_{\mathrm{v}=-\infty}^{0} \mathrm{vL}_{\mathrm{v}} \rho^{\mathrm{v}-1} & \sigma^{1-\mathrm{v}} \sum_{\mathrm{v}=0}^{\infty} \mathrm{a}_{\mathrm{v}} \rho^{\mathrm{v}} \sigma^{\mathrm{v}}= \\
& -\frac{\mathrm{k}_{1}}{\mathrm{G}_{1}}\left(\mathrm{~L}_{-1} \rho^{-2} \sigma^{2}+2 \mathrm{~L}_{-2} \rho^{-3} \sigma^{3}+3 \mathrm{~L}_{-3} \rho^{-4} \sigma^{4}+\cdots\right)\left(\mathrm{h}_{0}+\mathrm{h}_{1} \rho^{-1} \sigma^{-1}\right. \\
& \left.+\mathrm{h}_{2} \rho^{-2} \sigma^{-2}+\mathrm{h}_{3} \rho^{-3} \sigma^{-3}+\cdots\right)
\end{aligned}
$$

where the positive power of $\sigma$ in Equation (24) is obtained as:

$$
\begin{array}{ll}
\sigma^{1} & -\frac{\kappa_{1}}{G_{1}}\left(h_{1} L_{-1} \rho^{-3} \sigma+2 h_{2} L_{-2} \rho^{-5} \sigma+3 h_{3} L_{-3} \rho^{-7} \sigma+\cdots\right) \\
\sigma^{2} & -\frac{\kappa_{1}}{G_{1}}\left(h_{0} L_{-1} \rho^{-2} \sigma^{2}+2 h_{1} L_{-2} \rho^{-4} \sigma^{2}+3 h_{2} L_{-3} \rho^{-6} \sigma^{2}+\cdots\right) \\
\sigma^{3} & -\frac{\kappa_{1}}{G_{1}}\left(2 h_{0} L_{-2} \rho^{-3} \sigma^{3}+3 h_{1} L_{-3} \rho^{-5} \sigma^{3}+4 h_{2} L_{-4} \rho^{-7} \sigma^{3}+\cdots\right) \\
\ldots & \cdots
\end{array}
$$

The zero and negative powers of $\sigma$ in Equation (24) can be derived:

$$
\begin{array}{ll}
\sigma^{0} & -\frac{k_{1}}{\mathrm{G}_{1}}\left(\mathrm{~h}_{2} \mathrm{~L}_{-1} \rho^{-4}+2 \mathrm{~h}_{3} \mathrm{~L}_{-2} \rho^{-6}+3 \mathrm{~h}_{4} \mathrm{~L}_{-3} \rho^{-8}+\cdots\right) \\
\sigma^{-1} & -\frac{\mathrm{k}_{1}}{\mathrm{G}_{1}}\left(\mathrm{~h}_{3} \mathrm{~L}_{-1} \rho^{-5} \sigma^{-1}+2 \mathrm{~h}_{4} \mathrm{~L}_{-2} \rho^{-7} \sigma^{-1}+3 \mathrm{~h}_{5} \mathrm{~L}_{-3} \rho^{-9} \sigma^{-1}+\cdots\right) \\
\sigma^{-2} & -\frac{\kappa_{1}}{\mathrm{G}_{1}}\left(\mathrm{~h}_{4} \mathrm{~L}_{-1} \rho^{-6} \sigma^{-2}+2 \mathrm{~h}_{5} \mathrm{~L}_{-2} \rho^{-8} \sigma^{-2}+3 \mathrm{~h}_{6} \mathrm{~L}_{-3} \rho^{-10} \sigma^{-2}+\cdots\right)
\end{array}
$$

The second item on the right of Equation (22) can be expanded as follows:

$$
\begin{aligned}
\frac{\kappa_{1}}{G_{1}} \sum_{\mathrm{v}=1}^{+\infty} \mathrm{vL}_{\mathrm{v}} \rho^{\mathrm{v}-1} \sigma^{1-\mathrm{v}} \sum_{\mathrm{v}=0}^{\infty} \mathrm{h}_{\mathrm{v}} \rho^{-\mathrm{v}} \sigma^{-\mathrm{v}} \\
=\frac{\mathrm{K}_{1}}{\mathrm{G}_{1}}\left(\cdots+3 \mathrm{~L}_{3} \rho^{2} \sigma^{-2}+2 \mathrm{~L}_{2} \rho^{1} \sigma^{-1}+\mathrm{L}_{1}\right)\left(\mathrm{h}_{0}+\mathrm{h}_{1} \rho^{-1} \sigma^{-1}+\mathrm{h}_{2} \rho^{-2} \sigma^{-2}\right. \\
\left.+\mathrm{h}_{3} \rho^{-3} \sigma^{-3}+\cdots\right)
\end{aligned}
$$


There are only zero and negative powers of $\sigma$ in the above Equation (23), which can be derived as follows:

$$
\begin{array}{ll}
\sigma^{0} & \frac{\mathrm{K}_{1}}{\mathrm{G}_{1}} \mathrm{~h}_{0} \mathrm{~L}_{1} \\
\sigma^{-1} & \frac{\mathrm{K}_{1}}{\mathrm{G}_{1}}\left(\mathrm{~h}_{1} \mathrm{~L}_{1} \rho^{-1} \sigma^{-1}+2 \mathrm{~h}_{0} \mathrm{~L}_{2} \rho \sigma^{-1}\right) \\
\sigma^{-2} & \frac{\mathrm{K}_{1}}{\mathrm{G}_{1}}\left(\mathrm{~h}_{2} \mathrm{~L}_{1} \rho^{-2} \sigma^{-2}+2 \mathrm{~h}_{1} \mathrm{~L}_{2} \sigma^{-2}+3 \mathrm{~h}_{0} \mathrm{~L}_{3} \rho^{2} \sigma^{-2}\right) \\
\ldots & \cdots
\end{array}
$$

The general formula of Equation (29) are determined based on the expanded functions of Equation (25) as follows:

$$
\frac{\mathrm{k}_{1}}{\mathrm{G}_{1}} \sum_{\mathrm{k}=0}^{\infty} \mathrm{h}_{\mathrm{k}} \rho^{-2 \mathrm{k}-\mathrm{v}}(\mathrm{k}+\mathrm{v}-1) \mathrm{L}_{-\mathrm{k}-\mathrm{v}+1} \sigma^{\mathrm{v}}
$$

The general formula of Equation (30) is determined based on the expanded functions of Equations (26) and (28) as follows:

$$
-\frac{\kappa_{1}}{\mathrm{G}_{1}} \sum_{\mathrm{k}=0}^{\infty} \mathrm{h}_{\mathrm{k}} \rho^{-2 \mathrm{k}+\mathrm{v}}(\mathrm{k}-\mathrm{v}-1) \mathrm{L}_{-\mathrm{k}+\mathrm{v}+1} \sigma^{-\mathrm{v}} \mathrm{v}=0,1,2,3 \ldots
$$

Based on the power series of the complex variable method written by Chen [15], which have been presented in Equations (22)-(30), the other items of Equation (19) can be determined and separate the positive and negative exponents of Equation (19); the positive power system of Equation (19) can be expanded as follows:

$$
\begin{array}{rl}
\frac{\mathrm{k}_{2}}{\mathrm{G}_{2}} \sum_{\mathrm{k}=0}^{\infty} \mathrm{a}_{\mathrm{k}} \rho^{2 \mathrm{k}-\mathrm{v}}(\mathrm{k} & -\mathrm{v}+1) \mathrm{L}_{\mathrm{k}-\mathrm{v}+1} \sigma^{\mathrm{v}}-\frac{\mathrm{k}_{2}}{\mathrm{G}_{2}} \sum_{\mathrm{k}=1}^{\infty} \mathrm{b}_{\mathrm{k}} \rho^{-2 \mathrm{k}-\mathrm{v}}(\mathrm{k}+\mathrm{v}-1) \mathrm{L}_{-\mathrm{k}-\mathrm{v}+1} \sigma^{\mathrm{v}} \\
& -\frac{1}{\mathrm{G}_{2}} \sum_{\mathrm{k}=1}^{\infty} \overline{\mathrm{a}_{\mathrm{k}}} \rho^{2 \mathrm{k}+\mathrm{v}-2} \mathrm{~kL}_{\mathrm{k}+\mathrm{v}-1} \sigma^{\mathrm{v}}+\frac{1}{\mathrm{G}_{2}} \sum_{\mathrm{k}=1}^{\infty} \overline{\mathrm{b}_{\mathrm{k}}} \rho^{-2 \mathrm{k}+\mathrm{v}-2} \mathrm{~kL}_{-\mathrm{k}+\mathrm{v}-1} \sigma^{\mathrm{v}} \\
& +\frac{1}{\mathrm{G}_{2}} \sum_{\mathrm{k}=0}^{\infty} \overline{\mathrm{c}_{\mathrm{k}}} \rho^{-\mathrm{v}}(\mathrm{k}+\mathrm{v}-1) \mathrm{L}_{-\mathrm{k}-\mathrm{v}+1} \sigma^{\mathrm{v}} \\
& -\frac{1}{\mathrm{G}_{2}} \sum_{\mathrm{k}=1}^{\infty} \overline{\mathrm{d}_{\mathrm{k}}} \rho^{-\mathrm{v}}(\mathrm{k}-\mathrm{v}+1) \mathrm{L}_{\mathrm{k}-\mathrm{v}+1} \sigma^{\mathrm{v}} \\
& +\frac{\mathrm{k}_{1}}{\mathrm{G}_{1}} \sum_{\mathrm{k}=0}^{\infty} \mathrm{h}_{\mathrm{k}} \rho^{-2 \mathrm{k}-\mathrm{v}}(\mathrm{k}+\mathrm{v}-1) \mathrm{L}_{-\mathrm{k}-\mathrm{v}+1} \sigma^{\mathrm{v}} \\
& -\frac{1}{\mathrm{G}_{1}} \sum_{\mathrm{k}=0}^{\infty} \overline{\mathrm{h}_{\mathrm{k}}} \rho^{-2 \mathrm{k}+\mathrm{v}-2} \mathrm{~kL}-\mathrm{k}+\mathrm{v}-1 \sigma^{\mathrm{v}}+\frac{1}{\mathrm{G}_{1}} \sum_{\mathrm{k}=0}^{\infty} \mathrm{m}_{\mathrm{k}} \rho^{-\mathrm{v}}(\mathrm{k}-\mathrm{v}+1) \mathrm{L}_{\mathrm{k}-\mathrm{v}+1} \sigma^{\mathrm{v}} \\
& =0 \\
\mathrm{v} & 01,2,3 \cdots
\end{array}
$$

The negative power system of Equation (19) can be expanded as follows:

$$
\begin{aligned}
\frac{\mathrm{k}_{2}}{\mathrm{G}_{2}} \sum_{\mathrm{k}=0}^{\infty} \mathrm{a}_{\mathrm{k}} \rho^{2 \mathrm{k}+\mathrm{v}}(\mathrm{k} & +\mathrm{v}+1) \mathrm{L}_{\mathrm{k}+\mathrm{v}+1} \sigma^{-\mathrm{v}}-\frac{\mathrm{k}_{2}}{\mathrm{G}_{2}} \sum_{\mathrm{k}=1}^{\infty} \mathrm{b}_{\mathrm{k}} \rho^{-2 \mathrm{k}+\mathrm{v}}(\mathrm{k}-\mathrm{v}-1) \mathrm{L}_{-\mathrm{k}+\mathrm{v}+1} \sigma^{-\mathrm{v}} \\
& -\frac{1}{\mathrm{G}_{2}} \sum_{\mathrm{k}=1}^{\infty} \overline{\mathrm{a}_{\mathrm{k}}} \rho^{2 \mathrm{k}-\mathrm{v}-2} \mathrm{~kL}_{\mathrm{k}-\mathrm{v}-1} \sigma^{-\mathrm{v}}+\frac{1}{\mathrm{G}_{2}} \sum_{\mathrm{k}=1}^{\infty} \overline{\mathrm{b}_{\mathrm{k}}} \rho^{-2 \mathrm{k}-\mathrm{v}-2} \mathrm{~kL}_{-\mathrm{k}-\mathrm{v}-1} \sigma^{-\mathrm{v}} \\
& +\frac{1}{\mathrm{G}_{2}} \sum_{\mathrm{k}=0}^{\infty} \overline{\mathrm{c}_{\mathrm{k}}} \rho^{\mathrm{v}}(\mathrm{k}-\mathrm{v}-1) \mathrm{L}_{-\mathrm{k}+\mathrm{v}+1} \sigma^{-\mathrm{v}} \\
& -\frac{1}{\mathrm{G}_{2}} \sum_{\mathrm{k}=1}^{\infty} \overline{\mathrm{d}_{\mathrm{k}}} \rho^{\mathrm{v}}(\mathrm{k}+\mathrm{v}+1) \mathrm{L}_{\mathrm{k}+\mathrm{v}+1} \sigma^{-\mathrm{v}} \\
& +\frac{\mathrm{k}_{1}}{\mathrm{G}_{1}} \sum_{\mathrm{k}=0}^{\infty} \mathrm{h}_{\mathrm{k}} \rho^{-2 \mathrm{k}+\mathrm{v}}(\mathrm{k}-\mathrm{v}-1) \mathrm{L}_{-\mathrm{k}+\mathrm{v}+1} \sigma^{-\mathrm{v}} \\
& -\frac{1}{\mathrm{G}_{1}} \sum_{\mathrm{k}=0}^{\infty} \overline{\mathrm{h}_{\mathrm{k}}} \rho^{-2 \mathrm{k}-\mathrm{v}-2} \mathrm{~kL}-\mathrm{k}-\mathrm{v}-1 \sigma^{-\mathrm{v}} \\
& +\frac{1}{\mathrm{G}_{1}} \sum_{\mathrm{k}=0}^{\infty} \mathrm{m}_{\mathrm{k}} \rho^{\mathrm{v}}(\mathrm{k}+\mathrm{v}+1) \mathrm{L}_{\mathrm{k}+\mathrm{v}+1} \sigma^{-\mathrm{v}} \\
& =0 \\
& 0
\end{aligned}
$$


The positive power system of Equation (20) is determined as follows:

$$
\begin{aligned}
& \sum_{\mathrm{k}=0}^{\infty} \mathrm{a}_{\mathrm{k}} \rho^{2 \mathrm{k}-\mathrm{v}}(\mathrm{k}-\mathrm{v}+1) \mathrm{L}_{\mathrm{k}-\mathrm{v}+1} \sigma^{\mathrm{v}}-\sum_{\mathrm{k}=1}^{\infty} \mathrm{b}_{\mathrm{k}} \rho^{-2 \mathrm{k}-\mathrm{v}}(\mathrm{k}+\mathrm{v}-1) \mathrm{L}_{-\mathrm{k}-\mathrm{v}+1} \sigma^{\mathrm{v}} \\
& +\sum_{\mathrm{k}=1}^{\infty} \overline{\mathrm{a}_{\mathrm{k}}} \rho^{2 \mathrm{k}+\mathrm{v}-2} \mathrm{~kL}_{\mathrm{k}+\mathrm{v}-1} \sigma^{\mathrm{v}}-\sum_{\mathrm{k}=1}^{\infty} \overline{\mathrm{b}_{\mathrm{k}}} \rho^{-2 \mathrm{k}+\mathrm{v}-2} \mathrm{~kL}_{-\mathrm{k}+\mathrm{v}-1} \sigma^{\mathrm{v}} \\
& -\sum_{\mathrm{k}=0}^{\infty} \overline{\mathrm{c}_{\mathrm{k}}} \rho^{-\mathrm{v}}(\mathrm{k}+\mathrm{v}-1) \mathrm{L}_{-\mathrm{k}-\mathrm{v}+1} \sigma^{\mathrm{v}}+\sum_{\mathrm{k}=1}^{\infty} \overline{\mathrm{d}_{\mathrm{k}}} \rho^{-\mathrm{v}}(\mathrm{k}-\mathrm{v}+1) \mathrm{L}_{\mathrm{k}-\mathrm{v}+1} \sigma^{\mathrm{v}} \\
& +\sum_{\substack{\mathrm{k}=0 \\
\infty}}^{\infty} \mathrm{h}_{\mathrm{k}} \rho^{-2 \mathrm{k}-\mathrm{v}}(\mathrm{k}+\mathrm{v}-1) \mathrm{L}_{-\mathrm{k}-\mathrm{v}+1} \sigma^{\mathrm{v}}+\sum_{\mathrm{k}=0}^{\infty} \overline{\mathrm{h}_{\mathrm{k}}} \rho^{-2 \mathrm{k}+\mathrm{v}-2} \mathrm{~kL}-\mathrm{k}+\mathrm{v}-1 \sigma^{\mathrm{v}} \\
& -\sum_{\mathrm{k}=0}^{\infty} \mathrm{m}_{\mathrm{k}} \rho^{-\mathrm{v}}(\mathrm{k}-\mathrm{v}+1) \mathrm{L}_{\mathrm{k}-\mathrm{v}+1} \sigma^{\mathrm{v}} \\
& =2 \Gamma \sum_{\mathrm{k}=0}^{\infty} \mathrm{L}_{\mathrm{k}} \rho^{2 \mathrm{k}-\mathrm{v}}(\mathrm{k}-\mathrm{v}+1) \mathrm{L}_{\mathrm{k}-\mathrm{v}+1} \sigma^{\mathrm{v}} \\
& -2 \Gamma \sum_{\mathrm{k}=1}^{\infty} \mathrm{L}_{-\mathrm{k}} \rho^{-2 \mathrm{k}-\mathrm{v}}(\mathrm{k}+\mathrm{v}-1) \mathrm{L}_{-\mathrm{k}-\mathrm{v}+1} \sigma^{\mathrm{v}} \\
& -\Gamma^{\prime} \sum_{\mathrm{k}=0}^{\infty} \mathrm{L}_{\mathrm{k}} \rho^{-\mathrm{v}}(\mathrm{k}+\mathrm{v}-1) \mathrm{L}_{-\mathrm{k}-\mathrm{v}+1} \sigma^{\mathrm{v}} \\
& +\Gamma^{\prime} \sum_{\mathrm{k}=1}^{\infty} \mathrm{L}_{-\mathrm{k}} \rho^{-\mathrm{v}}(\mathrm{k}-\mathrm{v}+1) \mathrm{L}_{\mathrm{k}-\mathrm{v}+1} \sigma^{\mathrm{v}} \\
& \mathrm{v}=1,2,3 \ldots
\end{aligned}
$$

The negative power system of Equation (20) can be expanded as follows:

$$
\begin{aligned}
& \sum_{\mathrm{k}=0}^{\infty} \mathrm{a}_{\mathrm{k}} \rho^{2 \mathrm{k}+\mathrm{v}}(\mathrm{k}+\mathrm{v}+1) \mathrm{L}_{\mathrm{k}+\mathrm{v}+1} \sigma^{-\mathrm{v}}-\sum_{\mathrm{k}=1}^{\infty} \mathrm{b}_{\mathrm{k}} \rho^{-2 \mathrm{k}+\mathrm{v}}(\mathrm{k}-\mathrm{v}-1) \mathrm{L}_{-\mathrm{k}+\mathrm{v}+1} \sigma^{-\mathrm{v}} \\
&+\sum_{\mathrm{k}=1}^{\infty} \overline{\mathrm{a}_{\mathrm{k}}} \rho^{2 \mathrm{k}-\mathrm{v}-2} \mathrm{k} \mathrm{L}_{\mathrm{k}-\mathrm{v}-1} \sigma^{-\mathrm{v}}-\sum_{\mathrm{k}=1}^{\infty} \overline{\mathrm{b}_{\mathrm{k}}} \rho^{-2 \mathrm{k}-\mathrm{v}-2} \mathrm{~kL}_{-\mathrm{k}-\mathrm{v}-1} \sigma^{-\mathrm{v}} \\
&-\sum_{\mathrm{k}=0}^{\infty} \overline{\mathrm{c}_{\mathrm{k}}} \rho^{\mathrm{v}}(\mathrm{k}-\mathrm{v}-1) \mathrm{L}_{-\mathrm{k}+\mathrm{v}+1} \sigma^{-\mathrm{v}}+\sum_{\mathrm{k}=1}^{\infty} \overline{\mathrm{d}_{\mathrm{k}}} \rho^{\mathrm{v}}(\mathrm{k}+\mathrm{v}+1) \mathrm{L}_{\mathrm{k}+\mathrm{v}+1} \sigma^{-\mathrm{v}} \\
&+\sum_{\mathrm{k}=0}^{\infty} \mathrm{h}_{\mathrm{k}} \rho^{-2 \mathrm{k}+\mathrm{v}}(\mathrm{k}-\mathrm{v}-1) \mathrm{L}_{-\mathrm{k}+\mathrm{v}+1} \sigma^{-\mathrm{v}}+\sum_{\mathrm{k}=0}^{\infty} \overline{\mathrm{h}_{\mathrm{k}}} \rho^{-2 \mathrm{k}-\mathrm{v}-2} \mathrm{~kL}_{-\mathrm{k}-\mathrm{v}-1} \sigma^{-\mathrm{v}} \\
&-\sum_{\mathrm{k}=0}^{\infty} \mathrm{m}_{\mathrm{k}} \rho^{\mathrm{v}}(\mathrm{k}+\mathrm{v}+1) \mathrm{L}_{\mathrm{k}+\mathrm{v}+1} \sigma^{-\mathrm{v}} \\
&=2 \Gamma \sum_{\mathrm{k}=0}^{\infty} \mathrm{L}_{\mathrm{k}} \rho^{2 \mathrm{k}+\mathrm{v}}(\mathrm{k}+\mathrm{v}+1) \mathrm{L}_{\mathrm{k}+\mathrm{v}+1} \sigma^{-\mathrm{v}} \\
&-2 \Gamma \sum_{\mathrm{k}=1}^{\infty} \mathrm{L}_{\mathrm{k}} \rho^{-2 \mathrm{k}+\mathrm{v}}(\mathrm{k}-\mathrm{v}-1) \mathrm{L}_{-\mathrm{k}+\mathrm{v}+1} \sigma^{-\mathrm{v}}-\Gamma^{\prime} \sum_{\mathrm{k}=0}^{\infty} \mathrm{L}_{\mathrm{k}} \rho^{\mathrm{v}}(\mathrm{k}-\mathrm{v}-1) \mathrm{L}_{-\mathrm{k}+\mathrm{v}+1} \sigma^{-\mathrm{v}} \\
&+\Gamma^{\prime} \sum_{\mathrm{k}=1}^{\infty} \mathrm{L}_{-\mathrm{k}} \rho^{\mathrm{v}}(\mathrm{k}+\mathrm{v}+1) \mathrm{L}_{\mathrm{k}+\mathrm{v}+1} \sigma^{-\mathrm{v}} \\
& \quad \mathrm{v}=1,2,3 \cdots
\end{aligned}
$$

where $\rho$ is the radius of the circle, which equals 1 at the lining-rock interface.

The positive power system of Equation (21) is determined as follows:

$$
\begin{gathered}
\sum_{\mathrm{k}=0}^{\infty} \mathrm{a}_{\mathrm{k}} \rho^{2 \mathrm{k}-\mathrm{v}}(\mathrm{k}-\mathrm{v}+1) \mathrm{L}_{\mathrm{k}-\mathrm{v}+1} \sigma^{\mathrm{v}}-\sum_{\mathrm{k}=1}^{\infty} \mathrm{b}_{\mathrm{k}} \rho^{-2 \mathrm{k}-\mathrm{v}}(\mathrm{k}+\mathrm{v}-1) \mathrm{L}_{-\mathrm{k}-\mathrm{v}+1} \sigma^{\mathrm{v}} \\
+\sum_{\mathrm{k}=1}^{\infty} \overline{\mathrm{a}_{\mathrm{k}}} \rho^{2 \mathrm{k}+\mathrm{v}-2} \mathrm{~kL}_{\mathrm{k}+\mathrm{v}-1} \sigma^{\mathrm{v}}-\sum_{\mathrm{k}=1}^{\infty} \overline{\mathrm{b}_{\mathrm{k}}} \rho^{-2 \mathrm{k}+\mathrm{v}-2} \mathrm{~kL}_{-\mathrm{k}+\mathrm{v}-1} \sigma^{\mathrm{v}} \\
-\sum_{\mathrm{k}=0}^{\infty} \overline{\mathrm{c}_{\mathrm{k}}} \rho^{-\mathrm{v}}(\mathrm{k}+\mathrm{v}-1) \mathrm{L}_{-\mathrm{k}-\mathrm{v}+1} \sigma^{\mathrm{v}}+\sum_{\mathrm{k}=1}^{\infty} \overline{\mathrm{d}_{\mathrm{k}}} \rho^{-\mathrm{v}}(\mathrm{k}-\mathrm{v}+1) \mathrm{L}_{\mathrm{k}-\mathrm{v}+1} \sigma^{\mathrm{v}} \\
=0 \\
\mathrm{v}=1,2,3 \ldots
\end{gathered}
$$

The negative power system of Equation (20) can be expanded as follows: 


$$
\begin{gathered}
\sum_{\mathrm{k}=0}^{\infty} \mathrm{a}_{\mathrm{k}} \rho^{2 \mathrm{k}+\mathrm{v}}(\mathrm{k}+\mathrm{v}+1) \mathrm{L}_{\mathrm{k}+\mathrm{v}+1} \sigma^{-\mathrm{v}}-\sum_{\mathrm{k}=1}^{\infty} \mathrm{b}_{\mathrm{k}} \rho^{-2 \mathrm{k}+\mathrm{v}}(\mathrm{k}-\mathrm{v}-1) \mathrm{L}_{-\mathrm{k}+\mathrm{v}+1} \sigma^{-\mathrm{v}} \\
+\sum_{\mathrm{k}=1}^{\infty} \overline{\mathrm{a}_{\mathrm{k}}} \rho^{2 \mathrm{k}-\mathrm{v}-2} \mathrm{~kL}_{\mathrm{k}-\mathrm{v}-1} \sigma^{-\mathrm{v}}-\sum_{\mathrm{k}=1}^{\infty} \overline{\mathrm{b}_{\mathrm{k}}} \rho^{-2 \mathrm{k}-\mathrm{v}-2} \mathrm{~kL}_{-\mathrm{k}-\mathrm{v}-1} \sigma^{-\mathrm{v}} \\
-\sum_{\mathrm{k}=0}^{\infty} \overline{\mathrm{c}_{\mathrm{k}}} \rho^{\mathrm{v}}(\mathrm{k}-\mathrm{v}-1) \mathrm{L}_{-\mathrm{k}+\mathrm{v}+1} \sigma^{-\mathrm{v}}+\sum_{\mathrm{k}=1}^{\infty} \overline{\mathrm{d}_{\mathrm{k}}} \rho^{\mathrm{v}}(\mathrm{k}+\mathrm{v}+1) \mathrm{L}_{\mathrm{k}+\mathrm{v}+1} \sigma^{-\mathrm{v}} \\
=0 \\
\mathrm{v}=1,2,3 \cdots
\end{gathered}
$$

where $\rho$ is the radius of the circle, which equals 0.8633 at the lining-atmosphere interface.

Equations (31)-(36) can be written as a set of linear equations:

$$
[[\mathrm{A}][\mathrm{B}][\mathrm{C}][\mathrm{D}][\mathrm{H}][\mathrm{M}]]_{(6 \mathrm{v}+3) \times(6 \mathrm{k}+4)}\left[\begin{array}{l}
{[\mathrm{a}]_{(\mathrm{k}+1) \times 1}} \\
{[\mathrm{~b}]_{\mathrm{k} \times 1}} \\
{[\mathrm{c}]_{(\mathrm{k}+1) \times 1}} \\
{[\mathrm{~d}]_{\mathrm{k} \times 1}} \\
{[\mathrm{~h}]_{(\mathrm{k}+1) \times 1}} \\
{[\mathrm{~m}]_{(\mathrm{k}+1) \times 1}}
\end{array}\right]=[\mathrm{F}]_{(6 \mathrm{k}+3) \times 1}
$$

where $[A],[B],[C],[D],[H],[M]$ represent the coefficients of the $a_{k}, b_{k}, c_{k}, d_{k}, h_{k}$ and $m_{k}$ terms in the equations, respectively. [F] represents the coefficient of the right side of each equation; [a], [b], [c], [d], $[\mathrm{h}]$, and $[\mathrm{m}]$ represent the unknowns, respectively. The coefficients $\mathrm{a}_{\mathrm{k}}, \mathrm{b}_{\mathrm{k}}, \mathrm{c}_{\mathrm{k}}, \mathrm{d}_{\mathrm{k}}, \mathrm{h}_{\mathrm{k}}$ and $\mathrm{m}_{\mathrm{k}}$ required in the final stress function are obtained by Equation (37).

As Equation (26) has $6 \mathrm{k}+4$ unknowns and $6 \mathrm{v}+3$ conditions, where $\mathrm{v} \rightarrow \infty$ and $\mathrm{k} \rightarrow \infty$, there cannot be a unique solution. It can be easily seen that Equation (37) is linearly related when $\mathrm{k} \rightarrow \infty$ and $\rho=1$. The number of conditions in the equation set is reduced from $(6 \mathrm{v}+3)$ to $(6 \mathrm{v}+2)$. Considering that $\varphi_{0}(\infty)=0$ and $\psi_{0}(\infty)=0$, the coefficients $h_{k}$ and $m_{k}$ can take any value if $k \rightarrow \infty$. It is indicated that $h_{\infty}$ and $m_{\infty}$ can take zero. Since the coefficients $h_{\infty}$ and $m_{\infty}$ are known, the number of unknown coefficients in the equation set is reduced from $(6 k+4)$ to $(6 k+2)$. The number of unknown coefficients equals the boundary conditions, in which all the coefficients have been determined uniquely. Equations (5), (6), (11) and (12) are obtained by the calculated coefficients, and the stress and deformation of the non-circular deep tunnel are obtained through Equations (2)-(4).

\section{Implementation}

In this section, the new complex variable method is applied to an example and a comparison is provided with FLAC finite difference software in order to verify the formula.

\subsection{Fundamental Assumption}

(a) The tunnel is assumed to have an infinite length; the surrounding rock mass is homogeneous, isotropic and linear elastic and without creep or viscous behaviors. (b) The tunnel's length and depth are assumed to far outweigh its diameter; the surrounding rock mass conforms to the plane strain condition $(k=3-4 v)$.

\subsection{Comparison of the New Analytical Solution with That of the Numerical Simulation Results}

The tunnel distribution diagram is presented and a 3916 zones and 13887 grid-points finite mesh calculation model was used to simulate stress and displacement distribution in Figure 2. The horizontal displacement of the finite mesh calculation model is constrained by the left and right boundary, the vertical displacement is constrained by the bottom boundary, and the top boundary is free and unconstrained. The numerical model is concerned with continuity of deformation and the stress field 
across the lining-rock interface due to the no-slip condition. The tunnel lining inside should be entirely free of stress.

The calculation parameters are shown in Table 1.

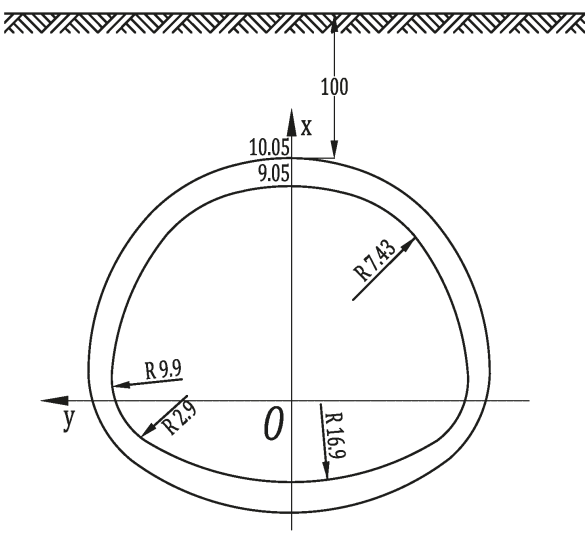

(a)

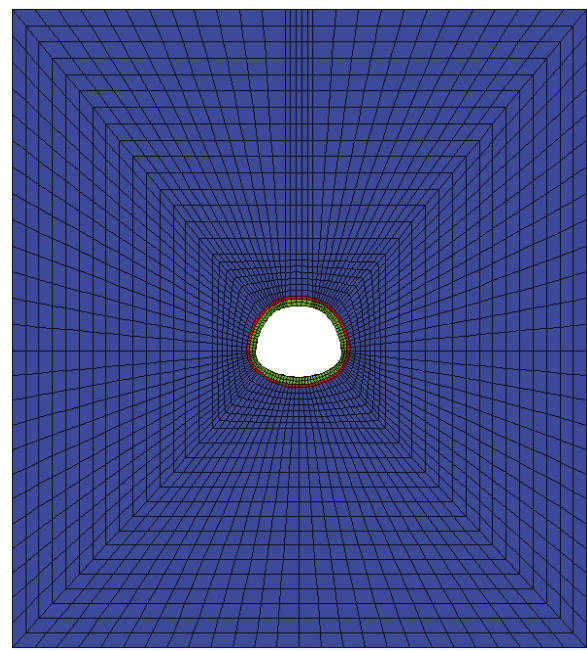

(b)

Figure 2. (a) Tunnel distribution diagram (Unit: $\mathrm{m}$ ) and (b) Finite mesh calculation model.

Table 1. Main physical parameter for tunnel calculation.

\begin{tabular}{ccccc}
\hline Material Type & $\begin{array}{c}\text { Elasticity Modulus E } \\
\mathbf{( M P a )}\end{array}$ & Poisson's Ratio v & Density (kN· m $\mathbf{~}^{\mathbf{3}}$ ) & $\begin{array}{c}\text { Lateral Pressure } \\
\text { Coefficient K }\end{array}$ \\
\hline Shale & 25,000 & 0.3 & 26 & 0.5 \\
Lining & 30,000 & 0.2 & 25 & 0.5 \\
\hline
\end{tabular}

According to Lv's [16,17] method and the geometry of the tunnel (in Figure 2), the conformal mapping function (Equation (1)) was determined and provided by a self-programming optimal design software as follows:

$$
\mathrm{w}(\zeta)=8.0339\left(\zeta+0.3121-0.0697 \zeta^{-1}+0.0338 \zeta^{-2}-0.0087 \zeta^{-3}-0.0068 \zeta^{-4}\right)
$$

where coefficient $\mathrm{k}=4$ is close enough to Equation (1). The radius $\rho=0.8633$ is related to $\zeta$ by $\zeta=\rho \sigma$, where $\sigma=\exp (i \theta)$. It is assumed that the tunnel lining inside $\left(\mathrm{L}_{1}\right)$ can be mapped conformally onto a circle $\left(R_{1}\right)$. When the radius $\rho=1$ the $L_{2}$ can be mapped conformally onto $R_{2}$.

As an example, the boundary condition across the lining-rock interface and tunnel lining inside can be determined by Equations (13)-(15). The boundary conditions at infinity can be expressed through Equations (9) and (10).

Based on Equation (37), a simple computer program written by MATLAB was applied to solve the problem.

As the coordinates of the analytical solution and numerical simulation are different, it is necessary to rewrite the results. The comparison of the rewritten results between the analytical solution and numerical simulation are shown below.

Figure 3 shows the circumferential stress along the rock-lining interface predicted by the new analytical solution and the FLAC finite difference software. It can be observed that the maximum 
circumferential stress happens at a position of an 85-degree angle (i.e., the widest part of the tunnel) and, moreover, the circumferential stress of the new analytical solution has not declined rapidly, but creates a stress concentration at $\alpha=115^{\circ}$. The circumferential stress along the inner lining periphery is presented in Figure 4a, demonstrating good agreement between the new analytical solution and the numerical simulation apart from an 85-degree angle. Normal stress and shear stress along the inner lining periphery is illustrated in Figure $4 b, c$; the maximum value of the analytical solution for normal stress and shear stress are about $200 \mathrm{KPa}$ and $100 \mathrm{KPa}$, respectively, and the maximum value of the numerical simulation of normal stress and shear stress are about $350 \mathrm{KPa}$ and $600 \mathrm{KPa}$. The analytical solution is smaller than the numerical simulation. The analytical solution is more accurate than the numerical simulation.

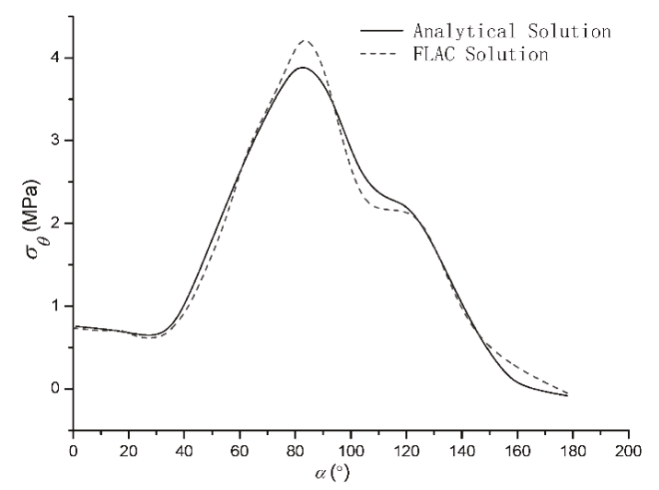

(a)

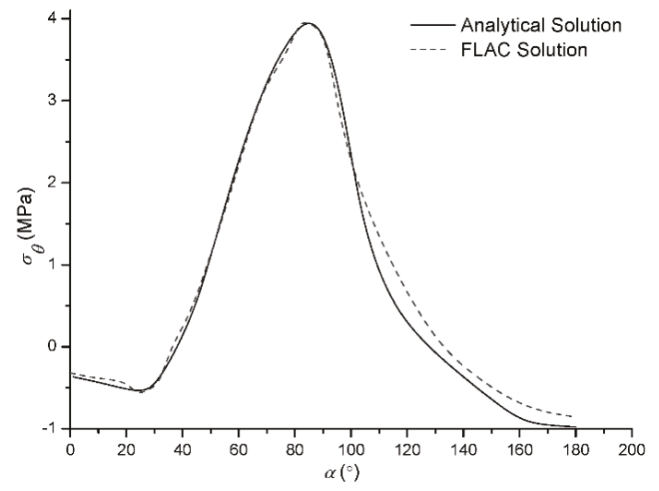

(b)

Figure 3. Circumferential stress along the rock-lining interface (a) from the lining side (b) from the rock side. 


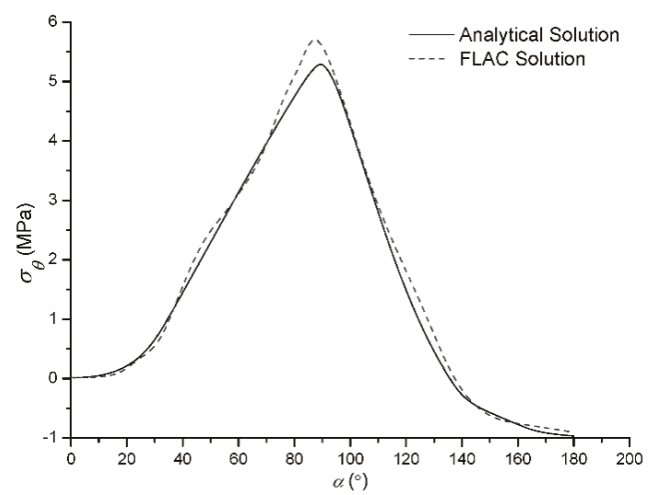

(a)

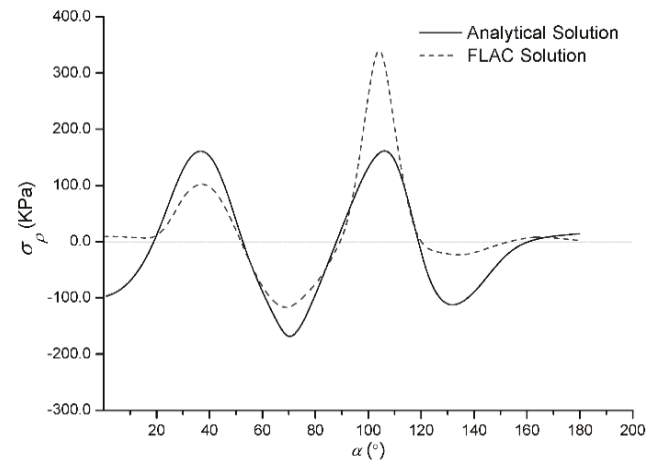

(b)

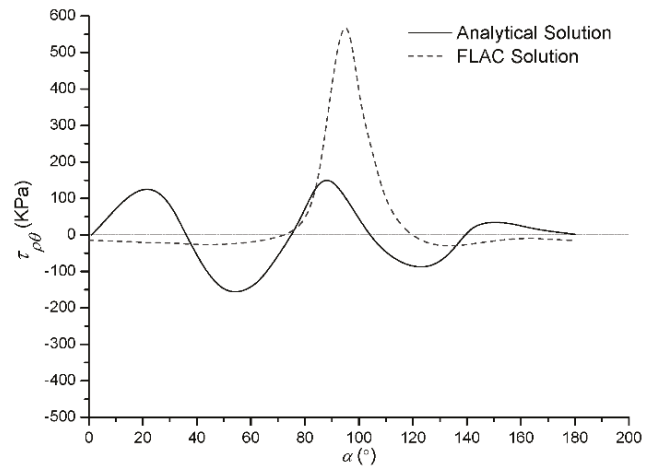

(c)

Figure 4. Stress along the inner lining periphery (a) circumferential stress (b) normal stress (c) shear stress.

Figure 5 shows the radial displacement of the tunnel along the rock-lining interface predicted by the analytical solution. It could be demonstrated that the radial displacement along the rock-lining interface was in good agreement with the displacement boundary condition, which proves its high accuracy. 


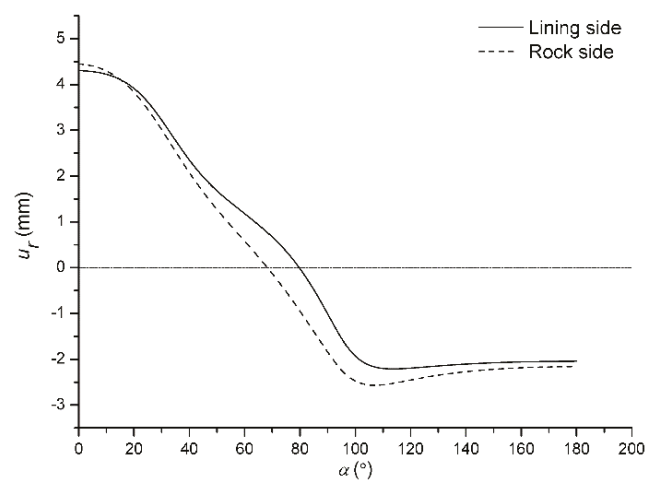

Figure 5. Radial displacement along the rock-lining interface predicted by the analytical solution.

The radial displacement of the tunnel along the inner lining periphery is illustrated in Figure 6, which shows that the radial displacement of the analytical solution and numerical simulation are all zero at $\alpha=75^{\circ}$. It was demonstrated that the numerical solution agrees well with the analytical solution. Considering the results of Figure 5, the analytical solution had good agreement with the displacement boundary condition, thus the analytical solution results were reasonable.

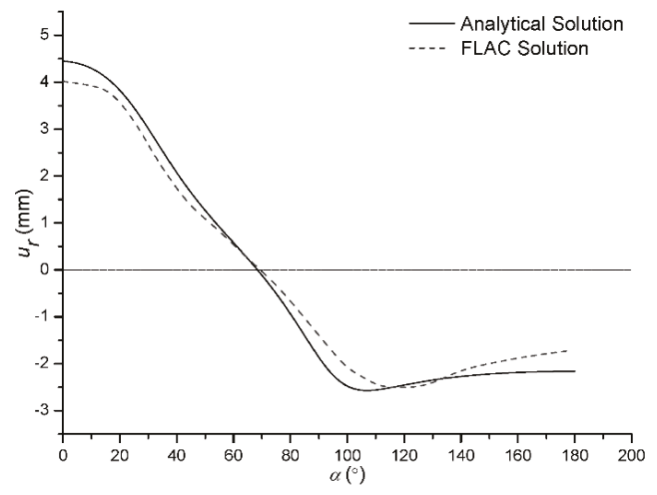

Figure 6. Radial displacement along the inner lining periphery.

The discrepancies between the new analytical solution and the numerical simulation described above, especially Figure 4, may be due to the fact that the grid size in the numerical modeling is not small enough to produce accurate results. The stress information of the numerical simulation is stored in zones not in grid points, and the stress cannot be accurately expressed along a specified boundary.

\section{Conclusions}

In this paper, the stress and displacement of a non-circular deep tunnel and within their lining supports were studied using a new analytical solution, which is based on the basic theory of complex variables and plane elasticity [17], and the following conclusions can be made.

The analytic functions were exactly established to predict the stress and displacement distribution of the non-circular deep tunnel within their lining supports, but it is obviously not entirely true that the stress and displacement value is only determined by the in-situ stress boundary conditions and coefficient of the elasticity modulus, Poisson's ratio, lateral pressure and material density. 
The analytical solution for radial displacement was smaller than the numerical simulation results, however, and further study will be needed to develop these functions.

Due to the fact that the grid size in modeling was not small enough to produce accurate results and stress information was stored in zones, not in grid points in the numerical simulation, the stress cannot be accurately expressed along a specified boundary. But the analytical solution results were not affected by grid size and zones in the numerical modeling.

The curves of the stress value showed that the new analytical solution and numerical simulation were in reasonable agreement. Both solutions predicted a normal stress concentration at the lower and upper corners of the tunnel, and both maximum circumferential stress results occurred in the widest part of the tunnels. The normal and shear stress values of the tunnel along the inner lining periphery were almost zero, which proved its high accuracy.

Although numerical simulation is the main tool for solving tunnel excavation problems, especially non-circular tunnels, the complex variable method can provide another way to solve non-circular tunnel excavation problems in a faster and more accurate way.

Author Contributions: Y.L. and S.C. conceived and theoretical research; Y.L. performed the formula derivation; Y.L. and S.C. verified results by numerical simulation; Y.L. wrote the paper; S.C. checked and proofread the paper.

Acknowledgments: We thank the Fundamental Research Funds for the Central Universities (SWJTU11ZT33) and the Innovative Research Team in University (IRT0955) for their sponsorship of this project, and thanks to Professor Chen, who guided and revised the paper.

Conflicts of Interest: The authors declare no conflict of interest.

\section{References}

1. Bobet, A. Analytical solutions for shallow tunnels in saturated ground. J. Eng. Mech. 2001, 127, 1258-1266. [CrossRef]

2. Bobet, A. Effect of pore water pressure on tunnel support during static and seismic loading. Tunn. Undergr. Space Technol. 2003, 18, 377-393. [CrossRef]

3. Lee, I.M.; Nam, S.W. The study of seepage forces acting on the tunnel lining and tunnel face in shallow tunnels. Tunn. Undergr. Space Technol. 2001, 16, 31-40. [CrossRef]

4. Timoshenko, S.P.; Goodier, J. Theory of Elasticity, 2nd ed.; McGraw-Hill: New York, NY, USA, 1951.

5. Muskhelishvili, N.I.; Radok, J.R.M. Some Basic Problems of the Mathematical Theory of Elasticity; Cambridge University Press: Cambridge, UK, 1953.

6. Exadaktylos, G.; Stavropoulou, M. A closed-form elastic solution for stresses and displacements around tunnels. Int. J. Rock Mech. Min. Sci. 2002, 39, 905-916. [CrossRef]

7. Exadaktylos, G.E.; Liolios, P.A.; Stavropoulou, M.C. A semi-analytical elastic stress displacement solution for notched circular openings in rocks. Int. J. Solids Struct. 2003, 40, 1165-1187. [CrossRef]

8. Verruijt, A. A complex variable solution for a deforming circular tunnel in an elastic half-plane. Int. J. Numer. Anal. Methods Geomech. 1997, 21, 77-89. [CrossRef]

9. Verruijt, A. Deformations of an elastic half plane with a circular cavity. Int. J. Solids Struct. 1998, 35, 2795-2804. [CrossRef]

10. Zhao, G.P.; Yang, S.L. Analytical solutions for rock stress around square tunnels using complex variable theory. Int. J. Rock Mech. Min. Sci. 2015, 80, 302-307. [CrossRef]

11. Kargar, A.R.; Rahmannejad, R.; Hajabasi, M.A. A semi-analytical elastic solution for stress field of lined non-circular tunnels at great depth using complex variable method. Int. J. Solids Struct. 2014, 51, 1475-1482. [CrossRef]

12. Li, Y.S.; Chen, S.G. A Complex Variable Solution for Stress and Deformation of Lining in a Non-Circular Tunnel. Scientific Research Report. Southwest Jiaotong University: Chengdu, China, 2018. (In Chinese)

13. Liu, F.S. Seepage Studies of Tunnel Water-Enriched Region Based on Fluid-Solid Coupling Theory and Complex Analysis. Ph.D. Thesis, South China University, Guangzhou, China, 2012. (In Chinese)

14. Wang, G.Q. Elastic Mechanics; China Railway Publishing House: Beijing, China, 2008. (In Chinese)

15. Chen, Z.Y. Analytical Method in Mechanical Analysis of Surrounding Rock, 1st ed.; China Coal Industry Publishing House: Beijing, China, 1994. (In Chinese) 
16. Lv, Z.A.; Zhang, L.Q. Complex Function Method for Mechanical Analysis of Underground Tunnel, 1st ed.; Science Press: Beijing, China, 2007. (In Chinese)

17. Brown, J.W.; Churchill, R.V. Complex Variables and Applications, 9th ed.; China Machine Press: Beijing, China, 2015. (In Chinese)

(C) (1) BY
(C) 2018 by the authors. Licensee MDPI, Basel, Switzerland. This article is an open access article distributed under the terms and conditions of the Creative Commons Attribution (CC BY) license (http://creativecommons.org/licenses/by/4.0/). 


\title{
4D Remeshing Using a Space-Time Finite Element Method for Elastodynamics Problems
}

\author{
Serge Dumont ${ }^{1}$, Franck Jourdan ${ }^{2, *}$ and Tarik Madani ${ }^{2}$ \\ 1 Institut Montpelliérain Alexander Grothendieck, University Montpellier, CNRS, 34090 Montpellier, France; \\ serge.dumont@umontpellier.fr \\ 2 Laboratory of Mechanics and Civil Engineering, University Montpellier, CNRS, 34090 Montpellier, France; \\ tarik.madani@umontpellier.fr \\ * Correspondence: franck.jourdan@umontpellier.fr; Tel.: +33-467-149-633
}

Received: 10 March 2018; Accepted: 22 May 2018; Published: 25 May 2018

\begin{abstract}
In this article, a Space-Time Finite Element Method (STFEM) is proposed for the resolution of mechanical problems involving three dimensions in space and one in time. Special attention will be paid to the non-separation of the space and time variables because this kind of interpolation is well suited to mesh adaptation. For that purpose, we have developed a technique of $4 \mathrm{D}$ mesh generation adapted to space-time remeshing. A difficulty arose in the representation of $4 \mathrm{D}$ finite elements and meshes. This original technique does not require coarse-to-fine and fine-to-coarse mesh-to-mesh transfer operators and does not increase the size of the linear systems to be solved, compared to traditional finite element methods. Space-time meshes are composed of simplex finite elements. Computations are carried out in the context of the continuous Galerkin method. We have tested the method on a linearized elastodynamics problem. Our technique of mesh adaptation was validated on elementary examples and applied to a problem of mobile loading. The convergence and stability of the method are studied and compared with existing methods. This work is a first implementation of $4 \mathrm{D}$ space-time remeshing. A stability criterion for the method is established, as well as a convergence rate of about two. Using simplex elements, it is possible to develop a technique of mesh adaptation able to follow a mobile loading zone.
\end{abstract}

Keywords: finite elements; space-time; elastodynamics; mesh adaptation

\section{Introduction}

The STFEM (Space-Time Finite Element Method) can be regarded as an extension of the classical finite element method, applied to a boundary problem resulting from a non-stationary problem. Currently, several approaches exist. One can quote for example the Large Time INcrement method (LATIN [1]), the discontinuous Galerkin method [2-4] and our method, which is a continuous Galerkin method [5,6]. In most publications on the discontinuous Galerkin method, like in [7], the interpolation functions are assumed to be a product of functions of space variables and functions of time variables. We will see in this paper that special attention will be paid to the non-separation of the space and time variables. The reason for this choice is not motivated by the accuracy of the numerical results, but rather by what constitutes the aim of our study: remeshing. We will see that this kind of interpolation is well suited to mesh adaptation. The space-time mesh adaptation we developed is based on a method of mesh generation not structured in space and time. The construction of 4D meshes collides with the limits of representation. To overcome this difficulty, we propose an automatic method of construction inspired by what can be achieved in 2D and 3D. Our technique of mesh adaptation was applied to a problem of mobile load like contact forces. Our approach makes possible the building of an evolutionary mesh able to follow the clamping zone. 
Moreover, this technique does not require a mesh-to-mesh transfer operator and allows the preservation of the exact sizes of the linear systems on each space-time slab.

Let us note that one of the drawbacks of the STFEM such as defined in the works of $[8,9]$ is the size of the linear systems to be solved, since it is necessary to solve the full 4D problem at once. The use of a laminated mesh allows us to avoid the assembling of the total matrix of the problem and permits us to consider only submatrices. This drastically reduces the size of the systems to be solved. The size of these linear systems is exactly the same as that obtained in the case of approaches coupling an incremental method of finite differences type to solve time integration, with the "classical" finite element method being used to solve the space problem.

Another large group of methods is based on semi-discretisation, whereby finite elements are used in space and finite differences are used in time. Even if this well-known technique is simpler to use in a classical framework, the remeshing required is expensive due to the necessity of the construction of interpolation/restriction operators between the grids.

The paper is organized as follows. In Section 2, the elastodynamics problem is formulated, and the space-time finite element method is developed. The $4 \mathrm{D}$ mesh generation is presented in Section 3, and a paragraph is specially devoted to adaptive mesh refinement. Numerical results are presented and discussed in Section 4.

\section{Principle of the Method}

We consider the motion of an elastic body within the small perturbations hypothesis. Let $\Omega$ be the set taken up by the body and $[0, T]$ a time interval. The body is submitted to volume force density $f_{d}$, boundary force density $F_{d}$ on its boundary part $\partial_{1} \Omega$ and imposed displacements $u_{d}$ on its boundary part $\partial_{0} \Omega\left(\partial \Omega=\partial_{0} \Omega \cup \partial_{1} \Omega, \partial_{0} \Omega \cap \partial_{1} \Omega=\varnothing\right)$. The dynamic problem is: seek the displacement $u$ and the Cauchy stress tensor $\sigma$ such that:

$$
\begin{cases}\operatorname{div}(\sigma(x, t))+f_{d}(x, t)=\rho \ddot{u}(x, t) & \forall(x, t) \in \Omega \times] 0, T[ \\ \sigma(x, t) n(x, t)=F_{d}(x, t) & \forall(x, t) \in \partial_{1} \Omega \times[0, T] \\ u(x, t)=u_{d}(x, t) & \forall(x, t) \in \partial_{0} \Omega \times[0, T] \\ u(x, t)=u_{0}(x) & \forall(x, t) \in \Omega \times\{0\} \\ \dot{u}(x, t)=\dot{u}_{0}(x) & \forall(x, t) \in \Omega \times\{0\} \\ \sigma(x, t)=a \varepsilon(x, t) & \forall(x, t) \in \Omega \times[0, T]\end{cases}
$$

where $\rho$ is the specific mass, $\ddot{u}$ is the second derivative of the displacement with respect to time, $u_{0}$ is the initial displacement, $\dot{u}_{0}$ is the initial velocity, $a$ is the Hooke tensor and $\varepsilon$ is the infinitesimal linear strain tensor. The aim of this study is to use a finite element method. Then, the previous dynamic problem has to be considered as a boundary problem on the time interval $[0, T]$. For that purpose, as in the cases of the discontinuous Galerkin method $[2,10,11]$ and the LArge Time INcrement (LATIN) method $[1,12]$, the variational formulation is written on the whole space-time domain $\Omega \times[0, T]$. The variational formulation of the previous boundary problem can be written as follows:

Find $u \in U_{a d}$ such that:

$$
\begin{aligned}
& \int_{0}^{T} \int_{\Omega}\left(\rho \ddot{u} v+a \varepsilon(u): \varepsilon(v)-f_{d} v\right) d x d t=\int_{0}^{T} \int_{\partial_{1} \Omega} F_{d} v d s d t \\
& \forall v \in U_{a d}^{0}
\end{aligned}
$$

where $U_{a d}$ is the set of displacements, regular enough, which verifies the boundary kinematic conditions and the initial conditions, $v$ is the virtual displacement and $U_{a d}^{0}$ is the set of virtual displacements, regular enough, which verify boundary kinematic conditions only. 
The first term on the left-hand side of Equation (2) is integrated by parts in time in order to determine the first derivative of $u$ and the initial velocity. It gives:

$$
\begin{aligned}
\int_{0}^{T} \int_{\Omega} \rho \ddot{u} v d x d t= & -\int_{0}^{T} \int_{\Omega} \rho \dot{u} \dot{v} d x d t \\
& +\int_{\Omega}\left[\rho \dot{u}_{T}(x) v(x, T)-\rho \dot{u}_{0}(x) v(x, 0)\right] d x
\end{aligned}
$$

where $\dot{u}_{T}(x)$ is the velocity at time $t=T$.

The space-time finite element method (STFEM) was firstly proposed in [5,6]. Their discretisation used structured space-time meshes obtained as the Cartesian product of spatial elements and a time interval, which is not generally suitable for space-time mesh adaptations. Since then, numerous papers on STFEM have been published. Most of them, like [11,13], deal with the discontinuous Galerkin method in time, but the discretisation also uses structured meshes obtained as the Cartesian product of spatial elements and a time interval. However, the STFEM proposed in [2] has been developed on unstructured meshes. It also employs the discontinuous Galerkin method in time and incorporates stabilizing terms of the least squares type. The space and time discontinuities of all variables are taken into account. In our study, we use a continuous Galerkin method. Classical Lagrange polynomials are used. The finite elements are isoparametric. On a space-time finite element $E_{e}$ (Figure 1), the displacement verifies:

$$
u(x, t)=\sum_{i=1}^{n_{e}} \varphi_{i}^{e}(x, t) u_{i}^{e}
$$

where $n_{e}$ is the total number of nodes of the element $E_{e}, \varphi_{i}^{e}$ are the interpolation functions and $u_{i}^{e}$ the nodal displacements. Using matrix notation, one has:

$$
u(x, t)=N_{e}(x, t) U_{e} \text { where } U_{e}=\left(u_{1}^{e}, \ldots, u_{n_{e}}^{e}\right)^{T} \text { and } N_{e}(x, t)=\left(\varphi_{1}^{e}(x, t), \ldots, \varphi_{n_{e}}^{e}(x, t)\right)
$$

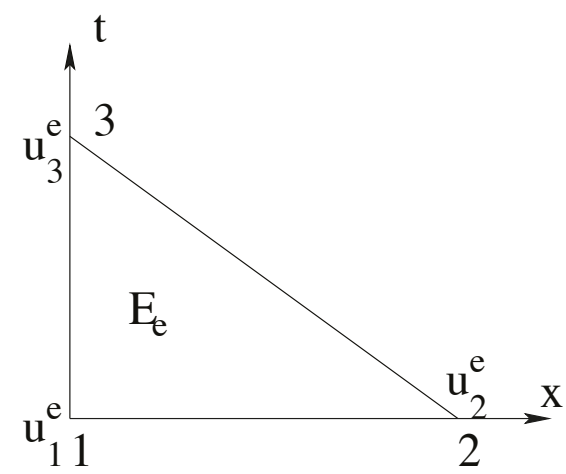

Figure 1. 2D space-time finite element.

The same interpolation is used for the virtual displacement $v$. Then:

$$
v(x, t)=N_{e}(x, t) V_{e} \text { where } V_{e}=\left(v_{1}^{e}, \ldots, v_{n_{e}}^{e}\right)^{T}
$$

Let $p$ be the total number of space-time elements; the previous discretization gives:

$$
\int_{0}^{T} \int_{\Omega} \rho \dot{u} \dot{v} d x d t=\sum_{e=1}^{p} V_{e}^{T} M_{\mathcal{e}} U_{e}
$$


where:

$$
M_{e}=\int_{0}^{T} \int_{E_{e}} \rho \frac{\partial N_{e}^{T}}{\partial t} \frac{\partial N_{e}}{\partial t} d x d t
$$

is the elementary matrix relative to the inertia forces. One can notice that $M_{e}$ is symmetric. Concerning the discretization of the initial and final impulses' contributions, one has:

$$
\int_{\Omega}\left[\rho \dot{u}_{T}(x) v(x, T)-\rho \dot{u}_{0}(x) v(x, 0)\right] d x=\sum_{e=1}^{p} V_{e}^{T} \Lambda_{e}
$$

where $\Lambda_{e}$ is the elementary vector relative to the initial and final impulses. It is defined by:

$$
\Lambda_{e}=\left[\int_{E_{e} \cap \Omega_{T}} \rho N_{e}^{T} \dot{u}_{T} d x-\int_{E_{e} \cap \Omega_{0}} \rho \dot{u_{0}} d x\right]
$$

where $\Omega_{0}$ is the domain at time $t=0$ and $\Omega_{T}$ is the domain at time $t=T$.

Similarly, let $B_{e}$ be the matrix such that:

$$
\varepsilon(u(x, t))=B_{e}(x, t) U_{e},
$$

the virtual works of internal and external forces are respectively discretized by:

$$
\int_{0}^{T} \int_{\Omega} a \varepsilon(u): \varepsilon(v) d x d t=\sum_{e=1}^{p} V_{e}^{T} K_{e} U_{e}
$$

and:

$$
\int_{0}^{T} \int_{\Omega} f_{d} v d x d t+\int_{0}^{T} \int_{\partial_{1} \Omega} F_{d} v d s d t=\sum_{e=1}^{p} V_{e}^{T} F_{e}
$$

where the elementary matrix $K_{e}$ relative to internal forces is:

$$
K_{e}=\int_{0}^{T} \int_{E_{e}} B_{e}^{T} a B_{e} d x d t
$$

and the elementary vector $F_{e}$ relative to external forces is:

$$
F_{e}=\int_{0}^{T} \int_{E_{e}} N_{e}^{T} f_{d} d x d t+\int_{0}^{T} \int_{E_{e} \cap \partial_{1} \Omega} N_{e}^{T} F_{d} d s d t .
$$

This space-time discretization leads to the following linear system:

$$
\left(\left[\widetilde{M_{u}}\right]+\left[\widetilde{K_{u}}\right]\right)\{U\}=\left\{F_{u}\right\}+\{\Lambda\}
$$

where $\left[\widetilde{M_{u}}\right]$ is the assembled matrix relative to the inertia forces, $\left[\widetilde{K_{u}}\right]$ is the assembled matrix relative to the internal forces, $\left\{F_{u}\right\}$ is the nodal vector of external forces, $\Lambda$ is the nodal vector of impulses and $\{U\}$ is the nodal vector of displacements. One can note that the matrices $\left[\widetilde{M_{u}}\right]$ and $\left[\widetilde{K_{u}}\right]$ are symmetric. In order to have band matrices, and because we have in mind making computations incrementally in time, the meshes are built to be stratified in time, as in Figure 2. Moreover, the node numbering is conducted in such a way that all nodes in a same stratum have close numbers, then the left-hand side of the system (16) verifies. 


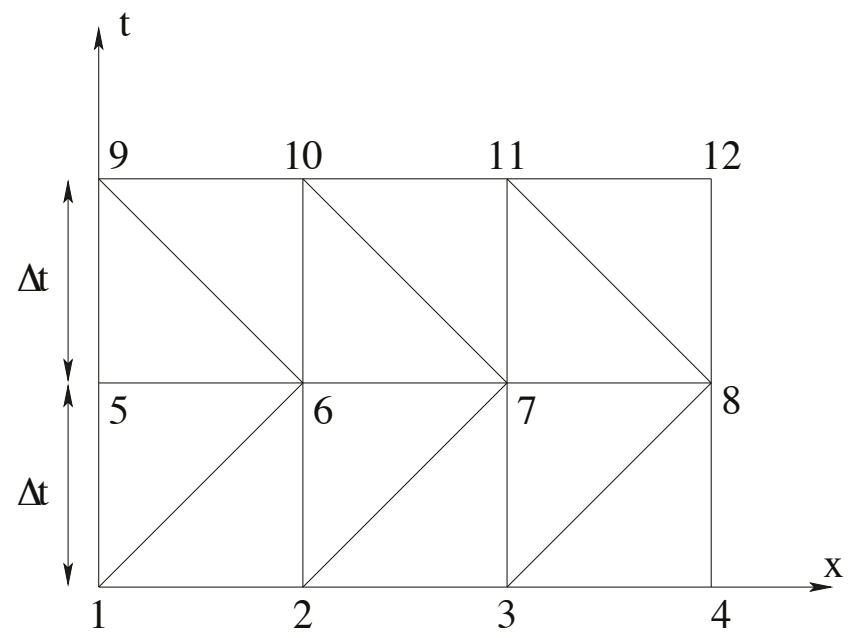

Figure 2. 2D regular space-time mesh.

$$
\begin{gathered}
\left(\left[\widetilde{M_{u}}\right]+\left[\widetilde{K_{u}}\right]\right)\{U\}=[T]\{U\}= \\
\left(\begin{array}{cccccc}
{\left[T_{11}\right]} & {\left[T_{12}\right]} & 0 & 0 & 0 & 0 \\
{\left[T_{21}\right]} & {\left[T_{22}\right]} & {\left[T_{23}\right]} & 0 & 0 & 0 \\
0 & {\left[T_{32}\right]} & {\left[T_{33}\right]} & {\left[T_{34}\right]} & 0 & 0 \\
\cdot & \cdot & \cdot & \cdot & \cdot & \cdot \\
0 & 0 & 0 & {\left[T_{n / n-1}\right]} & {\left[T_{n / n}\right]} & {\left[T_{n / n+1}\right]} \\
0 & 0 & 0 & 0 & {\left[T_{n+1 / n}\right]} & {\left[T_{n+1 / n+1}\right]}
\end{array}\right)\left(\begin{array}{c}
\left\{U_{0}\right\} \\
\left\{U_{1}\right\} \\
\cdot \\
\cdot \\
\cdot \\
\left\{U_{n}\right\}
\end{array}\right) .
\end{gathered}
$$

Using the space-time mesh described in Figure 2, one has:

$$
\left\{U_{j}\right\}=\left(\begin{array}{l}
u_{j+1} \\
u_{j+2} \\
u_{j+3} \\
u_{j+4}
\end{array}\right) \text { for } j=0, \ldots, n .
$$

With this numbering, the total matrix $[T]$ and the sub-matrices $\left[T_{i j}\right]$ are band matrices.

\section{Comments:}

- Choosing a Lagrange interpolation for displacements implies that displacements are continuous, but the velocities are discontinuous. As a consequence, integration by parts as in (3) is not totally rigorous, and it could be necessary to use the discontinuous Galerkin formulation, which amounts to writing the derivative of velocity within the theory of distributions. We will preserve the formulation in (3), knowing that the error here is of the same order as in the case of traditional finite elements in space. Indeed, with a Lagrange interpolation local displacements are continuous, whereas the global deformation is discontinuous.

- Even if it is not absolutely necessary, the advantage of using a laminated mesh such as defined here is that it becomes possible, rather than assembling the total matrix $[T]$, to only assemble the sub-matrices $\left[T_{i j}\right]$. This considerably reduces the size of the systems to be solved. More precisely, the size of these linear systems is exactly the same as that obtained in the case of approaches based on the coupling of finite incremental differences in time with finite elements in space. Moreover, the method is not limited to simplex elements, and the spatial position of each set of nodes can vary from one time plane to the other. It is one of the main advantages of the method. 
- We specify that the nodal vector relating the boundary conditions with velocity $\{\Lambda\}$ is written as:

$$
\{\Lambda\}=\left(\left\{\Lambda_{0}\right\}, 0, \ldots, 0,\left\{\Lambda_{n}\right\}\right)^{T}
$$

where $\left\{\Lambda_{0}\right\}$ is given starting from conditions of initial velocity while $\left\{\Lambda_{n}\right\}$ is unknown. Consequently, the resolution of System (16) is the following:

The first system of equations,

$$
\left[T_{11}\right]\left\{U_{0}\right\}+\left[T_{12}\right]\left\{U_{1}\right\}=\left\{F_{0}\right\}+\left\{\Lambda_{0}\right\}
$$

provides $\left\{U_{1}\right\}$; the system of equations:

$$
\left[T_{i / i-1}\right]\left\{U_{i-2}\right\}+\left[T_{i / i}\right]\left\{U_{i-1}\right\}+\left[T_{i / i+1}\right]\left\{U_{i}\right\}=\left\{F_{i-1}\right\} \quad 2 \leq i \leq n,
$$

provides the displacements $\left\{U_{i}\right\}$; and the last system of equations,

$$
\left[T_{n+1 / n}\right]\left\{U_{n-1}\right\}+\left[T_{n+1 / n+1}\right]\left\{U_{n}\right\}=\left\{F_{n}\right\}+\left\{\Lambda_{n}\right\},
$$

gives $\left\{\Lambda_{n}\right\}$.

- Finally, the matrices of resolution $\left[T_{i / i+1}\right]$ are generally non-symmetric, even if the total matrix $[T]$ is symmetric. Thus, for the algorithm presented above, a non-symmetrical solver should be used. This can appear penalizing in terms of computing time. However, since the final objective is to use this approach to deal with problems of contact with friction and since the nonlinear resolution we developed in [14] is of the Gauss-Seidel nonlinear type, asymmetries do not affect computing time.

\section{4D Mesh and Remeshing}

In order to propose a remeshing technique, it is firstly necessary to be able to build 4D meshes. Obtaining only one 4D finite element does not pose real problems, even if some difficulties in graphic representation arise (see Figure 3). On the contrary, building a 4D mesh, even the most elementary is far from being commonplace, except in the case of regular meshes formed by finite elements of multiplexing type (functions of interpolation obtained as the products of functions of space by functions of time). However, in the general case and in particular with the problem of remeshing, which is what is of interest here, the meshing remains an issue.

\section{1. $4 \mathrm{D}$ Mesh Generation}

Figure 3 identifies parts of elementary 2D, 3D and 4D meshes with their node numbering. One denotes by $n_{0}$ the total number of nodes at time $t=0$ of the entire space mesh, and we assume this total number is the same at time $t=h$.

For the 2D mesh, the connectivities are

$i, j, n_{0}+i$

$j, n_{0}+i, n_{0}+j$.

For the 3D mesh, the connectivities are

$i, j, k, n_{0}+i$

$j, k, n_{0}+i, n_{0}+j$

$k, n_{0}+i, n_{0}+j, n_{0}+k$.

Using the previous building of connectivities, obtained by circular permutations, we propose the following generalization of connectivities for the 4D mesh.

$$
\begin{aligned}
& i, j, k, l, n_{0}+i \\
& j, k, l, n_{0}+i, n_{0}+j
\end{aligned}
$$




$$
\begin{aligned}
& k, l, n_{0}+i, n_{0}+j, n_{0}+k \\
& l, n_{0}+i, n_{0}+j, n_{0}+k, n_{0}+l .
\end{aligned}
$$

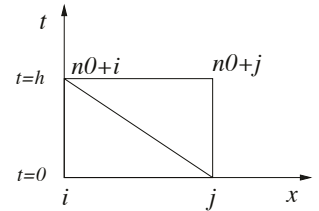

$2 \mathrm{D}$

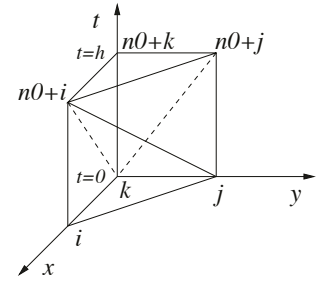

$3 \mathrm{D}$

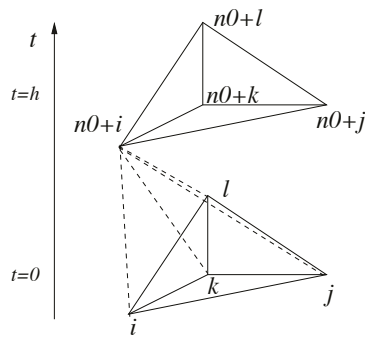

$4 \mathrm{D}$

Figure 3. 2D, 3D and 4D space-time mesh.

This $4 \mathrm{D}$ mesh is constituted by four hypertetrahedrons (The hypertetrahedron is the four-dimensional tetrahedron. Other names of this element type are simplex or pentatope). We propose to build 4D space-time meshes, resulting from unspecified 3D space meshes, by applying the building technique developed above, for each 3D finite element of the 3D space mesh. However, in this case, it must be checked that the total space-time volume is covered by the $4 \mathrm{D}$ mesh. For this purpose, we computed the sum of the volumes of the hypertetrahedrons of the 4D mesh and compared it with the total volume generated by the 3D object multiplied by the time interval.

For a 3D mesh made up of tetrahedrons, the interfaces between the elements are triangles. In 4D, these interfaces are tetrahedrons (see the diagram at the bottom of Figure 4). Therefore, we must thus check that for our technique of mesh generation, all couples of adjoining $4 \mathrm{D}$ finite elements have a common tetrahedron. As we use a building technique containing circular permutations, it is necessary to respect a particular order in the numbering of the nodes of each 3D finite element. A way of doing this is to arrange the nodes of each 3D element in ascending order. Table 1 gives an example of a table of connectivities for an elementary $4 \mathrm{D}$ mesh, resulting from the 3D space mesh represented by the left-hand diagram of Figure 4. Let us note that this $4 \mathrm{D}$ mesh contains eight finite elements, against two for the $3 \mathrm{D}$ mesh source, and that $n_{0}=5$. It is observed that the connectivities are arranged in ascending order. In this case, it is checked that the tetrahedra filling the space-time interface (diagram at the right-hand of Figure 4 ) are common to the adjoining elements. Indeed, Elements 1 and 5 contain the tetrahedron $(1 ; 2 ; 4 ; 6)$, and Elements 2 and 6 contain the tetrahedron $(2 ; 4 ; 6 ; 7)$. Lastly, Elements 4 and 7 contain the tetrahedron $(4 ; 6 ; 7 ; 9)$. Let us notice that the tetrahedron $(1 ; 2 ; 3 ; 4 ; 5)$ at time $t$ and the related tetrahedron $(6 ; 7 ; 8 ; 9 ; 10)$ (not represented) at time $t+\Delta t$ could also have different shapes and could be localized at different places (see for example Figure 5 ). In this case, the triangles $(1 ; 2 ; 4)$ and $(6 ; 7 ; 9)$ in the right part of Figure 4 can be different. 

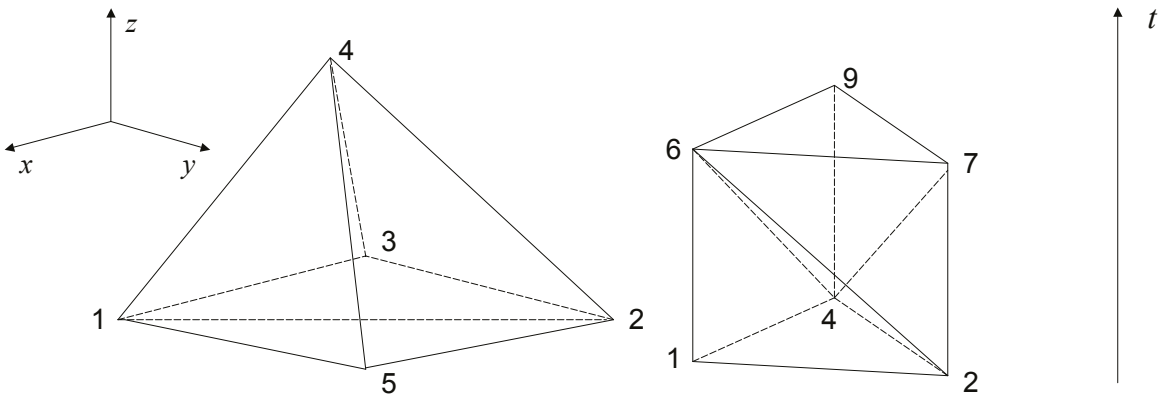

Figure 4. 3D initial mesh (scheme at the left); part of the $4 \mathrm{D}$ mesh generated by the triangle $(1 ; 2 ; 4)$ common to the two finite elements of the 3D initial mesh (scheme at the right).

Table 1. Table of connectivities of the $4 \mathrm{D}$ space-time mesh resulting from the elementary $3 \mathrm{D}$ space mesh of Figure 4.

\begin{tabular}{cccccc}
\hline Element Number & Node $\mathbf{1}$ & Node 2 & Node 3 & Node 4 & Node 5 \\
\hline 1 & 1 & 2 & 3 & 4 & 6 \\
2 & 2 & 3 & 4 & 6 & 7 \\
3 & 3 & 4 & 6 & 7 & 8 \\
4 & 4 & 6 & 7 & 8 & 9 \\
5 & 1 & 2 & 4 & 5 & 6 \\
6 & 2 & 4 & 5 & 6 & 7 \\
7 & 4 & 5 & 6 & 7 & 9 \\
8 & 5 & 6 & 7 & 9 & 10 \\
\hline
\end{tabular}

\subsection{Remeshing Technique}

In this section, we present our technique of space-time mesh adaptations. In the literature, many articles on mesh adaptations [2,3,15-21] can be found. Among these papers, a large number deals with space-time mesh adaptations. They use the discontinuous Galerkin method. In most of them, the approach is incremental, i.e., remeshing is carried out at given steps of time. Generally, the values of the unknown of the new mesh are obtained by approximation or interpolation from those of the old mesh, which we will call "mesh-to-mesh transfer". Moreover, the interpolation used is of a multiplexing type; the function of interpolation is defined by the product of a function of space by a function of time.

In [22], we proposed an incremental technique for mesh adaptation, which does not require mesh-to-mesh transfer. It was coupled with problems of rubbing contact (see [14]). In addition, we developed a non-incremental technique of mesh adaptation, based on non-structured space-time meshes.

Some teams have already worked on this problem. One can quote the works of Hugues and Hulbert [2,3], Tezduyar et al. [20,21] and Idesman et al. [9,23]. They use the continuous or discontinuous Galerkin method. In these approaches, calculations are carried out on the whole space-time domain $\Omega \times[0, T]$. Thus, for a field $\Omega$ of dimension $d$ and a total number $N$ of nodes of the space-time mesh, the dimension of the linear problem to be solved is $d \times N$, which quickly becomes large when $d=2$ or $d=3$. A solution to decrease the computational time is to use parallel computations. This is the option chosen in $[8,9]$.

In the context of the continuous Galerkin method, we suggested, in [22,24], a non-incremental solution, which substitutes the concept of a step of time by that of a "space-time front". Erickson et al. [16] have also proposed an advancing-front mesh generation, in the context of the discontinuous Galerkin method. This technique was successfully used by Miller et al. [25] in their multi-field space-time discontinuous Galerkin method, for $d=1$ and 2 in linearized elastodynamics 
applications. The advantage of this frontal resolution is that it decreases the size of the linear systems to be solved. Due to technical difficulties, the frontal resolution in the case of $d=3$ has not yet been implemented. Nevertheless, we propose a particular incremental remeshing technique based on the construction of $4 \mathrm{D}$ space-time meshes that are able to follow an evolutionary loaded zone. This technique of mesh generation uses simplex finite elements. Figure 5 gives an illustration of the technique. The principle is to maintain the same number of nodes during the simulation, but to locate a sufficiently large number of them under the loaded area. In this particular case, it is possible to preserve the matrices $T_{i / i-1}, T_{i / i}, T_{i / i+1}$ identical for all $i$, which involves a reduction of the computational time. An example of mechanical application is provided in the following section. This technique is aimed at applications in simulating problems of wear between two bodies in contact.

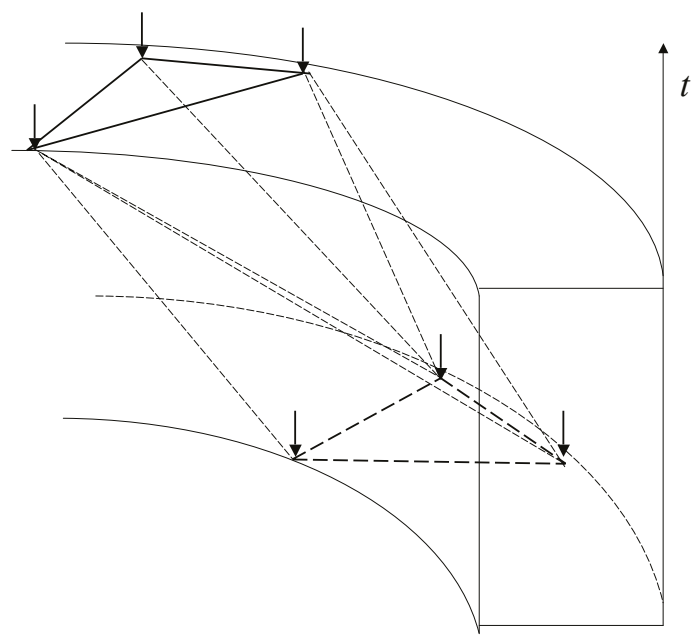

Figure 5. Space-time mesh generation by rotation of the loaded area. The loaded area is represented by arrows.

\section{Numerical Analysis}

Our space-time finite elements method was programmed using MATLAB software and was validated on elementary examples.

\subsection{Stability}

Preliminary results on the stability of the method have been established in [22] and compared with the Newmark integration scheme. Here, we summarize the main results. Let $\delta$ and $\theta$ be two real parameters; the Newmark integration scheme reads:

$$
\left\{\begin{array}{c}
\left\{\dot{U}_{i+1}\right\}=\left\{\dot{U}_{i}\right\}+\Delta t\left[(1-\delta)\left\{\ddot{U}_{i}\right\}+\delta\left\{\ddot{U}_{i+1}\right\}\right] \\
\left\{U_{i+1}=\left\{U_{i}\right\}+\Delta t\left\{\dot{U}_{i}\right\}+\Delta t^{2}\left[\left(\frac{1}{2}-\theta\right)\left\{\ddot{U}_{i}\right\}+\theta\left\{\ddot{U}_{i+1}\right\}\right]\right.
\end{array}\right.
$$

where $\Delta t$ is the time step of integration, $\left\{\dot{U}_{i+1}\right\}$ and $\left\{\ddot{U}_{i+1}\right\}$ are respectively the assembled vector of nodal velocities and accelerations at time $(i+1) \Delta t$. We showed in [22] that:

- $\quad$ For 1D space-time elastodynamic applications, the use of the STFEM method with linear simplex elements is similar to the use of the implicit Newmark integration scheme with $\delta=1 / 2$ and $\theta=1 / 3$. The method is then unconditionally stable.

- $\quad$ For 2D space-time elastodynamic applications, the use of the STFEM method with linear simplex elements is similar to the use of the explicit Newmark integration scheme with $\delta=1 / 2$ and $\theta=0$. 
The method is then conditionally stable. Classically, the time step has to verify the CFLcondition: $\Delta t \leq \min _{J} \frac{2}{\omega_{I}}$, where each $\omega_{J}$ is the frequency of a normal mode of vibration.

- $\quad$ For higher dimensions (3D and 4D), no direct relationship between the STFEM and the Newmark method has been established. Nevertheless, we noted that our method required sufficiently small space-time slabs, of the same order of the discretization time step necessary with explicit methods of integration.

- $\quad$ Furthermore, the use of the STFEM method with multiplex elements is similar to the use of the implicit Newmark integration scheme with $\delta=1 / 2$ and $\theta=1 / 3$, for 1D, 2D, 3D and 4D space-time applications. In this case, the method is unconditionally stable.

In the present study, a specific numerical investigation has been carried out to estimate the stability conditions for the STFEM method with linear simplex elements for 4D space-time elastodynamic applications. The stability was tested on a beam of length $L=0.1 \mathrm{~m}$ and a square section of $0.01 \times 0.01 \mathrm{~m}^{2}$ (see Figure 6). The Young modulus $E$ was equal to $1000 \mathrm{~Pa}$; the Poisson's ratio $v$ was equal to 0.3 ; and the density $\rho$ was equal to $680 \mathrm{~kg} / \mathrm{m}^{3}$.
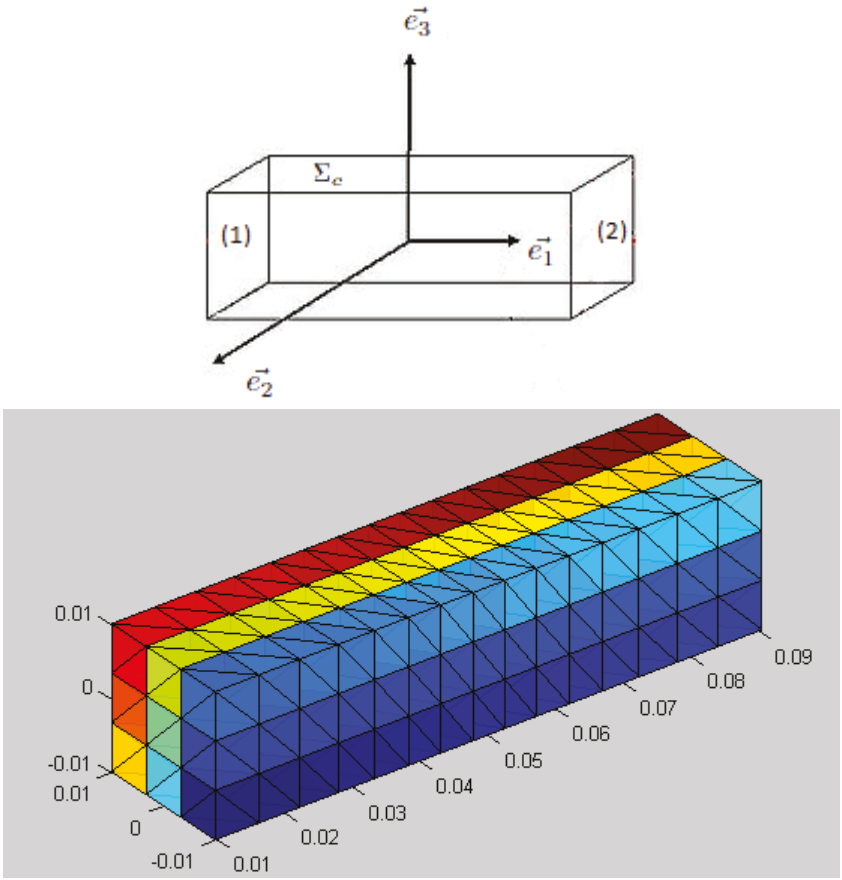

Figure 6. Geometry and 3D mesh of a beam with $h=6.6 \times 10^{-3} \mathrm{~m}$.

We built boundary conditions in order to obtain the following analytic solution:

$$
u(x, t)=\cos \left(\frac{\pi x_{1}}{L}\right) \cos \left(\frac{\pi c t}{L}\right) e_{1}
$$

where $c$ is the velocity of the wave propagation. On Faces (1) and (2), null Neumann conditions were imposed. On the other four faces $\left(\Sigma_{c}\right)$, Dirichlet conditions were imposed in order to satisfy the analytic 
solution (20). The volume external force density $f_{d}$ was assumed to be vanishing. This imposes that the wave velocity $c$ must verify:

$$
c=\sqrt{\frac{E}{\rho(1+v)}\left(1+\frac{v}{\rho(1-2 v)}\right)}
$$

Results plotted in Figure 7 give the dependence of the time discretization (size of the space-time slab) $\Delta t$ on the average size $h$ of the 3D finite elements, to ensure the stability of the method.

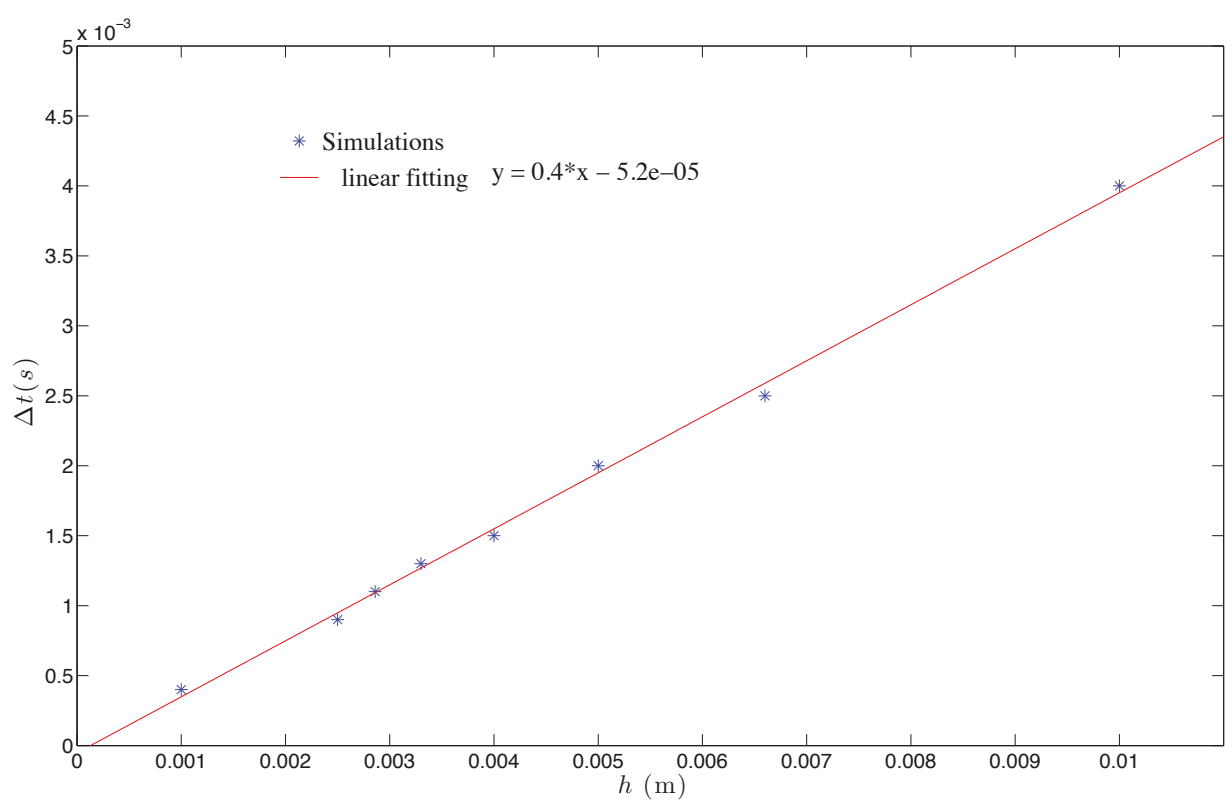

Figure 7. Size of the time step of discretization $\Delta t$ necessary for stability with respect to the average size $h$ of the 3D finite elements.

Linear fitting suggests that the stability criterion is:

$$
\Delta t \leq \alpha h
$$

with $\alpha \simeq 1 / 2 c$. Indeed, in our example, $c=1.407 \mathrm{~m} / \mathrm{s}$.

\subsection{Convergence}

In the case of simplex finite elements, the convergence with the STFEM method is comparable to the convergence with the Newmark scheme, for the 1D and 2D problem. Concerning the convergence analysis of the STFEM in $4 \mathrm{D}$, we used the previous example. The time step of discretization $\Delta t$ is scaled with respect to $h$, using the stability criterion obtained in the previous paragraph. We computed the maximum error over space at the last time step between the analytic solution and the numerical solution for each mesh size $h$. The results are plotted in Figure 8. 


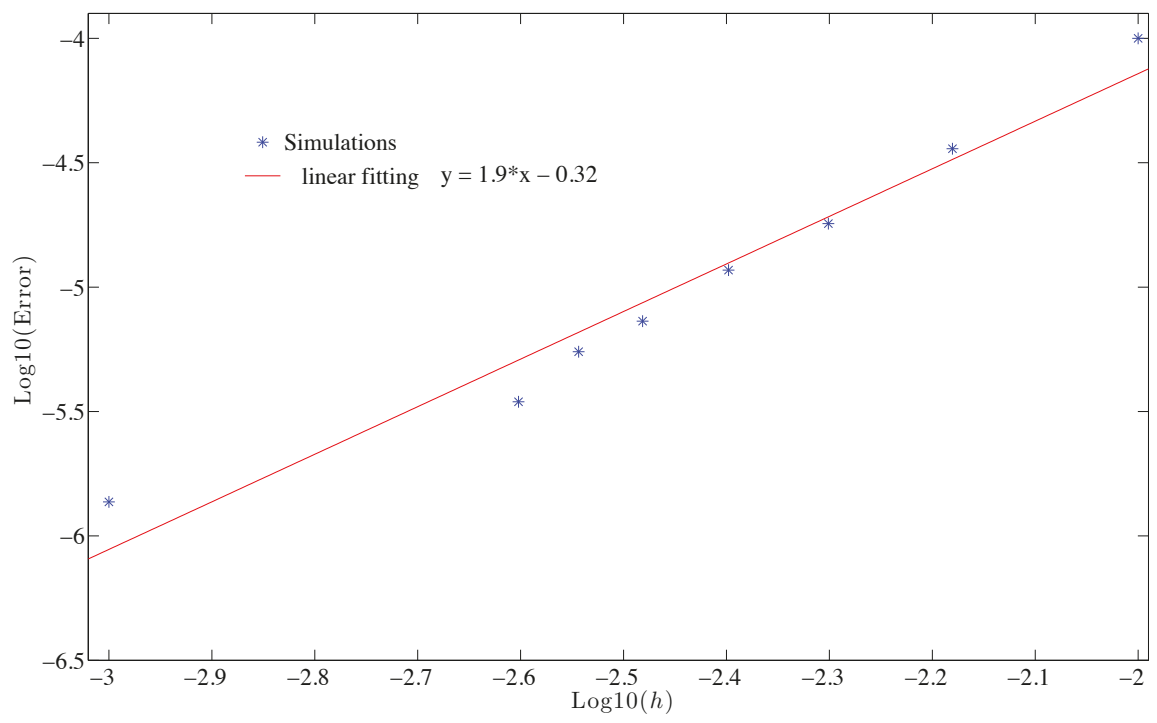

Figure 8. Maximum error over the space, at the last time step, between the analytic solution and the numerical solution for each mesh size $h$, in the logarithmic scale.

We can note that the convergence rate for the method is nearly quadratic.

\section{Numerical Results on Mesh Adaptation}

To illustrate our technique of mesh adaptation, we consider the example of a brake disc subjected to the clamping of a plate on one of its faces (see the source model with the coarse mesh in Figure 9) and blocked on the opposite face. The disc is made of steel with a Young's modulus equal to 210,000 MPa, a Poisson's ratio equal to 0.3 and a density equal to $7800 \mathrm{~kg} / \mathrm{m}^{3}$. The internal radius of the disc is equal to $40 \mathrm{~mm}$; its external radius is equal to $100 \mathrm{~mm}$; and its thickness is $10 \mathrm{~mm}$. Two 3D initial meshes have been tested: a coarse mesh, which contains 634 nodes and 1752 elements, refined only in the area of the clamping, and a fine mesh, which contains 6476 nodes and 25,856 elements and has a uniform mesh fineness over all the sample.

The clamping area is modeled by a constant pressure of $100 \mathrm{MPa}$. This area is moved along the circumference of the disc with a rotational speed equal to that of the propagating wave $V=\frac{1}{2 \pi R} \sqrt{\frac{E}{\rho}}$, where $R=70 \mathrm{~mm}$ is the average radius of the disc. For both 3D initial meshes, we built an incremental $4 \mathrm{D}$ space-time mesh, which preserves the 3D mesh at each time step, by imposing an axial rotation to keep the finest zone of the 3D mesh under the loading area. An illustration is shown schematically in Figure 5 .

The results of the calculations presented were obtained for space-time slabs of $10^{-7} \mathrm{~s}$ (this is equivalent to using a time step equal to $10^{-7} \mathrm{~s}$ ). The vertical displacements obtained with fine and coarse meshes have been compared for points located on a circle of control, of radius $R$ equal to $70 \mathrm{~mm}$ (see Figure 10). 


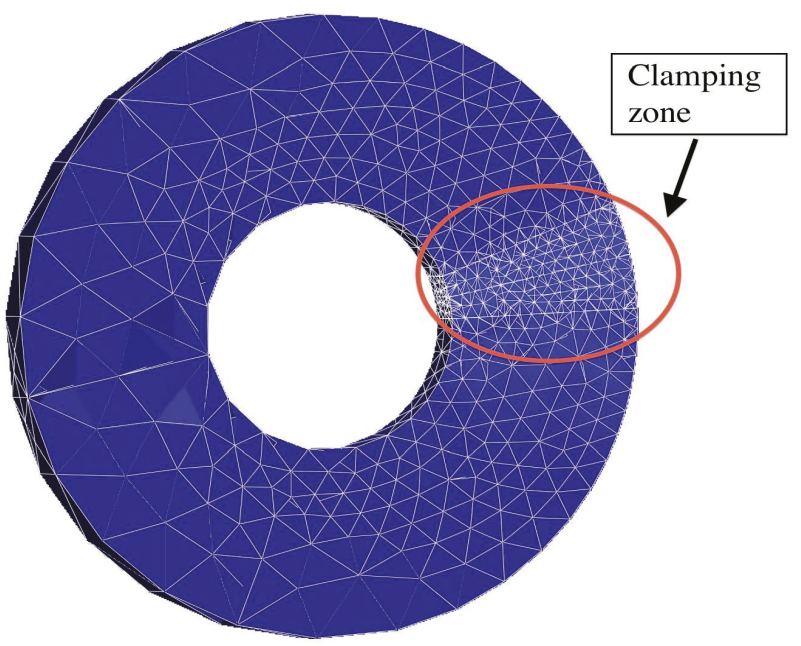

Figure 9. 3D initial coarse mesh: the mesh is finer under the clamping zone.

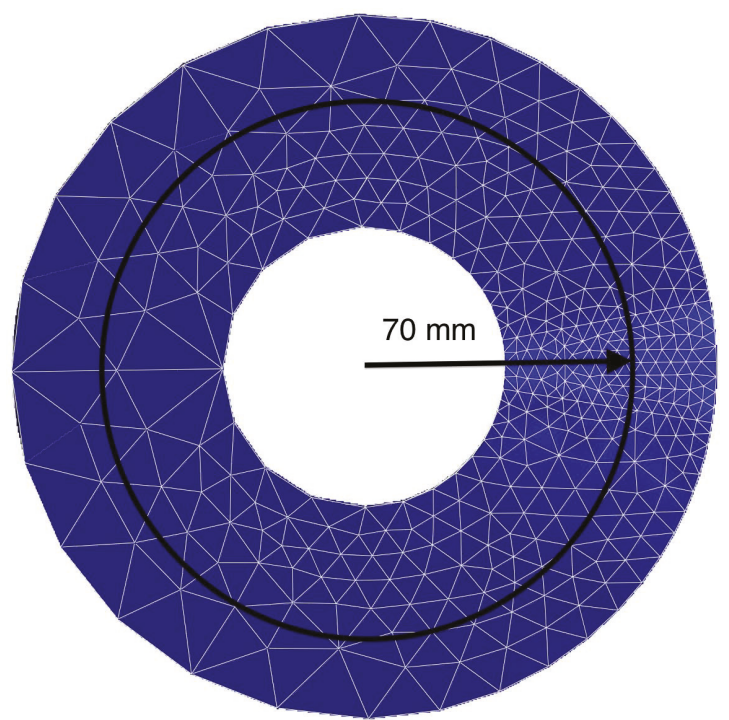

Figure 10. Location zone of checked displacements: circle of radius equal to $70 \mathrm{~mm}$.

Numerical values are gathered in Figure 11 for the results at time $t=2 \cdot 10^{-5} \mathrm{~s}$ and in Figure 12 for the results at time $t=4 \cdot 10^{-5} \mathrm{~s}$. Each check point is defined by its angle, in polar coordinates.

Numerical comparisons show that the coarse and fine meshes give similar results in the clamping zone. However, apart from this zone, the results are somewhat different. Let us note that nodal displacements cannot be identical because dynamic effects depend on the fineness of the mesh. 


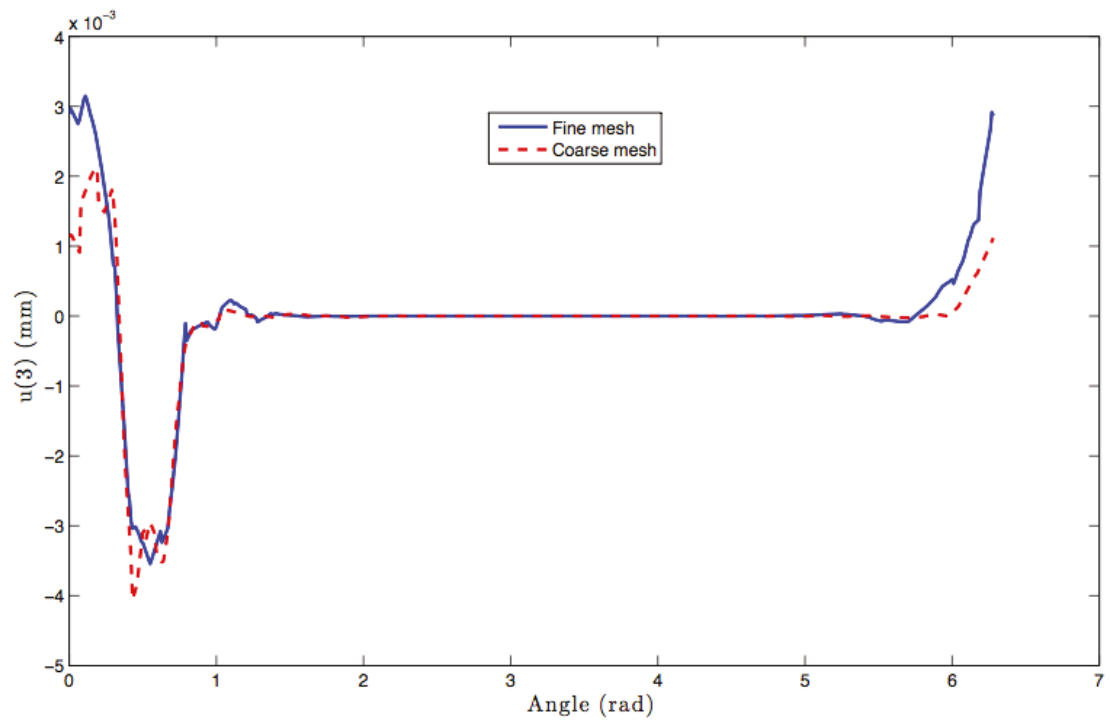

Figure 11. Comparison of vertical displacements for points situated on the circle of control, expressed in $\mathrm{mm}$, at time $t=2 \cdot 10^{-5} \mathrm{~s}$. Each check point is defined by its angular coordinate, expressed in radians.

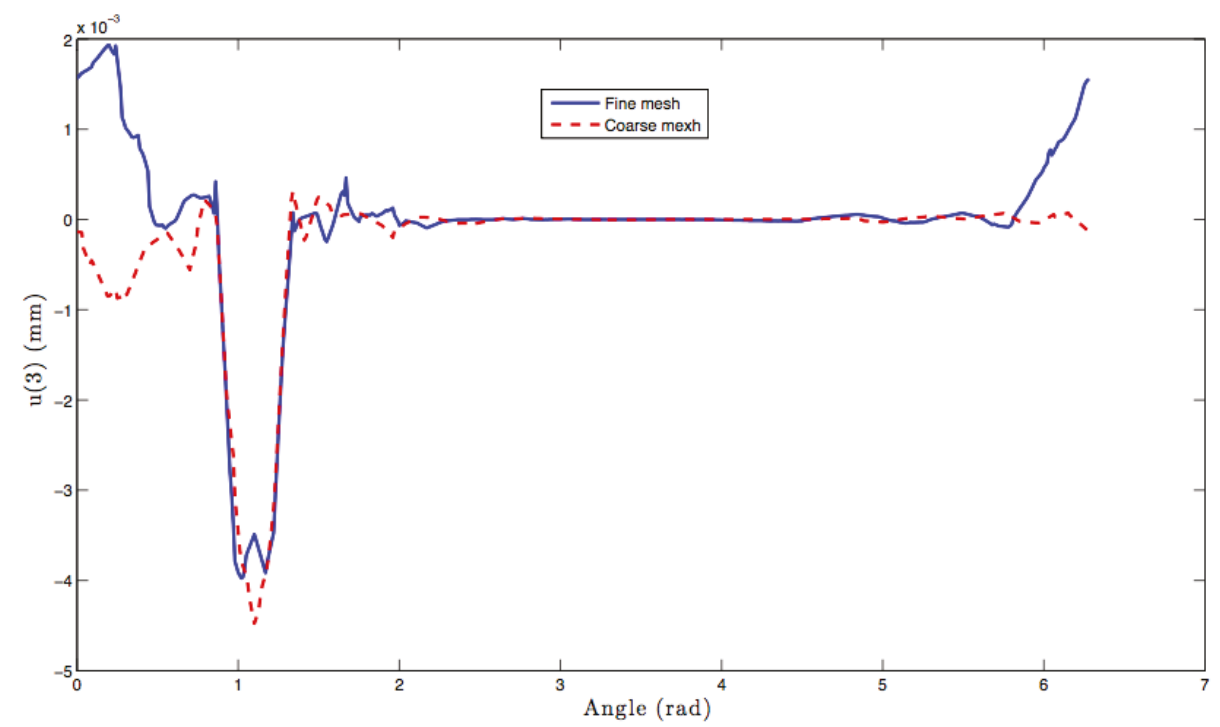

Figure 12. Comparison of vertical displacements for points situated on the circle of control, expressed in $\mathrm{mm}$, at time $t=4 \cdot 10^{-5} \mathrm{~s}$. Each check point is defined by its angular coordinate, expressed in radians.

Figures 13 and 14 show the norm of incremental displacements at time $t=2.10^{-5} \mathrm{~s}$ and $t=4.10^{-5} \mathrm{~s}$ for the coarse and the fine mesh, respectively. For the coarse mesh, we can observe that the refined zone really remains under the zone of clamping. The distribution of the norm of node displacements is similar for the two positions of the load. It is important to note that they are incremental displacements and not total displacements. 
Finally, it must be noted that the computational time is 6.6-times faster using the coarse mesh.
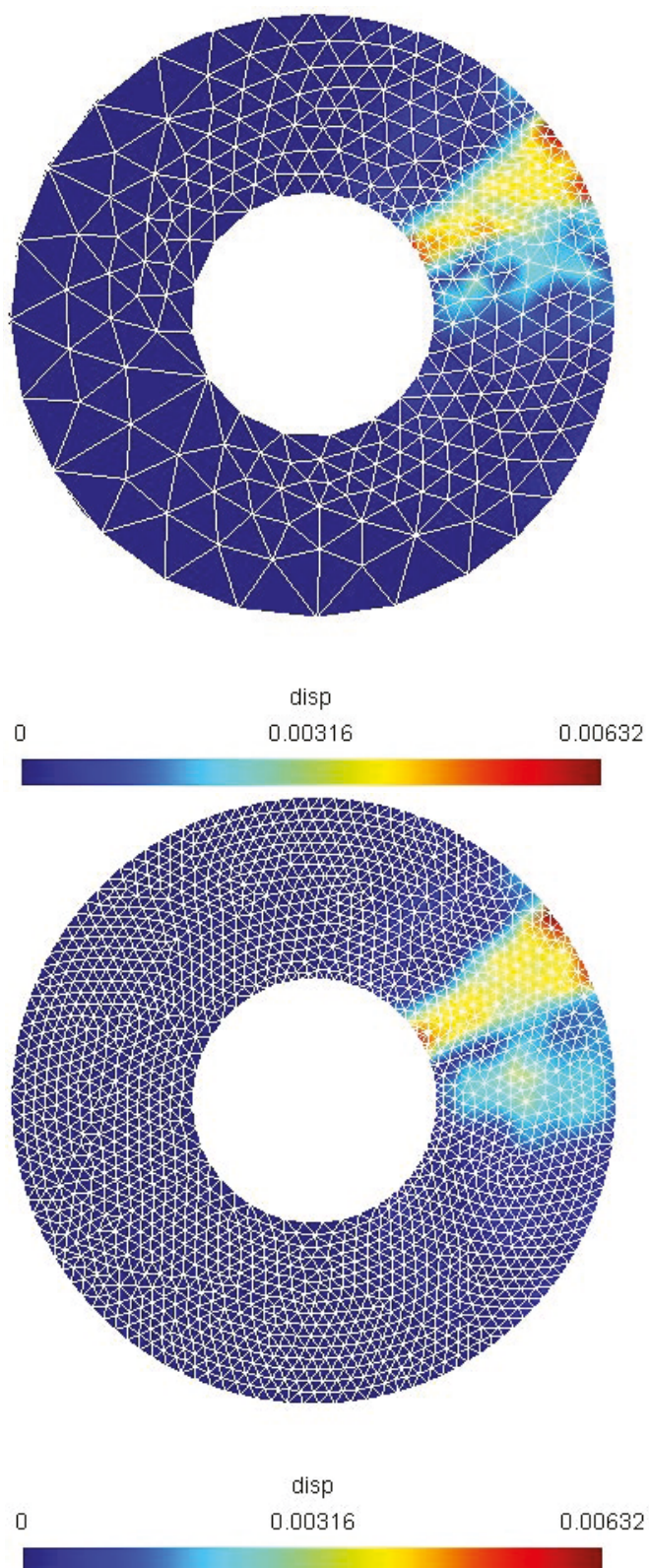

Figure 13. Isovalues of the norm of nodal displacements, expressed in $\mathrm{mm}$, at $t=2.10^{-5} \mathrm{~s}$ for the coarse mesh (image at the top) and for the fine mesh (image at the bottom). 

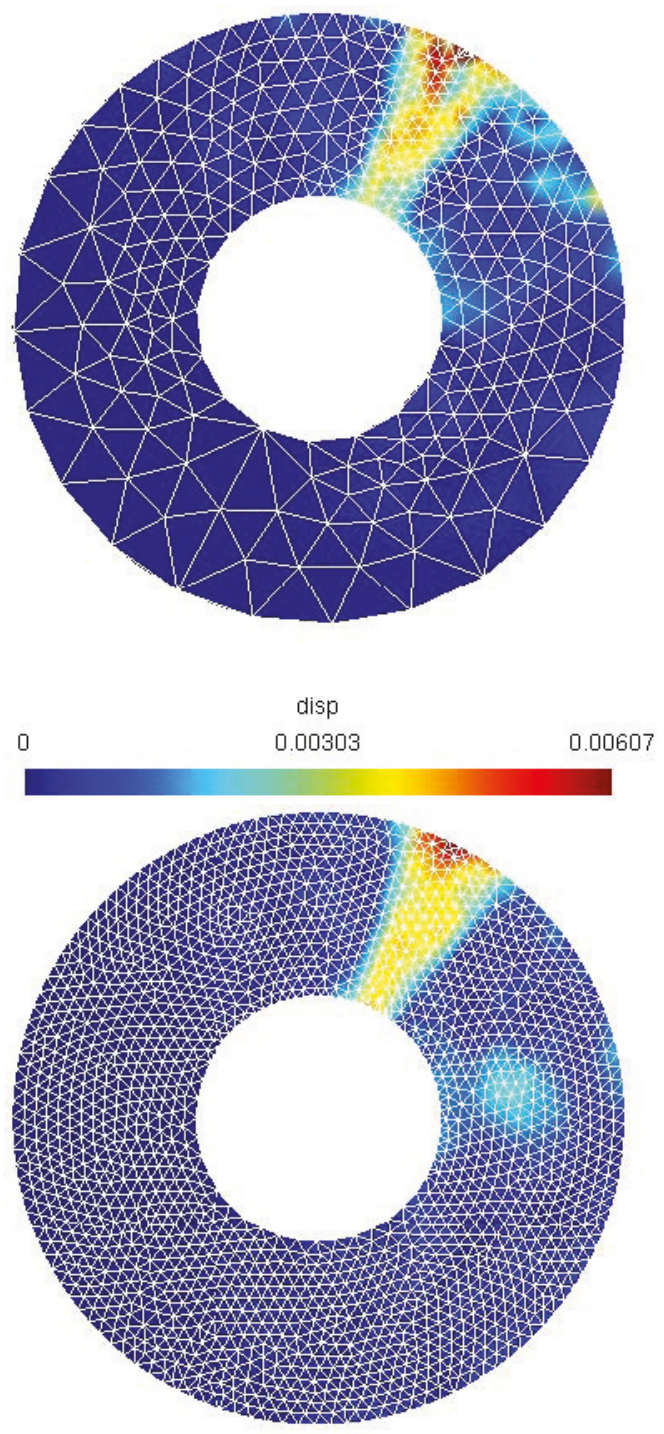

disp

0

0.00304

0.00607

Figure 14. Isovalues of the norm of nodal displacements, expressed in $\mathrm{mm}$, at $t=4.10^{-5} \mathrm{~s}$ for the coarse mesh (image on the top) and for the fine mesh (image on the bottom).

\section{Conclusions}

The method we have presented for space-time mesh generation for 4D domains, using simplex elements, made it possible to develop a technique of mesh adaptation able to follow a mobile loading zone. This original technique has been carried out to ensure a minimal computational time and does 
not require coarse-to-fine and fine-to-coarse mesh transfer operators. The convergence and stability of the method were studied and compared with existing methods. This approach opens the way to 4D remeshing. It allows, thanks to simplex elements, remeshing in both space and in time.

However, to demonstrate the all the capacities of this method, it is necessary to go much further in the mechanical applications and to propose a technique of $4 \mathrm{D}$ mesh adaptation using the frontal approach, as presented in $[14,16,22,25]$.

Author Contributions: Conceptualization, F. Jourdan; Software, S. Dumont, F. Jourdan; Validation, T. Madani; Original Draft Preparation, S. Dumont, F. Jourdan and T. Madani.

Conflicts of Interest: The authors declare no conflict of interest.

\section{References}

1. Ladevèze, P. Non Linear Computational Structural Mechanics, New Approaches and Non Incremental Methods of Calculation; Springer: New York, NY, USA, 1999.

2. Hughes, T.J.R.; Hulbert, G.M. Space-time finite element methods for elastodynamics: Formulations and error estimates. Comput. Methods Appl. Mech. Eng. 1988, 66, 339-363. [CrossRef]

3. Hulbert, G.M.; Hughes, T.J.R. Space-time finite element methods for second-order hyperbolic equations. Comput. Methods Appl. Mech. Eng. 1990, 84, 327-348. [CrossRef]

4. Khalmanova, D.K.; Costanzo, F. A space-time discontinuous Galerkin finite element method for fully coupled linear thermo-elasto-dynamic problems with strain and heat flux discontinuities. Comput. Methods Appl. Mech. Eng. 2008, 197, 1323-1342. [CrossRef]

5. Argyris, J.H.; Scharpf, D.W. Finite elements in time and space. Nucl. Eng. Des. 1969, 10, 456-464. [CrossRef]

6. Zienkiewics, O.C.; Parekh, C.J. Transient field problems-two and three dimensional analysis by isoparametric finite elements. Int. J. Numer. Methods Eng. 1970, 2, 61-71. [CrossRef]

7. Chien, C.C.; Yang, C.S.; Tang, J.H. Three-dimensional transient elastodynamic analysis by a space and time-discontinuous Galerkin finite element method. Finite Elem. Anal. Des. 2003, 39, 561-580. [CrossRef]

8. Anderson, M.; Kimn, J.-H. A numerical approach to space-time finite elements for the wave equation. J. Comput. Phys. 2007, 226, 466-476. [CrossRef]

9. Idesman, A.V. Solution of linear elastodynamics problems with space-time finite elements on structured and unstructured meshes. Comput. Methods Appl. Mech. Eng. 2007, 196, 1787-1815. [CrossRef]

10. Huang, H.; Costanzo, F. On the use of space-time finite elements in the solution of elasto-dynamic problems with strain discontinuities. Comput. Methods Appl. Mech. Eng. 2002, 191, 5315-5343. [CrossRef]

11. Karaoglan, L.; Noor, A.K. Space-time finite element methods for sensitivity analysis of contact/impact response of axisymmetric composite structures. Comput. Methods Appl. Mech. Eng. 1997, 144, 371-389. [CrossRef]

12. Jourdan, F.; Bussy, P. Large time increment method in dynamic regularization: Sheet cutting simulations. Comput. Methods Appl. Mech. Eng. 2000, 190, 1245-1259. [CrossRef]

13. Li, X.D.; Wiberg, N.-E. Implementation and adaptivity of space-time finite element method for structural dynamics. Comput. Methods Appl. Mech. Eng. 1998, 156, 211-229. [CrossRef]

14. Adélaïde, L.; Jourdan, F.; Bohatier, C. Frictional contact solver and mesh adaptation in Space-Time Finite Element Method. Eur. J. Mech. A/Solids 2003, 22, 633-647. [CrossRef]

15. Donea, R.; Huerta, A.; Sarrate, J. Arbitrary Lagrangian-Eulerian for fluid-rigid body interaction. Comput. Methods Appl. Mech. Eng. 2001, 190, 3171-3188.

16. Erickson, J.; Guoy, D.; Sullivan, J.M.; Üngör, A. Building spacetime meshes over arbitrary spatial domains. In Proceedings of the 11th International Meshing RoundTable, Sandia, Ithaca, NY, USA, 15-18 September 2002; pp. 391-402.

17. Farhat, C.; Degand, C. A three-dimensional torsional spring analogy method for unstructured dynamic meshes. Comput. Struct. 2002, 80, 305-316.

18. Feng, Y.T.; Peric, D. A time-adaptive space-time finite element method for incompressible Lagrangian flows with free surfaces: Computational issues. Comput. Methods Appl. Mech. Eng. 2000, 190, 499-518. [CrossRef]

19. Mohr, R.; Menzel, A.; Steinmann, P. A consistent time FE-method for large strain elasto-plasto-dynamics. Comput. Methods Appl. Mech. Eng. 2008, 197, 3024-3044. [CrossRef] 
20. Tezduyar, T.E.; Sunil, S. Enhanced-discretization space-time technique (EDSTT). Comput. Methods Appl. Mech. Eng. 2004, 193, 1385-1401. [CrossRef]

21. Tezduyar, T.E.; Sathe, S.; Keedy, R.; Stein, K. Space-time finite element techniques for computation of fluid-structure interactions. Comput. Methods Appl. Mech. Eng. 2006, 195, 2002-2027. [CrossRef]

22. Adélaïde, L.; Jourdan, F.; Bohatier, C. Méthode des éléments finis espace-temps et remaillage. Rev. Eur. Elem. Finis 2003, 12, 427-459. [CrossRef]

23. Idesman, A.; Niekamp, R.; Stein, E. Continuous and discontinuous Galerkin methods with finite elements in space and time for parallel computing of viscoelastic deformation. Comput. Methods Appl. Mech. Eng. 2000, 190, 1049-1063. [CrossRef]

24. Adélaïde, L.; Jourdan, F.; Bohatier, C. New results on mesh adaptation in space-time finite element method. In Proceedings of the ASME Engineering Technology Conference on Energy (ETCE 2002/STRUC-29042), Houston, TX, USA, 4-5 February 2002.

25. Miller, S.T.; Kraczek, B.; Haber, R.B.; Johnson, D.D. Multi-field spacetime discontinuous Galerkin methods for linearized elastodynamics. Comput. Methods Appl. Mech. Eng. 2009, 199, 34-47. [CrossRef]

(C) 2018 by the authors. Licensee MDPI, Basel, Switzerland. This article is an open access article distributed under the terms and conditions of the Creative Commons Attribution (CC BY) license (http:/ / creativecommons.org/licenses/by/4.0/). 
Article

\title{
Integration of Direction Fields with Standard Options in Finite Element Programs
}

\author{
Herbert Moldenhauer \\ Dipl.-Ing. H. Moldenhauer GmbH, Im Brückengarten 9 A, 63322 Rödermark, Germany; \\ hm@FEM-Moldenhauer.de; Tel.: +49-06074-1394
}

Received: 11 March 2018; Accepted: 4 May 2018; Published: 7 May 2018

\begin{abstract}
The two-dimensional differential equation $y^{\prime}=f(x, y)$ can be interpreted as a direction field. Commercial Finite Element (FE) programs can be used for this integration task without additional programming, provided that these programs have options for the calculation of orthotropic heat conduction problems. The differential equation to be integrated with arbitrary boundaries is idealized as an FE model with thermal 2D elements. Its orthotropic thermal conductivities are specified as $\mathrm{k}_{1}=1$ and $\mathrm{k}_{2}=0$. In doing so, $\mathrm{k}_{1}$ is parallel to $\mathrm{y}^{\prime}$, and $\mathrm{k}_{2}$ is oriented perpendicular to this. For this extreme case, it is shown that the isotherms are identical to the solution of $y^{\prime}=f(x, y)$. The direction fields, for example, can be velocity vectors in fluid mechanics or principal stress directions in structural mechanics. In the case of the latter, possibilities for application in the construction of fiber-reinforced plastics (FRP) arise, since fiber courses, which follow the local principal stress directions, make use of the superior stiffness and strength of the fibers.
\end{abstract}

Keywords: direction field; tensor line; principal stress; tailored fiber placement; heat conduction

\section{Introduction}

Orthotropic materials can have extremely different thermal conductivities, for example, in printed circuit boards. The tracks conducting current are made of copper $(\mathrm{Cu})$ and are electrically insulated against the plastic plate. Due to its low thermal conductivity $\left(\mathrm{k}_{2}\right)$, the $\mathrm{Cu}$ conducting track $\left(\mathrm{k}_{1}\right)$ is heated to a constant, relatively high temperature. It can be considered as an isothermal line. The thermal heat flow in the plastic due to conduction is very low. For example, if there are two $\mathrm{Cu}$ conducting tracks with constant but different temperatures, then a steady temperature gradient independent of $k_{2}$ prevails in the plastic between them, even if $\mathrm{k}_{2} \rightarrow 0$. The $\mathrm{Cu}$-tracks can be described by their position $y=y(x)$, and they control the temperature distribution in the insulator. Alternatively, the position can be described by $\mathrm{y}^{\prime}(\mathrm{x})$ together with their starting positions. If these tracks are infinitely densely distributed, the direction field of the Cu-conductance $k_{1}$ is described by $y^{\prime}=f(x, y)$. Insulation works perpendicular to the Cu-tracks when $\mathrm{k}_{2}<<\mathrm{k}_{1}$. Should the (infinitely densely) guided conduction paths be directed parallel to an arbitrary direction field $y^{\prime}=f(x, y)$, then the following hypothesis shall be mathematically verified:

Hypothesis: The isotherms of an orthotropic steady-state $2 D$ thermal conduction problem with the thermal conductivities $k_{1}$ and $k_{2}$ are tangential to an arbitrarily prescribed direction field $y^{\prime}=f(x, y)$ provided that the local orientation of $k_{1}$ follows the direction field $y^{\prime}$, and perfect insulation exists perpendicular to this $\left(k_{2}=0\right)$.

The integration method, which can be derived from the hypothesis, is the topic of the dissertation by the author [1]; however, this was published without mathematical verification. Two-dimensional direction fields of the type $y^{\prime}=f(x, y)$ can describe physically different processes. In fluid mechanics, the vectorial direction field $\mathrm{y}^{\prime}=\mathrm{v}(\mathrm{x}, \mathrm{y}) / \mathrm{u}(\mathrm{x}, \mathrm{y})$ of the velocity components $\mathrm{u}$ and $\mathrm{v}$ are tangential to the streamlines. The general solution of the direction field to be integrated provides the flow field in the 
form of streamlines. This task is solved by default in Computational Fluid Dynamics (CFD) codes and is therefore not pursued further here. In stress analysis, the visualization of principal stress (PS) lines is not present in commercial FE programs. The visualization of stream and tensor lines is the subject of numerous investigations. A representative selection is given by [2-6].

Beyer [2] investigated in his dissertation principal stress trajectories arising in civil engineering and in constructions of fiber-reinforced plastics. He established an algorithm for their visualization in two and three dimensions. The procedure is similar to the method shown in Figure 1; however, improved accuracy is gained with his variable and iterative step control.

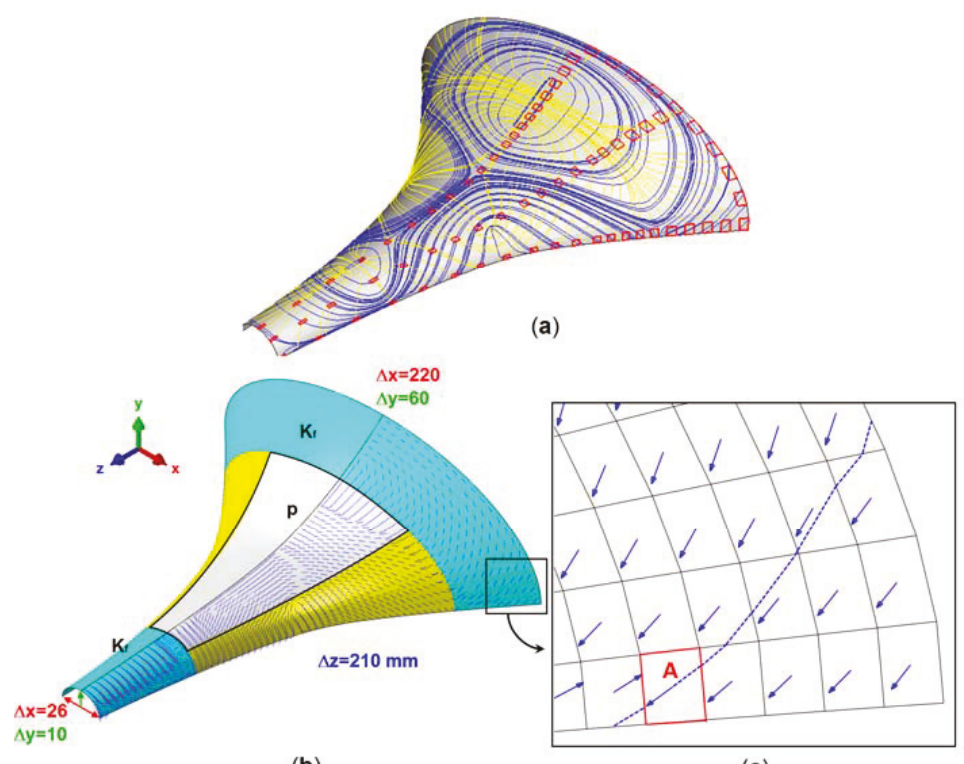

(b)

(c)

Figure 1. Shell under lateral pressure $\mathrm{p}=0.15 \mathrm{MPa}$, bedded elastically $\left(\mathrm{K}_{\mathrm{f}}=1000 \mathrm{~N} / \mathrm{mm}\right)$; typical dimensions $\Delta \mathrm{x}, \Delta \mathrm{y}$, and $\Delta \mathrm{z}$, modulus $\mathrm{E}=50 \mathrm{GPa}$, thickness $\mathrm{t}=2 \mathrm{~mm}$. (a) stress trajectories, standard integration begins in the red start elements; (b) PS directions from a linear static analysis; (c) detail, standard integration begins in element A. Improved accuracy is gained with mesh refinement.

Delmarcelle [3,4] investigated symmetric tensor fields by studying their topological structure. The basic constituents of tensor topology are the degenerate points where eigenvalues (or principal stress values) are equal to each other. Separatrices and degenerate points are the basic constituents of tensor field topology. A separatice is the tensor line (PS line) between two degenerate points. Knowledge of the topological structure of tensor fields is important, as standard integration methods need this information.

With standard integration methods the uniform distribution of tensor lines depends critically on the placement of starting points (elements), see Figure 1. Jobard's algorithm [5] for the placement of evenly-spaced PS lines (in his paper: streamlines) is based on a separating distance between adjacent PS lines to control their density. If a PS line is too close to another one, it is abruptly cut. With respect to fiber placement in FRP construction this may have adverse consequences. Trichoche [6] extends the work of Delmarcelle $[3,4]$ to broaden the scope of topology-based vector and tensor field visualization. One focus in his work is on turbulent flows. The streamline distribution is extremely complex for turbulent flows with its local, small-scale details. Another focus is instabilities in structural problems. 
Visualization of stress trajectories should permit detection and identification of bifurcation to provide insight into the time-dependent structural evolution. These issues are beyond the scope of this paper.

All these papers lead to specialized algorithms, which are not public. Therefore, it is desirable to investigate the possibilities of integrating vectorial and tensorial direction fields within FE programs without programming efforts or additional software. PS lines in FRP constructions are of particular interest since fiber courses, which follow the local PS directions, make use of the superior stiffness and strength properties of the fibers [1,7-10].

To demonstrate some disadvantages with conventional standard integration methods, Figure 1 shows an integration procedure developed by the author [1] (p. 16) applied to the bottom of a shell structure.

Figure $1 \mathrm{~b}$ shows the PS directions of the blue principal stress trajectories on the bottom of the shell. In the middle plane and on the top of the shell there are other PS directions since membrane and bending stresses occur simultaneously. These directions stem from an eigenvalue problem of the stress tensor, whose eigenvectors (principal stress directions) remain indefinite in the sign. The PS directions can therefore switch the sign from one element to its neighboring element. The integration pattern in Figure 1c takes this problematic situation into account by replacing the direction vector with a bidirectional element. The bidirectional element intersects two opposing element sides and thereby allows the integration to be continued in the two neighboring elements with their directional elements. Each start element (marked in red) in Figure 1a provides two individual fiber courses with the procedure just described, corresponding to the two PS directions. A regular placement of the start elements in no way guarantees a uniform distribution of the PS lines. A homogenization of the fiber courses is achieved only with considerable effort. A further disadvantage of this procedure is immediately apparent; each individual trajectory (fiber) must be calculated separately. Should the designer change the fiber volume ratio for reasons of strength, then the procedure must be repeated from the beginning.

This problem is substantially simplified through the hypothesis set out above, which can be modeled by means of the orthotropic heat conduction in FE programs. Since the two-dimensional direction field of the principal stresses describes a planar stress condition, shells with an arbitrary boundary can also be processed. An additional advantage is that all of the PS trajectories (fiber courses) can be calculated by means of a single thermal analysis. If the fiber volume ratio is subsequently increased, correspondingly more isotherms (fiber courses) are extracted from the existing continuous temperature field.

The recommended calculation alternative for the integration of $y^{\prime}=f(x, y)$ is based on the drastically changing properties of Fourier's law for $k_{1} / k_{2} \rightarrow \infty$. The following section provides a mathematical verification of the hypothesis set out above. The practicability of the integration method is based on independence from thermal boundary conditions; the isotherms always follow the direction field in nonsingular areas. However, the (weighted) distance of the isotherms from each other is influenced by the boundary conditions and can be controlled by them. In Section 3, the procedure is based on some rules and these are demonstrated practically through examples. The influence of the orthotropy ratio $\mathrm{k}_{1} / \mathrm{k}_{2}$, as well as the influence of singularities on the course of the isotherms, is investigated.

\section{The Integration of Direction Fields by Means of the Orthotropic Heat Equation}

The direction field is given through an ordinary differential equation of the first order in the Cartesian coordinate system $(x, y)$ :

$$
y^{\prime}=f(x, y)
$$

In accordance with the hypothesis in the previous section, it shall be demonstrated that orthotropic heat conduction can be used for the integration of this equation. 


\subsection{The Anisotropic Fourier Heat Conduction Law and the Heat Conduction Equation}

Verification of the hypothesis: In the two-dimensional case, Fourier's anisotropic heat conduction law with respect to a Cartesian coordinate system is given by Equation (2), and the steady heat balance without internal heat sources by Equation (3), [11]:

$$
\begin{gathered}
q_{x}=-\left(k_{x x} \frac{\partial T}{\partial x}+k_{x y} \frac{\partial T}{\partial y}\right), \\
q_{y}=-\left(k_{y x} \frac{\partial T}{\partial x}+k_{y y} \frac{\partial T}{\partial y}\right), \\
\frac{\partial q_{x}}{\partial x}+\frac{\partial q_{y}}{\partial y}=0 .
\end{gathered}
$$

These equations are combined, resulting in the anisotropic heat conduction equation:

$$
\frac{\partial}{\partial x}\left(k_{x x} \frac{\partial T}{\partial x}\right)+\frac{\partial}{\partial x}\left(k_{x y} \frac{\partial T}{\partial y}\right)+\frac{\partial}{\partial y}\left(k_{y x} \frac{\partial T}{\partial x}\right)+\frac{\partial}{\partial y}\left(k_{y y} \frac{\partial T}{\partial y}\right)=0 .
$$

Orthotropic (principal) thermal conductivities $k_{1}$ and $k_{2}$ being oriented according to the direction field of Equation (1) should be transformed into the Cartesian system. The transformation equations for the 2D heat conduction tensor are analogous to a $2 \mathrm{D}$ stress tensor, and can be illustrated using trigonometric relationships with Mohr's (Stress) Circle, as shown in Figure 2.

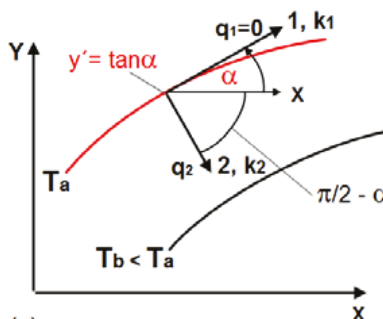

(a)

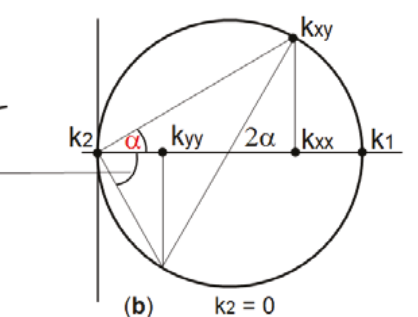

(b)

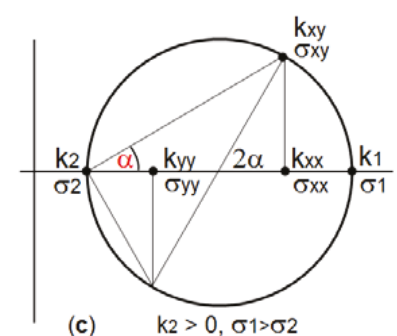

(c)

Figure 2. Transformation between local and global conductivity tensors $\mathbf{k}$ in the temperature field $T(x, y)$ : (a) Angle $\alpha$ and $(\pi / 2)-\alpha$ between the local system $(1,2)$ and the global $x$-axis, $\mathbf{q}=$ heat flux density; (b) Mohr's Circle for $k_{1}>0$ and $k_{2}=0$; (c) Mohr's Circle for $k_{1}>0$ and $k_{2}>0$ by analogy with Mohr's Stress Circle.

$$
\begin{gathered}
k_{x x}=k_{1} \cos ^{2} \alpha+k_{2} \sin ^{2} \alpha=\frac{k_{1}}{1+\tan ^{2} \alpha}+\frac{k_{2} \tan ^{2} \alpha}{1+\tan ^{2} \alpha}=\frac{k_{1}}{1+f^{2}}+\frac{k_{2} f^{2}}{1+f^{2}}, \\
k_{x y}=k_{y x}=\left(k_{1}-k_{2}\right) \sin \alpha \cos \alpha=\left(k_{1}-k_{2}\right) \frac{f}{1+f^{2}}, \\
k_{y y}=k_{1} \sin ^{2} \alpha+k_{2} \cos ^{2} \alpha=\frac{k_{1} f^{2}}{1+f^{2}}+\frac{k_{2}}{1+f^{2}} .
\end{gathered}
$$

The hypothesis in Section 1 is based on extreme orthotropic conductivity ratios, $k_{1} / k_{2} \rightarrow \infty$ and therefore can be validated only for $k_{2}=0$. Setting $k_{2}=0$ in Equation (5) and substituting the simplified expression in Equation (2) we have:

$$
q_{x}=-\left(k_{1} \frac{\partial T}{\partial x}+k_{1} f \frac{\partial T}{\partial y}\right) /\left(1+f^{2}\right)
$$




$$
q_{y}=-\left(k_{1} f \frac{\partial T}{\partial x}+k_{1} f^{2} \frac{\partial T}{\partial y}\right) /\left(1+f^{2}\right) .
$$

The heat conduction Equation (4) is solved in the FE programs. Substitution of Equation (5) into (4), together with $k_{2}=0$, results in:

$$
\frac{\partial}{\partial x}\left(\frac{k_{1}}{1+f^{2}} \frac{\partial T}{\partial x}\right)+\frac{\partial}{\partial x}\left(\frac{k_{1} f}{1+f^{2}} \frac{\partial T}{\partial y}\right)+\frac{\partial}{\partial y}\left(\frac{k_{1} f}{1+f^{2}} \frac{\partial T}{\partial x}\right)+\frac{\partial}{\partial y}\left(\frac{k_{1} f^{2}}{1+f^{2}} \frac{\partial T}{\partial y}\right)=0 .
$$

The solution $T(x, y)$ of Equation (7) can be visualized by isotherms, which represent a direction field $\mathrm{y}_{\mathrm{T}}{ }^{\prime}$. An isotherm is characterized by a constant temperature $C$. Its equation in implicit form is given by $F(x, y)=T(x, y)-C=0$. A directional element of this curve has the slope:

$$
y_{T}^{\prime}=-\frac{\partial F / \partial x}{\partial F / \partial y}=-\frac{\partial T / \partial x}{\partial T / \partial y}
$$

The question arises as to which properties Equation (6) shows if the direction fields from Equations (1) and (8) are equated:

$$
y^{\prime}=f(x, y)=f=-\frac{\partial T / \partial x}{\partial T / \partial y}=y_{T}^{\prime} \text {, i.e., } f=-\frac{\partial T / \partial x}{\partial T / \partial y} .
$$

Use of " $f$ " from Equation (9) in the numerators of Equation (6) results in:

$$
\begin{gathered}
q_{x}=-\left(k_{1} \frac{\partial T}{\partial x}+k_{1}\left(-\frac{\partial T / \partial x}{\partial T / \partial y}\right) \frac{\partial T}{\partial y}\right) /\left(1+f^{2}\right)=0 \\
q_{y}=-\left(k_{1}\left(-\frac{\partial T / \partial x}{\partial T / \partial y}\right) \frac{\partial T}{\partial x}+k_{1}\left(-\frac{\partial T / \partial x}{\partial T / \partial y}\right)^{2} \frac{\partial T}{\partial y}\right) /\left(1+f^{2}\right)=0 .
\end{gathered}
$$

The first and second term for $q_{x}$ and $q_{y}$ cancel out, and the right hand sides in Equations (10a) and $(10 \mathrm{~b})$ become zero. Back-substitution of $(\partial T / \partial x) /(\partial T / \partial y)=-f$ into Equation (10) results in:

$$
q_{x}=q_{y}=\frac{\partial T}{\partial x}+f \frac{\partial T}{\partial y}=0 .
$$

For this extreme case $\left(k_{2}=0\right)$, both heat flux components $\left[q_{x}, q_{y}\right]=\mathbf{q}$ are identical to zero. Therefore, the heat flux components $\mathrm{q}_{1}$ and $\mathrm{q}_{2}$ parallel to the orthotropic (principal) thermal conductivities $\left(k_{1}, k_{2}\right)$ in Figure 2a are also zero. The resulting isotherms do not contradict their definition; the heat flux along an isotherm is zero and, perpendicular to this, the heat flux is also zero due to $k_{2}=0$, and the equating of Equations (1) and (8) was justified: If the heat fluxes are zero for any direction field $y^{\prime}=f(x, y)$, then their divergence $\operatorname{div}(\mathbf{q})$ in the form of Equation (3) or (4) is also zero. The latter is the heat conduction equation, which is solved in FE programs for steady-state problems without internal heat sources. From Fourier's law with the unknowns T and $\mathbf{q}$, a directly solvable equation has been formed for $\mathrm{T}$ alone provided that $k_{2}=0$. Whether the orthotropic heat conduction problem in Equation (11) is solved, which is not generally available as a calculation option in FE programs, or Equation (7) is solved by means of an FE program, the same isotherms result and are tangential to the given direction field $y^{\prime}=f(x, y)$. Whenever $k_{2}>0$, the proof does not work.

2.2. The Relationship between the Isotherms of Extremely Orthotropic Heat Conduction and the Characteristics of a Partial Linear Differential Equation of the First Order

A linear partial differential equation of the first order is:

$$
A(x, y) \frac{\partial T}{\partial x}+B(x, y) \frac{\partial T}{\partial y}=0
$$


and is discussed in detail in the literature [12-14]. The required function $T(x, y)$ depicts a surface in three-dimensional space. The theory of the "characteristic method" [12-14] for solving Equation (12) implies that its characteristics are the contour lines of $T(x, y)$ and can be calculated with the ordinary differential equation:

$$
\frac{d y}{d x}=B(x, y) / A(x, y)
$$

with $A \neq 0$. The similarity of Equations (11) and (12) is obvious for $A=1$ :

$$
\frac{\partial T}{\partial x}+B \frac{\partial T}{\partial y}=\frac{\partial T}{\partial x}+f \frac{\partial T}{\partial y}=0 .
$$

Along the characteristics, the solution variable $T$ is constant. Therefore, they can be interpreted as isotherms. Equation (14) physically describes the convective transport of the T variable. Equation (12) is valid for a partial differential equation with two independent variables. If $x$ and $y$ are used, then there is a time-independent situation in 2D space. If $x$ is used and instead of $y$, then there is a time-dependent situation in 1D space, the latter associates the transport character of Equation (12). A simple transport equation reads [12]:

$$
\frac{\partial T}{\partial t}+u \frac{\partial T}{\partial x}=0
$$

with time $t$ and the convection velocity $u=d x / d t$. The variable $T$ represents temperature here; it could also stand for mass, momentum, or energy. In order to demonstrate the particular properties of Fourier heat conduction $\left(k_{2}=0\right)$ in a simple example, $f=y^{\prime}=1$ is substituted in Equation (11) and $u=1$ in Equation (15). Therefore, both equations are formally identical. The location variable $y$ in Equation (11) corresponds to the time variable $t$ in Equation (15), $y$ and $t$ are interchangeable. By simple integration of Equation (13), $d y / d x=f=1$, the isotherms are represented by the straight lines $y=x+C$. Figure 3 shows the corresponding solution for Equations (11) and (15), which was obtained with the FE program ABAQUS (Dassault Systèmes, Vélizy-Villacoublay, France).

The initial condition for $T\left(t=0, x_{0} \leq x \leq x_{1}\right)$ and $T\left(y=0, x_{0} \leq x \leq x_{1}\right)$ is given by a parabolic temperature distribution. The orientation of $k_{1}$ is given by the vector $(1,1)$ in the entire solution area. Extreme orthotropy is achieved by zero-setting of $k_{2}$ whereby $\mathrm{k}_{2}$ is perpendicular to $k_{1}$.

The isotherms in Figure 3 depict both the general solution for the two-dimensional Equation (11) in the $x-y$ plane as well as the time-dependent solution for the one-dimensional Equation (15) in the $x-t$ plane. The isotherms follow the direction field $y^{\prime}=1$, not only inside the rectangular area but also on the boundary.

The hypothesis in Section 1 restricts usage to steady state problems. If the FE user investigates the behavior of the (extreme) orthotropic heat conduction beyond this restriction also in the time domain (with three independent variables: $x, y, t$ ), then the isotherms are no longer parallel to the specified direction field for finite times. The parabolic start temperature distribution propagates, corresponding to the thermal diffusivity $a=k_{1} /(c \rho)$ with finite velocity ( $c=$ specific heat, $\rho=$ density). As can be seen from Figure 4, the illustratively plotted $0.01 \cdot \mathrm{T}_{\max }$-isotherms at time $t_{1}<t_{2}<t_{3}$ are not parallel to the direction field $y^{\prime}=1$. This only applies for the steady-state case when $t \rightarrow \infty$. 


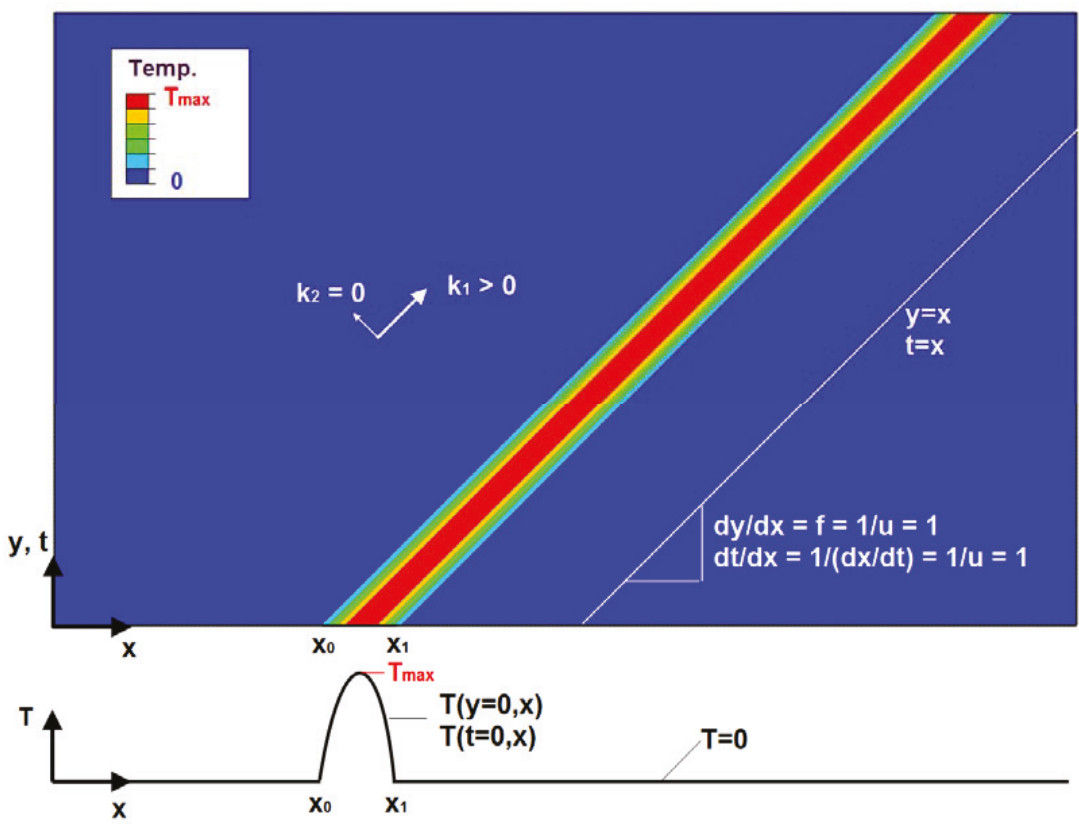

Figure 3. The Fourier heat conduction equation with $k_{1} / k_{2} \rightarrow \infty$ is hyperbolic and has transport character. The diagram is valid for the $x-y$ plane as well as for the $x-t$ plane. As an example, the direction field $y^{\prime}=f(x, y)=1$ is integrated with the orthotropic heat conduction $\left(k_{2}=0\right)$.

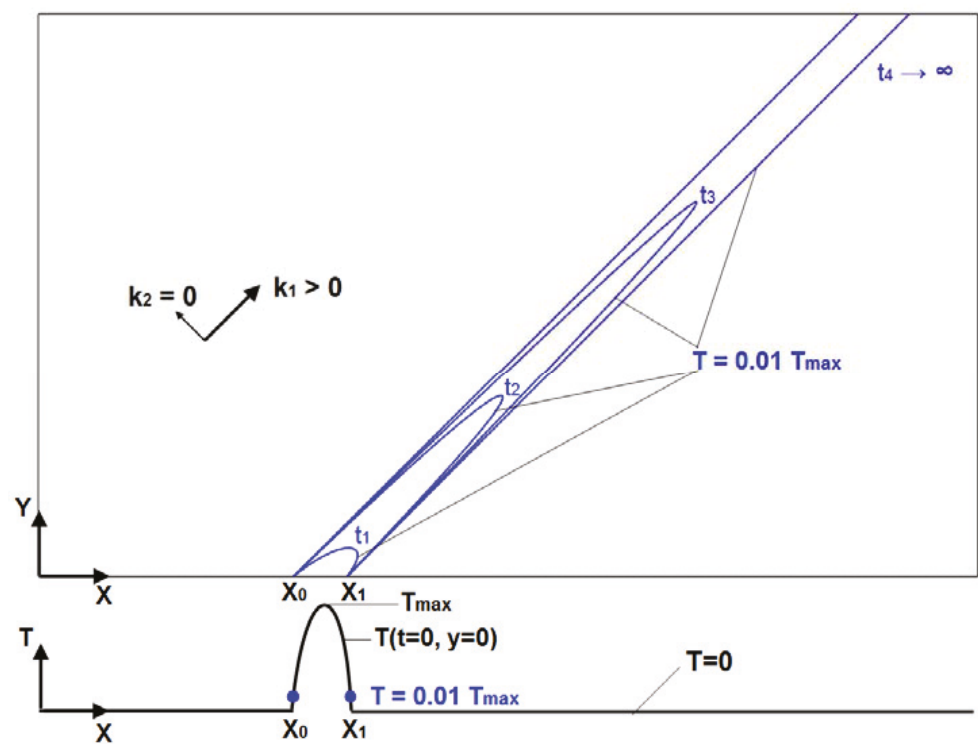

Figure 4. The steady-state problem from Figure 3 analyzed in the time domain. The isotherms are parallel to the given direction field $y^{\prime}=1$ only for the final steady state when $t \rightarrow \infty$. 


\subsection{Temperature Boundary Conditions for Extremely Orthotropic Heat Conduction}

The Equations (4) and (7) are solved in FE programs. These are second order and require Dirichlet or Neumann boundary conditions. Non-specified conditions at the boundary include $\partial T / \partial n=\mathbf{q}=\mathbf{0}$ ( $\mathbf{n}=$ normal vector perpendicular to the boundary). In case of extreme orthotropy when $k_{1}>0$ and $k_{2}=0$, the latter condition is always fulfilled according to Equation (11) both internally as well as on the boundary; therefore, in principle, no further conditions need to be specified. However, without specification of a reference temperature, the FE solution is singular. If a reference temperature $T_{\text {ref }}$ is specified at an arbitrary node, we only obtain the trivial solution $T(x, y)=T_{\text {ref }}$. Therefore, at least two different temperatures at two different nodes need to be specified in order to obtain a non-trivial solution. All temperature boundary conditions, which are specified at two or more nodes, provide isotherms that are tangential to the given direction field $y^{\prime}=f(x, y)$. The difference between a two-node and a multiple-node boundary condition manifests itself through different weightings with respect to the location of the isotherms. In order to obtain appropriate solutions, the direction field must be viewed in connection with the physical task, see Figure 5. This figure shows a disc (half-model) with an elliptical hole under pure bending.

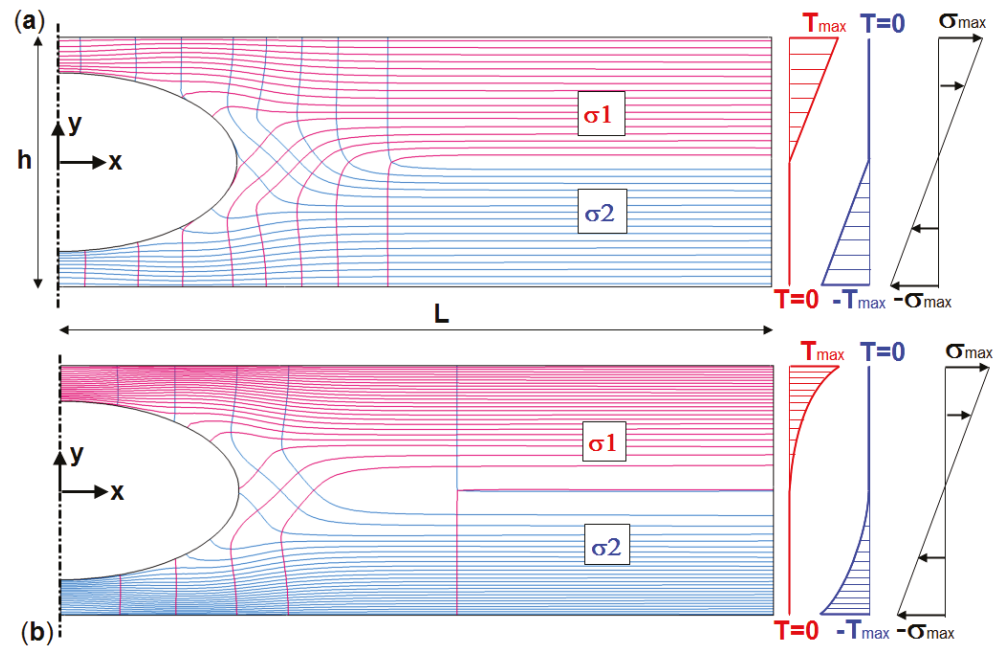

Figure 5. FRP disc with hole under bending: (a) Linearly increasing temperature boundary conditions for $\sigma_{1}$ and $\sigma_{2}$ trajectories yield a uniform distribution of fibers (isotherms); (b) parabolic temperature boundary conditions yield a weighted distribution of fibers, which carries a bending moment more effectively.

From a practical point of view, FRP constructions should have a uniform fiber placement. This is achieved with an orthotropic heat conduction analysis, specifying a linear temperature distribution for $y>0$ along the right boundary (Figure 5a). The isotherms, as representatives of the $\sigma_{1}$ trajectories, are then distributed equidistantly. For $y<0, T=0$ is defined. The $\sigma_{2}$ trajectories are calculated in a further orthotropic heat conduction analysis. These require a linear temperature distribution for $y<0$ and $T=0$ for $y>0$. Both isotherm images are superimposed and result in the two fiber layers in Figure 5a.

Should the objective be concentrated on load-related fiber placement, then a linear weighted distribution of isotherms is achieved by defining a parabolic temperature distribution for $y>0$ ( $\sigma_{1}$ trajectories) and $y<0\left(\sigma_{2}\right.$ trajectories) along the right boundary; see Figure $5 \mathrm{~b}$. 
The two-node temperature boundary condition is likewise possible. For example, to determine the $\sigma_{1}$ trajectories, specification of $T(L, h / 2)=T_{\max }$ and $T(L, 0)=0$ provides similar results, though the isotherms are not distributed equidistantly on the right boundary; see also Section 3.1.

In Section 2.1 it was shown that in cases of extreme orthotropy $\left(k_{1}>0, k_{2}=0\right)$, the differential Equation (7) of the second order and the Fourier heat conduction Equation (11) of the first order physically describe the same situation. In Section 2.2, it was shown that the Fourier heat conduction Equation (11) can be traced back to the general linear differential Equation (12). The integration of the corresponding characteristic Equation (13) provides characteristics identical to the isotherms in the Fourier heat conduction Equation (11). The theory of the characteristics also includes the treatment of boundary conditions [14], the rules of which can be applied completely to the Fourier heat conduction Equation (14). Figure 6 shows the most important rule.

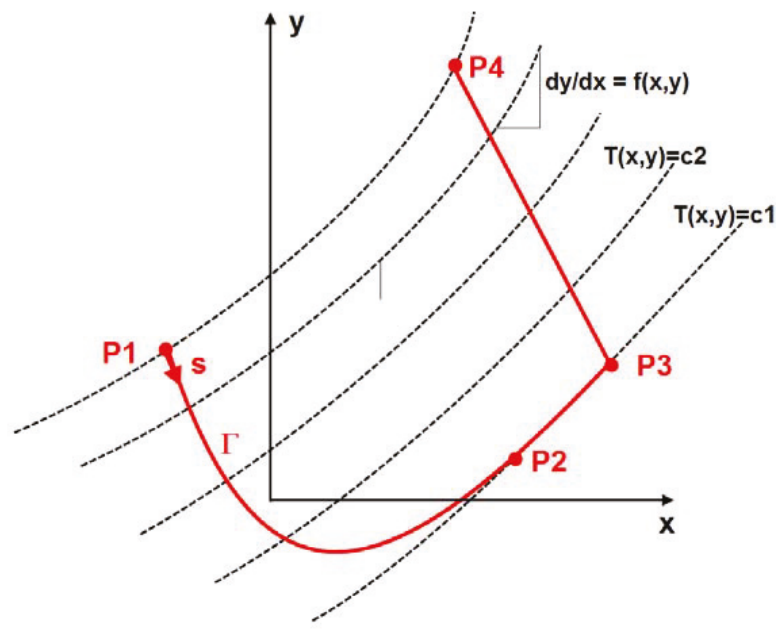

Figure 6. The solution curves $T(x, y)$ of the direction field $y^{\prime}=f(x, y)$ are simultaneously the characteristics of the Fourier heat conduction Equation (11). The specification of arbitrary boundary conditions along $\mathrm{P} 2 \mathrm{P} 3$ is not permitted since this curve is a characteristic or isoline $(y=$ const.).

According to this, Equation (12) is integrated through the specification of the solution variables $T$ along a curve $\Gamma$. However, only the specification on the P1P2 curve is permitted. Since a solution variable is constant on a characteristic, arbitrary specifications along P2P3 are not permitted. Furthermore, arbitrary specifications along P3P4 lead to contradictory definitions with the specifications along P1P2. The curve $\Gamma$ must not necessarily lie on the exterior boundary, but can also cross the integration area.

\section{Procedure and Application Examples}

The direction field $y^{\prime}=f(x, y)$ to be integrated very often originates from FE analysis in the form of PS directions. The procedure for their integration shall be determined with the help of some rules:

1. Calculation of the PS directions in all elements (disc, plate, or shell). If this is not provided by the respective FE program used, they can be calculated via the stress components $\left[\sigma_{x x} \sigma_{y y} \sigma_{x y}\right]$. For example, $y^{\prime}=d y / d x=\tan \alpha=\sigma_{x y} /\left(\sigma_{x x}-\sigma_{2}\right)$; see also Figure 2c. Plates and shells have variable stresses across the thicknesses. The direction field therefore must be evaluated for every "thickness integration point".

2. Replacement of the structural elements with thermal elements.

3. Transmission of the PS directions (Step 1) to local systems, depending on the FE program used, (ABAQUS: *Orientation). 
4. Assignment of orthotropic thermal conductivities $k_{1}$ and $k_{2}$ in these local systems with extreme ratios, e.g., $k_{1} / k_{2}>10^{4}$. ( $k_{2}=0$ is permitted in ABAQUS.)

5. Definition of thermal boundary conditions according to Section 2.3.

6. The ratios $k_{1} / k_{2}>10^{4}$ and $k_{2} / k_{1}>10^{4}$ provide the PS trajectories for the first and for the second principal stress, respectively.

It should be emphasized that the integration of the PS directions for the calculation of the real fiber course is a separate task, independent of the structural analysis. The PS direction field of the structural calculation is adopted as material orientation for the thermal conductivities in the thermal analysis. The boundary conditions are independent in both analyses and have nothing in common with each other.

\subsection{Influence of the Orthotropy Ratio $k_{1} / k_{2}$ on the Integration Accuracy}

The influence of the orthotropy ratio $k_{1} / k_{2}$ on the integration accuracy is demonstrated by the following example. Figure 7 shows a perforated disc under axial tension. Due to symmetry, a quarter model is sufficient $\left(U_{x}=0, U_{y}=0\right)$. The supplementary material accompanying this paper includes the ABAQUS input for this example.
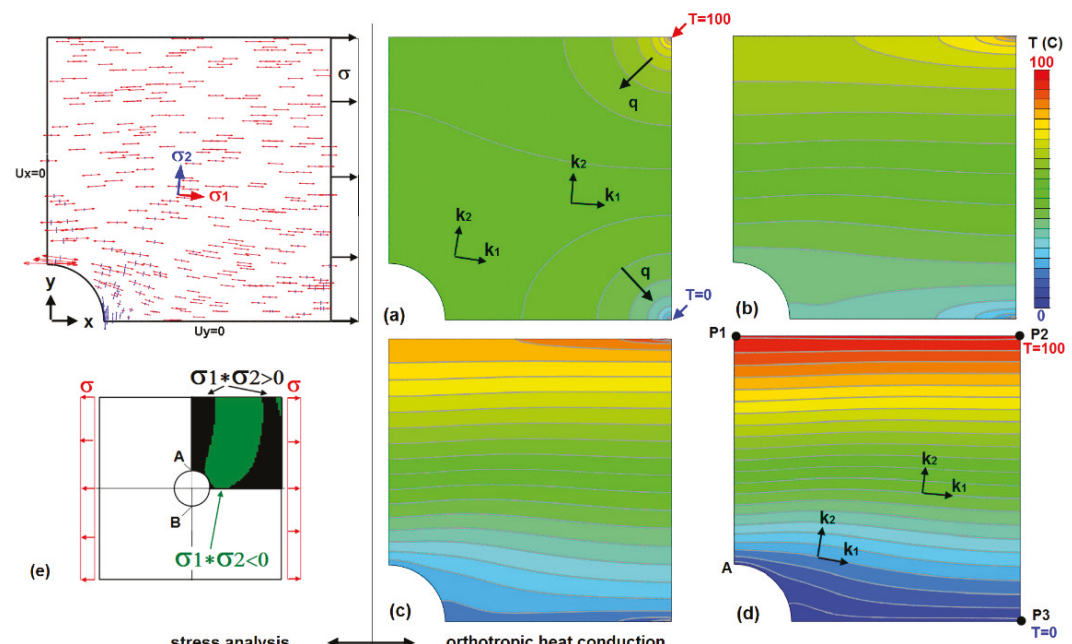

Figure 7. Perforated disc under axial tension and calculation of the PS lines (isotherms) dependent on heat conduction orthotropy $k_{1} / k_{2}$, where the orientation of $k_{1}$ and $k_{2}$ corresponds to the PS directions $\sigma_{1}$ and $\sigma_{2}$ : (a) $k_{1} / k_{2}=1$; (b) $k_{1} / k_{2}=16$; (c) $k_{1} / k_{2}=128$; (d) $k_{1} / k_{2}=4096$; (e) algebraic signs of the two principal stresses; this point is addressed in Section 3.4 .

The task is to place the fibers in the direction of the largest principal stress for an FRP construction. The PS directions portray the direction field $y^{\prime}=f(x, y)$, which shall be integrated. The elements of the mechanical model are replaced with heat conduction elements whose heat conduction $k_{1}$ is oriented parallel to the previously calculated PS directions of $\sigma_{1}$. The $k_{2}$ direction is orthogonal to $k_{1}$. Now the ratio $k_{1} / k_{2}$ is successively increased, thus the isotherms get more and more tangential to the PS direction of $\sigma_{1}$. The influence of the two-node temperature boundary condition at points P2 and P3 (Figure 7d) on the course of isotherms is no longer perceptible for $k_{1} / k_{2}>10^{4}$. Under such simple boundary conditions, it must be ensured that their positions are meaningful. If both temperatures are determined at points P1 and P2, then, practically, two different temperatures are defined on one isotherm. 
The relatively uniform distribution of the PS trajectories is remarkable, although only a very simple temperature boundary condition was selected. Should the desired outcome be a distribution of isotherms exactly equidistant between P2 and P3, the temperature along this line must be specified as linearly increasing. Since the isotherms represent the fiber course, the constant line load along P3P2 is uniformly carried by the fibers. The maximum load in the perforated disc at points A and B in Figure 7e is approximately three times the applied tension, which is well reflected by the fiber course.

At this point, the transport character of the Fourier heat conduction equation in Figure $7 \mathrm{~d}$ and Equations (14) and (15) shall be emphasized again. The specification of a linear temperature distribution on the boundary P3P2 is transported along its characteristics without diffusion effects, and these characteristics follow the user defined direction field $y^{\prime}=f(x, y)$.

\subsection{The Influence of Singularities on the Course of the Isotherms}

In cases of stress singularities, the following distinctions are made: An isotropic (or neutral) point with an undefined PS direction occurs if the two principal stresses $\sigma_{1}$ and $\sigma_{2}$ are identical. If both principal stresses are zero, then a classic singularity is present. Points with concentrated loads are neither one nor the other. Their isoclines are particularly concentrated at the point of force transmission. What does the course of stress trajectories (represented by isotherms) look like in the vicinity of these points? This can be studied using an example of a circular ring under diametrically opposed single forces. Figure 8 a shows the course of the isoclines and stress trajectories that were determined photoelastically by Frocht [15].
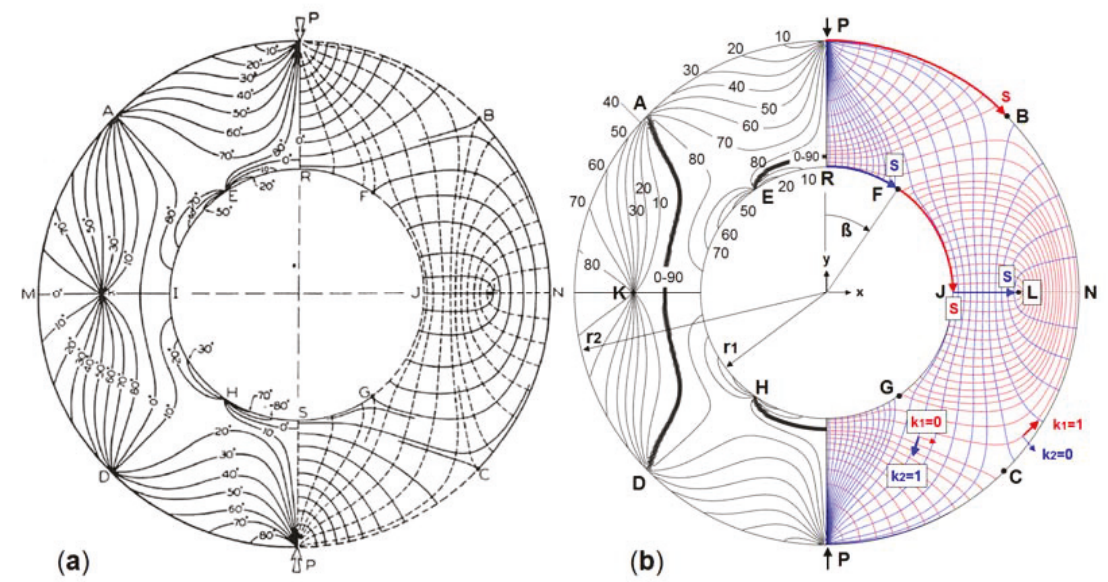

Figure 8. Circular ring under diametrically opposed single forces, $r_{2} / r_{1}=2$ : (a) Photoelastic isoclines (left) and trajectories (right) [15]; (b) Verification with ABAQUS, isoclines (left), $\sigma_{1}$ trajectories and $\sigma_{2}$ trajectories (right).

The experimental results were verified through an FE stress analysis (ABAQUS) followed by an orthotropic heat conduction analysis in order to determine the trajectories, as shown in Figure 8b. Modeling a quarter of the disc is sufficient due to the symmetry. The stress calculation provides the PS directions. In the subsequent heat conduction calculation, these PS directions serve as local systems for the thermal conductivities $k_{1}$ and $k_{2}$. Two of these systems are illustratively plotted in the fourth quadrant of Figure $8 \mathrm{~b}$. The system $k_{1}=1, k_{2}=0$ provides the isotherms as $\sigma_{1}$ trajectories. With $k_{1}=0$, $k_{2}=1$, the $\sigma_{2}$ trajectories are determined. The calculation of the isoclines is helpful in order to localize 
the singular points A, D, E, H, and K (Figure 8b, left). The isoclines result from Equation (16), i.e., the contour lines of the stress expression on the right side must be visualized; see also Figure 2c.

$$
y^{\prime}=f(x, y)=\tan \alpha=\frac{\sigma_{x y}}{\sigma_{x x}-\sigma_{2}}=\text { const. }
$$

In the trajectories image (right), the singular points appear with the designations $\mathrm{B}, \mathrm{C}, \mathrm{F}$, G, and L, and are either of the "non-interlocking" type (B, C, F, G) or "interlocking" type (L). This evaluation is useful since a uniform distribution of the isotherms (fibers) is desirable, which is only achievable with knowledge of the singularities. This is shown in Figure 9a for the $\sigma_{2}$ trajectories (with $\sigma_{2}<\sigma_{1}$ ). A uniform distribution is achieved through a linearly increasing temperature along RF, with continuation along JL.
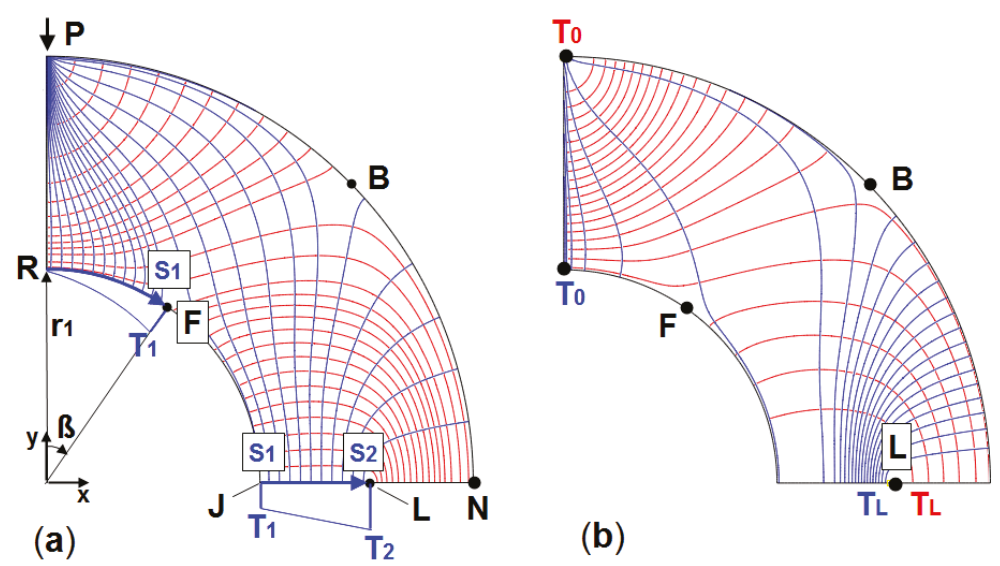

(b)

Figure 9. Circular ring analogous to Figure 8: (a) Determination of the $\sigma_{2}$ trajectory through specification of a linearly increasing temperature along RF and JL; (b) Simplified analysis with a two-node temperature boundary condition; $\mathrm{T}_{0}$ and $\mathrm{T}_{\mathrm{L}}$ for the $\sigma_{1}$ trajectories, $\mathrm{T}_{0}$ and $\mathrm{T}_{\mathrm{L}}$ for the $\sigma_{2}$ trajectories.

Specifying a linear increase in temperature from $\mathrm{R}$ to $\mathrm{J}$ is incorrect since the $\sigma_{2}$ trajectory along RF turns into the $\sigma_{1}$ trajectory at point $\mathrm{F}$ along $\mathrm{FJ}$. The FJ boundary segment itself is an isotherm and no variable temperature specification is allowed there.

The existence of a singular point on a load-free boundary of a 2D structure always results in this change of trajectories. This property is responsible for the fact that efforts need to be made regarding the specification of suitable temperature boundary conditions in order to obtain uniformly distributed isotherms. Should the temperature be defined only along RF, then only a part of the structure will show isotherms. A further boundary segment with temperature boundary conditions must then be applied in order to capture the rest of the structure. In Figure 9a, this is segment JL. The procedure is the same when looking for $\sigma_{1}$ trajectories. The temperature is defined as linearly increasing along PB with continuation along LN.

The aim of calculating strictly uniformly distributed isotherms (trajectories) can only be achieved locally. Figure 9 a shows uniformly distributed $\sigma_{1}$ trajectories along PB; however, these end up unevenly distributed on the PR boundary.

On the perforated disc (Figure 7), the trajectory change mentioned above does not occur along the right boundary of P2P3. This boundary is free from singularities. The specification of two temperatures at points P2 and P3 is sufficient. We can generally use this simple two-node boundary condition (Section 2.3) for all 2D discs and 3D shells and get-depending on the number 
of singularities-correspondingly weighted isotherm distributions. Figure $9 \mathrm{~b}$ shows this simple procedure for the circular ring with its singularities. The isotherms shown there are less uniformly distributed than in Figure 9a, but this is adequate for a first overview.

The circular ring problem (Figure 8) contains the most important forms of singularities: Isotropic points of the "interlocking" and "non-interlocking" type, as well as concentrated point loads. Errors in representation of isotherms are inevitable since they do not fulfil $y^{\prime}=f(x, y)$ in the near vicinity of the singularity. In fact, the isotherms in close proximity there evade in a sideways direction depending on the model mesh size. The model in Figure 8b, in comparison to Figure 8a, is clearly fine enough to minimize this error. The element size is virtually the same all over; 60 elements were used (with four nodes respectively) along JN. Through further mesh refinement in the area of singularities, the error can be kept arbitrary small.

\subsection{The Principal Stress Trajectories in Shell Structures}

A conventional method for the calculation of principal stress trajectories in a shell was shown in Figure 1. Disadvantages resulted from the uneven fiber placement, which originates from the separate calculation of each individual fiber. In integrating the direction field, it is not practically possible to select optimal start positions (start elements) beforehand.

In contrast to this is the method of integration by means of an orthotropic heat conduction calculation. Even with a two-node temperature boundary condition with $\mathrm{Q}_{0}: \mathrm{T}=0{ }^{\circ} \mathrm{C}, \mathrm{Q}_{1}: \mathrm{T}=1{ }^{\circ} \mathrm{C}$ in Figure 10a, a better fiber distribution can be attained than is depicted in Figure 1a. This temperature distribution, shown in Figure 10a, provides the approximate position of three local temperature extremes at the positions $\mathrm{P}_{0}, \mathrm{P}_{1}$ and $\mathrm{P}_{2}$.

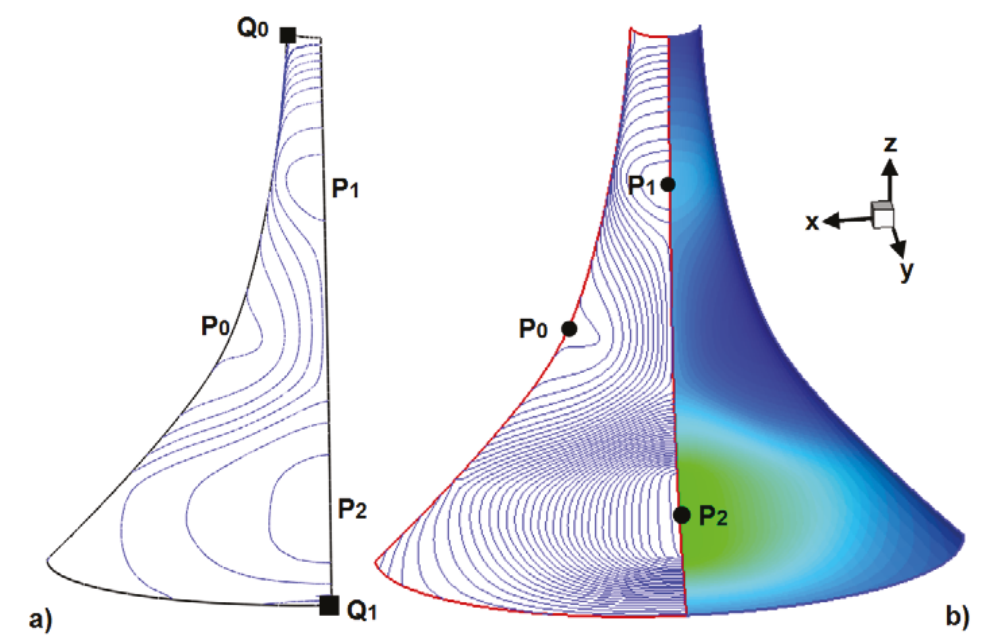

Figure 10. Shell structure analogous to Figure 1: (a) Isotherms with a two-node temperature boundary condition at $\mathrm{Q}_{0}$ and $\mathrm{Q}_{1} ;(\mathbf{b})$ Isotherms with a three-node temperature boundary condition at $\mathrm{P}_{0}, \mathrm{P}_{1}, \mathrm{P}_{2}$, right: Temperature field for extraction of any number of isotherms (fibers).

By repeating the heat conduction analysis with a three-node temperature boundary condition $\left(\mathrm{P}_{0}: T=0{ }^{\circ} \mathrm{C}, \mathrm{P}_{1}: T=1{ }^{\circ} \mathrm{C}, \mathrm{P}_{2}: T=2{ }^{\circ} \mathrm{C}\right.$, Figure $\left.10 \mathrm{~b}\right)$, the uniformity of the isotherms is improved. A specification of the linear varying temperatures along boundary segments analogous to the previous section would yield further improvement. However, in light of the result in Figure 10b, it does not appear to be crucial. 
The right half of Figure $8 \mathrm{~b}$ highlights another aspect: The continuous temperature field is infinitely dense with respect to the isotherms, so an arbitrary number of contours can be visualized, avoiding calculation of trajectories one after another (Figure 1).

\subsection{Optimization of Fiber Placement in FRP Constructions}

In order to demonstrate a useful application beyond the visualization of tensor lines, the fiber placement in FRP constructions is highlighted. Fiber courses, which follow local PS directions, use the superior stiffness and strength properties of the fibers. In plane stress structures the fiber courses due to $\sigma_{1}$ and $\sigma_{2}$ are perpendicular to each other, forming a curvilinear cross-ply (CCP) laminate. With regard to optimality, attention must be paid to the algebraic signs of principal stresses.

Fiber placement in the direction of principal stresses is optimal according to net theory [16], and this is also valid for CCP-laminates if ignoring the support of the matrix. To be on the safe side, the carrying capacity of the construction can be calculated by using a fiber failure criterion. A design based on an inter-fiber failure must additionally take into account the contribution of the matrix. In this case CCP-laminates are optimal only in areas with principal stresses showing different algebraic signs. However, if the algebraic signs are the same, then the curvilinear balanced angle-ply (CBAP) laminate is the better choice according to classical lamination theory [17]. This design differs from the (right-angled) CCP-laminate through a correction angle $\pm \beta$ regarding the $\sigma_{1}$ direction. The direction field $y^{\prime}$ for the largest principal stress $\sigma_{1}$ is then represented by two direction fields: $y^{\prime}+\beta$ and $y^{\prime}-\beta$, as shown in Figure 11.

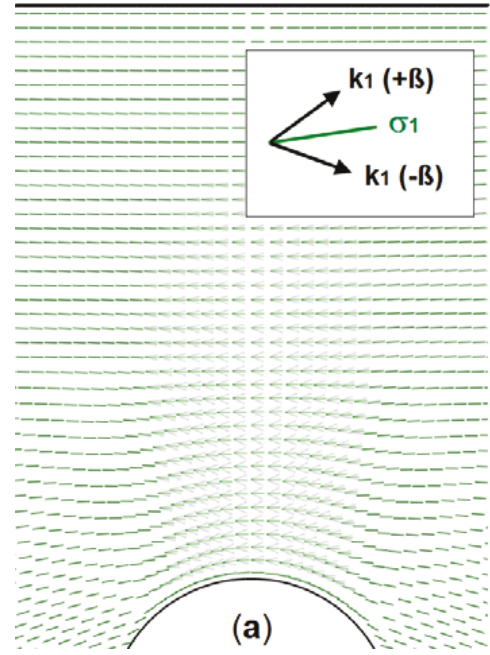

(a)

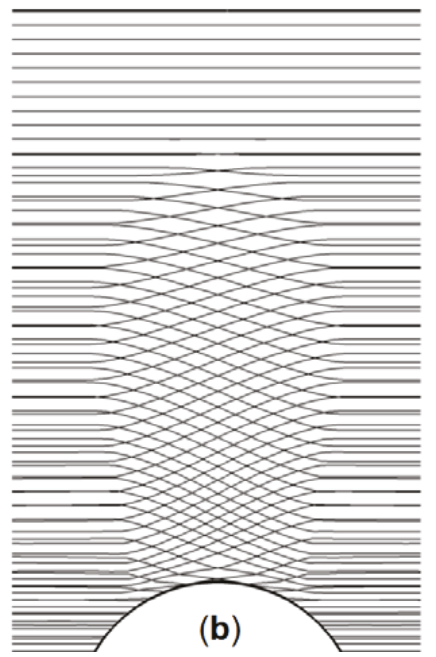

(b)

Figure 11. Perforated disc (detail) similar to Figure 7e. In areas with the same principal stress algebraic sign, the CBAP-laminate is optimal: (a) Correction angle $\pm \beta$ regarding the $\sigma_{1}$ direction; (b) Integration of the modified direction field.

For example, the perforated disc (Figure 7e) contains the areas $\sigma_{1} \cdot \sigma_{2}<0$ (CCP-laminate is optimal) and $\sigma_{1} \cdot \sigma_{2}>0$ (CBAP-laminate is optimal). In the latter case the modified direction fields can also be integrated without restriction by means of the orthotropic heat conduction analysis. The correction angle $\beta$ is dependent on the material and the principal stress ratio $\sigma_{1} / \sigma_{2}$ [17]. It should be noted that the orthotropic heat conduction is suitable for the integration of arbitrary direction fields even if they are modified for reasons determined by the engineer. Reference is made to Moldenhauer [1] (pp. 54-60] and [18] for further details and consideration of particular aspects of load-related optimization (layer 
thickness distribution, loading condition range, and nonlinearity of stress distribution over the shell thickness).

\section{Discussion and Conclusions}

The method presented in this paper is suitable for integrating arbitrary vectorial and tensorial 2D direction fields by means of commercial FE programs, provided that the FE programs have options for the analysis of orthotropic heat conduction. The calculated isotherms represent the integral curves of these direction fields inside and on the edge of the 2D structure (disc or shell). The procedure is based on some rules (Section 3), which concern only the standard input of the FE program. Additional programming is not required. Two temperatures at two different positions shall be specified as a minimum for thermal boundary conditions. The isotherms arising from this can still run in a non-uniform way. Uniformity is achieved while linearly varying temperatures along a boundary segment are specified (Figure 5a). Should the direction field or the differential equation $y^{\prime}=f(x, y)$ exhibit singularities on the boundary, then the definition of the boundary conditions must be carefully adjusted (Figure 9). In this case, a preliminary visualization of the isoclines, Equation (16), and/or an analysis with the two-node temperature boundary condition is helpful. The precision of the integration depends on the element size of the FE model.

In comparison with the traditional integration methods, significant improvements arise with the new procedure:

- The integration of the direction fields can take place with standard FE programs with options for an orthotropic heat conduction analysis. No additional programming is necessary.

- Any number of isotherms can be extracted from the integrated continuous temperature field.

- Quick and accurate results are obtained with a simple two-node temperature boundary condition; however, the distribution of isotherms may be non-uniform if singular points are present in the direction field.

- As the computed temperature field is continuous, valuable additional information can be extracted from this field. For example, the density of the isotherms (tensor lines) can be computed by displaying the temperature gradients, see [1] (p. 96).

- Applying the proposed integration method to stress tensors it should be noted that principal stress direction fields can originate from linear or nonlinear analyses, see [1] (p. 74). If principal shear stress (or strain) directions are evaluated in the plastic range then the trajectories can be regarded as slip-lines, see [1] (pp. 41-44).

- Civil engineering: Aligning reinforcement in concrete structures parallel to PS directions is a meaningful tool to effectively increase the low tension strength of concrete, see also [1] (p. 78).

Mathematical aspects were the focus of this paper. An important application seems to be on FRP constructions. Aligning fiber courses along PS lines make the most of the superior stiffness and strength properties of fibers. Calculation of these fiber courses by integrating the PS directions followed by the evaluation of this optimized FRP design can be done with one and the same FE program. To evaluate and optimize an FRP construction based on PS lines, some practical aspects should be highlighted.

As with all FRP problems, the optimization success is greatest when a special load case is investigated. In reality, loads and boundary conditions change frequently, and the principal stress directions change accordingly. However, the associated degradation of the optimization method affects alternative optimization strategies as well. If one load case dominates other load cases, the optimization effort can be worthwhile nevertheless. A CCP- or CBAP-laminate can be combined with textile preforms and can be stitched onto standard laminates. This technique is known as tailored fiber placement (TFP). The layer with the fiber course aligned to the trajectories covers the critical load case, and the standard laminate the other load cases. In 2D, the TFP stitching technique has been used for a long time [10]. In 3D, textile preforming or tape laying are possible options. 
The effort for the proposed method appears to be very high in the case of plates and shells, since the PS directions for superimposed membrane and bending stress vary across the thickness. The integration of the stress components $\left[\sigma_{x x} \sigma_{y y} \sigma_{x y}\right]$ over the thickness produces normal forces $N=\left[N_{x x} N_{y y} N_{x y}\right]$ and bending moments $\boldsymbol{M}=\left[M_{x x} M_{y y} M_{x y}\right]$. FE programs generally provide these section forces and moments $N$ and $M$, which are both constant across the thickness. Their principal directions $(1,2)$ can be computed in analogy to Equation (16), i.e., $\mathrm{y}_{1}{ }^{\prime}=N_{x y} /\left(N_{x x}-N_{2}\right), \mathrm{y}_{2}{ }^{\prime}=-1 / \mathrm{y}_{1}{ }^{\prime}$. It makes sense to arrange the M-layers on the inside and outside and the N-layers in the middle of the shell. Symmetric layers are then obtained with $\left[M_{1} / M_{2} / N_{1} / N_{2}\right]_{S}$, see also [1] (pp. 64-73).

When evaluating a TFP laminate, one is faced with the variable fiber volume fraction due to the diverging and converging streamline nature of the fiber course. The fiber volume fraction is an important input quantity to define laminate stiffness with dependence on local fiber volume and local fiber orientation. The gradient of the temperature field is a measure for the local fiber volume. Displaying the gradient of the temperature field is identical to displaying the isotropic heat flux, $\mathbf{q}=k \cdot \operatorname{grad}(\mathrm{T})$ with $k=k_{1}=k_{2}$. For extreme orthotropy $\left(k_{1} / k_{2} \rightarrow \infty\right)$ we have an orthotropic heat flux, which is zero everywhere, see Equation (11). If the temperature field is based on isotropic conduction with constant k, then the isotropic heat flux is the correct gradient. For this reason, the nodal temperatures from the orthotropic analysis must be used as prescribed nodal temperatures in an additional thermal run. More details can be found in [1] (p. 96).

Provided that the course of the isotherms (trajectories) shall be used for fiber placement in FRP constructions, the visualized isotherms must be converted into polylines with $\mathrm{x}-, \mathrm{y-}-$, and $\mathrm{z}$-coordinates. TECPLOT (Bellevue, WA, USA) is suitable as a commercial solution for this. Alternatively, a Fortran program can be used, for which the source code is contained in DeVries [19].

Supplementary Materials: The following are available online at http://www.mdpi.com/2297-8747/23/2/24/s1.

Funding: This research received no external funding.

Conflicts of Interest: The author declares no conflict of interest.

\section{References}

1. Moldenhauer, H. Die Orthotrope Wärmeleitung als Numerischer Integrator Allgemeiner Richtungsfelder mit Anwendung zur Optimalen Faserplatzierung und Kraftflussvisualisierung. Available online: https: / / publikationen.bibliothek.kit.edu/1000060087 (accessed on 4 May 2018).

2. Beyer, F.R. Hauptspannungstrajektorien in der numerischen Festkörpermechanik—Ein Algorithmus zur Visualisierung der Bauteilbeanspruchung in zwei und drei Dimensionen. Available online: http://nbnresolving.de/urn:nbn:de:bsz:14-qucosa-178497 (accessed on 4 May 2018).

3. Delmarcelle, T. The Visualization of Second-Order Tensor Fields. Ph.D. Thesis, Stanford University, Stanford, CA, USA, December 1994.

4. Delmarcelle, T.; Hesselink, L. The Topology of Second-Order Tensor Fields. Available online: https://graphics. ethz.ch/teaching/scivis_common/Literature/DelmarcelleHesselink94.pdf (accessed on 4 May 2018).

5. Jobard, B.; Lefer, W. Creating Evenly-Spaced Streamlines of Arbitrary Density. In Proceedings of the 8th Eurographics Workshop on Visualization in Scientific Computing, Boulogne-sur-Mer, France, 28-30 April 1997; pp. 44-55.

6. Tricoche, X. Vector and Tensor Field Topology Simplification, Tracking and Visualization. Ph.D. Thesis, University of Kaiserslauten, Kaiserslauten, Germany, April 2002.

7. Hyer, M.W.; Charette, R.F. Use of Curvilinear Fiber Format in Composite Structure Design. AIAA J. 1991, 29, 1011-1015. [CrossRef]

8. Mattheck, C. Design in Nature; Springer: Berlin, Germany, 1998.

9. Tosh, M.W.; Kelly, D.W. On the Design, Manufacture and Testing of Trajectorial Fibre Steering for Carbon Fibre Composite Laminates. Composites Part A Appl. Sci. Manuf. 2000, 31, 1047-1060. [CrossRef]

10. Spickenheuer, A. Zur fertigungsgerechten Auslegung von Faser-Kunststoff-Verbundbauteilen für den extremen Leichtbau auf Basis des variabelaxialen Fadenablageverfahrens Tailored Fiber Placement. Available online: http:/ / nbn-resolving.de/urn:nbn:de:bsz:14-qucosa-147748 (accessed on 4 May 2018). 
11. Hahn, D.W.; Özişik, M.N. Heat Conduction, 3rd ed.; John Wiley and Sons: Hoboken, NJ, USA, 2012; p. 625.

12. Hoffman, J.D. Numerical Methods for Engineers and Scientists, 2nd ed.; Marcel Dekker: New York, NY, USA, 2001; pp. 501-526.

13. Riley, K.F.; Hobson, M.P.; Bence, S.J. Mathematical Methods for Physics and Engineering, 3rd ed.; Cambridge University Press: Cambridge, UK, 2006; pp. 675-706.

14. Pinchover, Y.; Rubinstein, J. An Introduction to Partial Differential Equations, 3rd ed.; Cambridge University Press: Cambridge, UK, 2005; pp. 23-36.

15. Frocht, M.M. Photoelasticity; John Wiley and Sons: New York, NY, USA, 1946; Volume 1, pp. $209-210$.

16. Schürmann, H. Konstruieren mit Faser-Kunststoff-Verbunden, 2nd ed.; Springer: Berlin, Germany, 2007; pp. $451-476$.

17. Michaeli, W.; Huybrechts, D.; Wegener, M. Dimensionieren mit Faserverbundkunststoffen; Hanser: Munich, Germany, 1994; pp. 107-119.

18. Moldenhauer, H. Beanspruchungsgerechte Faserplatzierung in Faser-Kunststoff-Verbunden. Konstruktion 2017, 69, 68-74.

19. DeVries, P.L. Computerphysik; Spektrum Akademischer Verlag: Heidelberg, Germany, 1994; pp. 417-420.

(c) 2018 by the author. Licensee MDPI, Basel, Switzerland. This article is an open access article distributed under the terms and conditions of the Creative Commons Attribution (CC BY) license (http:/ / creativecommons.org/licenses/by/4.0/). 

MDPI

St. Alban-Anlage 66

4052 Basel

Switzerland

Tel. +41616837734

Fax +41 613028918

www.mdpi.com

Mathematical and Computational Applications Editorial Office

E-mail: mca@mdpi.com

www.mdpi.com/journal/mca

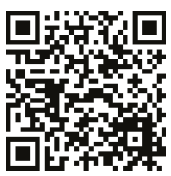



MDPI

St. Alban-Anlage 66

4052 Basel

Switzerland

Tel: +41 616837734

Fax: +41 613028918

www.mdpi.com 